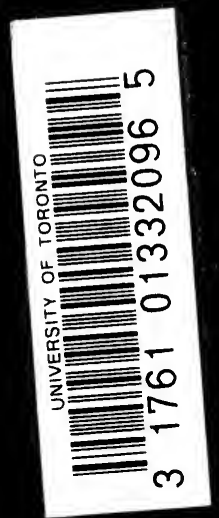


Digitized by the Internet Archive in 2007 with funding from Microsoft Corporation 
, 



\title{
NTS OF FOLK PSYCHOLOGY
}

OUTLINES OF A PSYCHOLOGICAL HISTORY OF THE DEVELOPMENT OF MANKIND

\author{
BY \\ WILHELM WUNDT
}

AUTHORIZED TRANSLATION

BY

EDWARD LEROY SCHAUB, Ph.D.

Professor of Philosophy in Northwestern University

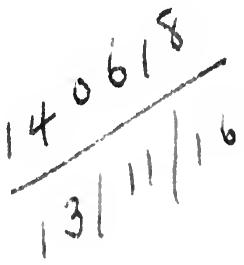

LONDON: GEORGE ALLEN \& UNWIN LTD. NEW YORK: THE MACMILLAN COMPANY 
First published in 1916 


\section{TRANSLATOR'S PREFACE}

THE keen interest which the present age is manifesting in problems connected with the interpretation of human experience is no less a result than it is a precondition of the fruitful labours of individual scholars. Prominent among these is the distinguished author of the volume which is herewith rendered accessible to English readers. The impetus which Professor Wundt has given to the philosophical and psychological studies of recent years is a matter of common knowledge. Many of those who are contributing richly to these fields of thought received their stimulus from instruction directly. enjoyed in the laboratory. and the classrooms of Leipzig. But even more than to Wundt, the teacher, is the world indebted to :Wundt, the investigator and the writer. The number and comprehensiveness of this author's publications, as well as their range of subjects, are little short of amazing. To gauge the extent of their influence would require an examination of a large part of current philosophical and psychological literature. No small measure of this influence, however, must be credited to those whose labours have made possible the appearance of Wundt's writings in other tongues. Of the English translations, we owe the first to Professors Creighton and Titchener. Succeeding their translation of the "Lectures on Human and Animal Psychology," came the publication, in English, of the first volume of the "Principles of Physiological Psychology," of the two briefer treatises, "Outlines of Psychology " and "Introduction to Psychology," and, in the meantime, of the valuable work on "Ethics." 
Though Professor Wundt first won recognition through his investigations in physiology, it was his later and more valuable contributions to physiological psychology, as well as to logic, ethics, epistemology, and metaphysics, that gained for him his place of eminence in the world of scholarship. One may hazard the prophecy, however, that the final verdict of history will ascribe to his latest studies, those in folk psychology, a significance not inferior to that which is now generally, conceded to the writings of his earlier years. The Völkerpsychologie is a truly monumental work. The analysis and interpretation of language, art, mythology, and religion, and the criticisms of rival theories and points of view, which occupy its five large volumes of over three thousand pages, are at once so judicial and so suggestive that they may not be neglected by any serious student of the social mind. The publication of the Volkerpsychologie made necessary a number of defensive and supplementary articles. Two of these, in a somewhat revised form, together with an early article on "The Aim and Methods of Folk Psychology," and an additional essay on "Pragmatic and Genetic Psychology of Religion," were published in I9I I under the title, Probleme der Volkerpsychologie. Finally, in 1912, there appeared the book which we are now presenting in translation, the Elemente der Völkerpsychologie. As regards the difference in method and character between the Elemente and the Volkerpsychologie, nothing need be added to what may be gleaned from the author's Preface and Introduction to this, his latest, work. Here, too, Professor Wundt indicates his conception of the nature and the problem' of folk psychology, a fuller discussion of which may be found both in the Volkerpsychologie and in the first essay of the Probleme.

He who attempts to sketch the "Outlines of a Psychological History of the Development of Mankind" necessarily incurs a heavy indebtedness, as regards his 
material, to various more specialized sciences. The success with which the data have been sifted in the present instance and the extent to which the author has repaid the special sciences in terms of serviceable principles of interpretation, must, to a certain extent, be left to the determination of those who are engaged in these specific fields. Human beliefs and institutions, however, as well as all products of art and modes of labour, of food-getting, of marriage, of warfare, etc.-in short, all elements of human culture-even though subject to natural conditions of various sorts, are essentially mental processes or the expression of psychical activities. Hence no theory relating to these phenomena is acceptable, or even respectable, that does violence to wellestablished psychological principles. The unpsychological character of many of the hypotheses that still abound in ethnological, sociological, and historical literature, in itself renders necessary such discussions as those comprised within the present volume. One of the very valuable, even though not novel, features of the "Elements," therefore, is its clear exposure of the untenability, of rationalistic and other similarly erroneous types of explanation.

The dependence of folk psychology, as conceived by Professor Wundt, upon general psychology-or, in this particular case, upon the author's system of physiological psychologywill be apparent. It should not be overlooked, however, that the examination of the mental processes that underlie the various forms in which social experience comes to expression involves a procedure which supplements, in an important way, the traditional psychological methods. More than this. Wundt's Völkerpsychologie is the result of a conviction that there are certain mental phenomena which may not be interpreted satisfactorily by any. psychology which restricts itself $X$ to the standpoint of individual consciousness. Fundamental to the conclusions of the present volume, therefore, is the assumption of the reality of collective minds. For Pro- 
fessor Wundt, however, this assumption is not in the least of a dogmatic character. On the contrary, its acceptance is necessitated by the failure of opposing theories, and its validity is sustained by the fact that it renders intelligible a large and important body of facts. If this be admitted, it follows that folk psychology, supplements not merely the methods of individual or physiological psychology, but also its principles and its laws. As yet, however, the prevailing tendency of psychologists, both in England and in America, is to retain the point of view of individual consciousness even when dealing with those phenomena which Wundt considers to be creations of the social group. That this occurs so frequently without any apparent thought of the necessity of justifying the procedure is-whether the position itself be right or wrong-an illustration of the barriers offered by a foreign language.

For the general reader who professes no acquaintance with the nature or the viewpoint of psychological science, it may not be amiss to remark that the author aims, in this book, to present, not a discussion of the philosophical validity of ideas or of the ethical or religious value of customs and institutions, but merely a descriptive account of human development. The "Elements" is an attempt to answer the question as to what beliefs and practices actually, prevailed at the various stages of human development and what psychological explanation may be given of them'. Such an investigation is quite distinct from an inquiry as to whether these beliefs and practices are justifiable. It is equally, foreign, moreover, to the question as to whether the ideas that are entertained may be held either to bring us into relation with trans-subjective realities or to acquaint us with a truth that is, in any significant sense, eternal. However sacred or profane, true or delusional, experiences may be to the philosopher, the theologian, or the man of practical affairs, to him who is psychologizing they all alike 
are mental phenomena demanding, not evaluation, but observation, analysis, and reduction to mental laws. Wundt explicitly emphasizes the fact that his psychological account neither represents nor renders unnecessary. a philosophy of history; similarly, it may be added, the present work is neither the equivalent nor the negation of ethics, jurisprudence, theology, epistemology, or metaphysics. Nevertheless, while the distinctions which we have suggested should be strictly kept in mind, a just appreciation of the significance of such books as the "Elements" demands that we recognize their notable value to all the various philosophical disciplines. Works of this sort succeed above all others in stimulating and sustaining a keen empirical interest on the part of philosophy, and they supply the latter with a fund of carefully, selected and psychologically. interpreted facts. Doubtless it is in connection with ethics and the science of religion that these services are most obvious. Even the epistemologist, however, will find much that is suggestive in Wundt's account of the origin and development of language, the characteristics and content of primitive thought, and the relation of mythological and religious ideas to the affective and conative life. That the Volkerpsychologie may contribute largely toward the solution of metaphysical problems has been strikingly demonstrated by Professor Royce in his profound volumes on "The Problem of Christianity."

The trials of the translator have been recounted too often any longer to require detailed mention. President G. Stanley Hall has suggested that the German proclivity to the use of long, involved sentences, loaded with qualifying words and phrases, and with compounds and supplementary clauses of every description, may perhaps be said to have the merit of rendering language somewhat correspondent with the actual course of thought. The significance of this statement can be appreciated by 
no one quite so keenly as by a translator, for whom the very. fact which President Hall mentions causes many German sentences to be objects of despair. In the present instance, the endeavour has been to reproduce as faithfully as possible both the meaning and the spirit of the original, while yet taking such liberties as seemed necessary either to clarify certain passages or to avoid any serious offence to the English language. In a number of cases, no absolutely satisfactory equivalent of the German term seemed available. The very expression 'folk psychology,' for example, may scarcely be said to commend itself in every respect. Its use seemed unescapable, however, in view of the fact that the author, in his Introduction, expressly rejects the terms Sozialpsychologie and Gemeinschaftspsychologie in favour of Völkerpsychologie. Bildende Kunst has been rendered 'formative art,' not in the belief that this translation is wholly unobjectionable, but because it seemed preferable to all possible alternatives, such as 'plastic,' ' shaping,' or ' manual' art. Those who are familiar with, or who will take notice of, the very precise meaning which the present author gives

) to the terms Märchen, Sage, Legende, and Mythus will understand without explanation our frequent use of the word 'saga' and the necessity of the term 'märchen' in the translation. Wundt has always attached great significance to the distinctions which he has drawn between the various forms of the myth, and, more especially, to his contention that the earliest and, in a sense, the progenitor of these was the märchen. The crying need of exact definition and of clear thinking in a field so confused as that of mythology led him, on one occasion, to enter a plea for a clear-cut and consistent terminology such as that which he was attempting to maintain (vide Völkerpsychologie, Band $V$, Zweiter Teil, Zweite Auflage, s. 33). In this instance again, therefore, it seemed best to give to the author's own terms a preference over words which, while more familiar 
to the English reader, are less suited to convey the precise meaning intended.

The most pleasant of the translator's duties consists in acknowledging the very material assistance which he has received from his wife, whose preparation of an enlarged index for this English edition is but the last of many services which she has rendered in connection with the present undertaking.

EDWARD LEROY SCHAUB.

NoRTHWESTERN UNIVERSITY,

Evanston, Illinors,

October 19I5. 



\section{P R E F A C E}

THIS volume pursues a different method, in its treatment of the problems of folk psychology, from that employed in my more extensive treatment of the subject. Instead of considering successively the main forms of expression of the folk mind, the present work studies the phenomena, so far as possible, synchronously, exhibiting their common conditions and their reciprocal relations. Even while engaged on my earlier task I had become more and more convinced that a procedure of this latter sort was required as its supplement. Indeed, I believed that the chief purpose of investigations in folk psychology must be found in a synthetic survey. The first prerequisite of such a survey is, of course, a separate examination of each' of the various fields. The history, of the development of the physical organism aims to understand not merely the genesis of the particular organs but primarily their co-operation and the correlation of their functions. An analogous purpose should underlie an account of the mental development of any human community and, finally, of mankind itself. In addition to the problem of the relations of the separate processes to one another, however, we must in this case face also the broader question as to whether or not mental development is at all subject to law. This it is, therefore, that the sub-title of the present volume is intended to suggest. That we can be concerned only with outlines, moreover, and not with an exhaustive presentation of details, 
follows from the very fact that our aim is a synthetic survey. An exhaustive presentation would again involve us in a more or less detached investigation of single problems. A briefer exposition, on the other hand, which limits itself to arranging the main facts along lines suggested by the subject-matter as a whole, is, without doubt, better adapted both to present a clear picture of the development, and to indicate its general amenability to law, the presence of which even the diversity of events cannot conceal.

This being my main purpose, I believed that I might at once reject the thought of giving the various facts a proportionate degree of attention. In the case of the better known phenomena, it appeared sufficient to sketch their place in the general development. That which was less familiar, however, or was still, perhaps, generally unknown, seemed to me to require a more detailed discussion. Hence the following pages deal at some length with the forms of original tribal organization and of the consummation of marriage, with soul, demon, and totem cults, and with various other phenomena of a somewhat primitive culture. On the other hand, they describe in barest outline the social movements that reach over into historical times, such as the founding of States and cities, the origin of legal systems, and the like. No inference, of course, should be drawn from this with regard to the relative importance of the phenomena themselves. Our procedure, in this matter, has been governed by practical considerations alone.

The above remark concerning the less familiar and that which is as yet unknown, will already have indicated that folk psychology in general, and particularly a history of development in terms of folk psychology, such as this book aims to give, are as yet forced to rely largely on suppositions and hypotheses, if they are not to lose the thread that unites the details. Questions similar to the ones which we have just mentioned regarding the beginnings of humatr 
society, or others, which, though belonging to a later development, nevertheless still fall within the twilight dawn of history-such, for example, as those concerning the origin of gods and of religion, the development of myth, the sources and the transformations in meaning of the various forms of cult, etc.-are, of course, as yet largely, matters of dispute. In cases of this sort, we are for the most part dealing not so much with facts themselves as with hypotheses designed to interpret facts. And yet it must not be forgotten that folk psychology rests on precisely the same experiential basis, as regards these matters, as do all other empirical sciences. Its position in this respect is similar, more particularly, to that of history, with which it frequently, comes into touch in dealing with these problems of origin. The hypotheses of folk psychology never refer to a background of things or to origins that are by nature inaccessible to experiential knowledge; they are simply assumptions concerning a number of conjectured empirical facts that, for some reason or other, elude positive detection. When, for example, we assume that the god-idea resulted from a fusion of the hero ideal with the previously existing belief in demons, this is an hypothesis, since the direct transition of a demon into a god can nowhere be pointed out with absolute certainty. Nevertheless, the conjectured process moves on the factual plane from beginning to end. The same is true, not merely of many of the problems of folk psychology, but in the last analysis of almost all questions relating to the beginning of particular phenomena. In such cases, the result is seldom based on actually given data-these are inaccessible to direct observation, leaving psychological probability, as our only guide. That is to say, we are driven to that hypothesis which is in greatest consonance with the sum total of the known facts of individual and of folk psychology. It is this empirical task, constituting a part of psychology and, 
at the same time, an application of it, that chiefly differentiates a psychologicial history of development, such as the following work aims briefly to present, from a philosophy of history. In my opinion, the basis of a philosophy, of history, should henceforth be a psychological history of development, though the latter should not intrude upon the particular problems of the former. The concluding remarks of our final chapter attempt, in a few sentences, to indicate this connection of a psychological history of development with a philosophy of historical development, as it appears from the point of view of the general relation of psychology to philosophical problems.

W. WUNDT.

LEIPZIG,

March 31, 1912. 


\section{CONTENTS}

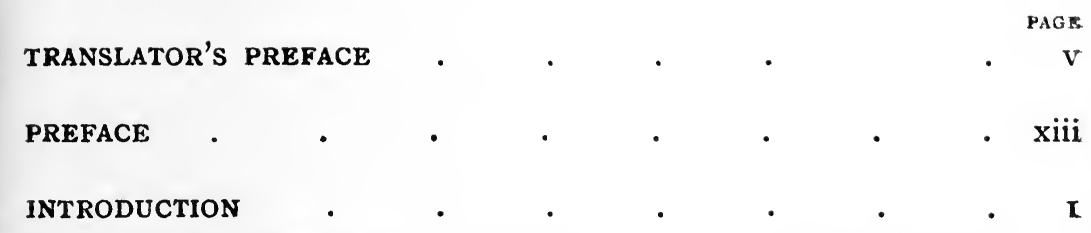

History and task of folk psychology-Its relation to ethnology-Analytic and synthetic methods of exposition-Folk psychology as a psychological history of the development of mankind-Division into four main periods.

\section{CHAPTER I}

\section{PRIMITIVE MAN}

I. THE DISCOVERY OF PRIMITIVE MAN

Early philosophical hypotheses-Prehistoric remains-Schweinfurth's discovery of the Pygmies of the Upper Congo-The Negritos of the Philippines, the inland tribes of Malacca, the Veddahs of Ceylon.

2. THE CULTURE OF PRIMITIVE MAN IN ITS EXTERNAL EXPRESSIONS

Dress, habitation, food, weapons-Discovery of bow and arrowAcquisition of fire-Relative significance of the concept 'primitive.'

3. THE ORIGIN OF MARRIAGE AND THE FAMILY .

Bachofen's "Mother-right" and the hypothesis of an original promiscuity-Group-marriage and the Malayan system of relationshipErroneous interpretation of these phenomena-Polygyny and polyandry-The monogamy of primitive peoples.

\section{PRIMITIVE SOCIETY}

The primitive horde-Its relation to the animal herd-Single family and tribe-Lack of tribal organization. 
5. THE BEGINNINGS OF LANGUAGE - $\cdot$ -

Languages of primitive tribes of to-day-The gesture-language of the deaf and dumb, and of certain peoples of nature-The signs of natural gesture-language-Its syntax-General conclusions drawn from gesture-language.

6. THE THINKING OF PRIMITIVE MAN - • •

The Soudan languages as examples of relatively primitive modes of thinking-The so-called 'roots' as words-The concrete character of primitive thought-Lack of grammatical categories-Primitive man's thinking perceptual.

7. EARLIEST BELIEFS IN MAGIC AND DEMONS • .

Indefiniteness of the concept 'religion'-Polytheistic and monotheistic theories of the origin of religion-Conditions among the Pygmies-Belief in magic and demons as the content of primitive thought-Death and sickness-The corporeal soul-Dress and objects of personal adornment as instruments of magic-The causality of magic.

\section{THE BEGINNINGS OF ART}

The art of dancing among primitive peoples-Its importance as a means of magic-Its accompaniment by noise-instruments-The dance-song-The beginnings of musical instruments-The bull-roarer and the rattle-Primitive ornamentation-Relation between the imitation of objects and simple geometrical drawings (conventionalization) -The painting of the Bushmen-Its nature as a memorial art.

9. THE INTELLECTUAL AND MORAL CHARACTERISTICS OF PRIMITIVE MAN .

Freedom from wants-Significance of isolation-Capacity for observation and reflection-No inferiority as to original endowment demonstrable-Negative nature of the morality of primitive man-Dependence upon the environment.

\section{CHAPTER II}

\section{THE TOTEMIC AGE}

\section{THE GENERAL CHARACTER OF TOTEMISM}

The word 'totem'-Its significance for cult-Tribal organization and the institution of chieftainship-Tribal wars-Tribal ownership of land -The rise of hoe-culture and of the raising of domestic animals. 
2. THE STAGES OF TOTEMIC CULTURE - •

Australian culture-Its low level of economic life-Its complicated tribal organization-Perfected weapons-Malayo-Polynesian cultureThe origin and migrations of the Malays-Celestial elements in MalayoPolynesian mythology-The culture of the American Indians and its distinctive features-Perfection of totemic tribal organization-Decline of totem cults-African cultures-Increased importance of cattle raising-Development of despotic forms of rulership-Survivals of totemism in the Asiatic world.

\section{TOTEMIC TRIBAL ORGANIZATION}

Similarity in the tribal organizations of the Australians and the American Indians-Totem groups as cult associations-Retrogression in America-The totem animal as a coat of arms-The principle of dual division-Systems consisting of two, four, and eight groups.

\section{THE ORIGIN OF EXOGAMY}

Unlimited and limited exogamy-Direct and indirect maternal or paternal descent-Effects upon marriage between relatives-Hypotheses concerning the origin of exogamy-Hygienic theoryMarriage by capture.

5. MODES OF CONTRACTING MARRIAGE • . •

Marriage by peaceful capture within the same kinship group-Exogamous marriage by barter-Marriage by purchase and marriage by contract-Survivals of marriage by capture.

\section{THE CAUSES OF TOTEMIC EXOGAMY}

Relation of clan division to totem groups-Totem friendshipsParental and traditional totem alliances-The rise of exogamy with direct and with indirect maternal or paternal descent.

7. THE FORMS OF POLYGAMY . . .

Origin of group-marriage-Chief wife and secondary wives-Polyandry and polygyny and their combination-The prevalence and causes of these forms of marriage.

8. THE DEVELOPMENTAL FORMS OF TOTEMISM .

Two principles of classification-Tribal and individual totemism-Conception and sex totemism-Animal and plant totemism-Inanimato totems (churingas)-Relation to ancestor worship and to fetishism.

\section{THE ORIGIN OF TOTEMIC IDEAS}

Theories based on names-Spencer and Lang-Frazer's theory of conception totemism as the origin of totemism-The animal transformations of the breath soul-Relations to soul belief-Soul animals as totem animals. 
10. THE LAWS OF TABOO

The concept 'taboo'- The taboo in Polynesia-The taboo of mother-in-law and father-in-law-Connection with couvade-The sacred and the impure-Rites of purification-Fire, water, and magical transference.

II. SOUL BELIEFS OF THE TOTEMIC AGE

The psyche as a breath and shadow soul-Its relation to the corporeal soul-Chief bearers of the corporeal soul-Modes of disposition of the dead.

12. THE ORIGIN OF THE FETISH - . - .

Fetishes in totem cult-Attainment of independence by fetishismFetishes as the earliest forms of the divine image-Retrogressive development of cult objects-Fetish cult as a cult of magic and demons -Amulet and talisman

\section{THE ANIMAL ANCESTOR AND THE HUMAN ANCESTOR}

The Mura-Mura legends of the Australians-The animal ancestorTransition to the human ancestor-Relation to disposal of the corpse and to cults of the dead-Surviving influences of totemism in ancestor cult.

14. THE TOTEMIC CULTS .

Customs relating to disposition tof the corpse and to sacrifices to the dead-Initiation into manhood-Vegetation cults-Australian Intichiuma festivals-Cults of the soil at the stage of hoe-culture-Underlying factor of community of labour-Unification of cult purposes and their combination with incipient deity cults.

I5. THE ART OF THE TOTEMIC AGE • • ceremonial dance-Instruments of concussion and wind instrumentsCult-songs and work-songs-The märchen-myth and its developmental forms.

\section{CHAPTER III}

\section{THE AGE OF HEROES AND GODS}

I. GENERAL CHARACTER OF THE HEROIC AGE • -

Significance of the individual personality-The hero an ideal human being, the god an ideal hero-Changes in economic life and in society -The rise of the State. 
2. THE EXTERNAL CULTURE OF THE HEROIC AGE

Folk migration and the founding of States-Plough-culture-Breeding of domestic animals-The wagon-The taming of cattle-The ox as a draught animal-The production of milk-Relation of these achievements to cult-Warfare and weapons-Rise of private propertyColonization and trade.

3. THE DEVELOPMENT OF POLITICAL SOCIETY - -

The place of the State in the general development of society-The duodecimal and the decimal systems in the organization of political society-The mark community and military organization.

4. FAMILY ORganization Within POLITICAL SOCIETY

The joint family-The patriarchal family-Paternal descent and paternal dominance-Reappearance of the monogamous family.

5. THE DIFFERENTIATION OF ClASSES - • -

Common property and private property-The conquering race and the subjugated population-Distinction in rank and property-The influence of State and of legal system.

6. THE DIFFERENTIATION OF VOCATIONS .

The priesthood as combining class and vocation-Military and political activity-Agriculture and the lower vocations-The gradual equalization of respect accorded to vocations.

7. THE ORIGIN OF CITIES .

The original development of the city-Castle and temple as the signs of a city-The guardian deity of city and State-Secondary developments.

8. THE BEGINNINGS OF THE LEGAL SYSTEM

Custom and law-Civil law as the original province of law-Political and religious factors-The council of elders and the chieftain-The arbitrator and the appointed judge-The religious sanction of legal practices.

9. THE DEVELOPMENT OF PENAL LAW - • -

Blood revenge and its replacement-Wergild-Right of sanctuaryDevelopment of imprisonment out of private custody of wrongdoerThe $\mathfrak{F}_{\text {us }}$ Talionis-Increase in complexity of rewards and punishments.

IO. THE DIFFERENTIATION OF LEGAL FUNCTIONS

Division of the judicial function-Influence of social organizationLogical classification of forms of the State lacking in genetic significance-Development of constitutions out of history and custom. 
II. THE ORIGIN OF GODS . $\quad$ - . $\quad$ -

Degeneration theories and developmental theories-Hypotheses of an original monotheism or polytheism-Theory based on nature-mythology-Demon theory of Usener-Characteristics distinguishing the god from the demon and the hero-The god as the result of a fusion of ideal hero and demon.

\section{THE HERO SAGA}

The hero of saga and the hero of märchen-The purely mythical and the historical hero saga-Magic in märchen and saga-The religious legend-The saint legend.

\section{COSMOGONIC AND THEOGONIC MYTHS}

The gods as demoniacal beings-Their struggle with the demons of earliest times-Myths of creation-Sagas of flood and of universal conflagration-Myths of world-destruction.

14. THE BELIEF IN SOULS AND IN A WORLD BEYOND -

Sequence of ideas of the beyond-The spirit-village-The islands of the blessed-Myths of the underworld-Distinction between dwellingplaces of souls-Elysium-The underworld and the celestial regionsPurgatory-Cults of the beyond-The conception of salvation-Transmigration of souls.

\section{THE ORIGIN OF DEITY CULTS}

Relation of myth and cult-Religious significance of cult-Vegetation cults-Union of cult purposes-Mystery cults.

\section{I6. THE FORMS OF CULT PRACTICES}

Prayer-Conjuration and the prayer of petition-Prayer of thanksgiving-Praise-The penitential psalm-Sacrifice-Purpose of sacrifice originally magical-Jewish peace-offering and sin-offering-Development of conception of gift-Connection between value and sacrificeVotive and consecration gifts-Sacrifice of the first fruits-Sanctification ceremonies-Means of lustration as means of sanctificationWater and fire-Baptism and circumcision-Magical sanctificationHuman sacrifice as a means of sanctification.

I7. THE ART OF THE HEROIC AGE $\cdot$. -Art as generic and as individualizing-The appreciation of the significant-Expression of subjective mood in landscape paintingThe epic-Its influence upon the cult-song-The drama-Music as an accessory and as an independent art. 


\section{CHAPTER IV}

\section{THE DEVELOPMENT TO HUMANITY}

I. THE CONCEPT 'HUMANITY' 470

Herder's idea of humanity as the goal of history-The concepts 'mankind' and 'human nature'-Humanity as a value-concept-The idea of a cultural community of mankind and its developmental forms.

2. WORLD EMPIRES - . . . . .

The empires of Egypt and of Western Asia-The monarch as ruler of the world-The ruler as deity-Apotheosis of deceased rulers-Underlying cause of formation of empires-Disappearance of world empires from history.

3. WORLD CULTURE

The world dominion of Alexander-Greek as the universal languageWriting and speech as factors of culture-Travel as symptomatic of culture-Hellenistic world culture and its results-The culture of the Renaissance-Cosmopolitanism and individualism.

\section{WORLD RELIGIONS}

Unity of the world of gods-Cult of Asculapius and cults of the beyond-Their transition into redemption cults-Buddhism and Christianity-Development of the idea of a superpersonal deityThe incarnate god as the representative of this deity-Three aspects of the concept 'representative.'

\section{WORLD HISTORY}

Twofold significance of the concept 'history'-History as selfconscious experience-The rôle of will in history-Prehistoric and historic periods-Influence of world culture and world religions on the rise of the historical consciousness-The philosophy of historyIts relation to a psychological history of the development of mankind. 


\section{ELEMENTS OF FOLK PSYCHOLOGY}

\section{INTRODUCTION}

THE word 'Völkerpsychologie' (folk psychology) is a new compound in our [the German] language. It dates back scarcely farther than to about the middle of the nineteenth century. In the literature of this period, however, it appeared with two essentially different meanings. On the one hand, the term 'folk psychology' was applied to investigations concerning the relations which the intellectual, moral, and other mental characteristics of peoples sustain to one another, as well as to studies concerning the influence of these characteristics upon the spirit of politics, art, and literature. The aim of this work was a characterization of peoples, and its greatest emphasis was placed on those cultural peoples whose civilization is of particular importance to us-the French, English, Germans, Americans, etc. These were the questions of folk psychology that claimed attention during that period, particularly, to which literary history has given the name "young Germany." The clever essays of Karl Hillebrand on Zeiten, Völker und Menschen (collected in eight volumes, I $885 \mathrm{ff}$.) are a good recent example of this sort of investigation. We may say at the outset that the present work follows a radically different direction from that pursued by these first studies in folk psychology.

Practically coincident with the appearance of these earliest studies, however, was a radically different use of the term 'folk psychology.' The mental sciences began to realize the need of a psychological basis; where a serviceable psychology did not exist, they felt it necessary to establish an independent psychological 
foundation for their work. It was particularly in connection with the problems of philology and mythology, and at about the middle of the century, that the idea gradually arose of combining into a unified whole the various results concerning the mental development of man as severally viewed by language, religion, and custom. A philosopher and a philologist, Lazarus and Steinthal, may claim credit for the service of having introduced the term 'folk psychology" to designate this new field of knowledge. All phenomena with which mental sciences deal are, indeed, creations of the social community. Language, for example, is not the accidental discovery of an individual; it is the product of peoples, and, generally speaking, there are as many different languages as there are originally distinct peoples. The same is true of the beginnings of art, of mythology, and of custom. The natural religions, as they were at one time called, such as the religions of Greece, Rome, and the Germanic peoples, are, in truth, folk religions; each of them is the possession of a folk community, not, of course, in all details, but in general outline. To us this fact has come to appear somewhat strange, because in our age these universal mental creations have already long transcended the limits of a single people. Though this is true, it does not imply that the folk community is not really the original source of these mental creations. Now, in the works of Lazarus and Steinthal and in the Zeitschrift für Volkerpsychologie und Sprachwissenschaft edited by them and appearing in twenty volumes from 1860 on, the conception had not as yet, it is true, received the precise definition that we must give it to-day. Nevertheless, a beginning was made, and the new venture was successfully launched along several different lines. Some uncertainty still prevailed, especially with regard to the relation of these studies to philosophy, and as to the method which psychology must follow when thus carried over into a new field. It was only gradually, as the psychological point of view gained ground in the special fields of research, that this condition was improved. To-day, doubtless, folk psychology may be 
regarded as a branch of psychology concerning whose justification and problem there can no longer be dispute. Its problem relates to those mental products which are created by a community of human life and are, therefore, inexplicable in terms merely of individual consciousness, since they presuppose the reciprocal action of many. This will be for us the criterion of that which belongs to the consideration of folk psychology. A language can never be created by an individual. True, individuals have invented Esperanto and other artificial languages. Unless, however, language had already existed, these inventions would have been impossible. Moreover, none of these languages has been able to maintain itself, and most of them owe their existence solely to elements borrowed from natural languages. How, again, could a religion have been created by an individual? There have, indeed, been religions whose founders were individual men : for example, Christianity, Buddhism, and Islamism. But all these religions rest on earlier foundations; they are elaborations of religious motives arising within particular folk communities. Thus, then, in the analysis of the higher mental processes, folk psychology is an indispensable supplement to the psychology of individual consciousness. Indeed, in the case of some questions the latter already finds itself obliged to fall back on the principles of folk psychology. Nevertheless, it must not be forgotten that just as there can be no folk community apart from individuals who enter into reciprocal relations within it, so also does folk psychology, in turn, presuppose individual psychology, or, as it is usually called, general psychology. The former, however, is an important supplement to the latter, providing principles for the interpretation of the more complicated processes of individual consciousness. It is true that the attempt has frequently been made to investigate the complex functions of thought on the basis of mere introspection. These attempts, however, have always been unsuccessful. Individual consciousness is wholly incapable of giving us a history of the development of human thought, for it is conditioned by an earlier history concerning which it cannot of itself give us any knowledge. 
For this reason we must also reject the notion that child psychology can solve these ultimate problems of psychogenesis. Among cultural peoples, the child is surrounded by influences inseparable from the processes that arise spontaneously within its own consciousness. Folk psychology, however, in its investigation of the various stages of mental development still exhibited by mankind, leads us along the path of a true psychogenesis. It reveals well-defined primitive conditions, with transitions leading through an almost continuous series of intermediate steps to the more developed and higher civilizations. Thus, folk psychology is, in an important sense of the word, genetic psychology.

In view of the general nature of the task of the science, objection has sometimes been raised to its being called folk psychology. For, the study is concerned, not merely with peoples but also with more restricted, as well as with more comprehensive, social groups. Family, group, tribe, and local community, for example, are more restricted associations; on the other hand, it is to the union and reciprocal activity of a number of peoples that the highest mental values and attainments owe their origin, so that, in this case, folk psychology really becomes a psychology of mankind. But it is self-evident that, if it is not to fade into indefiniteness, a term such as 'folk psychology' must be formulated with reference to the most important conception with which it has to deal. Moreover, scarcely any of - the proposed emendations are practicable. 'Gemeinschaftspsychologie' (community psychology) may easily give rise to the misconception that we are concerned primarily with such communities as differ from the folk community; 'Sozialpsychologie' (social psychology) at once reminds us of modern sociology, which, even in its psychological phases, usually deals exclusively with questions of modern cultural life. In an account of the total development of mental life, however-and this is the decisive consideration -the 'folk' is the most important collective concept and the one with which all others are associated. The 'folk' embraces families, classes, clans, and groups. These 
various communities are not excluded from the concept 'folk,' but are included within it. The term 'folk psychology' singles out precisely the folk as the decisive factor underlying the fundamental creations of the community.

When this point of view is taken, the question, of course, arises whether the problem thus assigned to folk psychology is not already being solved by ethnology, the science of peoples, or whether it ought not to be so solved. But it must be borne in mind that the greatly enlarged scope of modern ethnology, together with the increased number and the deepened character of its problems, necessarily precludes such a psychological investigation as falls to the task of folk psychology. I may here be allowed to refer to one who, perhaps more than any other recent geographer, has called attention to this extension of ethnological problems-Friedrich Ratzel. In his treatise on anthropography and in a number of scattered essays on the cultural creations of peoples, Ratzel has shown that ethnology must not only account for the characteristics and the habitats of peoples, but must also investigate how peoples originated and how they attained their present physical and mental status. Ethnology is the science of the origin of peoples, of their characteristics, and of their distribution over the earth. In this set of problems, psychological traits receive a relatively subordinate place. Apparently insignificant art products and their modifications may be of high importance in the determination of former migrations, fusions, or transferences. It is in this way that ethnology has been of valuable service to history, particularly in connection with prehistoric man. The central problem of ethnology concerns not only the present condition of peoples, but the way in which they originated, changed, and became differentiated. Folk psychology must be based on the results of ethnology; its own psychological interest, however, inclines it to the problem of mental development. Though of diverse origins, peoples may nevertheless belong to the same group as regards the mental ievel to which they have attained. Conversely, 
peoples who are ethnologically related may, psychologically speaking, represent very different stages of mental culture. The ' ethnologist, for example, regards the Magyars and the Ostiaks of $\mathrm{Obi}$ as peoples of like origin. Psychologically, they belong to different groups: the one is a cultural people, the other is still relatively primitive. To the folk psychologist, however, 'primitive' always means the psychologically primitive-not that which the ethnologist regards as original from the point of view of the genealogy of peoples. Thus, folk psychology draws upon ethnology, while the latter, in turn, must invoke the aid of the former in investigating mental characteristics. The problems of the two sciences, however, are fundamentally different.

In fulfilling its task', folk psychology may pursue different methods. The course that first suggests itself is to single out one important phenomenon of community life after another, and to trace its development after the usual pattern of general psychology in its analysis of individual consciousness. For example, an attempt is made to trace the psychological development of language by the aid of the facts of linguistic history. This psychology of language is then followed by a study of the development of art, from its beginnings among primitive races down to its early manifestations among cultural peoples, at which point its description is taken up by the history of art. Myth and religion are similarly investigated as regards the development of their characteristics, their reciprocal relations, etc. This is a method which considers in longitudinal sections, as it were, the total course of the development described by folk psychology. For a somewhat intensive analysis this is the most direct mode of procedure. But it has the objection of severing mental development into a number of separate phases, whereas in reality these are in constant interrelation. Indeed, the various mental expressions, particularly in their earlier stages, are so intertwined that they are scarcely separable from one another. Language is influenced by myth, art is a factor in myth development, and customs and usages are everywhere sustained by mythological conceptions. 
But there is also a second path of investigation, and it is this which the present work adopts. It consists-to retain the image used above-in taking transverse instead of longitudinal sections, that is, in regarding the main stages of the development with which folk psychology is concerned in their sequence, and each in the total interconnection of its phenomena. Our first task, then, would be the investigation of primitive man. We must seek a psychological explanation of the thought, belief, and action of primitive man on the basis of the facts supplied by ethnology. As we proceed to more advanced stages, difficulties may, of course, arise with regard to the delimitation of the various periods; indeed, it will scarcely be possible to avoid a certain arbitrariness, inasmuch as the processes are continuous. The life of the individual person also does not fall into sharply distinct periods. Just as childhood, youth, and manhood are stages in a continuous growth, so also are the various eras in the development of peoples. Yet there are certain ideas, emotions, and springs of action about which the various phenomena group themselves. It is these that we must single out if the content of folk psychology is to be classified, with any measure of satisfaction, according to periods. Moreover, it should be particularly noticed that, in starting our discussion with primitive man, as we naturally must, the term 'primitive' is to be taken relatively, as representing the lowest grade of culture, particularly of mental culture. There is no specific ethnological characteristic that distinguishes this primitive stage from those that are more advanced; it is only by reference to a number of psychological traits, such as are indicative of the typically original, that we may determine that which is primitive. Bearing in mind this fact, we must first describe the external traits of primitive culture, and then consider the psychological factors of primitive life.

Of the second period in the development of civilization, we may safely say that in many respects it represents a newly discovered world. Historical accounts have nothing to say concerning it. Recent ethnology alone has disclosed 
the phenomena here in question, having come upon them in widely different parts of the earth. This period we will call the totemic age. The very name indicates that we are concerned with the discovery of a submerged world. The word 'totem,' borrowed from a distant American tongue, proves by its very origin that our own cultural languages of Europe do not possess any word even approximately adequate to designate the peculiar character of this period. If we would define the concept of totemism as briefly as possible, it might perhaps be said to represent a circle of ideas within which the relation of animal to man is the reverse of that which obtains in present-day culture. In the totemic age, man does not have dominion over the animal, but the animal rules man. Its deeds and activities arouse wonder, fear, and adoration. The souls of the dead dwell within it ; it thus becomes the ancestor of man. Its flesh is prohibited to the members of the group called by its name, or, conversely, on ceremonial occasions, the eating of the totemanimal may become a sanctifying cult activity. No less does the totemic idea affect the organization of society, tribal division, and the forms of marriage and family. Yet the elements that reach over from the thought-world of this period into later times are but scanty fragments. Such, for example, are the sacred animals of the Babylonians, Egyptians, and other ancient cultural peoples, the prophetic significance attached to the qualities or acts of animals, and other magical ideas connected with particular animals.

Totemic culture is succeeded-through gradual transitions-by a third period, which we will call the age of heroes and gods. Initial steps towards the latter were already taken during the preceding period, in the development of a rulership of individuals within the tribal organization. This rulership, at first only temporary in character, gradually becomes permanent. The position of the chieftain, which was of only minor importance in the totemic age, gains in power. when the tribal community, under the pressure of struggles with hostile tribes, assumes a military organization. Society thus develops into the State. War, as also 
the guidance of the State in times of peace, calls out men who tower far above the stature of the old chieftains, and who, at the same time, are sharply distinguished from one another through qualities that stamp them as typical personalities. In place of the eldest of the clan and the tribal chieftain of the totemic period, this new age gives rise to the hero. The totemic age possesses only fabulous narratives; these are credited myths dealing, not infrequently, with animal ancestors who have introduced fire, taught the preparation of food, etc. The hero who is exalted as a leader in war belongs to a different world, a world faithfully mirrored in the heroic song or epic. As regards their station in life, the heroes of Homer are still essentially tribal chieftains, but the enlarged field of struggle, together with the magnified characteristics which it develops, exalt the leader into a hero. With the development of poetry, the forms of language also change, and become enriched. The epic is followed by formative and dramatic art. All this is at the same time closely bound up with the origin of the State, which now displaces the more primitive tribal institutions of the preceding period. When this occurs, different customs and cults emerge. With national heroes and with States, national religions come into being; "and, since these religions no longer direct the attention merely to the immediate environment, to the animal and plant world, but focus it primarily upon the heavens, there is developed the idea of a higher and more perfect world. As the hero is the ideal man, so the god becomes the ideal hero, and the celestial world, the ideally magnified terrestrial world.

This era of heroes and gods is finally succeeded by a fourth period. A national State and a national religion do not represent the permanent limits of human striving. National affiliations broaden into humanistic associations. Thus there begins a development in which we of the present still participate ; it cannot, therefore, be referred to otherwise than as an age that is coming to be. We may speak merely of an advance toward humanity, not of a development of humanity. This advance, however, begins immediately with 
the fall of the barriers that divide peoples, particularly with regard to their religious views. For this reason, it is particularly the transcendence of the more restricted folk circle on the part of religions that constitutes one of the most significant events of mental history. The national religions -or, as they are generally, though misleadingly, called, the natural religions-of the great peoples of antiquity begin to pass beyond their original bounds and to become religions of humanity. There are three such world religions-Christianity, Islamism, and Buddhism-each of them adapted in character and history to a particular part of mankind. This appears most clearly in the contrast between Christianity and Buddhism, similar as they are in their endeavour to be world religions. The striving to become a world religion, however, is also a symptomatic mental phenomenon, paralleled externally by the extension of national States beyond the original limits set for them by the tribal unit. Corresponding to this expansion, we find those reciprocal influences of cultural peoples in economic life, as well as in custom, art, and science, which give to human society its composite character, representing a combination of national with universally human elements. Hellenism and the Roman Empire afford the first and, for Occidental mental development, the most important manifestations of these phenomena. How immense is the chasm between the secret barter of primitive man who steals out of the primeval forest by night and lays down his captured game to exchange it, unseen by his neighbours, for implements and objects of adornment, and the commerce of an age when fleets traverse the seas, and eventually ships course through the air, uniting the peoples of all parts of the world into one great commercial community! We cannot undertake to delineate all aspects of this development, for the latter includes the entire history of mankind. Our concern is merely to indicate the outstanding psychological factors fundamental to the progression of the later from that which was original, of the more perfect from the primitive, partly under the pressure of external conditions of life and partly as a result of man's own creative power. 


\section{CHAPTER I}

\section{PRIMITIVE MAN}

\section{The Discovery of Primitive MaN.}

WHO is the primitive man? Where is he to be found? What are his characteristics? These are the important questions which here at once confront us. But they are questions to which, strangely enough, the answer has, up to very recent times, been sought, not in the facts of experience, history, or ethnology, but purely by the path of speculation. The search was not, for the most part, based on investigations of primitive culture itself, but took as its starting-point contemporary culture and present-day man. It was primarily by means of an abstract opposition of culture to nature that philosophy, and even anthropology, constructed natural man. The endeavour was not to find or to observe, but to invent him. It was simply by antithesis to cultural man that the image of natural man took shape; the latter is one who lacks all the attainments of culture. This is the negative criterion by means of which the philosophy of the Enlightenment, with its conceited estimate of cultural achievements, formed its idea of primitive man. Primitive man is the savage ; the savage, however, is essentially an animal equipped with a few human qualities, with language and a fragment of reason just sufficient to enable him to advance beyond his deplorable condition. Man in his natural state, says Thomas Hobbes, is toward man as a wolf. He lives with his fellow-beings as animal among animals, in a struggle for survival. It is the contrast of wild nature with peaceful culture, of ordered State with unorganized herd or horde, that underlies this conception. 
But this antithesis between the concepts of culture and of nature, as objectively considered, is not the only factor here operative ; even more influential is the contrast between the subjective moods aroused by the actual world and by the realm disclosed by imagination or reason. Hence it is that the repelling picture of primitive man is modified as soon as the mood changes. To an age that is satiated with culture and feels the traditional forms of life to be a burdensome constraint, the state of nature becomes an ideal once realized in a bygone world. In contrast to the wild creature of Thomas Hobbes and his contemporaries, we have the natural man of Jean Jacques Rousseau. The state of nature is a state of peace, where men, united in love, lead a life that is unfettered and free from want.

Alongside of these constructions of the character of natural man, however, there early appeared a different method of investigation, whose aim it was to adhere more closely to empirical facts. Why should we not regard those of our human institutions which still appear to be a direct result of natural conditions as having existed in the earliest period of our race? Marriage and the family, for example, are among such permanent cultural institutions, the one as the natural union of the sexes, the other as its necessary result. If marriage and family existed from the beginning, then all culture has grown out of the extension of these primitive associations. The family first developed into the patriarchal joint family; from this the village community arose, and then, through the union of several village communities, the State. The theory of a natural development of society from the family was first elaborated by Aristotle, but it goes back in its fundamental idea to legend and myth. Peoples frequently trace their origin to an original pair of ancestors. From a single marriage union is derived the single tribe, and then, through a further extension of this idea, the whole of mankind. The legend of an original ancestral pair, however, is not to be found beyond the limits of the monogamous family. Thus, it is apparently a projection of monogamous marriage into the 
past, into the beginnings of a race, a tribe, or of mankind. Wherever, therefore, monogamous marriage is not firmly established, legend accounts for the origin of men and peoples in various other ways. It thinks of them as coming forth from stones, from the earth, or from caverns; it regards animals as their ancestors, etc. Even the Greek legend of Deukalion and Pyrrha contains a survival of such an earlier view, combined with the legend of an original ancestral pair. Deukalion and Pyrrha throw stones behind them, from which there springs a new race of men.

The thought of an original family, thus represents simply a projection of the present-day family into an inaccessible past. Clearly, therefore, it is to be regarded as only an hypothesis or, rather, a fiction. Without the support which it received from the Biblical legend, it could hardly have maintained itself almost down to the present, as it did in the patriarchal theory of the original state of man to which it gave rise. The Aristotelian theory of the gradual origin of more comprehensive organizations, terminating in the State, is no less a fiction ; the social communities existing side by side in the period of Greece were arbitrarily represented as having emerged successively in the course of history. Quite naturally, therefore, this philosophical hypothesis, in common with the corresponding legend of the original family, presupposes primitive man to have possessed the same characteristics as the man of to-day. Thus, it gives no answer at all to the question concerning the nature of this primitive man.

When, therefore, modern anthropology made the first attempt to answer this question on the basis of empirical facts, it was but natural to assume that the characteristics of original man were not to be learned from a study of existing peoples, nor, indeed, from history, but that the data for the solution of the problem were of a prehistoric nature, to be found particularly in those human remains and those products of man's activity that have been preserved in the strata of the earth's crust. What we no longer find on the earth, so it was held, we must seek under the earth. 
And thus, about six decades ago, prehistoric anthropology began to gather material, and this has gradually grown to a considerable bulk. Upon the completion of this task, however, it appeared, as might, of course, have been expected, that psychology could gain but little in this way. The only source from which it might derive information lay in the exhumed objects of art. Then, however, the very disappointing discovery was made that, as regards implements of stone, drawings on the walls of caves which he inhabited, and pictures cut into horn or bone, the artistic achievements of the man of diluvial times did not differ essentially from those of the present-day savage. In so far as physical characteristics are concerned, however, the discovered remains of bones seemed to point to certain differences. While these differences, of course, were incapable of establishing any direct psychological conclusions, the fact that the measurements of the skeletal parts more closely resembled those of animals, and, in particular, that the measurements of the interior of the skull were smaller than those of the savages of our own time, offered indirect evidence of a lower development. Because of the close relation of cranial capacity to size of brain, moreover, a lower degree of intelligence was also indicated. Nevertheless, the remains that have been brought to light have not as yet led to any indubitable conclusions. There have been fairly numerous discoveries pointing to races that resemble the lower tribes among. contemporary peoples, and but a few cases in which uncertainty is possible, and concerning which, therefore, there exists a conflict of opinions. A typical instance is the history of one of the first discoveries made in Europe of the remains of a prehistoric man. It was in 1856 , in German territory, that there was discovered, in a grotto or cave in the Neander valley, near Duesseldorf, a very remarkable skull, though only, of course, the bones of the cranium and not the facial bones. All were at once agreed that these were the remains of a very primitive man. This was indicated particularly by characteristics which are still to be found, though scarcely in so 
pronounced a form, among certain lower races of men. Of special significance were the strongly developed, prominent bone-elevations above the eye-sockets. Some of the investigators believed that the long-sought 'homo primigenius' had perhaps at last been discovered. It was generally agreed that the form of the skull resembled most closely that of the modern Australian. In more recent years, however, anthropologists have developed somewhat more exact methods of measurement and of the reconstruction of a skeleton from parts only incompletely given. When Hermann Klaatsch, equipped with this knowledge, carried out such a reconstruction of the Neanderthal skull, he came upon the surprising fact that its capacity was somewhat greater than that of the present-day Australian. Little as this tells us concerning the actual intelligence of these primitive men, it nevertheless clearly indicates how uncertain the conclusions of prehistoric anthropology still are. A number of other recent discoveries in Germany, France, and elsewhere, have proved that several prehistoric races of men once lived in Europe. Some of these, no doubt, date back far beyond the last glacial period, and perhaps even beyond the period preceding this, for we now know that several glacial periods here succeeded one another. Nevertheless, no important divergencies from still existent races of men have been found. This, of course, does not imply that no differences exist; it means merely that none has as yet been positively detected, and that therefore the anatomy of prehistoric man can give us no information concerning the psychological aspect of the question regarding the nature of primitive man.

Considerably more light is thrown on this question when we examine the products of human activity, such as implements, weapons, and works of art. Traces of man, in the form of objects hammered out of flint and shaped into clubs, chisels, knives, and daggers, capable of serving as implements of daily use no less than as weapons, are to be found as far back as the first diluvian epoch, and, in their crudest forms, perhaps even as early as the tertiary period. 
The more polished objects of similar form belong to a later age. Still more remarkable are the works of art. in particular the cave pictures of prehistoric animals, such as the cave bear and the mammoth. Nevertheless, none of these achievements is of such a nature as to afford positive evidence of a culture essentially different from, or lower than, that of the primitive man of to-day. Two outstanding facts, especially, make a comparison difficult. On the one hand, wood plays an important rôle in the life of modern primitive man, being used for the construction of tools, weapons, and, in part, also of baskets and vessels. But the utensils of wood that may have existed in prehistoric times cquld not have withstood the destructive forces of decomposition and decay. All such utensils, therefore, that prehistoric man may have possessed have been lost. Thus, for example, it will be difficult ever to ascertain whether or not he was familiar with the bow and arrow, since the arrow, as well as the bow, was originally made of wood. Secondly, there is at the present time no primitive tribe, however much shut off from its more remote environment, into which barter, which is nowhere entirely absent, may not introduce some objects representing a higher form of civilization, particularly metals and metal implements. If, however, we bear in mind that, in the one case, products have suffered destruction and that, in the other, articles have been introduced from without, the impression made by prehistoric utensils and products of art-aside from certain doubtful remains dating back beyond the diluvial epoch-is not essentially different from that made by the analogous products of the Negritos of the Philippines or the inland tribes of Ceylon. Though the material of which the implements are constructed differs, the knives, hammers, and axes in both instances possess the usual form. Thus, the wooden knife which the Veddah of Ceylon still carves out of bamboo is formed precisely like some of the stone knives of the diluvial period. We find a similar correspondence when we examine the traces of dwellings and decorations that have been preserved, as well as certain remains that throw 
light upon customs. The oldest prehistoric people of Europe dwelt in caves, just as the primitive man of the tropics does to-day in the rainy season. In a rock cavern near Le Moustier, in France, there was discovered a skeleton whose crouching position points to a mode of burial still prevalent among primitive peoples, and one which is doubtless always a fairly positive indication of a belief in demons such as arises in connection with the impression made by death. The dead person is bound in the position that will best prevent his return. Thus, all these prehistoric remains suggest a culture similar to that of primitive tribes of to-day. But, just because they reveal conditions not essentially different from those of the present, these remains make another important contribution to our knowledge of primitive man. They indicate the great stability of primitive culture in general, and render it probable that, unless there are special conditions making for change, such as migrations and racial fusions, the stability increases in proportion to the antiquity. Though this may at first glance seem surprising, it becomes intelligible when we consider that isolation from his surroundings is an important characteristic of primitive man. Having very little contact 'with other peoples, he is in no wise impelled to change the modes of action to which his environment has led him from immemorial times.

Thus, the correspondence of the prehistoric with that which is to-day primitive indicates a high degree of permanence on the part of primitive culture. But, even apart from this consideration, it is apparent that we must really seek primitive man in the inhabited world of the present, since it is here alone that we can gain a relatively accurate knowledge of his characteristics. Our information concerning primitive man, therefore, must be derived from ethnology. We must not seek him under the earth, but on the earth. Just where, however, is he to be found? For decades the natives of Australia were believed to represent a perfect example of primitive culture. And, as a matter of fact, their material culture and some of their 
mythological ideas still seem to be of a very primitive character. Because of the conjecture that it was here dealing with a relatively primitive type of man, modern anthropology has for two decades applied itself with great partiality to the study of Australian tribes. English and German investigators have given us many works, some of them excellent, treating of the continent of Australia, which appears almost as unique with respect to its population as in its flora and its fauna. From these investigations, however, which are reported particularly in the volume by Howitt published in I900, in the works of Spencer and Gillen, and, finally, in those of Strehlow, a German missionary, it is apparent that the Australian culture is anything but primitive : it represents, rather, a stage of development already somewhat advanced. In certain respects, indeed, it may contain very primitive elements, such as are not to be found even among tribes that are, on the whole, on a lower level. Australian culture, however, possesses an enormously complex social organization, and this places it above that which may be called primitive. In its present form, it presupposes a development of probably thousands of years. Assuredly, therefore, the Australian should not be included in a chapter on primitive man. He will rather claim our attention in the next chapter, as a well-defined type of the totemic age. Indeed, he is beginning, in part, to lose even the characteristics of this age, mainly, no doubt, as a result of racial fusion, whose influence is here also in evidence.

Although the races of Australia are unquestionably not primitive, as was formerly believed and is still held in certain quarters, there are other parts of the earth which, in all probability, really harbour men who are primitive in that relative sense of the term which alone, of course, we are justified in using. If one were to connect the discovery of this primitive man with any single name, the honour would belong to a German traveller and investigator, George Schweinfurth. He was the first to discover a really primitive tribe-that is, one which remained prac- 
tically untouched by external cultural influences. When Schweinfurth, sailing up the Upper Nile in 1870 , listened to the narratives of the Nubian sailors in charge of his boat, he repeatedly heard accounts of a nation of dwarfs, of people two feet tall (so the exaggerated reports went), living in the impenetrable forests beyond the great lakes which constitute the source of the Nile. Schweinfurth was at once reminded of the old legends regarding pygmies. Such legends are mentioned even by Homer and are introduced also into the writings of Herodotus and of Aristotle. Aristotle, indeed, expressly says that these dwarf peoples of Central Africa exist in reality, and not merely in tales. When Schweinfurth arrived in the country of the Monbuttus, he was actually fortunate enough to gain sight of these pygmies. It is true, they did not exactly correspond to the fantastic descriptions of the sailors -descriptions such as are current here and there even to-day. The sailors represented the pygmies as having long beards, reaching to the earth, and gigantic heads; in short, they imputed to them the characteristics of the dwarf gnome, who appears also in German folk-lore. In reality, it was found that the pygmies are, indeed, small-far below the average normal size of man-but that they are of excellent proportions, have small heads, and almost beardless faces.

Subsequent to Schweinfurth's discovery, similar tribes were found in various parts of the earth. Emin Pasha, together with his companion StuhImann, had the good fortune to be able to observe the pygmies of the Congo more closely even than had been possible for Schweinfurth. In the Negritos of the Philippines a similar dwarf people was discovered. They also are of small stature, and, according to their own belief and that of the neighbouring Malays, are the original inhabitants of their forests. Besides these, there are the inland tribes of the Malay Peninsula, the Semangs and Senoi, and, finally, the Veddahs of Ceylon, studied particularly by the cousins Paul and Fritz Sarasin. All of the peoples just mentioned live in forests and have probably been isolated from civilization for thousands of 
years. The Bushmen of South Africa, of whom we have . long known, also belong to this group, although they have not to the same extent been free from the influence of surrounding peoples. In all these cases we have to do with tribes which at one time probably occupied wider territories, but which have now been crowded back into the forest or wilderness. In addition to these tribes, furthermore, there are remnants of peoples in. Hindustan, in Celebes, Sumatra, the Sunda Islands, etc. Concerning these, however, we as yet have little knowledge. In some respects, doubtless, the inhabitants of the Andaman Islands should also be here included, although they cannot, on the whole, be regarded as primitive in the strict sense of the word. This is precluded by their external culture, and especially by their legends, the latter of which point to the influence of Asiatic culture.

Observations of these relatively most primitive tribesand this is especially worth noting-show them to be remarkably similar. If we read a description of the characteristics, habits, and customs of the Negritos of the Philippines and then pass on to the Malaccans, to the Semangs and Senoi, or, further, to the Veddahs of Ceylon, we constantly meet with almost the same phenomena, there being but slight differences depending on the specific character of the natural environment. We are thus in possession of data that are now observable. The statements and conclusions which these enable us to make are more than mere speculations with regard to the past ; and they are more than inferences drawn from the silent fragments of the bones and from a few of the art products of primitive man. According as the phenomena are simpler in character and require fewer antecedent conditions for their explanation, may we be confident that we are really dealing with primitive conditions. This in itself implies that the criteria of primitive culture are essentially psychological in nature, and that racial characteristics and original tribal relationships are probably negligible so far as this question is concerned. A culture would be absolutely primitive if no antecedent mental development. whatsoever could be presupposed. Such an absolute concept can never be realized in 
experience, here any more than elsewhere. We shall, therefore, call that man primitive in the relative sense of the term -our only remaining alternative-whose culture approximates most nearly to the lowest mental achievements conceivable within the limits of universal human characteristics. The most convenient measure of these achievements, and the one lying nearest at hand, is that afforded by external culture, as expressed in dress, habitation, and food, in selfmade implements, weapons, and other productions serving to satisfy the most urgent needs of life.

\section{The Culture of Primitive Man in its External EXPRESSIONS.}

Following the above-mentioned criteria as to what may be regarded as primitive, the question concerning the external culture of primitive man may, in general, be briefly answered. Of dress there are only meagre beginnings: about the loins a cord of bast, to which twigs of trees are attached to cover the genitals-that is generally all, unless, through secret barter with neighbouring peoples, cotton goods, leather, and the like, have been imported. As regards personal decoration, conditions are much the same. On the next stage of development, the totemic, there is, as we shall later see, a desire for lavish decoration, especially as regards the adornment of the body by painting and tatooing. Little of this, however, is to be found among primitive tribes, and that which exists has probably been introduced from without. Some examples of such decoration are the scanty tatooing in single lines, the painting of the face with several red and white dots, and the wooden plug bored through the bridge of the nose. The Negritos of the Philippines bore holes through their lips for the insertion of a row of blades of grass. Other decorations found are necklaces and bracelets, fillets, combs, hair ornaments made of twigs and flowers, and the like.

What is true of his dress holds also of the dwelling of primitive man. Everything indicates that the first 
permanent dwelling was the cave. Natural caves in the hillsides, or, less frequently, artificially constructed hollows in the sand, are the places of refuge that primitive man seeks when the rainy season of the tropics drives him to shelter. During the dry season, no shelter at all is necessary; he makes his bed under a tree, or climbs the tree to gain protection from wild animals. Only in the open country, under the compulsion of wind and rain, does he construct a wind-break of branches and leaves after the pattern supplied by nature in the leafy shelter of the forest. When the supports of this screen are inclined toward one another and set up in a circle, the result is the original hut.

Closely connected with the real dwelling of primitive man, the cave, are two further phenomena that date back to earliest culture. As his constant companion, primitive man has a single animal, the $d o g$, doubtless the earliest of domestic animals. Of all domestic animals this is the one that has remained most faithful to man down to the present time. The inhabitant of the modern city still keeps a dog if he owns any domestic animal at all, and as early as primitive times the dog was man's faithful companion. The origin of this first domestic animal remains obscure. The popular notion would seem to be that man felt the need of such a companion, and therefore domesticated the dog. But if one calls to mind the dogs that run wild in the streets of Constantinople, or the dog's nearest relative, the wolf, one can scarcely believe that men ever had a strong desire to make friends of these animals. According to another widely current view, it was man's need of the dog as a helper in the chase that led to its domestication. But this also is one of those rationalistic hypotheses based on the presupposition that man always acts in accordance with a preconceived plan, and thus knew in advance that the dog would prove a superior domestic animal, and one especially adapted to assist in the chase. Since the dog possessed these characteristics only after its domestication, they could not have been known until this 
had occurred, and the hypothesis is clearly untenable. How, then, did the dog and man come together in the earliest beginnings of society? The answer to this question, I believe, is to be found in the cave, the original place of shelter from rain and storm. Not only was the cave a refuge for man, but it was equally so for animals, and especially for the dog. Thus it brought its dwellers into companionship. Furthermore, the kindling of the fire, once man had learned the art, may have attracted the animal to its warmth. After the dog had thus become the companion of man, it accompanied him in his activities, including that of the chase. Here, of course, the nature of the carnivorous animal asserted itself; as man hunted, so also did the animal. The dog's training, therefore, did not at all consist in being taught to chase the game. It did this of itself, as may be observed in the case of dogs that are not specifically hunting dogs. The training consisted rather in breaking the dog of the habit of devouring the captured game. This was accomplished only through a consciously directed effort on the part of man, an effort to which he was driven by his own needs. Thus, it is the cave that accounts for the origin of the first domestic animal, and also, probably, for the first attempt at training an animal. But there is still another gain for the beginnings of culture that may probably be attributed to the cave in its capacity of a permanent habitation. Among primitive peoples, some of whom are already advanced beyond the level here in question, it is especially in caves that artistic productions may be found. These consist of crude drawings of animals and, less frequently, of men. Among the Bushmen, such cave pictures are frequently preserved from destruction for a considerable period of time. Natural man, roaming at will through the forests, has neither time nor opportunity to exercise his imagination except upon relatively small objects or upon the adornment of his own body. But the semidarkness of the cave tends, as do few other places, to stimulate the reproductive imagination. Undisturbed by external influences, and with brightnesses and colours 
enhanced by the darkness, the memory images of things seen in the open, particularly those of the animals of the primeval forest, rise to consciousness and impel the lonely and unoccupied inhabitant to project them upon the wall. Such activity is favoured by the fact, verifiable by personal introspection, that memory images are much more vivid in darkness and semi-darkness than in the light of day. Thus, it was in the cave, the first dwelling-place of man, that the transition was made, perhaps for the first time, from the beginnings of a graphic art, serving the purposes of adornment or magic, to an art unfettered except by memory. It was an art of memory in a twofold sense : it patterned its objects after the memory of things actually observed, and it sought to preserve to memory that which it created.

From the consideration of dress and habitation we turn to that of food. Primitive man was not bound to fixed hours for his meals. Among civilized peoples, so close a connection has grown up between meals and definite hours of the day that the German word for meal, Mahlzeit, reminds us of this regularity by twice repeating the word for time-for Mahl also means time. Primitive man knew nothing of the sort. If he found food and was hungry, he ate; if he found none, he went hungry. Sometimes, moreover, in order to provide for the future, he gorged to such an extent as to injure his health. As concerns the food itself, there is an old theory which has led to misconceptions concerning primitive man. He was a hunter, we are told ; the chase supplied him with food ; only incidentally and occasionally did he enjoy parts of plants or fruits that he had gathered or accidentally discovered. It is scarcely correct, however, to assume that systematic hunting was practised by primitive man. Doubtless he did engage in this occupation. Yet this furnished him with only an incidental part of his food supply - a part with which, living as he did from hand to mouth, he satisfied only his momentary needs. It was with plant food, if at all, that he made provision for the future. Here may be found also the first traces 
of a division of labour: woman gathered the plant foodroots, bulbs, and berries-while man occasionally found it necessary to hunt. Plant food being capable of longer preservation, it was woman who first learned to economize and to make provision for the future. In part, indeed, the influence of these cultural beginnings persists even to-day. Moreover, just as mixed food, part plant and part animal, is by far the most common to-day, so also was it the original diet of man. The proportion, however, varied more than in later times, according as the external conditions of life were propitious or otherwise. Of this the Bushmen afford a striking illustration. Fifty years ago they were still by preference huntsmen. Armed with their bows, they dared to hunt the elephant and the giraffe. But after the surrounding peoples of South Africa-the Hottentots, Betschuans, and Herero-came into the possession of firearms, which the Bushman scornfully rejects, the game was, in part, exterminated, and to-day the Bushmen, crowded back into rocky wastes, derive but a small part of their living from the chase. They gather bulbs, roots, and other parts of plants, such as can be rendered edible by boiling or roasting. Their animal food, moreover, is no longer wild game, but consists, for the most part, of small animals found while gathering the plant food-frogs, lizards, worms, and even insects. Hunting, therefore, was never more than one of the customary means of providing food ; and primitive man, especially, was a gatherer rather than a hunter. The word 'gatherer' implies also that he took from nature only what it directly offered, and that he was familiar neither with agriculture nor with the raising of animals. In procuring his food, moreover, he was aided by a knowledge, often surprising, of the properties of the objects gathered. This knowledge, probably gained as a result of many disastrous experiences in his search for food, enabled primitive man to utilize even such roots and fruits as are not wholesome in their raw state, either because they are not edible until prepared by means of fire, or because they are poisonous. Primitive man learned to overcome the 
injurious effects of many of these plants. By reducing them to small pieces, washing them in a solution of lye, and heating them, he converted them into palatable food. The bulbs and roots were secured from beneath the surface of the ground by means of the most primitive of all agricultural implements and the progenitor of all succeeding ones, the digging-stick. This is a wooden stick, with a pointed end that has been hardened by fire.

Connected with the removal of poison, by means of water and fire, from parts of plants that are otherwise edible, is still another primitive discovery-the utilization of the poisons themselves. Only when the arrow is smeared with plant poisons does the bow become a real weapon. In itself the arrow wound is not sufficient to kill either game or enemy; the arrow must be poisoned if the wound is to cause death or even temporary disability. The Veddahs and the inland tribes of Malacca therefore use the juice of the upas-tree mixed with that of strychnos-trees. The best known of these arrow poisons, curare, used in South America and especially in Guiana, is likewise prepareid from the juice of strychnos-trees.

This brings us to the weapons of primitive man. In this connection it is highly important to note that all of the primitive peoples mentioned above are familiar with the use of bow and arrow, but we must also bear in minid that this is practically their only weapon. Contrary to what archæological excavations would suggest concerning the earliest age of peoples, primitive culture, in respect to implements and weapons, depended only to a small extent upon the working of stone. We might better speak of this period as an age of wood. Wood is not only decidedly easier to manipulate than stone, but it is always more easily obtainable in shapes suitable for constructive purposes. Possibly even the arrow-head was originally always made of wood, as it sometimes is even to-day. Only in later times was the wood replaced by a sharpened stone or by iron acquired through barter.

It is not difficult to see how wood, in the forms which 
it possesses by nature, came to be fashioned into clubs, axes, and digging-sticks, and how bones, horns, shells, and the like were converted into tools and objects of adornment. But how did primitive man acquire bow and arrow? The general belief seems to be that this weapon was invented by some resourceful mind of an early age. But an inventor, in the proper sense of the word, must know in advance what he wishes to invent. The man, therefore, who constructed the bow and arrow for the first time must already have had some previous idea of it. To effect a combination of existing implements, or to improve them in useful ways, is a comparatively easy matter. But no one can manufacture implements if he possesses nothing over and above material that is in itself somehow suitable for the purpose. The most primitive implements, therefore, such as the digging-stick, the club, and the hammer, are all products of nature, at most changed slightly by man as their use requires. But this is obviously not true of bow and arrow. We may, perhaps, find a suggestion for the solution of our problem in a hunting weapon which, though belonging, of course, to the later totemic culture, is in principle simpler than the bow and arrow-the boomerang of the Australians. The word is probably familiar to all, but the nature of the weapon is not so well known, especially its peculiarly characteristic form by virtue of which, if it fails to strike its object, it flies back to the one who hurled it. The boomerang, which possesses this useful characteristic, is, in the first place, a bent wooden missile, pointed at both ends. That this curved form has a greater range and strikes truer to aim than a straight spear, the Australian, of course, first learned from experience. The boomerang, however, will not return if it is very symmetrically constructed ; on the contrary, it then falls to the ground, where it remains. Now it appears that the two halves of this missile are asymmetrical. One of the halves is twisted spirally, so that the weapon, if thrown forward obliquely, will, in accordance with the laws of ballistics, describe a curve that returns 
upon itself. This asymmetry, likewise, was discovered accidentally. In this case, the discovery was all the more likely, for primitive weapons were never fashioned with exactitude. That this asymmetry serves a useful purpose, therefore, was first revealed by experience. As a result, however, primitive man began to copy as faithfully as possible those implements which most perfectly exhibited this characteristic. Thus, this missile is not a weapon that required exceptional inventive ability, though, of course, it demanded certain powers of observation. The characteristics, accordingly, that insured the survival of the boomerang were discovered accidentally and then fixed through an attentive regard to those qualities that had once been found advantageous. Now, can we conceive of the origin of bow and arrow in an analogous way? Surely this weapon also was not devised in all its parts at a single time. The man of nature, pressing his way through the dense underbrush of the forest and experiencing in person the hard blows of branches that he has bent back, gains a lively impression of the elastic power of bent wood. How easily the attention is forced to the observation that this effect increases when the wood is bent out of its natural shape, appears strikingly in the case of a kind of bow found in Asia and the Asiatic islands. The bow is here constructed out of a piece of wood bent by nature, not in such a way, however, that the natural curve of the wood forms the curve of the bow, but contrariwise. Thus arises a reflexive bow, whose elastic power is, of course, considerably increased. In order that such a bow may be bent back more easily, some people of a more advanced culture construct it out of several layers of wood, horn, sinew, or the like. Having first observed the powerful impulsive force which a rod gains through being bent, it was a simple matter to render this force permanently available by bending the rod back and binding its ends together with a cord of bast, or, if bamboo was used, with strips torn from the bamboo itself. Thus originated the common form of the bow. Next, it was, of course, easy to observe 
that the bowstring thus contrived would communicate a powerful impetus to a lighter piece of wood placed against it. In addition to the bow, we then have the arrow, which is hurled into the distance by the combined propelling power of the bow and its string. But at this point a new factor appeared, clearly indicating that several motives generally co-operated in the case of such so-called primitive inventions. In these inventions nature itself played no less a part than did the inventive genius of the individual. The arrow but rarely consists merely of a piece of wood one of whose ends is somehow pointed or provided with a stone head, or, at a later period, with an iron head. As is well known, the other end is feathered, either with genuine bird feathers or, as in the case of the pygmies of Central Africa, with an imitation of bird feathers made of palm-leaves. The feathers are usually supposed to have been added to insure the accurate flight of the arrow. And this accuracy is, indeed, the resultant effect. As in the case of the boomerang, however, we must again raise the question : How did man come to foresee this effect, of whose mechanical conditions he had, of course, not the slightest knowledge? The solution of this problem probably lies in the fact of an association of the discharged arrow with a flying bird that pierces the air by the movement of its feathers. Thus, in the arrow, man copied the mode of movement of the bird. He certainly did not copy it, however, with the thought that he was causing movement in a mechanical way. We must bear in mind that for primitive man the image of a thing is in reality always equivalent to the thing itself. Just as he believes that his spirit resides in his picture, and is, therefore, frequently seized with fright when a painter draws his likeness and carries it away with him, so also does the feathered arrow become for him a bird. In his opinion, the qualities of the bird are transferred by force of magic to the arrow. In this case, indeed, the magical motive is in harmony with the mechanical effect.

Nature directly supplies primitive man not only with the 
patterns of his implements and weapons, but also with those of the vessels which he uses. Of the primitive tribes none is familiar, at the outset, with pottery. In its stead suitable natural objects are utilized for storing what is gathered. The Negritos of the Philippines, for example, employ coconut shells. The inland tribes of the Malay Peninsula use bamboo, whose varying thicknesses, and, particularly, whose internodes enable it to be converted into the desired vessels by cutting the stem at the upper end of an internode and immediately below it, thus securing a vessel with a bottom. Wherever primitive peoples cut vessels out of wood, as occurs among the Veddahs and the Bushmen, we may be sure that this represents a comparatively late acquirement, following upon a knowledge of metals and the use of stone implements. Primitive man possesses no vessels for cooking purposes. He prepares his food directly in the fire or in hot ashes.

We are now confronted by a final and an especially interesting question of primitive culture, that of the acquisition of fire. This acquisition made a deep impression on the human mind, and one whose effects long survived in legend. The totemic age, as we shall see, is replete with legends of beneficent animals which brought fire to man. In the heroic age, the fire-bringing animal is displaced by the fire-bringing hero. We may call to mind Prometheus, who brought fire from heaven, and by so doing drew upon himself the vengeance of the gods. Nevertheless, the question concerning the original production of fire is a very simple one. As in the case of very many utensils and tools, we must look to natural conditions that present themselves in the course of experience. Man did not invent the art of kindling fire; it would be nearer the truth to say that he found it, inasmuch as he discovered it while making his utensils. In this connection, particularly, it is highly important to note that the first age, if we would designate it by its tools, was not an age of stone but an age of wood. We have already referred to the way in which bamboo was worked up into vessels for the 
storing of fruits and liquids. With a sharp sliver of bamboo, a bamboo-stem is sawed into pieces in order that its parts may be utilized. If this sawing occurs during dry weather, the wood is pulverized and the heated sawdust finally becomes ignited. As soon as it begins to glow, the worker blows upon it and the fire flames up. This mode of kindling fire has been called that of sawing, and is probably the oldest in origin. After fire was thus accidentally produced, it became possible to kindle it at will, and this developed into a skilful art. At a later stage, however, there came the further need of drilling holes into wood. This gave rise to a second method of kindling fire, that of drilling. A piece of wood is bored through with a sharpened stick of hard wood, and the same results occur as in the case of the sawing. The method of drilling is the more effective; it produces fire more quickly. Nevertheless, both methods are laborious and tedious, and we cannot blame the savage for regarding as a magician the European who before his very eyes lights a match by friction. Because of the difficulty in producing fire, its preservation plays an important rôle in the life of the savage. When he changes his dwelling-place, his first consideration, as a rule, is to take with him some live fire so that he will not be obliged to kindle it anew.

In conclusion, we may supplement these sketches of external culture by mention of a feature that is particularly characteristic of the relation of primitive man to his environment. Primitive man lives in close association with his fellow-tribesmen, but he secludes himself from other tribes of the neighbourhood. $\mathrm{He}$ is led to do so because they threaten his means of subsistence; indeed, he himself may fall a prey to them, as do the Pygmies of Central Africa to the anthropophagic customs of the Monbuttus. And yet, primitive man early feels the need of such useful articles as he cannot himself produce but with which he has, in some accidental manner, become acquainted. This gives rise to what is generally called 'secret barter.' An illuminating example of this 
occurs in the records of the Sarasin cousins as relating to the Veddahs. The Veddah goes by night to the house of a neighbouring Singhalese smith and there deposits what he has to offer in barter, such as captured game, ivory, etc. With this he places a representation of an arrow-head, made of palm-leaves. The next night he returns and finds real arrows of iron which the smith has laid out in exchange for the proffered goods. It might be thought that such a system of barter would imply an excessive measure of confidence. The smith, however, knows that, should he take away that which was brought to him without delivering the arrows, he would himself be struck by an arrow shot from some sheltered ambush. Thus, many things, especially iron, materials for clothing, and articles of adornment, come into the possession of primitive man. through secret barter, raising his external culture to a somewhat higher level.

A retrospective survey of this culture brings to notice especially the fact that the concept 'primitive' is never valid, as applied to man, except in a relative sense. Of an absolutely primitive man we know nothing at all. Moreover, the knowledge of such a being could hardly render explicable his further development, since he would really belong to the animal level and therefore to the prehuman stage of existence. Primitive man is relatively primitive, for, while he does possess certain beginnings of culture, these are in no respect more than mere beginnings, all of which are borrowed from nature and from the direct means of assistance which it offers. It is precisely these elementary acquisitions, however, that already differentiate primitive man from the animal. He has the beginnings of a dwelling and of dress, even though he does no more in either case than merely to utilize the means which nature offers, or than partly to imititate and partly to combine these means, as he does in the case of the leafy wind-break and of the weapons which doubtless represent the highest achievement of this age-namely, the bow and arrow. But these are all beginnings which already contain within themselves 
the possibilities of higher achievements. The development of the hut out of the wind-break, of the lance out of the staff and the arrow, of the woven basket out of the coconut or the gourd, severally represent easy steps in the advance from nature to culture. Next there comes the preparation of food by means of fire. This is closely connected with the discovery of the art of kindling fire, which, in its turn, was partly an accidental discovery connected with the manufacture of primitive tools out of wood and partly a real invention. Thus, the manufacture of tools, on the one hand, and the kindling of fire, which was connected with it, on the other, are the two primary features which from early times on distinguished primitive man from animals. Furthermore, there is the bow and arrow, which is the first real weapon and differs markedly from all other implements. Its construction also was dependent upon the assistance of nature. The fact that this was the only weapon of primitive society throws an important light on the nature of the latter. The bow and arrow continued to be used for a long time afterwards-indeed, even down to the appearance of firearms ; it served not only as a weapon of warfare but also as an implement for hunting. With it alone, however, no organized strife or warfare of any sort is possible. While, therefore, it is true that the archer appears on the earliest monuments of cultural peoples, it is only as the fellow-combatant of the warrior who is armed with shield and lance. With lance and shield it is possible to fight in closed ranks. The archer must fight single-handed. Primitive man, therefore, does not engage in tribal wars; he is familiar only with the strife of individual with individual. In fact, wherever the bow and arrow is used exclusively, open warfare is impossible. With it, primitive man slays his enemy from behind a sheltering bush. It is thus that the Veddah of nature serves the cultural Veddah, or the Singhalese who has deceived him in secret barter, or even the fellowtribesman who steals his wife. Just as secret barter is carried on in concealment, so also is warfare. This, how- 
ever, indicates that the bow and arrow was originally intended for hunting and not for warfare. From this consideration alone it is evident that primitive life was not a war of all against all, as it was described by Thomas Hobbes. On the contrary, there doubtless existed a state of peace, interrupted only occasionally by the strife of individual with individual-a strife that resulted from a conflict of interests, such as occurred even during this early period.

\section{The Origin of Marriage and the Family.}

That the origin of marriage and the family really constitutes a problem, long failed to be recognized. Because of the natural relations of the sexes it was supposed that man lived in a state of marriage from the very beginning. Furthermore, the monogamous marriage of the present was projected back into an indefinite past, where it found final termination in the idea of a primal pair of ancestors. But, even apart from this mythological belief,' there were also positive grounds for supposing an original state of monogamy. Do not many animals live in monogamous union? In addition to nest-building birds, monogamy prevails particularly among mammals, and, of the latter, among those that have the closest physical relationship to man. We might cite the gorilla, the primate that most resembles man, and probably also the chimpanzee, although in this case we lack positive proof. Why, then, should not man have carried over monogamous marriage from his animal state into his primitive culture? This theory, therefore, was regarded as almost self-evident until after the middle of the last century. But in $\mathrm{r} 86 \mathrm{I}$, a Swiss jurist and antiquarian, J. Bachofen, published a remarkable work on "Mother-right." In this book' Bachofen attempted to prove the falsity of the doctrine-previously almost uncontested-that monogamy was the original form of marriage, and to refute the view, regarded as equally selfevident, that within this marriage union man held the supremacy-in brief, the patriarchal theory. Bachofen 
started with a discussion of the Lycians as described by Herodotus. According to this writer, the kinship of the children, among the Lycians, was determined by the mother, not by the father. The sons and daughters belonged to the family of the mother, and descent was traced through her instead of through the father. Bachofen found similar indications among other peoples also. $\mathrm{He}$ called attention, for example, to Tacitus's reports in the Germania of some of the German tribes in which a son stands closer to the brother of his mother than he does to his father. Similar statements occur in Cæsar's Bellum Gallicum concerning the Britons. Bachofen collected other examples of the same nature, and also especially emphasized certain elements in myth and legend that seemed to indicate a like ascendancy of woman in early times. In his opinion, legend is esteemed too lightly if, as occurred in his day, it is regarded as entirely meaningless. Of course, legend is not history; yet it gives a picture, even though in fanciful terms, of the real conditions of earlier times. On the basis of these detached observations, Bachofen at once constructed a general theory. Preceding the patriarchal period of paternal rule, there was maternal rule, gynecocracy. In earliest times the mother was the head of the family. In romantic colours Bachofen pictures the era in which the fair sex guided the destinies of humanity. Later, man, with his rougher nature but greater intelligence, displaced her and seized the dominion for himself. Bachofen then asks, How did it come about that, in spite of this natural superiority of man, woman ruled the family earlier than he? To this he gives an extremely prosaic and realistic reply, contrasting sharply with his romantic ideas in connection with the dominance of woman. We must find a clue, he believes, in those cases of our own day in which mothers still determine the name, descent, etc., of their children. This happens when the children are born out of wedlock. Under such conditions, the child does not know its father, nor does, perhaps, even the mother. To understand the origin of maternal descent, therefore, we must suppose that 
children were universally born out of wedlock. Thus, prior to the ascendancy of woman, there existed a state of agamy, in which there was no marriage but only a promiscuous relation of the sexes. We thus have, as it were, a picture whose outlines are determined by contrast with the family, of civilized peoples, and which reminds us of Hobbes's account of the earliest political relations, there being in both cases an entire absence of order. But it is precisely in this fact, Bachofen believes, that we have a clue to the origin of gynecocracy if only we bear in mind the actual characteristics of woman. Woman's nature is such that this universal promiscuity of the sexes must have become repulsive, first of all, to her. Turning away all other men, she accepted but a single one. In so doing, woman proved herself the champion of chastity and morals which she has since remained. To her, and not to man, is due the honour of having founded the monogamous family. At the outset she was also its natural preserver and guardian. The children were counted to her kin; her kin determined descent; and, in Bachofen's view, this condition, which arose out of causes of a universal nature, long prevailed throughout the world generally. But why was it not maintained? It was not possible, so runs the answer, because, though woman alone was psychically fitted to establish' itman could never have instituted monogamy-she was not equally fitted to render it permanent. Woman is not born to rule. In intelligence, as well as in physical strength, she is inferior to man. Altogether, therefore, there are three periods of development : agamy or promiscuity, followed by female supremacy or mother-right, and, finally, by the dominance of man, or father-right.

These hypotheses of Bachofen created much dispute in succeeding years. Some of the facts could not be denied from the standpoint of the antiquarian. Nevertheless, the supposition of the universality of an early mother-right was quite rightly questioned, and its origin out of the completely unrestrained condition of the horde was even more vigorously contested. And so the theory of the Swiss jurist, 
which was based essentially on philologic-antiquarian arguments, gradually fell into the background, until, in the seventies of the nineteenth century, it suddenly, seemed to find important corroboration and a new basis from an entirely different quarter. It was ethnology that supplied the new facts, and these were again derived from a study of Australia, that field of ethnological observation which was generally regarded as more particularly exemplifying primitive culture. Bachofen believed to have demonstrated that maternal descent was originally a universal custom, even in the case of those who are now cultural peoples. Ethnology revealed the fact that this system of kinship is still very prevalent in Australia. Indeed, it is so prevalent that even to-day about three-fifths of the tribes trace descent through the mother and only two-fifths through the father. In some of the cases in which the system of paternal descent is now established', moreover, it is probable that the mother once determined the kinship of the children. It was on the basis of these facts that, in his volume on the natives of south-eastern Australia, Howitt, the most thorough investigator of the social conditions of the Australians, came to a conclusion similar to that previously reached by Bachofen on the basis of his antiquarian investigations. In Howitt's view, all family relations were originally based on the system of maternal descent. This system, though generally, restricted to narrower bounds than in Australia, is likewise to be found in America, Melanesia, Polynesia, and in several parts of the Old World, especially among the peoples of northern Siberia and among the Dravidian tribes in the southern part of Hindustan. These facts have more and more led present-day ethnologists to a view that is in essential agreement with Bachofen's theory. Again the question was raised how such a system of maternal descent was possible. The answer was that it could be possible only if the mother, but not the father, was. known to son and daughter-again an analogical conclusion from conditions prevailing in present-day society outside the marriage tie. Accordingly, the idea was again adopted that, ante- 
ceding marriage, there was an original state of promiscuity. It was believed that there was originally neither marriage nor family, but merely a condition in which there were sexual relations of all with all-a picture of the relations between man and woman suggested by the idea of an original state of natural rights and of freedom from political restraints, and forming, as it were, the counterpart of the latter.

But ethnology then discovered other phenomena also that seemed to favour this view. Two lines of argument, particularly, have here played an important rôle, and still retain a measure of influence. The first argument was again derived from the ethnology of Australia. This region possesses a remarkable institution, describable neither as monogamy nor as agamy, but appearing, at first glance, to be an intermediate form of association. This is the socalled group marriage; several men are united in common marriage with several women. Either a number of brothers marry a number of sisters, or a number of men belonging to one kinship group marry in common women of another. Group marriage, therefore, may seem to represent a sort of transitional stage between promiscuity and monogamy. At first, so we might picture it to ourselves, the union of all with all became restricted to more limited groups, and only later to the union of one man with one woman.

But had not a further argument been added, perhaps neither female descent alone nor group marriage would have attracted to this theory so many prominent ethnologists, including, besides Howitt, the two able investigators of Australia, Spencer and Gillen, the learned exponent of comparative ethnology, J. G. Frazer, and a number of others. This further argument was presented with particular thoroughness by the American ethnologist Lewes Morgan, in his history of primitive man, "Ancient Humanity". (1870). It is based upon what Morgan has termed the 'Malayan system of relationship.' We are not, of course, familiar with this as a system of actual relationship; it occurs only in the languages of certain peoples, as a system of names-in short, as a nomenclature-referring in part to 
relations of kinship, but chiefly to age-relations within one and the same kinship group. The name 'Malayan' is not entirely appropriate as applied to this system. The nomenclature is found particularly on the island of Hawaii, though it also occurs in Micronesian territory. Its essential characteristic may be very simply described. It consists, or consisted, in the fact that a native of Hawaii, for example, calls by the name of 'father,' not only his actual father but also every man of an age such that he could be his father-that is, every man in the kinship group of the next older generation. Similarly, he calls by the name ' mother,' not only his own mother but every woman who might possibly, as regards age, be his mother. He calls brother and sister the men and women of his own generation, son and daughter those of the next younger generation, and so on up to grandfather and grandmother, grandson and grand-daughter. The Hawaiian native does not concern himself about more distant generations; greatgrandfather is for him the same as grandfather, and greatgrandchild the same as grandchild. The terms, thus, are of the simplest sort. The brothers and sisters of a man, whom we designate in the accompanying diagram by $M$, are placed alongside of him in the same generation; above, as an older generation, are fathers and mothers; still

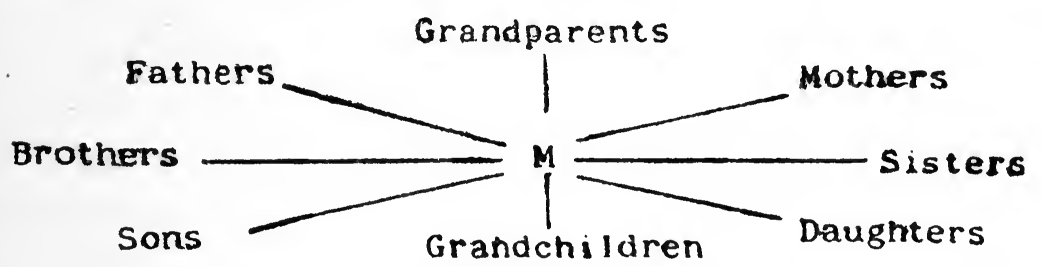

higher, are grandfathers and grandmothers; below, are sons and daughters and the grandsons and granddaughters. The same, of course, holds also for women. Thus, the system as a whole comprises five generations.

Now, it was maintained that this system could have arisen only out of a previous condition of general promiscuity. For, unless the actual father were universally 
unknown, how could it be possible that a person would call by the name of father every man within the same kinship group who might, as regards age, be his father? If, however, we propose this argument, we immediately strike a weak point in the hypothesis, since all women of the older generation are called mother just as its men are called father. W.e should certainly expect that the real mother would be known, because the child derives its nourishment from her during a period which is especially long among primitive peoples, and because it grows up close to her. And, furthermore, the hypothesis is hardly reconcilable with the fact that, for the most part, Malayo-Polynesian languages differentiate relations by marriage even more sharply than do our own. An Hawaiian man, for example, calls the brother of his wife by a different name than does a woman the brother of her husband. Thus, in place of our word 'brother-in-law' they have two expressions. In any event, the term 'brother-in-law' is applied to an individual, and therefore implies marriage. To meet this point, we would be obliged to fall back on the supposition that these terms represent later additions to the original nomenclature of relationship. But even then the fact would remain that, in their direct reference, these terms are merely names for differences in age. It therefore remains an open question whether the terms also designate relationship; to the extent of our observation, this is certainly not the case. The native of Hawaii, so far as we know anything about him, knew his father and mother: what he lacked was merely a specific name for them. Whenever he did not call his father by his given name, he evidently called him by the same name that he applied to the older men of his immediate group. Among European peoples also, the terms 'father' and 'mother' are sometimes used in connection with men and women outside this relationship. For example, the Russians, particularly, have a custom of addressing as 'little father' and 'little mother' persons who are not in the least related to them. That which makes it highly, probable that in the so-called Malayan system of relationship we are dealing not 
with degrees of relationship but with age-periods, is, in the last event, a different phenomenon-one that has hitherto been overlooked in connection with these discussions. In the very regions whose languages employ, this nomenclature, custom prescribes that the youths and men live in separation from the women and children from their earliest years on. This is the institution of the men's club with its agegroups. Its social rôle is an important one, crowding even the family association into the background. Under such circumstances, the individual is naturally interested first of all in his companions of the same age-group, for each of these usually occupies a separate apartment in the men's house. Thus, the so-called Malayan system of relationship is really not a system of relationship at all, but a nomenclature of age-groups based on social conditions. These conditions bring it about that companions of the same sex are more closely associated than are men and women. In the men's houses a companion of the same group is a brother, one of the next older group, a father. Together with these men the individual goes to war and to the hunt. Thus, these phenomena cannot be said to belong to the lowest stage of culture. Nor, obviously, does this terminology, which has reference to differences of age, exclude any particular form of marriage. In this case it is a mistake to associate the names 'father,' 'mother,' 'brother,' etc., with the concepts that we attach to these words.

The hypothesis that the family, whether of monogamous or of polygamous organization, was preceded by a state of unrestricted sexual intercourse, so-called agamy or promiscuity, is, however, as was remarked above, based not only on the fact of maternal descent and of the Malayo-Polynesian method of designating ages, but also on that of groupmarriage. In this form of marriage, a number of men marry in common a number of women. This is interpreted as a transitional stage between an unrestricted sexual intercourse within the tribe and the limited marriage unions of later times. At first glance, indeed, this might appear 
probable. In order, however, to decide whether such a transition could take place, and how it might occur, we must first of all consider the relation which group-marriage sustains, among the peoples who practise it, to the other forms of marriage. It then appears at once that it is a particular form of polygamy. True, it is not identical with the form of polygamy most familiar to us, in which one man possesses several wives. But there is also a second form, which, though less frequent, is of greatest importance for an interpretation of group-marriage. One woman may have several husbands. The two forms of polygamy may conveniently be called polygyny and polyandry, and these terms should always be distinguished in any attempt at a precise account of polygamous marriage. Polygyny is very prevalent even in our day, occurring particularly in the Mohammedan world, but also among the heathen peoples of Africa, and in other regions as well. It was likewise practised by the ancient Israelites, and also by, the Greeks, although the Indo-Germanic tribes for the most part adhered to monogamy from early times on. Polyandry is much less common, and is, indeed, to be found only among relatively primitive peoples. It occurs in Australia and, in the southern part of Hindustan, among the Dravidians, a tribe of people crowded back to the extreme end of the continent by peoples who migrated into India ; it is found also far in the north among the Esquimos of Behring Strait and among the Tchuktchis and Ghilyaks of Siberia, and, finally, here and there in the South Sea Islands.

If, now, we wish to understand the relation of these: two forms of polygamy to each other, we must first of all attempt to picture to ourselves the motives that underlie them, or, wherever the custom has become fixed through age, to bring to light the motives that were originally operative. In the case of polygamy, the immediate motive is evidently the sexual impulse of man, which is more completely satisfied by the possession of several wives than by that of a single one. This motive, however, does not stand alone; as a rule other contributing circumstances 
are present. Two such important factors, in particular, are property rights and the power of authority. Polygyny flourishes particularly wherever the general conceptions of property and of authority, and, connected with the latter, that of the supremacy of man within the family, have attained undue importance. Under the co-operation of these motives, the wife becomes the absolute property of the husband, and may, therefore, wherever polygyny prevails among barbaric peoples, be given away or exchanged. Bound up with this, moreover, is the fact that, wherever there are considerable social differences, dependent on differences in property and rank, it is principally the wealthy or the aristocratic man who possesses many wives. In the realm of Islam, the common man is, as a rule, content with a single wife, so that monogamy here prevails in the lowest stratum of society.

With polyandry the case is essentially otherwise. In it, entirely different motives are operative; it might, indeed, be said that they are the exact opposite of those that bring about polygyny. It is particularly significant that polyandry is found in regions where there is a scarcity. of women. This scarcity, however, is, in turn, generally due to an evil custom of barbaric culture, namely, infanticide. In Polynesia, where polyandry was very prevalent, this custom was at one time fairly rampant. Even today infanticide still appears to be practised by some of the Dravidian tribes of Hindustan. Similar conditions prevail among the Australians. In Polynesia, however, and probably in other localities as well, it was chiefly the female children who were the victims of infanticide. The natural result was a decrease in women and a striking numerical disproportion between the sexes. Thus, Ellis, one of the older English investigators of conditions in these territories, estimated the relation of men to women as about six to one. Under such circumstances the custom of polyandry is intelligible without further explanation. It was not possible for every one to possess a wife of his own, and so several men united to win one wife in common. 
We might ask why it was chiefly girls who fell victims to this murder. That children in general should be sacrificed, under the rough conditions of nature, is not inexplicable. It is due to the struggle for the necessities of life and to the indolence that shrinks from the labour of raising children. The desire is to preserve the lives of only a limited number ; the remainder are killed immediately after birth. In Polynesia, the murder was forbidden if the child had lived but a single hour. Occasionally, magical motives are operative, as in the case of the horror which the man of nature feels towards deviations from the normal and towards the birth of twins. That male children are more often spared than female, however, can scarcely, be explained otherwise than on the ground that a particular value is placed on men. The man is a companion in sport and in the chase, and is regarded as more valuable for the further reason that he aids in tribal warfare. This higher value reverts back even to the child. It is evidenced also in the fact that, in the case of women, the arrival of adolescence is not celebrated with the same solemn ceremonies as are held in the case of young men. Whereas great celebrations are held when the youth reaches the age of manhood, little notice is taken, as a rule, of the maiden's entrance into womanhood. By means of these celebrations, the youths are received into the society of men, and, together with companions of their own age, are initiated into the traditional ceremonies. In these ceremonies women are not allowed to participate.

Though the causes of polyandry are thus entirely different from those of polygyny, it does not at all follow that these forms of marriage are mutually exclusive. On the contrary, they may very, well exist side by side, as, indeed, they actually do in many. places. But how, then, is so-called group-marriage related to these two forms? It is obviously nothing but a combination of polyandry and polygyny. In fact, whenever a group of men marries a group of women, these two forms of polygamy are both involved. Every man has several wives, and every wife has several husbands. Only, indeed, on the basis of a 
purely external and superficial consideration could one look upon polygyny and polyandry as unconjoinable, because they are, in a certain sense, opposing ideas. As a matter of fact, they do not really exclude each other. If we bear in mind the causes mentioned above, it is obvious that under certain conditions of life, such as occur particularly in a more primitive environment, their combination is more probable than their mutual exclusion. If, especially among tribes who have not yet developed sharply defined distinctions based on property and power, as, for example, among the Australians, every man strives to obtain several wives (which is the state of polygyny), while, on the other hand, there actually exists a dearth of women (which means that motives to polyandry are present), the two forms naturally combine with each other. This is frequently verified, moreover, whenever we are able to gain any degree of insight into the particular conditions surrounding the origin of such group-marriages, and also whenever their forms undergo a modification of details. Among Australian tribes, for example, particularly in the southern part of the continent, there is a common form of group-marriage, in which a man possesses either one or several chief wives, together with secondary wives; the latter are the chief wives of other men, whereas his own chief wife is in turn the secondary wife of those men or of others. This custom is very similar to what is probably the most common form of polygyny, namely, the possession by a man of only one. chief wife in addition to several secondary wives-a form of marriage that is obviously derived from monogamy. One agency that is particularly apt to bring about such a form of marriage, transitional between monogamy and polygyny, is war. We know from the Iliad that in barbaric times woman was the booty of the conqueror, and became his slave or secondary wife. So also, according to the Biblical legend, Abraham possessed a chief wife, Sarah, who belonged to his own tribe, but also a secondary wife, Hagar, who was an Egyptian slave. Wherever the concept of property became prominent, the purchase of women 
proved to be a further source of polygyny. In this case also, there was generally one chief wife, wherever polyandry did not interfere. When the Mohammedan of modern times calls his chief wife 'favourite,' it is merely another indication that this form of polygyny developed from monogamy, since, according to the old custom, there was but one chief wife. Here, however, the chief wife is no longer necessarily the wife belonging to a man's own tribe, as was the case among the ancient Israelites; the favour of the master determines which wife shall be given the privileged place.

Thus, from whatever angle we view group-marriage, its polygyny and its polyandry seem to rest on monogamy. This is true also of forms of group-marriage other than those mentioned above. Where the theft of women still continues to be a practice more serious than are the somewhat playful survivals that occur in the marriage ceremonies of cultural peoples, the one who wishes to steal a wife not infrequently secures confederates for his undertaking. Custom then commonly gives these companions a certain right to the stolen woman. This right, of course, is for the most part temporary, but it may nevertheless come to approximate the conditions of group-marriage in case the first man assists his confederates in the same way in which they have aided him. There is still another and a related motive that may lead to the same result. When a woman enters into marriage with a man of a certain tribe, she at once enters into very close relations with the tribe itself. Where tribal association has gained a preponderant importance, custom sometimes grants to all the male members of the tribe certain transient rights with respect to the woman on the occasion of her marriage. This occurs particularly when the man and woman belong to different tribes-that is, in the case of exogamy, an institution characteristic of the totemic age and to be considered later. For, the lively consciousness of kinship differences naturally tends to strengthen the right of appropriation belonging to the entire tribe. A similar thought is reflected in the mediæval jus prima noctis of certain provinces of France and Scot- 
land, except that in place of the right of the kinship group to the possession of the individual we here find the authority of the lord over his vassals.

Thus, all these phenomena, belonging in part to the transitional stage between monogamy and polygamy and in part to a combination of the two forms of polygamy, namely, polygyny and polyandry, point to monogamy as the basal form of marriage, and that form from which, under the influence of particular conditions, all others have developed. Whether or not we regard it as probable that the system of maternal descent was at one time universal, no argument for the existence of an original promiscuity can be based upon it. If we call to mind the close association of the youths and men of the kinship group in the men's house, it will be apparent that such conditions of social intercourse make for a particularly intimate bond between mother and children. Before his entrance into the community of men, the boy lives in the company of the women. This close association between mother and children is sufficient to account for the origin of maternal descent. But, owing to the gradual change of cultural conditions, it is to be expected that maternal descent should pass over into paternal descent as soon as more positive conceptions of authority and property are formed. Moreover, the possibility also remains that among some tribes paternal descent prevailed from the very outset; positive proof is here not available. We cannot, of course, deny the possibility that under certain cultural conditions man exercised the decisive influence from the very beginning, as early, indeed, as one may speak of clan membership and hereditary succession. The most primitive stage of culture, as we shall see in the following discussion, lack's the conditions for either maternal or paternal descent, inasmuch as it possesses neither clearly defined clans nor any personal property worth mention.

Thus, the arguments based on the existing conditions of primitive peoples, and contending that the original condition of mankind was that of a horde in which both marriage and the family were lacking, are untenable. On 
the contrary, the phenomena, both of group-marriage, valued as the most important link in the chain of proof, and of the simpler forms of polygamy, everywhere point to monogamy as their basis. Furthermore, these arguments all rest on the assumption that the peoples among: whom these various phenomena occur, particularly the combination of polygyny and polyandry in group-marriage, occupy a primitive plane of social organization. This presupposition also has proven fallacious, since it has become evident that this organization, especially among the Australian tribes, is an extremely complicated one, and points back' to a long history involving many changes of custom.

Meanwhile, primitive man, in so far as we may speak of him in the relative sense already indicated, has really been discovered. But the Australian does not belong to this class, nor, even less, can many of the peoples of Oceania be counted within it. It includes only those tribes which, having probably been isolated for many centuries and cut off from the culture of the rest of the world, have remained on the same primitive level. We have become familiar with them in the preceding account of the external culture of primitive man. We find them to be forest peoples who have, for the most part, been crowdied back into inaccessible territory and who have entered but slightly into intercourse with the outside world, inasmuch as their needs are limited. They generally call themselves, whether rightly or wrongly we need not inquire, the original inhabitants of these regions, and they are regarded as such by their neighbours. They include, in addition to several tribes of Hindustan (as yet insufficiently studied), particularly the Semangs and Senoi of the interior of the Malay Peninsula, the Veddahs of Ceylon, the Negritos of the Philippines and Central Africa, and, finally, to some extent, also the Bushmen. This is certainly a considerable number of peoples, some of whom live at great distances from the others. In spite of this, however, even their external culture is largely the same. Considering the primitive character of their social institutions and customs, it would seem safe to 
say that without doubt they approach the lowest possible level of human culture. Besides bow and arrow they have scarcely a weapon, no vessels of clay, and practically only. such implements as are presented directly by nature herself. At this stage there is scarcely anything to distinguish man from the animal except the early, discovered art of kindling fire, with its influence on the utilization of the food that is gathered. Briefly summarized, these are the main traits of primitive culture that are known to us.

What, now, is the status of marriage and the family at this period? The answer to this question will come as a surprise to those who are imbued with the widespread hypotheses that presuppose the primitive state to be that of the horde. And yet, if these hypotheses be regarded in the proper light, our answer might almost be expected. Among the primitive tribes that we have mentioned, monogamy is everywhere found to be not only the exclusive mode of marriage, but that which is always, so to speak, taken for granted; and this monogamy, indeed, takes the form of single marriage. It is but rarely that related families live together more or less permanently, forming: the beginning of the joint family. The Bushmen alone offer something of an exception to this rule. Among them, polygyny, together with other practices, has been introduced. This is probably due to the influence of neighbouring African peoples, such as the Hottentots and the Bantus. Elsewhere conditions are different. This is true especially: of the Semangs and Senoi, whose isolation has remained more complete, and of the Veddahs of nature, as the Sarasin cousins call them in distinction from the surrounding Veddahs of culture. Among these peoples, monogamy-indeed, lifelong monogamy-has remained the prevailing form of marriage. Connected with it is found the original division of labour, which is based on sex. Man provides the animal food by hunting; woman gathers the vegetable food-fruits, tubers, and seeds-and, by the employment of fire, if necessary, renders both it and the game edible. This basis of division of labour, which appears natural and 
in harmony, with the endowment of the sexes, contrasts with the conditions of later culture in that it indicates an approximate equality, of the sexes. Furthermore, Rudolf Martin and the two Sarasins, investigators of the primitive Asiatic tribes of Malacca and Ceylon, commend the marriage of these peoples as being a union of husband and wife strictly guarded by custom. In forming: a moral estimate of these conditions, it should not be overlooked that the exclusive possession of the wife is probably due to jealousy as much as it is to mutual faithfulness. Among the Veddahs, the intruder who threatens this possession is struck' to earth by a well-aimed arrow shot from behind ambush, and custom approves this act of vengeance as a justifiable measure on the part of the injured man. Therefore, even though a French traveller and investigator may, to a certain extent, have confused cause and effect when he stated that the monogamy of these tribes had its origin in jealousy, the exercise of the right of revenge may, nevertheless, have helped to strengthen the custom. But, of course, in view of the primitive state of culture that here prevails, this custom of revenge is itself merely| an indication of the undisputed supremacy of monogamy. Even as the individual, and not the clan, exercises this vengeance, so also does marriage continue to be restricted to single marriage. Of the formation of joint families, which arise out of the union of immediate blood relations, we find at most, as has been remarked, only the beginnings.

\section{Primitive Society.}

The more extensive social groups generally result from the fact that during the rainy season families withdraw into caves among the hills. The larger caves are frequently occupied in common by a number of families, particularly by such as are most closely related. Yet the groups of co-dwellers are not so much determined by considerations of kinship as by the size of the places of refuge; a single family occasionally occupies a small cave by itself. 
Nevertheless, this community life plainly furnishes the incentive to a gradual formation of wider social groups. This, no doubt, accounts for the fact that during the favourable season of the year several families of the Veddahs claim for themselves a specific plot of ground, whose supply of game, as well as of the products of the soil, which the women gather, belongs exclusively to them. Thus, there is a division of the people into districts, and these are determined geographically rather than ethnologically. Every one is entitled to obtain his food, whether game or products of the soil, from a specified territory. Custom strictly guards this communal property, just as it protects the single marriage. The Veddah, for example, who encroaches upon the territory belonging to a group other than his own, is in no less danger of falling a victim to an arrow shot from an ambush than is the one who trespasses on marriage ties.

These various institutions form the beginnings of social organization, but as yet they do not represent developed clan groups or established joint families of the patriarchal type. On the contrary, as they arise through the free association of individuals, so also may they be freely dissolved. Each man has exclusive possession of his wife. Without interference on the part of his clan, moreover, he exercises absolute control over his children, who remain with the individual family, just as in the case of a developed monogamy. There is no trace of sex-groups, such as are later to be found in the case of the men's houses and the age-groups. Only temporarily, on the occasion of common undertakings, such as the hunting of large animals, which requires a considerable measure of strength, or when new hunting-grounds are being sought, is a leader appointed from among the older men. His leadership, however, ceases with the completion of the undertaking. There are no permanent chiefs, any more than there are clans or tribal organizations.

Thus, in summary, we might say: Whenever the social organization of primitive man has remained unin- 
fluenced by peoples of a higher culture, it consists in a firmly established monogamy of the form of single marriage-a mode of existence that was probably carried over from a prehuman stage resembling that of the presentday anthropoids. There are also scanty beginnings of social groups. If we consider these tribes as a whole, they still continue to lead the life of a horde, meaning by this an unorganized, in contrast to an organized, tribe of people. Indeed, it was through a curious change of meaning that this word acquired its present significance. It is supposed to have originated in a Mongolian idiom, whence it found its way first into the Russian and later into other European languages. The Tartars called a division of warriors a horda. First used in this sense, the word apparently did not receive its present meaning in Germany until the beginning of the eighteenth century. Having in mind the "Golden Horde" of the Tartars, a horde was understood to mean a particularly dreaded division of warriors. The furious force of these Asiatic hordes, and the terror which they spread, later caused the concept to be extended to all unorganized, wild, and unrestrained masses of men. Taking the word in this wider significance, we may now say that the horde, as a fairly large social group in which only, very meagre suggestions of an organized tribal system occur, is characteristic of primitive times, no less than are the isolated single family and the beginnings of the joint family. Thus defined, however, the horde does not differ essentially from the animal herd, in the meaning which the latter concept would possess when applied to human-kind. And it is not impossible that in the extension of the meaning of the term 'horde,' this association of the foreign word with the original Germanic word 'herd' played a part. A horde, we might say, is a human herd, but it is precisely a human herd. Between the members of a horde, therefore, there exists a relation that is lacking in the animal herd, in flock's of migratory birds, for example, or in herds of sheep and cattle. This relation is established and preserved through a community of language. Herder, 
therefore, truthfully remarks that man was from the beginning a 'herding animal,' in so, far as he possessed social instincts. Even in the formation of language these social instincts were operative. Without a community life, and, we may add, without the mental interaction of individuals, language would be impossible. Language, however, in turn, strengthened this community life, and elevated it above the status of the animal herd or of an association concerned merely with momentary needs.

Thus, these reflections concerning the social relations of primitive man lead us to a further field of phenomena which likewise affords a glimpse into the mental characteristics of primitive peoples. For that which differentiates the horde from the herd is the language of primitive man, together with the activity most closely bound up with language, namely, thinking.

\section{The Beginnings of Language.}

Our knowledge of those peoples whom we, avoiding the errors of the past, may now regard as primitive, led to the conviction that the Asiatic and African tribes described above were actually primitive, in the above-mentioned relative sense of the word. Naturally the question concerning the language of these peoples then began to arouse considerable attention, on the part, not only of ethnologists, but also of those interested in philology. The question is of equal importance, to say the least, for the psychologist. For language is bound up with thought. From the phenomena of language, therefore, we may draw inferences concerning the most general characteristics of thought. Such fundamental differences of language as exist, for example, between the Chinese and the Indo-Germanic tongues do not, of course, allow the direct conclusion that there are quantitative differences in mental culture. They do imply, however, that there are divergent directions and forms of thought. In their ceaseless change, the latter react upon language, and this in turn, again influences mental characteristics. We cannot 
suppose that, in the period of Old High German, much less in that of the original German, our ancestors employed the same forms of thought with which we of to-day are familiar. To a lesser degree, similar changes have undoubtedly transpired within much shorter spaces of time.

These considerations make the question concerning the language of primitive man of the utmost psychological importance. Linguistic investigations, however, so far as they, in their early attempts, had been able to survey. the field, had brought to light a fact which discouraged all efforts to discover an original language. Indeed, it was inevitable that at first glance this discovered fact should have appeared exceedingly strange, particularly when viewed in connection with the life of primitive man. It appeared that, for the most part, the original languages of primitive tribes no longer exist. It is true that in the vocabularies of the Semangs and Senoi of Malacca, of the Veddahs of Ceylon, of the Negritos of the Philippines, and in other vocabularies that have been collected, single words may be found which do not occur in the languages of the neighbouring tribes; and it is noteworthy that the bow and arrow are the objects most frequently designated by such words, a proof of the fact that these are really relatively primitive inventions. On the whole, however, the Veddahs speak the language of the Singhalese and Tamils; the Semangs and Senoi, as well as the Negritos of the Philippines, that of their neighbours, the Malays ; similarly, among the African tribes, the Pygmies of Central Africa have apparently appropriated the language of the Monbuttus and other negro races, and the Bushmen that of the Hottentots.

How may this remarkable fact be explained? That these tribes formerly possessed languages of their own can scarcely be doubted. For, as respects physical characteristics, they are absolutely distinct races. Considering. their characteristics as a whole, moreover, it is utterly impossible that they could have lacked language before coming into contact with the peoples who entered the country at a later period. How, then, did these people come 
apparently to lose their original language? To this we may briefly reply that there here transpired what always occurs when the well-known principle of the struggle for existence is applied to the field of mental phenomena. The stronger race crowded out the most important mental creation of the weaker, its language. The language of the weaker race, which was probably very meagre, succumbed to a language that was more highly developed. At first glance, this explanation would appear to contradict what we know concerning the life of these primitive tribes. With what anxiety they isolate themselves from their neighbours! A striking proof of this is offered by the practice of secret barter, in which primitive man sets out from the forest, if possible by night, and deposits his captured game at a place which custom has set apart for this purpose, returning the next night to take whatever the more civilized neighbouring tribes have left in exchange-iron implements and weapons, material for clothing, and especially articles of adornment. The participants in this barter do not see each other, much less speak with each other. But where such seclusion exists, how is it possible for a strange language to penetrate? This problem appears almost insoluble. Nevertheless, a solution that appears at least probable was suggested by the investigations of Kern, an able Dutch scholar. His studies were based mainly on the development of the various Malayan idioms. A remarkable exception to the rule that primitive tribes have adopted the language of their more civilized neighbours came to light in the case of the Negritos of the Philippines. Their neighbours, as well as those of the tribes of the interior of Malacca, belong to that much-migrating race, the Malayans. If we compare the Negrito word-formations that have been collected during the past forty years with the vocabulary of the neighbouring Malayans, it is evident that all the words are entirely different, or at least seem to be so with few exceptions. When, however, Kern traced the probable development of these words, and compared them, not with the present-day usage of the Malays but with older stages 
of their language, he found that the latter invariably, contained the counterparts of the Negrito words. Thus, while these Negritos have remained untouched by the present-day Malays, who probably entered the country at least several centuries ago, they have evidently derived their language from a Malayan influx that occurred much earlier still. To this may be added the demonstrable fact, gleaned from another source, that from very early times the Malayan tribes undertook migrations at widely separated intervals. Traversing the seas in their unsteady boats, they at various times peopled such islands, in particular, as were not too remote from the mainland. Now the testimony of language, to which we have referred, demonstrates that there were at least two such migrations to the Philippines, and that they occurred at widely different times. The original Malayan dialect, which has now become extinct or unknown tho the modern Malays, was assimilated by the Negrito peoples, who probably occupied this territory before the arrival of any of the Malays. But this leads to a further inference. If the language was appropriated in prehistoric times and if the conditions of the present are such as would make this scarcely possible, we must conclude that the interrelations of the immigrants and the original inhabitants were formerly not the same as those that now prevail. And, as a matter of fact, this seems altogether probable, if we call to mind the descriptions which modern travellers give of their experiences among: these primitive peoples. The traits of character that particularly distinguish them are fear and hatred of their more civilized neighbours ; corresponding to this, is the contempt felt by the latter, because of their higher culture, for the more primitive peoples. The only thing that restrains the immigrant people from waging a war of extermination against the original inhabitants is the fear of the poisoned arrow which the Negrito directs against his enemy from behind an ambush. In view of these facts it is not difficult to understand the almost universal isolation of primitive man at the present time. On the other hand, 
travellers who have been admitted into the lives of the primitive tribes of Malacca and Ceylon and have sought to gain their friendship, unanimously assure us that, whenever a person has once succeeded in coming close to these people and in overcoming their distrust, he finds their outstanding characteristics to be good nature and readiness to render assistance. We may, therefore, be justified in assuming: that the seclusion of primitive man was not an original condition, but that it grew up, here and elsewhere, as a result of the war of extermination to which he was exposed on the part of the races attempting to crowd him out of a large part of his territory. Before this state of affairs arose, barter also could scarcely have, possessed that character of secrecy which only fear and hatred could give it. In all probability the intercourse which necessarily took place in early times between the older inhabitants and the newer peoples, led to a competition of languages in which the poorer and less developed language of primitive man inevitably succumbed. Nevertheless, the primitive language may also have quietly exercised a reciprocal influence upon the more advanced language. An observation that we cannot escape, even on far higher stages of linguistic development, is the fact that, in such a struggle between a superior minority and a less civilized majority, the former determines the main stock of words, and even, under favourable conditions, the grammatical form, whereas the latter exercises a decisive influence on pronunciation. That a similar process occurred in connection with the displacement of primitive languages, the language of the Bushmen offers proof. This is essentially a Hottentot dialect, even though it is characterized by certain traits of primitive thought. The Hottentots, however, have derived their well-known clacking sounds from the Bushmen, who also gave these sounds to the languages of the Bantu peoples.

But are we deprived of all knowledge concerning the most primitive grammatical forms and concerning the related question of the origin of language, by virtue of the fact that the languages of primitive peoples have, with 
the exception of meagre remnants, apparently been lost? There is a consideration touching the question of primitive forms of thought and language that enables us, in spite of the difficulty suggested, to answer this question in the negative. The development of language does not at all keep pace with that of the other forms of culture. Primitive forms of thought especially, and the corresponding expression which they receive in language, may long persist after external culture is relatively advanced. And thus, among tribes that are in general far beyond the primitive stage, linguistic forms may still be found that are exact counterparts of phenomena that, from a psychological point of view, must be regarded as primitive. As regards this point, it is especially the African languages of the Soudan that offer a typical field for linguistic study. If we analyse the syntax of such a language and the forms of thought which the sentence structure allows us to infer, we gain the impression that it is hardly possible to imagine a form of human thought whose essential characteristics could be more primitive. This is clearly apparent from a consideration of the Ewe language of the peoples of Togo, a German colonial possession. This is a Soudan language, on whose grammar D. Westermann, a German missionary, has given us a valuable treatise. While the Ewe language does not contain all the essential features apparently characteristic of relatively primitive thought, it does exemplify some of them. We are led to this conclusion particularly when we compare it, together with other Soudan languages, with a form of language which, though it arises under highly advanced cultural conditions, may nevertheless be regarded as primitive, since it is actually formed anew before our very eyes. I refer to gesture-language. In this case, it is not sounds, but expressive movements, imitative and pantomimic, that form the means by which man communicates his thoughts to man. Though we may regard gesturelanguage as an original form of language, in so far as we can observe it at the moment of its creation, we must not, of course, forget that the genesis of the forms of 
gesture communication familiar to us belongs to a higher culture whose conditions differ widely from those of primitive thought.

Now, of the various forms of gesture-language, the one that is least subject to change is doubtless the means of communication employed by those who are bereft of hearing, and therefore of speech as well, namely, the deaf and dumb. A similar means of communication through signs and gestures may also be observed among peoples of low culture. Especially when they consist of tribes with markedly different dialects, do such peoples make use of gestures in communicating with one another. Investigations of the spontaneously arising gesture-language of deafmutes date largely from the first half of the nineteenth century. More recent studies have been made of the gestures of the North American Indian tribes, and similar, though less complete, observations have been reported concerning the Australians. In these cases, however, gestures sometimes serve also as a sort of secret language. This is even more true of certain signs that occur among some of the peoples of southern Europe, as, for example, among the Neapolitans. In considering the question before us, such cases must, of course, be excluded, since the motive of communicating ideas may here be entirely displaced by that of keeping them secret; instead of a language that arises spontaneously, we have a means which is, on the whole, consciously elaborated for purposes of mutual understanding. If we disregard these cases, which belong to an entirely different order of facts, and examine the data gathered from widely different parts of the earth and from very diverse conditions of culture, we find a remarkable agreement. In certain details, of course, there are differences. The ideas of the Indian are not in all respects like those of the civilized European or those of the Australian. Nevertheless, the gestures that refer to specific concrete objects are frequently so similar that many of the signs employed by the gesture-language of the deafmutes of Europe may be found among the Dakota Indians. 
Could we transfer one of these deaf and dumb persons to this group of Indians, he would probably have no difficulty at all in communicating with them. In more recent times the opportunity of investigating spontaneous gesture-language has not been so great, because deaf-mutes have become more and more educated to the use of verbal language. The principal material for the study of the natural gesturelanguage of deaf-mutes is, therefore, still to be found in the older observations of Schmalz (1838, 2nd ed. I848), a German teacher of people thus afflicted, and in the somewhat later reports of an Englishman by the name of Scott (1870).

What, now, do these observations teach us concerning the origin of gesture-language, and therefore probably also concerning the factors underlying the origin of language in general? According to the popular notion, a so-called impulse for communication or, perhaps, certain intellectual processes, voluntary reflections, and actions, account for the fact that the contents of one's own consciousness come to be communicated to other individuals. If, however, we observe gesture-language in its origin, we obtain an entirely different view. This mode of communication is not the result of intellectual reflections or conscious purposes, but of emotion and the involuntary expressive movements that accompany emotion. Indeed, it is simply a natural development of those expressive movements of human beings that also occur where the intention of communicating is obviously absent. As is well known, it is not only emotions that are reflected in one's movements, particularly in mimetic movements of the face, but also ideas. Whenever ideas strongly tinged with feeling enter into the course of emotions, the direct mimetic expressions of the face are supplemented by movements of the arms and hands. The angry man gesticulates with movements which clearly indicate the impulse to attack that is inherent in anger. Or, when we have an ideational process of an emotional nature, and ideas arise referring to objects that are present to us, we point to the objects, even though there be no intention of communicating the ideas. Directions in space, likewise, as well as past time and futurity, 
are involuntarily expressed by means of backward and forward pointing movements; ' large' and 'small' are expressed by the raising and lowering of the hands. When further movements are added, indicating the form of an object by describing its image in the air with the hands, all the elements of a gesture-language are complete. What is lacking is "only that the emotionally coloured idea be not a mere expression of one's own emotion, but that it evoke the same emotion and, through this, the same idea, in the minds of others. Under the influence of the emotion aroused within them, those addressed must then reply with the same, or slightly different, expressive movements. When this occurs, there is developed a common thinking in which impulsive movements are more and more displaced by voluntary actions, and ideational contents, together with the corresponding gestures, enter into the foreground of attention. By virtue of this ideational content, movements expressive of emotions come to be expressions of ideas; the communication of an individual's experiences to others results in an exchange of thought-that is, in language. This development, however, is influenced by that of all the other psychical functions, and especially by the transition of emotional and impulsive movements into voluntary actions.

Of what nature, now, is the content of such a gesturelanguage as arises independently within a community, and which may, in so far, be regarded as primitive? To this we may briefly reply that all elements of this language are perceptible to the senses, and therefore immediately intelligible. Hence it is that deaf-mutes, though of different nationalities, can make themselves understood without difficulty, even upon meeting for the first time. This intelligibility of gesture-language, however, rests upon the fact that the signs it employs-or, translated into the terminology of spoken language, its words-are direct representations of the objects, the qualities, or the events referred to. Whenever the object discussed is present, the gesture of pointing with the hand and finger is itself the clearest way of designating the object. Thus, for instance, 'I' and 'you' are expressed 
by the speaker's pointing to himself or to the other person. This suggests a similar movement to designate a "third person' who is not present. The sign in this case is a backward movement of the finger. Whenever the objects of conversation are present in the field of vision, the dumb person, as a rule, dispenses with every other form of representation but that of merely pointing to them.

Since the objects under discussion are, on the whole, only rarely present, there is a second and important class of gestures, which, for the sake of brevity, we may call graphic. The deaf-mute, as also the Indian and the Australian, represents an absent object by pictures outlined in the air. What he thus sketches in only very general outlines is intelligible to one practised in gesture-language. Moreover, there is a marked tendency for such gestures to become permanent within a particular social group. For the word ' house,' the outlines of roof and walls are drawn ; the idea of walking is communicated by imitating the movements of walking with the index and middle fingers of the right hand upon the left arm, which is held out horizontally; the idea of striking. is represented by causing the hand to go through the movements of striking. Not infrequently, however, several signs must be combined to make a gesture intelligible. In the German and English deaf and dumb language, the word 'garden,' for example, is expressed by first describing a circle with the index finger to indicate a place, and by then lifting the thumb and the index finger to the nose as the gesture for smelling. ' Garden,' thus, is, as it were, a place where there are flowers to smell. The idea 'teacher' cannot, of course, be directly represented or pictured; it is too complicated for a language of representation. The deaf and dumb person, therefore, is likely to proceed by first making the gesture for man. For this purpose, he singles out an incidental characteristic, his gesture being that of lifting his hat. Since women do not remove their hats in greeting, this gesture is highly typical. The distinctive sign for woman consists in laying the hands upon the breast. Now, in order to communicate 
the idea of 'male teacher', the hat is first lifted as the above gesture for man, and then the index finger is raised. This is done either because pupils in school raise the index finger to indicate their knowledge of a certain thing or, perhaps, because the teacher occasionally raises his finger when he wishes to command attention or to threaten punishment.

Pointing and graphic gestures thus represent the two means which gesture-language employs. Within the second of these classes of gestures, however, we may distinguish a small sub-group that may be called significant; in this case, the object is not represented by means of a direct picture, but by incidental characteristics-man, for example, is expressed by lifting the hat. The signs are all directly perceptible. The most important characteristic of gesturelanguage, as well as the most distinctive feature of an original language, is the fact that there is no trace of abstract concepts, there being merely perceptual representations. And yet some of these representations-and this is a proof of how insistently human thought, even in its beginnings, presses on to the formation of concepts-have acquired a symbolical meaning by virtue of which they become sensuous means, in a certain sense, of expressing concepts which in themselves are not of a perceptual nature. We may here mention only one such gesture, noteworthy because it occurs independently in the language of the European deaf and dumb and in that of the Dakota Indians. 'Truth' is represented by moving the index finger directly forward from the lips, while 'lie' is indicated by a movement towards the right or left. The former is thus held to be a straight speech and the latter a crooked speech, transcriptions which also occur, as poetical expressions, in spoken language. On the whole, however, such symbolical signs are rare if the natural gesture-language has not been artificially reconstructed; moreover, they always remain perceptual in character.

Corresponding to this feature is also another characteristic which all natural gesture-languages will be found to possess. In vain we search them for the grammatical 
categories either of our own or of other spoken languagesnone may be found. No distinction is made between noun, adjective, or verb ; none between nominative, dative, accusative, etc. Every representation retains its representative character, and that to which it refers may exemplify any of the grammatical categories known to us. For example, the gesture for walking may denote either the act of walking or the course or path; that for striking, either the verb ' to strike' or the noun 'blow.' Thus, in this respect also, gesture-language is restricted to perceptual signs expressing ideas capable of perceptual representation. The same is true, finally, of the sequence in which the ideas of the speaker are arranged, or, briefly, of the syntax of gesture-language. In various ways, depending on fixed usages of language, our syntax, as is well known, permits us to separate words that, as regards meaning, belong together, or, conversely, to bring together words that have no immediate relation. Gesture-language obeys but one law. Every single sign must be intelligible either in itself or through the one preceding it. It follows from this that if, for example, an object and one of its qualities are bath to be designated, the quality must not be expressed first, since, apart from the object, it would be unmeaning ; its designation, therefore, regularly occurs after that of the object to which it belongs. Whereas, for example, we say 'a good man,' gesture-language says ' man good.' Similarly, in the case of verb and object, the object generally precedes. When, however, the action expressed by the verb is thought of as more closely related to the subject, the converse order may occur and the verb may directly, follow. the subject. How, then, does gesture-language reproduce the sentence "The angry teacher struck the child'? The signs for teacher and for striking have already been described; 'angry' is expressed mimetically by wrinkling: the forehead; 'child' by rocking the left forearm supported by the right. Thus, the above sentence is translated into gesture-language in the following manner: First, there are the two signs for teacher, lifting the hat and raising 
the finger; then follows the mimetic gesture for anger, succeeded by a rocking of the arm to signify child, and, finally, by the motion of striking. If we indicate the subject of the sentence by $\mathrm{S}$, the attribute by $\mathrm{A}$, the object by $\mathrm{O}$, and the verb by $\mathrm{V}$, the sequence in our language is ASVO ; in gesture-language it is SAOV, 'teacher angry child strikes,' or, in exceptional cases, SAVO. Gesturelanguage thus reverses the order of sequence in the two pairs of words. A construction such as 'es schlug das Kind der Lehrer' (VOS), always possible in spoken language and occurring not infrequently (for example, in Latin), would be absolutely impossible in gesture-language.

If, then, gesture-language affords us certain psychological conclusions regarding the nature of a primitive language, it is of particular interest, from this point of view, to compare its characteristics with the corresponding traits of the most primitive spoken languages. As already stated, the so-called Soudan languages typify those that bear all the marks of relatively primitive thought. These languages of Central Africa obviously represent a much more primitive stage of development than do those of the Bantu peoples of the south or even those of the Hamitic peoples of the north. The language of the Hottentots is related to that of these Hamitic peoples. It is, in fact, because of this relationship, and also because of characteristics divergent from the negro type, that the Hottentots are regarded as a race that immigrated from the north and underwent changes by mixture with native peoples. If, now, we compare one of the Soudan languages, the Ewe, for example, with gesture-language, one difference will at once be apparent. The words of this relatively primitive spoken language do not possess the qualities of perceptibility and immediate intelligibility that characterize each particular gesture-sign. This is readily explicable as a result of processes of phonetic change, which are never absent, as well as of the assimilation of foreign elements and of the replacement of words by conceptual symbols that are accidental and independent of the sound. These changes occur in the 
history of every language. Every spoken language is the outcome of recondite processes whose beginnings are no longer traceable. And yet the Soudan languages, particularly, have preserved characteristics that show much more intimate connections between sound and meaning than our cultural languages possess. The very fact is noteworthy that certain gradations or even antitheses of thought are regularly expressed by gradations or antitheses of sound whose feeling tone plainly corresponds to the relation of the ideas. While our words 'large' and 'small,' 'here' and 'there,' show no correspondence between the character of the sound and the meaning, the case is entirely different with the equivalent expressions in the Ewe language. In this language large and small objects are designated by the same word. In the one case, however, the word is uttered in a deep tone, while in the other a high tone is used. Or, in the case of indicative signs, the deep tone signifies greater remoteness, the high tone, proximity. Indeed, in some Sudan languages three degrees of remoteness or of size are thus distinguished. 'Yonder in the distance' is expressed by a very deep tone; 'yonder in the middle distance,' by a medium tone ; and 'here,' by the highest tone. Occasionally, differences of quality are similarly distinguished by differences of tone, as, for example, 'sweet' by a high tone, 'bitter' by a deep tone, 'to be acted upon' (that is, our passive) by a deep tone, and activity (or our active) by a high tone. This accounts for a phenomenon prevalent in other languages remote from those of the Soudan. In Semitic and Hamitic languages, the letter ' $U$,' particularly, has the force of a passive when occurring either as a suffix to the root of a word or in the middle of the word itself. For example, in the Hebrew forms of the so-called 'Pual' and 'Piel,' as well as 'Hophal' and 'Hiphil,' the first of each pair is passive, and the second, active in meaning. It was frequently supposed that this was accidental, or was due to linguistic causes of phonetio change other than the above. But when we meet the same variations of sound and meaning in other radically different 
languages, we must stop to ask ourselves whether this is not the result of a psychological relationship which, though generally lost in the later development of language, here still survives in occasional traces. In fact, when we recall the way in which we relate stories to children, we at once notice that precisely the same phenomenon recurs in childlanguage - a language, of course, first created, as a rule, by adults. This connection of sound and meaning is clearly due to the unconscious desire that the sound shall impart to the child not merely the meaning of the idea, but also its feeling-tone. In describing giants and monsters, she who relates fairy-tales to the child deepens her tone; when fairies, elves, and dwarfs appear in the narrative, she raises her voice. If sorrow and pain enter, the tone is deepened; with joyous emotions, high tones are employed. In view of these facts, we might say that this direct correlation of expression and meaning, observed in that most primitive of all languages, gesture-language, has disappeared even from the relatively primitive spoken languages; nevertheless, the latter have retained traces of it in greater abundance than have the cultural languages. In the cultural languages they recur, if at all, only in the onomatopoetic word-formations of later origin. We may recall such words as sausen (soughing), brummen (growling), knistern (crackling), etc.

The question still remains how the other characteristics of gesture-language, particularly the absence of grammatical categories and a syntax which follows the principle of immediate and perceptual intelligibility, compare with the corresponding characteristics of the relatively primitive spoken languages. These characteristics, indeed, are of incomparably greater importance than the relations of sound and meaning. The latter are more strongly exposed to external, transforming influences. Word-formations, however, and the position of the words within the sentence, mirror the forms of thought itself; whenever the thought undergoes vital changes, the latter inevitably find expression in the grammatical categories of the language, and in the laws of syntax which it follows. 


\section{The Thinking of Primitive MaN.}

From the point of view just developed, the investigation of the grammatical forms of primitive language is of particular importance for the psychology of primitive man. True, as has already been remarked, the languages of the most primitive tribes have not been preserved to us in their original form. And yet it is in this very realm of grammatical forms, far more even than in that of sound pictures and onomatopoetic words, that the Soudan languages possess characteristics which mark them as the expression of processes of thought that have remained on a relatively primitive level. This is indicated primarily by the fact that these languages lack what we would call grammatical categories. As regards this point, Westermann's grammar of the Ewe language is in entire agreement with the much earlier results which Steinthal reached in his investigation of the Manda language, which is also of the Soudan region. These languages consist of monosyllabic words which follow one another in direct succession without any intermediate inflectional elements to modify their meaning. Philologists usually call such languages ' root-languages,' because a sound complex that carries the essential meaning of a word, apart from all modifying elements, is called by their science a verbal root. In the Latin word fero, fer, meaning " to bear,' is the root from which all modifications of the verb ferre (to carry) are formed by means of suffixal elements. If, therefore, a language consists of sound complexes having the nature of roots, it is called a root-language. As a matter of fact, however, the languages under discussion consist purely of detached, monosyllabic words; the conception 'root,' which itself represents the product of a grammatical analysis of our flectional languages, may only improperly be applied to them. Such a language is composed of detached monosyllabic words, each of which has a meaning, yet none of which falls under any particular grammatical category. One and the same monosyllabic word may denote an object, an act, or a quality, just as in gesture-language 
the gesture of striking may denote the verb 'to strike' and also the noun 'blow.' From this it is evident to what extent the expressions 'root' and 'root-language' carry over into this primitive language a grammatical abstraction which is entirely inappropriate in case they suggest the image of a root. This image originated among grammarians at a time when the view was current that, just as the stem and branches of a plant grow out of its root, so also in the development of a language does a word always arise out of a group of either simple or composite sounds that embody the main idea. But the component parts of a language are certainly not roots in this sense; every simple monosyllabic word combines with others, and from this combination there result, in part, modifications in meaning, and, in part, sentences. Language, thus, does not develop by sprouting and growing, but by agglomeration and agglutination. Now, the Soudan languages are characterized by the fact that they possess very few such fixed combinations in which the individual component parts have lost their independence. In this respect, accordingly, they resemble gesture-language. The latter also is unfamiliar with grammatical categories in so far as these apply to the words themselves; the very same signs denote objects, actions, and qualities-indeed, generally even that for which in our language we employ, particles. This agreement with gesture-language is brought home to us most strikingly if we consider the words which the primitive spoken languages employ for newly formed ideas-such, for instance, as refer to previously unknown objects of culture. Here it appears that the speaker always forms the new conception by combining into a series those ideas with which he is more familiar. When schools were introduced into Togo, for example, and a word for 'slatepencil' became necessary, the Togo negroes called it 'stone scratch something '-that is, a stone with which we scratch something. Similarly 'kitchen,' an arrangement unknown to these tribes, was referred to as 'place cook something'; 'nail,' as 'iron head broad.' The single word always 
stands for a sensibly perceptual object, and the new conception is formed, not, as epistemologists commonly suppose, by means of a comparison of various objects, but by arranging in sequence those perceptual ideas whose combined characteristics constitute the conception. The same is true with regard to the expressions for such thought relations as are variously indicated in our language by the inflections of substantive, adjective, and verb. The Soudan languages make no unambiguous distinction between noun and verb. Much less are the cases of the substantive, or the moods and tenses of the verb, distinguished; to express these distinctions, separate words are always used. Thus, 'the house of the king' is rendered as 'house belong king.' The conception of case is here represented by an independent perception that crowds in between the two ideas which it couples together. The other cases are, as a rule, not expressed at all, but are implied in the connection. Similarly, verbs possess no future tense to denote future time. Here also a separate word is introduced, one that may be rendered by 'come.' 'I go come' means 'I shall go'; or, to mention the preterit, 'I go earlier' means 'I went.' Past time, however, may also be expressed by the immediate repetition of the word, a sensibly perceptual sign, as it were, that the action is completed. When the Togo negro says 'I eat,' this means 'I am on the point of eating' ; when he says 'I eat eat,' it means 'I have eaten.'

But ideas of such acts and conditions as are in themselves of a perceptual nature are also occasionally expressed by combining several elements which are obtained by discriminating the separate parts of a perceptual image. The idea 'to bring,' for example, is expressed by the Togo negro as 'take, go, give.' In bringing something to some one, one must first take it, then go to him and give it to him. It therefore happens that the word 'go,' in particular, is frequently added even where we find no necessity for especially emphasizing the act of going. Thus, the Togo negro would very probably express the sentence, 
'The angry teacher strikes the child,' in the following way: 'Man-school-angry-go-strike-child.' This is the succession that directly presents itself to one who thinks in pictures, and it therefore finds expression in language. Whenever conceptions require a considerable number of images in order to be made picturabie, combinations that are equivalent to entire sentences may result in a similar manner. Thus, the Togo negro expresses the concept 'west' by the words 'sun-sit-place'-that is, the place where the sun sits down. $\mathrm{He}$ thinks of the sun as a personal being who, after completing his journey, here takes a seat.

These illustrations may suffice to indicate the simplicity and at the same time the complexity of such a language. It is simple, in that it lacks almost all grammatical distinctions ; it is complicated, because, in its constant reliance on sensibly perceptual images, it analyses our concepts into numerous elements. This is true not merely of abstract concepts, which these languages, as a rule, do not possess, but even of concrete empirical concepts. We need only refer to the verb 'to bring,' reduced to the form of three verbs, or the concept 'west,' for whose expression there is required not only the sun and the location which we must give it but also its act of seating itself. In all of these traits, then, primitive language is absolutely at one with gesture-language.

The same is true of the syntax of the two kinds of language. This also is no more irregular and accidental in the Soudan language than it is in gesture-language. As a rule, indeed, it is stricter than the syntax of our languages, for in the latter inflection makes possible a certain variation in the arrangement of words within a sentence according to the particular shade of meaning desired. In primitive language, the arrangement is much more uniform, being governed absolutely and alone by the same law as prevails in gesture-language-namely, the arrangement of words in their perceptual order. Without exception, therefore, object precedes attribute, and substantive, adjective. Less constant, however, is the relation of verb and object, in the 
Ewe language; the verb generally precedes, but the object may come first; the verb, however, always follows the subject whose action it expresses. This perceptual character of primitive language appears most strikingly when we translate any thought that is at all complicated from a primitive language into our own, first in its general meaning, and then word for word. Take an illustration from the language of the Bushmen. The meaning would be substantially this: "The Bushman was at first received kindly by the white man in order that he might be brought to herd his sheep ; then the white man maltreated the Bushman; the latter ran away, whereupon the white man took another Bushman, who suffered the same experience.' The language of the Bushmen expresses this in the following way: 'Bushman-there-go, here-run-to-white man, white man-give-tobacco, Bushman-go-smoke, go-fill-tobacco-pouch, white man-give-meat-Bushman, Bushman-go-eat-meat, stand-up-go-home, go happily, go-sit-down, herd-sheepwhite man, white man-go-strike Bushman, Bushman-cryloud-pain, Bushman-go-run-away-white man, white man-runafter-Bushman, Bushman-then-another, this one-herd-sheep, Bushman-all-gone.' In this complaint of the man of nature against his oppressor, everything is concrete, perceptual. He does not say, The Bushman was at first kindly taken up by the white man, but, The white man gives him tobacco, he fills his pouch and smokes; the white man gives him meat, he eats this and is happy, etc. He does not say, The white man maltreats the Bushman, but, He strikes him, the Bushman cries with pain, etc. What we express in relatively abstract concepts is entirely reduced by him to separate perceptual images. His thought always attaches to individual objects. Moreover, just as primitive language has no specific means for expressing a verb, so also are change and action overshadowed in primitive thought by the concrete image. The thinking itself, therefore, may be called cuncrete. Primitive man sees the image with its separate parts; and, as he sees it, so he reproduces it in his language. It is for this very reason that he is unfamiliar 
with differences of grammatical categories and with abstract concepts. Sequence is still governed entirely by the pure association of ideas, whose order is determined by perception and by the recollection of that which has been experienced. The above narrative of the Bushman expresses no unitary thought, but image follows upon image in the order in which these appear to consciousness. Thus, the thinking of primitive man is almost exclusively associative. Of the more perfect form of combining concepts, the apperceptive, which unites the thoughts into a systematic whole, there are as yet only traces, such as occur in the combination of the separate memory images.

Many analogues to the formal characteristics of primitive thought revealed in these linguistic phenomena may be met in child-language. There is a wide divergence, however, with respect to the very element which has already disappeared, with the exception of slight traces, from the language of primitive peoples. I refer to the close correlation of sound and meaning. As regards this feature, child-language is much more similar to gesture-language than is possible in the case of forms of speech that have undergone a long historical development. For, childlanguage, like gesture-language, is, in a certain sense, continually being created anew. Of course, it is not created, as is sometimes supposed, by the children themselves. It is a conventionalized language of the mothers and nurses who converse with the child, supplemented, in part, by the child's associates along the lines of these traditional models. The sound-complexes signifying animals, 'bow-wow' for the dog, 'hott-hott' for the horse, 'tuk-tuk' for the chicken, etc., as also 'papa' and 'mamma' for father and mother, are sounds that are in some way fitted to the meaning and at the same time resemble so far as possible the babbling sounds of the child. But this entire process is instituted by the child's associates, and is at most supplemented by the child himself to the extent of a few incidental elements. For this reason, child-language has relatively little to teach us concerning the development of speaking and thinking; 
those psychologists and teachers who believe that it affords an important source of information concerning the origin of thought are in error. Such information can be gained only from those modes of expressing thought which, like gesture-language, are originated anew by the speaker and are not externally derived, or from those which, like the spoken languages of primitive peoples, have retained, in their essential characteristics, primitive modes of thinking. Even in these cases it is only the forms of thought that are thus discoverable. The content, as is implied by the formal characteristics themselves, is, of course, also of a sense-perceptual, not of a conceptual, nature. And yet the particular character or quality of this content is not inherent in the forms of the language as such. To gain a knowledge of its nature we must examine the specific ideas themselves and the associated feelings and emotions.

Thus, then, the further question arises: Wherein consists the content of primitive thought? Two sorts of ideas may be distinguished. The one comprises that stock of ideas which is supplied to consciousness by the direct perceptions of daily life-ideas such as go, stand, lie, rest, etc., together with animal, tree (particularly in the form of individual animals and trees), man, woman, child, I, thou, you, and many others. These are objects of everyday perception that are familiar to all, even to the primitive mind. But there is also a second class of ideas. These do not represent things of immediate perception; briefly expressed, they originate in feeling, in emotional processes which are projected outward into the environment. This is an important and particularly characteristic group of primitive ideas. Included within it are all references to that which is not directly amenable to perception but, transcending this, is really supersensuous, even though appearing in the form of selisible ideas. This world of imagination, projected from man's own emotional life into external phenomena, is what we mean by mythological thinking. The things and processes given to perception are supplemented by other realities that are of a non-perceptible 
nature and therefore belong to an invisible realm back of the visible world. These are the elements, furthermore, which very early find expression in the art of primitive man.

\section{Earliest Beliefs in Magic and Demons.}

In entering upon a consideration of the development of primitive myths, we are at once confronted by the old question disputed by mythologists, ethnologists, and students of religion, Where and when did religion originate? For is not religion always concerned with the supernatural? Now, in certain cases, even primitive man supplements the sensuous world in which he lives and whose impressions he has not so much as elaborated into abstract concepts, with supersensuous elements, though he himself, of course, is unaware of their supersensuous character. The question, therefore, lies near at hand: Is religion already present at this stage, or is there at most a potentiality of religion, the germ of its future development? If the latter should be true, where, then, does religion begin? Now, our interest in the history of myth-formation derives largely from the very fact that the problem is intimately bound up with that of the origin of religion. Merely in itself the origin of the myth might have relatively little interest for us. The question, however, as to how religion arose acquires its great importance through its connection with the two further questions as to whether or not religion is a necessary constituent of human consciousness and whether it is an original possession or is the result of certain preconditions of mythological thought.

It is interesting to follow this ancient dispute, particularly its course during the last few decades. In I 880, Roskoff wrote a book entitled "The Religion of the Most Primitive Nature-Peoples." In this work he assembled all the available facts, and came to the conclusion that no peoples exist who have not some form of religion. About ten years ago, however, the two Sarasins, students of Ceylon and of the primitive Veddah tribes, summed up their conclusions in the proposition: The Veddahs have no religion. 
If, however, we compare Roskoff's facts concerning primitive peoples with those reported by the Sarasins concerning the belief of the Veddahs in demons and magic, it appears that the facts mentioned by these investigators are essentially the same. What the former calls religion, the latter call belief in magic; but in neither case is there a statement as to what is really meant by religion. Now, we cannot, of course, come to an understanding with reference to the presence or absence of anything until we are agreed as to what the thing itself really is. Hence, the question under dispute is raised prematurely at the present stage of our discussion; it can be answered only after we have examined more of the steps in the development of myth and of the preconditions of the religion of later times. We shall therefore recur to this point in our third chapter, after we have become acquainted with such religions as may indubitably lay claim to the name. Postponing the question for the present, we will designate the various phenomena that must be discussed at this point by the specific names attaching to them on the basis of their peculiar characteristics. In this sense, there is no doubt that we may speak of ideas of magic and of demons even in the case of primitive peoples; it is generally conceded that such ideas are universally entertained at this stage of culture. But the further question at once arises as to the source of this belief in magic and in demons, and as to the influences by which it is sustained. Now, in respect to this point two views prevail, even among the ethnologists who have made an intensive study of primitive peoples. The one view may briefly be called that of nature-mythology. It assumes that even far back under early conditions the phenomena of the heavens were the objects that peculiarly fascinated the thought of man and elevated it above its immediate sensible environment. All mythology, therefore, is supposed originally to have been mythology of nature, particularly of the heavens. Doubtless this would already involve a religious element, or, at least, a religious tendency. The second view carries us even farther in the 
same direction. It holds that the ideas of primitive man, so far as they deal with the supersensuous, are simpler than those of the more highly developed peoples. Just for this reason, however, it regards these ideas as more perfect and as approaching more nearly the beliefs of the higher religions. As a matter of fact, if we compare, let us say, the Semangs and the Senoi, or the Veddahs, with the natives of Australia, we find a very great difference as regards this point. Even the mythology of the Australians is undoubtedly much more complex than that of these peoples of nature, and the farther we trace this myth development the greater the complexity becomes. That which is simple, however, is supposed to be also the higher and the more exalted, just as it is the more primitive. The beginning is supposed to anticipate the end, as a revelation not yet distorted by human error. For, the highest form of religion is not a mythology including a multitude of gods, but the belief in one God-that is, monotheism. It was believed, therefore, that the very discovery of primitive man offered new support for this view. This theory, however, is bound up with an important anthropological consideration-the question concerning the place of the so-called Pygmies in the history of human development. It was on the basis of their physical characteristics that these dwarf peoples of Africa and Asia, of whom it is only in comparatively recent times that we have gained any considerable knowledge, were first declared by Julius Kollman to be the childhood peoples of humanity, who everywhere preceded the races of larger stature. Such childhood characteristics, indeed, are revealed not only in their small stature but in other traits as well. Schweinfurth observed that the entire skin of the Pygmies of Central Africa is covered with fine, downy hair, much as is that of the newly born child. It is by means of these downy hairs that the Monbuttu negro of that region distinguishes the Pygmy from a youth of his own tribe. The Negrito is primitive also in that his dermal glands are abnormally active, causing a bodily odour which is far 
greater than that of the negro, and which, just as in the case of some animals, increases noticeably under the stress of emotion. If, in addition to these physical characteristics, we consider the low cultural level of all these dwarf peoples, the hypothesis that the Pygmies are a primitive people does not, indeed, seem altogether strange. Starting with this hypothesis, therefore, William Schmidt, in his work, "The Place of the Pygmies in the Development of Mankind" (I910), attempted to prove the proposition that the Pygmies are the childhood peoples of humanity in their mental culture no less than in their physical development. This being their nature, they are, of course, limited intellectually ; morally, however, they are in a state of innocence, as is demonstrated among other things by the pure monogamy prevailing among them, as well as by their highest possession, their monotheistic belief.

Now, the supposition of moral innocence rests essentially on the twofold assumption of the identity of primitive man with the Pygmy and of the legitimacy of holding that what has been observed of one tribe of Pygmies is true of "the primitive condition generally. But this identity of primitive man with the Pygmy cannot be maintained. The most typical traits of primitive mental culture are doubtless to be found among the Veddahs of Ceylon. The Veddahs, however, are not really Pygmies, but are of large stature. Moreover, there are primitive people who are so far from being Pygmies that they belong rather to the tall races. We might cite the extinct Tasmanians, whose culture was probably a stage lower than that of the modern Australians. In most respects, many of the tribes of Central Australia exhibit traits of primitive culture, even though their social organization is of a far more complicated nature. Finally, all the peoples whose remains have been found in the oldest diluvial deposits of Europe belong to the tall races. On the other hand, there are peoples of small stature, the Chinese and the Japanese, who must be counted in the first ranks of cultured peoples. Thus, nental culture certainly cannot be measured in terms 
of physical size but only in terms of itself. Mental values can never be determined except by mental characteristics. It is true that W. Schmidt has sought to support his theory regarding the Pygmies by reference to the reports of $\mathrm{E}$. $\mathrm{H}$. Man, a reliable English observer. According to these reports, the Andamanese, one of these dwarf peoples, possess some remarkable legends that are doubtless indicative of monotheistic ideas. Since the Andamans are a group of islands in the Sea of Bengal and the inhabitants are therefore separated from other peoples by an expanse of sea, Schmidt regarded as justifiable the assumption that these legends were autochthonous; since, moreover, the legends centre about the belief in a supreme god, he contended that we here finally had proof of the theory of an original monotheism. The main outlines of the Andamanese legends as given by E. H. Man are as follows: The supreme god, Puluga, first created man and subsequently (though with regard to this there are various versions) he created woman. She was either created directly, as was man, or man himself created her out of a piece of wood, possibly a reminiscence of Adam's rib. Then God gave man laws forbidding theft, murder, adultery, etc., forbidding him, furthermore, to eat of the fruits of the first rainy season. But man did not keep the Divine commandments. The Lord therefore sent a universal flood, in which perished all living things with the exception of two men and two women who happened to be in a boat. In this story, much is naturally distorted, confused, and adapted to the medium into which the legend is transplanted. But that it points to the Biblical accounts of the Creation, Paradise, and the Flood, there cannot, in my opinion, be the slightest doubt. If it is objected that the Andamans are altogether too far separated from the rest of the world by the sea, and also that no missionaries have ever been seen on these islands, our answer would be : Whatever may be the ' when' and the 'how;' the fact that the Biblical tradition at some time did come to the Andamanese is proven by the legend itself. This conclusion is just as incontestable as is the 
inference, for example, that the correspondence of certain South American and Asiatic myths is proof of a transmission. Indeed, the two latter regions are separated by an incomparably wider expanse of sea than that which divides the Andamans from Indo-China and its neighbouring islands. It should also be added that the inhabitants of the Andaman Islands have obviously progressed far beyond the condition in which we find the inland tribes of Malacca, the Veddahs of Ceylon, or the Negritos of the Philippines. They practise the art of making pottery-an art never found among peoples who are properly called primitive; they have a social organization, with chiefs. These phenomena all characterize a fairly advanced culture. When, therefore, we are concerned with the beliefs of peoples who are really primitive, the Andamanese must be left out of consideration. According to the available proofs, however, these people possess a belief neither in one god nor in many. gods. Moreover, even far beyond the most primitive stage, no coherent celestial mythology may be found, such as could possibly. be regarded as an incipient polytheism. No doubt, there are ideas concerning single heavenly phenomena, but these always betray an association with terrestrial objects, particularly with human beings or animals. And, to all appearances, these ideas change with great rapidity. Nowhere have they led to the actual formation of myths. Among the Indians of the Brazilian forests, for example, the sun and moon are called leaves or feather-balls; by several of the Soudan tribes they are conceived as balls that have been thrown to the sky by human beings and have stuck there. Such ideas alternate with others in which the sun and moon are regarded as brothers or as brother and sister, or the sun is said to be chasing the moon-images influenced particularly by the phenomena of the moon's phases. As a matter of fact, this whole field of ideas reveals only one belief that is practically universal, appearing among peoples of nature and recurring even among civilized peoples. Because of the rare occurrence of the phenomenon, 
however, it has never led to a real mythology. I refer to the belief that in an eclipse of the sun, the sun is swallowed by a dark demon. This belief, obviously, is very readily suggested to the primitive imagination; it occurs in Central Africa, in Australia, and in America, and is found even in Indian mythology. Taken by itself, however, the notion is incapable of engendering a myth. It is to be regarded merely as an isolated case to be classed with a more richly developed set of demon-ideas that dominate the daily life of primitive man. At this stage, these ideas are the only elements of an incipient mythology that are clearly discernible and that at the same time exercise an important influence upon life. In so far as the mythology of primitive man gains a permanent foothold and influence, it consists of a belief in magic and demons. There are, however, two motives which engender this belief and give form and colour to the ideas and emotions springing from them. These are death and sickness.

Death I There are doubtless few impressions that have so powerful an effect upon the man of nature; indeed, civilized man as well is still very greatly stirred by the phenomenon of death. Let his companion meet with death, and even the outward actions of a primitive man are significant. The moment a person dies, the immediate impulse of primitive man is to leave him lying where he is and to flee. The dead person is abandoned, and the place where he died continues to be avoided for a long time-if possible, until animals have devoured the corpse. Obviously the emotion of fear is regnant. Its immediate cause is apparently the unusual and fear-inspiring changes which death makes in the appearance of a man. The suspension of movements, the pallor of death, the sudden cessation of breathing-these are phenomena sufficient to cause the most extreme terror. But what is the nature of the ideas that associate themselves with this fearsome impression? The flight from the corpse is evidence that man's fears are primarily for himself. To tarry in the presence of a dead person exposes the living man to the 
danger of being himself overtaken by death. The source of this danger is evidently identical with that which has brought death to the recently deceased person himself. Primitive man cannot think of death except as the sudden departure from the dying person of that which originally brought life. Nevertheless, there is evidently bound up with this conception the further idea that powers of life are still resident in the body; the latter remains firmly associated in the mind of primitive man with the impression of life. Here, then, we have the original source of the contradictory idea of a something that generates life and is therefore independent of the body, while nevertheless being connected with it. So far as we can gain knowledge of the impression which death makes on the mind of primitive man, two disparate motives are indissolubly united. $\mathrm{He}$ regards life as something that, in part, continues in some mysterious manner to dwell within the corpse, and, in part, hovers about, invisible, in its vicinity. For this reason, the dead person becomes to him a demon, an invisible being capable of seizing upon man, of overpowering or killing him, or of bringing sickness upon him. In addition to this primitive idea of demons, we also find the conception of a corporeal soul, meaning by this the belief that the body is the vehicle of life, and that, so long as it has not itself disappeared, it continues to harbour the life within itself. The corporeal soul is here still regarded as a unit which may, by separating itself from the body, become a demon and pass over into another person. No certain traces are as yet to be found of belief in a breath or shadowlike soul. As will appear later, this is a characteristic feature of the transition from primitive to totemic culture. When some investigators report that the soul is occasionally referred to by the Semangs of Malacca as a small bird that soars into the air at the death of a person, it is not improbable that we here have to do either with the Semangs of culture, who have undergone marked changes under Malayan influence, or with the presence of an isolated idea that belongs to a different cultural circle. For in no other 
case are ideas similar to that of the psyche to be found on the level of primitive culture. On the other hand, the burial customs of the Malays and of the mixed races living in the immediate vicinity of the primitive peoples of the Malay Peninsula, already exhibit a striking contrast to the flight of primitive man from the corpse.

The next group of ideas, those arising from the impression made by sickness, particularly by such sicknesses as attack man suddenly, are also restricted to the conception of a corporeal soul. For, one of the most characteristic marks of this conception is that magical, demoniacal powers are believed to issue from the body of the dead person. These powers, however, are not, as occurs in the above case, regarded as embodied in any visible thing-such as the exhalations of the breath or an escaping animal-that separates itself from the person. On the contrary, the demon that leaves the corpse and attacks another person in the form of a fatal sickness, is invisible. He is purely the result of an association between the fear aroused by the occurrence of death and the fright caused by an unexpected attack of sickness. The dead person, therefore, continues to remain the seat of demoniacal powers; these he can repeatedly direct against the living persons who approach him. Primitive man believes that the demon may assume any form whatsoever within the body, and deceitful medicine-men take advantage of this in ostensibly removing the sickness in the form of a piece of wood or of a stone. But it is precisely these ideas that are totally unrelated to that of a psyche and its embodiments. Though the corpse is perhaps the earliest object that suggests sickness-demons, it is in no wise the only one. Indeed, the attack of sickness is in itself sufficient to arouse fear of a demon. Thus, the Semangs and Senoi distinguish a vast number of different sickness-demons. Such ideas of demons, however, as we find among the Malays and the Singhalese, where demons are regarded as counter-agents to sickness-magic and usually take the form of fantastical animal monsters, never occur except at a later cultural stage. Any resemblance of 
these demons to 'soul animals,' which, as we shall find in our next chapter, are always actual animals, is confined to the fact that they have some similarity to animals. Obviously they are creations of the imagination, due to fear and terror. Their only difference from the monsters of similar origin that are projected into the outward world is that they are reduced to proportions which fit the dimensions of the human body.

Closely connected with the magic of sickness is countermagic, an agency by which disease is removed or the attack of sickness-demons warded off. Even primitive man seeks for such modes of relief. Hence, probably, the original formation of a special group of men, which, though not, of course, at the very first a fixed professional class, was nevertheless the precursor of the latter. Among the American Indians, these were the 'medicine-men'; the peoples of northern Asia called them 'shamans'-more generally expressed, they were magicians. The name 'medicine-man,' indeed, is not inappropriate. The medicine-man of the savages is, in truth, the predecessor of the modern physician, and also, in a certain sense, of the modern priest. He not only ministers to the individual whom he restores to health by means of his counter-magic, but he can himself directly practise magic. Since he has power over demons, he can exorcise them from the body; but he can also magically cause them to enter it. Thus, the medicine-man has a twofold calling. He is feared, but he is also valued as a helper in need. His position differs according as the one or the other emotion predominates. He was the first to investigate the effect of herbs on man. He probably discovered the poisons, and, by rendering the arrow poisonous, gained a still higher authority in the cyes of the savage. For the arrow, too, is a means of magic. But he also discovered methods of removing poisons, and thereby transformed poisonous plants into articles of food. His calling, then, is a supremely important one, though also at all times dangerous for the one who practises it. $\mathrm{He}$ is not only exposed to persecution if he fails to accomplish 
what is expected of him or if he is suspected of evil magic, but the magician, when pressed by need, also becomes a deceiver. The deception of the medicine-man, indeed, apparently dates back to the very earliest times. Koch-Grünberg tells us that among the Central Brazilians the medicine-men expel disease by carrying about with them a piece of wood, which they bring forth, after various manipulations, as the alleged seat of the demon. If the suggestion thus given is effective, the patient may, of course, feel himself improved. At any rate, we must not think that the mass of the people is led to lose belief in magic; in most cases, perhaps, the medicine-man himself remains a deceived deceiver.

Nevertheless, on the primitive stage, death and sickness are the main sources of belief in magic and in demons. From this as a centre, the belief radiates far out into all departments of life. The belief in magic, for example, assumes the form of protective magic, of magical defence against demoniacal influences. In this form, it probably determines the original modes of dress, and, more obviously and permanently still, the adornment of the body. In fact, in its beginnings, this adornment was really designed less for decoration than for purposes of magic.

In connection with the external culture of primitive man we have already noted his meagre dress, which frequently consisted merely of a cord of bast about the loins, with leaves suspended from it. What was the origin of this dress? In the tropical regions, where primitive man lives, it was surely not the result of need for protection; nor can we truthfully ascribe it to modesty, as is generally done on the ground that it is the genital parts that are most frequently covered. In estimating the causes, the questions of primary importance are rather those as to where the very first traces of dress appear and of what its most permanent parts consist. The answer to the latter question, however, is to be found not in the apron but in the loin-cord, which is occasionally girt about the hips without any further attempt at dress. Obviously this was not a means of pro- 
tection against storm and cold; nor can modesty be said to have factored in the development of this article, which serves the purposes both of dress and of adornment. But what was its real meaning? An incident from the life of the Veddahs may perhaps furnish the answer to this question. When the Veddah enters into marriage, he binds a cord about the loins of his prospective wife. Obviously this is nothing else than a form of the widely current ' cordmagic,' which plays a not inconsiderable rôle even in presentday superstition. Cord-magic aims to bring about certain results by means of a firmly fastened cord. This cord is not a symbol, but is, as all symbols originally were, a means of magic. When a cord is fastened about a diseased part of the body and then transferred to a tree, it is commonly believed that the sickness is magically transplanted into the tree. If the tree is regarded as representing an enemy, moreover, this act, by a further association, is believed to transfer sickness or death to the enemy through the agency of the tree. The cord-magic of the Veddah is obviously of a simpler nature than this. By means of the cord which he has himself fastened, the Veddah endeavours to secure the faithfulness of his wife. The further parts of primitive dress were developments of the loin-cord, and were worn suspended from it. Coincidentally with this, the original means of adornment make their appearance. Necklaces and bracelets, which have remained favourite articles of feminine adornment even within our present culture, and fillets about the head which, among some of the peoples of nature, are likewise worn chiefly by the women, are further developments of the loin-cord, transferred, as it were, to other parts of the body. And, as the first clothing was attached to the loin-cord, so also were the bracelet and fillet, and particularly the necklace, employed to carry other early means of protective magic, namely, amulets. Gradually the latter also developed into articles of adornment, preferably. worn, even to-day, about the neck.

The assumption that the present purpose of clothing 
is also the end that it originally served led naturally to the theory that when the loin-cord alone is wornas a mere indication, seemingly, of the absence of clothing -this is to be regarded not as an original custom but as the remnant of an earlier dress now serving solely as an adornment. But this supposition is contradicted, in the first place, by the fact that the loin-cord occasionally occurs by itself precisely amidst the most primitive conditions, and, in the second place, by the general development not only of clothing as such but also of certain means of adorning the surface of the body, particularly painting and tattooing. Now, there is a general rule that development proceeds not from the composite to the simple, but, conversely, from the simple to the complex. Moreover, indications of the influence of magical ideas are generally the more marked according as the stages on which the phenomena occur are the earlier. The loin-cord, particularly, is occasionally put to certain magical uses which are scarcely intelligible without reference to the widely prevalent cord-magic. If the binding of a cord of bast of his own weaving about the hips of his prospective wife signifies a sort of marriage ceremony for the Veddah, as it undoubtedly does, this must imply that the cord is a means of magic that binds her for life. Instances have been found of another remarkable and complex custom that substantiates this 'magical' interpretation. A man binds a loin-cord of his own weaving about the woman and she does the same to him-an exchange of magic-working fetters which is a striking anticipation of the exchange of rings still customary with us upon betrothal or marriage. For the exchange of rings, to a certain extent, represents in miniature the exchange of cords practised by primitive man, though there is, of course, this enormous difference that, in the primitive ceremony the binding has a purely magical significance, whereas the later act is merely symbolical. All these phenomena indicate that even the beginnings of clothing involve ideas of magic. Later, of course, a number of other motives also enter in, gradually 
leading to a change in meaning and to a wide departure from the idea originally entertained. Owing to the influence of climatic changes, there arises, in the first place, the need of protection; and the greater this becomes, the more does magic recede. And so, even among primitive tribes, the loin-cord is gradually replaced by the apron proper, which no longer requires a special cord for its support. In the course of this transition into a means of protection, the feeling of modesty more and more enters into the development as a contributing factor. According to a law operative everywhere, even under very different conditions, modesty is always connected with such parts of the body as are required by custom to be kept covered. To do what custom forbids arouses the feeling of shame, particularly in such cases as this, where the violation is so direct and apparent. It is for this reason that the feeling of shame may be aroused by the exposure of very different parts of the body. Thus, the Hottentot woman wears an apron in front and also one behind. The latter covers a cushion of fat over the seat, which is greatly developed in the case of the Hottentot woman and is regarded by these tribes as a particular mark of beauty. To a Hottentot woman it is no worse to have the front apron removed than for some one to take away the rear apron. In the latter case, she seats herself on the ground and cannot be made to get up until the apron has been restored t'o her. When Leonard Schultze was travelling in the Hottentot country of Namaqua Land, he noticed a certain Hottentot custom which strictly prescribes that the legs must be stretched out when one sits down upon the ground-they are not to be bent at the knees. When one of his companions, unfamiliar with the custom, sat differently, a Hottentot struck him on the knees so that they straightened out; when the reason was asked, the answer was that "this manner of sitting brings misfortune." The reply is significant, particularly because it shows how the feeling of shame, which arises at a later period in the development of the original idea of magic and is due to the influence of custom, itself, in turn, reacts asso- 
ciatively on the older magical ideas. The violation of custom is regarded as dangerous, and as a matter requiring, wherever possible, the employment of protective magic. The reasons for guarding against a violation of custom are not merely subjective, but also objective, for guilt is followed by punishment. Thus, there is here an intertwining of motives.

The necklace, bracelet, finger-ring, and sometimes the head-fillet, occur as specific means of magic, in addition to, and in substitution for, the loin-cord. In more restricted localities we find also earrings and nose-rings, the boring through of the lips, and combs to which twigs and leaves are attached. Of these, the necklace has maintained itself far down into later culture, for it is the necklace that gives support to the amulet. The latter is supposed to afford protective magic against all possible dangers; the fingerring, on the other hand, is the favourite vehicle of an active magic, changing things in accordance with the wishes of the owner-that is to say, it is a talisman. Similar in its powers to the necklace, furthermore, is the bracelet-found even in primitive culture-and also the head-fillet, which encircles the forehead and the back part of the head. The Semangs and Senoi of the Malaccan forests are invested with the head-fillet by the medicine-man, who exchanges it for another at particularly important turningpoints of life, such, for example, as the entrance of the youth into manhood, or of the woman upon marriage. The head-fillets that have been removed are preserved in the house of the medicine-man. If the woman is widowed, her former fillet is placed on her head. This signifies the annulment of the magical union that existed throughout the period of marriage. Evidently this magic custom is closely connected with the strict observance of monogamy. These ceremonial changes in dress are accompanied by a similar change in name. On entering the married state a woman changes her name, as does also the youth who passes into manhood. Moreover, this change is not in the least a mere symbol, but represents a magical act. With the change in name, the individual himself becomes another 
person. The name is so closely connected with the person that even the speaking of it may exercise a magical influence upon him.

But the magical ideas radiating from death and sickness come to be associated also with other external objects -objects not attached to the individual's person, as are clothing and adornment. Examples of this are implements, and, in particular, the weapon of primitive man, namely, the bow and arrow. The magical significance has, of course, frequently disappeared from the memory of the natives. The Sarasins saw the Veddahs execute dances about an arrow that had been set upright. On inquiring the reason, they were told: "This was done even by our fathers and grandfathers; why should we not also do it?' A similar answer could be given in the case of many, indeed, of most of these magical ceremonies. Those ceremonies particularly that are in any way complicated are passed down from generation to generation, being scrupulously guarded and occasionally augmented by additional magical elements. It is for this reason that, in the presence of the extraordinarily complicated dances and magical ceremonies of primitive peoples, we sometimes ask in amazement : How could such a wealth of connected ideas possibly arise and become expressed in action? To this it might briefly be replied that they did not arise at all as creations of a single moment. The meaning of the ceremonies has for the most part long been lost to the participants themselves, and was probably unknown even to their ancestors. The general reason for the various acts that are executed according to ancient usage is that they serve a magical purpose. The performers firmly believe that the acts will secure that which is desired, whether it be good fortune or protection from evil, and that the greater the care and exactitude with which the act is performed, the more certainly will the magical purpose be attained. The conditions here are really not essentially different from those that still prevail everywhere in the cult ceremonies of civilized peoples. It is the very fact that the motives are forgotten that leads to the enormous 
complexity of the phenomena. Even in the case of the above-mentioned dance about the arrow, there may have entered a considerable number 'of motives that were later forgotten. Of them all, nothing was eventually remembered except that, to insure the welfare of the individual and that of the group, the act prescribed by custom must be performed at siated times or under particular conditions.

Quite secondary to these numerous irradiations of magical ideas among primitive peoples are the general notions connected with natural phenomena. A cloud may, no doubt, occasionally be regarded as a demon. And, as already stated, an unusual natural phenomenon, such as an eclipse of the sun, is likewise almost everywhere regarded as a demoniacal event. But, on the whole, celestial phenomena play a passing and an exceedingly variable rôle in the beliefs of primitive man. Moreover, while the ideas and the resultant acts engendered by death and sickness are, on the whole, of a uniform character, the fragments of celestial mythology vary in an irregular and self-contradictory manner. For this reason the latter cannot be regarded as having any important significance on the earliest plane of culture. This flatly contradicts a theory, still prevalent in the scientific world, to the effect that all mythological thinking is due to the influence of celestial phenomena, whether it be the moon in its changing phases, or the sun, the thunderstorm, or the clouds. This theory is certainly not valid as regards primitive man. It can be maintained only if we distinguish-as has, indeed, sometimes been done-between two completely disparate realms, a 'higher' mythology, exemplified by the above, and a 'lower' mythology. We shall return to this point later. We are here concerned with the standpoint of naturemythology only in so far as it has exercised a decisive influence on the interpretation of the earliest manifestations of the 'lower' mythology. With respect to the ultimate psychological motives of mythology as a whole, including that of primitive man, the idea is even to-day widely current that mythological thought was from the very beginning a 
naive attempt at an interpretation of the phenomena which man encounters in nature or in his own life. That is to say, all mythology is regarded as a sort of primitive science, or, at any rate, as a precursor of philosophy. This innate need for explanation is then usually associated with an alleged a priori principle of causality inherent in the mind. The mythological view of nature, therefore, is supposed to be nothing but an application-imperfect as yet, to be sureof the causal law to the nexus of phenomena. But if we call to mind the condition of natural man as revealed in his actions, no trace can be found of any need for explanation such as requires the initial employment of the concept of causality. Indeed, as regards the phenomena of daily life and those that surround him on all hands and constantly recur in a uniform manner, primitive man experiences no need at all for explanation. For him everything is as it is just because it has always been so. Just as he dances about an arrow because his father and his grandfather practised this custom in the past, so also does he hold that the sun rises to-day because it rose yesterday. The regularity with which a phenomenon recurs is for him sufficient testimony and explanation of its existence. Only that which arouses his emotion and calls forth particularly fear and terror comes to be an object of magical and demoniacal belief. The primitive level of mythological thought differs from the more developed stage in also another respect. In the former case, the phenomena that are most apt to arouse ideas of magic and of demons are those that concern man himself and that arouse fear and terror. But here again death and sickness are of greatest importance. True, a thunderstorm may occasionally find a place in the nexus of magical ideas, or an eclipse of the sun, or some other natural phenomenon -and this occurs the more readily according as the phenomenon is the more unusual and striking. The regularly recurring features of the primitive myth, however, have their source in the immediate environment and in the facts of personal experience, in fear and terror. Thus, it is not intelligence nor reflection as to the origin and inter- 
connection of phenomena that gives rise to mythological thinking, but emotion; ideas are only the material which the latter elaborates. The idea of a corporeal soul, present in the corpse yet also capable of abandoning it and of becoming a dangerous demon, is a creation of the emotion of fear. The demons who possess the sick man and cause his death, or who depart from him in convalescence, are products of emotion. They are supersensible, as is the soul, because they are born purely of emotion. Nevertheless, they always tend to assume a sensible nature, being imaged either as men, or as external things, such as animals, plants, weapons, and implements. Only in the course of later development are the demons themselves equipped with relatively permanent qualities that differ from the characteristics of the vehicles in which they are regarded as embodied.

Thus, then, we utterly confuse primitive thinking with our own scientific standpoint when we explain it by the need for the interpretation of phenomena. Causality, in our sense of the word, does not exist for primitive man. If we would speak of causality at all on his level of experience, we may say only that he is governed by the causality of magic. This, however, receives its stamp, not from the laws that regulate the connection of ideas, but from the forces of emotion. The mythological causality of emotional magic is no less spasmodic and irregular than the logical causality arising out of the orderly sequence of perceptions and ideas is constant. That the former preceded the latter is, nevertheless, of great importance. For the causality of natural law, as we know it, would hardly have been possible had not magical causality prepared the way for it. Yet the later arose from the earlier just at that moment in which the attention of men ceased to be held by the unusual, the startling, and the fearful, and occupied itself with the orderly, the regular, and commonplace. For this reason the very greatest advance in the investigation of natural laws was made by Galileo, when he took as the object of his research that which was 
the most commonplace, the falling of a body to the earth. Primitive man did not reflect about this phenomenon nor, until a long time afterwards, did civilized man. That a body should fall to the earth when thrown upwards 'is selfevident' because it is thus that bodies have always acted. An echo of this primitive view remains even in the older physics, which, following Aristotle, tells us that a body falls because the centre of the earth is its natural point of rest-that is, to put it otherwise, it must behave as it does because it has always done so.

\section{The Beginnings of ART.}

Though mythological thinking, particularly on the level of belief in demons and magic, has but slight connection with later science, it stands in close relation to the beginnings of art. This relation appears, among other things, in the fact that the simplest forms of the one are connected with the simplest forms of the other. This connection is twofold. Ideas of magic are, in a certain sense, projected into the products of art; art, on the other hand, being the means whereby mythological thinking finds expression, reacts upon magical ideas and brings about an enhancement of their motives. This is particularly apparent, in the beginnings of art, in the fact that, as viewed by civilized man, primitive peoples have brought but one art to a high degree of perfection, the art of dancing. For no other form of artistic expression is early man better endowed. His body is incomparably more supple than that of civilized races. The life of the forest, the climbing of trees, and the capturing of game qualify him for performances that would prove difficult to a modern art-dancer. All who have witnessed the dancing of men of nature have marvelled at their great skill and dexterity, and especially at their wonderful ability in respect to postures, movements, and mimetic expression. Originally, the dance was a means for the attainment of magical ends, as we may conjecture from the fact that even at a very early stage it developed 
into the cult dance. Nevertheless, from the very beginning it obviously also gave rise to pleasure, and this caused it to be re-enacted in playful form. Thus, even the earliest art ministered not only to external needs but also to the subjective life of pleasure. The direct source of the latter is one's own movements and their accompanying sensations. The dance of the group enhances both the emotion and the ability of the individual. This appears clearly in the dances executed by the inland tribes of Malacca. These peoples do not seem to have any round dances. The individual dancer remains at a fixed spot, though he is able, without leaving his place, to execute marvellous contortions and movements of the limbs. These movements, inoreover, combine with those of his companions to form an harmonious whole. They are controlled, however, by still another factor, the attempt to imitate animals. It is true that, on the primitive level proper, the animal does not play so dominant a rôle as in later times. Nevertheless, the initation of animals in the dance already foreshadows the totemic period. Some individuals are able, while remaining at a fixed spot, to imitate with striking life-likeness the movements of even small animals, and this is regarded as art of the highest order. Yet the animal-mask, which is later commonly used in cult and magic, is here as yet entirely lacking. These very mimic and pantomimic dances, however, unquestionably bear the traces of magic. When the Veddah imitates game-animals while executing his dance about the arrow, the arrow is without doubt regarded as a means of magic, and we may conjecture that the gameanimals that are struck by an arrow are supposed actually to succumb as a result of this mimetic performance.

Among primitive peoples, the dance is not, as a rule, accompanied by music. At most, means of producing noise are introduced, their purpose being to indicate the rhythm. The simplest of these noise-instruments consists of two wooden sticks that are beaten together. The drum is also common at a very early time ; yet it was probably introduced from without. The real musical accompaniment 
of the dance is furnished by the human voice in the dancesong. It would, of course, be wrong to suppose that because the dance originally served purposes of magic, the dancesong was a sort of primitive cult-song. Of such songs as the latter no traces occur until later. The contents of the early songs are derived from the most commonplace experiences of life. The songs really consist of detached fragments of purely descriptive or narrative prose, and have no inner connection with the motives of the dance. That which characterizes them as songs is the refrain. One might say without qualification that this poetic form of speech begins with the refrain. The song has grown up out of selected natural sounds. Anything that has been done or observed may serve as content of the song. After such material has once been employed, it is continually repeated. Thus it becomes a folk-song that is sung particularly during the dance. The melody is of a very monotonous character; could it be translated into our notes, we would find that in the songs of the Veddahs or of the inland tribes of Malacca, the melody moves at most within the range of a sixth. Moreover, there is an absence of harmonic intervals, so that, not having been phonographically recorded, the songs cannot be reproduced in our notes except with great uncertainty. Of their content, the following illustrations may give us some idea. One of the songs of the Veddahs runs as follows :--

The cioves of Taravelzita say kuturung.

Where the talagoya is roasted and eaten, there blew a wind, Where the memmina is roasted and eaten, there blew a wind, Where the deer is roasted and eaten, there blew a wind.

On a somewhat higher level stands the following song of the Semangs. It refers to the ring-tailed lemur (macaco), a monkey species very common in the forests of Malacca; by the Semangs it is called ' $\mathrm{kra}$ ' :-

He runs along the branches, the kra, $\mathrm{He}$ carries the fruit with him, the kra, He runs to and fro, the kra; Over the living bamboo, the kra, Over the dead bamboo, the kra; 
He runs along the branches, the kra,

He leaps about and screams, the kra,

He permits glimpses of himself, the kra,

He shows his grinning teeth, the kra.

As is clear, we have here simply observations, descriptions of that which the Semang has seen when watching the lemur in the forest. This description, of course, serves only as the material for the music of speech; that which is really musical is the refrain, which in this case consists simply of the one word kra. This music of speech exalts and supplements the dance; when all parts of the body are in motion the articulatory organs also tend to participate. It is only the modern art-dance which has substituted an instrumental accompaniment for the voice and has thus been able to suppress the natural expression of emotions. But, even in our culture, the emotions receive active, rocal expression in the folk-dances of our villages.

Musical instruments, in the strict sense of the word, are almost unknown to primitive man. Where somewhat complex forms occur, they appear to have been imported. Such, for example, is the bamboo nose-flute, occasionally found among the inland tribes of the Malay Peninsula. The nose-flute is similar to our flutes, except that it is blown from above instead of from the side, and is not played by means of the mouth, but is placed against one of the nostrils, so that the side of the nose serves as the tone-producing membrane. It has from three to five holes that may be covered with the fingers. This instrument is a genuine product of Melanesia, and was doubtless acquired from this region by the Malayan tribes. Of earlier origin, no doubt, are stringed instruments. These are to be found even among primitive peoples. The forms that occur in Malacca have, in this case also, obviously come from Oceania. But, on the other hand, an instrument has been found among the Bushmen and the neighbouring peoples which may be regarded as the most primitive of its kind and which throws important light on the origin of musical instruments of this sort. A bow, essentially similar to that 
which he employs in the chase, affords the Bushman a simple stringed instrument. The string of the bow now becomes the string of a musical instrument. Its tones, however, cannot be heard distinctly by any one except the player himself. He takes one end of the bow between his teeth and sets the string into vibration with his finger. The resonance of the bones of his head then causes a tone, whose pitch he may vary by holding the string at the middle or at some other point, and thus setting only a part of the string into vibration. Of this tone, however, practically no sound reaches the external world. On the other hand, the tone produces a very strong effect on the player himself, being powerfully transmitted through the teeth to the firm parts of the skull and reaching the auditory nerves through a direct bone-conduction. Thus, then, it is a remarkable fact that music, the most subjective of the arts, begins with the very stringed instruments which are the most effective in arousing subjective moods, and with a form in which the pleasure secured by the player from his playing remains purely subjective. But, from this point on, the further development to tone-effects that are objective and are richer in gradations is reached by simple transitions effected by association. The one string, taken over from the bow used in the chase, is no longer sufficient. Hence the bridge appears, which consists of a piece of wood whose upper side is fastened at the middle of the bow and whose lower side is toothed for the reception of several strings. The strings also are perfected, by being made of threads detached from the bamboo of which the bow is constructed. Then follows a second important advance. Instead of taking the end of the bow in his mouth and using his own head as a resonator, the player makes use of a hollow gourd and thus renders the tone objectively audible. The best and most direct point of connection between the gourd and the bow proves to be the end of the stick that carries the bridge. It is now no longer the head of the player that furnishes the resonance, but the substituted calabash. In its external appearance the calabash re- 
sembles the head-indeed, upon other occasions also, it is sometimes regarded as a likeness of the head, and eyes, mouth, and nose are cut into its rind. Thus, the association of the gourd with the head may possibly have exerted an influence upon this step in the development of the musical instrument. Perhaps the inventor himself did not realize until after the artificial head came into use that he had made a great advance in the perfection of his instrument. $\mathrm{His}$ music was now audible to others as well as to himself.

Another instrument also, the bull-roarer, dates back to the beginnings of music, though its development, of course, differed from that of the zither. The bull-roarer, indeed, is an instrument of tone and noise that is to be found only among relatively primitive peoples. True, it does not reach its highest development among those peoples who, from a sociological point of view, occupy the lowest plane of culture ; it becomes an instrument of magic, as we shall see, only within the totemic culture of Australia. Nevertheless, there has been discovered, again among the Bushmen, a form of bull-roarer of an especially primitive character. Doubtless that which led primitive man to the invention of the zither was the tone which he heard in his everyday experience in war or in the hunt when he applied an arrow to his bow. No doubt, also, it was the whirring noise of the arrow, or that, perhaps, of the flying bird which the arrow imitates, that led him to reproduce this noise in a similar manner. Indeed, in South Africa, the bull-roarer, though, of course, used only as a plaything, occurs in a form that at once reminds one of a flying bird or arrow. The feather of a bird is fastened at right angles to a stick of wood. When the stick is vigorously swung about in a circle, a whistling noise is produced, accompanied, particularly when swung with great rapidity, by a high tone. This tone, however, is not capable of further perfection, so that no other musical instrument developed from the bull-roarer. The contrary, rather, is true. In other forms of the bull-roarer in which the feathers were displaced by a flat wooden board-whose only resem- 
blance to a bird was a slight similarity in form-the noise was more intense but the tone less clear. For this reason the bull-roarer soon lost its place in the ranks of musical instruments and became purely an instrument of magic, in which function also it was used only temporarily. In many parts of the world, moreover, there is a similar primitive implement, the rattle, whose status is the same as that of the bull-roarer.

It was in connection with ideas of magic and of demons that formative art, or, as it would perhaps be truer to say, the elements from which this art proceeded, was developed. Such art was not unknown even to the primitive peoples of the pre-totemic age. If anywhere, it is doubtless among the primitive tribes of Malacca and Ceylon that we can, in some measure and with some certainty, trace formative art to its earliest beginnings and to the causes back of these. The Bushman must here be excluded from consideration, since, as we shall see, he was clearly affected by external influences. The Veddahs, as well as the Senoi and Semangs, are familiar with only the simplest forms of linear decoration. Yet this - makes it evident that simple lines, such as can be produced by cutting or by scratching, form the starting-point of almost all later development. Here again it is the bamboo that is utilized, its wood being a material suitable for these simple artistic attempts. Its connection with art is due also to the fact that it is used in the manufacture of implements and weapons, such as the bow and the diggingstick, and, later, the blow-pipe and the flute. As important objects of adornment, we find the combs of the women, which, among the Malaccan tribes, are extremely rich in linear decorations. At first, the dominant motive is the triangle. Just as the triangle is the simplest rectilinear figure of geometry, so also is it the simplest closed ornamental pattern. The weapons not infrequently have a series of triangles included within two parallel straight lines. This illustrates in its simplest form the universal characteristic of primitive ornaments, namely, uniform repetition. The pattern later becomes more 
complicated; the triangles are crossed by lines between which there are spaces that are also triangular in form. Such figures are then further combined into double triangles having a common base, etc. These are followed by other forms, in which simple arcs take the place of straight lines. For example, an arc is substituted for the base of each triangle, again with absolute uniformity. Finally, the arc, in the form of the segment of a circle, is utilized independently, either in simple repetition or in alternation. These simple designs then become increasingly complex by the combination either of the forms as a whole or of some of their parts. This multiplication of motives reaches its most artistic development in the women's combs found among the tribes of the Malay Peninsula. The comb, in some form or other, is a very common article of adornment among peoples of nature. But it is just in the form in which it occurs among the Senoi and Semangs that the comb gives evidence of having originally been, at most, only incidentally an article of adornment and of having only gradually come to be exclusively a decoration. In shape, it is like the women's combs of to-day. The teeth are pointed downwards, and serve the purpose of fastening the hair. The upper part forms a broad crest. But among these peoples the crest is the main part of the comb, the function of the teeth being merely to hold it to the head. For the crest is decorated in rich profusion with the above-mentioned ornamentations, and, if we ask the Semangs and the Senoi what these mean, we are told that they guard against diseases. In the Malay Peninsula, the men do not wear combs, evidently for the practical reason that, because of their life in the forest and their journeys through the underbrush, they cut their hair short. In other regions which have also evolved the comb, as in Polynesia, such conditions do not prevail ; the comb, therefore, is worn by both men and women. In this, its earliest, use, however, the comb as such is clearly less an object of adornment than a means of magic. It serves particularly. as a sort of amulet, to protect against sickness- 
demons. For this reason the ornamental lines in their various combinations are regarded as referring to particular diseases. The marks which a Semang woman carries about with her on her comb are really magical signs indicating the diseases from which she wishes to be spared. The head would appear to be a particularly appropriate place for wearing these magical signs. It is to magical ideas, therefore, that we must probably look for the origin of this very common means of adornment. In Malacca, indeed, the combs are carefully preserved; the drawings made upon them render them, as it were, sacred objects. But it is impossible to learn directly from the statements of the natives just how primitive articles of adornment came to acquire the significance of ornaments. Our only clue is the fact that the decorations on the bows and blow-pipes are supposed to be magical aids to a successful hunt; for, among the representations, there are occasionally those of animals. This fact we may bring into connection with observations made by Karl von den Steinen among the Bakairi of Central Brazil. This investigator here found remarkable ornamentations on wood. All of these were of a simple geometrical design, just as in the case of other primitive peoples, yet they were interpreted by the natives not as means of magic but as representations of objects. A consecutive series of triangles whose angles were somewhat rounded off, was interpreted as a snake, and a series of squares whose angles touched, as a swarm of bees. But the representations included also other things besides animals. For example, a vertical series of triangles in which the apexes pointed downwards and touched the bases of the next lower triangles, was regarded as a number of women's aprons-the upper part was the girdle, and, attached to this, the apron. In a word, primitive man is inclined to read concrete objects of this kind into his simple ornamental lines. That we also can still voluntarily put ourselves into such an attitude, is testified to by Karl von den Steinen himself, when he tells us that he succeeded without particular effort in discovering similar objects in certain simple 
ornamentations. We here have a case of the psychical process of assimilation. This is characteristic of all consciousness, but, as might be supposed from the fact that primitive peoples live continuously in the open, it is more strongly in evidence among them than among civilized races.

But the question now arises, Which came first? Did the Bakairi really wish to represent snakes, bees, women's aprons, etc., and reduce these to geometrical schematizations? Or did he, without such intention, first make simple linear decorations, and later read into them, through imaginative association, the memory images of objects? The latter is doubtless the case. For it is much easier first to draw simple lines and then to read complicated objects into them than it is, conversely, to reduce these pictures at the outset to abstract geometrical schemata. Indeed, when the Bakairi wishes to draw real objects, he proceeds just as our children do: he copies them as well as he can. For example, the Bakairi occasionally draws fishes in the sand for the purpose of marking out a path, or he attempts to reproduce men and animals in a way strikingly similar to our children's drawings. Evidently, therefore, it was not inability to draw the objects themselves that gave rise to these primitive geometrical decorations. The decorations came first, and the memory images of the objects of daily perception were then read into them. The answer, however, to the question as to why primitive man produces decorations at all, is easily found by calling to mind the motives discernible in such uniform and simple series of figures as the triangles and arcs which the Senoi and the Semangs cut into bamboo. Because of the character of his locomotor organs, primitive man repeats the movements of the dance at regular intervals, and this rhythm gives him pleasure. Similarly, he derives pleasure even from the regularly repeated movements involved in making the straight lines of his drawings, and this pleasure is enhanced when he sees the symmetrical figures that arise under his hand as a result of his movements. The earliest resthetic stimuli are symmetry and rhythm. We learn this even from the most 
primitive of all arts, the dance. Just as one's own movements in the dance are an æsthetic expression of symmetry and rhythm, so also are these same characteristics embodied in the earliest productions of pictorial art-in the beginning, indeed, they alone are to be found. The primitive song comes to be a song only as a result of the regular repetition of a refrain that in itself is unimportant. As soon as primitive man produces lines on wood, his pleasure in rhythmic repetition at once leads him to make these symmetrical. It is for this reason that we never find decorations that consist merely of a single figure-a single triangle, for instance-but always find a considerable number of figures together, either above one another, or side by side, or both combined, though the last arrangement occurs only at a somewhat more advanced stage. If, now, these decorations are more and more multiplied by reason of the increasing pleasure in their production, we naturally have figures that actually resemble certain objects. This resemblance is strengthened particularly by the repetition of the figures. A single square with its angles placed vertically and horizontally would scarcely be interpreted as a bee, even by a Bakairi; but in a series of such squares we ourselves could doubtless imagine a swarm of bees. Thus there arise representations resembling animals, plants, and flowers. Because of their symmetrical form, the latter particularly are apt to become associated with geometrical designs. Yet on the whole the animal possesses a greater attraction. The animal that forms the object of the hunt is carved upon the bow or the blowpipe. This is a means of magic that brings the animal within range of the weapon. It is magic, likewise, that affords the explanation of the statement of the Senoi and the Semangs that the drawings on the combs of their women are a means of protection against diseases. These two sorts of purposes illustrate the two forms of magic that are still exemplified on higher cultural levels by the amulet, on the one hand, and the talisman, on the other-protection from danger, and assistance in one's personal undertakings. Now 
it is easy to understand how especially the complicated decorations on the combs of the Malaccan tribes may, through the familiar processes of psychical assimilation, come to be regarded as living beings, in the form either of animals or of plants, and how these forms in turn may come to be interpreted as sickness-demons. For, these demons are beings that have never been seen; hence the terrified imagination may all the more readily give them the most fantastic shapes. Indeed, we still find examples of this in the more elaborate pictures of the art of some semicultural peoples. Thus also are explained many of the masks used among the most diverse peoples. It is almost always grotesque animal or human masks that are employed to represent fear-demons. The freer the sway of the imagination, the easier it is to see the figure of a demon in any decoration whatsoever. The multiplicity of the ornamental drawings, moreover, meets the need for distinguishing a great number of such demons, so that a woman of the Senoi or the Semangs carries about on her head the demoniacal representation of all known diseases. For, according to an ancient law of magic, the demon himself has a two-fold rôle-he both causes the sickness and protects against it. Just as a picture is identified with its object, so also is the drawing that represents or portrays the sickness-demon regarded as the demon itself. Whoever carries it about is secure against its attack. Both magic and counter-magic spring from a common source. The medicine-man who exercises counter-magic must also be familiar with magic. The two are but divergent forms of the same magical potency that has its birth in the emotions of fear and terror.

In summary of what we have thus far learned with regard to the art of drawing among primitive men, it may be said that this art is throughout one of magic and adornment. These are the two motives from which it springs, and which, apparently, co-operate from the outset. The mere drawing of lines in regular and symmetrical repetition is due to that regularity of movement which also finds expression in the dance, and, even prior to this, 
in ordinary walking and running. But the artist himself then attributes a hidden meaning to that which he has created. Astonishment at his creation fuses with his pleasure in it, and his wonder at the picture that he has produced makes of it, when animated and retransformed by the imagination, a magical object. The pictures carried about on the person, or wrought on an object of daily use, assist in guarding against diseases and other injuries, or they assure the success of the weapon and the implement.

In view of these characteristics of a purty magical and decorative art, it may perhaps at first glance cause surprise that there should be a people which, although primitive in other essential respects, has far transcended this stage in artistic attainment, and has, apparently, followed an entirely different direction in its pathway to art. Such are the Bushmen. The primitive tribes mentioned above show no traces of an art of drawing ; beyond suggestions of a single object, it is absolutely impossible to find representations of objects and their groupings such as are common in the pictures of the Bushmen, which portray particularly animals and, to a less extent, men. This is all the more significant in view of the fact that, while the Bushmen also decorate their weapons and utensils with magical and ornamental designs, these are of far less importance than in the case of the primitive tribes referred to above. The painting of the Bushmen, however, is obviously neither magical nor decorative in character. Originally these pictures seem to have been drawn in caves ; at any rate, it is here that many of them have been found. We have already indicated the importance of this primitive dwelling for the beginnings of a memorial art. When external impressions are absent, as in the cave, the imagination is all the more impelled to preserve memories in self-created pictures. The simpler of these resemble, in their characteristics, the drawings and paintings of present-day children. But we can plainly distinguish the more primitive work from that which is more advanced; the latter frequently reproduces its objects with accuracy, particularly animals, 
such, for example, as the elk' and also the giraffe, which is a favourite object, probably because of its long neck. Occasionally, indeed, a quadruped is still represented in profile with only two legs, but most of the pictures are certainly far beyond this childish mode of drawing. In general, mineral pigments were used from the very outset, particularly red iron ore, blue vitriol, etc. We also find mixtures of pigments, so that almost all colours occur. Now it might, of course, be supposed that such a picture of an animal has the same significance as attaches to the drawing occasionally executed on the bow of a primitive man for the purpose of magically insuring the weapon of its mark. But the very places where these paintings occur, far removed as they are from chase and battle, militate against such a supposition. An even greater objection is the fact that the more perfect pictures represent scenes from life. One of them, for example, portrays the meeting of Bushmen with white men, as is evident partly from the colour and partly from the difference in the size of the figures. Another well-known picture represents the way in which the Bushmen steal cattle from a Bantu tribe. The Bantus are represented by large figures, the Bushmen by small ones; in a lively scene, the latter drive the animals away, while the far-striding Bantus remain far in the rear. The picture reveals the joy of the primitive artist over the successful escapade. This is not magical art, but plainly exemplifies the first products of a memorial art. The one who painted these pictures desired first of all to bring before his memory that which he had experienced, and he doubtless also wished to preserve these scenes to the memory of his kinsmen. This is memorial art in a twofold sense. Memory renews the experiences of the past, and it is for memory that the past is to be retained. But this art also must still be classed as primitive, for it has not as yet attained to the level of imitative art. It is not an art that reproduces an object by a direct comparison of picture with copy. This is the sense in which the present-day portrait or landscape painter practises imitation. Even 
where the primitive era transcended a merely magical or decorative art, it did not advance beyond memorial art. The Bushman did not have the objects themselves before him, but created his pictures in accordance with his memory of them. Moreover, suited as the cave is to the development of a memorial art, it of itself makes imitative art impossible. But how can we account for the fact that the primitive tribe of Bushmen attained to a level of art whose exclusion of magical motives ranks it as relatively advanced, and which must be estimated all the more highly because it is not shared by the neighbouring African tribes? The Hottentots, for example, no less than the Bechuanas and the Bantus, are inferior in artistic accomplishments to the Bushmen, although the culture of the latter is in other respects far below the level of that of the former. May we say of this memorial art what seems probable as regards the magical and decorative art of the inland tribes of Malacca and of Ceylon, namely, that it arose independently from the same original motives as the dance? The answer to this question depends primarily upon the antiquity of these art productions. Do they date back to an immemorial past, as we may suppose to be the case with the decorations of the Veddahs and the Malaccan tribes? There are two considerations, principally, that prove the contrary, namely, that they are relatively recent creations. In the first place, the paintings present the pictures of animals, in particular of the horse and the sheep, with which the Bushman has been acquainted at farthest since the latter part of the eighteenth century. True, these animals were brought into Cape Colony as early as the seventeenth century; it was clearly not until later, however, that the Bushmen became familiar with them. A second consideration is the remarkable circumstance that these primitive painters employ essentially the same tools as the Europeans. This art has now, indeed, almost disappeared, the race having been crowded back and depleted. But the remains show that the painters possessed a stone plate on which they mixed their paints and also a stone pounder with which the mixing was done-that is, a palette and a pestle. Indeed, 
for applying the colours they occasionally utilized a paintbrush made of fine splinters of bone, though some, no doubt, were content to do this with the fingers.

These are all signs which certainly suggest a not very distant past. Moreover, art products cannot resemble each other in so many respects without having some connection in origin. Added to this is the fact that the very character of such pictures as are still in existence scarcely allows us to regard them as more than sixty to seventy years old. From all of this we must conclude that this art is not primitive at all, but was imported, resembling in this many other things that gain entrance into the life of a primitive tribe. If the essential elements of the Biblical account of the Creation reached the Andamanese, who in other respects are primitive, why may we not also suppose that a wandering European artist at one time came to the Bushmen, even before any other elements of European culture had become accessible to them? Nevertheless, the fact that this painting exists indicates the presence of a remarkable talent. This brings us to our last problem in the psychology of primitive man, to the question concerning his mental equipment in general.

9. The Intellectual and Moral Characteristics of PRIMITIVE MAN.

For a general estimate of the mental characteristics of a race or a tribe, the observation of a single individual or of several individuals is not adequate. Judgment can be based only on the totality of the various mental phases of culture-language, custom, myth, and art. But, if we would also obtain a conception of the mental capacities of a people or a tribe, we must take into further consideration the mental endowment of the individual. For, in the case of mental capacity, we must consider not merely that which has actually been achieved but also everything within the possibility of attainment. Here, again, the standpoint differs according as we are concerned (to limit our- 
selves to the two most important and typical aspects) with an intellectual or a moral estimate. These two aspects, the intellectual, taken in its widest sense, and the moral, are not only of supreme importance, but, as experience shows, they in no wise run parallel courses. For an understanding of mental development in general, therefore, and of the relation of these its aspects, the early conditions of human culture are particularly significant.

If, now, we consider the general cultural conditions of primitive man, and recall the very meagre character of his external cultural possessions as well as his lack of any impulse to perfect these, we may readily be led to suppose that his intellectual capacities also have remained on a very low plane of development. How, some have asked, could the Bushman have dispensed for decades with firearms-just as accessible to him as to the surrounding tribes - unless he possessed a low degree of intelligence? Even more true is this of the Negritos of the Philippines or the Veddahs of Ceylon. How, unless their mental capacities were essentially more limited than those of their neighbours, could they have lived in the midst of highly cultivated tribes and have remained for decades on an unchanged mental level? But we need to bear in mind two considerations that are here decisive. The first of these is the limited nature of the wants of primitive man, a condition fostered, no doubt, by his relatively small intercourse with neighbouring peoples. Added to this is the fact that up to very recent times-for here also many changes have arisen-the primitive man of the tropics has found plenty of game and plant food in his forests, as well as an abundance of material for the clothing and adornment to which he is accustomed. Hence he lacks the incentive to strive for anything beyond these simple means of satisfying his wants. It is agreed, particularly by the investigators who have studied those tribes of Malacca and Ceylon that have remained primitive, that the most outstanding characteristic of primitive man is contentment. He seeks for nothing further, since he either finds all that he desires 
in his environment, or, by methods handed down from the ancient past, knows how he may produce it out of the material available to him. For this reason the Semangs and Senoi, no less than the Veddahs, despise as renegades those mixed tribes that have arisen through union, in the one case, with the Malays, and, in the other, with the Singhalese and Tamils. All the more firmly, therefore, do they hold to that which was transmitted to them by their fathers. Together with this limited character of their wants, we find a fixity of conditions, due to their long isolation. The longer a set of customs and habits has prevailed among a people, the more difficult it is to overturn. Prior to any change we must, in such cases, first have mighty upheavals, battles, and migrations. To what extent all deeper-going changes of culture are due to racial fusions, migrations, and battles we shall presently see. The tribes that have remained relatively primitive to this day have led a peaceful existence since immemorial times: Of course, the individual occasionally slays the man who disturbs his marriage relations or trespasses upon his hunting-grounds. Otherwise, however, so long as he is not obliged to protect himself against peoples that crowd in upon him, primitive man is familiar with the weapon only as an implement of the chase. The old picture of a war of all with all, as Thomas Hobbes once sketched the natural state of man, is the very reverse of what obtained. The natural condition is one of peace, unless this is disturbed by external circumstances, one of the most important of which is contact with a higher culture. The man of nature, however, suffers less from an advanced culture than he does from the barbarism of semi-culture. But whenever a struggle arises for the possession of the soil and of the means of subsistence which it furnishes, semi-culture may come to include more peoples than are usually counted as belonging to it. The war of extermination against the red race was carried on by the pious New England Puritans with somewhat different, though with scarcely better, weapons than the Hottentots and Herero to-day turn 
against the Bushmen, or the Monbuttus against the Negritos of Central Africa.

It is characteristic of primitive culture that it has failed to advance since immemorial times, and this accounts for the uniformity prevalent in widely separated regions of the earth. This, however, does not at all imply that, within the narrow sphere that constitutes his world, the intelligence of primitive man is inferior to that of cultural man. If we call to mind the means which the former employs to seek out, to overtake, and to entrap his game, we have testimony both of reflection and, equally so, of powers of observation. In order to capture the larger game, for example, the Bushman digs large holes in the ground, in the middle of which he constructs partitions which he covers with brush. An animal that falls into such a hole cannot possibly work its way out, since two of its legs will be on one side of the partitional division and two on the other. Smaller animals are captured by traps and snares similar to those familiar to us. The Negritos of the Philippines, furthermore, employ a very clever method for securing wild honey from trees without exposing themselves to injury from the bees. They kindle a fire at the foot of the tree, causing a dense smoke. Enveloped by this, an individual climbs the tree and removes the object of his lesire, the smoke rendering the robber invisible to the scattering swarm. It is thus that the Negritos secure honey, their most precious article of food. How great, moreover, is the inventive ability required by the bow and arrow, undoubtedly fashioned even by primitive men! We have seen, of course, that these inventions were not snatched from the blue, but that they were influenced by all sorts of empirical elements and probably also by magical ideas, as in the case of the feathering of the arrow. Nevertheless, the assembling and combining of these elements in the production of a weapon best suited to the conditions of primitive life is a marvellous achievement, scarcely inferior, from an intellectual point of view, to the invention of modern firearms. Supplementing this, we have the testimony of observers concerning the 
general ability of these races. A missionary teacher in Malacca, whose school included Chinese, Senoi, and Malays, gave first rank to the Chinese as regards capacity, and second place to the Senoi, while the Malays were graded last, though they, as we know, are held to be a relatively talented race. Now, this grading, of course, may have been more or less accidental, yet it allows us to conclude that the intellectual endowment of primitive man is in itself approximately equal to that of civilized man. Primitive man merely exercises his ability in a more restricted field; his horizon is essentially narrower because of his contentment under these limitations. This, of course, does not deny that there may have been a time, and, indeed, doubtless was one, when man occupied a lower intellectual plane and approximated more nearly to the animal state which preceded that of human beings. This earliest and lowest level of human development, however, is not accessible to us.

But what, now, may be said concerning the moral characteristics of primitive man? It is clear that we must here distinguish sharply between those tribes that have hitherto remained essentially unaffected by external influences and those that have for some time past eked out a meagre existence in their struggle with surrounding peoples of a higher culture. The primitive man who still lives uninfluenced by surrounding peoples-typical examples are, in general, the natural Veddahs of Ceylon and the inland tribes of the Malay Peninsula-presents an entirely different picture from that of the man who seeks in the face of difficulties to protect himself against his environment. In the case of the tribes of Ceylon and Malacca, the somewhat civilized mixed peoples constitute a sort of protective zone, in the former case against the Singhalese and Tamils, in the latter, against the Malays. These mixed peoples are despised, and therefore they themselves hesitate to enter into intercourse with the primitive tribes. Thus they offer an outer buttress against inpressing culture. The result is that these primitive peoples continue to live their old life essentially undisturbed. Now, the 
testimony of unprejudiced observers is unanimous in maintaining that primitive man is frank and honest, that lying is unknown to him, and that theft does not exist. He may, of course, be strongly moved by emotion, so that the man who disturbs the Veddah's marriage relation may be sure of a poisoned arrow, as may also the strange huntsman who encroaches unbidden upon his hunting-grounds. This reprisal is not based upon legal enactments-of such there are none; it is custom that allows this summary procedure. Many investigators have believed that these various characteristics exhibited by unmixed primitive culture indicate a high state of morality. In this they agree with Wilhelm Schmidt, for whom primitive men are the infant peoples of the world, in that they possess the innocence of childhood. It is not only man's moral outlook, however, but also his moral character, as this very illustration shows, that depends upon the environment in which he lives. Since the primitive man who lives undisturbed by external conditions has no occasion to conceal anything, his honesty and frankness ought scarcely to be counted to his particular credit; so far as theft is concerned, how can there be a thief where there is no property? It may, of course, happen that an individual takes the weapon of his companion for a short time and uses it. This action, however, is all the more permissible since each man makes his own bow and arrow. The same is true of clothing and articles of adornment. Thus, the rather negative morality of primitive man also has its origin in his limited wants, in the lack of any incentives to such action as we would call immoral. Such a positive situation, however, is, no doubt, afforded by the strict monogamy, which probably originated in the prehuman natural state and was thenceforth maintained.

Quite different is the moral picture of primitive man wherever he is at strife with surrounding peoples. Here, as was noted particularly by Emin Pasha and Stuhlmann in the case of the Negritos of the Upper Nile, the outstanding characteristics are, in the first place, fear, and then deception and malice. But can we wonder at 
this when we learn that the flesh of the Pygmies is especially prized by the anthropophagic Monbuttus of that region, and that the pursuit of this human game on the part of the latter is absolutely unrestrained, except by the fear of the arrows which the Pygmies shoot from behind ambush? Here, of course, innocence, frankness, and honesty are not to be expected; under these circumstances, theft also comes to be a justifiable act. Wherever the Negrito finds something to take, he takes it. The same is true of the South African Bushmen, who occupy a similarly precarious position with respect to the Bantus and Hottentots. The Bushmen are the most notorious thieves of South Africa. Of this we have striking evidence in the abovementioned picture of the Bushman who glorifies and preserves to memory the theft of cattle. The Bushman is crafty and treacherous, and steals whenever there is opportunity. But what else could be expected, when we consider that, by killing off the game with their firearms, the Hottentots and Bantus deprive the Bushman of that which was once his source of food, and that they shoot the Jushman himself if he resists?

To summarize: The intelligence of primitive man is indeed restricted to a narrow sphere of activity. Within this sphere, however, his intelligence is not noticeably inferior to that of civilized man. His morality is dependent upon the environment in which he lives. Where he lives his life of freedom, one might almost call his state ideal, there being few motives to immoral conduct in our sense of the word. On the other hand, whenever primitive man is hunted down and hard pressed, he possesses no moral principles whatsoever. These traits are worth noting, if only because they show the tremendous influence which eternal life exerts, even under the simplest conditions, upon the development of the moral nature. 


\section{THE TO'TEMIC AGE}

I. The General Character of Totemism.

THE expression 'totemic age' involves a widened application of the term 'totem.' This word is taken from the language of the Ojibways or, as the English call them, the Chippewa Indians. To these Indians of the Algonquin race, the 'totem' signified first of all a group. Persons belong to the same totem if they are fellow-members in a group which forms part of a tribe or of a clan. The term ' clan,' suggested by the clan divisions of the Scottish Highlanders, is the one usually employed by English ethnologists in referring to the smaller divisions of a tribe. The tribe consists of a number of clans, and each clan may include several totems. As a rule, the totem groups bear animal names. In North America, for example, there was an eagle totem, a wolf totem, a deer totem, etc. In this case the animal names regularly refer to particular clans within a tribe ; in other places, as, for example, in Australia, they designate separate groups within a clan. Moreover, the totem animal is also usually regarded as the ancestral animal of the group in question. 'Totem,' on the one hand, is a group name, and, on the other, a name indicative of ancestry. In the latter connection it has also a mythological significance. These various ideas, however, interplay in numerous ways. Some of the meanings may recede, so that totems have frequently become a mere nomenclature of tribal divisions, while at other times the idea of ancestry, or, perhaps also, the cult significance, pre- 
dominates. The idea gained ground until, directly or indirectly, it finally permeated all phases of culture. It is in this sense that the entire period pervaded by this culture may be called the 'totemic age.'

Even in its original significance-as a name for a group of members of a tribal division or for the division itselfthe conception of the totem is connected with certain characteristic phenomena of this period, distinguishing it particularly from the culture of primitive man. I refer to tribal division and tribal organization. The horde, in which men are united purely by chance or at the occasional call of some undertaking, only to scatter again when this is completed, has disappeared. Nor is it any longer merely the single family that firmly binds individuals to one another; in addition to it we find the tribal division, which originates in accordance with a definite law of tribal organization and is subject to specific norms of custom. These norms, and their fixed place in the beliefs and feelings of the tribal members, are connected with the fact that originally, at all events, the totem animal was regarded, for the most part, as having not merely given its name to a group of tribal members but as having actually, been its forefather. In so far, animal ancestors apparently preceded human ancestors. Bound up with this is the further fact that these animal ancestors possessed a cult. Thus, ancestor cult also began with the cult of animals, not with that of human ancestors. Aside from specific ceremonies and ceremonial festivals, this animal cult originally found expression primarily in the relations maintained toward the totem animal. It was not merely a particular animal that was to a certain extent held sacred, but every representative of the species. The totem members were forbidden to eat the flesh of the totem animal, or were allowed to do so only under specific conditions. A significant counter-phenomenon, not irreconcilable with this, is the fact that on certain occasions the eating of the totem flesh constituted a sort of ceremony. This likewise implies that the totem animal was held sacred. When this conception came into the foreground, the totem idea 
became extended so as to apply, particularly in its cult motives and effects, to plants, and sometimes even to stones and other inanimate objects. This, however, obviously occurred at a later time.

From early times on, the phenomena of totemism have been accompanied by certain forms of tribal organization. Every tribe is first divided, as a rule, into two halves. Through a further division, a fairly large number of clans arise, which, in turn, eventually split up into subclans and separate totem groups. Each of these groups originally regarded some particular totem animal or other totem object as sacred. The most important social aspect of this totemic tribal organization, however, consists in the fact that it involved certain norms of custom regulating the intercourse of the separate groups with one another. Of these norms, those governing marriage relations were of first importance. The tribal organization of this period was bound up with an important institution, exogamy, which originated in the totemic age. In the earliest primitive period every tribal member could enter into marriage with any woman of the tribe whom he might choose ; according to the Veddahs, even marriage between brother and sister was originally not prohibited. Thus, endogamy prevailed within the primitive horde. This, of course, does not mean that there was no marriage except within the narrow circle of blood relationship, but merely that marriage was permitted between close relatives, more particularly between brothers and sisters. The exogamy characteristic of totemic tribal organization consists in the fact that no marriages of any kind are allowed except between members of different tribal divisions. A member of one particular group can enter into marriage only with one of another group, not with a person belonging to his own circle. By this means, totemic tribal organization gains a powerful influence on custom. Through marriage it comes into relation with all phenomena connected with marriage, with birth and death and the ideas bound up with them, with the initiation ceremonies in which the youths are received into the 
association of men, etc. As a result of the magical significance acquired by the totem animal, special associations are formed. These become united under the protection of a totem animal and give impetus to the exoteric cult associations, which, in their turn, exercise a profound influence upon the conditions of life. Though it is probable that these associations had their origin in the abovementioned men's clubs, their organizing principle was the totem animal and its cult.

Besides its influence on matters connected with the relations of the sexes, the totem animal was the source of several other ideas. After the separate tribal group has come to feel itself united in the cult of the totem animal, a single individual may acquire a particular guardian animal of his own. Out of the tribal totem there thus develops the individual totem. Then, again, the different sexes, the men and the women of the tribe, acquire their special totem animals. These irradiations of the totemic conception serve partly to extend it and partly to give it an irregular development. Of the further phenomena that gradually come to the foreground during the totemic age, one of the most important is the growing influence of dominant individual personalities. Such personalities, of course, were not unknown even to the primitive horde, on the occasion of important undertakings. But tribal organization for the first time introduces a permanent leadership on the part of single individuals or of several who share the power. Thus, totemism leads to chieftainship as a regular institution-one that later, of course, proves to be among the foremost factors in the dissolution of the age that gave it birth. For chieftainship gives rise to political organization; the latter culminates in the State, which, though destroying the original tribal organization, is, nevertheless, itself one of the last products of totemic tribal institutions.

With the firmer union of tribal members there comes also tribal warfare. So long as primitive man remains comparatively unaffected by other peoples, and particularly by those of a different cultural level, he lives, on the whole, in 
a state of peace. An individual may, of course, occasionally raise his weapon against another person, but there are no tribal wars. These do not appear until the period of totemism, with whose firm social organization they are closely connected. The tribe feels itself to be a unit, as does likewise each subordinate clan and group. Hence, related tribes may unite in common undertakings. More frequently, however, they fall into dissension, and warfare must decide their claims to the possession of territory or to a disputed hunting-ground. This warfare finds contributory causes in tribal migrations. New peoples, some of them perhaps from strange tribes, enter into a territory and crowd out its inhabitants. Thus, war and migration are closely connected. Strife between tribes and peoples-that is, warfare-begins with culture in general, particularly with the most primitive social culture, as we may, doubtless designate totemism in distinction from the still more primitive life of the horde.

This leads to a number of further changes. Tribal ownership of the land becomes more firmly established, as does also the custom of allotting a particular share to the clan. Personal property, moreover, comes to be more and more differentiated from the possessions of the group. Trade, which in primitive times was almost entirely restricted to secret barter, becomes public, and is finally widened into tribal commerce. When this occurs, great changes in external culture are inaugurated. Implements, weapons, and articles of dress and of adornment are perfected. This stage having been attained, the totemic age advances to a utilization of the soil in a way that is unknown to primitive man. The land is cultivated by means of agricultural implements. Of these, however, the hoe long continues to be the only one ; though it supplants the digging-stick, its use depends on human power alone. The care and breeding of animals is also undertaken ; the herdsman's or, as it is usually called, the nomadic, life is inaugurated. The breeding of useful domestic animals, in particular, is very closely connected with totemism. The animal, which at the beginning of 
the period was regarded as sacred, acquires the status of a work animal. It loses its dominion over mankind ; instead, it becomes a servant, and, as a result, its cult significance gradually vanishes. The very moment, however, that marks the passing of the sacred animal into the useful animal also signalizes the end of the totemic era and the beginning of the age of heroes and gods.

These various traits are far from giving us a complete picture of the wide ramifications of totemic ideas and customs. Enough has been said, however, to indicate how the totemic conception first widens and deepens its influence, permeating the external social organization no less than the separate phases of society, and then finally leads on to its own dissolution. It is precisely this that justifies us in calling the entire period the totemic age. Yet the boundaries of this period are naturally much less clearly defined, or sharply demarcated as to beginning and end, than are those of the preceding primitive age. Man is primitive so long as he is essentially limited in his immediate means of support to that which nature directly offers him or to the labour of his own hands. But even in its beginnings the totemic age transcends these conditions. Tribal organization and the connected phenomena of war, migration, and the beginnings of open trade relations are cultural factors which from the outset represent an advance beyond the primitive state. But the lower limit of the age cannot be definitely fixed; still less can we determine the point at which it terminates. The chieftain of the totemic age is the forerunner of the ruler who appears in the succeeding period. Similarly, totem animals are even more truly the precursors of the later herd, and of agricultural animals. Thus, it is not at all permissible to speak merely of $a$ culture, as one may do in the case of the primitive age. There are a number of different cultures-indeed, several levels of culture, which are in part co-existent but in part follow upon one another. Their only similarity is the fact that they all exhibit the fundamental characteristics of the totemic age. Consider the Veddahs of Ceylon, the Negritos of the 
Philippines, the inland and forest-dwelling tribes of Malacca. When we have described the general cultural conditions of one of these tribes, we have given the essential features of all. This, however, is far from true in the case of totemism, for this includes many forms of culture and various periods of development. Even in speaking of levels of culture we may do so only with the reservation that each level in its turn includes within it a large number of separate forms of culture, of numerous sorts and gradations. Moreover, the external culture, reflected in dress and habitation, in personal decoration, in implements and weapons, in food and its preparation, does not in the least parallel the social phenomena represented by tribal organization, marriage relations, and forms of rulership. Though the general character of the Polynesian peoples permits their inclusion within the totemic age, their tribal organization exhibits the characteristics of totemic society only imperfectly. In other aspects of their culture, however, they rank far higher than the Australians or some of the Melanesian tribes; these possess a very complex social organization, but are, nevertheless, only slightly superior, on the whole, to primitive peoples. Thus, the various phases of totemic culture may develop in relative independence of one another, even though they are in constant interaction. This is true particularly in the sense that the more developed totemic customs and cults occur even on low cultural levels, whereas, on the other hand, they more and more disappear with the progress of culture.

\section{The Stages of Totemic Culture.}

We cannot undertake to describe the extraordinarily rich external culture attained by those groups of peoples who may, in the main, be counted as belonging to the domain of totemism. This is the task of ethnology, and is not of decisive importance for folk psychology. True, in the case of primitive man, the conditions of external culture were described in some detail. This was necessary because 
of the close connection between these conditions and the psychical factors fundamental to all further development. The beginning of the totemic period marks a great change. New forces now come into play, such as are not to be found among the universal motives that have controlled the life of man from its very beginning. Of these forces there is one in particular that should be mentioned-one that is practically lacking among primitive tribes. This consists in the reciprocal influences exercised upon one another by peoples who occupy approximately the same plane of culture but who nevertheless exhibit certain qualitative differences. Migrations are also an important factor in the totemic age, as well as is the tribal warfare with which migrations are connected.

If we disregard these qualitative differences and attempt to introduce a degree of order into the profusion of the totemic world solely on the basis of general cultural characteristics, we may distinguish three great cultural stages, of which the third, again, falls into two markedly different divisions. We may ignore certain isolated remnants of peoples that are scattered over almost all parts of the world and exhibit very unlike stages of civilization, in order to give our exclusive attention to those forms of culture that belong to compact groups. In this event we shall find that the lowest stage is unquestionably exemplified in the Australian region, as well as by some of the Melanesian peoples. Above this, we have a second level of culture, the Malayo-Polynesian. Wide as is the difference between these cultures, they are nevertheless connected by numerous transitional steps, to be found particularly in Melanesian and Micronesian regions. The third stage of totemic culture itself falls into two essentially different divisions, the American, on the one hand, and the African, on the other. These divisions, of course, include only the so-called natural peoples of these countries, or, more accurately expressed, those tribes which, as regards the characteristics of their social and particularly of their religious development, still belong to totemic culture.

The fact that Australian culture, in spite of its highly 
complex tribal organization, occupies the lowest plane of all, itself indicates how great may be the discrepancy between totemism in general and the direct influence which it exerts upon tribal organization and external culture. This explains why the Australian native was regarded, up to very recent times, as the typical primitive man. As a matter of fact, his general culture differs but slightly from that of primitive races. The Australian also is a gatherer and a hunter, and shows no trace of a knowledge of agriculture nor, much less, of cattle-raising. Even his faithful domestic animal, the dog, is rarely used for hunting, but is regarded solely as the companion of man. Among the Australians, therefore, the woman still goes about with digging-stick in hand, seeking roots and bulbs for food. Man's life still centres about the chase, and, when one hunting-ground becomes impoverished, he seeks another. Likewise, there is no systematic care for the future. The food is prepared directly in the ashes of the fire or between hot stones-for cQoking is not yet customary-and fire is produced by friction or drilling just as it is by primitive man. His utensils also are in essential harmony with his general culture.

But there is one important difference. There has come a change of weapon. This change points to a great revolution inaugurated at the beginning of the totemic age. Primitive man possesses only a long-distance weapon; for the most part he uses bow and arrow. With this weapon he kills his game; with it the individual slays his enemy from ambush. On the other hand, war between tribes or tribal divisions, in which large numbers are opposed, may scarcely be said to exist. This would not be possible with bow and arrow. Thus, the very fact that this is the only weapon indicates that relatively peaceful conditions obtained in primitive culture. Quite otherwise with the Australian ! His weapons are markedly different from those of primitive man. Bow and arrow are practically unknown to him; they are found only among the tribes of the extreme north, having probably entered from Melanesia. The real weapons of the Australian are the wooden missile and the javelin. 
The wooden missile, bent either simply or in the form of a boomerang, whose above-mentioned asymmetrical curve is designed to cause its return to the thrower, is a long-distance weapon. For the most part, however, it is employed only in hunting or in play. The same remains true, to some extent, also of the javelin. The latter has reached a perfected form, being hurled, not -directly from the hand, but from a grooved board. The pointed end of the javelin extends out beyond this groove; at its other end there is a hollow into which is fitted a peg, usually consisting of a kangaroo tooth. When the spear is hurled from the board this peg insures the aim of the shot, just as does the gun-barrel that of the bullet; the leverage increases the range. There are also other weapons which are designed for use at close range-the long spear, the club, and, what is most indicative of battle, the shield. The latter cannot possibly be a hunting implement, as might still be the case with the spear and the club, but is a form of weapon specifically intended for battle. The shield of the Australian is long, and usually raised toward the centre. It covers the entire body, the enemy being attacked with spear or club. Thus, the weapons reflect a condition of tribal warfare.

The second great stage of culture, which we may call, though somewhat inaccurately, the Malayo-Polynesian, offers a radically different picture. To a certain extent, the relation between tribal organization and external culture is here the reverse of that which obtains in the Australian world. In Australia, we find a primitive culture alongside of a highly developed tribal organization; in the Malayo-Polynesian region, there is a fairly well developed culture, but a tribal organization which is partly in a state of dissolution and partly in transition to further political and social institutions, including the separation of classes and the rulership of chiefs. Evidently these latter conditions are the result of extensive racial fusion, which is incomparably greater in the Malayo-Polynesian region than in Australia. True, we no longer harbour the delusion that Australia is inhabited 
by a uniform population. It also has been subject to great waves of immigration, particularly from New Guinea, from whence came the Papuans, one of the races which itself attained to the Malayo-Polynesian level of culture. Naturally the Papuan influx affected chiefly the northern part of Central Australia. The Tasmanian tribe, now extinct, was probably a remnant of the original Australian population. But migrations and racial fusions have caused even greater changes among those peoples who, culturally, must be classed with the Malayo-Polynesians. Here likewise there are many different levels, the lowest of which, as found among the Malayo-Polynesian mixed population, was yet but slightly higher, in some respects, than Australian culture, whereas the culture of the true Malays and Polynesians has already assumed a more advanced character. Ethnology is not yet entirely able to untangle the complicated problems connected with these racial fusions. Much less, of course, can we undertake to enter into these controversial points. We here call attention merely to certain main stages exhibited by the external culture of these peoples, quite aside from considerations of race and of tribal migrations. The Negritos and the Papuans of various parts of Melanesia possess a culture bordering on the primitive-indeed, they may even be characterized as primitive, since they possess characteristics of pretotemic society. Of these tribes, the Papuans of New Guinea and of the islands of the Torres Straits clearly manifest totemic characteristics, while yet possessing special racial traits that are exceptionally pronounced. They differ but little from primitive man, however, so far as concerns either their method of securing food or their dress, the latter of which is exceedingly scanty and is made, for the most part, of plant materials. But these peoples, just as do the Australians, have weapons indicative of battles and migrations; moreover, they exhibit also other marks of a somewhat developed culture. The Papuans are the first to change the diggingstick into the hoe, a useful implement in tilling the soil. In this first form of the hoe, the point is turned so as to form 
an acute angle with the handle to which it is attached. Hence the soil is not tilled in the manner of the later hoeculture proper; nothing more is done than to draw furrows into which the seeds are scattered. In many respects, however, this primitive implement represents a great advance over the method of simply gathering food as practised when the digging-stick alone was known. It is the man who makes the furrows with the hoe, since the loosening of the ground requires his greater strength ; he walks ahead, and the woman follows with the seeds, which she scatters into the furrows. For the first time, thus, we discern a provision for the future, and also a common tilling of the soil. The gathering of the fruits generally devolves upon the woman alone. But even among the Papuans this first step in the direction of agriculture is found only here and there. The possibility of external influences therefore remains.

Far superior to the Papuan race is the Micronesian population, which, as regards its racial traits, is intermediate between the Melanesians and the Polynesians. Migration and racial fusion here become increasingly important cultural factors. In their beginnings, these factors already manifest themselves in the wanderings of the Papuan and Negrito tribes. One of the most striking discoveries of modern ethnology is the finding of distinct traces of Papuan-Negritic culture in regions, such as the west coast of Africa, which are very remote from the original home of the culture in question. The Papuan races likewise wandered far across the Indian Ocean. Obviously there were Papuan migrations, probably in repeated trains, from New Guinea across the Torres Strait to Northern Australia, where they seem to have influenced social institutions and customs as well as external culture. Above the level of the Negrito and Papuan peoples, who, in their numerous fusions, themselves form several strata, we finally have the Malayo-Polynesian population. The Malayo-Polynesians are widely spread over the tropical and sub-tropical regions of the earth. Because of their significance for the particular stage of totemism now under 
discussion, we have called the entire cultural period by their name. The fragments of the Negrito and Papuan races, which are scattered here and there over limited sections of the broad territory covered by the wanderings of these tribes, apparently represent remnants of the original inhabitants. As the result of long isolation, certain groups of these peoples have remained on a very primitive plane, as have, for example, the above-described inland tribes of Malacca, or the peoples of Ceylon and of other islands of the Indian archipelago. Others have mingled with the Malays, who have come in from the mainland of India, and with them have formed the numerous levels and divisions of the Malayo-Polynesian race. This accounts for the fact that this Oceanic group of peoples includes a great many forms of culture, which are not, however, susceptible of any sharp demarcation. The culture of the Negritos and the Papuans, on the one hand, is as primitive as is that of the Australians -indeed, isolated fragments of perished races were even more primitive than are the Australians; on the other hand, however, some of the Malayo-Polynesian peoples are already decidedly in advance of any other people whose culture falls within the totemic age.

The chief ethnological problem relating to these groups of peoples concerns the origin of the Malays, who, without doubt, have given the greatest impetus to the cultural development of these mixed races. This problem is as yet unsolved, and is perhaps insolvable. The Malay type, however, particularly on its physical side, points to Eastern Asia. The resemblance to the Mongolians as regards eyes, skull, and colour of skin is unmistakable. At the same time, however, the original Malays probably everywhere mixed with the native inhabitants, remnants of whom have survived in certain places, particularly in the inaccessible forest regions of the Malayan archipelago. Now, the Malays were obviously, even in very early times, a migratory people. Their wanderings, in fact, were far more extensive than any other folk-migrations with which we are familiar in the history of Occidental peoples. Start- 
ing, as we may suppose, in Central Asia, that great cradle of the human race, they spread to the coasts, particularly to Indo-China, and then to the large islands of Sunda, Sumatra, and Borneo, to Malacca, and, farther, over the entire region of Oceania. Here, by mixture with the native population, they gave rise to a new race, the Polynesians proper. But the Polynesian portion of the race also preserved the migratory impulse. Thus, the Malayans were the first to create a perfected form of boat, and to it the Poly:nesians added many new features. Thenceforth the Malay was not restricted to dangerous coast voyages, as was the case with the use of such boats as those of the Australians or the Papuans of New Guinea. It was a boat of increased size, equipped with sails and oars and often artistically fitted out, in which the Malay traversed the seas. With the aid of these boats-which were, at best, small and inadequate for a voyage on the open sea-and at a time when the compass was as yet unheard of and only the starry heavens could give approximate guidance to their course, the Malays and Polynesians traversed distances extending from the Philippines to New Zealand. Of course, these expeditions advanced only stage by stage, from island to island. This is shown by the legends of the Maoris of New Zealand, who were clearly the first of the Polynesians to migrate, and who therefore remained freest from mixture with strange races. The same fact is attested by the great changes in dialect which the Malayan language underwent even in the course of the migrations of the Malays-changes which lead us to infer that to many of the island regions settled by these peoples there were repeated waves of immigration separated by intervals of centuries.

Connected with this is a further important factor-one which exercised a destructive influence upon the original totemism, only a few traces of which have survived among. these tribes. The boatman, alone on the broad seas, with only the starry firmament to direct his course, turns his gaze involuntarily to the world of stars which serves as his guide. Thus, particularly in Polynesia, there sprang up 
a celestial mythology. This, in turn, again reacted upon the interpretation of terrestrial objects. By breaking up tribes and their divisions, furthermore, the migrations destroyed the former tribal organization and, through the influence gained by occasional bold leaders on such expeditions, gave rise to new forms of rulership. An added factor was the change of environment, the effect of which was noticeable even at the beginning of totemic culture in the influence which the Papuan migration exercised upon the northern parts of Australia-the parts most accessible to it. The Oceanic Islands are as poor in animal life as they are rich in plants. The totemic ideas prevalent in these regions, therefore, came more and more to lose their original basis. This accounts for the fact that the entire domain is characterized by two phenomena which are far in advance of anything analogous that may be found on similar cultural levels in other parts of the earth. One of thesenamely, the development of a celestial mythology-scarcely occurs anywhere else in so elaborate a form. Of course, we also find many clear traces of the influence of celestial phenomena in the mythological conceptions of the Babylonians and Egyptians, of the Hindoos, the Greeks, the Germans, etc. But the elements of celestial mythology have here been so assimilated by terrestrial legend-material and by heroic figures as to be inseparable from them. Thus, the celestial elements have in general become secondary features of mythological conceptions whose characteristic stamp is derived from the natural phenomena of man's immediate environment. Even the celestial origin of these elements has been almost entirely lost to the popular consciousness which comes to expression in the legend. The case is entirely different with the celestial mythology of the Polynesians, particularly as it occurs in the legends of the Maoris. In the latter, the celestial movements, as directly perceived, furnish a large part of the material for the mythical tales. These deal with the ascent of ancestors into the heavens or their descent from heaven, and with the wanderings and 'destinies of the original ancestors, who are regarded 
as embodied in the sun, moon, and stars; thus, they differ from the mythologies of most cultural peoples, in that they are not simply deity legends that suggest celestial phenomena in only occasional details. Moreover, no mention of ancestral or totem animal occurs in Polynesian mythology. There are only occasional legends, associated with the mighty trees of this island-world, that may perhaps be traceable to the plant totems of Melanesia. Such being the conditions, it might seem that, in any case, we are not ijustified in including the entire Malayo-Polynesian culture within the totemic age. Nevertheless, quite apart from the fact that the other phases of external culture are all such as indicate the totemic stage of development, the obviously primitive character of the celestial legends themselves-for they have not as yet developed true hero and deity conceptions-marks this culture as one of transition. Its totemic basis has almost disappeared; yet the earlier manner of securing food, the modes of dress, the decoration, and the belief in spirits and magic have essentially remained, even though decoration and weapons, particularly, have undergone a far richer development. Thus, the external decoration of the body reached its highest perfection in the artistic dotpatterns exemplified in the tattooing of the Polynesians. The origin of this bodily adornment is here again probably to be traced to magical beliefs. The Polynesians also possess carved wooden idols and fantastically shaped masks. To the bow and the lance they have added the knife and the sword ; to the long shield, the small, round shield, which serves for defence in the more rapid movements of single combat. Many localities also have a peculiar social institution, likewise bound up with the development of warfare initiated by migration and strife. This institution consists in an exclusive organization comprising age-groups and the men's club. The latter, in turn, are themselves symptomatic of the disintegration of the original totemic tribal divisions. There is, moreover, one further custom, taboo, which has grown up under totemic influences and has received its richest development with manifold transformations and ramifica- 
tions within this very transitional culture of Polynesia. The earliest form of taboo, which consists in the prohibition of eating the flesh of the totem animal, has, it is true, disappeared. But the idea of taboo has been transferred to a great number of other things, to sacred places, to objects and names, to the person and property of individuals, particularly of chiefs and priests. The tremendous influence of these phenomena, whose origin is closely intertwined with totemism, clearly shows that this entire culture belongs essentially to the totemic age.

Very different is the third stage of totemic culture. As was remarked above, this falls into two essentially distinct divisions of apparently very different origin. American culture, on the one hand, represents a remarkable offshoot of totemic beliefs; besides this there is the African culture, which, because of peculiar conditions, again connected with racial fusion, is, in part, far in advance of the totemic age, though in some details it clearly represents a unique development of it. To one who wishes to gain a coherent picture of totemic culture, nothing, indeed, is more surprising than the fact that foremost among the peoples who may be regarded as the representatives of this great epoch are the Australians. Strange to say, the condition of the Australians approximates to that of primitive man. On the other hand, the North American Indians, particularly those of the Atlantic Coast regions, may be classed among semicultural peoples, and yet they seem, at first glance, to have made exactly the same social application of totemic ideas as have the Australians. The typical tribal organization of the Australians and that of the Iroquois tribes who formerly lived in the present state of New York, are, in fact, so very similar that a superficial view might almost cause them to appear identical. This is all the more surprising since we have not the slightest ground for supposing any transference of institutions. That which makes the similarity so striking is primarily the fact that the single groups or clans are designated by animal names, that they entertain the conception of an animal ancestor, and that the regular tribal 
organization is based on the principle of dual division. Nevertheless, the more advanced culture of the Iroquois has already led to certain changed conditions. The animal ancestor recedes to some extent. In its stead, there are associated with the animal other conceptions, such as are connected with more systematically conducted hunting. The American Indian, in contrast to the Australian, no longer regards the totem animal as a wonderful and superior being, to be hunted only with fear and not to be used for food if this can possibly be avoided. He requires for his subsistence all the game available. Hence he does not practise the custom of abstaining from the flesh of the totem animal. On the other hand, he observes ceremonies of expiation, such as are unknown to the Australian. The totem ceremonies of the latter are chiefly objective means of magic designed to bring about the increase of the totem animals. This idea appears among the Indians likewise. Their totem ceremony, however, has also an essentially subjective significance and is concerned with the past no less than with the future. Its object is to obtain forgiveness for the slaying of the animal, whether this has preceded or is to follow the act of expiation. Connected with these customs is a further difference, which is seemingly insignificant but which is nevertheless characteristic. Whereas the Australian, in many regions, thinks of the totem animal as his ancestor, the Indian of the prairies speaks of the buffaloes as his elder brothers. Thus, among the Indian tribes, man and animal still stand on an equal footing. Hence the animal must be conciliated if it is to serve as food for man. In many of the myths of the American Indians, a man is transformed into an animal or, conversely, an animal assumes the human form. Hand in hand with this change in cult ideas and customs appear the richer forms of external culture. The weapons are perfected; dress becomes more complete; decoration of the body itself, though it does not disappear, more and more finds its substitute in the rich embellishment of the clothing. Social organization becomes stable, and advances beyond the 
original tribal limits. The tribes choose permanent chieftains and, in times of war, enter into group alliances with one another. Thus, tribal organization paves the way for the formation of States, though fixed rulership has not as yet been established. In so far, the democratic organization of North America later instituted by the Europeans, shows a trace of similarity to the free tribal alliances of the natives who had inhabited the country for centuries. For the most part, moreover, the Indians were familiar with agriculture, though, of course, in the primitive form of hoeculture. Man himself tilled his field with the hoe, since plough and draught animals were wanting. But a firmer organization is revealed in the fact that the individual did not go to the field alone, followed by the woman who scatters the seed, but that the land was prepared by the common labour of the clan. This caused the rise of great vegetation festivals, with their accompanying ceremonies. In external details also these far surpassed the cult festivals which the Australians hold in connection with the adolescence of the youths or for the purpose of multiplying the animal or plant totems which serve as human food.

The conditions differ in the southern and, to some extent also, in the western portion of the great American continent. Closely related as the various tribes are, the old hypothesis that they migrated from Asia across Behring Strait is untenable. Moreover, in spite of their physical relationship and, in part also, of their linguistic similarities, their culture shows important differences. In the southern and central parts of America particularly, we find widely different cultural levels, ranging from the forest Indians of Brazil, who have made scarcely any essential advance beyond the primitive culture of the Veddahs or of the natives of Malacca, to the tribes of New Mexico and Arizona, who have obviously been influenced by the cultural peoples of the New World, and, under this influence, have undergone an independent development. All advances that they have made, however, clearly depend upon the development of agriculture. In addition to numerous elements of celestial 
mythology that have found their way from Mexico, we find vegetation cults and agricultural ceremonies. The latter are often closely fused with the borrowed mythology, particularly among the semi-cultural peoples of the central region of America. These cults-sometimes governed by totemic conceptions, while in other cases dominated by celestial mythology-underlie the development of art throughout the whole of America. Whereas the chief expression of the æsthetic impulse in Polynesia is the decoration of the body, particularly by means of tattooing, this practice is secondary, in the case of the American Indian, to the possession of external means of adornment. It is primarily the beautiful plumage of the bird kingdom that furnishes the decorations of the head and of the garment. At the ceremonies of the Zunis and other New Mexican tribes, the altars are decked with the feathers of birds. These festivals exhibit a wealth of colour and a complexity of ceremonial performances that have always aroused the astonishment of the strangers who have been able to witness them. The decoration of garments, of altars, and of festal places is paralleled in its development by that of the pictorial decoration of clay vessels. Here for the first time we have a developed art of ceramics which employs ornamentations, pictures of totemic animals, and combinations of the two or transitional forms. Originally, no doubt, these ornamentations were intended as means of magic, but they came more and more to serve the purposes of decoration. All of these factors exert an influence on the numerous cult dances. All over America, from the Esquimos in the north far down to the south, a very important part of the equipment of the dancers is the mask. This mask reproduces either animal features or some fantastic form intermediate between man and animal. Thus, this culture is of a peculiar nature. Even externally it combines the huntsman's culture with that of the tiller of the soil, although in its agriculture it has not advanced beyond the level of hoe-culture. As compared with Malayo-Polynesian culture, however, it presents an important additional factor. This consists in the com- 
munity of labour, which is obviously connected with the more stable tribal organization and with the development of more comprehensive cult associations. It is this factor that accounts for those great cult festivals that are associated with sowing and harvest and that extend far down into the higher civilizations, as numerous rudimentary customs still testify.

The changes which we likewise find in mythological conceptions also carry us beyond the narrow circle of original totemism. Again there appear elements of a naturemythology, particularly of a celestial mythology. These supplant the animal cult, but nevertheless retain some connection with the totem animal; the culture is one in which the totem animal never entirely loses its earlier significance. Thus, the vegetation festivals, especially those of North and Central America, exhibit many cult forms in which ideas that belong to a celestial mythology combine with the worship of animals and of ancestors. The conceptions of ancestors and of gods thus play over into one ariother, and these godancestors are believed to have their seat in the clouds and in the heavens above. However constantly, therefore, totemic ideas may be in evidence within the field of external phenomena, a much superior point of view is attained, by the American races, as regards the inner life.

Among the African peoples we find the second important form of culture belonging to this third stage-a culture which in many respects diverges from the one which we have just described. More clearly even than in the case of America has the idea been disproven that the inhabitants of the interior of Africa are essentially a homogeneous race that has developed independently of external influences. Even more than other peoples, the Africans show the effects of great and far-reaching external influences. Hamitic and Semitic tribes entered the country from the north at an early time; even from the distant south of Asia, probably from Sumatra and its neighbouring islands, great waves of immigration, crossing Madagascar in the 
distant past, swept on towards the west even to the Gold Coast, introducing elements of Papuan-Negritic culture into Africa. There were frequent fusions between these tribes and the negro peoples proper, as well as with the Hamites, the Semites, and also with those who were probably the original inhabitants of this region, remnants of whom are still to be found in the Bushmen. The negro race, which, relatively speaking, has remained the purest, lives in the Soudan region; the Bantus inhabit the south of Africa ; the north is occupied mostly by Hamitic tribes, whose advent into this region was followed by that of a people of related origin, the Semites. Corresponding to the racial mixtures that thus arose, there are various forms of culture. As regards the Bantus, it is highly probable that they are a mixed people, sprung from a union of the Soudan negroes with the Hamites. That the Hamites pressed on, in very early times, into southern Africa, is proved by the Hottentot tribe, whose language exhibits Hamitic characteristics, and the colour of whose skin, furthermore, is lighter than that of the negro proper or that of the Bantu. The language of the Bantus shows traits resembling partly the negro idioms of the Soudan and partly Hamitic-Asiatic characteristics. The element of culture, however, which is peculiar to the Hamites and which was introduced by them into the northern part of the continent, is the raising of cattle and of sheep. There can be scarcely any doubt that the African cattle originally came from Asia. Probably, however, cattle were brought to Africa on the occasion of two different Hamitic migrations; this is indicated by the fact that two breeds of cattle are found in Africa. Moreover, it is clear that, at the time of their introduction, cattle were not totem animals, but had already gained a position intermediate between the totem and the breeding animal. The Hottentot, as well as the Bantu, prizes his cattle as his dearest possession. Since, however, he slaughters them only in times of extreme necessity, he has progressed only to the point of obtaining a milk supply. Yet even this represents 
an important advance. Owing to his efforts, the cow no longer merely provides the calf with milk, as in the natural state, but, long after the time of suckling has passed, places the milk at man's disposal. Everywhere in the interior of Africa the cow is still a common milk animal. As such, it is a highly prized source of nourishment, but it is not used for agricultural purposes. Thus, its position is midway between that of the original totem animal of cult and that of the draught animal. For the Hottentot, cattle are objects of supreme value. As such, they are accorded a certain degree of reverence. They are not utilized as beasts of burden nor for slaughter, but only as a source of such means of nourishment as do not cost their lives. South Africa, therefore, has remained on the level of hoe-culture. The boundary between these southern districts in which hoe-culture and the nomadic life prevail and the northern regions into which the Hamites and Semites have introduced plough-culture is, practically speaking, the desert of Sahara. It is only, when the animal is used to draw the plough that it becomes in all respects a useful animal. Thenceforth it no longer merely gives its milk for food, but it performs the work that is too hard for man, and, finally, as an animal of slaughter, it takes the place of the gradually, disappearing wild animal of the chase. Coincident with this development, totemic ideas and customs disappear. Though these have still left distinct traces in the south, particularly, among the Bantus, it is, at most, isolated survivals that remain among the Hamitic population of the north.

Thus, the animal has come to be a breeding and a work animal throughout the whole of Africa, though this is particularly the case wherever the cultural influences of the immigrant peoples from the East have been operative. The relations of man to man have likewise undergone a change in this locality, due, in part, to migrations and tribal wars. No region so much as Africa has become the centre of despotic forms of government. It is this factor, together with the potent influence of ideas of personal property associated 
with it, that has contributed, on the one hand, to the origin of polygyny, and, on the other, to the rise of slavery. Long before Africa became the slave market of the New World it harboured an intertribal traffic in human beings. These changes in culture undermined the older cults, so that, with the dissolution of the totemic tribal organization, the original totem conceptions disappeared from all parts of this region. All the more marked was the progress of animism and fetishism, of which the former is closely connected, in its origin, with totem belief, while the latter is a sort of degenerate totemism. In certain regions, furthermore, as among the Bantus and the Hamitic tribes, another outgrowth of the cult of the dead-namely, ancestor worshiphas gained great prominence alongside of elements of a celestial mythology.

To a far greater extent than in Africa, totemic culture has almost entirely disappeared throughout the entire Asiatic world. Only in the extreme north among the Tchuktchis, the Yakutes, and Ghilyaks, and in the far south among the Dravidian tribes of Hindustan who were pushed back by the influx of Hindoos, have remnants of totemic institutions survived. In addition to these, only scanty fragments of totemism proper may be found in Asia-the home of the great cultural peoples of the Old World. Surviving effects of totemic culture, however, are everywhere apparent, no less in the sacred animals of the Babylonians, Egyptians, Hindoos, Greeks, and the Germanic peoples, than in the significance attached by the Romans to the flight of birds and to the examination of entrails, and in the Israelitic law which forbids the eating of the flesh of certain animals.

In the light of all these facts, the conclusion appears highly probable that at some time totemic culture everywhere paved the way for a more advanced civilization, and, thus, that it represents a transitional stage between the age of primitive man and the era of heroes and gods. 


\section{TOtemic TRibal Organization. ${ }^{\text {I }}$}

As has already been stated, the beginning of the totemic age is not marked by any essential change in external culture. As regards dress, decoration, and the acquisition of food, the conditions that we meet, particularly among the natives of Central Australia, differ scarcely at all from those of the primitive races of the pretotemic age. It is only in the weapons, which are already clearly indicative of tribal warfare, that we find an unmistakable external indication of deeper-going differences in social culture. At the same time, however, the totemic age includes peoples whose general manner of life we are accustomed to call semicultural. The greatest contrast occurs between the natives of Australia and of some of the portions of Melanesia, on the one hand, and those of North America, particularly of the eastern part, on the other. While the former still live the primitive life of the gatherer and the hunter, the latter possess the rudiments of agriculture, as well as the associated cult festivals, the beginnings of a celestial mythology, and richer forms of legend and poetry. Nevertheless, as regards the most universal characteristic of totemic culture, namely, the form of tribal organization, the two groups of peoples differ but slightly, although conditions in Australia have on the whole remained more primitive. This is most clearly shown by the fact that, among the Australian natives, the totem animal possesses the significance of a cult object, whereas in America, and particularly among the Atlantic tribes, whose totemic practices have received the most careful study, the totem animal has obviously come to be a mere coat of arms. The difference might, perhaps, be briefly stated thus: In Australia, the totem names signify groups of cult members within a clan; in America, they are the

The survey presented in this and in the following section aims to give only a general outline of the relations between totemism and tribal organization, as based particularly on several tribes of Central Australia. For a more detailed account of the conditions and of their probable interpretation, I would refer to a paper on "Totemism and Tribal Organization in Australia," published, in 1914, in Anthropos, an international journal. 
designations of clans themselves, but these as such possess no cult significance. In both regions, however, tribal organization follows the principle of dual division. The tribe first divides into two tribal halves (I and II); then each of these separates into two clans (A and B, C and D); finally, the latter again break up into subclans, so that eventually we may have eight tribal divisions. In certain cases, the division has not advanced beyond the dual form ; the upper limit, on the other hand, seems to be eight distinct groups. The schemata representing tribal organization in Australia and in America are so similar that it is easy to

\section{$\frac{\mathrm{Kam} \mathrm{i} \text { l a } r o \mathrm{i}}{\text { (central Australians) }}$}

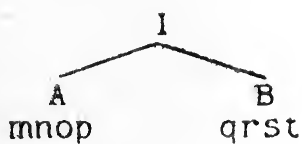

mnop qrst

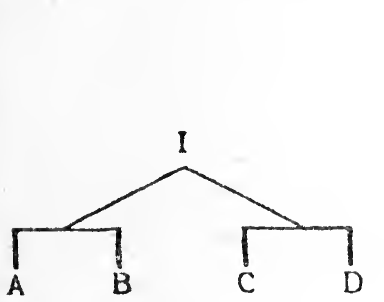

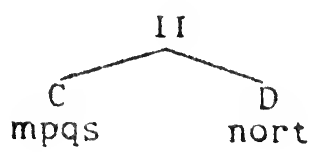

$\frac{\mathrm{sen} e \mathrm{e} a}{\text { (Iroquois) }}$

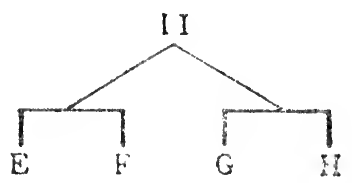

understand how most authors have come to regard conditions in the two countries as essentially identical. Yet the divergence in the nomenclature of the tribal divisions points to significant differences. The fact is that the clan names of the Australians are entirely different from the totem names. The former have, as a rule, become unintelligible to the present-day native, and, since many of them recur among distinct tribes who now speak different dialects, they probably derive from an older age. Words such as Ipai, Kumbo, Murri, Kubbi, etc., may originally, perhaps, have possessed a local significance. At any rate, clan names but rarely consist of the names of animals. On the other hand, such words as emu, kangaroo, opossum, eagle-hawk, and others, 
are the regular designations of the clans composing the totem groups. The case is otherwise among the North American Indians. Here the clans all have animal names. Nor can we anywhere find alongside of the clans any particular totem groups which might be regarded as cult alliances. The schema shown on p. I 4 I exhibits these relations. 'The tribal halves are designated by I and II, the clans by A, B, C, etc., and the independent totem groups existing within the individual clans by $m, n, o, p$, etc.

Owing to the external similarity of the tribal organizations, it has generally been thought that the totem groups of the Australians are merely clans or subclans, such as are, doubtless, the social groups of the American Indians, designated by similar totem names. This interpretation, however, has unquestionably led to serious confusion, particularly in the description of the tribal organization of the Australians. A study of the detailed and very valuable contributions of Howitt and of other early investigators of the sociological conditions of Australia, inevitably leaves the impression that, particularly as regards the interpretation of the various group names, the scholars were labouring under misconceptions which caused the relations to appear more complex than they really are. Such'misconceptions were all the more possible because the investigators in question were entirely ignorant of the languages of the natives, and were therefore practically dependent upon the statements of their interpreters. Under these circumstances we may doubtless be allowed a certain degree of scepticism as to the acceptance of these reports, especially when they also involve an interpretation of phenomena; and we may be permitted an attempt to discover whether a different conception of the significance of the various group names may not give us a clearer picture of the phenomena, and one that is also more adequate when the general condition of the inhabitants is taken into account. The conditions prevalent among the American Indians are in general much easier to understand than are those of the Australians, particularly where the old 
tribal organization has been preserved with relative purity, as among the Iroquois. In this case, however, the totem names have obviously become pure clan designations without any cult significance. Now this has not occurred among the Australians; for them, the totem animal has rather the status of a cult object common to the members of a group. The fact that the Australians have separate names for the clans, as was remarked above, whereas the American Indians have come to designate clans by totem names, provides all the more justification for attributing essentially different meanings to the two groups that bear totem names. In attempting to reach a more satisfactory interpretation of totemic tribal organization, therefore, we shall consider those totem groups which are obviously in a relatively early stage of development-namely, the Australian groups-simply as cult associations which have found a place within the tribal divisions or clans, but whose original significance is of an absolutely different nature. In the above schema, therefore, A, B, C, D, etc., represent tribal divisions or clans, $m, n, o, p$, etc., cult groups. The latter are lacking in the part of the diagram which refers to the American Indians, since these have no cult associations that are independent of the tribal divisions; indeed, the old totem names have lost their former cult significance and have become mere clan names. Thus, the conception here advanced differs from the usual one in that it gives a different significance to the totem names on the two levels of development. In the case of the Australians, we regard them as the names of cult groups ; in America, where the totemi cult proper has receded or has disappeared, we regard them as mere clan names. But the extension of totem names to the entire clan organization in the latter case is not, as it were, indicative of a more developed totemism', but rather of a totemism in the state of decline. The totem animal, though here also at one time an object of cult, is such no longer, but has become a mere coat of arms. In support of this view of American totem names, we might doubtless also refer to the so-called totem poles. Such a pole consists 
of a number of human heads representing the ancestors of the clan, and is crowned by the head of the totem animal. This is obviously symbolic of the idea that this succession of generations has as its symbol the totem animal that surmounts it-that is, the totem pole is an enlarged coat of arms.

Because of the great regularity of its occurrence, the dual form of tribal division must be regarded as everywhere due to the same cause. Concerning its origin there can scarcely be any doubt. Obviously it has no real connection with totemism itself. This explains why the tribal divisions originally derived their names, not from the totem, but from localities or from other external sources, as the conditions among the Australians would seem to indicate. A phenomenon which recurs in widely distant regions with such regularity as does dual division, is scarcely intelligible except by reference to the general conditions attendant upon the spread of peoples. A tribe leading the unsettled life of gatherers and hunters must of inner necessity separate as its numbers increase or as the food-supply begins to fail. It is but natural that the tribe should first separate into two divisions on the basis of the hunting-grounds which the members occupy; the same process may then repeat itself in the case of each division. The fact that when deviations from the principle of dual division are found, they äre most likely to occur in the subordinate groups, is also in harmony with the view that the divisions are due to the natural conditions of dispersion. For, in the case of the subordinate groups, one of the smaller units might, of course, easily disintegrate or wander to a distance and lose its connection with the tribe.

\section{The Origin OF EXogamy.}

Though the dual organization of the tribe seems to admit of a comparatively simple and easy explanation, the totemic exogamy which is closely bound up with it offers great difficulties. As we have already seen, totemic exogamy is characterized by the fact that a member of one specific clan, or of a totem group belonging to the clan, may enter into marriage 
only with a member of another clan or totem group. This restriction of the marriagie relationship is generally known as 'exogamy,' a term first introduced by the Scottish ethnologist and historian, McLennan. In order to distinguish this custom from later regulations of marriage, such, for example, as exist in present law, in the prohibition of the union of relatives by blood or by marriage, we may call it more specifically 'totemic exogamy.' Totemic exogamy clearly represents the earliest form of marriage restriction found in custom or law. The phenomena bound up with it may be regarded as having arisen either contemporaneously with the first division of the tribe or, at any rate, soon thereafter, for some of the Australian and Melanesian tribes practise exogamy even though they have not advanced beyond a twofold division of the tribe. On the other hand, the primitive horde of the pretotemic age remains undivided, and, of course, shows no trace of exogamy. True, marriages between parents and children seem to have been avoided as early even as in pretotemic times. But this could hardly have been due to the existence of firmly established norms of custom. Such norms never developed except under the influence of totemic tribal organization, and they are closely related to its various stages of development.

Taking as the basis of consideration the above-mentioned conditions in Australia, where an approximate regularity in the successive stages of this development is most clearly in evidence, we may distinguish particularly three main forms of exogamy. The first is the simplest. If we designate the two divisions of the tribe between which exogamic relations obtain, by $A$ and $B$, and the various subgroups of A by $l, m, n, o$, and of B by $p, q, r, s$, we have, as this simplest form, unlimited exogamy. It corresponds to the following schema :-

I. Unlimited Exogamy.

A

$l m n o$

$\mathrm{B}$

$p q r s$ 
This means : A man belonging to Class A may take in marriage a woman from any of the subgroups of Class B, and conversely. Marriage is restricted to the extent that a man may not take a wife from his own class; it is unrestricted, however, in so far as he may select her from any of the subgroups of the other class. This form of exogamy does not appear to occur except where the divisions of the tribe are not more than two in number. The marriage classes, $A$ and $B$, then repnesent the two divisions of the tribe; the subgroups $l, m, n, o, p, \ldots$. are totem groups-that is to say, according to the view maintained above, cult groups. For the most part, marriage relationships between the specific cult groups meet with no further restrictions. A man of Class A may marry a woman belonging to any of the totem groups $p, q, r, s$, of Class $B$-it is only union with a woman belonging to one of the totem groups of Class $A$ that is denied him. Nevertheless, as we shall notice later, we even here occasionally find more restricted relations between particular totem groups, and it is these exceptions that constitute the transitional steps to limited exogamy. Such transitions to the succeeding form of exogamy are to be found, for example, among the Australian Dieri, some of whose totem groups intermarry only with some one particular group of the other tribal division.

The second form of exogamy occurs when a member of Class A is not allowed to take in marriage any woman he may choose from Class $B$, but only one from some specific subgroup of B. For example, a man of group $n$ is restricted to a woman of group $r$.

\section{Limited Exogamy with Direct Maternal or Paternal Descent.}

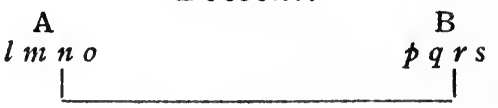

Both forms of exogamy, the unlimited and the limited, observe the same law with respect to the group affiliation of children. If, as universally occurs in Australia, A and B are clans having exogamous relations, and $l, m, n, o, p, \ldots$ 
are totem groups within these clans, then, if maternal descent prevails, the children remain both in the clan and in the totem of the mother ; in the case of paternal descent, they pass over to the clan and to the totem of the father. Of these modes of reckoning descent, the former is dominant, and was everywhere, probably, the original custom. One indication of this is the connection of paternal descent with other phenomena representing a change of conditions due to external influences-the occurrence of the same totem groups, for example, in the two clans, A and B, that enjoy exogamous relations. The latter phenomenon is not to be found under the usual conditions, represented by diagrams I and II. In the case of unlimited exogamy (I), no less than in that of limited exogamy, we find that if, for example, maternal descent prevails, and the mother belongs to clan $\mathrm{B}$ and to totem group $r$, the children likewise belong to this group $r$. This condition is much simplified in the case of the American Indians. With them, totem group and clan coincide, the totem names having become the names of the clans themselves. The particular totem groups, $l, m, n, o, p, \ldots$ do not exist. Exogamous relations between clans $\mathrm{A}$ and $\mathrm{B}$ consist merely in the fact that a man of the one clan is restricted in marriage to women of the other clan. Wherever maternal descent prevails, as it does, for example, among the Iroquois, the children are counted to the clan of the mother; in the case of paternal descent, they belong to the clan of the father.

In the Australian system, howrever, which distinguishes clan and totem, and therefore, as we may suppose, still exemplifies, on the whole, an uninterrupted development, we find also a third form of exogamous relationship. This last form of exogamy seems to be the one which is most common in Australia, whereas, of course, it has no place in the pure clan exogamy of the American Indians. The system indicated in diagram II, in which children belong directly to the clan of the mother in maternal descent. and to that of the father in paternal descent, may be designated as limited exogamy with direct maternal or paternal 
descent. There developed from this a third system, in which, while the children are counted to the clan of the parent who determines descent, they nevertheless become members of a different totem group. Thus arises a limited exogamy with indirect maternal or paternal descent, as represented in diagram III.

\section{Limited Exogamy with Indirect Maternal or Paternal Descent.}

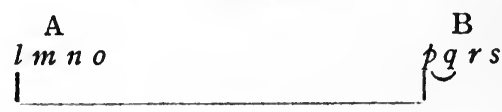

A man of clan A and totem group $l$ may marry only a woman of clan B and totem group $p$; the children, however, do not belong to the totem $p$, but to another specifically defined totem group, $q$, of clan B.

The way in which these various forms of exogamy affect the marriage relations of the children that are born from such unions is fairly obvious. Turning first to form Iunlimited exogamy-it is clear that, in the case of maternal descent, which here appears to be the rule, none of the children of the mother may marry except into the clan of the father; in paternal descent, conversely, they may marry only into the clan of the mother. Marriage between brothers and sisters, thus, is made impossible. Nor may a son marry his mother where maternal descent prevails, or a daughter her father in the case of paternal descient. In the former case, however, the marriage of father and daughter would be permitted, as would that of mother and son in the latter. The marriage of a son or daughter with relatives of the mother who belong to the same clan is not allowed in the case of maternal descent. The son, for example, may not marry a sister of his mother, nor the daughter a brother of the mother, etc. Since it is maternal descent that is dominant in the case of unlimited exogamy, the most important result of the latter is doubtless its prevention of the marriage of brother and sister, in addition to that of a son with his mother. The system of paternal 
descent, of course, involves a corresponding change in marriage restrictions.

What, now, are the results of form II-limited exogamy with direct maternal or paternal descent? It is at once clear that such exogamy prohibits all the various marriage connections proscribed by unlimited exogamy. Marriage between brothers and sisters is rendered impossible, as is also, in the case of maternal descent, that between a son and his mother or the relatives in her clan. Marriage between father and daughter, however, is permitted. Where paternal descent prevails, these latter conditions are reversed. Although forms I and II are to this extent in complete agreement, they nevertheless show a very important difference with respect to the prohibitions which they place on marriage. In unlimited exogamy, a man is at liberty to marry into any totem that belongs to the clan with which his own has exogamous relations; in limited exogamy, however, he may marry into only one of the totems of such a clan. Thus, the circle within which he may select a wife is very materially reduced. Limited exogamy with direct maternal or paternal descent, accordingly, means a reapproach to endogamy. The wife must be chosen from an essentially smaller group, narrowed down, in the case of maternal descent, to the more immediate relatives of the father, or, in paternal descent, to those of the mother. Such a condition is not at all a strict form of exogamy, as is maintained by some ethnologists, but is, on the contrary, something of a return to endogamy. This point is of decisive importance in determining the motives of the remarkable institution of exogamy.

What are the conditions, finally, which obtain in form III-limited exogamy with indirect maternal or paternal descent? It is at once obvious that marriage between brother and sister is here also excluded. Furthermore, another union is prohibited which was permitted' in form II. For son and daughter, in the case of maternal descent, no longer belong to the totem group of the mother, $p$, but pass over into another group, say $q$. Not only, 
therefore, is a son prevented from marrying his mother because they both belong to the same clan, but a father is forbidden to marry his daughter because he may take only a woman of group $p$, to which his wife belongs. No less true is this of the son, who now likewise belongs to group $q$, and may therefore no longer marry a female relative of his father's, since the group $q$ into which he has entered has exogamous connections with another totem group of the paternal clan, say with $m$. With this change a step to a stricter exogamy is again taken; the earlier restrictions on marriage remain, and the possibilities of marriage between relations are further reduced by changing the totem of the children. Cousins may not marry each other. Thus, the limits of exogamy are here narrower than those, for example, which obtain in Germany. It is evident that such limitations might become a galling constraint, particularly where there is a scarcity of women, as is the case, for the most part, in Australia. This has led some of the Australian tribes to the remarkable expedient of declaring that a man is not to be regarded as the son of his father, but, in the case of maternal descent, as the son of his paternal grandfather-a step which practically amounts to transferring him into the totem of his father and allowing him to enter into marriage with his mother's relatives. This circumvention, reminding one of the well-known fictions of Roman law, may have its justification in the eyes of the Australians in the fact that they draw practically no distinction between the various generations of ancestors.

The three forms of exogamy, accordingly, agree, in prohibiting the marriage of brothers and sisters and, in so far as maternal descent may be regarded as the prevailing system, the marriage of a son with his mother. Both these prohibitions, doubtless, and especially the latter, reflect a feeling which was experienced by mankind at an early age. The aversion to the marriage of a son with his mother is greater than that to the marriage of brother and sister or even that of father and daughter. Consider the tragedy of CEdipus. It might, perhaps, be less horrible were it 
father and daughter instead of son and mother who were involved in the incestuous relation. Marriages between brothers and sisters have, of course, sometimes occurred. Thus, as has already been remarked, the Peruvian Incas ordained by law that a king must marry his sister. In the realm of the Ptolemies, likewise, the marriage of brother and sister served the purpose of maintaining purity of blood, and even to-day such marriages occur in some of the smaller despotic negro states. The custom is probably always the result of the subjugation of a people by a foreign line of rulers. Indeed, even the Greeks permitted marriage between half-brothers and half-sisters.

Though these natural instincts were less potent in early times than in later culture, they may not have been entirely inoperative in the development from original endogamy to exogamy. Nevertheless, one would scarcely attempt to trace to the blind activity of such instincts those peculiar forms of exogamy that appear particularly among the Australian tribes. On the contrary, we would here also at once be inclined to maintain that the reverse is true, thus following a principle that has approved itself in so many other cases. The aversion to marriage with relatives has left its impress on our present-day legislation, not so much, indeed, in the positive form of exogamy, as in the negative form which forbids endogamy within certain limits. This aversion, however, is not the source so much as it is the effectat least in great measure-of the exogamous institutions of early culture. All the more important is the question concerning the origin of these institutions. This question, in fact, has already received much attention on the part of ethnologists, particularly since the beginning of the present century, when it has become more and more possible to study the tribal organization of the Australians. Here, however, we must distinguish between the general theories that have been advanced concerning the causes of exogamy as such-theories which date back in part even to a fairly early period-and hypotheses concerning the origin of the various forms of exogamy. 
Exogamy as such has generally been approached from a rationalistic point of view. It has been regarded as an institution voluntarily created to obviate the marriage of relatives, and is supposed to have arisen contemporaneously with another institution of like purpose, namely, tribal division. This view is championed, among other scholars, by the able American sociologist, Lewes Morgan, in his book "Ancient Society" (1870), and even by Frazer in his comprehensive work "Totemism and Exogamy" (1910), which includes in its survey all parts of the earth. Frazer says explicitly: 'In the distant past, several wise old men must have agreed to obviate the evils of endogamy, and with this end in view they instituted a system that resulted in exogamous marriage.' Thus, the determinant motive is here supposed to have been aversion to the marriage of relatives. According to Morgan's hypothesis-an extreme example of rationalistic interpretation-the aversion was due to a gradually acquired knowledge that the marriage of relatives was injurious in its effects upon offspring. The entire institution, thus, is regarded as a eugenic provision. We are to suppose that the members of these tribes not only invented this whole complicated system of tribal division, but that they foresaw its results and for this reason instituted exogamous customs. Were people who possess no name's for numbers greater than four capable of such foresight, it would indeed be an unparalleled miracle. Great social transformations, of which one of the greatest is unquestionably the transition from the primitive horde to totemic tribal organization, are never effected by the ordinances of individuals, but develop of themselves through a necessity immanent in the cultural conditions. Their effects are never foreseen, but are recognized in their full import only after they have taken place. Moreover, as regards the question of the injurious effects resulting from the marriage of relatives, authorities even today disagree as to where the danger begins and how great it really is. That the Australians should have formed definite convictions in prehistoric times with reference to these matters, is absolutely inconceivable. At most, they 
might have felt a certain instinctive repugnance. Furthermore, if these institutions were established with the explicit purpose of avoiding marriage between relatives, the originators, though manifesting remarkable sagacity in their invention, made serious mistakes in their calculations. For, in the first place, the first two forms of exogamy only partially prevent a union which even endogamous custom avoids, namely, that between parents and children; in the second place, the transition from unlimited to limited exogamy with direct maternal or paternal descent does not involve an increased restriction of marriage between relations, but, as we have already seen, marks a retrogression, in the sense of a reapproach to endogamy.

The above view, therefore, was for the most part abandoned in favour of other, apparently more natural, explanations. Of these we would mention, as a second theory, the biological hypothesis of Andrew Lang. This author assumes that the younger brothers of a joint family were driven out by the stronger and older ones in order to ward off any want that might arise from the living together of a large number of brothers and sisters, and that these younger brothers were thus obliged to marry outside the group. Even this, however, is not an adequate theory of exogamy, since it does not explain how the custom has come to apply also to the older members of the family group. As a final hypothesis, we may mention one which may perhaps be described as specifically sociological. In its fundamental aspects it was proposed by MacLennan, the investigator who also gave us the word 'exogamy.' MacLennan does not regard exogamy as having originated in times of peace, nor even as representing voluntarily established norms of custom. $\mathrm{He}$ derives it from war, and in so doing he appeals to the testimony both of history and of legend. As is well known, even the Iliad, the greatest epic of the past, portrays as an essential part of its theme a marriage by capture. The dissension between Achilles and Agamemnon arose from the capture of Briseis, for whom the two leaders of the 
Achæans quarrelled with each other. According to MacLennan, the capture of a woman from a strange tribe represents the earliest exogamy. The rape of the Sabines is another incident suggesting the same conclusion. True, this is not an event of actual history. Nevertheless, legend reflects the customs and ideas of the past. Now, in the case under discussion, it is clear that marriage by capture involves a foreign and hostile tribe, for this is the relation which the Sabines originally sustained to the Romans. A significant indication of the connection between marriage by capture and war with hostile tribes occurs also in Deuteronomy (ch. xxi.), where the law commands the Israelites: "If in war you see a beautiful woman and desire her in marriage, take her with you. Let her for several weeks bewail her relatives and her home, and then marry her. But if you do not wish to make her your wife, then let her go free; you shall not sell her into slavery.' This is a remarkable passage in that it forbids the keeping and the selling of female slaves, but, on the other hand, permits marriage with a woman of a strange tribe. A parallel is found in Judges (ch. xxi.), where it is related that the elders of Israel, being prevented by an oath to Jahve from giving their own daughters in marriage to the children of Benjamin, advised the latter to fall, from ambush, upon a Canaanitic tribe and to steal its maidens.

In spite of all these proofs, exogamy and the capture of women from strange tribes differ as regards one feature of paramount importance. In both legend and history the captured woman is universally of a strange tribe, whereas totemic exogamy never occurs except between clans of the same tribe. Added to this is a further consideration. The above-mentioned passage from Deuteronomy certainly presupposes that the Israelite who captures a wife in warfare with a strange tribe already possesses a wife from among his own tribe. This is his chief wife, in addition to whom he may take the strange woman as a secondary wife. We may refer to Hagar, the slave, and to Sarah, Abraham's rightful wife, who 
belonged to his own tribe. The resemblance between exogamy and the capture of women in warfare is so far from being conclusive that exogamy is permitted only between clans of the same tribal group; hence, in cases where there are four or eight subgroups, it is not even allowed: between members of the two tribal halves. Indeed, the essential characteristic of exogamous tribal organization, marriage between specific social groups, is entirely lacking in the marriage by capture that results from war. Moreover, the woman married under exogamous conditions is either the only wife or, if she is the first, she is the chief wife; in the case of marriage by capture in war, the captured woman is the secondary wife.

\section{Modes of Contracting Marriage.}

Though the theory that exogamy originated in the capture of women in warfare is clearly untenable, it has without doubt seized upon one element of truth. Marriage by capture may also occur within one and the same tribe, and under relatively savage conditions this happens very frequently. Indeed, it is precisely in the case of the Australians, to judge from reports, that such marriage is probably as old as the institution of exogamy itself, if not older. Early accounts, in particular, give abundant testimony to this effect. That later writings give less prominence to the phenomenon does not imply its disappearance. The decreased emphasis is due rather to the fact that in more recent years the attention of investigators has been directed almost exclusively to the newly discovered conditions of tribal organization. Even on a more advanced and semi-cultural stage we find struggles for the possession of a wife. The struggle, however, is regularly carried on, not between members of different groups, much less between entirely strange peoples of widely differing language and culture, but between members of one and the same tribe. Two or more members of a tribe fall into a quarrel for the possession of a woman who, though 
not belonging to their own clan, is nevertheless a member of a neighbouring clan of the same tribe. Such conditions are doubtless to be traced back to earliest times. The victor wins the woman for himself. The custom of marriage by capture has left its traces even down to the present, in practices that have for the most part assumed a playful character. Originally, however, these practices were without doubt of a serious nature, as were all such forms of play that originated in earlier customs. Just as ancient exogamous restrictions are still operative in the prohibitions which the statutes of all cultural peoples place on the marriage of relatives, so the influence of marriage by capture is reflected in some of the usages attending the consummation of marriage, as well as in various customs, such as the purchase of wives and its converse, the dowry, which succeeded marriage by capture. Moreover, the fact that marriage by capture occasionally occurs even in primitive pretotemic culture and that it is practised beyond that circle of tribal organization whose totemic character can be positively proved, indicates that it is presumably older than an exogamy regulated by strict norms of custom. It is just in Australia, that region of the earth where, to a certain extent, the various stages of development of exogamy still exist side by side, that we find other cultural conditions which make it practically impossible to hold that marriage by capture originated in warfare between tribes. Though the woman who is here most likely to become an object of dissension between brothers or other kinsmen may not belong to the same clan and the same totem as the latter, she is nevertheless a member of one of the totems belonging to one of the most closely related clans. A, woman of their own clan is too close to the men of the group to be desired as a wife; a woman of a strange tribe, too remote. In the ordinary course of events, moreover, there is no opportunity for meeting women of other tribes. The slave who is captured in war and carried away as a concubine appears only at a far later stage of culture. The original struggle for the possession of a woman, therefore, was not carried on 
with members of a strange tribe, as though it were to this that the woman belonged. Doubtless also it was only to a slight degree a struggle with the captured woman herself-this perhaps represents a later transference that already paves the way for the phenomena of mere mock-struggles. The real struggle took place between fellow-tribesmen, between men of the same clan, both of whom desired the woman. There is a possibility, of course, that the kinsmen of the woman might oppose her capture. This aspect of the struggle, however, like the opposition of the woman herself, was probably unknown prior to the cultural stage, when the female members of the clan came to be valued, as they are among agricultural and nomadic peoples, because of the services which they render to the family. The theory just outlined, moreover, readily explains the further development of the conditions that precede the consummation of marriage, whereas the theory that marriage by capture originated in warfare is in this respect a complete failure. Valuable information concerning the later stages in the development of the marriage by capture which originates during a state of tribal peace, is again furnished by Australian ethnology. Among these peoples, the original capture has in many instances passed over into an exchange in which the suitor offers his own sister to the brother of the woman whom he desires for himself. If this proposal for exchange is accepted and he has thereby won the kinsmen of the woman to his side, his fellow-contestants may as well give up the struggle. Thus, exogamous marriage by capture here gives way to exogamous marriage by barter, an arrangement in entire harmony with the development of trade in general, which always begins with barter. At the same time, the form of this barter is the simplest conceivable : a woman is exchanged for a woman; the objects of exchange are the same and there is no necessity for estimating the values in order to equalize them.

There may be some, however, who do not possess sisters whom they may, offer in exchange to the men of other clans. What then occurs? In this case also it is in 
Australia that we find the beginnings of a new arrangement. In place of offering his sister in exchange, the suitor presents a gift to the parents of the bride, at first to the mother. Gift takes the place of barter. Since there is no woman who may be bartered in exchange, a present is given as her equivalent. Thus we have exogamous marriage by gift, and, as the custom becomes more general and the gift is fixed by agreement, this becomes exogamous marriage by purchase. The latter, however, probably occurs only at a later stage of culture. The man buys the woman from her parents. Sometimes, as we know from the Biblical example of Jacob and from numerous ethnological parallels, he enters into service in order to secure her-he labours for a time in the house of her parents. In an age unfamiliar with money, one who has possessions purchases the woman with part of his herd or of the produce of his fields. Whoever owns no such property, as, for instance, the poor man or the dependent son, purchases the woman with his labour.

Marriage by purchase, however, does not represent the terminus of the development. On the contrary, it prepares the way for marriage by contract, an important advance that was already, to a certain extent, made by the Greeks, and later particularly by the Romans. Not purchase, but a contract between him who concludes the marriage and the parents of the woman-this is an arrangement which still finds acceptance with us to-day. Now, the marriage contract determines the conditions for both bride and groom, and eventually also the marriage portion which the man brings to the union, as well as the dowry of the wife. As soon, therefore, as property considerations come to be dominant within the field of marriage, marriage by contract opens the way for a twofold marriage by purchase. The man may either buy the woman, as was done in the case of the earlier marriage by purchase, or the woman may buy the man with the dowry that she brings. At first, in the days of marriage by capture, the struggle with fellowclansmen or with strangers was of decisive importance; 
at a later time, however, differences in property, rank, and occupation came to be the determining factors in the case of marriage. Thus, if we regard marriage by gift as a mode of marriage by purchase, though, in part, more primitive, and, in part, more spontaneous, our summary reveals three main stages: marriage by capture, marriage by purchase, and marriage by contract. Between these modes of marriage, of course, there are transitional forms, which enable us to regard the course of development as constant. The fact, however, that the entire development bears the character of a more or less-thorough-going exogamy, is due to the oldest of these modes of marriage - a mode which, as we may assume, was prevalent at the beginning of the totemic age. This is a form of marriage by capture in which the woman belonged, not to a strange tribe, but to a neighbouring clan of the same tribe, or to one with which there were other lines of intercourse. When capture disappeared, the exogamy to which it gave rise remained. The old customs connected with the former passed over, though more and more in the form of play, into the now peaceful mode of marriage by purchase ; their survivals continued here and there even in the last form of marriage, that by contract.

\section{The Causes of Totemic Exogamy.}

How does this general development of the modes of marriage account for those peculiar laws of exogamy which are universally characteristic of totemic culture, representing strict norms of custom that forbid all marriage except that between specific clans of a tribe, or even only between pairs of totem groups of different clans? Were these marriage ordinances, which have evidently arisen in various places independently of one another, intentionally invented? Or are they the natural outcome of totemic tribal organization, resulting from its inherent conditions, just as did the laws of dual tribal division from the natural growth and partition of the tribes? 
Now, the forms of totemic exogamy unmistakably con: stitute a developmental series. In the simplest arrangement, there are no restrictions whatever upon marriage between members of one clan and those of another with which marriage relations exist. Such exogamy, however, is relatively rare in Australia, the land in which the developmental forms of exogamy, are chiefly to be found. It seems to be limited to tribes that have merely a dual organization, in which event the clan coincides with one-half the tribe. Even in such cases we find transitions to the next form of exogamy. In this second system, exogamy is restricted to particular totems of the two clans of one and the same tribal division; and, just as in the first case, the children are, as a rule, born directly into the totem group of the mother, or, less commonly, into that of the father. Following this exogamy with direct maternal or paternal descent and undeniably proceeding out of it, we finally have, as the third main form, exogamy with indirect maternal or paternal descent. In this form of exogamy, as in the preceding ones, the children belong to the totem of the mother or to that of the father so far as birth is concerned; as respects their exogamous totem relation, however, they pass over into another totem of the same clan. Thus, birth-totem and marriage-totem are here distinct, and every member of a group belongs to two totems that differ in significance. Now, in the case of a marriage by capture in which the individuals belong to different clans, the question of the totem does not enter. When, therefore, this mode of marriage remains undisturbed by further conditions, we have exogamy of the first form. When a suitor seeks to win the favour of the clan by means of a gift presented to the parents or the kin, marriage by capture passes over directly and without further change into the simple marriage by purchase. The two more exclusive forms of exogamy, on the other hand, are obviously connected with the rise of totemism; they are the result both of the clan divisions which follow. from tribal partition and of the accompanying separation 
into totem groups. The question, therefore, concerning the development of these forms of exogamy, dependent as they are both upon clan divisions and upon totem groups, is essentially bound up with the question concerning the temporal relation of the two important phenomena last mentioned. An unambiguous answer to the latter question, however, may be gathered precisely by a study of Australian conditions, at least so far as the development in these regions is concerned. If we recall our previous schema (p. I $4 \mathrm{I}$ ), representing the tribal organization of the Kamilaroi, and here, as there, designate the totemic groups (emu, kangaroo, opossum, etc.) comprised within the clan by $m n \circ p$..., it is apparent that the totems must be at least as old as the division into the two tribal halves. Unless this were the case, we could not explain the fact that, with very minor exceptions, precisely the same totems exist in the two tribal divisions. The condition might be represented thus :-

\section{I}

- $m n \circ p q$
II

$o p m s n$

It is also evident, however, that the totems could not have influenced this first division, otherwise their members would not have separated and passed over into the two tribal divisions, as they did in almost every case. Remembering that the totemic groups are also cult associations, we might express the matter thus : At the time of the first tribal division, the cult groups were not yet strong enough to offer resistance to the separation of the tribal divisions, or to determine the mode of division; therefore, members of totem $m$, for example, went here or there according as other external conditions determined. Conditions were quite different at the time of the second division, when the tribal half I separated into clans $\mathrm{A}$ and $\mathrm{B}$, and II into $\mathrm{C}$ and $\mathrm{D}$, according to the schema :-

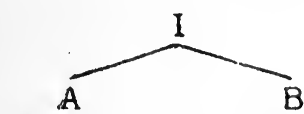

mnop

qrst

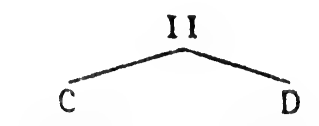

mpqs nort 
These clans, as we see, separated strictly according to totems. The bond of cult association had now become so strong that all members of a particular totem regularly. affiliated themselves with the same clan, though the grouping of the totem divisions within the clans of the two tribal halves proceeded along absolutely independent lines, as may be concluded from the fact that the totems composing the clans within the two tribal divisions are grouped differently. The formation of such cult or totem groups, thus, may already have begun in the primitive horde. At that time, however, these cult groups were probably loosely knit, so that when the horde split up, its members separated, each of the two tribal divisions, generally speaking, including individuals of all the various tribes. Not so in the case of those divisions of the tribe which originated later, after mankind had advanced further beyond the condition of the horde. By this time the totem unions must have become stronger, so that the members of a cult group no longer separated but, together with other similar groups, formed a clan. When the growth of the tribe, together with the conditions of food supply and the density of population, led to a separation of the tribe, certain totem groups invariably joined one division and others, the other, but the more firmly organized groups remained intact.

A further phenomenon of great importance for the development of exogamous marriage laws must here be mentioned-one that occurs throughout the entire realm of totemic culture but is particularly prominent among the Australian totem groups. This phenomenon consists in totem friendships. Certain totem groups regard themselves as in particularly close relations with certain other groups. Friendships similar to these, in a general way, are to be found even in connection with the highest forms of political organization. For modern States themselves enter into political alliances or friendships, and these, as is well known, are subject to change. Such alliances occur from the beginnings of totemism on up to the advanced plane of modern international culture. Though these affiliations eventually come 
to be determined primarily by the commercial relations of peoples, the determining factors at the outset were faith and cult. In both cases, however, the friendships are not of a personal nature, but are relations based on common interests. This common interest may consist, for example, in the fact that, as has been observed among some of the Australian totem alliances, the member of a totem may slay the totem animal in the hunt, but may not eat of it, though the member of the friendly totem may do so. Thus, the interest in cult becomes also a means for the satisfaction of wants, as well as a bond that unites more closely the particular totem groups.

These facts help to explain how the unlimited exogamy which first arises from marriage by capture comes to pass over into a 'limited exogamy,' as it does immediately upon the appearance of conditions that regulate the forceful capture and substitute for it the friendly exchange of women. These factors, however, always come into play whenever the intercourse between tribal members becomes closer, and particularly when the struggle with strange tribes keeps in check the strife between individuals of the same tribal association. In such cases, exchange, or, in later development, purchase, proves the means of putting an end to force. Thus, blood revenge, which persists into far later times, is displaced by the wergild which the murderer pays to the kin of his victim. This transition is precisely the same, in its own field, as that which occurs in the institution of marriage, for in the former case also the strife involves members of the same tribe. The passion, however, which causes the murder and which creates the demand for vengeance, sometimes prevented the introduction of peaceful means of settlement. In the case of marriage by capture, however, a marriage relationship, unrestricted and friendly in character, was doubtless first developed between the two clans, particularly wherever tribal division and clan were identical. And though marriage by capture was for a time still occasionally practised-since all changes of this sort are gradual-such marriages, nevertheless, more and more assumed a playful character. The actual capture everywhere finally gave 
way to exchange and later to the gift. When, however; the totem groups, and with them the cult associations that established a bond between clan and clan, gained the ascendancy, the totem groups naturally displaced the clan in respect to marriage arrangements ; those totems who maintained close cult relations with one another, entered also into a marriage 'relationship. Thus, exogamy became limited ; the members of a totem of clan A married only into the friendly totem of clan B, and this usage became an established norm whose violation might result in the death of the guilty person, unless he escaped this fate by flight. This transition of exogamy from clan to totem group, and from the unlimited to the limited form, came only gradually. This is clearly shown by the conditions among the Dieri. Certain of their totems have already entered upon the stage of limited marriage relationship, whereas others have not advanced beyond unlimited exogamy.

But even after the development had reached its final form and limited totemic exogamy was completely established, further changes ensued. For the basis of such exogamy, we may conjecture, is the fact that certain totem groups of associated clans enjoy particularly close relations with one another. Even on these primitive levels, however, the friendships of such groups are not absolutely permanent any more than are the political friendships of modern civilized states, though their degree of permanence is probably greater than that of the latter. Migrations, changes in hunting-grounds, and other conditions, were doubtless operative also in totemic culture, loosening the bonds between friendly totems and cementing others in their stead. This led to changes in the exogamous relations of totem groups. Instead of groups $n$ and $r$ of clans $\mathrm{A}$ and $\mathrm{B}, n$ and $q$ might then come to have exogamous connections (see diagram III on p. I 48). But the severance of the old connection did not immediately obliterate the tradition of the former relationship. The influence of the latter would naturally continue to be felt, not in connection with acts of a transitory nature, such as wooing and marriage, but in matters permanent in 
character and thus affecting the traditional organization of the tribe. Such a permanent relation, however, is totem affiliation. This explains how it happens that, even after the old totem connection gave way to the new, it nevertheless continued to exercise a claim on the totem membership of the children born under the new marriage conditions; hence also the recognition of the claim on the part of custom. In one respect, indeed, such recognition was impossible. More firmly established than any form of exogamy was the law that children belonged to the mother, or, in the case of paternal descent, to the father. This law could not be violated. Hence exogamous and parental tribal membership became differentiated. The latter ordained that children in every case belong to the totem of the parent who determines descent; the tradition of the former decreed that children belong, not to the parental totem, but to some other totem of the same clan. Such a condition of dual totem membership might, of course, arise from a great variety of conditions, just as may the similarly overlapping social relations within our own modern culture-such, for example, as the military and the so-called civil station of a man. The customary designation of the first two forms of limited exogamy as exogamy with direct maternal descent, and of the third as exogamy with indirect maternal descent, is plainly inappropriate and may easily give rise to misunderstandings. For it may suggest that the maternal totem disposes of its rights in respect to marriage arrangements to another totem group, and that eventually this even occurs in accordance with a definite agreement. But this is certainly not the case. For maternal descent or, speaking more generally, the fact that children belong to the parents, obtains invariably. It would be preferable, therefore, simply to distinguish the parental totem connection from the traditional exogamous connection, or one system in which the exogamous and the parental connections coincide, from a second in which they differ.

The conjecture, therefore, that a traditional marriage relation, differing from that based on parentage, grew up 
out of a previous totem friendship, is based primarily on the importance which totemic cult alliances in general possessed within the totemic tribal organization. Other causes, of course, may also have co-operated. Two further points must be noticed. In the first place, it is not at all likely that the transition from the parental exogamous relation to the traditional form occurred at the same time in all the totem groups. This is not only highly improbable in itself, but is also absolutely irreconcilable with the fact, shown by the example of the Dieri, that the earlier transition from unlimited to limited exogamy was gradual. Moreover, one must bear in mind that the transition from parental to traditional exogamy, represented by diagram III (p. I 48), not only underwent several repeated transformations, but that, due to the power which tradition always exerts, a traditional exogamous union of two totems, after it once arose, may have persisted throughout several changing cult friendships. An existing marriage relation may not at all have corresponded to the cult friendship that immediately preceded it ; it may have been based on any earlier friendship whatsoever that had been favoured by conditions and that had received a firm place in tradition. These facts show that the hypothetical 'wise ancestors' of the present-day Australians-sages who are said to have invented this complicated organization in the immemorial past for the purpose of avoiding endogamy-are just as superfluous as they are improbable. The phenomena arose in the course of a long period of time, out of conditions immanent in the life and in the cult of these tribes. The various forms of exogamy appearing in the course of this period were not the causes but the effects of the phenomena in question.

\section{The Forms of POlygamy.}

Unless external influences have changed his mode of life, primitive man, as we have seen, is both monogamous and endogamous, the latter term being used in a relative sense as denoting a condition in which marriages are permitted between blood relations as well as between non-relations, 
As a result of the external conditions of life, however, particularly the common habitation of the same protective cave and the use of adjacent hunting-grounds, unions within a wider joint family generally predominate. Following upon the rise of exogamy, polygamy also regularly appears. These two practices give to the marriage and family relations of totemic society an essentially different character from that which they possess under primitive conditions. Even in the totemic era, indeed, polygamy is not universal ; monogamy continues to survive. Monogamy, however, ceases to be a norm of custom. It is everywhere set aside, to a greater or less extent, in favour of the two forms of polygamy-polygyny and polyandry.

Now it is apparent that precisely, the same conditions that underlie the development of the various forms of exogamy also generate polygyny and polyandry. From the standpoint of the general human impulses determining the relations of the sexes, both sorts of polygamy are manifestly connected very closely with the origin of exogamy. Here, again, the fact that exogamy originated in marriage by capture from within the tribe is of decisive importance. It is precisely this friendly form of the capture of brides, as we may learn from the example of the Australians and of others, that is never carried out by the individual alone, whether the custom be still seriously practised or exists only in playful survivals. The companions of the captor aid him, and he, in turn, reciprocates in similar undertakings. Thereby the companion, according to a view that long continued to be held, gains a joint right to the captured woman. Hence the original form of polygamy was probably not polygyny-the only form, practically, that later occurs-but polyandry. At first this polyandry, which originates in capture, was probably only temporary in character. Nevertheless it inevitably led to a loosening of the marriage bond, the result of which might easily be the introduction of polygyny. The man who has gained a wife for his permanent possession seeks to indemnify himself, so far as possible, for the partial loss which he suffers through his companions. Here, then, two motives 
co-operate to introduce the so-called 'group-marriage'-the dearth of women, which may also act as a secondary motive in the claim of the companions to the captured woman, and the impulse for sexual satisfaction, which is, in turn, intensified by the lack of women. Similarly, the right to the possession of a woman, even though only temporarily, also has two sources. In the first place, the helper demands a reward for his assistance. This reward, according to the primitive views of barter and exchange, can consist only in a partial right to the spoils, which, in this case, means the temporary joint possiession of the woman. In the second place, however, the individual is a member of the clan, and what he gains is therefore regarded as belonging also to the others. Thus the right of the closest companions may broaden into a right of the clan. Indeed, where strict monogamy does not prevent, phenomena similar to marriage by capture persist far beyond this period into a later civilization. Thus, in France and Scotland, down to the seventeenth century, the lord possessed the right of jus prima noctis in the case of all his newly married vassals. In place of the clan of an earlier period we here find the lord; to him has been transmitted the right of the clan. At the time when these phenomena were in their early beginnings, the temporary relation might very easily have become permanent. It is thus that group-marriage originates-an institution of an enduring character which not only survives the early marriage by capture but which is reinforced and probably first made permanent by its substitute, namely, marriage by purchase. In this instance again, Australian custom offers the clearest evidence. In the so-called 'Pirrauru marriage' of Australia, a man, M, possesses a chief wife, CI, called 'Tippamalku.' Another man, $\mathrm{N}$, likewise has a chief wife, $\mathrm{C}^{2}$. This wife, $\mathrm{C}^{2}$, is, however, at the same time a secondary wife, $\mathrm{S}^{\mathrm{I}}$, or 'Pirrauru' of $\mathrm{M}$. In like manner the chief wife, Cr, may, in turn, be a secondary wife, $\mathrm{S}^{2}$, of $\mathrm{N}$. This is the simplest form of group-marriage. Two men have two wives, of whom one is the chief wife of $M$ and the secondary wife of $N$, and the 
other is the chief wife of $\mathrm{N}$ and the secondary wife of $\mathrm{M}$. Into such a group yet a third man, $\mathrm{O}$, may occasionally enter with a chief wife, $\mathrm{C}_{3}$, whom he gives to $\mathrm{M}$ as a secondary wife, $\mathrm{S} 3$, and eventually to $\mathrm{N}$ as a secondary wife, S4, without himself participating further in the group. In this way there may well be innumerable different relations. But the marriage is a 'Pirrauru marriage' whenever a man possesses not only a chief wife but also one or more secondary wives who are at the same time the wives of other men. 'Pirrauru marriage?' is a

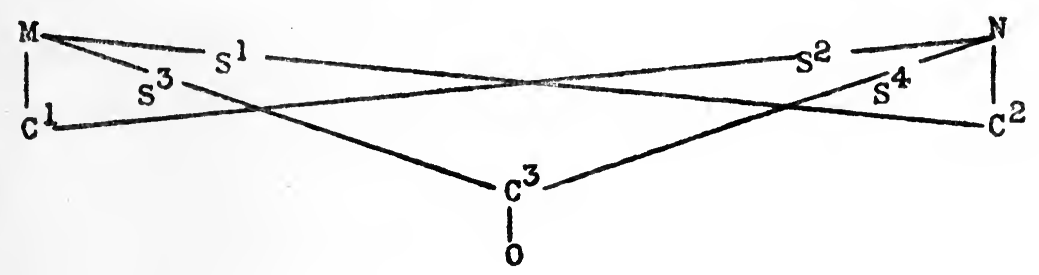

form of group-marriage, for it involves an exchange of women between the men of a group according to the reciprocal relation of chief and secondary wives. The very manner in which 'Pirrauru marriage' originates, however, indicates that in all probability its basis is monogamy, and not, as is supposed by many ethnologists and sociologists, 'promiscuity,' or the total absence of all marriage. In harmony with this interpretation is the fact that in numerous regions of Australia, especially in the northern districts, it is not group-marriage but monogamy that prevails. There is also, of course, a form of group-marriage that differs from 'Pirrauru marriage,' and is apparently simpler. In it, the differences between chief and secondary wives disappear; several men simply possess several wives in common. Because this form of group-marriage is the simpler, it is also usually regarded as the earlier. This view, however, is not susceptible of proof. The supposition rests simply and alone upon the consideration that, if a state of absolutely promiscuous sexual intercourse originally prevailed, the transition to an undifferentiated group-marriage without distinction of chief and secondary wives would be the next 
stage of development. The reverse, however, would obtain were monogamy the original custom. For the groupmarriage with chief and secondary wives is, of course, more similar to monogamy than is undifferentiated groupmarriage. Moreover, this order of succession is also in greater consonance with the general laws underlying social changes of this sort. As a matter of fact, it would scarcely be possible to find grounds for a transition from undifferentiated group-marriage to the 'Pirrauru system.' If we assume that there was a growing inclination for single marriage, it would be difficult to understand why the circuitous path of 'Pirrauru marriage' should have been chosen. On the other hand, it is very easy to see that the distinction between chief and secondary wives might gradually disappear. Indeed, this is what has almost universally happened wherever pure polygyny prevails. Wherever polygyny may be traced back to its beginnings, it always seems to have its origin in the combination of a chief wife with several secondary wives. Later, however, when the wife comes to be regarded as property, we find a formal co-ordination of the wives. Or, there may be a distinction that arises from the accidental preference of the husband, as in the case of the Sultan's favourite wife, though in modern times such choice has again been displaced by a law of more ancient tradition. The latter change, however, was the result of the external influence of the culture of Western Europe. Such a retrogressive movement, in the sense of a reapproach to monogamy, is foreign to the motives immanent in the development itself. Furthermore, 'Pirrauru marriage' is very easily explicable by reference to the same condition that best explains the origin of exogamy, namely, the custom of marriage by capture as practised between groups enjoying a tribal or cult relationship. The captured wife is the Tippamalku, or chief wife, of the captor ; to the companions who assist the latter she becomes a Pirrauru, or secondary wife. This latter relation is at first only temporary, though it later becomes permanent, probably as a result, in part, of a dearth of 
women. By rendering his companions a similar service, the original captor in turn gains the chief wives of the former as his secondary wives. As frequently happens, the custom which thus arises outlives the conditions of its origin. This is all the more likely to happen in this case, because the general motives to polyandry and polygyny persist and exercise a constant influence.

Proof that this is the forgotten origin of groupmarriage may perhaps be found in a remarkable feature of the customs of these tribes-one that is for the most part regarded as an inexplicable paradox. Marriage with the chief wife is not celebrated by ceremonies or festivals, as is the union with the secondary wife. Thus, the celebration occurs, not in connection with that marriage which is of primary importance even to the Australian, but, on the contrary, on the occasion of the union which is in itself of less importance. The solution of this riddle can lie only in the origin of the two forms of marriage. And, in fact, the two result from radically different causes, if it be true that capture from a friendly clan is the origin of the Tippamalku marriage and that assistance rendered to an allied companion underlies Pirrauru marriage. Capture is an act which precludes all ceremony ; alliance with a companion is a contract, perhaps the very first marriage contract that was ever concluded-one that was made, not with the woman or with her parents, but with her husband. The consummation of such a contract, however, is an act which in early times was always accompanied by ceremonial performances. These accompanying phenomena may also, of course, persist long after their source has been lost to memory. Thus, the difference between the two forms of primitive group-marriage also indirectly confirms the supposition that monogamy lies at the basis of group-marriage in general.

After a man has won one or more secondary wives in addition to his chief wife, in Pirrauru marriage, there will doubtless be a tendency for him to seek additional chief wives. This will be particularly apt to occur where, on the 
one hand, marriage by capture gives way to marriage by barter and later to marriage by purchase, and where, on the other hand, group-marriage is on the wane. Custom may then either recur to monogamy, or it may advance to a polygyny which is pure and not, as in the case of groupmarriage, combined with polyandry. Whether the former or the latter will occur, will depend, now that marriage by purchase has become predominant, upon might and property. Since these are also the factors which insure man's supremacy within the family, the older forms of combined polyandry and polygyny almost universally (with few exceptions, conditioned by the dearth of women) give way, with the advance of culture, to simple polygyny, which is then practised alongside of monogamy. This polygyny, in turn, also finally recedes in favour of monogamy. The circle of development, accordingly, may be represented by the following diagram :-

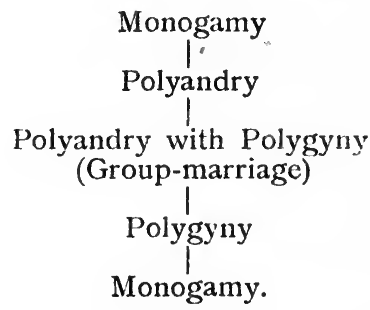

As an intermediate stage between monogamy and groupmarriage, pure polyandry, it should be remarked, is doubtless a very transitory phenomenon. Nevertheless, it has a priority over polygyny in so far as it first furnishes the motives for the additional practice, and thus for the very origin, of the latter.

As a matter of fact, the ethnological distribution of the forms of marriage entirely confirms, as a general rule, the truth of this diagram. Even in Australia the phenomena of Pirrauru and of group-marriage are confined particularly to the southern regions. In the northerly regions, where immigration and racial fusion have played a greater rôle, both monogamy and polygyny may be found. The same 
is true of America and of Africa, monogamy decidedly predominating in the former and polygyny in the latter. The influence of marriage by purchase then constantly becomes stronger, with the result that the woman comes to be regarded from the point of view of property. The rich man is able to buy more wives than the poor man. In all polygynous countries and fields of culture, therefore, even in the present domain of Islamism, the poor man, as a rule, lives in monogamy, the rich man in polygyny. Only the wealthiest and most aristocratic allow themselves a real harem with a considerable number of wives.

Linked with these influences is yet a further change. Its beginnings are to be found as early as Australian culture ; in America, it has progressed somewhat farther; in the other regions of totemism, it has finally succeeded in crowding out the original conditions with the exception of meagre remnants and survivals of customs. The change to which I refer is the transition from maternal descent, which, in all probability, was originally universal, to paternal descent. Maternal descent is in direct harmony with the natural feeling that the children who are born of the mother, and whose early care rests with her alone, should also belong to her. In this sense, mother-right represents the earliest of all conceptions of property. At the same time it precludes the possibility of that marriage which was avoided even by primitive man, and which, on higher cultural levels, is abhorred beyond all the other unions forbidden by the exogamous norms of custom-marriage between son and mother. The decisive external factor in connection with maternal descent, however, is the subordinate position of the family as compared with the association of the age-companions of the same sex, particularly the men's club. Because of its tribal struggles, whose increasing importance is externally reflected in the character of the weapon, it is precisely the totemic era that tends to loosen the natural family ties of the preceding primitive age, and, as a result, to allot the child to the mother. This tendency is clearly expressed in certain transitional phenomena that may occasionally be observed; they 
occur more frequently in Melanesia and America, however, than in Australia. The child, in these cases, inherits the totem of the mother as well as that of the father; or the son, though continuing to inherit the totem of the mother, nevertheless passes over into the clan of the father. These are intermediate phenomena, preparatory to the general transition from maternal to paternal descent. At the same time, the fact that membership is inherited in the paternal clan, in spite of the custom whereby the mother determines the totem, directly suggests that the bond uniting the men may become a force which counteracts maternal descent and then readily leads to paternal descent. This transition is bound to occur, particularly under the co-operation of other favouring conditions. Such conditions, as a matter of fact, are present ; for social organization gains an increasing influence upon the whole of life's relations. There are primarily three factors that militate against the original custom of maternal descent. The first of these consists in the increasing authority of the man over his family, particularly over the son, who was generally subject to stricter regulations than, was the daughter. This authority begins to manifest itself at that time, especially, when the man's relations with his family again become closer, and the associations which originally embraced, without exception, all the men of the clan, are displaced by family groups subject to the control of a family elder. Coincident with these changes and with the resulting transition to a patriarchal order, there occurs also the gradual dissolution of the general system of totemic tribal organization. Now, the system of maternal descent was closely bound up with totemic tribal organization from the very beginning. With the disappearance of the latter, therefore, the former loses its power of resistance against the forces making for its destruction. Finally, as a third factor, there is the gradually increasing prominence of personal property. Just as the wife becomes the property of the man, so also does the child. So great was this emphasis of the property conception, combined with the notion of authority, that even among the Romans the pater familias 
had power extending over the life of his children. Beginnings of such conceptions, however, are to be found even in more primitive societies. Polynesian custom, for example, permitted the murder of new-born children, and free advantage was taken of the permission. Only after the child had lived for a short time was infanticide prohibited. The decision, however, as to whether or not the child should be allowed to live rested primarily with the father.

\section{The Developmental Forms of Totemism.}

Our discussions thus far have been restricted to those aspects of totemism which are directly related to tribal organization. But however important these phases may be, particularly in so far as they affect marriage regulations, they are, after all, but an external indication of the all-pervading influence of totemism upon life as a whole. Moreover, tribal totemism leaves many things unexplained, especially the origin of totemic belief. At any rate, the fact that totem groups were originally cult associations unmistakably points to inner motives of which the influence of totemism upon tribal organization and upon exogamy is but the outer expression. To answer the question concerning the nature of these motives, however, we must first call to mind the various sorts of totemic ideas. An analysis of these ideas may proceed in either of two directions. It may concern itself either with the social unit that regards itself as in relation to the totem or with the nature of the object that constitutes the totem. So far as the social unit is concerned, it may be a particular group of individuals-whether constituting a cult association independent of the real tribal organization, as in Australia, or, as in America, representing one of the tribal divisions themselves -that takes the name of a particular animal or, less frequently, of a plant for its totemic designation. The individual, however, may also possess a personal totem. Furthermore, the totemic idea may be associated with the birth of an individual, conception being regarded as an act 
in which the totem ancestor passes over into the germ as a magic being. This particular form of totemic belief is generally known as conception totemism. It supposes either that the totem ancestor co-operates with the father in the begetting of the child or that the father has no connection with procreation, the child being the direct offspring of the mother and the totem ancestor. There is, finally, also a fourth, though a relatively uncommon, form of totemism, generally called 'sex totemism.' Sex totemism also is social in nature, though in this case it is not different cult or tribal associations that possess separate totems, but the sexes, the men and women of a tribe or clan. The men have a totem, as have also the women, or there may be several totems for each sex.

Intercrossing with this classification based on the social factor, on whether the totem is associated with the tribe, the individual, or the procreation of the individual, there is a second classification. The latter concerns itself with the nature of the objects that are regarded as totems. These objects are of various sorts. Here again, moreover, we must doubtless recognize a development in totemic conceptions. The original totem, and the one that is by far the most common, is the animal. Numerous peoples possess no totems except animals. In many communities, however, plant totems have been adopted, and in certain regions they have gradually become predominant. Of the plant totems, the most important are the nutritious plants. In addition to these two classes of totemic objects, there is, finally, another, though an exceedingly rare, sort of totem. The totem that is conceived as an animal ancestor may give way to other fanciful ancestral ideas or may intercross with them. Various forms of such phenomena are to be found, particularly in Australia. In this region, such ancestors, which; doubtless, are for the most part regarded as anthropomorphic, are sometimes called Mura-mura or also Alcheringa. They are apparently imaged as mighty human beings possessed of magic powers. They, are believed to have introduced totemism and to have instructed 
the forbears of the Australians in magic ceremonies. Muramura is the name that occurs especially in Southern Australia; the term, Alcheringa, prevails in the north, where the age of these mythical ancestors is often directly referred to as the Alcheringa age. At times, apparently, it is believed that these ancestors merely singled out as totems certain already existing animals. In other cases, however, animals, as well as mankind, are held to have been created by the magic-working beings out of formless matter, doubtless earth. It is commonly believed that the creatures that were thus created were at first lifeless, but became animals and men when placed in the sun. These various ideas are for the most part so intertangled in Australian legend that no coherent history of creation is anywhere discoverable. The legends plainly embody merely a number of detached fanciful ideas.

Closely connected with these original ancestors there is a third sort of totem or of totemic objects which we may briefly designate as inanimate. The objects are regarded as possessing magical powers and as having been bequeathed by the original ancestors, thus representing a legacy of the magical Alcheringa age. It is particularly stones and pieces of wood that are held to be the abode of these totemic spirits and that are represented by legend as having at one time been entrusted to the custody of the forefathers. These ideas abound particularly in northern Australia, where the magical objects are called churingas (or tjurungas). Churingas play an important rôle in the ceremonies of the totem festivals. For the most part, they consist of symmetrically shaped stones, somewhat similar to the boomerang; yet other objects also may be found, particularly such as are somehow striking in form. These churingas are also associated with other totemic ideas, particularly with conception totemism. The original ancestor is supposed to continue his existence, as it were, in the churinga, so that when this comes into contact with the mother he may pass over directly into the child.

If, now, we compare with each other the two extreme forms of the first class of totemic ideas-namely, tribal 
and individual totemism-we at once face the question, Which is the earlier, the original form? The ideas connected with the individual totem are certainly much more widely disseminated than is tribal totemism. Guardian spirits, particularly demoniacal, protective animals, may be found in many regions of the earth where there is little or no trace of the tribal totem. This is true especially of many regions of North America and of southern Africa, and likewise of numerous islands of Oceania. In these localities the individual totem is sometimes regarded as a sort of double of the individual person. If the totem animal dies, the man whose totem it is must also die. Closely related to this conception are a vast number of ideas reaching far down into later mythology, particularly into Germanic lore-ideas according to which the soul of a man lies hidden in some external object, perhaps in a plant or in an animal, and, when this vehicle of the soul is destroyed, the man, or the god or demon who has assumed human form, must die.

In these various modifications, individual totemism is doubtless more widespread than is tribal totemism. Nevertheless, this by no means implies that the latter developed from the former. On the contrary, both may possibly be equally original, grounded as they are in universal human motives that run parallel and independent courses. For this very reason, however, it is also possible that tribal totemism is the older form, for on somewhat higher cultural levels it recedes in favour of the belief in protective spirits of individuals. In questions such as this it is helpful to adduce parallels from later cults whose mode of origin is more familiar. In the present instance, leaving out of account the animal ideas, the two forms of totemism are closely analogous to the Roman Catholic worship of saints. The saints also are regarded partly as guardians of communities and partly as personal protectors. Thus, on the one hand, we have the patrons of cities, of monasteries, of vocations, and of classes; on the other hand, the individual also may possess a particular patron saint. He knew of a 
certainty, however, that the patron saints of individuals did not antecede those of the Church itself. It was this most inclusive community that first elected the saints, whereupon smaller groups and finally individuals, guided by motives that were frequently quite external, selected specific patron saints from among the number of ecclesiastical saints. When the Church set apart a certain day of the year for the particular worship of one of its saints, this day was called by the name of the saint ; to those individuals who were named after him, the day became sacred. Thus, the patron saint of the individual appeared later than the more universal saint. This order of development, moreover, is in harmony with the general nature of custom, language, and myth, according to which the individual succeeds the universal ; only secondarily may the process occasionally be reversed. Usually, however, it is cult associations and their common cult objects that are first in origin. Our contention is unaffected by the fact that individual cult objects, as well as individual totems, may continue to survive after tribal cults and tribal totems have disappeared. For the need of a personal protector is generally much more permanent than are the social conditions that gave it birth. Again we may find verification in the analogous development of saint worship. Nowadays the patron saints of the vocations, classes, and cities have more and more passed into oblivion. Among the Roman Catholic rural population, however, the individual still frequently has his patron saint, and, even where the saint has disappeared, the celebration of the 'name-day' has been retained. It is particularly in the religious realm that personal need gains a greater and greater ascendancy over community need. Everything seems to indicate that such a change took place even within totemism, especially under the influence of the gradual dissolution of the original totemic tribal organization-a change analogous to that which occurred in the case of saint worship as a result of the decay of mediæval guilds. These arguments, of course, cannot lay claim to more than probability. No one can show how the individual totem developed out of the group totem. 
Certain indications, however, suggest that the above was the course of development. In Australia, the stronghold of original tribal totemism, a youth is frequently given a personal totem, in addition to the tribal totem, upon the occasion of his initiation into manhood. The personal totem is frequently a matter of secrecy, being known only to the medicine-men or to the elders of the tribe. The fact that this is true indicates that such a personal totem possesses no public significance and, moreover, that it is probably bound up with the idea that the real essence of a man is contained in his name, just as it is in his picture, so that the mere speaking of the name might bring harm to the person. It is doubtless probable, therefore, that, after groups came to be formed within the primitive horde, they were at once bound together by relations of cult. As Australian conditions indicate, the origin of totems in the sense of cult groups is at least as old as tribal organization, if not older.'

The same cannot be said of the much more remarkable, though also rarer, forms of totemism, conception and sex totemism. The former of these may be regarded as a modification of individual totemism, inasmuch as it relates to the procreation of the individual. However, it also forms a sort of intermediate stage between tribal and individual totemism. A woman receives the totem of the child on a specific occasion, of which she usually has knowledge. Among the Aranda, the conception may occur at any place whatsoever; among the Warramunga, the woman retires to a certain spot, the totem place, where the ancestral spirits dwell. Either during the day or, especially, during the night and in sleep, the spirit of the ancestor passes over into her. The word 'spirit,' which is employed by English writers, is not, of course, an accurate rendering of the Australian term, and may easily lead to a misconception. The German missionary Strehlow has probably done better in using the word 'germ.' The germ of the child is thought to pass over into the body of the mother independently of any act of the father, or, at most, the participation of the latter is held to be merely secondary, and not essential. 
Adherents of the theory of original promiscuity have interpreted these ideas also as a survival of unrestrained sexual conditions, and thus as indicative of the fact that paternity was at one time unknown. A closer acquaintance with the phenomena, however, shows that this can scarcely be the case. Thus, the idea of the Warramunga that it is the totem ancestors of a woman's husband and not those of any other man that pass over into her, clearly presupposes a state of marriage, as does also the further fact that these same tribes reckon descent in the line of the father and not in that of the mother. Moreover, the passing of the totem ancestor into the woman is generally accompanied by magical ceremonies, such as the swinging of bull-roarers, or contact with churingas. Or, the totem ancestor may appear to the woman in sleep or in a waking vision. On the Banks Islands, strange to say, we find conception totemism without any trace of tribal totemism. The manner of reception of the totem ancestor also differs; the woman eats of the flesh of her husband's totem animal, which, since there is no tribal totemism, is in this case a personal, protective totem. Thus, conception totemism represents something of an exception in that the eating of the totem is not forbidden, as it generally is, but rather constitutes a sort of cult act, as it also does in certain other cases. In Australia, moreover, conception totemism is to be found only among several of the northern tribes, to whom it may at one time have come from Melanesia. Because of the primitive nature of the ideas connected with conception totemism, particularly when, as among the Aranda, the husband is ignored and it is believed that conception is mediated only by the totem ancestor, the northern tribes just referred to have sometimes been regarded as the most primitive. There are some writers, on the other hand, by whom the possibility of such ideas is denied on the ground that these very tribes must be familiar with the process of procreation in the animal world. But this does not prove the case. When, however, we learn that the older men of the tribe themselves no longer entertain the belief 
in magical generation, particularly as the exclusive factor, whereas, on the other hand, this is still taught to the young men, and especially to the children, we may well call to mind our own childish notions about the stork that brings the babies. Why might something similar not occur among the Australians, and the belief possibly retain credence somewhat beyond the age of childhood?

Sex totemism, similarly to conception totemism, is also of somewhat limited distribution, and seems to occur principally in those regions where tribal totemism proper is lacking or is at least strongly recedent. Among the Kurnai of southern Australia, for example, no tribal totemism has been discovered, though sex totemism occurs and actually forms the basis of certain marriage ceremonies. Sex totemism probably has its origin in the individual totem, especially in the appearance of this totem in dreams. If, after such a totem has appeared to an individual man or woman, it is then adopted by others of the same sex, specific sex totems may well come into being, particularly under the influence of the separate associations of men and women. It is also significant that in the case of sex totemism nocturnal animals predominate. The totem of the women is usually the bat; that of the men, the owl. This fact is indicative of a dream origin and of a genesis from the individual totem. Diurnal birds may, of course, also appear in dreams. Whether or not this occurs depends solely upon concomitant circumstances. At the stage of culture, however, when man is accustomed to sleep in the open, it is probable that the nocturnal birds which circle about him will also appear in his dreams. A further characteristic phenomenon of the regions where sex totemism prevails, is the manner in which marriage is consummated. In this case also, the woman eats of the totem of the man. This causes a struggle between the man and the woman, which is really a mere mock-fight ending with an offer of reconciliation on the part of the man. With this, the marriage is concluded. Such customs likewise point back to individual totemism as their original source, and probably also to marriage by capture. 
The fact that tribal totemism everywhere receded with the dominance of individual totems, explains why sex and tribal totemism seem to be mutually exclusive. Of the two rare forms of totemism, accordingly, it is probable that conception totemism was the earlier, and that sex totemism belongs to a relatively late stage of development. A further indication of the primitive nature of conception totemism is to be found in the fact that the Aranda possess a tribal organization in which the grouping of totems to form clan divisions follows a principle which elsewhere obtains only in the case of the two tribal halves. Two clans, A and B, that enjoy exogamous relations with each other, do not have different totem groups, as they do among all other tribes ; their totem groups are largely the same. Among the Aranda, therefore, a man of one totem may, under certain circumstances, marry a woman of the same totem, provided only she belongs to the other clan. True, phenomena are not lacking-such particularly as those of plant totems, to be mentioned below, and the ceremonial festivals connected with them-which indicate that these northern tribes were affected by Papuan immigrations and by race-mixture. But influences of this kind are the less apt to lead to the submergence of primitive views and customs according as they are instrumental, particularly when they are operative at an early age, in maintaining conditions which might otherwise possibly disappear as a result of further development.

The second mode of classifying the forms of totemism is based on the objects which are used as totems and leads to an essentially different analysis of totem beliefs. Each of the forms which the classification distinguishes is, of course, also subsumable under one of the kinds of totemism already discussed. The earliest totem objects, as has already been mentioned, are without doubt animals. In America, as in Australia, there are practically no totems except animals ; in other places also it is the animal that plays the principal rôle in totemic mythology. In part, the animal continues to remain predominant even after the age of actual totemism has passed. Nevertheless, plant totemism has 
found its way into certain regions. Here also the facts are most clearly traceable in Australia, our most important source of information regarding the history of the development of totemic ideas. In southern Australia, there are no totems except animals; towards the north, plant totems gradually begin to make their appearance, until finally, among the most northerly peoples of central Australia, such totems have the dominance. Plant totems, moreover, are also found particularly in Melanesia, from which place they might easily have come to Australia across the chain of islands which extends from New Guinea to the north coast of the island-continent. That plants play an unusually large rôle in the regions of Oceania, in connection with totemism as well as otherwise, is directly due to external conditions. These islands are poor in fauna; true, they possess great numbers of birds, but these are of little value to the hunter. On the other hand, they have a luxuriant flora. From early times on, therefore, it is chiefly the plant world that has been the centre of interest and that has left its stamp upon myth and custom. Clearly, plant totemism had its origin on these islands. From them it was introduced into Australia, where it combined with animal totemism. But the regions into which plant totemism was introduced underwent a great change in their totemic cults. It is probably only with the appearance of plant totems that those cult ceremonies arose which are celebrated, not, as the festivals of tribal totemism originally were, mainly at the adolescence of youths, but primarily for the sake of effecting a multiplication of the totems. Annually, at stated times, the members of allied clans unite in magical ceremonies and cult dances, the wellknown 'corroborees,' as they are called by those who practise them. The primary aim of such cults is to bring about by. magical means an increase of the totem plants and animals. Doubtless we may regard it as highly probable that this ceremony represents a borrowing on the part of animal totemism from plant totemism. For the hunter, similarly, desires that there be a very great abundance of game animals. Yet is is mainly plants that are the object of concern-a concern caused 
by the changes in weather, with its incalculable oscillations between life-bringing rain and the withering glare of the sun. These are the motives that find expression in the festivals designed for the multiplication of the totems, the 'Intichiuma' festivals. The motives to these ancient cults still frequently find their counterparts in the customs of the cultural peoples of the present. When, in times of a long drought, processions pass over the fields and supplicate Heaven for rain, as occurs even to-day in some regions, we certainly have an analogous phenomenon. The only difference is that the Australian tribes invoke their totems instead of Heaven; they call upon the plants which are to increase and upon the animals which are to be available for hunting, with the aim of thus exercising a magical influence upon them.

In connection with the Australian ceremonies designed to multiply the food plants and game animals, we come upon still a third kind of totem objects. They differ from those of the two preceding classes in that they are not regarded as independent totems, but merely as vehicles of the same sort of magical power as is possessed by animal and plant totems. In distinction from the latter, we may briefly call them inanimate totems. They consist of stones and sticks. These are utilized as magical objects in the Australian Intichiuma festivals, and also, under the above-mentioned name of ' churingas,' in connection with conception totemism. They differ from animate totems in that the latter are in themselves endowed with magical properties, whereas the former are always held to derive these powers from living magicians, from the anthropomorphic or zoömorphic ancestors of antiquity. These magicians are thought to have transmitted the objects to later generations for the use of the latter in the practice of magic. Thus, the churingas have a peculiar status, intermediate between magical beings and magical implements. They are carefully preserved because-as is indicated by their use in connection with conception totemism-they are regarded as legacies left by ancestors; moreover, they are also sup- 
posed to harbour the demoniacal power of these ancestors. One of the factors determining the selection of these objects is doubtless generally their shape, which is frequently of a striking nature, such as to arouse astonishment. Ejected into the object itself, this astonishment becomes a wonderworking power. Later, the desire to secure such magical means of aid may become a supplementary factor in the selection of these objects, and, as widespread phenomena of a similar nature show, may eventually suffice of itself to constitute an object the bearer of magical powers. Thus, it is these inanimate vehicles of a magic derived from totem ancestors, that form the transition from the totem object to the so-called fetish.

Each of the three kinds of totem objects just described, the plant totem, the animal totem, and the totemic fetish, may assert itself in connection with the three abovementioned social forms of totemism. Moreover, the three kinds of objects may also, to a certain extent, combine with one another. For, though the animal is very commonly the only totem, plant totems never occur except in connection with animal totems, even though there are certain conditions under which they attain the dominance. Finally, the totemic fetish is always associated in totemic regions with animal and plant totems, and is also closely connected with the idea, even here permeating totemic belief, that there were anthropomorphic ancestors who left these fetishes as magic-working legacies. Thus, totemism passes over, on the one hand, into ancestor-worship, and, on the other, into fetishism, with which it combines, particularly in the 'Intichiuma' festivals, to form a composite cult. Tribal totemism is the source of the individual totem; the latter, probably as a result of animistic ideas that displace tribal totemism, gives rise, as an occasional offshoot, to the sex totem. This is the conclusion to which we are led by, the fact that the choice of the sex totem is influenced by the dream. The last important product of individual totemism, in combination with tribal totemism, is an incipient ancestor worship, which is accom- 
panied by peculiar forms of fetishism. In view of its origin, we may perhaps refer to this cult as 'totemic fetishism.' The following diagram illustrates this genetic relationship :-

Tribal and Animal Totemism

Tribal Totemism-Animal and
Plant Totemism

\section{The Origin of Totemic Ideas.}

We have attempted to trace the succession of the various forms of totemism by reference to the characteristics which these forms reveal. Closely connected with this problem is the question concerning the origin of totemic ideas. With respect to this question, however, widely different hypotheses have been proposed. Of these, those that belong to an earlier stage of our ethnological knowledge concerning this subject can here receive but brief mention. Herbert Spencer held that the entire institution of totemism arose out of the totem names of individuals, such, for example, as wolf, deer, eagle, or, among the Australians, emu, kangaroo, etc. These animal names, according to him, were at first perhaps nicknames, such as are occasionally to be found even to-day. Out of the individual totem arose the tribal totem. The name then became identical with the thing itself-that is, with the animal, which thus became a protective and ancestral animal. Though rejecting the idea that the origin of totemism is to be found in nicknames and epithets, Andrew Lang retained the belief that the name was primary, and that the substitution of the animal or the plant for the name occurred only later. This theory is not so strange as it might appear. As a matter of fact, it is quite characteristic of primitive thought closely to associate a name and its object. Primitive man regards his name as a part of himself; this idea is similar to that which underlies 
the terror that he sometimes manifests when a sketch is made of him, a terror due to the belief that a part of his soul is being carried away in the picture of the artist. And yet there is prima facie little probability that a phenomenon so widely prevalent and so highly ramified as totemism could have its source in a fact of this kind, which is, after all, only incidental. Moreover, in one of the chief centres of tribal totemism, in the eastern part of North America, as, for example, among the Iroquois, we find very clearly defined personal names. These names, however, are never identical with those of the totems, nor even, as a rule, with those of animals. Sometimes they are borrowed from the names of flowers, although there are no plant totems in America ; or, they are flattering appellatives such as we still find in higher civilizations. Moreover, there is no indication that they ever came to be used for the designation of totems.

The view held by Howitt and by Spencer and Gillen, scholars deserving of high esteem for their knowledge of Australian totemism, is an essentially different one. In their opinions, it is the conditions of a hunting life that are reflected in totemic beliefs. They maintain that the animals of the chase were the first to become totem animals. Wherever plant food gained great importance, plant totems were then added. The evidence for this view is based mainly on those Intichiuma ceremonies and festivals by means of which the Australians aim to secure a multiplication of the totems. In these festivals, for example, grass seed is scattered broadcast by members of the grass seed totem, or a huge lizard is formed of clay by the members of the lizard totem, and pieces of it are strewn about. These are magic ceremonies that, in a certain sense, anticipate the sowing and harvest festivals of later times. The only difference consists in the fact that these primitive magic usages are not directed to the rain-bringing clouds or to celestial deities in petition for a blessing upon the crops, but to the objects themselves, to the animals and plants. Magic powers are ascribed to the latter; by virtue of these powers they are to multiply themselves. In regions where sowing and harvest do not 
as yet exist, but where man gains his food solely by gathering that which the earth of itself brings forth, such festivals and ceremonies are to a certain extent the natural precursors of the later vegetation festivals.

In view of these facts, the hypothesis of the abovementioned investigators seems to have much in its favour. There is a very important consideration, however, that obviously speaks against it. It is highly probable that these very ceremonies for the multiplication of totem objects are not indigenous to Australia, the chief centre of totemism, but that they, along with the plant totem, were introduced from without. These plant totems, as was remarked above, appear to have come from the Melanesian Islands, where the animal totem plays a small rôle, because the fauna is meagre and man is dependent in great measure upon plant food. Besides animal and particularly bird totems, therefore, which also occur on the Melanesian Islands, we find plant totems throughout the whole of northern Australia. These totems, as we may suppose, are the result of Papuan immigrations, to which are due also other objects of Melanesian culture to be found in the Australian continent. In the south, where there are no totems other than animals, Intichiuma ceremonies receive small emphasis. In entire harmony with our contentions are the conditions in America, where no festivals of this sort are connected with the totems themselves; an analogous significance is gained only later by the great vegetation festivals, and these presuppose agriculture, together with the beginnings of a celestial mythology.

In more recent times, therefore, Frazer, whose great work, "Totemism and Exogamy," has assembled the richest collection of facts concerning totemic culture, has turned to an essentially different theory. He traces all forms of totemism back to conception totemism. Since the latter, as we have already stated, probably arose out of individual totemism, we are again confronted by an individualistic view, much as in the hypothesis of the origin from names. Frazer derives conception totemism from the dreams which 
mothers are supposed occasionally to have experienced before the birth of a child. The animal appearing in such a dream is thought to have become the totem or guardian animal of the child. But, though conception totemism, as well as sex totemism, may possibly have some connection with such phenomena-the fact that the animals here concerned are chiefly nocturnal animals suggests that such may be the case-totemism as a whole may, nevertheless, scarcely be derived from dreams. Still less can this hypothesis be harmonized with the fact that conception totemism is an anomaly. The ideas centred about it are but of rare occurrence within the system of totemic culture as a whole. Moreover, as Frazer also has assumed, they never appear except as an offshoot of individual totemism, and this in turn, when viewed in all its phases, cannot be regarded otherwise than as a product of tribal totemism. In its reference to the dream, however, this hypothesis may perhaps contain an element of truth, inasmuch as it involves ideas that obviously play an important rôle in totemism. This is shown particularly by reference to the totem animals that are found most commonly in Australia, and that suggest a relation between totemism and animistic ideas of the soul.

As a matter of fact, the totem is already itself the embodiment of a soul. Either the soul of an ancestor or that of a protective being is regarded as incorporated in the animal. The other totems, such as plants or totem fetishes (churingas), are obviously derivative phenomena, and the same is true of those legendary beings that inhabit the churingas as spirits, or that gave them to the ancestors for the purposes of magic. Now, originally, the totem was probably always an animal. But a survey of the great mass of animistic conceptions prevalent in all parts of the world shows that in this case also it is particularly the animal that is represented as capable of becoming the receptacle of a human soul after death. Animals, of course, are not all equally suited to this purpose. Some are more apt than others to be regarded as soul animals, particularly such as are characterized by rapid movement, flight through the air, or by other features that 
arouse surprise or uncanny dread. Thus, even in the popular belief of to-day, it is especially the snake, the lizard, and the mouse, in addition to the birds, that are counted among the soul animals. If, now, with these facts in mind, we cast a glance over the list of totem animals, we are at once struck by the fact that the most common among them are soul animals. In Australia, we find the hawk, the crow, and the lizard ; in America, the eagle, the falcon, and the snake.

In respect to these ideas, the totemic age marks an important turning-point in the history of soul conceptions. Primitive man regards that which we have succinctly called the 'corporeal soul' (p. 82) as the principal, and perhaps originally as the only, soul. At death, the soul is believed to remain in the body, wherefore primitive man flees in terror from the corpse. Even at this stage, of course, we occasionally find traces of a different idea. The soul may also be regarded as active outside of the body, in the form of a demoniacal being. But as yet these ideas are generally fluctuating and undefined. There then comes a change, dependent, just as are the other cultural transformations, on the strife and warfare arising as a result of tribal migrations. This change, as we may suppose, is due to the fact that tribal struggles bring with them the impressive spectacle of sudden death. One who is killed in battle exhibits the contrast between life and death so directly that, even though the belief in the continued existence of the soul within the body still survives, it nevertheless permits the co-presence of other more advanced conceptions. Thus two sets of ideas come to be developed. On the one hand, the soul is believed to depart with the blood. In place of the entire body, therefore, the blood comes to be the chief vehicle of the soul. Blood magic, which by itself constitutes an extensive chapter in the history of magic beliefs, and which is prevalent in all periods of culture, has its source in this conception. Further factors then enter into the development. In addition to the blood, the inner parts of the body, which are exposed in cases of violent death, become vehicles of the soul. The idea of the sudden departure of the soul 
is then transferred from the one who is killed to the dying person in general. With the exhalation of his last breath, his soul is thought to depart from him. The soul is therefore conceived as a moving form, particularly as an animal, a bird, a rapidly gliding snake, or a lizard.

In dealing, later, with the soul conceptions of the totemic age, we will consider these several motives in their independent influence as well as in their reciprocal action upon one another. Here we can touch upon them only in so far as they harbour the sources of totemism itself. But in this connection two facts are of decisive importance. In the first place, the original totem, and the one which continues to remain most common, is the animal ; and, secondly, the earliest totem animals are identical with soul animals. But in addition to soul animals, other animals also may later readily come to be regarded as totems, particularly such as continually claim man's attention, as, for example, game animals. Thus, the soul motives are brought into interplay with other influences, springing in part from the emotions associated with the search for daily food, though primarily with success or failure in the chase. As a result, the soul motives obviously become less prominent, and the totem animal, freed from this association, acquires its own peculiar significance, which fluctuates between the ancestral idea and that of a protective demon. The concern for food, which was at first operative only as a secondary motive, was heightened in certain localities where the natural environment was poor, and, with the influx of immigrant tribes, it assumed ever greater prominence. In this way, plant totems came to be added to animal totems; finally, as a result of certain relations of these two totems to inanimate objects, there arose a fetishistic offshoot of totemism. This again brought totemism into close connection with ancestor ideas, and contributed also towards the transition from animal to human ancestors.

Thus, then, totemic ideas arise as a result of the diremption of primitive soul ideas into the corporeal soul and the breath-and shadow-soul. That the two latter are 
associated, is proven also by the history of totemism. Folk belief, even down to the present, holds that the soul of the dying person issues in his last breath and that it possesses the form of an animal. The soul of one who has recently died, however, appears primarily in dreams and as a phantom form. Now, the totem animal has its genesis in the transformation of the breath-soul into an animal. The shadowsoul of the dream, moreover, exercises an influence on individual totemism, as it does also on conception totemism and on sex totemism.

Thus, totemism is directly connected with the belief in souls-that is to say, with animism. It represents that branch of animism which exercised a long-continuing influence on the tribal organization as well as on the beliefs of peoples. But before turning to these final aspects of totemism and their further developments, it is necessary to consider another group of ideas which, in their beginnings, occupied an important place within the circle of totemic beliefs. The ideas to which I refer are those connected with the custom of taboo.

\section{Io. The Laws of Taboo.}

It is a significant fact that 'totem' and 'taboo' are concepts for which our cultural languages possess no adequate words. Both these terms are taken from the languages of so-called natural peoples, 'totem' from an idiom of the North American Indians, and 'taboo' from the Polynesian languages. The word 'totem' is as yet relatively uncommon in literature, with the exception of books on ethnology and folk psychology; the word 'taboo,' on the other hand, is much in use. A thing is called taboo when it may not be touched, or when it must be avoided for some reason, whether because of its peculiar sanctity or contrariwise because its harmful influence renders it 'impure,' defiling every one who comes into contact with it. Thus, two opposing ideas are combined in the conception of taboo: the idea of the sacred as something to be avoided because of its sanctity, and that 
of the impure or loathsome, which must be avoided because of its repulsive or harmful nature. These ideas combine in the conception of fear. There is, indeed, one sort of fear which we call awe, and another termed aversion. Now, the history of taboo ideas leaves no doubt that in this case awe and aversion sprang from the same source. That which aroused aversion at a later age was in the totemic period chiefly an object of awe, or, at any rate, of fear-that is, of a feeling in which aversion and awe were still undifferentiated. That which is designated by the simplest word [Scheu] is also earliest in origin; awe [Ehrfurcht] and aversion [Abscheu], developed from fear [Scheu].

If, now, we associate the term ' taboo' in a general way with an object that arouses fear, the earliest object of taboo seems to have been the totem animal. One of the most elemental of totemic ideas and custom's consists in the fact that the members of a totem group are prohibited from eating the flesh of the totem, and sometimes also from hunting the totem animal. This prohibition, of course, can have originated only in a general feeling of fear, as a result of which the members of a totemic group are restrained from eating or killing the totem animal. In many regions, where the culture, although already totemic, is, nevertheless, primitive, the totem animal appears to be the only object of taboo. This fact alone makes it probable that totemism lies at the basis of taboo ideas. The protective animal of the individual long survived the tribal totem and sometimes spread to far wider regions. Similarly, the taboo, though closely related to tribal organization in origin, underwent further developments which continued after the totemic ideas from which it sprang had either entirely disappeared or had, at any rate, vanished with the exception of meagre traces. This accounts for the fact that it is not in Australia, the original home of the totem, that we find the chief centre of taboo customs, nor in Melanesian territory, where the totem is still fairly common, nor in North America, but in Polynesia.

It is in Polynesia, therefore, that we can most clearly trace the spread of taboo ideas beyond their original start- 
ing-point. The taboo of animals is here only incidental; man himself is the primary object of taboo-not every individual, but the privileged ones, the superiors, the priest, the chieftain. Closely related to the fact that man is thus held taboo, is the development of chieftainship and the gradual growth of class differences. The higher class becomes taboo to the lower class. This fear is then carried over from the man himself to his possessions. The property of the nobleman is taboo to every other person. The taboo has not merely the force of a police law, similar to that whereby, in other localities, men of superior rank prohibit entrance to their parks ; it is a religious law, whose transgression is eventually punished by death. It is particularly the chief and his property that are objects of taboo. Where the taboo regulations were strict, no one was allowed to venture close to the chief or even to speak his name. Thus, the taboo might become an intolerable constraint. In Hawaii, the chief was not allowed to raise his own food to his mouth, for he was taboo and his contact with the food rendered this also taboo. Hence the Hawaian chief was obliged to have a servant feed him. The objects which he touched became taboo to all individuals. In short, he became the very opposite of a despotic ruler, namely, the slave of a despotic custom.

From the individual person, the taboo was further extended to localities, houses, and lands. A member of the aristocratic class might render taboo not only his movable property but also his land. The temple, in particular, was taboo, and, together with the priests, it retained this character longer than any other object. The taboo concerned with the eating of certain animals, however, also remained in force for a long time. Though these animals were at first avoided as sacred, the taboo of the sacred, in this case, later developed into that of the impure. Thus, this conception recurs, in a sense, to its beginning. For the fear that is associated with the animals which the totem group regards as sacred, is here combined with the fear that the eating of the flesh is harmful. Sickness or even 
death is believed to follow a transgression of such a taboo regulation. Even in its original home, however, the taboo assumes wider forms. It subjects to its influence the demonideas that reach back even to pretotemic times. The corpse particularly, and the sick person also, are held taboo because of the demoniacal magic proceeding from them'. Likewise the priest and the chief are taboo, because of their sacredness. Thus, the taboo gains a circle of influence that widens according as totemic ideas proper recede. The taboo which the upper classes placed upon their property had come to be such a preponderant factor in Polynesian custom that the first investigators of these regions believed the taboo in general to be chiefly an institution whereby the rich aimed to protect their property by taking advantage of the superstition of the masses.

One of the most remarkable extensions of the scope of taboo is the taboo which rests on relations by marriage. The history of exogamy, whose earliest stages are represented by the totemic marriage laws of the Australians, clearly teaches that the aversion to marriage between blood relations was not the cause but at most, to a great extent, the effect of exogamous customs that everywhere reach back into a distant past. But there is a second class of marriage prohibitions, and this likewise has found a place even in present-day legislation-the prohibition of unions between relations by marriage. Such prohibitions are from the very beginning outside the pale of exogamous laws. Indeed, it is clear that all unions of this sort-such, for example, as are forbidden by our present laws-were permitted by the totem and clan exogamy of the Australians and that of the American Indians. In the case of maternal descent, the group from which a man must select his wife included his mother-in-law as well as his wife. Similarly, in the case of paternal descent, the husband and father-inlaw were totem associates. There is another set of customs, however, which is generally connected with even the earliest forms of exogamy, and which fills out in a very remarkable way the gap that appears in the original totemic exogamy 
when this is compared with present-day legislation. These customs are no other than the laws of taboo. One of the earliest and most common of these regulations is the taboo of the mother-in-law. Corresponding to it, not so common and yet obviously a parallel phenomenon occasionally connected with it, is the taboo of the father-in-law. The relative distribution of the two taboos is analogous to that of maternal and paternal descent in the primitive condition of society, for it is maternal descent that is dominant. This is not at all meant to imply that there is any casual relation between these phenomena. Rather is it true, probably, that they are based upon similar motives, and that these motives, just as in the case of marriage between relations, are more potent in the case of the mother than in that of the father. In general, however, the taboo of parents-inlaw signifies that the husband must so far as possible avoid meeting his mother-in-law, and the wife, her father-in-law. Now, it is evident that in so far as this avoidance excludes the possibility of marriage, the custom is, in a way, supplementary to exogamy. Wherever maternal descent prevails, no one may marry his mother; and, where taboo of the mother-in-law exists, no one may marry his mother-in-law. The same holds of father and daughter, and of father and daughter-in-law, in the case of paternal descent. This analogy, may, possibly indicate the correct clue to the interpretation of the phenomena. It would certainly be erroneous to regard the taboo of the mother-in-law as a regulation intentionally formulated to prevent unions between direct relations by marriage. Yet there is evidence here of a natural association by virtue of which the fear of marriage with one's own mother, which, though not caused by the exogamous prohibition, is nevertheless greatly strengthened by it, is directly carried over to the motherin-law. Between a woman and the husband of her daughter there thus arises a state of taboo such as is impossible between mother and son because, from the time of his birth on, they are in close and constant relation with each other. In consequence of the above-mentioned associa- 
tion, mother and mother-in-law, or father and father-inlaw, form a unity analogous to that which obtains between man and wife. What is true of the husband, is also true in the case of the wife; similarly, what holds for the mother of the husband holds no less for the mother of the wife.

Striking evidence of the effect of an association of ideas that is perfectly analogous to the one underlying the taboo of the mother-in-law, is offered by a custom which is doubtless generally only local in scope and yet is found in the most diverse parts of the earth, thus showing plainly that it is autochthonous in character. I refer to the custom of socalled father-confinement or ' couvade.' This custom prevails in various places, occurring even in Europe, where it is practised by the Basques of the Pyrenees, a remarkable fragment of a pre-Indo-Germanic population of Europe. Due, probably, to the heavier tasks which these people impose upon women, it here occasionally occurs in an exaggerated form. Even after the mother has already begun to attend to her household duties, the father, lying in the bed to which he has voluntarily retired, receives the congratulations of the relatives. Custom also demands that he subject himself to certain ascetic restrictions, namely, that he avoid the eating of certain kinds of food. The custom of couvade is clearly, the result of an ideational association between husband and wife-one that is absolutely, analogous to that between the two mothers of the married couple. The child owes its existence to both father and mother. Both, therefore, must obey, the regulations which surround birth, and thus they, are also subject to the same taboo. Just as there is very commonly a taboo on the mother and her new-born child, so also, in the regions where couvade exists, is this transferred to the husband.

As is well known, the last vestiges of the taboo of the mother-in-law have not yet disappeared, though they survive only in humour, as do many other customs that were once seriously practised. In fact, there is no other form of relationship, whether by blood or by marriage, that is so subjected to the satire of daily life as well as to the 
witticisms and jokes of comic papers as is that of the unfortunate mother-in-law. Thus, the primitive taboo resting on the mother-in-law and also, even though in lesser degree, on the father-in-law, has registered itself in habits that are relatively well known. Graver results of the regulations of ancient custom are doubtless to be found in those prohibitions of union between relatives by marriage that still constitute essential elements of present-day laws. This, of course, does not mean that these prohibitions are unjustifiable or that they do not reflect natural feelings. They but exemplify the fact that every law presupposes a development which, as a rule, goes back to a distant past, and that the feelings which we to-day regard as natural and original had a definite origin and assumed their present character as the outcome of many changes.

Alongside of these later forms of the taboo, and outlasting them, we have its most primitive form. This is the taboo which rests on the eating of certain foods, particularly the flesh of certain animals, though less frequently it applies also to occasional plants. The latter, however, probably represents a transference, just as does plant totemism. A particular example of such a taboo is the avoidance of the bean by the Grecian sect of Orphians and by the Pythagoreans whom they influenced. The taboo of certain animals survived much longer. But it was just in this case that there came an important shift of ideas which gave to the taboo a meaning almost the opposite of that which it originally possessed. Proof of such a change is offered by the Levitical Priests' Code of Israel. The refined casuistry of the priests prescribed even to details what the Israelite might eat and what was taboo for him. For the Israelite, however, this taboo was not associated with the sacred but with the unclean. The original taboo on the eating of the flesh of an animal related, in the totemic period, to the sacred animal. This is the taboo in its original form. The Australian shrinks from eating the flesh of his totem animal, not because it is unclean, but because he fears the revenge of demons if he consumes the protec- 
tive animal of his group. In the Priests'. Code, the sacred object has become entirely transmuted into an unclean object, supposed to contaminate all who eat of it. It is a striking fact, however, that the animals which are regarded as unclean are primarily the early totem animals-the screech-owl, the bat, the eagle, the owl, etc. Of the animals that live in or near the sea, only those may be eaten that have scales, that is, only, fish proper, and not the snake-like fish. The snake itself and the snake-like reptiles are taboo, as well as numerous birds-all of which were at a very early period totem animals. Heading the list of the animals that may be eaten, on the other hand, are the ox, the sheep, the goat-in short, the animals of an agricultural and sheep-raising culture. Thus, as the original magical motives of taboo disappear, their place is taken by the emotion of fear, which causes the object arousing it to appear as unclean. Whoever touches such an object is polluted in a physical as well as a moral sense, and requires a cleansing purification according to rites prescribed by cult. We cannot avoid the impression, accordingly, that the unclean animals held to be taboo by the Priests' Code, are the same as those which this same people regarded as sacred soul and totem animals at an earlier stage of culture. Thus, these prohibitions with reference to food are analogous to the impassioned preaching against false idolatry-both refer back to an earlier cult. In this category belongs also the prohibition of consuming the blood of animals in the eating of their flesh. This likewise is the survival of a very common belief-certainly prevalent also among the Israelites at one time-that with the blood of an animal one might appropriate its spirit-power. The priestly law transforms this motive into its direct opposite. For the text expressly says: "In the blood is the life; but ye shall not destroy the life together with the flesh."

Thus, the significance of the taboo shifts from the sacred, which evokes man's fear, to the unclean and demoniacal, which also arouse fear but in the form of aversion. Closely related to this change is a group of views and customs resulting 
from this last form of taboo and reaching down, as its after-effects, far into the later religious development. These are the purification rites connected with the ideas of clean and unclean. The word lustratio, by which the Romans designated these rites, is really more appropriate than the German word Reinigung, since it suggests more than merely the one aspect of these usages. Indeed, the idea of purification is not even primary, any more than the conception of the unclean is the initial stage in the development of the taboo. On the other hand, the idea that a man might be exposed to demoniacal powers by touching an object or by eating a certain food, such, for example, as the flesh of certain animals, is in entire accord with such primitive notions as are expressed in the fear of the corpse and of sickness, as well as in other similar phenomena. The essential thing is to escape the demon who is harboured in the particular object of concern. This impulse is so irresistible that, whenever the idea of taboo arises, the conception of lustration, of a magic counteraction to the demoniacal power, is also evolved. Thus, magic and counter-magic, here, as everywhere, stand in antithesis. The means of such counter-magic are not only very similar throughout the most remote parts of the earth, but externally; they remain the same even throughout the various stages of culture. There are only three means by which an individual may free himself from the effects of a violation of taboo-water, fire, and magical transference.

Of these means, the one which is the most familiar to us is water. Just as water removes physical uncleanness, so also does it wash away soul or demoniacal impuritynot symbolically, for primitive man has no symbols in our sense of the word, but magically. As water is the most common element, so also is it the most common magical means of lustration. Besides water, fire also is employed; generally it is regarded as the more potent element-in any event, its use for this purpose anteceded that of water. Fire, no less than water, is supposed to remove the impurity or the demoniacal influences to which a man has been exposed. 
It is especially peculiar to fire, however, that it is held not only to free an individual from an impurity which he has already contracted, but also to protect him from the possibility of contamination. This preventive power, of course, later came to be ascribed to water also. Indeed, all the various means of lustration may come to be substituted for one another, so that each of them may eventually acquire properties that originally belonged exclusively to one of the others. The third form of purification, finally, consists in a magical transference of the impurity from man to other objects or to other beings, as, for example, from a man to an animal. Closely associated with such a transference are a considerable number of other magic usages. These have even found their way down into modern superstition. We need but refer to the above-mentioned cordmagic, by which a sickness, for example, is transferred to a tree by tying a cord around it.

In the primitive cult ceremonies of the .Australians, lustration is effected almost exclusively by fire. In America also fire still plays an important rôle, particularly in the cult ceremonies of the Pueblo peoples. They kindle a great fire, about which they execute dances. In the initiation ceremonies of the Australians, the youths must approach very close to the fire or, at times, leap over it. In this way they are made proof against future attacks. Such fire-magic reaches down even into later civilizations. A survival of this sort is the St. John's fire still prevalent in many regions of Europe and, in view of its origin, still frequently called 'solstice fire' in southern Germany. On these occasions also, the young. men and maidens leap over the fire and expose themselves to the danger of its flames, in the belief that whatever they may wish at the time will come to pass. Here again, as in the Australian initiation ceremonies, lustration by fire signifies a magic act having reference to the future.

Water is a far more common means of lustration than fire. It everywhere gained the ascendancy and at the same time very largely preserved its original significance. From 
early times on it combined the power of removing the impurities resulting from the violation of a taboo, or, more widely applied, of cleansing from guilt, with the power of protecting against impending impurity and guilt. Thus, even in the beginnings of taboo usages, the bath, or ablution, was a universal means of purification. The sprinkling with water, on the other hand, which has held its place even in Christian cult, is a means of purification directed primarily to the future. In the so-called Jordan festivals of the Greek Catholic Church, ordinary water is changed into Jordan water by the magic of the priest. The believer is confident that if he is sprinkled with this water he will commit no $\sin$ in the course of the following year.

Less common, on the whole, is the third form of lustration, that by magical transference. Israelitic legend affords a striking example of such lustration in the goat which, laden with the sins of Israel, is driven by Aaron into the wilderness. He takes the goat, lays both his hands on its head, and whispers the sins of Israel into its ear. The goat is then driven into the wilderness, where it is to bury the sins in a distant place. An analogous New Testament story, moreover, is related in St. Matthew's Gospel. W.e are here told that, in Galilee, a man who was possessed of many demons was freed from them by Jesus, who commanded them to pass into a herd of swine that happened to be near by. Since the demons had previously begged Jesus not to destroy them, they were banished into these animals. The swine, however, plunged into an adjacent sea, and thus the demons perished with them.

Totem, taboo, lustration, and counter-magic, accordingly, were originally closely related to one another, though each of them proved capable of initiating new tendencies and of undergoing a further independent development. The totem, for example, gave rise to numerous sorts of protective demons; the taboo was transferred to the most diverse objects, such as aroused feelings of fear and aversion; lustration led to the various counter agencies that freed men's minds from the ideas of contamination and guilt. 
These institutions, however, were themselves based upon certain more elementary ideas whose influence was far from being exhausted in them. On the one hand, totemic belief grew out of the belief in souls; on the other hand, totemic ideas were the precursors of further developments. The activity of totem ancestors was associated with certain inanimate objects, such as the Australian churingas, to which magical powers were held to have been transmitted. Inasmuch as the totem animal was also an ancestral animal, it formed the transition to the elevation of human ancestors into cult objects, first on a par with animal ancestors and later exalted above them. Thus, there are three sets of ideas which, in part, form the bases of totemism, and, in part, reach out beyond it, constituting integral factors of further developments of the most diverse character. These ideas may be briefly designated as animism, fetishism, and ancestor worship. Animism, as here used, refers to the various forms of the belief in souls. By fetishism, on the other hand, is universally meant the belief in the demoniacal power of inanimate objects. Ancestor worship, finally, is the worship in cult of family or tribal ancestors. The original totemism passes over into the higher ancestor worship, which, in turn, issues in hero cult, and finally in the cult of the gods.

\section{1. Soul Beliefs of the Totemic Age.}

Soul ideas, as we have already noted, constitute the basis of totem belief, and may thus be said to date back into the pretotemic age, even though it is obviously only within the totemic period that they attain to their more complete development. If we include the whole of the broad domain of soul belief under the term animism, the latter, in its many diverse forms, may be said to extend from the most primitive to the highest levels of culture. It is fitting, however, to enter upon a connected account of animism at this point, because the development of the main forms of soul belief and of their transformations takes place within the totemic age. Moreover, not only is totemism 
closely dependent from the very beginning upon soul conceptions, but the development of soul conceptions is to an equal degree affected by totemism.

Soul belief, thus, constitutes an imperishable factor in all mythology and religion. This accounts for the fact that there are some mythologists as well as certain psychologists of religion who actually trace all mythology and religion to animism, believing that soul ideas first gave rise to demon and ancestor cults, and then to the worship of the gods. This view is maintained by Edward Tylor, Herbert Spencer, Julius Lippert, and a number of others. Undeniable as it is that soul belief has exerted an important influence upon mythological and religious thought, it nevertheless represents but one factor among others. For this very reason, however, we must consider separately its own peculiar conditions, since it is thus alone that we can gain an understanding of its relation to the other factors of mythological thought. The fittest place for examining this general interconnection is just at this point, where we are in the very midst of totemic ideas, and where we encounter the transformations of soul ideas in a specially pronounced form. Everything goes to show that the most important change in the history of the development of soul belief falls within the totemic period. This change consists in the distinction between a soul that is bound to the body, and which', because of this permanent attachment, we will briefly call the corporeal soul, and a soul which may leave the body and continue its existence independently of it. Moreover, according to an idea particularly peculiar to the totemic age, this latter soul may become embodied in other living beings, especially in animals, but also in plants, and even in inanimate objects. We will call this soul psyche, the breath or shadow soul. It is a breath soul because it was the exhalation of the breath, perhaps, that first suggested these ideas; it is a shadow soul since it was the dream image, in particular, that gave to this soul the form of a shadowy, visible but intangible, counterpart of man. As a fleeting form, rapidly appearing and again disappearing, the shadow 
soul is a variety of breath soul. The two readily pass over into each other, and are therefore regarded as one and the same psyche.

There is ground for the conjecture that the distinction between these two main forms of the soul, the corporeal and the breath or shadow soul, is closely bound up with the changed culture of the totemic age. Primitive man flees from the corpse-indeed, even from those who are sick, if he sees that death' is approaching. 'The corpse is left where it lies, and even the mortally ill are abandoned in their helpless condition. The living avoid the places where death has entered. All this changes in an age that has become familiar with struggle and death, and particularly with the sudden death which follows upon the use of weapons. This is exemplified even by the natives of Australia, who are armed with spear and shield. The warrior who falls before the deadly weapon, whose blood flows forth, and who expires in the midst of his fellow-combatants, arouses an entirely different impression from the man of the most primitive times who dies in solitude, and from whose presence the living flee. In addition to the original ideas of a soul that is harboured in the body, and that after death wanders about the neighbourhood as an invisible demon, we now have a further set of ideas. The soul is believed to leave the body in the form of the blood. But it may take an even morel sudden departure, being sometimes supposed to leave in the last breath. In this case, it is held to be directly perceptible as a small cloud or a vapour, or as passing over into some animal that is swift of movement or possesses such characteristics as arouse an uncanny feeling. This idea of a breath soul readily leads to the belief that the psyche, after its separation from the body, appears in the dream image, again temporarily assuming, in shadowy form, the outlines of its original body.

Now the most remarkable feature of this entire development is the fact that the idea of the corporeal soul in no wise disappears, as one might suppose, with the origin of the breath or shadow soul. On the contrary, both continue 
to exist without any mutual interference. This is noticeable particularly in the case of death in war. The belief that the soul leaves the body with the blood may here be directly combined with the belief that it departs with the breath, though the two ideas fall under entirely different categories. Even in Homer this combination of ideas is still clearly in evidence. The breath soul is said to descend to Hades, there to continue its unconscious existence as a dreamlike shadow, while at the same time the corporeal soul is thought to inhere not only in the blood but also in other parts of the body. Certain particular organs of the body are held to be vehicles of the soul; among these are the heart, the respiratory organs, and the diaphragm, the latter probably in connection with the immediately adjacent kidneys, which these primitive soul ideas usually represent as an important centre of soul powers. The believer in animism was not in the least aware of any contradiction in holding, as he did for a long time, that these two forms, the corporeal soul and the breath soul, exist side by side. His concern was not with concepts that might be scientifically examined in such a way as to effect a reconciliation of the separate ideas or a resolution of their contradictions. Even the ancient Egyptians, with their high civilization, preserved a firm belief in a corporeal soul, and upon this belief they based their entire practice of preserving bodies by means of embalmment. The reason for leaving the mouth of the mummy open was to enable the deceased person to justify himself before the judge of the dead. That the mummy was very carefully enclosed in its burial chamber and thus removed from the sphere of intercourse of the living, indicates a survival of the fear of demoniacal power which is characteristic of the beginnings of soul belief. The Egyptians, however, also developed the idea of a purely spiritual soul. The latter was held to exist apart from the body in a realm of the dead, from which it was supposed occasionally to return to the mummy. It was by this simple expedient of an intercourse between the various souls that mythological thought here resolved the contradiction between unity and multi- 
plicity as affecting its soul concepts-a contradiction which even later frequently claimed the attention of philosophy.

When, on a more advanced cultural level, the structure of the body came to be more closely observed, a strong: impetus was given towards a progressive differentiation of the corporeal soul. Certain parts of the body, in particular, were singled out as vehicles of the soul. Those that are separable from the body, such, for example, as certain secretions and the products of growth, received a sort of intermediate position between the corporeal soul proper and the breath soul. Chief among these was the blood. Among some peoples, particularly the Bantus of South Africa, the saliva rivals the blood in importance, possibly because of the readily suggested association with the soul that departs in the vapour of the breath. The blood soul, however, is by far the most universal and most permanent of these ideas. In its after-effects it has survived even down to the present. For, when we speak of a 'blood relationship' uniting those persons who stand close to one another through ancestry, the word 'blood' doubtless represents a sort of reminiscence of the old idea of a blood soul. To the dispassionate eye of the physiologist, the blood is one of the most unstable elements of the body, so that, so far as the blood is concerned, the father and mother certainly transmit nothing of a permanent nature to their descendants. More stable parts of the organism are much more likely to be inherited. But, in spite of the fact that blood is one of the most transitory of structures, it continues to be regarded as the vehicle of the relationship existing between members of a family, and even between tribally related nations. More striking expressions of the idea of a blood soul are to be found on primitive levels. In concluding the socalled blood brotherhood, the exchange of blood, according to prevalent belief, mediates the establishment of an actual blood relationship. In accordance with a custom which probably sprang up independently in many different parts of the earth, each of the two parties to the compact, upon entering this brotherhood, took a drop of blood from a 
small, self-inflicted wound and transferred it to the corresponding wound of the other. Since the drop of exchanged blood represents the blood in general--not merely symbolically, as it were, but in real actuality-the two who have entered into the alliance have become nearest blood relatives, and thus brothers.

The idea that a soul exists in the blood, however, has also a converse aspect. This consists in the fear of shedding blood, since the wounded person would thus be robbed of his soul. The belief then arises that one who consumes the blood of a sacrificed person or animal also gains his soul powers-an idea which likewise comes to have reference to other parts of the body, particularly to the specific bearers of the soul, such as the heart and the kidneys. Thus, between fear, on the one hand, and this striving for power, on the other, a conflict of emotions may arise in which the victory leans now to the one and now to the other side. But the striving to appropriate the soul which is contained in the blood tends to become dominant, since the struggle which enkindles the passion for the annihilation of the enemy is also probably the immediate cause for acting in accordance with this belief concerning the blood. To drink the blood of the slain enemy, to consume his heartthese are impulses in which the passion to annihilate the foe and the desire to appropriate his soul powers intensify each other. These ideas, therefore, also probably represent the origin of anthropophagy. Anthropophagy is not at all a prevalent custom among primitive tribes, as is generally believed. On the contrary, it is just among primitive peoples that it seems to be entirely lacking. It appears in its primary forms, as well as in its modifications, only where weapons and other phenomena point to intertribal wars, and the latter do not occur until the beginning of the totemic age. The totemic age, however, is the period which marks the development not only of the idea of the blood soul but of other soul ideas as well. Accordingly, anthropophagy is, or was until recently, to be found, not among the most primitive peoples such as have not attained to the level of totemism, 
but precisely within the bounds of totemic culture, and, in part, in connection with its cults. In these cults, man, as well as the animal, becomes an object of sacrifice in the blood offering. Human sacrifice of this sort continues to be practised under conditions as advanced as deity cult. In the latter, anthropophagy even finds a temporary religious sanction, inasmuch as the priest, particularly, is permitted to eat of the flesh of the sacrifice. Of course, the perpetuation and extension of anthropophagy was not due merely to magical motives; even at a very early period, the food impulse was a contributing factor. The very fact of the relatively late origin of the custom, however, makes it highly improbable that the food impulse would, of itself and apart from magical and cult motives, ever have led to it, though such an explanation has been offered, especially as regards the regions of Oceania where animals are scarce.

In the course of religious development, human sacrifice gave way to animal sacrifice, and cult anthropophagy was displaced by the eating of the flesh of the sacrificial animal. Inasmuch as the latter cult was not only more common than the former but everywhere probably existed prior to the rise of human sacrifice, this later period involved a recurrence of earlier conditions. Nevertheless, there were phenomena which clearly indicated the influence of the fear of the blood, and this militated against the appropriation of the blood soul. Of extreme significance, for example, was the injunction of the Israelitic Priests' Code against partaking of the blood of animals. The original motive for drinking the blood became a motive for abstaining from it-a counter-motive, in which the prohibition, as in many other cases, may also indicate an intentional abandonment of an earlier custom. Among the Israelites, as among many other Semitic tribes, the blood of the animals was poured out at the sacrificial altar. That which was denied man was fitly given to the gods, to whom the life of the animal was offered in its blood.

In early ages, reaching down probably into the beginnings of totemic culture, two organ complexes, in addition to the 
blood, were held, in an especial degree, to be vehicles of the corporeal soul-the kidneys with their surrounding fat, and the external sexual organs. The fact that, in many languages, kidneys and testicles were originally denoted by the same name, indicates that these two organs were probably regarded as essentially related, a view that may possibly be due to the position of the urethra, which apparently connects the kidneys with the sexual organs. The Bible also offers remarkable testimony in connection with the history of the belief that soul powers are resident in the kidneys and their appended organs. In the earlier writings of the Old Testament, the kidneys, as well as the heart, are frequently referred to as bearers of the soul. It is said of God that he searches the heart and tries the reins; and Job, afflicted with sorrow and disease, complains, " $\mathrm{He}$ cleaveth my reins asunder and doth not spare." The sacrificial laws of the Israelites, therefore, state that, in addition to the blood, the kidneys with their surrounding fat are the burnt offering which is most. acceptable to God. Rationalistic interpretation has sometimes held that man retains the choice parts of the flesh of the sacrificial animal for himself and devotes the less agreeable parts to the gods. Such motives may have played a rôle when sacrificial conceptions were on the wane. The original condition, however, was no doubt the reverse. The most valuable part belonged to the gods, and this consisted of the organs that were pre-eminently the vehicles of the soul. Though man first aimed to appropriate the soul of the sacrifice for himself, the developed religious cult of a later period made this the privilege of the deity.

It was only in early custom and cult, however, that the kidneys played this rôle. Indeed, as already indicated, it is not improbable that they owe their importance to the fact that their position led to the belief that they are a central organ governing particularly the sexual functions. That this is the case is corroborated by the fact that, in the further development of these ideas of a corporeal soul, the kidneys more and more became secondary to the external 
sexual organs, and that the latter long continued to retain the dominant importance. Thus, the phallus cult, which was prevalent in numerous Oriental countries and which penetrated from these into the Greek and Roman worlds, may doubtless be regarded as the last, as well as the most permanent, expression of those ideas of a central corporeal soul that were originally associated with the kidneys and their surrounding parts. At the outset, the representation of the phallus was held to be not a mere symbol, as it were, but the very vehicle of masculine power. As a productive, creative potency, it was regarded as very especially characteristic of the deity, and, just as the attributes of deities were supposed to be vested in their images, so also was this divine power thought to be communicated to the phallus. In addition to and anteceding these ideas relating to gods, the phallus was held to be the perfect embodiment of demons, particularly of fielddemons, who cause the ripening and growth of the seed. The belief in phallus-bearing demons of fertility probably dates back to the totemic age. The cults, however, to which such ideas of the corporeal soul gave rise, reached their mature development only in the following period. It was then that deity belief was elaborated, and it was in connection with the latter that the phallus became a universal magic symbol of creative power. With the decline of these cults, the symbol, according to a law observable in the case of other phenomena also, was again relegated, for the most part, to the more restricted field of its origin.

Vestiges and survivals of the primitive forms of the corporeal soul extend far down into "later culture. Nevertheless, the second main form of soul-belief, that of the psyche, comes to gain the prepondering influence, at first alongside of the corporeal soul, and then more and more displacing it. In this case, the earliest form of the belief, that in a breath soul, proves to be also the most permanent. The idea that the soul leaves the dying person in his last breath, and that the breath, therefore, exercises animating or magical effects, or that in it the soul may 
pass over from one person to another, is a very common belief. Probably, moreover, it arose independently in many different localities. Some primitive tribes have the custom of holding a child over the bed of a dying person in order that the soul may pass over into it; or, a member of the family stoops over the expiring one to receive his soul. Virgil's AEneid contains an impressive account relating that upon Dido's death her sister attempted to catch the soul, which, as she assumed, roams about as an aerial form, while she also carefully removed the blood from the wound in order that the soul might not remain within the body. Thus, the blood soul and the breath soul are here closely connected.

In the further destinies of the breath soul, a particularly important incident is its passage into some swiftly moving animal, perhaps a bird hovering in the air, or, again, some creeping animal, such as the lizard or the snake, whose manner of movement arouses uncanny fear. It is these animals, chiefly, that are regarded as metamorphoses of the psyche. Remarkable evidence that the bird and snake in combination were regarded as vehicles of the soul may be found in the pictorial representations of the natives of northwestern America. The escape of the soul from the body is here portrayed as the departure of a snake from the mouth of a human figure seated in a birdlike ship. This picture combines three ideas, which occur elsewhere also, either singly or in combination, in connection with the wandering of the soul. There is, in the first place, the soul-bird; then the soul-ship, readily suggested by association with a flying bird, and recurring in the ship which was thought in ancient times to cross the Styx of the underworld; finally, the soul-snake, representing the soul in the act of leaving the body. This very common idea of the soul as a snake and, by further association, its conception as a fish, may be ascribed not only to the fear aroused by the creeping snake, but also to the circumstances attending the decomposition of the corpse. The worm which creeps out of the decaying body is directly perceived as a snake. Thus, corporeal soul and psyche are again 
united; in this union they mediate the idea of an embodied soul, which, in a certain sense, of course, is a psyche retransformed into a corporeal soul.

With the appearance of these ideas of : an embodied soul, totemism merges directly into soul-belief. Under the influence of the remaining elements of totemism, however, the soul-ideas come to be associated with more and more animals. The soul is no longer held to be embodied merely in the earliest soul-animals-bird, snake, and lizard-but other animals are added, such particularly as those of the chase, which have a closer relation to the life of man. Following upon this change are also the further developments mentioned above. When interest in the production of vegetable food is added to that of the chase, the same ideas become associated with plants. Their sprouting and growth continue to suggest soulpowers; and, even though the ancestor idea characteristic of the animal totem cannot attain to prominence because of the greater divergence of plants from man, this very fact causes the phenomena of sprouting and growth all the more to bring into emphasis the magical character of these vegetable totems. Hence it is mainly the plant totem that gives rise to those ceremonies and cult festivals which are designed for the magical increase of the totems. With the wane of the soul-beliefs connected with animal totemism, it is not only plants to which demoniacal powers are ascribed. Even inanimate objects come to be associated with magical ideas, either because of certain peculiar characteristics or because of the function which they perform. It is in this way that the introduction of the plant into the realm of totemic ideas mediates the transition from the totem to the fetish. On the other hand, as the totem animal comes more and more to be an ancestral animal, and as the memory of human forefathers gains greater prominence with the rise of culture, the animal ancestor changes into the human ancestor. Thus, fetishism and ancestor worship are logical developments of totemism. Though differing in tendency, they nevertheless constitute developmental forms which are not at all mutually exclu- 
sive, but which may become closely related, just as is the case with the animal and the plant totems from which they have proceeded.

Before turning to these later outgrowths of totemic soul-belief, however, we must consider their influence upon the important customs relating to the disposition of the dead. These customs give expression to the ideas of death and of the destiny of the soul after death. Hence the changes that occur at the beginning and in the course of the totemic age as regards the usages relative to the disposal of the corpse, mirror the important transformations which the latter undergoes. Primitive man, as we have seen, flees from the corpse. Dominated solely by his fear of escaping demons, he allows the dead to lie where they have died. Thus, no attempt whatsoever is made to dispose of the dead, or at most there are but slight beginnings in this direction. It is not the dead who vacate the premises in favour of the living, but the latter accommodate themselves to the dead. Totemic culture, accustomed to armed warfare and sudden death, begins from the outset gradually to lose its fear of the dead, even though not the fear of death, and this reacts upon the disposal of the corpse. Of course, the early custom of depositing the corpse in the open air near the place where death has occurred, does not entirely disappear. This locality, however, is no longer avoided; on the contrary, anxious expectation and observation are now fixed upon the corpse. Just as totemic man drinks the blood of those who are slain in battle, in order to appropriate their power, so also in the case of those who die of disease does he wish to acquire their souls the moment they leave the body. Traces of such a custom, indeed, occur even in much later times, as is shown in Virgil's above-mentioned account of the death of Dido. Within the sphere of totemic ideas, however, where the belief in a corporeal soul is still incomparably stronger, though already intercrossing with the belief in animal transformations of the psyche, the custom of depositing the dead in the open indeed continues to be practised, yet the disposition of the corpse 
changes, becoming, in spite of an external similitude, almost the very opposite. The corpse is no longer left at the place of death, but is stretched out on a mound of earth. This is the so-called 'platform' method of disposal, which, as is evident, forms a clear transition to burial, or interment. Before the mound of earth covers the body, it forms a platform upon which the corpse is laid out to be viewed, a primitive catafalque, as it were. This manner of disposing of the corpse has been regarded as a custom characteristic of the dominance of totemic culture. This is going entirely beyond the facts, since other modes of disposal are also to be found even in Oceania and Australia, the chief centres of totemism. Nevertheless, the phenomena connected with exposure on a platform indicate that a fusion with soul-ideas has now taken place. Decomposition follows relatively soon after death, particularly in a damp, tropical climate. On the one hand, the liquid products of decomposition that flow from the corpse are interpreted as a departure of the soul analogous to that which occurs, in the case of death by violence, in the loss of blood. As the blood is drunk to appropriate the soul of the deceased, so also do the relatives now crowd in to partake of the liquid products of decomposition-a transference similar to that which sometimes occurs when the powers of the blood are ascribed to the saliva or to other secretions. On the other hand, the first worm of decomposition to leave the corpse is held to be the bearer of the soul. Thus, corporeal soul and psyche are here closely fused. The liquid products that leave the body are in themselves elements of the corporeal soul, but in their separation from the body they resemble a psyche incorporated in an external object ; conversely, the worm of decomposition is an embodiment of the psyche, which is itself represented as proceeding directly from the corporeal soul.

This interplay of soul-forms appears also when we consider the other modes of disposing of the dead that are practised in regions where totemic culture or its direct outgrowths prevail. Among some of the North American 
Indian tribes, for example, the corpse is buried, but a small hole is pierced in the mound of earth over the grave, in order to allow the psyche an exit from the body or also a return to it. This view of the relation between body and psyche passed down, in a more developed form, even into the other-world mythology of the ancient Egyptians. The mummification practised in Egypt was also anticipated, for the idea of the connection of the soul with the body early led to the exsiccation of the corpse in the open air. According to another usage, observed particularly in America, the corpse was first buried, but then, shortly afterwards, exhumed for the purpose of preserving the skull or other bones as vehicles of the soul. The fundamental idea seems to have been that the soul survives in these more permanent parts of the body; in the case of the skull, an appreciation of the importance which the various organs of the head possess for the living person may also have played a rôle. Possibly these ideas likewise lie at the basis of the discreditable head-hunting practised by the Indians, even though it be true that the skull, which is preserved and utilized as a favourite adornment of the exterior of the hut, and also the representative of the skull, the scalp, have long been mere trophies of victory, similar to the antlers of the stag and the deer with which our huntsmen decorate their dwellings. Of the various forms of disposing of the dead that are peculiar to the totemic age, however, it is interment, the very opposite of platform disposal, that finally comes to be adopted in many places. The reason is evidently the same as that which impelled primitive man to flee from the corpse. The demons of the dead are to be banished into the earth, so that the living may pursue their daily activities undisturbed. That this is the aim is shown by many accompanying phenomena-such, for example, as the custom of firmly stamping down the earth upon the grave, or of weighting the burial-mound with stones. Moreover, the custom of burying the corpse as soon as possible after death-ordained even at the time of the Israelitic law-can hardly have originated as a hygienic provision. It is 
grounded in the fear of demons. When the living themselves no longer flee from the dead, this fear all the more necessitates the speedy removal of the corpse to the secure protection of the earth. The fear of demons is likewise expressed in the fact that prior to burial the arms and legs of the corpse are bound to the body. This obviously points to a belief that the binding constrains the demon of the dead, which is thereby confined to the grave just as is the fettered corpse. Herein lies the origin of the so-called 'crouching graves,' which are still to be found among the Bushmen, as well as among Australian and Melanesian tribes. Gradually, however, a change took place in that the binding was omitted, though the position was retained-doubtless a sign that fear of the demon of the dead was on the wane.

Under the influence of the profuse wealth of old and new soul-ideas, therefore, the totemic age developed a great number of modes of disposing of the dead. Of these modes, interment alone has survived. It is simpler than the others and may be practised in connection with the most diverse ideas of the destiny of the soul. Cremation was the only form of disposing of the dead that was unknown, at least in large part, to the totemic age. And yet the motives underlying cremation belong to the same circle of ideas as those that find expression in the customs of taboo and lustration. It is not impossible, therefore, that cremation may itself date back to the totemic age. Yet interment is universally the earlier mode of disposal; in most parts of the earth, moreover, it has also enjoyed a greater permanence. Only in isolated districts has interment been displaced by cremation. Even in early times it was chiefly among Indo-Germanic peoples that cremation was practised, whereas the Semites everywhere adhered to interment. If, therefore, cremation occurred in ancient Babylonia, as it appears to have done, it probably represents a heritage from the Sumerian culture preceding the Semitic immigration. But even among. Indo-Germanic peoples interment was originally universal. In Greece, it existed as late as the period of Mycenian culture. By the time of Homer, on 
the other hand, cremation had already become the prevalent mode of disposition of the corpse. Cremation was likewise practised very early by the Germans, the Iranians, and the peoples of India. But it was always conditioned by one fact which, as a rule, would seem to carry us beyond the boundaries of the totemic era. It is significant that prehistoric remains show no traces of cremation prior to the beginning of the bronze age-a period in which man was capable of utilizing the high degrees of heat necessary to melt metals. The tremendous heat required for the melting of bronze might well have suggested the idea of also melting man, as it were, in the fire. Nevertheless, external circumstances such as these played but a secondary rôle. They leave unanswered the decisive question regarding the motives that led to the substitution of cremation for interment. This, then, remains our unsolved problem, inasmuch as the economic motives at the basis of the present endeavour to reintroduce cremation were certainly not operative at the time of its origin. With reference to the origin of cremation, only psychological probabilities are possible to us. These are suggested particularly by the ceremonies which accompany cremation in India-the country where this custom has continued to preserve an important cult significance down to the very present. Indeed, even in our own day it has hardly been possible to eradicate from India the custom of burning the widow of the deceased. In particular, two different motives to the custom suggest themselves. In the first place, as we shall presently see, sacrificial usages, and especially the more advanced forms of the sacrifice to the deceased, are closely connected with the taboo and purification customs. Purification from a taboo violation, however, was attained primarily by two means, water and fire. The latter of these means was employed even in very primitive times. Now, the corpse, above all else, was regarded as taboo; contact with it was thought to bring contamination and to demand the rites of lustration. The one who touched a corpse was likewise held to be taboo, and as a result he him- 
self might not be touched before having undergone lustration. By one of those associative reversals which are common in the field of mythology, this then reacted upon the corpse itself. The corpse also must be subjected to a lustration by which it is purified. Such a purification from all earthly dross is mediated, according to the ideas of India, by fire. When the body is burned, the soul becomes pure. But connected with this belief, as we may conjecture, is still a second idea. The soul or psyche departs in the smoke which ascends from the body as this is burned. The body remains below in the ashes, while the soul soars aloft to heaven in the smoke. In this way, the burning of the corpse is closely connected with celestial mythology, which, indeed, was likewise developed relatively early among the Indo-Germanic peoples, with whom cremation had its centre. The customs of the Semitic peoples were different. They adopted the idea of a celestial migration of the soul only at a late period, probably under IndoAryan influences; but even then they continued to practise the ancient custom of burial. Amid these differences, however, there is a certain similarity. For, the Semitic peoples believed that the celestial migration of the soul would occur only after its sojourn under the earth, following upon its resurrection, which, it was thought, would take place only at the end of time. It was in this form, as is well known, that Christianity took over into its resurrection belief the ideas developed by Judaism, and, with them, the custom of interment.

\section{I2. The Origin OF the Fetish.}

If, as is customary, we employ the term 'fetish' to mean any natural object to which demoniacal powers are ascribed, or, as the word itself (Fr. fétiche from Lat. facticius, artificially constructed) indicates, an artificial, inanimate object of similar powers, a wide gulf appears at first glance to separate the fetish from the psyche. Nevertheless, the two are very closely related, as. is indicated by the totemic origin of certain primitive forms of fetishes. 
In the cults of totemic clans, magic stones and pieces of wood are reverenced and preserved, being regarded as powerful instruments that were originally fashioned, according to legend, by magic beings of a distant past. Into the objects has passed the magic power of these ancestors. By their agency, the plants and animals which man utilizes as food may be increased; through them, evils may be averted and, in particular, diseases may be cured. The universal characteristic of the fetish, however, over and above this special mode of origin, is the fact that it is supposed to harbour a soul-like, demoniacal being. In fact, most of the phenomena of so-called fetishism, and those which are still regarded as typical of it, are to be found outside of totemic cult. It is primarily African fetishism, a cult form which is apparently independent of totemism, that has given its characteristic stamp to the conception of the fetish. Among the Soudan negroes, fetishes generally consist of artificially fashioned wooden objects, not infrequently bearing a grimacing likeness of a human face. As regards the possession of magical powers, however, they do not differ from the so-called churingas of the Australians, although the latter are, as a rule, natural objects that have been picked up accidentally and that differ from ordinary stones and pieces of wood only in their striking form. It is clearly the form, both in the case of the artificial as well as of the natural fetish, that has caused the inanimate object to be regarded as a demoniacal vehicle of the soul. Yet it is not a lifeless object as such that constitutes a fetish, but the fact that a demoniacal, soul-like being is believed to lurk within it as an agency of magical activities.

At the time of its origin, which was probably totemic, fetishism possessed a more restricted meaning than that just given. Defined in this broader way, however, fetishism may be said to be disseminated over the entire earth. It is a direct offshoot of the belief in a corporeal soul, according to which magical powers are resident in certain parts of the human body. In Australia and elsewhere, the kidneys, particularly, are held to possess magical powers. The same, 
however, is true of the blood-also of the hair, which, as the Biblical legend of Samson serves to show, was supposed to be an especial centre of demoniacal power, and is still regarded by modern superstition as a means of magic. Thus, the transference of the properties of the soul to inanimate objects of nature appears, on the one hand, to be closely related to the activity of the soul in certain parts of the body; on the other hand, it is closely connected with the fact that certain independent beings, particularly such as arouse the emotions of surprise or fear by their form and behaviour, were believed to embody souls. The greater the difference between the object in which such a demon takes up his abode and the familiar sorts of living beings, the more does its demoniacal activity become a pure product of the emotions, which control the imagination that ascribes life to the object. Thus, while the characteristics of the totem animal and, to a certain extent, even those of the totem plant, continue to be determined by their own nature, the fetish is solely the product of the mental activities of the fetish believer. Whereas the totem, particularly the totem animal, retains in great part the nature of a soul, the fetish completely assumes the character of a demon, differing from the demons resident in storms, solitary chasms, and other uncanny places only in the fact that it is inseparable from the discovered or artificially fashioned object. Hence it all the more becomes the embodiment of the emotions of its possessor, of his fears and of his hopes, ever adapting itself to the mood of the moment.

The development of magical ideas is in an especial measure due to the incorporation of demoniacal beings in inanimate objects. Such objects circulate freely and may even survive the individual who owns them, gaining by their permanence an advantage over the animate objects to which soul-like demoniacal powers are ascribed. Inanimate objects may embody the magical beliefs of whole generations. This is exemplified even in the age of deity beliefs, for a sanctuary acquires increasing sacredness with age. And yet the fetish is never valued on its own account, as 
is the totem animal-in part, at least-or the organ containing the corporeal soul. The fetish is merely a means for furthering purposes of magic. It is especially the fetish, therefore, that represents the transition from soul-beliefs to pure magic-beliefs. For this reason we may speak of a 'cult of the fetish' only in so far as external ceremonies are employed for the purpose of arousing the fetish to magical activity. Such a fetish cult does not include expressions of reverence and thanksgiving, as do the soul and totem cults and later, in a greater measure, the deity cult. A fetishism of this sort, purely magical in purpose, may be found particularly in the Soudan regions of Africa. Fetishistic magic-cult here prevails in its most diverse forms, having, to all appearances, practically displaced the original soul and totem beliefs, though traces of the latter are everywhere present. Frequently it is an individual who calls upon his fetish, perhaps to free him from a sickness, or to protect him from an epidemic, or also to aid him in an undertaking, to influence distant objects, injure an enemy, etc. But an entire village may also possess a fetish in common, committing it to the care of the medicine-man. When exigencies arise, a threatening war or a famine, such a village fetish is particularly fêted in order that he may be induced to avert the disaster.

Among cult objects the fetish occupies a low place. Nevertheless, it is precisely because the demoniacal powers were supposed to be harboured in an inanimate object that the fetish prepared the way for the numerous transitions that led to the later cult-objects in the form of divine images. The fetish, as it were, was a precursor within the totemic age of the divine image of later times. For in the case of the latter also, the deity was supposed to be present and immediately operative ; the image, therefore, was called upon for assistance just as was the god himself. Originally, all worship involved an image that was supposed to embody the deity. The divine imałge, of course, differed in essential respects from the fetish, for it incorporated, as the personal characteristics of the god, those traits that were 
gradually developed in cult. The fetish, on the other hand, was impersonal ; it was purely, a demon of desire and fear. Because its activity resembled that of human beings, it was generally given anthropomorphic features, though occasionally it was patterned after animals. Sometimes no such representation was attempted, but, as in the case of the Australian churingas, an object was left just as it was found, particularly if it possessed a striking form. Nor did the divine image come of a sudden to its perfected form. Just as it was only gradually, in the development of the religious myth, that the god acquired his personal characteristics, so also did art search long in every particular case for an adequate expression of the divine idea. In so doing, art not merely gave expression to the religious development, but was itself an important factor in it. The development, however, had its beginning in the fetish. Moreover, so long as the god remains a demoniacal power without clearly defined personal traits, the divine image retains the indeterminate character of the fetish image. Even among the Greeks the earliest divine images were but wooden posts that bore suggestions of a human face; they were idols whose external appearance was as yet in nowise different from that of fetishes. The same is true of other cultural peoples in so far as we have knowledge of their earliest objects of religious art.

But there may be deterioration as well as advance. Wherever artistic achievement degenerates into the crude products of the artisan, the divine image may again approximate to the fetish. Religious cult may suffer a similar relapse, as is shown by many phenomena of present-day superstition. When religious emotions are restricted to very limited desires of a magical nature, the cult also may degenerate into its earliest form, so that the image of the deity or saint, reverting into a fetish, again becomes a means of magic. It is primarily such degenerate practices, or, as they might also be called, such secondary fetish-cults, that give the phenomena of so-called fetishism their permanent importance in the history of religion. The complexity 
of this course of development has led psychologists of religion to conflicting views in their interpretations of fetishism. On the one hand, the primitive nature of fetishes, and the fact that the earliest divine images resemble fetishes, have led to the assertion that fetishism is the lowest and earliest form of religion. On the other hand, fetishism has been regarded as the result of a degeneration, and as universally presupposing earlier or contemporary religious cults of a higher character. The latter of these views particularly, namely, the degeneration theory, is still maintained by many historians of religion, especially by those who believe that monotheism was the original belief of all mankind. The evidence for this theory is derived mainly from cultural phenomena of the present. The image of a saint, as is rightly maintained, may still occasionally degenerate into a fetish, as occurs when it is regarded as the seat of magical powers, or when its owner believes that he possesses in it a household idol capable of bringing him weal or woe. It was particularly Max Müller who championed the degeneration theory. Even in his last writings on mythology he held firmly to the view that fetishism is a phenomenon representing the decay of religious cults. But if we take into account the entire course of development of the fetish, this view collapses. Though substantiated by certain events that occur within higher religions, it leaves unconsidered the phenomena that are primitive. The earliest fetishistic ideas, as we have seen, go far back into the period of soul and demon beliefs. Developing from the latter, they were at first closely bound up with them, though they later attained a relative independence, as did so many other mythological phenomena. To think of fetishism as a degeneration of religious cults is inadmissible for the very reason that, in so far as such cults presuppose deity ideas, they cannot as yet be said to exist. A striking proof of this contention is offered particularly by that form of fetish cult, the churingal ceremony of the Australians, in which the connection with related primitive ideas may be most clearly traced. The churingal ceremony falls entirely 
within the development of totemism, and arises naturally under certain conditions; it is no more the product of degeneration than is the appearance of plant totemism in place of animal totemism. The basal step in the development of the fetish is the incorporation of soul-like demoniacal powers in inanimate objects, whether these be objects as they are formed by nature or whether they are artificially constructed. Such objects may result from a deterioration of religious art, but this is by no means the only alternative. In their original forms, they are allied to far more primitive phenomena, such as antedate both religious art and even religion itself, in the true sense of the word. For, of the many forms of the fetish, the most primitive is obviously some natural object that has been accidentally discovered. Such are the churingas of the Australians, and also many of the fetishes of the negroes, although others are artificially fashioned. The selection of such a fetish is determined in an important measure by the fact that it possesses an unusual form. The man of nature expects to find symmetry in animals and plants, but in stones this appears as something rare. Astonishment, which, according to circumstances, may pass over into either fear or hope, causes him to believe some soul-like being to be resident in the inanimate object. This accounts for the existence of such legends as those that have survived among some of the Australian tribes, in which fetishes, or churingas, are represented as the legacy of certain fantastically conceived ancestors. From the natural to the artificial fetish is but a short step. When natural objects are not to be found, man supplies the want. He constructs fetishes, intentionally giving them a striking form resembling that of a man or of some animal. Such fetishes are then all the more regarded as abodes of soul-like beings.

Hence we must also regard as untenable that theory which, in contrast with the degeneration theory, represents fetishism as a primitive mythology or even as the startingpoint of all mythology and religion. The fetish is not at all an independent cult-object characteristic of some primitive or more advanced stage of development. It 
always represents a secondary phenomenon which, in its general significance as an incorporation of demoniacal powers of magic, may occur anywhere. If, however, we inquire as to when fetishistic ideas make their first appearance, and where, therefore, they are to be found in their relatively primitive form, we will find that they are rooted in totemic ideas. Hence it is as a particular modification of such ideas that fetishism must be regarded. In the metamorphosis, of course, some of the essential traits of the original totem disappear. The fetish, consequently, acquires a tendency toward independence, toward becoming, apparently, a separate cult-object. This is illustrated by the fetish cult of many negro tribes. To however great an extent such independent cults may frequently have displaced the totemism from which they sprang, they nevertheless belong so properly to the totemic world of demons and magic that fetishism, in its genuine form, may unquestionably be regarded as a product of the totemic age.

Further verification of this contention may be found in the history of certain incidental products of fetishistic ideas, the amulet and the talisman. These occur at all stages of religious growth, but their development falls principally within the totemic period. The two objects are closely related, yet they differ essentially both from one another and from their parent, the fetish. It has, of course, been denied that a distinction may be drawn between these various objects of magic belief. From a practical point of view, this may doubtless sometimes be true, one and the same object being occasionally used now as a fetish and then again as an amulet or a talisman. But it is precisely their use that distinguishes these objects with sufficient sharpness from one another. The amulet and talisman are purely magical objects, means by which their possessor may produce magical effects. The fetish, however, is a magic-working subject, an independent demoniacal being, which may lend aid but may also refuse it, or, if hostilely. disposed, may cause injury. The amulet, on the other hand, always serves the purpose of protection. Not infrequently 
amulets are held to ward off merely some one particular disease; others are designed to avert sickness in general. In a broadened significance, the amulet then comes to be regarded as a protection against dangers of every sort, against the weapon no less than against malicious magic. Nevertheless, the amulet is always a means of protection to its possessor. It is its passive function, that of protection, which differentiates the amulet from the talisman. The latter, which is far less prominent, particularly in later development, and which is finally to be found only in the world of imaginal tales, is an active means of magic. By means of a talisman, a man is able to perform at will either some one magical act or a number of magical feats. The philosopher's stone of mediæval superstition exemplifies such a means of magic. In this case, the ancient talismanidea captured even science. The philosopher's stone was supposed to give its possessor the power to unlock all knowledge, and thus to gain control over the objects of nature. This illustrates the talisman in its most comprehensive function. In its restriction to a particular power, it makes its appearance in hero and deity legend, and even to-day in the fairy-tale. Such an active means of magic is represented by the helmet of invisibility, by the sword which brings death to all against whom it is turned, or, finally, by the Tischlein-deck-dich.

The two magical objects are generally also sharply distinct in their mode of use. The amulet is designed to render protection as effectively as possible against external dangers; it must be visible, for every one must see that its bearer is protected. Hence almost all amulets are worn about the neck. This was true of primitive man, and holds also of the survivals of the ancient amulets-women's necklaces, and the badges of fraternal organizations worn by men. The fact that a simple cord was used among primitive peoples and still prevails in present-day superstition, makes it probable that the original amulet was the cord itself, fastened about the neck or, less frequently, about the loins or the arm. Later, this cord was used to 
support the amulet proper. Even the Australians sometimes wear a piece of dried kidney suspended from a cord of bast-we may recall that the kidney is one of the important seats of the corporeal soul. The hair, teeth, and finger-nails of the dead likewise serve as amulets, all of them being parts of the body which, because of their growth, might well give rise to the idea that they, particularly, possessed soul-like and magical powers. The custom of attaching hair, or a locket containing hair, to a necklace, has survived even down to the present, though, of course, with a far-reachilg change of meaning. The magical protection of earlier ages has become a memorial of a loved one who has died. But here likewise we may assume that the change was gradual, and that the present custom, therefore, represents a survival of the primitive amulet. There are other objects also that apparently came to be amulets because of their connection with soul-ideas. Of these, one of the most remarkable is the scarab of the ancient Egyptians, which likewise continues to be worn even to-day. This amulet is a coloured stone shaped like a beetlemore specifically, the scarab. This beetle, with its red wingcoverings, has approximately the form of a heart; for this reason, both it and its representation were thought to be wandering hearts. As an amulet, however, its original significance was that of a vehicle of the soul, designed to protect against external dangers.

Whereas the amulet is worn so as to be visible, the talisman, on the contrary, is hidden so far as possible from the observing eye. It is either placed where it is inconspicuous, as is, for example, the finger ring, or it possesses the appearance of a familiar object. The magical sword gives no visible evidence of its unusual power; the helmet of invisibility resembles an ordinary helmet; the Tischlein-deck-dich of the fairy-tale is in form not unlike any other fable. It is with much the same idea that the Soudan negro who sets out upon an undertaking still takes with him some peculiar and accidentally discovered stone, in the hope that it will assist him in danger. This also is an example of a talisman, and not of a fetish. 


\section{The ANImAL ANCESTOR AND The Human ANCESTOR.}

The ideas fundamental to the cult of human ancestors, though also connected with soul-beliefs, are radically different from those that gave rise to the fetish. Whereas some mythologists have been inclined to regard fetishism as the primitive form of religion, others have made this claim for ancestor worship. The latter have believed that ancestor worship could be traced back to the very beginnings of culture, and that the god-ideas of the higher religions were a metamorphosis of ancestor ideas. This is corroborated, in their opinion, by the fact that in the age of natural religions the ruler or the aristocracy very generally claimed descent from the gods, and that the ruler and the hero were even worshipped as gods. The former is illustrated by the genealogy of Greek families; the latter, by the Roman worship of emperors, which itself but represented an imitation of an Oriental custom that was once very common. All these cases, however, are clearly secondary phenomiena, transferences of previously existing god-ideas to men who were either living or had already died. But even apart from this, the hypothesis is rendered completely untenable by the facts with which the history of totemism and of the earlier, more primitive conditions has made us familiar. Not a trace of ancestor worship is to be found among really primitive men. We have clear proof of this in their manner of disposing of the dead. So far as possible, the dead are left lying where they happen to be, and no cult of any kind is connected with them: Totemism, moreover, gives evidence of the fact that the cult of animal ancestors long anteceded that of human ancestors. Thus, then, the theory that ancestor worship was the primitive religion belongs essentially to an age practically ignorant of totemism and its place in myth development, as well as of the culture of primitive man. This era of a purely a priori psychology of religion still entertained the supposition, rooted in Biblical tradition, of an original state of pure 
monotheism. In so far as this view was rejected, fetishism and ancestor worship were generally rivals as regards the claim to priority in the succession of religious ideas. The only exception occurred when these practices were regarded as equally original, as they were, essentially, in the theories of Herbert Spencer, Julius Lippert, and others. In this event, the original form of the fetish was held to be an ancestral image which had become an object of cult.

True, along with the totemic ideas of animal ancestors we very early find indefinite and not infrequently grotesque ideas of human ancestors. In the 'Mura-mura' legends of southern Australia these ideas are so interwoven that they can scarcely be untangled. These Mura-mura are fanciful beings of an earlier age, who are represented as having transmitted magical implements to the generations of the present era and as having instructed the ancestors of the Australians in magical ceremonies. A few of the legends relate that the Mura-mura also created the totem animals, or transformed themselves into the latter. Here, then, we already find a mutual interplay between ideas of human and conceptions of animal ancestors. As yet, however, no clearcut idea of a human ancestor has been formed. This never occurs-a fact of prime importance as concerns its development-until the totem ancestor has lost his significance, and the original tribal totemism has therefore become of subordinate importance, even though totemism itself has not as yet completely disappeared. Under such circumstances the totem animal becomes the protective animal of the individual; the animal ancestor is displaced by the demon which mysteriously watches over the individual's life. This transition has already been touched upon in connection with the development of totemic ideas. Coincident with it, there is an important change with respect to the character of the totem animal. The tribal totem is an animal species. The Australian, whose totem, let us say, is the kangaroo, regards all kangaroos which he meets as sacred animals; he may not kill them, nor, above all, eat of their flesh. In the above-mentioned development of totemism (which is at the 
same time a retrogression) the totem animal becomes individualized. The protective animal-or the animal of destiny, as we might refer to it, in view of its many changes in meaning-is but an individual animal. A person may possibly never have seen the animal that keeps guard over him ; nevertheless, he believes that it is always near at hand. The unseen animal which thus accompanies him' is therefore sometimes also called his 'bush soul' ; it is hidden somewhere in the bushes as a sort of animal double. Whatever befalls the person likewise happens to it, and conversely. For this reason it is very commonly believed that, if this animal should be killed, the person also must die. This makes it clear why the North American Indian calls the animal, not his ancestor, but his 'elder brother.'

In South African districts, especially among the Bantus where the bush soul is common, and in North America, where the tribal totem has become a coat of arms, and fable and legend therefore continue all the more to emphasize the individual relation between a person and an animal, the idea of a human ancestor receives prominence. The totemic tribal organization as a whole, together with the totemic nomenclature of the tribal divisions, may continue to exist, as occasionally happens among the Bantus and in North America, even though the tribal totems proper have disappeared and become mere names, and the animal itself possesses no live importance except as a personal protector. But since the totemic tribal organization perpetuates the idea of a succession of generations, the human ancestor necessarily comes to assume the place of the animal ancestor. This change is vividly represented by the totem poles of the Indians of northwestern America. These totem poles we have already described. The head of the animal whose representation has become the coat of arms here surmounts a series of faces of human ancestors. Such a monument tells us, more plainly than words possibly could: These are the ancestors whom I revere and who, so far as memory reaches back, have found the symbol of their tribal unity in the animal which stands at their head. But totem poles do more than 
merely to directly perpetuate this memory. Though probably without the conscious intention of the artists who fashioned them, they also suggest something else, lost to the memory of living men. In the belief of earlier ages, this human ancestor was preceded by an animal ancestor to whom the reverence which is now paid to the human ancestors was at one time given. Thus, the animal ancestor was not only prior to the human ancestor from an external point of view, but gave rise to him through a neciessity immanent in the course of development itself.

The transition from animal to human ancestors, furthermore, is closely bound up with coincident transformations in tribal organization. Wherever a powerful chieftainship arises, and an individual, overtowering personality obtains supremacy over a tribe or clan-such supremacy as readily tends to pass down to his descendants-it is particularly likely that a cult will be developed in his honour, and, upon his death, to his memory. Since the memory of this personality outlasts that of ordinary men, the individual himself is held to live on after death, even in regions where there is no belief in a universal immortality. Hence, according to a belief prevalent particularly among the negro peoples, the ordinary man perishes with death; the chieftain, however, or a feared medicine-man, continues to live at least until all memory of him has vanished. In some parts of Africa and Oceania, moreover, the cult of the living chieftains not only involves manifestations of a servile subjection but, more characteristically still, causes even his name to be tabooed. No one is allowed to speak it, and whoever bears the same name must lay it aside when the chieftain assumes control.

As a result of the change in totemic tribal organization induced by the growing significance of chieftainship, the cult of living ancestors, as we may conclude from these phenomena, takes precedence over that of the just deceased, and still more over that of the long departed. In comparison with the importance which the man of nature 
attributes to living persons, that attaching to the dead is but slight, and diminishes rapidly as the individuals fade from memory. Individual rulers, whose deeds are remembered longer than those of ordinary men, may lay the foundations for an historical tradition. Nevertheless, the present long continues to assert a preponderating claim in belief as well as in cult. So long as man himself lives only for the present, having little regard for the future and scarcely any at all for the past, his gods also-in so far as we may apply this name to the supersensuous powers that shape his life-are gods of the present. True, the totem animal is secondarily also an animal ancestor. And yet it is only the living totem animal that is the object of cult and is believed to possess protective or destructive powers; compared with it, the ancestor idea fades into nebulous outlines, gaining a more definite significance only in so far as it is an expression of the tribal feeling which binds the members of the community to one another.

A further important factor enters into this development. This is the cult ceremony connected with the disposition of the dead. In this case, the departed one to whom the ceremony is dedicated is still directly present to memory. He holds, as it were, an intermediate position between the realm of the living and that of the dead. The memorial ceremony held in his honour also restores to memory older generations of the departed, even though this may cause theiri specific features to fade into indefiniteness and to assume outlines whose vagueness renders them similar. The American totem poles furnish a concrete portrayal of such a series of ancestors in which individual characteristics are totally lacking. Nevertheless, even under very diverse circumstances, we find that the ceremony in honour of one who has just died comes to develop into a general festival of the idead, and thus to include more remote generations. The circle of those who are honoured is likewise extended; the cult comes to be one that commemorates not merely chieftains but all tribesmen. As the wider tribal bonds dis- 
solve, the clan, and then later the family, pay their homage to the departed on the occasion of his funeral, and to earlier generations of the dead on specific days dedicated to such memories. This is the course of development in which the ancestor festivals of the Chinese and Japanese have their origin, as well as the cults of the Roman dii manes; it has introduced elements, at least, of ancestor worship into the beginnings of all religions, even though this cult but rarely attained the pre-eminent importance which it possessed among the cultural peoples of the Orient.

But whatever may have been the character of this earlier strain of ancestor worship in religious development, the beginning of a true ancestor cult is closely bound up with the universalization due to its having become the cult of the hearth and the family. As it is the human ancestor who displaces the animal ancestor in this cult, so the transition by which the family comes to be the central factor in social organization is an external indication of the dissolution of totemic culture and the dawning of a new era. In view of the predominant mythological and religious creations of this period, it might be called the age of heroes and gods. Ancestor worship itself is at the turning-point of the transition to the new era. In origin, it belongs to totemic culture: in its later development, it is one of the most significant indications of the dissolution of totemism, preparing the way for a new age in which it continues to hold an important place. At the same time, ancestor worship, no less than its rival, fetishism, constitutes but one factor among others in the development of mythological thought as a whole. In certain localities, as in the civilizations of eastern Asia, it may become sufficiently prominent to be one of the principal elements of religious cult. But even in such cases, ancestor worship is never able entirely to suppress the remaining forms of cult; still less can it be regarded as having given rise to the other fundamental phases of religious development-these rest on essentially different motives. Moreover, in connection with the relation of totemism to 
the ancestor worship which is rooted in the former and at the same time displaces it in one line of development, it is important to notice that in a certain sense the two follow opposite paths. As we have seen, the original totemthat is, the tribal totem-is the animal species in general ; the last form of totem is the protective animal, which is an individual animal. Ancestor worship, on the other hand, begins with the adoration of humanly conceived benefactors and prominent tribesmen. It ends with a worship in which the individual ancestor gives way to the general idea of ancestor, in whom the family sees only a reflection of its own unity and an object in terms of which reverence is paid to past generations. The fact that ancestor cult centres about impersonal beings betrays a religious defect. Herein also is evidenced the continuing influence of the totemic age, for it was in this period that ancestor worship had its rise. The defect just mentioned was first overcome with the origin of god-ideas. One of the essential characteristics of gods is precisely the fact that they are personal beings; each of them is a more or less sharply defined individuality. This of itself clearly indicates that ancestor worship is at most a relatively unimportant factor in the origin of gods.

\section{The TOTemic Cults.}

The primitive stage of human development, discussed in the preceding chapter, possessed no real cults in the strict sense of the term. Occasional suggestions or beginnings of cult acts were to be found, in the form of a number of magical customs. Such, particularly, were the efforts to expel sickness demons; also, the ceremonial dances designed to bring success to joint undertakings, as, for example, the above-mentioned dance of the Veddah about an arrow, whose purpose, perhaps, was to insure a successful hunt, if we would judge, among other things, from the fact that the dancers imitated the movements of animals.

In contrast to these meagre magical usages, which, 
for the most part, served individual purposes, the totemic age developed a great variety of cults. Just as the totemic tribal organization is an impressive phenomenon when compared with the primitive horde, so also do we marvel at the rich development of cults with which we meet as we pass to the totemic age. These cults are associated not only with the most important events of human life but also with natural phenomena, though, of course, only in so far as the latter affect the interests of man, the weal or woe that is in store for the individual or for the tribal community. Generally speaking, therefore, these cults may be divided into two great classes. Though these two classes of cults are, of course, frequently merged and united-for the very reason that both spring from the same emotions of hope, of desire, and of fear-they are nevertheless clearly distinguishable by reference to the immediate purpose which the magic of the cult aims to serve. The first of these classes includes those cults which relate to the most significant events of human life; the second, those concerned with the natural phenomena most important to man.

Human life furnishes motives for cult acts in its origin as in its decline, in birth and in death. Other motives are to be found in significant intervening events, such primarily as the entrance of the youth into manhood, though in the case of the maiden, ceremonies of this sort are very secondary or are entirely lacking. Of these most important events of life, that of birth is practically removed from present consideration. No ceremony or cult is connected with it. Not infrequently, however, the idea prevails that the child becomes capable of life only on condition that its parents endow it with life a second time, as it were, by an express act of will. Thus, many Polynesian tribes allow parents to put to death a new-born infant. Only after the child has lived several hours has it gained a right to existence and does the duty of rearing it devolve upon the parents. There is a survival of similar ideas in the older usages of cultural peoples, though they have not led to the widespread evils of infanticide as they have among many peoples of nature. 
But even among the early Germans, Romans, and Greeks, the life of a new-born child was secure only after the father had given recognition to it in a symbolical act-such, for example, as lifting it from the earth. On the other hand, the previous act of laying the child on the ground frequently came to be symbolical of the idea that it, as all living things, owes its existence primarily to mother earth. With this act of an express recognition of the child, moreover, there is also bound up the unconditional obedience which the child, even down to a late period, was held to owe to its parents.

The fewer the cult acts connected with entrance upon life, the greater is the number that attend departure from it. Almost all cults of the dead, moreover, originate in the totemic age. Wherever traces of them appear at an earlier stage, one can hardly avoid the suspicion that these are due to the influences of neighbouring peoples. Now, the totemic cults of the dead are closely interrelated with the abovedescribed usages relating to the disposition of the corpse. They make their appearance particularly when the original signs of fear and of flight from the demon of the dead begin to vanish, and when reverence comes into greater and greater prominence, as well as the impulse to provide for a future life of the dead-a life conceived somehow as a continuance of the present. The clansmen solemnly accompany the corpse to its burial ; death lamentations assume specific ceremonial forms, for whose observance there is very commonly a special class of female mourners. The cries of these mourners, of course, still appear to express the emotion of fear in combination with that of grief. The main feature of the funeral ceremonies comes to be a sacrifice to the dead. Not only are the usual articles of utility placed in the grave-such, for example, as a man's weapons-but animals are slaughtered and buried with the corpse. Where the idea of rulership has gained particular prominenceas, for example, among the Soudan and Bantu peoples of Africa-slaves and women must also follow the deceased chieftain into the grave. Evidently these sacrifices are in- 
tended primarily for the deceased himself. They are designed to help him in his further life, though in part the aim is still doubtless that of preventing his return as a demon. In both' cases, these usages are clearly connected with the increased importance attached to the psyche, for they first appear with the spread of the belief in a survival after death and in soul migration. These sacrifices are doubtless regarded partly as directly supplying the necessary means whereby the soul of the dead may carry on its further existence and partly as magical instruments that make it possible for the deceased to enjoy a continuance of life. Thus, these sacrifices already involve ideas of a beyond, though, generally speaking, the latter did not as yet receive further development.

At this point, sacrifice to the dead undergoes further modifications, as a consequence of which there are also changes in the accompanying cult acts. The sacrifice of food dedicated to the use of the deceased and the bloody sacrifice designed to equip him with magical power, are no longer offered merely to the departed. As soon as godideas begin to emerge, the sacrifice is brought, in first instance, to these higher beings, who are implored to furnish protection to the deceased. As this latter motive gains the ascendancy, the slaughtered animals are no longer placed in the grave along with the deceased, but their blood is poured out upon it ; of their flesh, moreover, only a part is thrown upon the grave as the portion of the dead, while the rest is consumed by the mourners. The feelings of reverence, thus expressed, issue, in the later development of these cults to the dead, in general ancestor worship. Not only the deceased himself and those who have assembled, but particularly the gods under whose protection the deceased is placed, receive a portion of the sacrifice. When this occurs, the offering, which had been devoted to the deceased, becomes sacrifice proper. The offering was given solely to the one who had died; at first, its purpose was to keep him in his grave, later, to afford him aid in his further life. Real sacrifice to the dead involves three 
parties-the deceased person, the deity, and the survivors. The deceased gains new life from the blood and flesh of the sacrificial animal; the deity is subjected to a magical influence which is to incline him favourably toward the departed; those who bring the sacrifices participate in this favour, since they enter into a magical union both with the deceased and with the protecting deity. In part, these developments extend on beyond the totemic age; their beginnings, however, are already everywhere present. True, in this early sacrifice to the dead the attempt to exercise a magical influence upon the deity-later, as we shall see, the essential feature of the sacrificial idea-is still in the background. Nevertheless, this magical feature, which characterizes sacrifice at the height of its development, has already made its appearance. Because of it, the original sacrifice to the dead possesses a significance intermediate between the two distinct concepts of a gift which sacrifice has been held to embody. Though originally a gift to the deceased, an offering laid beside him, sacrifice became a means of protective magic for him and for the survivors. When the deity came to constitute a third member of this magical group, and as he gradually gained the dominant place, the idea of a gift again began to displace the purely magical idea. The gift, however, was now a gift to the deity. This was the final stage in the development of sacrifice and represents the basis of the ordinary rationalistic interpretation. Originally, however, sacrifice possessed a different significance. It was purely a magical act, as is shown by the further circumstance that it is precisely the sacrifice to the dead which was already practised at a time when there were as yet no gods but merely a belief in demons. Additional evidence may be found in the nature of the sacrificial gifts which are deposited in the graves, particularly where ancestor worship prevails-as, for example, in the realms of East Asiatic culture. In these regions, it is not the objects themselves with which the deceased is to be equipped for his future life that are buried, but miniature paper representations of them. These representa- 
tions are really not symbols, as is generally held-or, at any rate, this is only a later and retrogressive form of the idea-but they are sensuously embodied desires originally regarded as means of magic. In this case also, we may detect the influence of soul-ideas, which lie at the basis of all beliefs of this sort. As the psyche of the dead is supposed to reincarnate itself in a new organism, so likewise are the object-souls incorporated in these representative miniatures to transform themselves, by means of the magical power attaching to their shape, into corresponding real objects. But in this instance again, the further modifications in the sacrifice to the dead lead on into deity cult. Hence it is not until our next chapter, when we discuss deity cults, that we will deal with the sacrificial idea in its total development.

Connected with another life-event to which this age attaches particular importance is a further significant group of totemic cults. This consists in the celebration of the adolescence of youths in the so-called initiation ceremonies. In a period such as this, when intertribal struggles are a matter of increasing concern, the reception of a youth into the association of men, into the community of the hunt and of war, represents the outstanding event of his life. Beginnings of such celebrations were transmitted by the primitive age to the totemic era, but it is only at this later period that they are developed into great cult festivals. It is these festivals, particularly, which everywhere recur in essentially the same form among all the tribes of Australia. They are great folk festivals, frequently assembling the clans of friendly tribes. Their celebration consists of dances and songs, though primarily of ceremonies centring about the youths who are reaching the age of maturity. For a considerable period these youths have been prepared for the festival by the older men. They have been subjected to a strict asceticism for weeks beforehand; meanwhile they have also been trained in the use of weapons, and instructed in certain matters of which the young are kept in ignorance. The actual celebration, which always occurs 
at night, includes ceremonies which, in part, involve extreme pain to the novices. The youths are obliged to stand very close to a fire kindled in the centre of the ceremonial ground. The older men, with painted faces, then execute dances, in which the women are forbidden to participate. An important feature of these dances is the imitation of totem animals. This also provides an opportunity for humorous episodes. During these pranks, however, the youths are compelled to remain serious. Moreover, they must give evidence of fortitude by fearlessly leaping over the fire. In many of these regions, there is a further ceremony, which is extremely peculiar and of uncertain significance. This consists in the knocking out of teeth. Generally the operation is performed by the medicine-man or, as he ought perhaps to be called in this capacity, the priest. The latter presses the teeth of his own lower jaw against one of the incisors of the upper jaw of the novice, thus loosening the tooth so that it may easily be knocked out with a stone hammer. This is the most primitive form of tooth deformation, a practice common to numerous peoples of nature as a means of beautification. That the original purpose was not cosmetic is clear. Whatever other end it was intended to serve, however, is uncertain, though it was doubtless connected with cult. Perhaps its meaning is suggested in the fact that, before marriage, girls also were frequently deprived of a front tooth, and that the idea prevailed, possibly in connection with this custom, that the exchange of breath, and thus the breath-soul, may play a part in the act of procreation. It is not unreasonable to suppose that these ideas may represent the origin of the kiss. At any rate; as Preusz has pointed out, ancient Mexican pictures represent two deities engaged, apparently, in the act of kissing while (perhaps in reminiscence also of the blood-soul) red smoke passes from the mouth of the one to that of the other. Moreover, it may well be that this exchange of souls in the kiss has its analogue in many regions, particularly in Melanesia, in the exchange of breath through the nose-the so-called 
nose-greeting which might therefore better be called the nose-kiss. That this exchange is mediated through the nose may be due to the fact that among many of these tribes kissing with the lips is impossible because of mouth-rings, lip-blocks, and other deformations, doubtless originally intended as means of magic. Similar ideas concerning the mouth and the nose, moreover, and their relation to the psyche, are suggested even by the Biblical history of the Creation, according to which God rouses Adam to life by breathing a soul into him through his nose. Through the mouth, man breathes out his soul; through the nose, he received it.

Though the festival of initiation into manhood was once associated with magical acts of cult, as the above ceremony seems to show, the meaning of this magic has for the most part been lost to the memory of the natives. For this reason they generally regard the ceremonies, including that of striking out the teeth, as a means of testing the fortitude of the young men. This was doubtless a secondary motive even at a very early time, and when the magical significance dropped out, it remained as the sole purpose. Nevertheless, the character of these alleged tests is much too peculiar to be intelligible on the hypothesis that they were originally intended merely to arouse fear or pain. And so, in view of the widely prevalent use of fire as a means of lustration, we may be allowed to regard also the fire-test, which occupies a central place in these cult forms, as having originally been a means of magical purification.

The second class of ceremonial festivals and cults, as above remarked, is associated with certain objective natural phenomena which exercise a decisive influence upon human life. The natural phenomena most likely to originate a cult, because representing the most important objects of desire and fear, are those connected with the need for food, with the growth of plants, and with the increase of animals, particularly the animals of the chase. For this reason vegetation cults date back to the very beginnings of the 
totemic period. Very probably they originated in the desire for plant food. Under relatively primitive conditions there was seldom a lack of game, though there was probably a scarcity of the vegetables necessary to supplement the food derived from animals. For plants frequently suffer from unfavourable weather, whether it be from the heat of the sun and from drought, as in tropical and subtropical regions, or from deluging rains, as in the temperate zones. Our interpretation of vegetation cults is supported particularly by the conditions prevailing in the original home of totemism, Australia. These cults here occur chiefly in the northern districts, into which there were early Melanesian immigrations; towards the south, they have gained but a relatively small foothold. The more northerly regions, as we have seen, are the very ones in which plant totems also are numerous, whereas they are lacking in the south. The cults of which we have been speaking are called Intichiuma ceremonies-an expression of Australian derivation. These ceremonies, moreover, involve the magical use of churingas, the Australian fetishes.

The character of these vegetation festivals is always very much the same. They include dances, in which, in essential distinction from those of the initiation ceremonies, women are generally allowed to participate; their central feature consists of specific magical acts designed to effect an increase of the food supply. In Australia, these acts, in part, take the form of ceremonies in which pieces of artificial animals are strewn about. We speak of them as artificial, of course, only from our own standpoint; to the Australian the material that is scattered represents an actual living being. Thus, for example, a heap of sand is moulded into the form of a large lizard, and, of this, various parts are thrown into the air by those who participate in the festival. The animal germs thus scattered are supposed to effect an increase in the animals of the lizard totem. These vegetation festivals, therefore, are also totem festivals, and their celebration has the secondary significance of a cult dedicated to the totem. The celebration connected with a fish 
totem is similar to the above, though somewhat more complicated. A member of the clan, whose arms or other parts of the body have been bored through with bone daggers, descends into the water and allows his blood to mingle with it. The totem germs that are to bring about an increase in fish are supposed to emanate from the blood.

In the case of plant totems, the cults are of a simpler nature. The plants themselves, or sometimes their seeds, which, moreover, also serve directly as food, are strewn to the winds. The grass-seed totem, for example, is particularly common in Australia. The seeds of the Australian grasses are gathered in large quantities and constitute an important part of the vegetable food. Thrown into the air, they are supposed to bring about an increased supply of these grasses. Externally regarded, this magical ceremony, primitive as it is, completely represents an act of sowing. It would be incorrect, however, as yet to speak of it as such, in the sense of the later tiller of the soil ; the significance of the ceremony is purely magical. An age which merely gathers wild seeds and fruits does not prepare the soil in the way that sowing presupposes. Nevertheless, the magical cult involves an act which later forms an important part of agricultural tasks. Indeed, it is not at all improbable that these magical ceremonies, which in any event already involve the recognition that the strewing of seed conditions the increase of plants, have elsewhere constituted a preparatory step to the development of agriculture. In general it may be said that the ceremony probably originated in connection with plant totems, where the idea of such an increase is very especially apt to suggest itself ; doubtless it was only later connected, through a process of external association, with animal totems. In harmony with such a view is the fact that Intichiuma festivals are chiefly. prevalent in the regions of plant totemism.

The vegetation cults which preceded the rise of agriculture were finally superseded by true cults of the soil. The latter presuppose the preparation of the soil by the efforts of man. This is clear from the fact that they 
occur more regularly, and at definite seasons of the year; moreover, they are of a more complex character, serving in part a number of other purposes. Typical of the transition are the vegetation festivals of the natives of Central America. These festivals are unique in that they embody elements of celestial mythology; thus they constitute important transitional stages between the demon cults of the totemic era and deity cults. The relation which the seeds are supposed to bear to the sprouts of the various grains is now no longer merely of a magical nature. The hoe-culture, to which the American Indian has attained, has taught him the dependence of the growth of plants upon the act of sowing. But here also there can be no cult until there is community labour. The original hoe-culture carried on by the individual about his hut no more tends to originate a cult than does the erection of the hut, the weaving of baskets, or the other tasks set by the needs of daily life. Individuals, however, frequently till the soil even prior to the rise of systematic agriculture, as occurs in certain regions of Melanesia, among the prairie peoples of North America, and elsewhere. Besides leading to more advanced ideas concerning the processes of germination and growth, these beginnings of agriculture, which still form part of the household duties of individuals, serve to engender what proves to be a permanent and basal factor in all further development-namely, provision for the future. However primitive may be the hoe-culture which the individual carries on about his hut, it is not concerned exclusively with the immediate present, as is the mere gathering of food, but it aims to satisfy a future need. True, even in this case, the beginnings may be traced back to the preceding age. Even such ceremonies as the Intichiuma festivals, in which the totems are strewn about in order magically to influence their growth and increase, are already thoroughly inspired by a regard for the future. Perhaps all human action concerned with the distant future was at first magical in aim.

The establishment of a cult, however, is due not merely to the foresight which provides for a future harvest by 
the tilling of the soil ; it is conditioned also by a second factor-namely, community labour. Just as entrance into manhood gives rise to initiation cults only when it becomes of tribal importance, precisely so is the development of cults of the soil dependent upon the association of members of a tribe or a mark in common labour. Moreover, initiation into manhood early came to be of common concern because of the community life of age-associates and of the need for military training created by tribal warfare; the same is true, though at a later stage and, of course, for essentially, different reasons, of the tilling of the soil. The most important factor in the latter case is the fact that because the natural conditions are common to all, all are obliged to select the same time both for the sowing and later for the harvest. This is of little moment so long as the population is sparse and the property of one individual is separated from that of the others by wide stretches of uncultivated land. The more closely the members of the mark live together, however, the more do they share in common labour. Whenever a migrating tribe takes possession of a new territory, moreover, there is a further decisive consideration, namely, the fact that at the outset the soil is common property. In this case, not merely the natural conditions, but also the very ground on which the work of the field is performed, is identical for all the members of a mark. Added to this objective factor there more and more comes to be one of a subjective nature. In common labour, the individual determines his activities by reference to a common end ; moreover, he regulates these activities, as to rhythm, tempo, and the accompanying expressive movements, so as to conform to the group in which he finds himself. Since, moreover, the activity of sowing and the subsequent growth of the crop preserve the magical character acquired in an earlier period, the work itself comes to be a cult activity. Just as initiation rites are not merely a declaration of manhood but a cult, designed magically to equip the novice with manly power and fortitude, so the tilling of the soil becomes a cult act through whose inherent magical power the 
prosperity of the crop is supposed to be secured. There are two factors which are of prime importance for the beginning of agricultural cults, and which give to their further development its peculiar stamp. In the first place, the labour whose performance in common engenders the cults of the soil is always connected with hoe-culture, the initial stage of agriculture. It is only because they work with the hoe that the members of the mark come into such close relations that they easily fuse into a cult community. When the plough, which is drawn by an animal, comes into use, the individuals are again separated. For the field which is tilled is larger, and, furthermore, the activity of the ploughman is confined to the guidance of his animals and implements, so that he personally is no longer directly concerned with the soil as in the case of hoe-culture. 'Moreover, since hoe-culture demands a very much greater expenditure of human energy, it arouses stronger emotions. The plough trains to reflection and brooding; the hoe stirs violent emotions. Furthermore, it is only when hoe-culture becomes common labour on a common field that the sexes are brought together. The early hoe-culture carried on about the hut of the individual generally devolves upon the woman alone, who thus merely continues the duty of foodgetting which rested with her, as the gatherer of food, under still more primitive economic conditions. With the appearance of more intensive hoe-culture the labour is divided. Man cuts up and loosens the soil with his hoe; woman follows after, strewing the seed between the clods. With the invention of the plough, agriculture finally becomes the exclusive concern of man. The furrowing and loosening of the soil is now done by means of an implement, and man, freed from this labour, assumes the duty of strewing the seed.

This twofold community of labour, that on the part of the holders of common property and that of the two sexes, undoubtedly underlies the peculiar character which the cults of the soil continue to preserve long after the period of their origin. On the one hand, the work of the field itself assumes the character of a cult act ; combined with it, on the 
other hand, there come to be additional ceremonies. That which brings the men and women together and converts the labour into a cult act is primarily the dance. The fertilization and growth of plants are regarded as processes resembling the procreation of man. When the cult members give themselves up to ecstatic and orgiastic dances, therefore, they believe that they are magically influencing the sprouting and growth of the seeds. According to their belief, sprouting and growth are due to the demons of the soil. These demons the orgiastic cult arouses to heightened activity, just as the labourers and dancers mutually excite one another to increased efforts. In this ecstasy of the cult, man feels himself one with external nature. His own activity and the processes of nature become for him one and the same magical potency. In addition to the terrestrial demons of growth, there are the celestial demons, who send fructifying rains from the clouds to the soil. Particularly in regions such as New Mexico and Arizona, where a successful harvest depends in large measure upon the alternation of rains with the withering heat of the sun, these vegetation festivals are combined with elements of celestial cults. The latter, of course, are also essentially demon cults, yet they everywhere exhibit distinct traces of a transition into deity cults. Particularly typical are the cults of the Zuni and Hopi, described in detail by various American scholars. The direction of these cult festivals is vested in a body of rain-priests, in conjunction with other associations of priests, named for the most part after animals, and with secret societies. In the vegetation ceremonies of the Hopi, the members of the rain-group, naked and with faces masked to represent clouds, parade through a neighbouring village and thence to the festival place. In their procession through the village, the women throw water over them from the windows of the houses. This is a magical ceremony intended to secure the blessings of rain upon the crops. The investigations of $\mathrm{W}$. Mannhardt concerning the field cults of ancient and more recent times have shown that survivals of such conceptions are still present in the sowing and 
harvest usages of modern Europe. Mannhardt's collection of customs deals particularly with East Prussia and Lithuania. In these localities it is customary for the maid-servants to return from the harvest earlier than the men, and to drench the latter with water as they enter the house. Though this custom has become a mere form of play, it nevertheless still vividly recalls the very serious magical ceremonies of earlier vegetation cults. But over and above this change from the serious to the playful, of which there are beginnings even in the festival celebrations of early cultural peoples, there is still another important difference between the earliest vegetation cults and their later recrudescences. The former are connected particularly with sowing, the latter primarily with the harvest. This again reflects the difference between hoe-culture and plough-culture. Hoe-culture unites the members of the mark in the activity of sowing, whereas labour with the plough separates them and imposes the work exclusively on the men. Harvesting the grain, on the other hand, long continues to remain a task in which individuals work in groups, women and men together. Moreover, as the magical beliefs associated with the activity of sowing gradually disappear, their place is taken by joy over the assured harvest. This also factors towards changing the time of the main festival from the beginning to the end of the season.

Since both earth and heaven must co-operate if the sowing is to be propitious and the harvest bountiful, vegetation festivals are intermediate between demon cults and celestial cults. In respect to origin, they belong to the former; in the degree in which more adequate conceptions of nature are attained, they give rise to the latter. In many cases, moreover, elements of ancestor cult still exercise an influence towards bringing about this transition. The cloud that bestows rain and blessing is regarded as dependent upon a controlling will. Back of the clouds, therefore, according to the ideas of the Zuni and other Pueblo tribes, dwell the ancestors. The prayer of the priests to the clouds 
is also a prayer to the ancestors for protection and aid. The procession of the rain-priesthood through the village is a representation of the ancestors who are hidden behind the mask of clouds, and is supposed to exercise a magical influence. These cult festivals also include invocations to the sun, whose assistance is likewise necessary to the prosperity of the crop. Thus, in the ceremonial customs of the Navajos, who occupy the same territory, the yellow sand that covers the festival place represents the coloured expanse of the rainbow, the sun, and the moon. All the heavenly forces are to co-operate in bringing about the ripening of the harvest. In this wise it is possible to trace an advance, stage by stage, from the cults of terrestrial demons, who dwell within the growing grain itself, to celestial cults. The fact that the aid of the heavens is indispensable draws the attention upwards. If, now, there are other causes such as give rise to the idea of a celestial migration of the souls of departed ancestors, the cloud demons become merged with ancestor spirits, and there are combined with them the supra-terrestrial powers that are conceived as inherent in the other celestial phenomena.

It is due to this synthesis of vegetation cults with celestial cults that these festivals, which are the most highly developed of any in the totemic age, continue to become more and more complex. They gradually incorporate other cults in so far as these are not associated with specific, undeferable circumstances, as are the death cults. Among the Zuni and Navajos, the most important ceremony thus incorporated into these festivals is the initiation of youths into manhood and their subsequent reception into the community of men. There are analogous ceremonies for the women. In this complex of cult elements, the emphasis more and more falls on the celestial phenomena, of which the more important force themselves upon the observation and therefore determine the time at which these festivals are held. Instead of at seedtime and harvest, which vary somewhat with weather conditions, the two main festivals are held at fixed dates corresponding to the summer and 
winter solstices. Thus, the cults become independent of variable circumstances. All the more are they able to assimilate other cults. Among the Zuni, for example, there is a ceremony which, though analogous to the declaration of manhood, is not held at the time when the youths reach manhood or the maidens arrive at the age of puberty, but occurs much earlier, and signifies reception into the cult community. This first consecration, which might be compared to our baptism, does not take place immediately after birth, but when the child is four or five years of age. Following upon this consecration, in the course of the same festival, comes the celebration of the adulthood of fully matured youths and maidens, set for the fourteenth or fifteenth year of life. In this ceremony the youths and maidens are beaten with consecrated rods. The present generation, which has no knowledge concerning the origin of this practice, generally regards these blows as a test of hardihood and courage. But the fact that specially consecrated rods are used by the priests shows unmistakably that their original purpose was to exercise a magical influence upon those who were being initiated. Indeed, the fact that many adults crowd in to receive some of the blows, in the belief that these possess a protective influence, proves that the original meaning of the ceremony has maintained itself to a certain extent even down to the present. In addition to these features of the cult-celebration, which are connected in general with the tribal or mark community as such, there are other ceremonies that are designed for the satisfaction of the wants of individuals. Sick persons drag themselves painfully to the festival, or are brought to it by their relatives, in search of healing. In America, the desire for magical healing has very commonly given rise to so-called sweat-lodges, which are located near the festival places. These lodges serve a twofold purpose. The primary aim of the sweat cure is to expel sickness demons. But healthy persons also subject themselves to the treatment. In this case the sole purpose of the sweating is obviously that of lustration. Just as we ourselves occasionally experience relief 
from the flow of perspiration, so also may the one who has passed through the ceremony of the sweat-lodge feel himself reborn, as it were. This would tend to strengthen the naturally suggested association between this ceremony and lustration by water. The ceremony, therefore, serves the same purpose as the other forms of lustration. The individual wishes either to purify himself from a guilt which he has incurred, or, if there is no particular element of guilt, to protect himself against future impurities. The custom thus acquires the significance of a sanctification ceremony, similar to baptism or to the bath of the Brahman. Because of the combination of these various cult motives and cult forms, the cult association which unites in the performance of the vegetation festivals comes to be the representative of the cult, as well as of the belief, of the tribal community in general. This likewise prepares the way for the transition from totemic to deity cults, as is indicated, among other things, by the sacrificial activities of these cult festivals. Sacrifice itself, as has already been mentioned, probably originated as sacrifice to the dead. Its further development occurs primarily in connection with the higher forms of vegetation cults. The Zuni and Navajos erect altars for their festivals. These they adorn with gaily coloured cloths and with the gorgeous plumage of birds. On them they place the plants and grains which the cult is designed to prosper. This is the typical form of the vegetable sacrifice as it passes on from these early practices into all higher cults. The sacrifice consists in offering the particular plants and grains whose increase is desired. At the outset, its character is exclusively magical; it is not a gift to the deity. Just as rain-magic is supposed to result from drenching the rain-association with water, so this offering of grains is held to have a magic effect upon the prosperity of the same sorts of grains. There is no indication or suggestion that the sacrifice represents an offering to the gods. This idea arises only later, when the magical sacrifice of grains, as well as that of animals, is connected with a further conception whose origin is apparently also to be found in 
sacrifice to the dead. The dead are presented with gifts, which they carry along into a world beyond. Similarly, the magical sacrifice connected with vegetation festivals and their associated cults more and more ceases to be regarded as purely magical in nature and comes to be an offering to the deity whose favour is thereby sought.

Coincident with these changes in sacrificial usages, the cult community which develops in the course of the transitional stages of cult-the best representatives are the semicultural peoples of America-undergoes a more thorough organization. Separate associations are formed within the wider circle of cult membership. These severally assume the various functions involved in the cult; as a rule, they are under the guidance of priests. Even apart from their connection with these cult festivals, the priests serve as magicpriests and magic-doctors, and it is they who preserve the traditions of the general cult ceremonies as well as of the means requisite on the part of the individual for the exercise of this twofold profession. This represents the typical figure of the medicine-man. $\mathrm{He}$ is to be found even in primitive culture, but his function more and more changes from that of the ordinary magician into that of the priest. As such, he attains to a position of authority that is publicly acknowledged and protected. Associated with him is a restricted group of those cult members who are most familiar with the secrets of the cult, and are his immediate assistants in the festal ceremonies. It is these individuals that compose the secret societies. These societies occur even among the tribes of the northern parts of America, and have their analogues particularly on the semi-cultural level which forms the threshold of the totemic age. Presumably they derive from the more primitive institution of men's clubs, within which the male members of a clan are united into age-groups. Membership in secret societies also continues to be limited to men, more especially to such as have reached a mature age. As tribal organization developed, and particularly as family bonds became firmer, age associations were dissolved. The association which 
originally included all men gave way to more restricted societies. Besides this numerical limitation, there was naturally also a qualitative restriction. In the first place, those who thus deliberately segregated themselves from the total body were the privileged members of the tribal community, or at least such as laid claim to special prerogatives ; these associations, furthermore, were formed for certain more specialized purposies connected with the particular needs of their members. The first of these considerations accounts for the respect, occasionally mingled with fear or reverence, which was accorded to these societies, a respect which was heightened by the secrecy in which they shrouded themselves. The fact that certain customs and traditions were surrounded with secrecy caused every such association to be organized into various ranks, graded according to the extent with which the individuals were familiar with the secret doctrines. This type of organization occurs as early as the associations of medicine-men among the Africans and the American Indians ; later, it is to be found in connection with the Eleusynian and Orphic mysteries; it is represented also by the Christian and Buddhistic orders, and by their various secular counterparts, such as the Rosicrucians and the Freemasons. Not infrequently these societies, in contradiction to their secrecy, have special emblems indicative of membership and of rank. Among the American Indians, this purpose is generally served by special drawings on the body; in other places, by specific tattooings as well as by the wearing of distinctive dress. The second restriction of membership on the part of the secret society is connected with the limited purpose which the society serves. The men's club includes all the interests of the clan or tribal community; the secret society is held together by a specific aim or by a limited circle of related task's. Here also it is universally true that these tasks are connected with cult, and are thus of a religious nature. Even the Greek phratries underwent a change of purpose analogous to that which occurred in the transition from the age-group to the secret society, for, after 
losing their earlier political significance, they continued to exist as cultural associations.

The men's group belongs exclusively to the totemic age. Secret societies, however, are organizations which, together with the cults that they maintain, belong to a stage transitional between totemic and deity cults. The emblems worn by the cult members are for the most part totemic ; totemic also are the cult usages, and likewise, particularly. among the American Indians, the name which the group adopts. The feathers of birds and the hides of other totem animals-the same as those which also adorn the festival altars-constitute a chief part of the dress. In addition to the general tribal festival in which they cooperate, these societies also maintain their special cults. It is particularly in these latter cults that ancient totemic survivals are in evidence. A remarkable example of such a totem group is the snake society of the Hopi Indians, who dwell, as do the Zuni and Navajos, in the regions of New Mexico. The totem animal of this society is the rattlesnake. In the snake festival, a procession is formed in which every member participates; each carries a rattlesnake in his mouth, holding it in his teeth directly back of its head. It is firmly believed that no snake will kill a member of the society which holds it sacred. Of course, as observers of the festival have noticed, an ingenious expedient is employed to avert the danger. Each snakebearer is followed by an associate who diverts the attention of the snake by continually, tickling its tail with a small stick. If a snake-bearer is bitten, as rarely occurs, his companion always sucks out the wound, by which act, as is well known, the snake-bite is rendered relatively innocuous.

\section{The ARt of the Totemic Age.}

The most prominent of the artistic activities of the totemic age is formative art. In this field, the lowest stages of totemic development show little advance beyond the achievements of primitive man. True, even Australia pos- 
sesses cave drawings which perhaps have some sort of cult significance. As yet, however, we have not succeeded in interpreting these drawings. With this exception, the formative art of the totemic period is limited to carvings upon weapons or other implements-obviously thought, just as in primitive times, to possess magical potencies-and to the painting of the face on the occasion of cult festivals.

In the regions of Oceania, particularly the Polynesian Islands, we find a far richer development of that form of pictorial art which aims at the adornment of the body, or, as we ought rather to say with reference to the beginnings of this artistic practice, at the exercise on the part of the body of a magical influence upon external things. Polynesia is the chief centre of artistic tattooing. Throughout these regions this practice has universally taken the form of prick tattooing. By means of separate, close-lying prick points filled with colour, various symmetrical designs are formed. This tattooing is the only art whose highest perfection is reached at the beginning of culture. As soon as clothing appears, the decoration of the body itself gives way to that of dress. On particular occasions, as, for example, in connection with certain cult practices of the American Indians, custom may continue to demand entire nakedness. Under these circumstances, there is a sort of retrogressive development in which the painting necessitated by the festivals takes the place of tattooing. This occurs even among the Australians. Moreover, even after clothing has appeared, it long remains a favourite custom to tattoo certain exposed parts of the skin, particularly the face and the arms and hands. Even to-day, indeed, the arms are sometimes tattooed. The fact that tattooing is now practised almost exclusively by criminals and prostitutes, and, occasionally, by sailors, finds its explanation in a circumstance which was also of influence at the time when tattooing was in its first flower, namely, in the interruption of occupational activity by long periods of leisure.

There is an additional factor which obviously favours the development of the art of tattooing, particularly in the 
territory of the Polynesian Islands. I refer to the combination of totemism with celestial mythology, which is peculiar to these peoples, and to the consequent recedence of totemism. Particularly illuminative as regards this point is the tattooing of the Maoris. The mythology of this people gives an important place to the sun, and their bodily decorations frequently include pictures of this celestial body, in the form of spiral ornamentations. Some two years ago travelling investigators brought back copies of the tattooing of other islanders, particularly those of the Marquesas group. These tattoo-patterns contain many significant elements of a celestial mythology; those of to-day, however, in so far as the custom has not been entirely effaced by the Europeans, consist almost entirely of simple geometrical ornamentations. The tattooings of early times frequently included also representations of animals. Plants were less common, as might be expected from the fact that it was only later that they acquired importance for totemic cults. At the same time, it is evident that a sort of reversal took place as regards the pictorial representation of objects. This is even more striking in the tattooing of the American Indians, a tattooing restricted to certain parts of the body. In the preceding chapter the fact has already been noted that, among the primitive peoples of the pretotemic age, as, for example, the Semangs and Senoi of Malacca, the multiplication of simple parallel lines, triangles, ar'cs, etc., gives rise to plant-like and animal-like forms. Doubtless the primitive artist himself discovers such figures in his drawings and then sometimes consciously sets about to imitate more closely the actual forms of the natural objects. At the stage of development now under discussion, we find, conversely, that animal forms, particularly; are retranslated into geometrical objects in that they become, as we would to-day express it, more and more conventionalized. Since only the simplest outlines of the objects are retained, it may eventually become a matter of doubt whether these really are schematic representations of natural objects, and whether they are not, even from the very beginning, geo- 
metrical ornamentations. Nevertheless the fact that there are continuous transitions from the developed animal form to the geometrical ornament, as occurs particularly in America, is incontrovertible proof that such a conventionalization took place, though in many cases, doubtless, very slowly. This process of conventionalization, however, may be more clearly traced in connection with a different art, one that is related to tattooing but whose development is not limited, as is that of the latter, and destined from the very outset to become obsolete. I refer to ceramics, the art of decorating the vessels which were at first intended for the preservation, and later for the preparation, of food.

Even though the art of making pottery is not to be found in primitive culture proper, it nevertheless dates back to a very early age. It is not impossible that this age coincides approximately with the beginning of the totemic period. At any rate, it was totemic cult which, from earliest times on, furnished the motives for the decoration or-as is here also doubtless generally true of the early beginnings-for the magical protection of the vessels, or for the imparting of magical potencies to their contents. Doubtless the clay vessel was originally modelled partly after the natural objects that were used for storing food, and partly after the woven basket. The latter, in turn, may, in its beginnings, have been copied from the bird's nest. When it was discovered, probably accidentally, that clay is hardened by fire, the clay vessel came to be used not merely for the preservation of food but also for its preparation by means of fire. Or, perhaps it would be truer to say that the attempt to accomplish this latter purpose with the unhardened clay vessel led to the art of baking clay. Now, even before the art of making pottery was known, implements, weapons, women's combs, and even the body itself were marked with simple and regular linear drawings to which a magical significance was attached. These geometrical forms, which arose semi-accidentally, were, even from very early times, apperceived as the outlines of animal or plant forms, and it was under the influence 
of these ideas that they attained a further development. Precisely the same process was repeated in the case of ceramics, only, as it were, upon a broader scale, challenging a richer play of imagination. It is precisely here, however, particularly in the ceramics of the American Indians, that we can trace the ascending and the descending developments of primitive linear drawings, first into completely developed animal designs with meagre suggestions of attempts at plant ornamentation, and then regressively, through a continued conventionalization, into purely geometrical figures. At the same time, it was ceramics, especially, that developed a combination of these two designs, the systematic arrangement of which marks the perfection of this art. Thus arose representations of natural objects framed in by geometrical ornamentations. In this respect also, tattooing furnished a preparation, even though imperfectly, for ceramics. In inner significance, moreover, the latter was a direct outgrowth of the former. By tattooing, man originally guarded his own person with protective magic ; in ceramics, this magic was brought into connection with man's utensils, with the food necessary for his life, and with its preparation. In ceramics, therefore, just as in tattooing, the animals represented were at first primarily totem animals. Among them we find particularly snakes, fish, and birds, and, in America, the alligator. Especially characteristic of the totemic age is the fact that the decorations scarcely ever include the representation of the human figure. It is by this mark that the art products, even of the earliest age of Greece, may be distingushed at first glance from those of totemic culture. In the former case, the human figure is introduced, either along with that of the animal or even alone ; in the latter case, only animal representations occur. Strange to say, it is in only one respect that the ceramics, more particularly of the American Indians, copy man-the vessel as a whole represents a head or a skull. Doubtless this is connected with the obnoxious custom of head-hunting. Just as the Indian adorns the roof of his hut with the heads of his conquered foes, so he perpetuates 
the memory of his feats of war in his ceramic objects. No portrayal of activities in which human beings participate, is to be found in the totemic age.

Connected with this, no doubt, is the lack of any real sculpture, with the exception of crude idols representing: animal or human forms. These idols, on the whole, are of the nature of fetishes, and as such may, of course, be regarded as the precursors of the divine images of a later period. As there is no sculpture, so also is there, strictly speaking, no architecture. In this respect, again, there is a wide difference between this age and the succeeding one. In its higher forms, architecture presupposes gods who are worshipped in a temple. In the totemic period, however, there are no temples. True, the Australian preserves his magic wands and pieces of wood, the churingas, in caves or huts, but the latter differ in no wise from other huts. In the totemic age, therefore, man alone has a dwelling-place. Of such structures there are, in general, two types, the conical and the spherical. The conical hut apparently had its origin in the tent. The rounded or beehive hut, as it has been called in Africa, may originally have been copied from a natural cave built in the sand. The two forms, moreover, are not always mutually exclusive. In winter, for example, the Esquimo of Behring Strait lives in a round hut made of snow; in summer, he pitches a tent. In Melanesia, Polynesia, and other regions, the erection of dwelling-places on the seashore or on the shores of large rivers led to the pole-hut, a modification which came to resemble the houses of later times. This hut, which is generally occupied jointly by several families, is erected on poles that are firmly driven into the ground and reach far up into the air. Such a pole-hut, even at this early age, develops the typical form of a commodious dwelling. One of the factors here operative is the institution of men's clubs, which is prevalent in these regions: the necessity that many individuals live together leads to the erection of buildings of considerable size. In this connection, we note a characteristic difference between the beginnings of architectonic art and that of the 
other arts. The latter, whether in the case of tattooing, ceramics, or the fetishistic precursors of sculpture, always originate in mythological and, primarily, in magical motives : the sole impetus to architecture is furnished by the immediate needs of practical life. Thus, then, it is not to religious impulses but to the social conditions which require that many individuals shall live together, that we must trace a more perfected technique of building than that of primitive times.

Much more nearly parallel to the development of the other forms of art is that of the musical arts, meaning by this all those arts which consist in the direct activity of man himself. The musical arts include the dance, poetry, and music, as well as the various combinations into which these enter with one another. Since it is the third of these arts, music, that manifests a particular tendency to combine with and to supplement the other two, all three may be comprehended under its name. This will also serve to suggest the fact that, just as the formative arts are closely related in that they give objective embodiment to the creations of the imagination, so also are the musical arts allied by virtue of their reliance on subjective expression. Of all these various arts, the dance preserves the closest connection with the more primitive age. In the cult dance of the totemic period, however, the dance receives an extraordinarily rich development, reaching a stage of perfection comparable to that to which formative art attains in the external adornment of the body-that is, in tattooing. The dance and tattooing, indeed, are closely related, since nowhere else is the personal body so directly the object and the means of artistic activity. To the dances of the primitive period, however, the totemic dance adds one external featurethe mask-whose origin is directly due to totem belief. Even the Australians, of course, are not familiar with the mask-dance. They sometimes paint the face or mark it with single lines, and this may be regarded as the pretcursor of the mask; the mask itself, however, appears only in the later development of totemism, and continues far 
into the succeeding age. Moreover, as regards its distribution, there are considerable differences. It plays its most important rôle in American and Polynesian regions, a less prominent one in Africa. In America, the mask-dance and the elevation of mask's into cult objects, to which the maskdance occasionally gives rise, extend from the Esquimos of the north far down to the south. Koch-Grünberg has given a clear picture of the mask-dances and the mask-cult of the natives of the Brazilian forests. Here the masks are not a secondary means of magic, as it were-much less an occasional object of adornment. Every mask is a sort of sacred object. When the youth attains to manhood, he receives a mask, which is sacred to him throughout his entire life. After the great cult festivals, which are celebrated with mask-dances, the masks are carefully preserved. In the mask there is supposed to reside the demon who is represented by it, and the fear of the demon is transferred to the mask. The dancing of this period consists primarily of the animal dance, which is a rhythmic imitation, often wonderfully skilful, of the movements of an animal. The mask also, therefore, always represents, in a more or less altered or grotesquely exaggerated form, an añimal's head, or a being intermediate between animal and man, thus vividly calling to mind certain totemic legends whose heroes are sometimes animals and sometimes human beings. On the more advanced stages of totemic culture, there are also masks representing objects of external nature. Mention has already been made of the cloud masks used in the vegetation festivals of the Hopi and Zuni. The rain-priests of these tribes, with these masks on their heads and with pictures of zigzag lightning on their garments, are the living representatives of storm demons. Thus, the mask imparts to its wearer the character of the demon represented by it. The characteristics of face-masks, such as enormous beards and teeth, huge eyes, noses, etc., cause them, particularly, to be the living embodiments of the fear of demons, and thus to be themselves regarded as demoniacal beings. Whatever may be their more specific nature, whether, for example, 
they represent demons of sickness or of fertility, they always present the same fear-inspiring features. A certain diversity of expression is much more likely to come as a result of the external character of the dance in which the masks are used. This may give rise to expressions portraying surprise and astonishment, or the more lively emotions of fear, terror, or exalted joy. In the latter case, we must bear in mind that representations of grinning laughter differ in but a few characteristic marks from those of violent weeping.

Corresponding to these differences in the character of the masks that are worn, are two main forms of the dance, particularly of the cult dance. The first of these is the ceremonial dance, which moves in slow and solemn rhythm. This is the dance that generally inaugurates the great cult festivals of the semi-cultural peoples of totemism or that accompanies certain of the chief features of the festivalsuch, for example, as the entrance and procession of the cloud-masked ancestral spirits in the vegetation festivals of New Mexico. Contrasting with the ceremonial dance are the ecstatic dances, which for the most part form the climax of the festival. Only the men are allowed to take part in the ceremonial dances, and the same is generally true also of the ecstatic dances. The women, if not altogether excluded from the ceremonies, are either silent witnesses or accompany the dance with songs or screams. It is only in the more extreme form of the ecstatic-orgiastic dance that both sexes participate. The mixed dances probably arose in connection with the vegetation festivals, as a result of the relation which was thought to exist between the sexual emotions and the creative forces of nature. It was doubtless because of this late origin that the Greeks long continued to regard the dances of the Dionysian festivals, which were borrowed from Oriental cults and executed by women alone or by women and men together, as in part a degeneration of good custom. In the drama, whose origin was the mimetic dance, the rôle of women was taken by men.

Closely connected with the dance is music, the preparatory stage of which is constituted by the participation 
of the voice in the rhythm of the external movements of the body. These articulatory movements, which form a part of the mimicking activity of the face, supplement the dynamic rhythm of the dance with the melodic rise and fall of tones. The emotion which finds its outlet in the dance itself, then seeks a further enhancement through objective means. These means also involve the activity of the bodily organs; noises are produced by clapping the hands, by stamping on the ground, or by the rhythmic clash of sticks. In the latter case, the transition from instruments of noise to those of tone is easily made. The earliest forms of tone instruments are of two sorts, according as they copy the production of sound by external means, on the one hand, or by the vocal organs, in the accompanying tones, on the other. Thus, the two original forms of musical instruments are instruments of concussion and wind instruments. In origin, these are directly connected with the dance. They are natural means of intensification created directly by the emotion, though later modified by systematic invention. The later development of musical art continues to remain in close relation to the two main forms of the dance, the solemn ceremonial and the ecstatic dance, between which there come to be numerous transitions. From the most primitive to the highest stages of music, we continually find two sorts of musical expression, the sustained and the animated. These correspond to the contrasting feelings of rest and excitement, which are experienced even by animals, and which man therefore doubtless carried with him from his natural state into his cultural life. With the progress of culture, these feelings constantly become more richly differentiated.

The totemic age may be said to include only the first few advances beyond the simple emotions already expressed in the dance. Nevertheless, there are ethnological differences that register in a very characteristic way those specific musical talents of the various races which are obscured on higher levels of culture because of the increasing complexity of international relations. Thus, 
Africa is apparently the chief centre, if not the original home, of instruments of concussion and of the great variety of stringed instruments that develop from them. America, on the other hand, is the region in which wind instruments, in particular their original form, the flute, have attained their chief development. The flute of the American Indians is not, of course, like our own; it is blown, not with the lips, but with the mouth. It therefore resembles a shawm or a clarinet. As regards production of tone, however, it is a flute, for the tone is produced by the extension of one lip over the other in a manner similar to that of the flute-pipes of our organs. That which distinguishes the sound of the flute and of its shorter form, the fife, from that of stringed instruments is primarily the greater intensity and the longer duration of the tone. Corresponding to the difference in musical instruments is that of the noise instruments which characterize the two regions. Africa possesses the drum. This it employs not only for purposes of accompaniment in cult ceremonies, but also as a means of signalling, since it renders distant communication possible by use of the so-called drum-language. In America, we find the rattle. Though this, of course, is not entirely lacking in Africa, it nevertheless occurs primarily within the cultural realm of the North American Indians. Here it is employed as an instrument of noise and magic, similarly to the bull-roarer of the Australians. As between the rattle and the drum, the difference is again one of the longer duration of sound in the case of the American instrument.

The tones produced by these early musical instruments, however, even those of the stringed instruments and their vocal accompaniment, by no means, of course, form harmonic music. On the contrary, harmony is an achievement of the succeeding age; it is here foreshadowed in only imperfect beginnings. Such beginnings, however, may everywhere be discerned in the records that we have of the melodies of the Soudan negroes and the American races. Nevertheless, most of the records that are as yet available are still of doubtful value. The auditor is too prone to 
find in them his own musical experiences. For reliable data we must wait until, following the beginnings that have already been made, a greater number of such natural songs will have been objectively recorded by the aid of the phonograph. As yet we can only say that, if we may judge from their musical instruments, the Africans surpass all other natural peoples in musical talent. Their melodies ordinarily move within the range of about an octave, whereas those of the North American Indians seldom pass beyond a sixth. The fact of this small tonal compass will itself indicate that the melody of all natural peoples tends to very constant rhythms and intervals. The latter, moreover, show some similarity to those with which we are familiar. The chief characteristic of these songs, however, is their tendency toward repetition. One and the same motive frequently recurs with tiresome monotony. The melodies thus reflect certain universal characteristics of primitive poetry as they appear in the songs of the Veddahs and of other pretotemic tribes.

Nevertheless, the forms of poetry exhibit an important advance over those of the more primitive peoples just mentioned. Particularly in the case of the song, we find that the simple expression of the moods directly aroused by nature is supplemented by a further important feature. This feature is closely bound up with that more lively bodily and mental activity of totemic culture which is reflected likewise in its use of implements and weapons. Karl Bücher was the first to point out that common labour gives rise to common songs, whose rhythm and melody are determined by the labour. The increasing diversity of the work results in a wider range of content and also in a richer differentiation of forms. Such work-songs are to be found throughout the entire totemic era, whereas, of course, they are lacking in the preceding age, in which common labour scarcely exists. Contemporaneously with the work-song, the cult-song makes its appearance. The latter is essentially conditioned by the development of totemic ceremonies. As these become more numerous, the cult-song likewise gradually grows richer and more manifold, in close reciprocal relations with the dance 
and music. In the case of the cult-song, as well as of the work-song, the above-mentioned repetition of motives comes to exercise an important influence on the accompanying activity. Though different causes are operative in the two cases, these causes nevertheless ultimately spring from a single source-namely, the heightening of emotions. In the cult-song, man aims to bring his petitions and, as we may say for the earlier age, the magic which his words exercise, as forcibly as possible to the notice of the demons or, at a later period, of the gods whom he addresses. For this reason the same wish is repeated again and again. The most primitive form of cult-song generally consists of but a single wish repeated in rhythmic form. In the worksong, on the other hand, it is the constantly recurring rhythm of the work that leads directly to the repetition of the accompanying rhythmic and melodic motives. When one and the same external task becomes associated time and again with these accompanying songs, the two mutually reinforce each other. The song is a stimulus to the work, and the work heightens the emotion expressed in the song. Both results vary with the degree in which the song is adapted to the work and thus itself becomes a poetic representation of it. Here again neither plan nor purpose originally played the least part; the development was determined by the rhythmic and melodic motives immanent in the work.

Several brief illustrations may serve to give us a clear picture of what has been said. The first is a cult-song of American origin. Again we turn to the cult usages of one of the tribes of New Mexico, the Sia. The motif of the song, which is rain-magic, furnishes the material for very many of the ceremonies of these regions. The song of the rain-priests is as follows :-

All ye fluttering clouds,

All ye clouds, cherish the fields,

All ye lightnings and thunders, rainbows and cloud-peoples,

Come and labour for us. 
This song is repeated again and again without change of motif-it is a conjuration in the form of a song.

The snake society of the Hopi, to which we have already referred, has a similar song, which it sings with musical accompaniment. It runs as follows :-

Oh, snake society of the North, come and labour for us,

Snake society of the South, of the West, snake society of the Zenith and of the Nadir,

Come hither and labour for us.

The fact that the snake societies of the Zenith and Nadir are invoked makes it clear that this song is not, as it were, an appeal addressed to other societies of human beings. There are, of course, none such at the Zenith or the Nadir. The song is obviously directed to a demon society conceived as similar to human cult associations. It petitions for assistance in the preparation of the field and for a successful harvest.

The repetitions in such cases as these are always due to the fact that the songs are conjurations. Not so with the work-song. This is generally the expression of a greater diversity of motives, as is shown by the following lines taken from a song of the Maoris of New Zealand. The song is one which they sing while transporting trunks of trees to the coast :-

Give more room,

Joyous folk, give room for the totara,

Joyous folk,

Give me the maro.

Slide on, slide on !

Slip along, slip along !

Joyous folk ! etc.

'Totara' and 'maro' are the names of trees that they have felled. In its rhythm and its repetitions, the song gives us a direct portrayal of the work itself.

These song-forms are still entirely the product of external motives and never arise under the independent and immediate influence of subjective moods. Far superior 
to them is another field of literary composition, the narrative. The totemic age, particularly, has produced a great variety of forms of narrative. Predominant among these is the märchen-myth, a narrative which resembles the fairy-tale and which, as a rule, continues during this period to be of the nature of a credited myth. It is a prose narrative circulated by word of mouth, in which manner it sometimes traverses wide regions. With occasional changes or in connection with different mythical ideas it may survive many generations. So far as these general characteristics are concerned, the märchen, indeed, is the most permanent of all forms of literary composition. It extends from the most primitive levels of culture down to the present. In the form of the märchen-myth, however, it is especially characteristic of the totemic age. We now possess numerous collections of such tales from the most diverse regions of totemic as well as of later civilizations. An Englishwoman, Mrs. Parker, has brought together a number of Australian tales, and these have been augmented in more recent times, particularly through the labours of the German missionary Strehlow. Strehlow has a great advantage over most of the other Australian investigators in being familiar with the languages of the tribes among whom he lives. Valuable material regarding America and Africa has been gathered particularly by American and English travellers ; data, furthermore, are not lacking concerning the natural and cultural peoples of other parts of the earth. Moreover, comparative research has for some time past studied the märchen with the primary purpose of determining to what localities the materials of the märchen and the fable have spread, and thus, in turn, of learning the early cultural relations of peoples. This investigation of the märchen, however, has, for the most part, suffered from a false preconception. The criterion by which we judge present-day tales of this sort was applied to märchen-fiction in general. The märchen-myths of primitive peoples, therefore, were regarded either as creations of individuals and as never having been credited, or, at best, as retrogressive forms of higher types of myth-particularly of nature myths-adapted 
to the needs of childlike comprehension. A closer investigation of the märchen-myths of relatively primitive peoples has rendered this theory absolutely untenable. True, retrogressive forms occasionally occur in this as well as in most other sorts of myth and of literary composition. Nevertheless, there is no longer any room for doubt that, on the one hand, the earliest products of narrative composition were all of the nature of the märchen, and that, on the other hand, most primitive märchen-fictions were credited myths. An attempt to arrive at the sources of the most common motifs of the märchen of different peoples and ages will reveal the fact that the majority of them must undoubtedly be traced to the totemic age. Such was the environment, certainly, in which the earliest narrative had its setting, particularly in so far as it was believed to report truths of history.

The early myth narrative was of the general character of the märchen primarily in that it was not, as a rule, restricted to a specific time or place. This also differentiates the folk märchen of to-day from the saga. An occasional exception is offered by the anthropogenic legends of peoples of nature, although these also are in other respects of the nature of the märchen. A second essential characteristic of the märchen is the fact that magical agencies play a rôle in the determination of events. This is true even of present-day folk märchen, and is due to the circumstance that the primitive märchen arose in an age which was still entirely under the dominance of magical beliefs. These beliefs, which influenced all phases of the activity of primitive man, also caused the magical märchen to be credited either in their entirety or at least in great part. All the narratives of this age, however, bear the characteristics of the märchen, as these have just been indicated, or, at any rate, it is at most only occasionally, in the primitive legend, that they approximate to the saga. It follows, therefore, that the development of the myth' in general begins with the märchen-myth. Here also the development proceeds from below upwards, and not the reverse.

But even though the beginnings of the märchen-myth 
doubtless date back to primitive man, the flower of the development is undeniably to be found in the totemic age. For it is to this age that all those characteristics point that are still to be found, as survivals of the totemic period, in present-day märchen and children's fairy-tales. Of such characteristics, we might mention primarily the magical causality which the action involves - a point to which we have already referred-and also the rôle assigned to the animal, which is portrayed either as the helper and benefactor of man or, at the least, as like him in nature. The latter resemblance appears particularly in the fact that marriages are frequently represented as taking place between man and animals; furthermore, transformations of men into animals are said to occur, and retransformations of the latter into men. In these totemic märchen we very seldom find man to the exclusion of animals ; just as little, moreover, do animals appear alone. Both the animal fable and the märchen which deals exclusively with human beings, are products of a later development and belong to a period in which the märchen is no longer credited. Even more truly, however, do these primitive märchen lack the moral lessons which are taught by, the stories of later times, particularly by the fable. Nevertheless, those fable märchen which are generally called ' explicative' because they explain the traits of certain animals, still generally bear the marks of the totemic age, even though they apparently belong to one of its somewhat later periods. An example of this is the tale of the American Indians of the North-west, according to which the crow became black through being burned by the sun while stealing celestial fire; or the tale of the Bantus, which explains that the rabbit acquired the cleft in his lip as the result of a blow once dealt him by the man in the moon.

The most primitive märchen lacks all such intellectualistic motives. It recounts an event without any discernible purpose or without bringing the action to any natural conclusion. The following Australian märchen may serve as an illustration: 'Several women go out into the 
field with their children to gather grass seed. There they meet a magpie. It offers to watch the children while the women are gathering the seeds. They leave the children with the magpie. When they return, however, the children have disappeared. The magpie has hidden them in a hollow tree. The women hear the children crying, but do not know where they are, and return home without them. The magpie has disappeared.' Such a narrative is strikingly similar, in its lack of aim, to the songs of primitive peoples. Markedly superior is the märchen-fiction found among other natural peoples of totemic culture. These tales gradually develop a closer connection between the events. It is now that the märchen hero makes his appearance, and it is with him, particularly, that the events are associated. This hero is not of course, similar to the one of the later hero saga, who gains distinction by his strength, cleverness, and other qualities. $\mathrm{He}$ is a magic-hero, in control of magical forces. The latter are frequently represented as communicated to him by an animal which he meets, or by an old woman ; more rarely, he is said to receive them from a male magician. A further characteristic of the childhood period of the märchen-fiction is the fact that the hero himself is almost always a child. A youth sets forth on adventure, meets with magical experiences, returns home, and generally benefits his tribe through certain possessions that the has acquired on his journey. Here, again, animals play a supporting rôle. Rich collections of such märchen have been gathered, particularly in America. One of the tales of the Pawnee tribe of prairie Indians runs as follows: 'A young man did not join his companions in their sports, but went alone into the forest. One day he returned with a buffalo cow which had become his wife and had borne him a buffalo calf. But the very moment that the wife and calf entered the hut of the man they were transformed into human beings. Nevertheless, a cloud of magic hung over the man. If the child were to fall to the floor, it would be changed back into a buffalo calf. Now, this misfortune actually came to pass, and the mother was also again changed into a buffalo cow. Sadly the young man 
then went with them into the forest, where he himself became a buffalo and for a time lived quietly with the buffalo herd. Suddenly he again returned home, transformed into a man. But he had learned from the buffaloes how one must set about to lure them forth in order to hunt them. This secret he imparted to his fellow-tribesmen, and since that time the tribe has enjoyed plenty of buffalo meat.' This is a buffalo legend which tells of a sort of compact between the tribe and the buffaloes. That the legend, moreover, is not a mere märchen in our sense of the term, has been strikingly shown by Dorsey, to whom we owe the collection of Pawnee tales from which this story is taken. The tale is still recounted by the Pawnees when they wish the buffalo to appear for the hunt. Thus, it is a magical märchen, not only in that it deals with magical events but also in that its narration is supposed to exercise magical powers. This naturally presupposes that it is credited.

To trace the further development of the totemic märchen-myth is to find the gradual emergence of characteristic changes. The relation between man and the animal is slowly altered. This is most clearly apparent in connection with the transformation of human beings into animals. This change is no longer held to be one in which man, because of the magical powers which he acquires, is the gainer, and not the loser. The transformation now more and more comes to be regarded as a degradation. The man who has changed into an animal is portrayed by the märchen as denounced and persecuted by his fellowtribesmen. He is compelled to withdraw into solitude or to live exclusively with the animal herd, because he is no longer regarded by his fellows as an equal. Later, near the end of the totemic period, the change is conceived, not as degradation but as the result of an evil magic from which an innocent person suffers, and, eventually, as a punishment which overtakes a person because of some misdeed or other. Of these notions, that of malevolent magic again apparently antedates that of punishment. When the latter 
appears, the relation which was characteristic of totemism at its height becomes practically reversed. Quite naturally, therefore, the idea that transformation into an animal is a punishment arises long after the close of the totemic age. Indeed, it is to be found far into the period of ideas of requital, which are a relatively late product of deity cult, and whose development is largely influenced by philosophical reflection. Thus considered, the doctrine of metempsychosis developed by the Brahmans of India and by the Pythagorean sect of the Occident is the last metamorphosis of a very ancient totemic animal tale. These changes, however, have had practically no influence on the development of the märchen itself. This is shown by the fact that the folk märchen of to-day have universally retained the idea that the transformation of men into animals is the result of malevolent magic. The latter, indeed, is the form in which these survivals of a distant totemic past are even to-day most easily comprehensible to the child mind.

Thus, the animal märchen is an important product of totemic culture, directly embodying the views that dominate the life of this age. In addition to such tales, however, and, in part, in combination with them, there are several other forms of the märchen-myth, consisting chiefly of ideas concerning nature and, to some extent, of magical ideas sustained by the human emotions of fear and of hope. Two sorts of märchen, especially, should here be mentioned, celestial tales and tales of fortune, both of which owe their development to totemic culture. The celestial märchen, however, disappears comparatively early, mainly, no doubt, because it is displaced or assimilated by the celestial mythology of the post-totemic age. The märchen of fortune, on the other hand, remains as a permanent form of märchen-fiction, and all later narrative composition has been influenced by it.

The celestial märchen affords a direct record of the impression made by celestial phenomena on the consciousness of an age whose ideas were as yet circumscribed by the environment. By the environment, however, must as 
yet be understood the entire visible world-sun, moon, and stars, as well as hills and valleys, animals and men. The distant, moreover, was always likened to that which was near at hand and immediately accessible. Animals and men were supposed to inhabit the clouds and the heavenly bodies, precisely as they do the earth, and the relations which they were there held to sustain to one another are identical with those described in the animal tale. When the new moon appears, a wolf is devouring the moon; in an eclipse of the sun, the sun is swallowed up by a black monster; and when, in the evening, the sun disappears behind a dark cloud, it likewise is overpowered by a monster, and the red glow of the sunset is the blood which it sheds. Three themes in particular are dominant in the most primitive celestial tales: the ascension of man into the heavens, his descent from heaven, and the devourment of the great heavenly bodies, in particular of the sun, at sunset. One of the earliest of these conceptions is the journey to heaven. This is indicated by the very fact that the means for this journey are always derived directly from nature, or consist of the weapons and implements of primitive culture. There is a conception current in Australia and Oceania that beings have climbed to heaven by means of high trees, or have allowed themselves to be raised up by the branch of a tree that had been bent down to the earth. Where the bow and arrow exist, as in Melanesia and America, the arrowladder is frequently employed for the celestial journey. A hunter shoots an arrow into the heavens, where it remains fixed; he then sends a second arrow which catches into the notch of the first, then a third, a fourth, etc., until the ladder reaches to the earth. The downward journey is not so difficult. This is generally accomplished by means of a basket or a prope sustained by cords ; it is thus that the celestial inhabitant is enabled to descend to the earth. Many märchen relate that the sun and the moon were originally human beings who journeyed to the heavens. Here they are thought to remain, or occasionally, perhaps, to return to the earth while other human beings take their place. 
Besides the märchen telling of the interrelations of human and celestial beings, there are also a number of other sorts. Of them we may here single out, as a particularly characteristic type, those which deal with devourment. Obviously, as has already been noticed, it is the setting of the sun that very frequently constitutes the central theme of these tales. These märchen of devourment, however, differ from those that deal with celestial journeys in that they clearly exemplify narratives in which only one of the elements consists of a celestial phenomenon ; in addition to it, there are regularly also other elements borrowed from the terrestrial environment. Indeed, the latter may of itself originate märchen, independently of the influence of celestial phenomena. We must distinguish at the outset, therefore, between those märchen of devourment that contain celestial elements and others in which these elements are apparently lacking. A familiar example of märchen of devourment is the Biblical legend of Jonah. In its traditional rendering, this is clearly of a relatively late origin, though it is probably based on much older tales. Many of the tales of devourment, which are common to all parts of the earth, centre about a hero, who is generally a courageous youth seeking adventure. The hero is devoured by a monster; he kindles a fire in the belly of the monster, and, by burning up its entrails, rescues himself. The fact that fire figures so prominently in these tales makes it highly probable that they took shape under the influence of observations of the setting sun. Other tales make no mention of fire, but relate that the belly of the monster is extremely hot, and that the heat singes the hair of the one who has been swallowed. In an old illustrated Bible which was recently discovered, Jonah is pictured as having a luxuriant growth of hair at the moment when he is being swallowed; in a second picture, when he comes forth from the belly of the whale, he is entirely bald. But even though this reference to fire and to heat indicates an influence on the part of the sunset, this type of celestial märchen is none the less entirely different from that which deals with 
journeys to heaven and the return to earth. In the latter, the heaven is itself the scene of action upon which men and animals play their rôles. In the märchen of devourment, the celestial phenomenon imparts certain characteristics to the terrestrial action that is being described, but the latter continues to preserve its terrestrial nature. The narrator of the märchen or legend, therefore, may be wholly unconscious of any reference to the heavens. The psychological process of assimilation causes elements of a celestial phenomenon to be fused into an action of the terrestrial environment and to communicate to the latter certain characteristics without, however, thereby changing the setting of the action. The shark and the alligator are animals capable of devouring men, though this occurs less frequently in reality than in story. Yet because thoughts of this sort arouse strong emotions, they may of themselves very well come to form themes of märchen of devourment. This has frequently been the case. It seems to have happened, for example, in the Jonah legend. The above-mentioned picture in which the prophet is represented as hairless after having been in the belly of the fish, may very well have its source in some other märchen of devourment. In thus combining numerous elements of different origins, the märchen is truly representative of myth development. It shows clearly that the main theme of the myth is usually taken from man's terrestrial environment. True, celestial elements may enter into its composition and may sometimes give to the mythological conception its characteristic features. Even in such cases, however, a consideration of the tale as a whole will show that the celestial elements are completely absorbed by the terrestrial theme; their very existence may be completely unknown to the narrators of the tale. In a similar manner, celestial elements have probably been involved in the formation of other widely current märchen. Thus, the märchen theme underlying the legends of the Babylonian Sargon, the Israelitic Moses, and the Egyptian Osiris, as well as other tales in which a child, secreted in a chest, is borne away 
by the waves and lands on a distant shore, is generally regarded as having been suggested by the temporary disappearance and reappearance of the sun in a cloudy sky. In this case, however, the supposition is doubtless much more uncertain than in the case of the märchen of devourment. The theme relating to fire in the belly of the monster may be regarded as fairly unambiguous evidence of the influence of celestial phenomena, precisely because it is related only externally and apparently accidentally to the action. It should further be said that the märchen of the floating chest, at least in its connection with the personalities of the saga and of history, does not appear until the posttotemic age. It is probably an old märchen-theme which was assimilated by these legends of origin because the origin of a hero or a god was unknown and demanded explanation. Once appropriated, it underwent a number of changes in form.

Thus, the celestial märchen transcends the ideas characteristic of the totemic age. No less do the tales of fortune or adventure generally mark the transition from the supremacy of the animal to the dominance of man. These tales, however, exhibit but a gradual and continuous development. In the earliest märchen-myths, of which several examples have already been mentioned, the narrative describes an event with entire objectivity, without any apparent colouring derived from the emotional attitude of the narrator. Later, however, even the totemic animal märchen more and more betrays a love of the adventurous and of shifting fortunes. This change varies with the degree in which man steps into the centre of action, and animals, though not entirely disappearing, receive a place, similarly to monsters and other fantastic beings, only in so far as they affect the destinies of the hero of the tale. The main theme of the narrative then consists of the adventures of the hero, who is represented as experiencing many changes of fortune, always, however, with a happy ending. But even at this stage of development the hero is a boy; at a somewhat later period, a young girl sometimes assumes the 
rôle, or a youth wins a maiden after numerous adventures. At this point, the tale of fortune ceases to be a true märchenmyth. Just as the dance changes from a cult ceremony into a direct expression of lively emotions of pleasure, themselves heightened by the joy in the rhythm of the bodily movements, so also does the märchen develop into a narrative that ministers to the mere delight in fluctuations of life-events and in their happy outcome.

Thus, the beginnings of the tale of fortune go back to early totemic culture, though its more perfect development is to be found only among the semi-cultural peoples of the totemic era. The hero of the märchen then gradually passes over into the hero of the saga and of the epic. Instead of the boy who sets forth upon magical adventures, we find the youth who has matured into manhood and whose mighty deeds fill the world with his fame. The preliminary steps to this transition are taken when the märchen hero, particularly in the tale of fortune, acquires a more and more personal character. Thus, even at a very early age, we find that two types of hero appear side by side-the strong and the clever. These types, portrayed by the märchen, survive also in the heroes of the epic. Moreover, in addition to the strong and the clever, the Achilles and the Ulysses, the märchen introduces also the malevolent, quarrelsome, and despicable hero, the Thersites. 


\section{THE AGE OF HEROES AND GODS}

\section{General Character of the Heroic Age}

THE expression 'the age of heroes and gods' may meet with objection no less than may 'totemic age.' The latter has an air of strangeness, because the conceptions of totem and totemism, borrowed from modern ethnology, have as yet remained unfamiliar to historians, and especially to the historians of civilization. The former expression may be objected to on the ground that the conceptions 'heroes' and 'gods' are altogether too familiar to be extended beyond their specific meaning and applied to an entire age. The word 'hero' suggests to us perhaps the Homeric Achilles, or Siegfried of the Niebelungen saga-those mighty, victorious warriors of epic song who, as we have already seen, gradually evolved out of the heroes of primitive märchen. It is self-evident, however, that, when applied to a great and important period of culture, the expression 'hero' must not be limited to the narrow meaning which it possesses in hero-lore. True, we must not go so far as does Carlyle when, in his "Heroes and Hero Worship," he begins the race of heroes with Odin of the Northmen and ends it with Shakespeare and Goethe, thus extending the heroic age from prehistoric times down to the present. Nevertheless, if we would do justice to the significance of the conception ' heroic' as applied to an important period of human development, we must be permitted to include under the broader conception 'heroic age,' not merely the heroic hero but also the hero who has factored in the spiritual realm, as the founder of cities or states, or the creator of 
religions. These latter heroes were gradually evolved, in the course of political and religious development, out of the ancient epic heroes; in them, the heroic age continues its existence after the heroes of the powerful and crafty types have disappeared. In this broader significance of the word, a hero is any powerful individuality whatsoever, and the general characteristic of this new age, therefore, is the predominance of the individual personality. Externally, this expresses itself primarily in the fact that the age regards even all past events as the deeds of individual persons. Bound up with this is a progressive individualization of human personalities, and a constant refinement of the crude distinctions that characterize the tale of adventure and the older hero-lore.

The gods of this age are likewise patterned entirely after powerful human personalities. They are anthropomorphic in every respect-human beings of a higher order, whose qualities, though found only among men, are magnified to infinitude. Just as the hero is a man endowed with more than ordinary human capacities, so the god is a hero exalted above the measure of earthly heroes. This itself implies that the hero necessarily precedes the god, just as man antedates the hero. Any fairly detailed account of this period, therefore, must deal with the hero before considering the god. The god is created after the image of the hero, and not, as traditional mythology still believes, the hero after the image of the god. It would, indeed, be a strange procedure for man first to create the ideal conception of his god and only subsequently to transform this into human outlines, and thus produce the hero. In the advance from man to the anthropomorphic god, the hero would surely already have been encountered. This, of course, does not imply that gods may not occasionally be transformed into heroes ; it simply means that in the development as a whole the hero must have preceded the god. The relation here is precisely the same as that found everywhere else in connection with the development and degeneration of mythological conceptions. The fact of sequence, however, must 


\section{THE AGE OF HEROES AND GODS 283}

not be interpreted to mean that we can point to a time in which there were heroes but no gods. Hero and god belong together. Both reflect an effort to exalt human personality into the superhuman. In this process, no fixed line may be drawn separating the hero, whose activity still falls within the human sphere, from the god, who is exalted above it. In fact, the differences between hero and god are by no means merely quantitative, measurable in terms of the elevation above the plane of human characteristics; the differentiating marks are essentially qualitative. The hero remains human in all his thought and action. The god, on the other hand, possesses not merely human capacities raised to their highest power, but also characteristics which are lacking in man and therefore also in the hero. Especially noteworthy among the latter is the ability through his own power to perform magical acts, and thus to interfere at will in the course of nature as well as in human life. True, the hero of saga and poetry also employs magical agencies. The means of magic which he controls, however, have been bestowed upon him by some strange demoniacal being, either by one of those demons which, in the form of a man, an animal, or a fantastic monster, are recognized even by the early mythical tales as magical beings, or by a god, who, as such, combines the highest qualities of the hero with those of the demon. The conception of an anthropomorphic god, therefore, results from a fusion of hero with demon. Of these, the hero is a new creation, originating in the mental life of this later age. He was long foreshadowed, however, first by the animal ancestor (especially in so far as the latter brought blessings and good fortune), and then by the subsequent cult of human ancestors. But the figure of the hero is not completely developed until the human personality enters into the very forefront of mythological thought; then, through regular transitions, the value placed on personal characteristics is enhanced until the ideal of the hero is reached. Doubtless the hero may still incidentally be associated with the ancestor, yet personality as such has now come so to 
dominate the interest of the age that in comparison with it the genealogical feature is but secondary.

Not so with the demon-idea. Though it has come down from very remote times and has assumed many forms as a result of varying cultural conditions, the demon has always remained a magic being, arousing now hope, now fear and terror. This was its nature up to the very time when the ideal of the hero arose. This new idea it then appropriated, just as it did, in earlier times, the ideas of a soul that survives the deceased, of the totem animal, of the ancestor, and of other mythological figures. The very nature of the demon has always been constituted by such incorporated elements. From this point of view, the god also is only a new form of demon. In its earlier forms, however, as spirit-demon, animal-demon, and, finally, even as ancestor-demon, the demon was an impersonal product of the emotions, and possessed characteristics which underwent constant transformations. When it became a hero, it for the first time rose to the level of a personal being. Through the enhancement of the qualities of the hero it was then elevated into the sphere of the superhuman. Thus it came to constitute a human ideal far transcending the hero. This accounts for the uniqueness of the god-conception, and for the fact that, though the god assumes the essential characteristics of the demon, the two are nevertheless more widely distinct than were any of the earlier forms of demon conceptions from those that anteceded them. The rise of the god-idea, therefore, ushers in a new epoch of religious development. Just because of the contrast between personal god and impersonal demon, this epoch may be designated as that of the origin of religion, in the narrower and proper sense of the word. The various forms of pure demon-belief are preparatory to religion; religion itself begins with the belief in gods. The relation which the belief in demons sustains to the belief in gods is another evidence that hero and god must be grouped together, for there can be no clearly marked temporal difference in the origin of these two ideals of personality. Just as soon as 
the figure of the human hero arises, it assimilates the demonconception, which was already long in existence and which continually underwent changes as a result of the various ideas with which it came into contact. Alongside of the being that arose from this fusion, however, there continued also the hero in his purity, as well as the demon, whose various forms were at most crowded into the background by the appearance of the gods. To however great an extent, therefore, the age of heroes and gods may introduce a completely new spiritual movement that proves fundamental to all future culture and religion, it nevertheless also includes all the elements of previous development. These elements, moreover, are not merely present in forms that have been altered and in part completely changed by the processes of assimilation ; side by side with such forms, there are always also the original elements, which may be traced back to the earliest beginnings of mythological thought. The dominant factor determining the character of this new age, however, is the hero. The ideal of human personality which the hero engenders in the folk consciousness conditions all further development, and especially the origin of the god. For this reason the 'age of heroes and gods' might also, and more briefly, be called the heroic age.

As the direct incarnation of the idea of personality, it is the hero about whom the new development of myth and religion centres. Similarly, the hero also stands in closest relation to the transformations that occur in all other departments of human life. Enormous changes in economic conditions and in the forms of life dependent upon them, new social institutions, with their reactions upon custom and law, transformations and creations in all branches of art -all give expression to the new development upon which this age has entered. Here also, just as at the beginning of the anteceding age, there are numerous reciprocal relations between these various factors. The hero and the god cannot be conceived apart from the State, whose founding marks the beginning of this period. Custom and law are just as much results of the new political society as they are them- 
selves essential factors in its creation. Neither the State nor the worship of gods protected by it could survive apart from the great changes in economic life that took place at the beginning of this period, and that were further established and perfected in the course of time. Thus, here also each element reinforces every other; all the factors of life are in constant interaction. At the beginning of the totemic period, as we have seen, it was the new creations of mythological thought that constituted the centre from which radiated all the other elements of culture. At the beginning of the age of heroes and gods it is the creative power of the religious consciousness whose activities most accurately mirror the various spiritual achievements of the period.

\section{The External Culture of the Heroic Age.}

The heroic era is so comprehensive and comprises so large a part of human history that any attempt to arrive at even the barest outlines of its external culture makes it clear that this culture is even less unitary than is that of the preceding period. The differentiation of phenomena naturally increases with advancing development. Even the various forms of totemic culture manifest wide differences in detail ; indeed, when taken as a whole, they represent distinct stages. When we come to the heroic age, however, whose beginning is practically coincident with the beginnings of history in the usual sense of the term, and which includes within itself a large part of the succeeding course of events, the multiplicity and diversity of the forms of culture are incomparably greater. Every nation has its particular heroes, even though there are also certain general hero-types which everywhere recur. Even more does each nation have its gods. Heroes and gods are ideals created in the image of men, and therefore they always reflect-if possible, in a heightened degree-the characteristic differences of peoples. Nevertheless, amid all these differences of times and peoples, there are certain constant features that distinguish the heroic period both from the preceding age and from the era that follows. Most important of all these features is the establishment of the State. It was a long step from 
totemic tribal organization to political institutions. In the surge and press of the folk migrations which occurred at the beginning of the heroic period, traces of the preceding tribal organization were still everywhere present. Tribes did not change suddenly into States. Nevertheless, along with the emergence of the heroic age and its concomitant phenomena, there was a noticeable tendency towards the formation of a political order. This development pursued different courses, depending on the character of the nations or of their heroes and gods. It is primarily the resultant differences in political organization which, when considered in connection with the parallel changes in mythological and religious development, clearly show that in this period, just as in the totemic age, all other aspects of culture were closely dependent upon mythological and religious, ideas. 'Totemism ' connotes not imerely a complex of mythological beliefs in which a certain stage of culture had its setting, but also a unique form of tribal organization, which, in spite of many differences of detail, remained constant in its general features. Similarly, political society, in the original form in which it long survived, was closely bound up with the heroic age, even though the increasing differences between national cultures led, from the very outset, to a greater diversity of forms than were to be found in the case of totemism. In spite of these differences, however, the factor fundamental to political society remained the same. The formation of States was always conditioned by individual rulership. This itself is indicative of the character of the age as a whole : its typical expression is to be found in the personalities of heroes and of gods. Again it was the migrations and wars of peoples that brought about the dissolution of the old tribal organization and the creation of political society. But these migrations and wars were on an incomparably broader scale and had more intimate interconnections than had previously been the case. This gave them a correspondingly greater significance, both intensively and extensively. As a matter of comparison, we may refer to the migrations of the Malayan race during the totemic age. It would be difficult to conceive of more 
extensive migrations. But they took place gradually, in separate waves, and left no traces, for the most part, beyond changes in the physical characteristics and in the languages of peoples. These migrations, which frequently involved long voyages across the sea, were carried on by but small numbers of people, who set out from restricted groups. It cannot be doubted that these migrations exercised an influence on the character and the culture of the resulting mixed races. They were never able, however, completely to transform the culture as a whole. Even when these tribal migrations occurred in oft-repeated waves, they never resulted in more than such imperfect beginnings of a political organization as we find among the Polynesians or, in other parts of the earth, among many of the semi-cultural peoples of America and Africa.

Quite different are the folk migrations that occur at the very dawn of the history of the great cultural peoples. The difference between tribal and racial migrations is an important one. When a race migrates, it retains its peculiar characteristics, its traditions, its heroes, and its gods, and transplants these into the new territory. True, these various elements do not remain unchanged. They inevitably become fused with the culture of the original inhabitants, and it is from these fusions, when they are at all deep-going, that new peoples arise. None of the great cultural nations that mark the beginning of this age of heroes and gods, from the Babylonians down to the Greeks, the Romans, and the Germans, is homogeneous. Indeed, recent Babylonian investigations have shown that the Semitic immigration into Babylon was preceded by that of other peoples who were probably of different origin-namely, the Sumerians. We know of the latter only through linguistic traces in Babylonian inscriptions, of which, however, the religious parts, especially, show that the Sumerians exercised a great influence upon later civilization. Similarly, the settlement of the Greeks, Romans, and Germans in the territory which they eventually occupied, followed upon great earlier migrations to these regions. The people that finally formed the Greek race left the mountain country of 
Thrace and Thessaly in prehistoric times; wandering towards the sea, they fused with the original inhabitants of the regions into which they entered. In view of these migrations of early history, the theory of the desirability of racial purity, which has recently been so ardently championed in many quarters, is scarcely tenable. Political organization, on the one hand, and mythology and religion, on the other, represent important creations which for the most part sprang into existence only in the wake of migration and of the resultant fusion of peoples of different races.

Though political organization has been mentioned as the first important feature distinguishing the heroic age from the preceding era, there is a second and not less significant differentia. This relates to the material conditions of life. Two things are of outstanding importance for the new culture. The first of these consists in what we ordinarily call agriculture-that is, the tilling of the soil by the aid of the plough, or, as it is therefore more properly called in contrast to the earlier hoe-culture, ploughculture. In addition, there is the breeding of domestic animals, particularly of food-supplying cattle, and, later, of sheep and goats.

It is even to-day widely believed that, of the various modes of procuring food, hunting came first. The hunter is thought to have been seized, one fine day, with an impulse to domesticate animals instead of hunting them. He tamed the wild creatures, and thus turned from a hunter into a nomad. In the course of time, the nomad is then supposed to have tired of his wandering life and to have settled down in permanent habitations. Instead of obtaining milk by herding his cattle, he hitched the ox to the plough, after having (with that wisdom and foresight which such theories always attribute to primitive man) invented the plough. This theory is an impossible fiction from beginning to end. It is just as intrinsically improbable as is the above-mentioned hypothesis that in prehistoric times the Australians invented totemic tribal organization and exogamy for the purpose of preventing the marriage of relatives. We have 
seen, on the contrary, that the prohibition of such marriages was a consequence of exogamy, and that the latter, in turn, was not a deliberate invention but the natural result of certain conditions inherent in the culture of the age. All these institutions were originally due to influences whose outcome could not possibly have been foreseen. The same is true of the subject under discussion. In the first place, the assumed order of succession of the three stages of life is contradicted by facts. It is hardly correct to speak of a hunting life which is not supplemented by a certain amount of agriculture in the form of hoe-culture-an industry which, as a rule, is carried on by the woman in the immediate vicinity of the hut. This primitive agriculture existed even at a very early age. We find it widely prevalent among the American aborigines, who possessed no domesticated animal whatever except the dog, and the dog, as was above observed, was never tamed at all, but domesticated itself at the very dawn of prehistoric times. The supposition that the nomadic life followed upon that of the hunter is impossible, in the second place, because the animals that are hunted are not identical with those that form the care of the nomad. Cattle were never objects of the chase ; the closely related buffalo, on the other hand, was never domesticated, but has remained exclusively a game animal down to the present day. Game animals have never been domesticated and utilized for the purpose of supplying milk and drawing the plough. No doubt the domestic animals of the nomad at one time existed in a wild state. Wild cattle, of course, preceded tame cattle. But the latter did not develop from the former by the indirect way of the hunted animal. Nor does agriculture at all presuppose a nomadic life. There are vast stretches of the Old World, as, for instance, all of China, Indo-China, and Indonesia, where the production of milk was never engaged in but where agriculture in the form of plough-culture has existed, in part, since early times. Agriculture, however, involves the raising of cattle, particularly of oxen. These male cattle are c̀astrated, usually when very young. They are thus made 
tractable, so that they may be hitched to the plough and used for agricultural purposes more easily than is possible in the case of bulls, which are never completely manageable. What, then, were the motives which led to the raising of cattle, an occupation which, in many places at least, is carried on solely in the interests of agriculture? What motives led to the castration of male cattle, a practice which everywhere obviously serves agricultural purposes?

The traditional mode of explanation would lead us to suppose that man foresaw the effects of castration, that he knew beforehand that if the bull were subjected to this operation he would become an animal fitted to draw the plough. The impossibility of this supposition is evident. Such an effect could be learned only from experience, prior to which, therefore, it could not have been known. The problem relating to the cultivation of the soil by means of the plough, therefore, divides into two questions : How may we account for the ox? How for the plough? These questions are closely related, and yet they lead us back to divergent explanations. For in all probability the plough was originally drawn by man. Moreover, the plough was not the first implement to be thus drawn; it was anteceded by the wagon. Even on the early Babylonian and Assyrian monuments there were figures of a wagon bearing either an image of a god or else the king or chief priest, both of whom were probably regarded as uniting in one person the function of their offices with that of representative of the deity. Thus, the question as to the origin of the plough carries us back directly to that of the origin of the wagon. Now, the earliest wagon had but two wheels; the four-wheeled wagon came as a later discovery or as an improvement. The two-wheeled wagon, however, presupposes the wheel. But how did the wheel come to be recognized as a useful object of locomotion? The first traces of a wheel or of wheel-like objects are to be found in the latter part of the stone age. A number of such objects have been discovered in Europe; in their centre is a hole, and there are spokes that radiate to the 
circumference. The fact that these wheels are of small size indicates that they may have been worn about the neck as amulets. But even in early culture the wheel was also put to an entirely different use. Widely prevalent over the earth and probably connected with ancient sun worship, is the custom of kindling a fire to celebrate the festival of the summer solstice. In ancient Mexico, tradition tells us, this fire was started by turning a notched disk of wood about a stake until the heat thus generated gave rise to firethe same method of producing fire by friction that is still in use among primitive peoples. This fiery wheel was then rolled down a hill as an image of the sun, and later, when the custom had lost its original magical significance, as a symbol of the sun moving in the heavens. According to the report of W. Mannhardt, a remarkably similar custom existed in East Prussia not so very long ago. Perhaps the wheel that was worn about the neck as an amulet or article of adornment likewise had some connection with the idea that the sun was a celestial wheel rolling across the heavens. After the early sun cults had once created the rolling wheel in imitation of the sun and its movements, it was but a short step to the idea of securing regular, continuous movements by means of which some sort of work might be performed. An early application of this idea is to be found in the practice of spinning with distaff and whorl. This invention was credited even by the ancients to prehistoric times. Doubtless its origin belongs to the beginnings of the heroic age. This same early period, however, probably also used the wheel for transporting heavy articles. This was the original purpose of the one-wheeled barrow. It alone enabled the ancient Babylonians and Egyptians to overcome the difficulties of transporting by human agencies the mighty blocks of stone required for their temples and pyramids. From this it was not a far advance to the two-wheeled wagon. The barrow was pulled or pushed by men. The wagon, in contrast to the barrow, was apparently from the beginning an aristocratic mode of transit, never used by the common people. The two-wheeled wagon was in the first instance a 
vehicle of the gods. Later it served as the vehicle of the ruler, the terrestrial counterpart of the deity. Finally, the nobleman employed it in war, in going forth to battle. A vivid portrayal of battles in which such two-wheeled wagons played a part is presented in the Iliad. True, the wagon is here also, as a rule, only a means for carrying the hero to the scene of combat. The fighting itself is seldom done from it. Upon its arrival at the appointed place, the warrior dismounts, to try his strength, shield against shield, with his opponent. The general populace, however, always goes on foot.

This sketch gives us the main outlines of the history of the wagon. But how did the animal, first the ox and later the horse, come to be hitched to the wagon? Originally, the wagon bearing the image of the god was very probably drawn by men, as was likewise, in imitation of this, the chariot of the king. But the breeding of animals soon changed matters. Oxen were used for the purpose of drawing wagons much earlier than were horses. The horse did not appear until late in the history of civilization. There are no Egyptian pictures of horses that date back farther than the fifteenth dynasty, whereas those of cattle occur considerably earlier. In Oriental civilization, furthermore, the ass antedates the horse. In harmony with ancient custom, the ass even to-day continues, in the Orient, to be a favourite beast of burden as well as a riding animal. The horse seems to make its first appearance in history along with the Indo-Germanic tribes, who were probably indebted for it to the Turanian peoples of the Asiatic steppes. As a result of its superior speed, it then superseded its rivals in all the civilized countries of the ancient world. The Assyrian king went forth to the chase and the Homeric hero proceeded to battle in a chariot drawn by steeds. It was only later that the Greeks used the horse for saddle purposes, and not merely to draw the chariot. When this took place, equestrian combat came into favour among the aristocracy.

This development, however, was preceded not only by the taming of cattle but probably also by the use of the ox for drawing the wagon. How the latter came about may, 
of course, only be conjectured. . The bull has remained unmanageable even to the present day; the attempt to hitch him to a wagon, therefore, must always have failed. The cow was not forced into this service-at least, not in those places where milk was valued. On the other hand, the castrated male animal is thoroughly suited to the task of drawing the wagon. It is stronger than the cow, and also more tractable. It is inconceivable, however, that castration was originally performed with the purpose of engendering these characteristics. Before there could be such a purpose, the results must already have been known-that is, the operation must already have been performed for other purposes. Eduard Hahn has offered a suggestion with reference to our problem. He has called attention to the ancient Asiatic cults of the Phrygian Cybele and the Syrio-Phœnician Astarte. These cults are similar to the vegetation festivals which, as was mentioned in the preceding chapter, may be found among the Pueblo peoples of America. Similar orgiastic phenomena recur wherever peoples are primarily concerned with agriculture and are anxious for the welfare of the grain. The beginnings of vegetation cults, found in the earlier period of hoe-culture, were succeeded by more developed deity cults, connected with plough-culture. The ecstatic motives associated with the tilling of the soil then extended their influence beyond the limits of vegetation cults proper and became universal elements of the deity, cults. The powers shared by the numerous demoniacal beings of the more primitive cults were now centralized in a single goddess mother. The life-giving activity of the deity in connection with human procreation came to be of focal interest. The exaggerated development of cult ecstasy caused the orgy to become a form of self-mortification. The cult associates, especially the priests, lacerated and emasculated themselves in the fury of religious excitement. By becoming a permanent custom, this gave rise to a group of eunuchs consecrated to the service of the deity. These were doubtless the earliest eunuchs of history. In the guardians of Turkish harems 
and in the singers of the Sistine Chapel, survivals of these unrestrained cults of the past still exist. Now, when the group of emasculated priests paced beside the chariot of the goddess, they might easily have hit upon the idea of hitching a castrated animal to the wagon. But, however plausible this hypothesis may appear, in that it avoids the impossible assumption of an invention, it nevertheless leaves one question unanswered. Even though the castration of the priest may be understood as the result of the well-known effects of extreme religious excitement, the castration of the bull is not yet accounted for. Are we to suppose that the priest merely aimed to render the animal similar to himself? Neither ecstasy nor reflection could account for such a purpose. But there is another factor which has always been significant for cult, and which attained to increased importance precisely in the worship of the deity. I refer to sacrifice. In its highest stages, sacrifice assumes new forms, in that man offers either himself or parts of his own body, his blood, his hair, or a finger. A late survival of such sacrifices is to be found in a custom that is still prevalent in Catholic countries. Here it frequently occurs that a sick man lays a wax replica of the diseased part of his body upon the altar of the saint. This idea of sacrificing parts of one's own body is also exemplified in the self-emasculation practised by the Russian sect of Skopzi even in our own Christian age. Such sacrifice, moreover, may receive a wider application, so as to include, among the sacrificial objects, parts of the animal. Now at one time the kidneys with their connected organs were regarded as vehicles of the soul, and, as such, were sacrificed to the gods. The castration of the bull, therefore, may originally well have been regarded as the sacrifice of the most readily accessible of the favourite vehicles of the soul. Thus, it may have been in the case of the animal whose generative organs had been sacrificed to the deity that man first observed the change of characteristics which fitted the animal to be hitched to the chariot of the deity, and finally, through an extension of its sphere of usefulness, to draw the plough 
across the fields. This hypothesis, which presupposes the joint influence of orgiastic vegetation cults and ancient sacrificial usages, is, of course, not susceptible of positive demonstration. Nevertheless, to one concerned with the transition from ancient field cults to the agriculture of later times, the combination of conditions just indicated may reasonably be regarded as affording the basis of an hypothesis that is psychologically not improbable.

Whether the raising of the milch cow was coincident with the taming of the ox for the purposes of agriculture, and whether it came about as the result of a similar transformation of motives, it is hardly, possible to determine. Though such changes are of more importance for the development of culture than are many of the campaigns and ancient folk wars of which history has preserved a record, no positive clue as to their origin has anywhere survived. All that we know with certainty is that the taming of the ox to draw the plough and the raising of the milch cow are not necessarily bound up with one another. For plough-culture and the milk industry are by no means always to be found together. In spite of his highly developed agriculture, the Chinaman loathes milk, whereas the Hindoo regards it as a valuable gift of civilization, prizing it not only because of the butter which he secures from it but especially as a food and as a sacrifice to the gods. The Israelites received the promise that Canaan was to be a land " that floweth with milk and honey." The latter expression suggests the cultural conditions of two widely different periods. Milk represents the most valuable product of later culture, while even primitive man regarded the honey which he gathered from the hives of wild bees as his most precious article of food.

Whatever may be the relation of the two factors in the domestication of cattle, whether the taming of the ox preceded the raising of cows or vice versa, the production of milk, at any rate, represents the more difficult and slower task. The taming of the ox is essentially an act that affects only the particular animal in question; even to-day 
it must be repeated in the case of every male calf ; the inheritance of acquired characteristics is here not operative. The cow, just as all female mammals in their natural condition, produces very little milk' except during the period of suckling, and then only so much as is necessary. for the support of her young. Only through efforts continued throughout generations and as a result of the inheritance of acquired characteristics could she be brought to that tremendous over-production of her secretion of which she has become capable. In this case, therefore, there must from the very outset have been a systematic striving toward the desired goal. It is not absolutely essential to assume a change of motives such as occurred in the taming of the ox ; from the very beginning there may have been an attempt to make personal use of the milk which Nature intended for the calf. Nevertheless, it is not impossible that religious motives here also played a part. This is made all the more probable by the fact that the cow, no less than the bull and the ox, was worshipped by many peoples even in the earliest period of deity cults. Such worship is particularly noteworthy, inasmuch as cattle were never favourite totem animals as was, for instance, the buffalo among the hunting peoples of the American prairies. Even though the general idea of animal cult was carried over from the totemic period to the beginnings of the agrarian deity cults, this animal cult was essentially changed, and it became associated with different objects. The latter are now no longer connected with the old totem beliefs that sprang, in part, from primitive animism; they are determined entirely by the conditions of a later culture, one of whose essential elements is the clomestication of cattle. The two fundamental constituents of this later culture, agriculture and the milk industry, are not everywhere equally prized. Hence there is a difference as regards the relative importance of the male and the female member of the species in the cult worship that is accorded to the most valued domestic animal of the new economic era. In the Opis-worship of the Egyptians, as well as in the Persian cult of Mithra, the bull was regarded as an incarna- 
tion of the supreme deity. In many sections of Northern Europe it is even to-day customary, at harvest-time, to bedeck an ox with ribbons and wreaths of flowers and to lead him in a festal procession. On the other hand, we find that the Vedas and the Avesta, in harmony, with the high value which the ancient Indian and Iranian peoples place on milk; extol the cow as the most sacred of animals. In the first stages of the domestication of cattle, it was possible to gain only a small supply of milk, since its over-production could be developed but slowly; just for this reason, however, milk was all the more valuable. This may probably also throw light on the high value which was long placed on butter as a sacrificial gift. The attempt to secure this valuable product for sacrificial purposes may then itself in turn have reacted upon the milk industry. Thus, the two great advances in material culture that attend the heroic age-the tilling of the soil with the plough and the systematic endeavour to secure milk and its products-seem to be, in part, directly due to, and, in part, closely bound up with, motives of cult. External culture and inner religious impulses have always attested themselves to be elements of a totality all of whose parts are interrelated.

Of the new forms of industry which thus arose, the cultivation of the soil by means of the plough led to a further important change. This change was just as much an effect of the new conditions of life as it was an expression of the altered spirit of the times. The guidance of the plough is a task which prevents the field work from being any longer done in common, as it was at the height of hoe-culture and during the time of the origin of the great vegetation festivals of totemism. The individual must guide his own plough. The appearance of plough-culture individualizes labour. Just as the individual comes to the fore in political development and is extolled in legend as the founder of cities and States, so also is it the individual who cultivates the land. This individualistic tendency also gradually makes itself felt in the raising of domestic animals. Plough-culture gives rise to private property as regards both the soil and its products. 
Here again, however, the new social order influences economic life, and both together produce further changes in external culture. Individual activity receives emphasis not alone in the cultivation of the soil but also in warfare. Primitive man was not at all familiar with war. He slew his enemy from an ambush, attacking him but seldom in open combat. In the totemic age, when actual weapons of war first made their appearance, tribal war was a strife of many against many. As yet the individual combatants were not sharply differentiated from one another. The masses clashed with each other in unregulated strife, without definite leadership or fixed system. Only with the dawn of the political era do we find regulated single combat. Such combat then becomes the decisive factor in warfare. Consider the Homeric description of the battles before the walls of Troy. The battle is decided by champions (promachoi). These alight from their chariots of war and fight, man against man. The masses stand in the background, hurling lances or stones. Their actions, however, have little importance. They flee as soon as their champion falls. The result of the battle thus depends upon individuals and not upon the masses. The weapons also conform to these altered conditions. In earlier times, practically none but long-distance weapons were used-the sling, the hurled spear, or the bow and arrow, weapons similar to those employed in the chase. Single combat necessitated weapons of close range-the axe, held fast in the hand, the lance, used as a thrusting weapon, and the sword. Instead of the long shield, covering almost the entire body-shields such as even the Australians and also the earliest Greeks carried-a small round shield was demanded by reason of the use of swords in fighting. Of the various weapons found at the zenith of the heroic age, therefore, the sword is the most characteristic. It is also the most typical creation of this period. It obviously originated through a gradual shortening of the lance, thus becoming a weapon specifically adapted for individual combat at close range. Thus, the tendency toward the assertion of individual personality made itself 
felt in warfare and in weapons, just as it did in the State, in agriculture, and in the cult of personal gods.

Similar fundamental factors underlie the last great cultural change. This we have already touched upon in our discussion of agriculture, namely, the rise of private property. Following inevitably upon the appearance of private property are distinctions in wealth; these lead to differences in social position. In the totemic age, the contrasting conditions of rich and poor are, on the whole, not in particular evidence; even towards the decline of the period, indeed, they are only beginning to arise. Every man is the equal of the other. Only the chiefs and a small number of the older men have a superior rank. This rank, moreover, is not due to property but to the services which ability and experience enable them to render; or to the reverence which custom metes out to them. It is not until the heroic age that a propertied class becomes differentiated from a class owning little or nothing. This change is due in an important measure to the folk migrations that inaugurate the beginning of the new age. The propertied class derives from the victorious conquerors; the original inhabitants are without property. In the warfare connected with these migrations, slaves are captured; these are employed particularly in the cultivation of the soil. Thus, the more aristocratic are exalted by their greater possessions above those who have less property. As free individuals, however, both of these classes are superior to the slaves, who, similarly to the animals used in agriculture, are themselves regarded as the possession of the free and the rich.

Bound up with these social distinctions is the division of labour which now arises. The landowner no longer himself manufactures the tools which he needs or the weapons with which he goes to war. A class of artisans is formed, consisting partly of those who have little property, and partly of slaves. This differentiation of labour leads to two phenomena which long continue to influence the development of culture. I refer to trade and colonization. The former consists in the transmission of the products of labour; the 
latter, in the migration of a part of the people itself into distant places, where the same conditions that led to the founding of the mother State result in daughter States. In the totemic age, there were no colonies. Extensive as were the wanderings of the Papuans, the Malays, the Polynesians, and of some of the American and African tribes, these peoples never established colonies; moreover, the group which settled in distant places always lost its connection with the mother group. True, new living conditions were sought and found, and, through mixture with the native populations, new races were produced. Nevertheless, it was not until the political age that those parts of a particular people which settled down in foreign lands continued to retain a consciousness of connection with the mother race.

Of the two above-mentioned elements of the newer culture, commerce naturally preceded colonization. Of all civilized peoples, the Semitic race was the first to open up great channels of trade. Phœnician commerce dates back to the earliest records of history. Even the Mycenian graves of Greece contain gold jewelry of Phœnician workmanship. Spacially, the trade relations of the ancient Phœnicians extended over the whole of the known Occident. It is characteristic of the Semitic race, however, that they rarely undertook actual colonization. Trade and all that is connected with it, the industrial ardour necessary to supply the objects of trade and to exchange them for grain and other natural products, has always been their chosen sphere. The IndoGermanic races, on the other hand, have naturally inclined to colonization from early times on. In the foremost rank' were the Greeks, with their colonies in Thrace, Asia Minor, Southern Italy, and Sicily. These colonial groups, moreover, always retained their connection with the mother people. Thus, the earliest culture of the Greeks was that of the colonies in Asia Minor. Later, the colonies of southern Italy exercised a strong reaction on the mother country in science and art. It was not until relatively late that the highest cultural development of the mother country followed upon that of these outposts of Greek culture. 


\section{The Development of Political Society.}

The fundamental characteristics of totemic society appear to be purely a product of nature. This is especially true of totemic tribal organization. Its simple regularity and the constant recurrence of essentially the same characteristics are the natural result of original conditions of life that were universally prevalent. A horde split up into two halves. In the simplest cases, such as we have noticed in our account of the Australians, tribal organization remained limited to this dual division. The condition that brought about this organization arose as soon as a horde that spoke the same language spread out over a fairly broad territory. The same process of division might then repeat itself in the case of each of the two halves. This gave rise to a clan organization of four or eight divisions, as found among most of the Australian tribes, and frequently also in Melanesia. Such an organization was developed also by the original inhabitants of North America, although the totemic basis here degenerated and became essentially an external form. Totemic tribal organization is unquestionably a phenomenon that arises with immanent necessity; indeed, one might almost say that its appearance involves no co-operation on the part of man himself. The division takes place of itself; it is a result of the natural conditions underlying the propagation and growth of society.

From the very beginning of the heroic age on, the development of political society gave rise to phenomena that were fundamentally different from those of earlier times. The irreconcilability of this fact with the view, still held by historians and philosophers, that the State represents the earliest form of an ordered community life, is evident. Such theories were possible only when the whole of totemic culture was as yet a terra incognita. Totemic tribal organization cannot possibly be interpreted as an incomplete and undeveloped form of the State. Rather is it true that totemic and political societies are completely different in kind. Essentially different characteristics and conditions of origin 


\section{THE AGE OF HEROES AND GODS 303}

demarcate them from one another, even though there are certain hybrid forms, representing primarily a partial survival of older tribal customs within the newly established political society. Now, in so far as mental history always involves a regular order of development, one would, of course, be justified in maintaining that human society also necessarily eventuates in the State-that is, in a political society. Indeed, this may perhaps be the meaning of Aristotle's statement that man is a "political animal." This statement may be interpreted to refer to a predisposition rather than to an inherited characteristic. Nevertheless, Aristotle's view that the State gradually developed out of the family and the village community is in contradiction with the actual facts. To read back a tendency toward political development into the very beginnings of human society, moreover, results in a failure to give proper emphasis to those essential differences which distinguish the great periods of this developmentdifferences which at the crucial points assume the form of antitheses. Furthermore, we must not overlook the fact that there are peoples who have even as yet not progressed beyond totemic tribal organization and who will very possibly never advance to the formation of a State, particularly in case this depends upon their own initiative. On the other hand, it is doubtless to be assumed that those peoples who later acquired a political organization at one time possessed a totemic tribal structure. The higher stage of political organization, however, obviously differs fundamentally from that which preceded it. The older motives have been superseded by such as are connected with the great folk migrations and tribal fusions, and with the changes consequent upon them. True, when the time was ripe, these migrations and fusions of peoples came to pass with the same necessity as did the original division of the primitive horde into two halves. Nevertheless, a new set of conditions became operative. These, of course, arose in a regular course of development out of the most primitive modes of life, and yet they were not directly derived from them. The creative 
power characteristic of all mental activity here manifested itself, not in the performance of miracles, but in a constant engenderment of new motives out of the interaction of existing motives with changing external conditions of life. In consequence of this constant change of motives and of existing conditions, even totemic culture made numerous attempts in the direction of political organization. Such steps were taken particularly by the semi-cultural peoples of America, who possess a relatively high civilization. It is precisely in the case of these peoples that it is instructive to notice the contrast between this political tendency and the original tribal organization.

The difference between the two fundamental forms of society, the totemic and the political, is most strikingly evident in the case of their most external characteristicnamely, in the numbers according to which society as a whole, as well as in its parts, is organized and divided. These numbers are the expression of inner motives; hence they form a basis from which we may draw conclusions concerning the latter. In the case of totemic tribal organization, these motives are apparently very simple; natural expansion over a broader territory leads to separation into groups, and this of itself gives rise to the customary division into two, four, and eight parts. How different and more complicated from its very beginnings is the organization of political society! Here also the development proceeds according to law, and yet there is not a constant recurrence of the same motive as is in the case of totemic tribal organization. On the contrary, we find a continuous fluctation between contradictory phenomena, and the frequent appearance of new motives. Early, and still partly legendary, tradition tells us of an organization of society on the basis of the number twelve. This mode of organization seems to have emanated from the Babylonians. They were the people who first attempted to govern human affairs in accordance with celestial phenomena. These they observed, not in the unsystematic, imaginative, mythological manner of the natural peoples 


\section{THE AGE OF HEROES AND GODS 305}

of Polynesia and America, but with the aid of astronomical instruments. True, the science of the Babylonians was also still based on mythological foundations. These mythological features, however, were combined with the idea of an all-embracing, divine rule of law. The endeavour to find this law and order in the starry sky, the greatest and most sublime sight that the human eye may behold, resulted in observations that were scientific and exact. Thus, the union of the two ideas led with a sort of inner necessity to the acceptance of the number twelve as a norm. The application of this norm to human relations was a direct result of the belief that it was of divine origin. The Babylonian calendar, whose fundamental principles, in spite of numerous reforms, have retained their authority even down to the present, was the first to emphasize the principle of bringing the courses of the sun and moon into an ordered numerical relation for the purpose of reckoning time. Taking as their point of departure the position of the sun at the vernal equinox, and following the movements of the moon until the sun returned to the same position, the Babylonians found that twelve revolutions of the moon were equivalent to one of the sun. While this observation is in reality, of course, only approximately true, to the first astronomers it might have appeared sufficiently exact to be regarded as the law of a divine world order. Thus, the year came to be divided into twelve months; and, since the moon presents four phases in each month, first quarter, full moon, last quarter, and new moon-an observation which long antedates astronomical calculation-the month was at once divided into four parts. Since the month has approximately twenty-eight days, the result was a week, comprising seven days. This number, therefore, was not, as has sometimes been erroneously assumed, derived from the seven planets. Rather is it true, conversely, that the number of the planets was, with a certain arbitrariness, first fixed at seven after this number, as well as twelve, had come to be regarded as sacred, because of its relation to the movements of the sun and moon. These numbers were believed to be 
written by the gods themselves in flaming letters on the sky. To the Babylonian, the sky furnished a revelation of the laws that should govern terrestrial life. The number twelve, especially, was adopted as the basis of the organization of human society. Of this oldest form of division, however, only meagre and occasional survivals have remained. We may refer to the legendary twelve tribes of pre-exilic Israel-later a source of much difficulty to Talmudic scholars, inasmuch as these tribes are not to be found in history-and also to the twelve gods of Greece, the twelve Apostles, etc. But the number twelve has not merely left its traces in legend; it has also inscribed itself in the records of history. Thus, the Athenian population originally comprised twelve divisions, there being four clans (phyles), each of which was composed of three phratries. Similarly, the colonial territory of the Greeks in Asia Minor is said to have included twelve Ionic cities. Moreover, even in later times, the Amphictyonic League, which undertook the protection of the Delphic oracle, consisted of twelve amphictyons, though this, it is true, was also connected with the division of time, each of the twelve tribal groups being entrusted with the guardianship of the shrine for one month in the year. With few unimportant exceptions, however, the number twelve, which was at one time probably very widely regnant, has lost its influence. Its place in the organization of society as well as in the regulation of other aspects of human life has been taken by a numerical system that still dominates our entire culture-the decimal system. Even prior to the age of Columbus, the decimal system made its appearance in certain more civilized parts of the Western world where the duodecimal system was never known. That the former originated independently, in different places, is rendered all the more likely by the fact that even primitive man used his ten fingers as an aid in counting, in spite of the fact that he had not as yet formed words for numbers greater than three or four. But, however natural this method of counting may be, its application to the organization of the group and the division of peoples nevertheless represents a 
deliberately adopted plan. If possible, this is even more true here than in the case of the duodecimal system. We are now face to face with the wide difference that separates political society from totemic tribal organization. In developing on the principle of dual division, the latter resembles a natural process which runs its own course apart from any operation of conscious intention, even though directly influenced, of course, by the general conditions of human life. The organization of society according to the number ten, on the other hand, can be interpreted only as an intentional act. Hence history not infrequently brings this form of organization into direct association with the names of individual lawgivers, with Clisthenes of Athens or Servius 'Tullius of Rome. No doubt, a basis for this new order had been prepared by the general conditions of a society which had progressed beyond the totemic stage. Its systematic introduction, however, and the series of decimal subdivisions that ensued, are only conceivable as a legislative act emanating from a personal will. In the formation of social groups, no less than in the classification and enumeration of external objects of nature; there may at times have been some vacillation of choice between the duodecimal and the decimal systems. In its application to human society, however, the decimal system finally prevailed. Indeed, the simple means of counting afforded by our ten fingers supplanted the system suggested by the firmament in every field of use, except in connection with celestial phenomena themselves and with the reckoning of time, which was directly based on the observation of these phenomena. That the victory of the decimal principle was due merely to the practical necessity of choosing the principle that was simplest and most convenient, is shown by the fact that ten was never a sacred number, as was twelve. It has a purely terrestrial and human origin. In the field of the practical necessities of life, man was victorious over the gods. Perhaps, therefore, the organization of society on the decimal principle reflects also the triumph of the secular State over theocracy. The decimal principle likewise cxercised a certain in- 
fluence upon the division of time, and it is surely not accidental that such influence coincides with epochs that are strongly characterized by a secularization of human interests. As early as the sixth century B.C., the great political organizer of Athens, Clisthenes, made an attempt to divide the year into ten months instead of twelve. The attempt miscarried, just as did the analogous one on the part of the first French Republic to introduce a week of ten days. As a matter of fact, objective measurements of time are derived from the heavens and not from man. On the other hand, our measurement of terrestrial spaces and our grouping of populations depend entirely upon ourselves, and therefore naturally conform to human characteristics. In these cases, it is the decimal system that is used. In view of the fact that the number ten was deliberately adopted, this number has been thought to represent an idea that emanated from a single source. Since the organization effected by Clisthenes and that of Servius Tullius in Rome fall approximately within the same century, it has been believed that in these cases, especially, we may assume this fundamental idea of division to have been borrowed. The very extensive distribution of the decimal system, however, militates against the probability of this supposition. Thus, the Book of Exodus no longer speaks of the legendary twelve tribes of Israel but tells of only ten tribes. We likewise hear of groups of one hundred, and of more extensive groups consisting of one thousand. These divisions also recur among the Germanic peoples, and in the far-distant realm of the Peruvian Incas. Among the latter, however, there are also distinct traces of a totemic tribal organization that antedated the invasion of the Incas. This was the foundation upon which the Inca kings and their officials finally reared an organization consisting of groups of ten, one hundred, and one thousand-indeed, the latter were even brought together to form groups of ten thousand. In certain cases, such systems may perhaps have been introduced from without or may, in part, have been acquired through imitation. Nevertheless, the supposition 


\section{THE AGE OF HEROES AND GODS 309}

that they all emanated from a single region is doubtless just as improbable as is the view that the decimal system in general had but a single origin. This new grouping of the population is closely bound up with the conditions of political society. It is dependent upon two motives, which, though not universally operative at first, became so the very moment that political society took its rise. The first motive is of a subjective nature. It consists in an increased facility in the use of the decimal mode of counting, as a result of which larger groups, consisting of multiples of ten, are formed : besides the single group of ten, it must have become possible to conceive of groups of one hundred, one thousand, and, in rare cases, even of one hundred thousand. The other motive is objective in character. There are changes in the external conditions of life such as to demand more comprehensive and at the same time more highly organized divisions than prevailed in the natural tribal organization of the preceding age. In two distinct directions does the decimal system prove readily applicable. One is in the distribution of landed property. With the appearance of plough-culture, land gradually came to be largely. converted into personal property. It was all the more necessary, therefore, for the individual to unite with others for the sake of protection and aid. Thus arose the markcommunity. This naturally centred about that part of the territory which, because it was not put under the plough but was reserved for common use as well as common care, temporarily remained common property-namely, the pasture and woodland. Thus, the mark-community was inevitable: it resulted from the new method of cultivating the soil, which brought with it a combination of personal property with common ownership. The size of the community was, of course, determined by the relation which these two forms of ownership sustained to each other, being dependent upon the fact that the amount of common property had to correspond with the number of individual owners who shared its use. The right proportion of these two sorts of property could be determined only by experience and reflection. Once 
ascertained, it was but natural to adopt this proportion more generally, in connection with more extensive groups of people. Here the decimal organization into groups of tens and hundreds, to which subjective influences naturally tended, promised to be convenient also from the standpoint of objective conditions.

Independently of other factors the mark-community might have permitted certain diversities in size. The groups were rendered uniform, however, through the influence of another organization, whose divisions, on the one hand, were necessarily identical with the mark-communities but, on the other hand, possessed by their very nature a strong inherent tendency toward regularity of size. I refer to the military organization, which was created by the political society in the interest of self-protection. In the early part of the heroic period, the individual champion was doubtless of such preeminent importance that the masses formed but a somewhat unorganized background. Homer presents such a picture, though his account is perhaps not so much a faithful representation of actual conditions as the result of the individualizing tendency of poetic narrative. But just as the masses very soon gain greater prominence in political life, so also do they in warfare. This encourages tactical organization. At this stage of political and military development, therefore, companies of one hundred, and soon afterwards groups of one thousand, are formed, and are organized as the chief divisions of the army. That these groups be always of approximately equal size is required by military tactics; that the group of one hundred is the tactical unit of which the other divisions are composed, is due to the circumstance that such a group is not too large to permit of being directed by a single leader; that the number is an even one hundred results solely from the tendency toward decimal enumeration. Since the political society is composed of individuals who are, as a rule, both mark-associates and companions in war, the two groups coalesce. The distribution of property and territorial and military organization are the determining factors in political society. 
Political society thus acquires a new basis. The conditions determining its character are very different from those that underlie totemic tribal organization. Quite naturally, therefore, the tribal system disappears with the rise of the State; it is at best but fragments of it that survive in names, cult-alliances, or in bits of custom. On the other hand, the new organization exercises an influence upon all the relations of life. In part, it effects changes in existing institutions; in part, it creates new institutions, which unite to give the political age its characteristic stamp. We have spoken of the peaceful arts of agriculture, which provide for the maintenance of society, and of the military organization, reared upon agriculture to assure safety and protection from without. There are primarily three additional features that characterize political society, especially at its inception. The first of these is a reorganization of the family. The other two are genuinely new creations, if we except certain sporadic beginnings that occur in the transitional culture. They consist, on the one hand, in the differentiation of classes and of occupationsboth of which arise in one and the same course of development-and, on the other, in the foundation of cities. Doubtless this order of sequence also approximately indicates the successive steps in the establishment of the new political organization. The reorganization of the family inaugurates this development; it is terminated by the founding of cities, for cities are the centres from which the management of the State is conducted and which mediate intercourse between the separate regions; following upon the former and preceding the latter, is the differentiation of classes and of occupations-a result of property conditions and of military organization.

\section{Family Organization within Political Society.}

Wherever primitive man has been protected against foreign influences, as we have seen, he apparently always lives in monogamy. This mode of marriage is continued 
in the totemic age, and is the fundamental mode from which all others are deviations. These deviations we found to be the two forms of polygamy-polyandry and polygyny. In the presence of these various marriage practices, firmly established family bonds are impossible. Striking evidence of the recedence of the family as compared with the social bond, is offered by the men's club, that widely prevalent institution of the totemic age. True, the individual member of the men's club may have his own wife who lives in her particular hut, but there is no common life of husband and wife such as is essential for a true family. In certain cases, of course, marriage conditions approximate somewhat more closely to a true family life, yet the development is hindered by the overshadowing polygyny. But the beginning of the political age mark's the rise of a new form of monogamy. The enlarged monogamous family, the so-called ancient or joint family, makes its appearance. The joint family, which' is characteristic of the heroic era, takes the place of the clan. Though the latter also survives for a time, it more and more loses its importance and finally disappears altogether. Now the clan, as well as the joint family, is composed of individuals of the same ancestry-that is, of blood relations, in the wider sense-even though, in exceptional cases, it also includes members of other clans or even tribal strangers. The recedence of the clan in favour of the joint family must therefore be regarded as a process in which a limited number of closer blood relatives separate from the clan and gradually attain the dominant influence within society. Such a development presupposes first of all a sharper demarcation of the individual family. Hence the joint family directly impresses one as being an extension of the individual family. As a rule, for example, a joint family includes three generations : father, son, and grandchild. This series of generations terminates with the third, because the oldest male member retains the authority over the joint family only so long as there is no generation younger than grandchildren. Though a great-grandfather is honoured as the 
oldest member of the family, the authority over the joint family passes down to the son who has become a grandfather. Moreover, nature allows such cases as this but rarely. The life-span of three generations is approximately a century; and the average life of man is such that it happens but seldom that those who are living at any one time will outspan a century. Thus, the fact that the ancient family comprised three generations may be due to the natural limit of life, which does not seem to have changed essentially since the beginnings of civilization. The family organization under discussion, therefore, is characterized, in the first place, by monogamy; secondly, by the dominance of the man within the single family; and thirdly, by the inclusion of three generations under the authority of the oldest member of the family. This third characteristic has frequently caused the typical joint family to be called the 'patriarchal family.' Since it was true even of the clan that the older men exercised the decisive influence, the clan may be regarded as preparing the way for a patriarchal order. Such clan alliances, for example, as the Germanic kinship groups, in which the fact of the blood relationship of the members receives particularly strong emphasis, form a sort of transition between the clan and the joint family. In the joint family, it is no longer the older generation as such that is dominant, but the oldest individual. This change, as a result of which authority becomes vested in an individual, is paralleled by that which leads to individual rulership within the State. Thus, totemic tribal organization is doubly exposed to disintegration, from below and from above. On the one hand, the patriarchal joint family undermines the leadership of the clan-elders. On the other hand, the clans, together with the tribes whose divisions they form, are shorn of their power; they become fused into one group which, with the rise of political society, passes under the rulership of a single chieftain. It is particularly important to notice that, when the joint family emerges and clan organization is consequently dissolved, one of the most important functions of the more restricted 
clan alliances, so far as concerns the inner life of society, passes from the clan to the joint family. I refer to bloodrevenge. Not until it underwent many changes did retribution come to be an affair of the State. Thus, the patriarchal family brings to completion a twofold series of changes, whose gradual beginnings may be discerned as early as the previous age. These are, in the first place, the displacement of maternal descent by paternal descent, and, secondly, the development of chieftainship. The latter at once concludes and annuls totemic tribal organization. The motives to the former show how untrue to the real nature of the difference between the two social institutions it is to speak of the contrast between mother-right and father-right, or even between maternal rule and paternal rule, instead of referring to the transition as one from maternal descent to paternal descent. Mother-right is to be found at most in a limited sense, as applying to certain rights of the kinship community and, connected with these, at a later time, to the inheritance of property ; mother-rule never occurs, or at most is an abnormal and exceptional phenomenon having scarcely any connection with maternal descent as such. The motives to maternal descent, as we have seen, are totally unrelated to the question of dominance within the family; they are the direct result of a separation of the sexes, which manifests itself likewise in the men's clubs. Paternal descent, on the other hand, is from the very outset based on paternal rule. In the form of father-right, paternal rule prevails even in the case of the primitive monogamous family. Its original source is the natural physical superiority of man; later, it derives its main strength from the fact-reflected also in the origin of chieftainship-that the general affairs of peace, as well as of war with hostile tribes, become subject to the authority of leaders. This latter factor comes to reinforce the former at that stage of development, particularly, which is characterized by the dissolution of totemic institutions and the re-emergence of the monogamous family. It is this change, together with the growing influence of chieftainship, that marks the beginning of the political age. 
Thus, the restoration of the monogamous family came as a result of political organization. The general course of development was the same everywhere, though the particular steps varied greatly. It was especially in connection with the rise of the patriarchal joint family, which is intermediate between the kinship group and the individual family, that obstructing influences sometimes manifested themselves. In such cases, the course of development was at once deflected directly towards the individual family. A patriarchal family organization of a sharply defined character appeared very early among many of the Semitic tribes, particularly among the Israelites. Of the Indo-Germanic peoples, it was especially the Romans who long preserved the patriarchal system ; among the Greeks and the Germanic peoples, it had already disappeared in early times in favour of the single family. That which preserved the joint family was probably the force of tradition, coupled with reverence of age; the single family reflects a sense of freedom on the part of individuals. This brings out clearly the essential difference between the original monogamy, which was due to natural instinct and the simple conditions of primitive life, and the monogamy that was reinstituted as a result of the new tendencies of political society. In the former case, no progress was made beyond the natural starting-point, namely, the single family; in the latter case, the joint family mediated the transition between the dissolution of clans and the establishment of political society. Inasmuch as the acts of primitive man were largely determined by instincts, the original monogamy is not to be interpreted as conformity to a norm. The reason for the almost universal occurrence of monogamous marriage is to be found in the uniformity of the conditions of life and of the social impulses. The monogamy of the political age, on the other hand, is confronted by all those conflicting tendencies which had previously given rise to the various polygamous marriage-unions of totemic society. One of these modes of marriage especially, namely, polygyny, finds favourable conditions of development in the new political 
order. It receives fresh impetus as a result of that very dominance of man which brought about the transition from the maternal descent of earlier times to paternal descent. Polyandry and group-marriage, on the other hand, have by this time disappeared, either entirely or, at least, with rare exceptions. Moreover, the character of polygyny has changed. This is apparent from the distinction between chief wife and secondary wife-a distinction which has, indeed, an analogy in certain phenomena of the totemic period, but which, as a result of the conditions of public life, now rests upon an entirely different basis. The chief wife is taken from one's own tribe; the secondary wife belongs to a strange tribe, being, in many cases, a slave captured in war. Thus, these changes in polygyny reflect the warlike character of the age, as well as a growing tendency toward a return to monogamy. On the other hand, however, we also discern certain tendencies of a retrogressive nature. These occur particularly within Islamitic culture, whenever the difference between chief and secondary, wives is either annulled or is subordinated to the will of the husband. Such deviations from the general trend of development are usually attributed to the influence of personalities. It is not impossible, however, that they are due in this case to the fact that Islamism spread to peoples of totemic culture. But in other departments of life also, remnants and traces of totemic culture have passed down to the heroic era. A striking example appears in the case of the Spartan State. The fact that the men lived in the city, engaged in military drill and political affairs, while the women, together with the slaves, cultivated the fields outside of the city, clearly betrays the influence of the ancient institution of the men's club.

\section{The Differentiation of Classes.}

We have seen that the family assumes a new status within political society. It comes to be a compact unit, contrasting markedly with the groups composed of the same sexin particular, the men's clubs-that dominated the preceding period. The differentiation of classes was a no less potent 
factor in the development of political society. Its beginnings, no doubt, go back to the declining period of totemic tribal institutions, but only in the political age does it become an important influence in social organization. This is due to two conditions, which are themselves the direct result of the folk migrations that mark the beginning of the political age. The first of these conditions consists in changes affecting property rights; the other, in the subjection of the native populations by the more energetic immigrants. The origin of property, as is well known, is even to-day generally traced, from an abstract juristic point of view, to the occupancy of an ownerless piece of land. This theory, however, is too abstract to be generally true. Above all, it presupposes the existence of ownerless land. But this is seldom to be found. Even when a migrating: people occupies new lands, it, as a rule, conquers a territory that was previously in the possession of other tribes. If, therefore, we have in mind the sort of property that was most significant for the development of political culture, we should trace its origin to an expropriation of earlier owners rather than to an occupation of ownerless land. Contradicting the abstract theory, moreover, is the fact that it is not the individual who becomes the owner of property through such occupation, but the entire tribe, the people that has immigrated and has dispossessed the original inhabitants. Property, therefore, was originally common property. True, even in early times, it was no longer all of the land that was held in common ownership. Nevertheless, the conditions of ownership that have emerged in the course of the development of political society. give unmistakable evidence of having originated in common ownership. Even up to fairly recent times, woodland and meadow have remained, either entirely or in part, common property; usually there is also a special templeproperty set apart for purposes of cult. Everything goes to show that these cases are to be regarded as remnants of a common property that was at one time more comprehensive, and not as the result of joining pieces of property 
that were at one time owned by individuals. The latter hypothesis is contradicted by the whole direction of development of private property. Interacting with changes in property rights are racial differences. The conquering immigrant peoples subjugate the native races or crowd them back. All the cultural peoples that possess a political organization are the product of folk mixtures. The subjugation of an original population may lead to varying results, depending on the racial difference between the peoples involved. If this difference is very great and the numerical relation makes the absorption of the one by the other impossible, there develops a distinction of castes, as in India, where the lower castes are clearly distinguishable from the higher, even as to physical characteristics. The situation is radically different where there is less divergence between the two populations. In such cases, racial distinctions do not occur, or at least only to a small extent ; in their stead, we find differences with respect to property and power. 'The conquering race becomes a privileged class; those who are subjugated form a class of dependents who possess fewer rights. There is no impassable barrier between the two classes, however, as there is in the caste system. The more a fairly unitary folk-type emerges from the racial fusions, and the more other factors than descent come into prominence-such as common interest in internal order and external defence, or a remarkable personal ability on the part of individual leaders of the lower classes-the greater the tendency, on the one hand, towards the abolition of traditional differences, and, on the other, towards an increased recognition of personal achievement as the basis of social standing. Such social struggles as occurred in the history of Greece and Rome from their early days on, are particularly illuminating as regards this point, for they exhibit clearly the motives that were originally involved -motives that later everywhere become more complicated.

From the very outset these motives exert a potent influence on property relations. The occupied territory first becomes the common property of the separate divisions of 
the immigrant tribe. The individual, however, vies with his tribal associates for the possession of the territory, and the new agricultural conditions connected with the introduction of cattle and of the plough favour division of the land. In addition to the superior ability of an immigrant race, it is its superior civilization that assures to it the supremacy over the native races. This superior civilization, however, involves a strong tendency toward individual industry, and thus toward the differentiation of personal property from common property. The success which the individual owner enjoys in his labour develops in him a consciousness of freedom, and this leads him to compete with his tribal associates both in the acquisition of property and in the attainment of power over the native population. Thus, the division of common property is succeeded by an inequality of personal property-an inequality which, from the very beginning, shows an unconquerable tendency to increase. This tendency is fostered by the fact that political organization makes it possible for individuals to exercise a certain control over common affairs. Property considerations become more and more decisive as regards class distinctions. In addition to descent from privileged ancestors, it is property that gives the individual his social position. An individual belonging to a people that at one time formed a class without rights, may rise to the ranks of the privileged classes, or, if the significance attached to birth continues to be maintained, he, together with those like him, may at any rate attain to an independent influence in public life. Property, however, not only affords increased rights; it also entails greater obligations. The wealthy possess a better military equipment, and are therefore enlisted in the more efficient, but also the more dangerous, divisions of the army. They are entrusted with leadership in war as well as with authority in times of peace. Individual initiative makes itself felt, and this, coupled with the opportunity for the exercise of such initiative, causes political development to appear, from an external point of view, as a series of separate voluntary acts on the part of individual personal 
leaders. This, however, is not the real truth of the situation so far as its inner motives are concerned. The heroic age is the epoch in which the action of the masses, impulsive and under the sway of environmental conditions, is more and more subjected to the direction of individual leaders who have become clearly conscious of the tendencies inherent in the social body. For this reason the heroic age is preeminently the era of personalities. Just as the personal god is dominant in mythology and religious cult, so the human personality plays the leading rôle in the State, and particular, outstanding individuals determine the conditions that regulate external life.

As personality comes into prominence, however, conflicts inevitably arise between individuals who feel themselves called to be the vehicles of this personal power. Political society was not only created by war, but it also continues to remain a theatre where conflicts are fought with changing fortunes. Together with the effort to abolish class distinctions, moreover, there gradually comes a demand for equality of rights. As a result, the influence of dominating personalities, even though never eliminated, is more and more subject to changing conditions. Thus regarded, the general course of events is indicated by, reference to two phenomena: firstly, by the development of the State and of the judicial system, and, secondly, by the transformations which the character of the hero undergoes in the course of history. The first of these phenomena will presently be discussed in some detail; the second, which puts its stamp upon the particular periods of history in question, consists in the gradual displacement of the warriorhero by the hero of peace. Even legend indicates that this is the sequence of the qualities that are supremely prized in personality. Thus, in the legend of the kings of Rome, the warlike Romulus, founder of the city, is followed by. Numa Pompilius, the organizer of religious cult, who is succeeded in due time by the secular lawgiver, Servius Tullius. The warrior-hero appears first; he suggests the origin of political society, in warfare. The founder 
THE AGE OF HEROES AND GODS 321

of deity cults is his immediate successor. The lawgiver, or the political hero in the true sense of the word, stands at the zenith of the age. The warrior initiates, whereas the legislator completes the organization of society. Then commences the age of citizenship, which no longer entertains a hero-ideal as such but, instead, prizes civic virtues. On this plane of culture, the general demands of political life and of cult are augmented by the particular duties which grow out of the position which the individual occupies within society. The position itself is conditioned primarily by the rise of differences of vocation.

\section{The Differentiation of Vocations.}

The above discussion will already have indicated the general significance of the differentiation of vocations in the development of political society. While the origin of classes is coincident with the rise of the State, separate vocations appear only at its zenith. At first there were no distinctions of vocation. The pursuits of war and politics were common to all free men ; and, while admitting of class distinctions, they allowed no vocational differences. The priesthood alone represented a class which followed a specific vocation, while also engaging in other occupations, particularly in politics. The earliest forms of specialized vocations were foreshadowed even in the totemic age. In the heroic period, they merely adapt themselves to the new social order resulting from the rise of a ruling class and the consequent class distinctions. Under the influence of deity cults, moreover, the social position of the priesthood changes, as do also its vocational practices. The transformations in cult are an important factor in elevating the class and the profession concerned in its administration, securing for them a more or less important, and in some cases a dominant, influence upon political life. In contrast with this, all forms of human labour not connected with politics and warfare are degraded. This results in occupational differences, which are henceforth closely bound up with class distinctions. The 
depreciation of which we speak, however, is not of sudden occurrence, nor does it appear everywhere to the same extent. The conditions that give rise to political society also involve a participation in the pursuits of politics and warfare on the part of the freeman, who, as an agriculturist, breeds his own domestic animals and guides his plough over the fields. Due to these same conditions, moreover, agriculture maintains a respected position even in later times, partly, no doubt, as a result of the fact that the free farmer continues to enjoy the privilege of participating in political and military affairs. Various accessory vocations come to be sundered out from the tasks of the early agriculturist, who, originally, himself manufactured the implements required for his work and was thus the primitive artisan. Political activity and the equally esteemed military vocation come more and more to be given the place of highest honour. The occupation of the farmer and that of the wealthaccumulating merchant, however, are also held in high regard, doubtless because of the growing desire for property. The independent task of the artisan, as well as art-the latter at first scarcely distinguishable from artisanship-are either left to the dependent population and slaves or, after class distinctions are well developed, are given over to the lower class of citizens as occupations of less esteem.

But in the case of vocational distinctions, just as in that of class differentiation, the process of depreciation is succeeded by a tendency toward equalization. This is due to a general shift in values. The rhapsodist of Homeric times, though welcomed as a guest by the superior classes, was not himself regarded by them as a companion of equal rank. It is only gradually that the value placed on an art becomes transferred to the artist himself. That this occurs is due in an important measure to the fact that the arts of outstanding significance-gymnastics, poetry, and music-are not practised merely by a specific profession, but are also favourite occupations of the warrior or the statesman in his hours of leisure. The respect accorded the artist is gradually extended to such other arts as already constitute vocational 
labour; as external culture becomes more refined, even the artisan wins a growing esteem, through his decoration of weapons, implements, and clothing. In the case of the arts that require a particularly high degree of vocational training, it is significant to note that, in spite of the high estimate placed on his product, the artist himself is able to rise but slowly above the plane of the mere artisan. Thus, the measure of esteem accorded to the arts gradually diminishes, according as we pass from those that spring up spontaneously, solely from inner impulse, to those that minister to the satisfaction of needs. The immediate cause for this gradation of values probably lies in the fact that political activity, which here forms the mediating link, is itself of the nature of a free vocation, requiring the exercise particularly of mental capacities. For this reason, however, the regard in which the various occupations are held tends to be equalized according as class distinctions disappear. The latter, however, occurs in proportion as all citizens come to acquire equal privileges in the exercise of political rights. To the majority, indeed, political activity remains but a secondary vocation, being overshadowed by the main occupation, which requires the greater amount of attention. Because of its political character, however, it is the secondary vocation that primarily determines the social position of the individual. The fact that all citizens come to participate in political activity, therefore, even though failing to equalize the esteem in which the various occupations were held, nevertheless caused the disappearance of the distinctions in personal status which occupational differences originally involved.

\section{The ORigin of Cíties.}

The differentiation of classes and vocations is conditioned, in a large measure, by a change in the spacial distribution of the population. This change is a result of the rise of political society, and comes to be the outstanding external characteristic of the State as soon as the latter begins to assume definite form. I have in mind the foundation of cities. In the totemic age, 
there were no cities, but at most fair-sized groups of huts or houses, forming villages. These village settlements were all equally independent; they. differed at most as regards spacial extent. But the city, in its original form, always exercised control over a smaller or larger stretch of territory, consisting either of separate farms or of villages with the territory belonging to them. As the seat of political power, the city was an infallible indication of the existence of the State. Hence it is that those who discuss the original forms of political society are not infrequently led to regard State and city as identical. Such an identification, however, is not at all justifiable. Even in their beginnings the Greek States and the Roman State were not mere city States; all that may be said is that the political power was centred in the city. This is true, also, of the original city. as it existed in the Orient and in the ancient civilizations of Mexico and Peru. The same characteristic distinguishes the early city from the many later sorts of cities that arose in response to the needs of intercourse and trade. The original city was the abode of the political and military leaders of the people who occupied the new territory and thus formed a State. This appears most strikingly in the case of Sparta-the State which preserved most fully the features of an earlier form of social organization. One might almost be inclined to say that the men's club developed by totemic tribal organization was here present in the form of a city of men established within a political order. But even in Athens and in the other Greek States the city was only the seat of the political power, whereas the State embraced the adjacent territory as well. The centre of the city, therefore, was the castle. This constituted the military defence of the State, and was the dwelling of the king or, in republican forms of government, of the highest officials. Connected with the castle was the temple of the guardian deity of the city. The immediate environment of the temple was the meeting-place of those who inhabited the territory protected by the castle and its temple. Here they assembled, partly for trade and partly for deliberative 
or popular gatherings. The economic and political intercourse which centred about the castle fostered the growth of a larger city, inasmuch as numbers of the rural inhabitants gradually settled down under the close protection of the castle. Directly connected with this development was the separation from agriculture of the occupations of art, handicraft, trade, and eventually of political office. Because of their enormous extent, the great Oriental realms included a number of city centres. Yet even here the original conditions maintained themselves, inasmuch as one of these cities continued to be not only the political seat of the State but also the chief centre of cult. The guardian deity of the leading city was likewise the guardian deity of the State, and, as such, was supreme among the gods. Cult was thus patterned after the political order. This influence of the city upon cult was reflected in temple construction. The totemic age possessed no cities, and it likewise lacked temples. Temples, therefore, are not only indicative of deity cult, whose development is bound up with political society, but they also signalize the existence of cities. The temple itself was characterized by a very rich architecture. In Babylonia it was the mighty tower, in Egypt the pair of obelisks at the entrance, which proclaimed to the surrounding neighbourhood the dwelling-place of the deity and the seat of political power. The two were identical, for it was in the name of the guardian deity of the city that the State was originally governed and that justice was meted out. In Oriental realms, the ruler was the representative of the deity, and the priests were the State officials, as well as the devotees of science and art. Tradition, together with numerous usages preserved in custom and laws, testify to the same original unification of religious and political authority in Greece and Rome. Although the State here became secularized at a comparatively early time, and art and science likewise freed themselves from theocratic dominance, the idea of a guardian deity of the city and State was long maintained. It was this that invested the secularized legal system with a halo of 
sanctity. If the course of development in Greece and Rome differed from that of the Oriental realms, this may be due, in an important measure, to the fact that they very early broke up into a considerable number of independent city States. Herein, of course, is expressed the character of Indo-Germanic peoples. Even in very ancient times they manifested a disposition to allow free play to the assertion of the individual personality; this differentiates them from the Semitic race, with its strong inclination to hold fast to traditional norms. Hence it is that, while the cult of the various Greek cities remained practically the same, the cities themselves became distinct political communities. The status of the Delphic priesthood, in whom this unity of cult very early found its expression, was therefore naturally reduced to that of an advisory council. In the individual States, the dominance of political interests and the struggle for power, which was heightened by the personal inter-relationships within the narrow circle of the city, deprived the priesthood of all authority except over cult. True, in the case of Rome, the original union of political order and religious cult was firmer and more permanent, due to the fact that one city early gained the supremacy over the other Italian cities and States. And yet, hand in hand with the extension of political dominance, went the adoption of cults that were previously strange. This led to a number of competing priest-associations, none of which could gain the leadership, since all alike were but servants of the political power.

Thus, in spite of considerable diversity as to incidental conditions, city and State were closely bound up with each other in the development of political society. We find no city apart from a State, and it is doubtful whether there was a State without a city as the seat and centre of its political power. But this correlation obtained only during the period of the genesis of States and of the attendant rise of the original city. Once States have come into existence, many other conditions may lead to the establishment of a community which, as regards extent and relative political independence, is of the nature of a city. Such phenomena may 
be referred to as the secondary foundation of cities ; they are possible only on the basis of a previously existing political society. An approximation to original conditions occurs when a victorious State either establishes cities in the conquered provinces, centralizing in them the power over the respective territories, or transforms cities that already exist into political centres. Occurrences of this sort were frequent during the extension of Alexander's world-dominion and at the time of the Roman Empire. The same fact may be observed at a later period, in connection with the occupation of the Italian cities by the Goths and Lombards. The German cities founded during the Middle Ages differ still more widely from the original type. These cities first arose as market centres, and then gradually acquired political privileges. Thus, the process of the original foundation of cities was, as it were, reversed. In the latter case, the castle came first and the market followed; the mediæval city began as a market and reached its completion with the building of a castle. In mediæval times, however, leadership was not originally vested in the city but in rulers who occupied isolated estates scattered here and there throughout the country. Yet these secondary phenomena and their further development do not belong to our present problem of the origin of political society.

\section{The Beginnings of the Legal System.}

The social regulations which we have thus far considered find their consummation in the legal system. This possesses no content independent of the various social institutions, but merely provides certain norms of action with a social sanction. As a result, these norms are protected against violation or are designated as regulations which, whenever necessary, are defended against violators by the use of external force. Thus, the legal system does not involve the outright creation of a social order. It consists primarily in the singling out, as definite prescriptions, of certain regulations that have already arisen in the course of social life, and that are for the most 
part already maintained by custom. The enforcement of these regulations is expressly guaranteed by society, and means are established whereby this pledge is to be redeemed. Thus, the most important social institutions-the family, the classes, the vocations, village settlements and cities, and also the relations of property, intercourse, and contract, which these involve-were already in existence before becoming constituent parts of a legal system. Moreover, the advance beyond custom and the settlement of difficulties case by case was not made suddenly or, much less, at the same time in all regions, but came only very gradually. The formulation of laws did not, as a rule, begin in connection with the political community and then pass down to the more restricted groups, ending with the single individual. On the contrary, law began by regulating the intercourse of individuals ; later, it acquired authority over family relations, which had remained under the shelter of custom for a relatively long period; last of all, it asserted itself also over the political order. That is to say, the State, which is the social organization from which the legal system took its rise, was the very last institution in connection with which objective legal forms were developed. We may account for this by reference to a factor which played an important rôle from the very outset. After the legal system had once grown up out of custom and had subjected many of the important fields of the latter to its authority, it was able of itself to create regulations, which were thus from the very beginning legal prescriptions. Such primarily legal regulations arose in connection with conditions in which, frequently, the fact that there be some law was of more importance than the precise character of the law. But even in these cases the regulations were always connected with the larger body of law that was rooted in custom. This larger body of law was but supplemented by ordinances that were called into being by temporal and cultural conditions.

The transition from custom to law reflects the joint influence of two factors, which, particularly at the outset, 
were themselves closely connected. The first of these factors consists in the rise of firmly established forms of rulership, which are indicative also of the transition leading to States; the other is the religious sanction which was attached to those regulations that were singled out by the law from the broader field of custom. Both factors indicate that the heroic age properly marks the origin of the legal system, even though it be true that all such changes are gradual and that occasional beginnings of the legal system, therefore, may be found at an earlier period, in connection with the very ancient institution of chieftainship. As regards the external social organization and the religious life of the heroic age, these are characterized, respectively, by the development of strict forms of rulership and by the origin of a deity cult. Each of these social phenomena reinforces the other. The kingdom of the gods was but the terrestrial State projected into an ideal sphere. No less was the development of the legal system dependent upon the union of the two factors. Neither the external force of the political authority governing the individual nor the inner constraint of religious duty sufficed in itself to establish the tremendous power characteristic of the legal system from early times on. It is true that, at a later period, the feeling that law represents a religious duty gave way to the moral law of conscience. The latter, however, itself owes its origin to the increasing influence of the political authority which is at the basis of the legal system ; moreover, as an inner motive reinforcing the external compulsion of the law, it continued to preserve a similarity to the religious source from which it sprang. True, a significant change occurred. During the early stages of legal development, the weight of emphasis fell on the religious aspect of law, whereas it later more and more shifted to the political side. At first, the entire body of law was regarded as having been given directly by the deity, as was the case, for example, with the Ten Commandments of Moses and with the Israelitic Priests' Code, which clothes even the most external modes of life in the garb of religious 
commands. Sometimes a twofold credit is given for the introduction of the legal system, in that the one who wields the power is regarded as administering justice both in his own name and as commissioned by the gods. An illustration of this is the Babylonian code of Hammurabi. It is, naturally, when the priests wield the authority that the laws are most apt to be ascribed exclusively to the gods. The tendency, on the other hand, to give the ruler a certain amount of credit for legislative enactments, is greatest whenever the ruler occupies also the position of chief priest. The direct impetus to such a union of priesthood and political authority is to be found in the rise of the legal system itself, for this resulted from a fusion of religious and political motives. The idea that the earthly ruler is the terrestrial representative of a world-governing deity, or, as occurs in extreme cases, that he is the world-governing deity himself, is, therefore, a conception that is closely bound up with the rise of political society and that receives pregnant expression in the earliest forms of the legal system. No trace of such a conception was associated with the chiefs of the totemic period. Their position was entirely distinct from that of the magicians, the shamans, and the medicinemen, who were the original representatives of the priestly class that later arose in the age of deity cults. But it is for this very reason that the mandates of the totemic chief cannot be said as yet to have constituted a legal system; they were commands which were given as occasion demanded, and which were determined partly by the will of the chief and partly by transmitted customs. Secular and religious motives are to be found in similar combination elsewhere, even among tribes that are usually regarded as peoples of nature, as, for example, particularly those of Polynesia. In cases such as these, however, there are present also the beginnings of a legal system, as well as its correlates, the fundamentals of a political organization and of a deity cult. Whether these are the remnants of a culture brought by these migratory peoples from their original Asiatic home, or whether they represent an inde- 
pendently achieved culture that has fallen into decay, we need not here inquire.

That the development of the legal system is dependent upon the first of these phenomena-that is, upon political organization-is directly apparent from the fact that the administration of justice in general presupposes two sources of authority. Here again the beginnings are to be found in the totemic age. During this period, the administration of justice was vested, in the first place, in a relatively restricted group of the older and experienced men, such as exercised authority over the older members of the horde even in pretotemic times. Judicial powers were assumed, in the second place, by individual leaders in the chase or in war. The authority of the latter, it is true, was temporary, frequently shifting with changing circumstances; it was all the more effective, however, for the very reason that it was centred in single individuals. Now, the initial step in the formation of a legal system-which, as already. remarked, was at first concerned merely with what we would call civil justicewas taken when the quarrels of individuals came to be settled in the same way as were matters of common concern to the clan or tribe-namely, by the decisions of the two longestablished authorities, the 'council of elders,' as they later continued to be called among many civilized peoples, and the individual leader or chieftain. Even in relatively primitive times, fellow-tribesmen or clansmen who disagreed as to the ownership of an object or perhaps as to whether or not some mutual agreement had been kept, and who preferred a peaceful decision to settlement by combat, were accustomed to seek the decision of the elders or of a man of commanding respect. Thus, these initial stages of legal procedure indicate that the earliest judge was an arbitrator; he was freely selected by the disputants, though he constantly became more firmly established in his position as a result both of his authority in the general affairs of the tribe and of tradition. We next find the appointed judge, who owes his office to political authority, and who decides particular controversies, not because he has been asked to do so by 
the parties themselves but 'of right' and as commissioned by the State ; supported as he is by the political power, his decision has compelling force. As soon as the State assumes the function of deciding the controversies of individuals, the judge becomes an official. Indeed, he is one of the first representatives of officialdom. For, in the early stages of political organization, all matters other than the quarrels of individuals are regulated by ancient customs, except in so far as war and the preparation for war involve conditions that necessarily place authority of an entirely different sort in the hands of particular individuals. Thus, together with the offices of those who, though only gradually, come to have charge of the maintenance of the military organization even in times of peace, the office of the judiciary represents one of the earliest of political creations. In it, we find a parallel to the division of power between the ruler and a separate council of experienced men, an arrangement that represents a legacy from the period of tribal organization, but that only now becomes firmly established. The individual judge and the college of judges both occur so early that it is scarcely possible to say whether either antedated the other. Affecting the development just described are two other conditions, capable of bringing about a division of judicial authority at an early time. One of these conditions is the connection of the state with deity cult, as a result of which the secular power is limited by the authority of the priesthood, whose chief prerogative comes to be penal justice. The second factor in the differentiation of judicial functions consists in the institution of chieftainship, one of the two characteristic features of political society. Chieftainship involves a tendency towards a delegation of the supreme judicial authority to the ruler. This is particularly the case during the first stages of political organization, which still reflect the fact that the external political power of the chieftain grew up out of the conditions attendant upon war. Even though the secular judiciary, which originated in the council of elders, or, in certain cases, the judicial office of the 
priest, also continues to be maintained, the ruler nevertheless reserves for himself the authority over the most important issues. Particularly in doubtful cases, in which the ordinary judge has no traditional norms to guide his decision, the 'king's court" intervenes in order, if necessary, to secure a recognition of the claim of reasonableness. This is especially apt to occur in connection with capital crimes. Hence it is that, even after penal law has once become a matter of general governmental control-which, as a rule, occurs only at a later stage of legal development -the final decision in criminal cases usually rests with the ruler. Generally, moreover, it is the ruler alone who has sufficient power to put an end to the bloodrevenge demanded by kinship groups. Owing to the fact that, in his capacity of military leader, the ruler possesses power over life and death during war with hostile tribes, he comes to exercise the same authority in connection also with the feuds of his fellow-tribesmen. Modern States have retained a last remnant of this power in the monarch's right to pardon, an erratic phenomenon of a culture that has long since disappeared.

Thus, the State, as such, possesses an external power which finds its most direct expression-just as does the unity of the State-in the exercise of judicial authority on the part of the ruler. In the beginnings of legal development, however, law always possesses also a religious sanction. True, the above-mentioned unification of the offices of priest and judge or of the authority of priest and ruler-the latter of which sometimes occurs in connection with the former-may be the result of particular cultural conditions. This, however, but indicates all the more forcibly how permanent has been the religious sanction of law. Such a sanction is evidenced by the words and symbolisms that accompany legal procedure even in the case of secular judges and of the relations of individuals themselves. Not without significance, for example, is the solemnity manifested in the tones of those who are party to a barter, a contract, or an assignment of property. Indeed, their words are 
usually accompanied by express confirmations resembling the formulas of prayer and imprecation; the gods are invoked as witnesses of the transaction or as avengers of broken pledges. Because of the solemnity of the spoken word, speech was displaced but slowly by writing. Long after the latter art had been acquired, its use continued to be avoided, not only in the case of legal formulas, such as the above, but occasionally even in connection with more general legal declarations. In the Brahman schools of India, for example, the rules of legal procedure, as well as the hymns and prayers, were for centuries transmitted purely through memory; we are told, moreover, that in ancient Sparta it was forbidden to put the laws in writing. To an age, however, which is incapable of conceiving even a legal transaction except as a perceptual act, the spoken word by itself is inadequate to give the impression of reality. As an indication that he has acquired a piece of land, the purchaser lifts a bit of soil from the earth, or the vendor tosses a stalk of grain to him-a ceremony which is imitated in the case of other objects of exchange and which has led to the word 'stipulation' (from the Latin stipulatio, throwing of a stalk). Another symbol of acquisition is the laying on of the hand. Similar to it is the clasp of right hands as a sign of mutual agreement. By this act the contracting parties pledge their freedom in case they break the promise which they are giving. When the fact that the two parties lived at some distance from each other rendered the hand clasp impossible, the Germans were accustomed to exchange gloves. One who challenged another to a duel likewise did so by the use of a glove, even though his opponent was present. By throwing his glove before his opponent the challenger gave expression to the distance which separated him in feeling from his enemy. In this case, the symbol has changed from a sign of agreement to the opposite. All the symbols of which we have been speaking agree in having originally been regarded, not as symbols, but as real acts possessing certain magical potencies. When an individual, who is acquiring a piece of land, picks up a bit 
of soil while speaking the appropriate words, he intends to produce a magical effect upon the land, such that disaster will come to any one who may seek to deprive him of it. $\mathrm{He}$ who offers his hand in sealing a compact signifies that he is prepared to lose his freedom in case he fails to keep his word. For this reason the shaking of hands is sometimes supplemented by the extension of a staff-a special use of the magical wand which occurs particularly when the pledge is administered by a judge. In a second stage of development, the act loses the status of reality, but it remain's associated with religious feelings. At a third stage, it becomes a mere matter of form, though the solemnity with which it envelops the transaction adds to the impressiveness of the latter and fixes it more firmly in memory.

Combined with the word, thus, is a gesture that faithfully reflects its meaning. Moreover, other individuals are summoned to witness the legal transaction. This is done, not so much that these persons may later be able to give definite testimony, as that they, too, shall hear the word and see the gesture, and so, in a sense, enhance the reality of that which is transpiring. Besides this oldest form of witness, who is not to testify regarding that which he has experienced, as occurs in later times, but who is merely present on the occasion of the legal transaction, there is the compurgator, who substantiales the oath of the man involved. The latter fortifies his statements by invoking the gods as witnesses. Now, the oath of the compurgator does not relate to the testimony of his companion, but merely to the companion himself ; it is a pledge to share the punishment of the latter in case he swears falsely. As in battle, so also in calling upon the terrible powers whose vengeance is to fall upon the perjurer, companion stands protectingly by the side of companion. Thus, the oath itself is a ceremony both of cult and of magic. As a cult activity, the oath was originally given at the place where the cult was administered-that is, in the immediate presence of the gods ; the method of procedure was to raise the fingers and to point them directly to the gods, who were regarded as 
witnesses of the act. The magical nature of the oath appears in the fact that the latter involved the conjuration of an object, which was to bring disaster upon him who took the oath in case he swore falsely. Thus, the Germans swore by their battle-steeds or their weapons, and, in so doing, they laid their hands upon these objects; or, instead of the latter, they used an oathstaff-one of the numerous metamorphoses of the magical wand-which was extended toward him who received the oath, whether the opposing party or the judge. This oath signified that the object by which the individual swore would bring ruin upon him in case he committed perjury. The oath, therefore, came to be a fixed and definitely prescribed means of judicial procedure, though this occurred only after deity cult effected a union of the two factors, cult and magic. Nevertheless, the beginnings of this development are to be found as early as the totemic age, and they approximate to the cult-oath particularly in those regions that practise ancestor worship. The Bantu, for example, swears by the head of his father or the cap of his mother, as well as by the colour of his ox. In all these cases, the intention is that the perjurer shall suffer the vengeance which the demon of the deceased or of the animal visits upon him who swears falsely.

Closely related in its motives to the oath is another legal institution, the ordeal. In the earliest form of the ordeal, the strife of individuals was settled by a duel. Such an ordeal was very similar to the sword-oath, at least among. Indo-Germanic peoples. Just as the man who swore by his weapons invoked death by their agency in the indefinite future, so each of the participants in the duel sought to bring these magical powers into immediate effect in the case of his opponent. Not to him whose arm is the stronger, but to him who has the stronger cause, will the gods grant victory through the magic of his weapon. Like the oath, therefore, the ordeal was originally a method of legal procedure in civil cases. Like the oath, furthermore, it was, in its beginnings, a means whereby individuals settled their 
controversies independently of a judge. It is at this point that the punitive action of individuals gives way to public legal procedure. Originally, crimes against life and property were dealt with by individuals; the endeavour to secure the judgment of the gods by means of the duel was doubtless one of the earliest steps by which the penal process became a public procedure, and the punishment itself, therefore, became raised above the plane of mere revenge. Blood revenge involved an unexpected attack in the open or from ambush. To renounce this custom in favour of the duel, therefore, was in harmony with the character of the heroic age. For this was the period in which the ideal of manly honour was rapidly gaining strength, and in which, therefore, it was regarded as unworthy under any circumstances to take the life of a defenceless man. The principle accepted as self-evident in war, namely, that the person attacked have an opportunity to defend himself, became, in a warlike age, a maxim applying also to times of peace. Moreover, even though it be true of the ordeal as of the oath that, at the outset, cult was secondary to magical conjuration, nevertheless, the dominance of the latter varied with the degree in which the State freed penal justice from the passion for revenge on the part of individuals. The ordeal thus came to be more than merely a combat between the accuser and the accused. The judge in charge of the combat acquired the duty of determining guilt or innocence, and, as a result, the ordeal assumed other forms. Only the one who was accused was now involved. The ordeal changed from a magic combat into a magic test, which came to be regarded as a direct revelation of the decision of the deity. This led to the adoption of means of proof other than combat. It was obviously cult that caused penal justice as such to be taken out of the hands of private individuals. For this reason it was particularly sacrilege that demanded a magical judgment independent of the combat of individuals. In cases of sacrilege, the deity himself tested the assertions of the one who endeavoured to free himself 
from the charges of religious crime. The means for determining guilt or innocence were fire and water-the same agencies that had long been employed by religious cult for purposes of lustration. That the tests by water and by fire used in connection with the witchcraft cases of mediæval times still possessed a magical significance is unmistakable. If the witch sank in the water-that is, if she was received by the purifying element-she was guiltless. If the accused was not injured by holding a glowing iron in his hand or by walking barefooted over coals, this also was regarded as indicative of innocence. Apparently the underlying conception was that the deity who gave to water and fire the power of purifying a sinner from his guilt also communicated to them the power of freeing the innocent from an accusation and of withholding assistance from the guilty. Hence it is that while these modes of divine judgment were not, indeed, as common as was purification by means of water and fire, they nevertheless appeared again and again, so far as their fundamental characteristics are concerned. They were resorted to by the Germanic peoples, and were prevalent also in Græco-Roman antiquity, and in India ; trial by water was likewise a custom in Babylonia, where it was prescribed by Hammurabi as a means by which a suspected person might free himself. We have noticed how, in the case of the ordeal and particularly of its earliest form, judicial combat, the legal controversies of individuals concerning rights relating to property, buying and selling and other agreements, came to be considered from the standpoint of punishment. This process is characteristic of the development of penal law in general.

\section{The Development of Penal Law.}

As an institution protected by the State, the administration of penal law everywhere grew up out of civil law. The judge who was appointed by the State to arbitrate personal controversies developed into a criminal judge. Still later these two judicial offices became distinct. This separation began in connection with the most serious offences, 
such as seemed to demand a separate tribunal. The determining feature, in this instance, was, at the outset, not any qualitative characteristic of the offence but its gravity. Now, at the time when deity cults were at their zenith, the most serious crimes were held to be those connected with religion, namely, temple sacrilege and blasphemy. Only at a relatively late period were crimes against life and limb classed along with those affecting religion; to these were added, shortly afterwards, violations of property rights. That murder, though the most frequent crime of early culture, should not be penalized by political authority until so late a period, is directly due to the fact that it has its origin in the strife of individuals. In such a strife, each man personally assumes all consequences, even though these consist in the loss of his life. Even to slay a man from ambush is regarded as justifiable by primitive society if an individual is avenging a crime from which he has suffered. As family and kinship ties become stronger, the family or kin participates as a group in the quarrels of its individual members, just as it does in war against hostile tribes. A murder, whether or not it be an act of vengeance, is avenged by a fellow-member of the victim, either upon the murderer or upon some one of his kin, inasmuch as in this case also the group is regarded as taking the part of the individual. This is the practice of blood-revenge, a practice which antedates the heroic age but which nevertheless continues to exercise a powerful influence upon it. Blood-revenge is so closely bound up with totemic tribal organization that it was probably never lacking wherever any such system arose. Its status, however, was purely that of a custom, not that of a legal requirement. It was custom alone, and not political authority, that compelled one kinsman to avenge the death of another. It was custom also that sought to do away with the disastrous results of a continuous bloodfeud by means of an arrangement that came to take the place of blood-revenge. This substitute was the 'wergild,' which was paid as an indemnity by the malefactor to the family of the one who had been murdered, and which thus 
maintained precisely the same relation to blood-revenge as did marriage by purchase to marriage by capture. In the former case, however, the substitution of a peaceful agreement for an act of violence gave the political authority its first occasion to exercise its regulative power. This first manifestation of power consisted in the fact that the political authority determined the amount which must be paid in lieu of the blood-guilt. With the institution of wergild the entire matter becomes one of civil law. Only one further step is necessary, and the law of contract will indirectly have established the penal authority of the State. This step is taken when the State compels the parties to enter into an agreement on the basis of the wergild. The advance, however, was not made at a single bound, but came only through the influence of a number of intermediate factors. That which first demanded a legal determination of the amount of expiation money was the neciessity of estimating the personal value of the one who had been murdered, according as the individual was free-born or dependent, of a high or of a low class, an able-bodied man or a woman. Such a gradation in terms of general social status suggested the proprietv of allowing temporary and less serious injuries to life and limb to be compensated for on the basis of their magnitude. But the estimation of damages in such cases again made civil jurisdiction absolutely necessary.

Closely interconnected with this complex of social factors, and imposing a check upon the impulse for vengeance that flames up in blood-revenge, was a religious influence-the fear of contaminating by a deed of violence a spot that was sanctified by the presence of invisible gods. No violence of any kind was allowed within sacred precincts, particularly in places set apart for sacrifice or for other cult ceremonies ; least of all was violence tolerated in the temple, for the temple was regarded as the dwelling of a deity. Such places, therefore, afforded protection to all who fled to them from impending blood-revenge or other sources of danger. The sacred place also stood under the protection 


\section{THE AGE OF HEROES AND GODS}

of the community; any violation of it brought down upon the offender the vengeance of the entire group, for the latter regarded such sacrilege as a source of common danger. Thus, the protection of the sanctuary came to be a legal right even at a time when retribution for the crime itself was left to the vengeance of individuals. The right of protection afforded by the temple, however, was sometimes held to exist also in the case of the dwellings of persons of distinguished power and esteem, particularly the dwellings of the chief and of the priest. Indeed, prior to the existence of public temples, the latter were doubtless the only places of refuge. In this form, the beginnings of a right of refuge date back even into the totemic age. At that early time, however, the protection was apparently due, not so much to directly religious factors, as to the personal power of the individual who afforded the refuge, or also, particularly in Polynesia, to the 'taboo' with which the upper classes were privileged to guard their property. But, since the taboo was probably itself of religious origin, and since the medicineman, and occasionally also the chief, could utilize demoniacal agencies as well as his own external power, even the very earliest forms of refuge were of the general nature of religious protection. In some cases, the right of refuge eventually became extended so as to be connected not only with the property set apart for the chief or the priest but also with the homes of inferior men. This, however, was a relatively late phenomenon. Its origin is traceable to the cult of household deities, first of the ancestral spirits who guard domestic peace, and then of the specific protective deities of the hearth by whom the ancestral spirits were supplanted. As a rule, it was not the criminal but the visiting stranger who sought the protection of the house. The right to hospitality thus became also a religiously sanctioned right to protection. The guest was no less secure against the host himself than against all others. The right of protection afforded by the house, therefore, should probably be interpreted as a transference of the right of refuge inherent in sacred precincts. The protective right of the 
chief was doubtless the beginning of what in its complete development came to be household right in general.

The divine protection afforded by the sanctuary obviously offers but a temporary refuge from the avenger. The fugitive again encounters the dangers of blood-revenge as soon as he leaves the sacred precincts. Nevertheless, the time that is thus made to elapse between the act and its reprisal tempers the passion of the avenger, and affords an opportunity for negotiations in which the hostile families or clans may arrange that a ransom be paid in satisfaction of the crime that was committed. Moreover, the chief or the temple priest under whose protection the fugitive places himself, is given a direct opportunity for mediating in the capacity of an arbitrating judge, and later, as the political power gradually acquires greater strength, for taking the measures of retribution into his own hands. Revenge, thus, is changed into punishment, and custom is displaced by the norm of law, which grows up out of repeated decisions in the adjudication of similar cases.

Sojourn in a place of refuge resembles imprisonment in that it limits personal freedom. One might, therefore, be inclined to suppose that, through a further development other than that described above, the sanctuary led to a gradual moderation of punishment by introducing the practice of imprisonment. Such a supposition, however, is not borne out by the facts. At the time when the transition from the place of refuge into the prison might have taken place, the idea of reducing the death penalty to the deprivation of freedom was still remote. The value which the heroic age placed on the life of the individual was not sufficiently high to induce such a change, and the enforcement of prison penalties would, under the existing conditions, have appeared difficult and uncertain. Hence imprisonment was as yet entirely unknown as a form of punishment. Though the State had suppressed blood-revenge, it showed no less an inclination than did ancient custom to requite not only murder but even milder crimes with death. Indeed, inasmuch as the peaceful mode of settlement by ransom gradually disappeared, 
it might be truer to say that the relentlessness of the State was even greater than that of blood-revenge. The oldest penal codes were very strongly inclined to impose death penalties. That the famous Draconian laws of Athens became proverbial in this respect was due merely to the fact that other ancient legal codes, though not infrequently more severe, were still unknown. The law of King Hammurabi punished by death any one who stole property belonging to the court or the temple, or even to one of the king's captains; the innkeeper who charged her guests extortionate prices was thrown into the water, and the temple maiden who opened a wine-shop was burned to death. Whoever acquired possession of stolen goods, or sheltered a runaway slave, was put to death', etc. For every crime that was judged to be in any way serious, and for whose expiation a money ransom was not adequate, the law knew only the one penalty, death. The earliest law made no use of custody except in connection with civil justice. The debtor was confined in the house of the creditor. This simply enforced the pledge involved in the shaking of hands at the time when the debt was contracted-an act by which the debtor vowed to be responsible for his debt with his own person.

The confinement of the debtor was at first a matter that was left to individuals, and its original sanction was custom ; later, however, it came under the supervision of the legal system of the State. This suggested the adoption of confinement in connection with other crimes, in which the death penalty appeared too severe a punishment and the exaction of money one that was too light, as well, primarily, as too dependent upon the wealth of the guilty individual. Contributory to this change, was a practice which, similarly to confinement, was also originally an arrangement between individuals, and was rooted in custom. I refer to the holding of individuals as pledges, to the hostage, who gave security with his own person for the promise of another. The hostage is of the nature of a forfeit, guaranteeing in advance the fulfilment of the obligation. For this reason the holding 
of hostages came to be practised not merely in the case of property contracts but in connection with every possible obligation of a private or a public nature. This development was furthered by the fact that hostages came to be held in times of war, and, as a result, were given also upon the assumption of public duties. In both cases, custody changed from a private arrangement into a public concern. This change made it possible for a judge to impose the penalty of imprisonment whenever the transgression did not appear to warrant death. Imprisonment is a penalty that admits of no fewer degrees than does a fine, and has the advantage of being independent of the irrelevant circumstance of the wealth of the one who is condemned. Moreover, the restriction of arbitrary deprivations of freedom in favour of custody on the part of the political power, makes it possible to hold a suspect whose case requires examination before a judicial verdict can be given. Thus arises the practice of confinement during investigation, an incidental form of legal procedure which is influenced by, and in turn reacts upon, the penalty of imprisonment. Such confinement makes it possible to execute the penalty of imprisonment in the case of those whom investigation shows to be guilty. But this is not its only important result. It also leads to those barbarous methods which, particularly during the early stages of this development, are connected with the infliction of the punishment itself as well as with the preceding inquisitorial activities. The public administration of justice is still affected by the passion for vengeance which comes down from the earlier period of blood-revenge. To this coarser sense of justice a merely quantitative gradation of punishment is not satisfactory; the punishment must rather be made to correspond qualitatively with the crime that has been committed. Hence the many different modes of prison punishment-more numerous even than the modes of inflicting the death penalty-and of the means of torture, which are often conceived with devilish cunning. These means of torture come to be uesd also in the inquisitional 
procedure ; the endeavour to force a confession causes them to become more severe, and this in turn reacts upon the punishment itself. On the whole, the ultimate tendency of imprisonment was greatly to restrict the death penalty and thus to contribute to more humane methods of punishment. Nevertheless, it is impossible not to recognize that this result was preceded by an increasing cruelty. The fact that the prisoner was under the control of the punitive authority for a longer period of time led to a multiplication of the means of punishment. How simple, and, one might say, how relatively humane, was blood-revenge, satisfied as it was to demand life for life, in comparison with the penal law of the Middle Ages, with its methods of forcing confession by means of the rack and of various forms of physical suffering and of death penalties!

The same is true of a further change inaugurated by the passing of blood-revenge into punishment. This change likewise led to a decided restriction of the death penalty, yet it also, no less than the forcing of confession, brought upon penal justice the stigma of systematic cruelty. The assumption of penal power on the part of the public judiciary, in conjunction with the possession of unlimited control over the person and life of the malefactor, led to the adoption of a principle which long continued to dominate penal justice. This principle was drastically expressed in the Priests' Code of the Israelites, "Eye for eye, tooth for tooth." True, this jus talionis was already foreshadowed in the custom of blood-revenge, and yet the simple form which it here possessed, 'a life for a life,' made it a principle of just retribution, and not a demand sharpened by hate and cruelty. In the case of blood-revenge, moreover, the emotions of revenge were moderated by virtue of the fact that considerations of property played a rôle. Requital was sought for the loss which the clan sustained through the death of one of its members. Hence the clan might be satisfied with a money compensation, or, occasionally, with the adoption either of a fellow-tribesman of the murderer, or, indeed, even of the murderer himself. In 
contrast with this, even the most severe physical injuries, so long as they did not result in death, were originally. always left to the retaliation of the individual. This retaliation was sought either in direct combat, or, in the heroic age proper, in a duel conducted in accordance with regulations of custom. All this is changed as soon as the State abolishes blood-revenge and assumes jurisdiction over cases of murder. In the event of personal injuries, the judge determines the sentence, particularly if the individual is unable for any reason to secure retaliation-having been rendered helpless, for example, through his injury, or being prevented by the fact of class differences. Under such circumstances it is but natural that the principle, 'a life for a life,' which has been borrowed from the institution of bloodrevenge and has been applied to the punishment for murder, should be developed into a scale of physical punishment representing the more general principle 'like for like.' He who has destroyed the eye of another, must lose his own eye; whoever has disabled another's arm, must have his arm cut off, etc. Other injuries then came to be similarly punished, even those of a moral character to which the principle " eye for eye, tooth for tooth" is not directly applicable. The hand which has been implicated in an act of sacrilege, such as the commission of perjury; is to be cut off; the tongue which has slandered, must be torn out. Originally, the death penalty was employed all too freely. Hence this substitution of a physical punishment which spared the life of the offender was doubtless in the direction of moderation. But, since this substitution gave rise to cruelties that resulted in the infliction of various sorts of death penalties, preceded and accompanied by tortures, its original effect became reversed, just as in the case of imprisonment. Moreover, the two forms of punishment-imprisonment and death-and the degree to which these were carried to excess differed according to civilization and race. The jus talionis was the older principle of punishment. It is more closely bound up with man's natural impulse for retaliation, and therefore recurs 
even within humane civilizations, sometimes merely in suggestions but sometimes in occasional relapses which are of a more serious sort and are due to the passion for revenge. In fundamental contrast with the Mosaic law, Christianity repudiated the requital of like with like. Perhaps it was the fear of violating its own principle that led it, in its later development, to seek in the cruelties of severe prison penalties a substitute for the repressed impulse to revenge which comes to expression in coarser conceptions of justice. Nevertheless, this substitution was superior to the inflexible severity of the jus talionis in that it more effectively enabled milder customs to influence the judicial conscience.

But there is still another respect in which the recedence of the principle of retaliation gradually led to an advance beyond the legal conceptions characteristic of the heroic age. The command for strict retribution takes into consideration merely the objective injury in which a deed results; to it, it is immaterial whether a person destroys another's eye accidentally or intentionally. The same injury that he has caused must befall him. Whoever kills a man must, according to the law of Hammurabi, himself suffer death; if he kills a woman, he is to be punished by the death of his daughter. If a house collapses, the builder who constructed it must suffer death. For a successful operation, the physician receives a compensation ; if the operation fails, the hand that has performed it is cut off. The same law determines both reward and punishment. Moreover, it includes within its scope even intellectual and moral transgressions. The judge who commits an error is to be dismissed from office in disgrace; the owner who neglects his field. is to be deprived of it.

Io. The Differentiation of Legal Functions.

The direct impetus to overcoming the defects that were inherent in penal justice as a result of its having originated in the conflicts of individuals, did not come from a clear 
recognition of differences in the character of the crimes themselves, but primarily from the fact of a gradual division of judicial functions. This is shown particularly by the development of Græco-Roman as well as of Germanic law. It is in the criminal court, which supersedes blood-revenge, that public authority is most directly conscious of its power over the individual. Hence the criminal court appears to be the highest of the courts, and the one that most deeply affects the natural rights of man. Its authority is vested solely in the ruler, or in a particularly sacred tribunal. This is due, not so much to the specific character of the crimes over which it has jurisdiction, as to the respect which it receives because it assumes both the ancient duty of blood-revenge and the function of exacting a requital for religious guilt. Similarly, other offences also gradually pass from the sphere of personally executed revenge or from that. of the strife of individuals, and become subject to the penal authority of the State. The division of judicial authority, to which these tendencies lead, is promoted by the differentiation of public power, as a result of which the administration of justice is apportioned to various officials and magistrates, as well as are the other tasks of the State. It is for this reason that, if we consider their civilization as a whole, the constitutional States of the Occidental world were led to differentiate judicial functions much earlier than were the great despotic monarchies of the Orient. These monarchies, as the code of Hammurabi shows, possessed a highly developed husbandry and a correspondingly advanced commercial and monetary system, whereas they centralized all judicial functions in the ruler.

Thus, the State gains a twofold power, manifested, in the first place, in the very establishment of a judicial order, and, secondly, in the differentiation of the spheres of justice in which the authority of the State over the individual is exercised. This finally prepares the way for the last stage of development. The state itself becomes subject to an established legal order which determines its various functions and the duties of its members. There thus originates an 
officialdom, organized on fixed principles and possessing carefully defined public privileges. The people of the State, on the other hand, are divided into definite classes on the basis of the duties demanded of them as well as of the rights connected with these duties. These articulations of political society, which determine the organization of the army, the mode of taxation, and the right of participation in the government of the State, develop, as we have already seen, out of totemic tribal organization, as a result of the external conditions attendant upon the migrations and wars connected with the rise of States. But they also exhibit throughout the traces of statutes expressing the will and recording the decisions of individual rulers, though even here, of course, universal human motives are decisive. After the political powers of the State have been divided and have been delegated to particular officials and official colleges, and after political rights have been apportioned to the various classes of society, the next step consists in rendering the organization of the State secure by means of a Constitution regulating the entire political system. In the shaping of the Constitution, it cannot be denied that individual legislators or legislative assemblies played a significant rôle. Nevertheless, it must be remembered that it is solely as respects the form of State organization that the final and most comprehensive legal creation appears to be predominantly the result of the will acts of individuals. The content of the Constitution is in every respect a product of history ; it is determined by conditions which, in the last analysis, depend upon the general culture of a nation and upon its relations with other peoples. These conditions, however, are so complex that, though every form of Constitution and all its modifications may be regarded as absolutely involved in the causal nexus of historical life, the endless diversity of particular conditions precludes Constitutions from being classifiable according to any universal principle. Constitutions can at most be classified on the basis of certain analogies. The most influential attempt at a genetic classification of the various historical 
forms of government was that of Aristotle. But his classification, based on the number of rulers. (one, a few, many, all) and on the moral predicates of good and evil (monarchy and tyranny, aristocracy and oligarchy, etc.), offers a purely logical schema which corresponds but partially with facts. True, it not infrequently happens that the rule of all-that is, democracy-gives way to the evil form of individual rulership-namely, tyranny. An aristocracy, however, or even a monarchy, may likewise develop into a tyranny. What the change is to be, depends upon historical conditions. Nor are monarchy, aristocracy, or the rule of the middle class forms of government that are ever actually to be found in the purity which logical schematization demands. Even in the Homeric State there was a council of elders and an assembly of freemen-an agora-in addition to the king. Indeed, if we go back still farther and inquire concerning those more primitive peoples of nature who are merely on the point of passing from tribal organization to a political Constitution, it might perhaps be nearer the truth to assert that democracy, and not monarchy, was the form of the early State. The fact is that the organization characteristic of the State as a whole is the product of historical factors of an exceedingly variable nature, and that it never adequately fits into any logical system that is based on merely a few political features. Even less may a logical schema of this sort be regarded as representing a universal law of development.

Thus, the State is indeed the ultimate source of all the various branches of the legal system. So far as the fundamental elements of its own Constitution are concerned, however, it is really itself a product of custom, if we take this term in its broadest sense, as signifying an historically developed order of social life which has not yet come under the control of political authority. The course of development is the very opposite of that which rationalistic theories have taught, ever since the time of the Sophists, concerning the origin of the State. These theories maintain that the 
legal system originated in connection with the State, and that it then acquired an application to the separate departments of life. The reverse is true. It is with the determination of the rights of individuals and with the settlement of the controversies arising from these rights that the legal power of the State takes its rise. It is strengthened and extended when the custom of personal retribution comes to be superseded by penal law. Last of all comes the systematic formulation of the political Constitution itself. The latter, however, is never more than a $d e-$ velopment; it is not a creation in the proper sense of the word. Even such States as the United States of North America and the new German Empire were not created by lawgivers, but were only organized by them in respect to details. The State as such is always a product of history, and so it must ever remain. Every legal system presupposes the power of a State. Hence the latter can never itself originate in an act of legislation, but can only transform itself into a legal order after it has once arisen.

\section{I. The Origin OF Gods.}

At first glance it may seem presumptuous even to raise the question as to how gods originated. Have they not always existed? one is inclined to ask. As a matter of fact, this is the opinion of most historians, particularly of historians of religion. They hold that the belief in gods is underived. Degenerate forms may arise, the belief may at times even disappear altogether or be displaced by a crude belief in magic and demons, but it itself can in no wise have been developed from anything else, for it was possessed by mankind from the very beginning. Were it true that the belief in gods represents an original possession of mankind, our question concerning the origin of gods would be invalidated. The assumption, however, is disproved by the facts of ethnology. There are peoples without gods. True, there are no peoples without some sort of supersensuous beings. Nevertheless, to call all 
such beings ' gods'-beings, for example, such as sicknessdemons or the demons which leave the corpse and threaten the living-would appear to be a wholly unwarranted extension of the conception of deity. Unbiased observation goes to show that there are no peoples without certain conceptions that may be regarded as precursors of the later god-ideas. Nevertheless, there can be no doubt that there are some peoples without gads. The Veddahs of Ceylon, the so-called nature-Semangs and Senoi of Malacca, the natives of Australia, and many other peoples of nature as well, possess no gods, in our sense of the word. Because all of these primitive peoples interpret certain natural phenomena-such as clouds, winds, and stars-in an anthropomorphic fashion, it has been attempted time and again to establish the presence of the god-idea of higher religions. Such attempts, however, may be straightway characterized as a play with superficial analogies in which no thought whatsoever is taken of the real content of the god-conception.

Accepting the lead of ethnological facts, then, let us grant that there are stages in the development of the myth in which real gods are lacking. Even so, two opposing views are possible concerning the relation of such 'prereligious' conditions to the origin of the god-ideas essential to religion. Indeed, these views still actively compete with each other in the science of religion. On the one hand, it is maintained that the god-idea is original, and that belief in demons, totemism, fetishism, and ancestor worship are secondary and degenerate derivatives. On the other hand, the gods are regarded as products of a mythological development, and, in so far, as analogous to the State, which grew up in the course of political development out of the primitive forms of tribal organization. Those who defend the first of these views subscribe to a degeneration theory. If the ancestors reverenced in cult are degenerated deities, and if the same is true of demons and even of fetishes, then the main course of religious development has obviously been downward and not upward. The representatives of 
the second view, on the contrary, assume an upward or progressive tendency. If demons, fetishes, and the animal or human ancestors worshipped in cult antedate gods, the latter must have developed from the former. Thus, the views concerning the origin of gods may be classified as theories of degeneration and theories of development.

But the theories of degeneration themselves fall into two classes. The one upholds an original monotheism, the basis of which is claimed to be either an innate idea of God or a revelation made to all mankind. Obviously this assumption is itself more nearly a belief than a scientific hypothesis. As a belief, it may be accounted for in terms of a certain religious need. This explains how it happens that, in spite of the multiplication of contradictory facts, the theory has been repeatedly urged in comparatively recent times. Only a short time ago, even a distinguished ethnologist, Wilhelm Schmidt, attempted to prove that such an original monotheism was without doubt a' dominant belief among the so-called Pygmies, who must, in general, be classed with primitive peoples. The argument adduced in support of this view, however, unquestionably lack's the critical caution otherwise characteristic of this investigator. One cannot escape the conviction that, in this case, personal religious needs influenced the ethnological views, even though one may well doubt whether the degeneration theory is a theory that is suited to satisfy such needs. ${ }^{\mathrm{I}}$ The second class of theories adopts the view that the basis of all religious development was not monotheism' but primitive polytheism. This polytheism is supposed to have originated, at a very early age, in the impression made by the starry heavens, particularly by the great heavenly bodies, the sun and the moon. Here for the first time, it is maintained, man was confronted by a world far transcending his own realm of sense perception ; because of the multiplicity of the motives that were operative, it was not the idea of one deity but the belief in many deities that was evoked. In essential contrast with the preceding view, this class of theories regards

' Concerning this alleged monotheism among primitive peoples, cf. supra. pp. 78 f. 
all further development as upward. Monotheism is held to be a refined religious product of earlier polytheistic conceptions. In so far, the hypothesis represents a transition to developmental theories proper. It cannot be counted among the latter, however, for it holds to the originality of the god-idea, believing that this conception, which is essential to all religion, was not itself the product of development, but formed an original element of man's natural endowment. Moreover, the theory attaches a disproportionate significance to the transition from many gods to a single god. It is doubtful, to say the least, whether the intrinsic value of the god-idea may be measured merely, in terms of this numerical standard. Furthermore, the fact is undeniable that philosophy alone really exhibits an absolute monotheism. A pure monotheistic belief probably never existed in the religion of any people, not even in that of the Israelites, whose national deity, Jahve, was not "at all the sole god in the sense of a strict monotheism. When the Decalogue says, "Thou shalt have no other gods before me," this does not deny the existence of gods other than Jahve, but merely prohibits the Israelites from worshipping any other deity. These other gods, however, are the national gods of other peoples. Not only do these other tribal gods exist alongside of Jahve, but the patriarchal sagas centre about individuals that resemble now demonic and now divine beings. The most remarkable of these figures is Jacob. In the account of his personality there seem to be mingled legends of differing origin, dating from a time probably far earlier than the developed Jahve cult. The scene with his fatherin-law, Laban, represents him as a sort of crafty märchenhero. He cheats Laban through his knowledge of magic, gaining for himself the choicest of the young lambs by constructing the watering troughs of half-peeled rods of wood-a striking example of so-called imitative magic. On the other hand, Jacob is portrayed as the hero who rolls from the well's mouth the stone which all the servants of Laban could not move. And finally, when he 
wrestles with Jahve by night on the bank of the stream and is not overcome until the break of day, we are reminded either of a mighty Titan of divine lineage, or possibly of the river demon who, according to ancient folk' belief, threatens to engulf every one who crosses the stream, be it even a god. But what is true of the figures of the patriarchal sagas applies also, in part, to Jahve himself. In the remarkable scene in which Jahve visits Abraham near the terebinths of Mamre, he associates with the patriarch as a primus inter pares. He allows Sarah to bake him' a cake and to wash his feet, and he then promises Abraham a numerous posterity. $\mathrm{He}$ appears as a man among men, though, of course, as one who is superior and who possesses magical power. Only gradually does the god acquire the remoteness of the superhuman. Abraham is later represented as falling down before him, and as scarcely daring to approach him. Here also, however, the god still appears on earth. Finally, when he speaks to Moses from the burning bush, only his voice is perceptible. Thus, his sensuous form vanishes more and more, until we come to the Jahve who uses the prophets as his mouthpiece and is present to them only as a spiritual being. The purified Jahve cult, therefore, was not an original folk-religion. It was the product of priests and prophets, created by them out of a polytheism which contained a rich profusion of demon conceptions, and which was never entirely suppressed.

If an original monotheism is nowhere to be found, one might be tempted to believe, conversely, that polytheism represents the starting-point of all mythology. In fact, until very recently this was doubtless the consensus of opinion among mythologists and historians of religion, and the idea is still widely prevalent. For, if we hold in any way to the view that the god-idea is underived, there is but one recourse, once we abandon the idea of an original monotheism. The polytheistic theory is, as a rule, connected with the further contention that god-ideas are directly due to celestial phenomena. In substantiation of this view, it is pointed out that, with the exception of 


\section{ELEMENTS OF FOLK PSYCHOLOGY}

the gods of the underworld, the gods are usually supposed to dwell in the heavens. Accordingly, it is particularly. the great heavenly bodies, the sun and the moon, or also the clouds and storms, to which-now to the one and now to the other, according to their particular tendency-these theories trace the origin of the gods. Celestial phenomena were present to man from the beginning, and it is supposed that they aroused his reflection from earliest times on. Those mythologists who champion the celestial theory, of the origin of religion, therefore, regard god-ideas as in great measure the products of intellectual activity; these ideas are supposed to represent a sort of primitive explanation of nature, though an explanation, of course, which, in contrast to later science, is fantastical, arbitrary, and under the control of emotion. During the past century, moreover, this class of hypotheses has gradually placed less emphasis on emotional as compared with rational factors. In the first instance, it was the phenomena of storms, clouds, thunder, and lightning that were thought to be the basis of deity belief; later, the sun came to be regarded as the embodiment of the chief god; the present tendency is to emphasize particularly the moon, whose changing phases may easily give rise to various mythological ideas. Does not the proverbial ' man in the moon' survive even to-day as a well-known fragment of mythological conceptions of this sort? Similarly, the crescent moon suggests a sword, a club, a boat, and many other things which, though not conceived as gods, may at any rate be regarded as their weapons or implements. The gods, we are told, then gradually became distinguished from celestial objects and became independent personal beings. The heroes of the hero saga are said to be degenerated gods, as it were. When the myth attributes a divine parentage to the hero, or allows him to enter the realm of the gods upon his death, this is interpreted as indicative of a vague memory that the hero was once himself a god. The lowest place in the scale of heroes is given to the märchen-hero, though he also is supposed in the last analysis to have originated as a celestial 
deity. The märchen itself is thus regarded as the last stage in the decline of the myth, whose development is held to have been initiated in the distant past by the celestial myth. Accordingly, the most prevalent present-day tendency of nature mythology is to assume an orderly development of a twofold sort. On the one hand, the moon is regarded as having been the earliest object of cult, followed by the sun and the stars. Later, it is supposed, a distinction was made between gods and celestial objects, though the former were still given many celestial attributes. On the other hand, it is held that the gods were more and more anthropomorphized; their celestial origin becoming gradually obscured, they were reduced to heroes of various ranks, ranging from the heroic figures of the saga to the heroes of children's märchen. These theories of an original polytheism are rendered onesided by the very fact that they are not based upon any investigations whatsoever concerning the gods and myths actually prevalent in folk-belief. They merely give an interpretation of hypothetical conceptions which are supposed to be original, and it is from these that the gods of actual belief are derived. Those who proceed thus believe that the task of the psychologist of religion and of the mythologist is completed with the demonstration that back of every deity of myth there lurks a celestial phenomenon. It has been maintained, for example, that every feature of the Biblical legend of Paradise had its origin in ideas connected with the moon. Paradise itself is the moon. The flaming sword of the angel who guards Paradise is the crescent moon. Adam is either the halfmoon or the familiar man in the moon. Finally, Adam's rib, out of which Eve was created, is again the crescent moon.

We need not raise the question whether such a mode of treatment ever correctly, interprets any actual mythological conception, or whether it represents nothing other than the creation of the mythologist's imagination. This much is clear, that it leaves out of consideration precisely those mythological ideas and religious views that really live in folk-belief. Doubtless we may 
assume that celestial phenomena occasionally factored as assimilative elements in the formation of mythological conceptions. But such conceptions cannot possibly have been due exclusively to celestial factors, for the very, reason that, even where these are indubitably present, they are inextricably interwoven with terrestrial elements derived from man's immediate environment. Consider, for example, the figure of Helios in Greek mythology. His very name so inevitably suggests the sun that this connection remained unsevered throughout later development. Nevertheless, the Greeks no more identified the god Helios with the sun than they did Zeus himself with thunder and lightning. On the contrary, these celestial phenomena were all only attributes of deities. The god stands in the background, and, in the idea which man forms of him, the image of human heroes plays no less a part than do the impressions made by the shining heavenly bodies. These various interpretations of nature mythology, therefore, overlook an important psychological factor which is operative even in elemental experiences, but which attains increasing significance in proportion as the psychical processes become more complicated, and especially, therefore, in the formation of mythological conceptions. I refer to the assimilative fusion of psychical elements of differing. origins. No external object is perceived precisely, as it is immediately given in reality. In the experience of it, there are fused numerous elements whose source is within ourselves; these partly reinforce and partly suppress the given elements, thus producing what we call the "perception' or the 'apprehension' of the object. The process of assimilation is greatly, influenced by the emotions that may be present. To the frightened person, thunder and lightning suggest a god who hurls the lightning. Such a person believes that he really sees this god. Either the surrounding portions of the sky. assume, in his imagination, the form of an immense anthropomorphic being, or the thunder and lightning lead his gaze to the canopy of clouds, hidden back of which he thinks that he discovers, at least in vague outline, the thundering Zeus. To gain some apprecia- 
tion of the tremendous potency of assimilative processes, one need but recall certain situations of ordinary life, such as are experienced even apart from the influence of fear or ecstasy. Consider, for example, the vivid impression that may be aroused by theatrical scenery, which in reality consists of little more than suggestive outlines. A particularly striking: illustration is offered also by the familiar puzzle pictures. In a picture of the foliage of a tree there are sketched the outlines of a human face or of the head of a cat. An uninitiated observer sees at first only the foliage. Not until his attention has been directed to it does he suddenly discover the head. Once, however, he has seen the latter, he cannot suppress it, try as he may. Here again it is sometimes but a few indistinct outlines that evoke the picture. The truth is that to a very great extent the observer reads the head into the drawing through the activity of his imagination. Now, it is but natural that such an assimilation should be immeasurably enhanced under the influence of the emotions which excite the mythological imagination. As is well known, Apollo, as well as Helios, was represented by the image of the sun. This image, however, was even less adequate to embody the idea of the Greek in the former case than it was in the latter. The Greek was able, however, to imagine the radiant sun as an attribute of the deity or as a manifestation of his activity. He could see in the sun the shield or chariot of the god; in the sun's rays, his missiles. Here again, however, he had in mind the indefinite outlines of a powerful anthropomorphic god, who could become independent of the natural phenomenon according as his name was free from connection with it.

Thus, even those nature gods who might appear to be purely celestial deities, as, for example, Helios, or the lightning-hurling Zeus, are the products of a psychological assimilation of perceptual elements, the most important of which have their ultimate source in terrestrial life. Hence it is that, wherever the nature myth has reached its complete development, the gods appear in human form. It is only 
in an age still influenced by totemic ideas that zoömorphism occurs alongside of anthropomorphism, or in combination with it. Of such figures, the one which maintained itself longestas is shown by the history of ancient Egypt-was that of a human body with the head of an animal. After this connection of an incipient deity cult with the ideas of the preceding age had disappeared, the only remaining trace of totemism was the fact that an animal was represented as accompanying the deity. Eventually the animal became a mere symbol used by art in its pictorial representations of the god. Doubtless the lamb, as a symbol of Christ, may be regarded as a late survival of a stage of deity belief which was still semitotemic, and under the influence of the sacred animals of older cultural religions. The expression 'sacred animals,' moreover, points to the fact that the worship and veneration paid to the god influenced also the attitude taken toward the animal. But however far this development of the god-idea may have advanced, the essential elements of the conception nevertheless remained of terrestrial origin. In the mythological assimilation-complexes that gave rise to gods, celestial phenomena furnished but a part of the elements. At best, they were the exciting stimuli; in many cases, it is doubtful whether they exercised any influence whatsoever upon the origin of mythological conceptions. Whether, for example, the crescent moon has actually any connection with the flaming sword of the angel of Paradise, or whether it suggested the club of Hercules, this and much else is possible, but is incapable of demonstration. Even where this influence upon mythological conceptions is incontestable, celestial phenomena are subordinate to terrestrial factors, and in most cases they have left no trace in consciousness. Proof of the dominant importance of the terrestrial environment is not far to seek. Even the celestial gods are conceived as men or as anthropomorphic beings, and it is usually the earth that is regarded as the scene of their activity.

The theories maintaining the originality of the godidea have more and more been displaced by the contrary 
view, namely, that the gods developed out of lower forms of mythological thought. Here there are two distinct interpretations. The first and the older is the ancestor theory. This represents a particular form of animism, for the soul of the ancestor is thought to become a god. The worship of the god, therefore, is held to have been originally a reverence paid to the ancestor. The main evidence for this view is found in the ancestor worship which is actually being practised, among many peoples, even at the present time. Prior to the Jahve religion, such a cult is supposed to have prevailed even among the Israelites. Do not the patriarchs Abraham, Isaac, and Jacob appear as the ancestors of the later tribes of Israel? More significant still are the ancestor cults that have prevailed in China and Japan since very ancient times. It should be remembered, however, that these cults, wherever they occur, represent but more or less prominent elements of more extensive mythological and religious conceptions. Hence the ancestor theory, also, is an arbitrary construction based on a presupposition which is in itself very improbable, namely, that all mythology and religion must eventually be traceable to a single source. The contention, for example, that a Zeus or a Jahve was a human ancestor elevated into a deity is a completely arbitrary supposition, lacking the confirmation of empirical facts.

Finally, there is another theory which, like the ancestor hypothesis, seeks to derive gods, or at least the beings generally regarded as gods, from more primitive mythological ideas. This theory, which was developed by Hermann Usener, the most prominent student of the science of religion among recent classical philologists, might perhaps be referred to, in distinction from the soul and ancestor hypothesis, as the demon theory of the origin of gods. Usener agrees with the rival hypothesis in assuming that the exalted celestial deities were not the first of the higher beings who were feared or worshipped in a cult, but that there were other more temporary gods. Though these many temporary gods are 
described as demoniacal beings, they are nevertheless regarded as gods of a primitive sort. Usener distinguishes three stages in the development of gods. First, there was the 'god of the moment.' Some phenomenon-such, for example, as a flash of lightning or a clap of thunderwas felt to be divine. But, inasmuch as the impression was vanishing, the mythological idea in question was that of a ' god of the moment.' Then followed a second stage, in which a demoniacal power was associated with a particular place. Following upon these local gods came other gods, representing the guardian powers of a tribe, a vocation, or some other social group. At the third stage of development, the 'particular god' acquired a personal nature, and thus finally became a god proper. The gods of this final stage are called by Usener 'personal gods.'

Although this theory is doubtless in greater consonance with certain general characteristics of myth development than is the ancestor theory, we would urge, as one chief objection, the fact that its god-concept unites mythologicalreligious elements of a very different nature. In particular, the so-called 'god of a moment' is neither a god, in the proper sense of the word, nor even a demon, but either a particular impression arousing fear, or, on a higher plane, a single manifestation of the activity of a demon or god. The Greeks referred the flash of lightning to Zeus, the lightning-hurler. On a more primitive level, the North American Indian sees in the lightning and thunder the acts of a demon hidden in the clouds. In neither case are the momentary phenomena identified with gods or demons themselves. There is not a shadow of proof in the entire history of myth that such acts or attributes as these, which were attributed to gods and demons, ever existed as independent realities of even but a moment's duration. The so-called 'particular gods,' on the other hand, are in every respect demons and not gods. They are not personal in nature; this also implies that they are not conceived as having a particular form, for somehow the latter always leads to personalization. As a matter of 
fact, these 'particular gods' are only objectified emotions of fear and terror. Spirits, in the sense of magical agents of disease conceived as invisible beings, or occasionally imaged in the form of fantastic though ever-changing animal shapes, are not gods, but demons. The same holds true of the multitude of nature demons that infest field and forest and the vicinity of streams and gorges. Wherever myth has given these spirits definite forms, they reveal no evidence of traits such as would constitute them individual personalities. This, of course, does not imply that there are no cases at all in which the indeterminate traits ascribed to them are so combined as to result in individual beings. When this occurs, however, we have already transcended the stage of so-called 'particular gods.' Such beings as the Greek Pan or the Germanic Hel must already be classed with gods proper, even though they exhibit traits indicative of a demoniacal past ; for the narrowness of character which they manifest results from the fact that they originated directly in a particular emotion. Surely, therefore, the decisive emphasis in the case of deity ideas in general must be placed on the attribute of personality. Gods are personal beings, whose characters reflect the peculiarity of the people who have created them. We see in the god Jahve of the Israelites the clear-cut lines of the stern god who threatens the disobedient, but who also rewards the faithful. More impressive still is the uniqueness of personality in those cases in which a multiplicity of gods causes the development of diverse and partly opposed characteristics in the various gods. How individual are the gods of the Greeks with respect to one another.! Under the influence of poetry every god has here become a clearly defined personality, whose individuality was fixed by, formative art. Thus, the error of the demon theory or, as it might also be called, the three-stage theory, lies in the fact that it effaces the essential distinctions between god and demon, retaining as the chief characteristic of the multitude of resulting deity-conceptions only the most external quality, that of permanence. For the 'god of a moment' is characterized merely by his extreme transitoriness; the 
'particular god' is the 'god of a moment' become somewhat more enduring but not yet possessed of sufficient stability to develop personal traits; the true or personal god, finally, owes his distinctive attribute solely to the permanence of his characteristics. Because of this confusion of the concepts 'god' and 'demon,' there is lacking precisely that which is of most importance for a psychological investigation-namely, an answer to the question as to the intrinsic marks that differentiate a god, in the proper and only true sense of the word, from demons, ancestors, and soulsin short, from all other creations of mythological thought.

Herewith we come to a question which will bring us closer to an answer respecting the origin of gods. By what characteristic marks is a mythological conception to be distinguished as that of an actual god? The question might also be stated in a more concrete form. What characteristics differentiate a god from a demon, who is not yet a god

- because he lacks personality, and from a hero, who is regarded by the age in which gods originate as somewhat approximating a god but as nevertheless still a man? Or, briefly expressed, how does the god differ from the demon and from the ideal man? The criteria thus demanded are to be found in the traits that are universally ascribed to gods wherever any complete deity mythology and a corresponding religion have been developed. The god is always distinguished by three characteristics. The first of these is that his place of abode is other than that of man. He may. occasionally visit man on the earth, but this occurs only rarely. So far as he himself is concerned, the god lives in another world. In this sense, the idea of a 'beyond' is closely bound up with that of gods. As a rule, the 'beyond' is the heavenly world. But gods may dwell also in the regions of the air and clouds between the heaven and the earth, on high mountains, on distant islands, or, finally, under special circumstances, in the depths of the earth. Secondly, the gods lead a perfect life, free, on the whole, from the evils and infirmities of earthly existence. A perfect life, however, is always regarded as primarily a 
life without death and without sickness. There then develops, though doubtless gradually, the idea of something even more perfect than is involved in this merely negative conception of immortal and painless existence. But at this point ideas begin to differ, so that, in reality, the most universal characteristics of the gods are that they know neither death nor sickness. There are occasional exceptions, however, just as there are with respect to the supra-mundane place of abode. The Greek as well as the Germanic deity sagas represent the gods as possessing a particular food and a particular drink, an idea connected with that of the anthropomorphic nature of these gods. The Germanic gods, especially, are described as capable of maintaining their perfect life only by far exceeding the human measure of food and drink. This, however, is but a subordinate feature. More important is the fact that if, by any unfortunate circumstance, food and drink are lacking, the gods waste away and meet the universal lot of human existence-death. But, even apart from this connection, the Germanic sagas, or at any rate the poetry inspired by them, tell of a decline of gods and of the rise of a new divine hierarchy. It is not to be assumed, of course, that this represents an original element in Germanic mythology. All records of Germanic deity sagas, as we know, date from Christian times. Even though the ancient skalds, as well as those historians who regarded the saga as a bit of actual history, may have made every effort to preserve for posterity. the memory of this departed world, they could, nevertheless, hardly have avoided mingling certain Christian ideas with tradition. In view of the actual decline of the former gods, the thought of a Götterdämmerung, in particular, must almost inevitably have forced itself upon them. At any rate, inasmuch as this particular conception represents the gods as subject to death, it contains an element that is bound up with the anthropomorphic nature of the divine beings, though this, of course, is irreconcilable with the immortality originally conceded to them. We are thus brought to the most important characteristic of gods, which is connected 
with this very fact of their similarity to man. The god is a personality; he has a specific personal character, which gives direction to his will and leads him to send blessings or misfortunes to mortals. These purely human characteristics, however, he possesses in an exalted and complete measure. His will-acts, as well as the emotion from which they spring and the insight by which they are guided, are superhuman in power. But this power is not equivalent to omnipotence. This it cannot be by very reason of the multiplicity of gods, each of whom has a particular sphere of activity. Frequently, moreover, omnipotence is rendered impossible by the idea-likewise carried over from the terrestrial to the supermundane world-of a destiny an impersonal power behind the wills of gods no less than those of men. This is a conception which deity beliefs inherit from the earlier demon beliefs. True, polytheistic myth itself takes a step in the direction of transcending this limitation when it here also transfers the conditions of the human order to the divine world, and creates for the latter a monarch, a supreme deity ruling over gods and men. But this very projection of human relations into the divine realm prevents the chief deity from being an unlimited ruler. On the one hand, he shares authority with a deliberative assembly consisting of the remaining gods; on the other hand, even behind him there lurk those demoniacal powers which, to a certain extent, continued to assert themselves even after they had been superseded by the gods. For here also it holds true that whatever lives in folk-belief must retain a foundation in myth. The advent of gods nowhere led to the complete banishment of demons. What occurred was that, due to the power of the gods, certain of the demons likewise developed into mighty forces of destiny, though continuing to remain impersonal.

Thus, the god possesses three characteristics : a special dwelling-place, immortality, and a superhuman, though at the same time a human, personality. Leaving out of regard the tribute exacted even of the gods by the last-mentioned 
of these characteristics, human nature, we have before us the marks which distinguish the god both from the demon and from the hero. The demon, however powerful he may be, lacks the attribute of personality; the hero, as thoroughly human, shares the universal lot of man as regards dwelling-place, length of life, and liability to sickness and death. This places the god midway between the demon and the hero, though, of course, by combining the attributes of both, he is really exalted above them. The demon, in the sense in which the Greeks employed this term, is a fundamental element in the development of all mythologies. There can be no doubt, moreover, that demons appeared far earlier than gods, if we exclude from among the latter those indefinite and transitory personifications of natural phenomena that have wrongly been classed with themsuch personifications as those of rocks, hills, clouds, stars, etc., which were widely current even among peoples of nature. According to a belief which has not entirely disappeared even among cultural peoples, the soul leaves the corpse in the form of a demon; the wandering ghost is a demon; demons dwell in the depths, in the neighbourhood of streams, in solitary ravines, in forests and fields, upon and beneath the earth. They are usually threatening, though sometimes beneficent, powers. In every instance, however, they are absolutely impersonal embodiments of the emotions of fear and hope, and it is these emotions, under the assimilative influences of impressions of external nature, that have given rise to them. Thus, demons are usually, mundane beings, or, at any rate, have their abode near the surface of the earth ; with few exceptions, the most distant realm which they occupy is that of the clouds, particularly the dark rain and thunder clouds. True, the heavenly bodies may manifest demoniacal powers, just as may also the gods. As a rule, however, celestial phenomena are far from belonging to the class of demons proper ; they are too constant and too regular in their changes and movements to be thus included. The activity of demons relates exclusively to the welfare of man. Hence it is but natural that idemons should be primarily. 
man's co-inhabitants on earth. Usually invisible, they assume sensuously perceptible forms only in the darkness of night, or, more especially, under the influence of heightened emotions. Sometimes they are audible even when invisible. Only in those narratives which tell of demoniacal beings that are not immediately present do demons acquire fairly definite forms. Thus, even soul beliefs-which the fear of the uncanny activity of the departed soul transforms directly, into a sort of demon belief-represent the soul in the form of a bird, a snake, or of other specific 'soul animals.' The demons of sickness lurking within the diseased body are usually portrayed as fantastic animals, whose monstrous forms reflect the terrible distress and the torturing pains of sickness. These animals hinder respiration and bore into and lacerate the intestines. Thus, they objectify both the pain of the sickness and the fear aroused in the community by the behaviour of the sick person. No less, however, can the impression of the desert, the dark forest, the lonely ravine, or the terror of an approaching storm cause demons, which are in first instance invisible, to assume definite shapes. Where there is a more highly developed sense of nature, such as begins to manifest itself in the heroic age, this objectification of impressions occurs not only under the influence of strong excitement but also in connection with the peaceful landscape. Here it gives rise to more friendly, beings, in the case of whom those characteristics, at least, which made the original demon an object of terror, are moderated so as to find expression in magic of a playful sort. This is the origin of satyrs, sylphs and fauns, of gnomes, giants and dwarfs, elves, fairies, etc., all of whom are debarred from personality by their very multiplicity, while their generic character accurately reflects the mood which led to their creation. The individualization of certain of these beings is, in general, due to poetry. But even poetry does not entirely succeed in freeing the demon from the generic character which once for all represents its nature. Thus, it is the contrast between genericalness and individual personality that differentiates the demon from the god. 
Every gnome resembles every other, and all nymphs are alike; hence these beings are generally referred to in the plural. Their multiplicity is such that they are imaged in only indefinite forms, except in cases where particularly strong emotions excite a more lively imagination. Indeed, they may be present to consciousness solely as a peculiar feeling associated with particular places or occasions, such as is the case with the Lares, Manes, and Penates of the Romans, and with the similar guardian spirits of the house and the field common among many peoples. Some of these guardian spirits are not very unlike the ancestors of cult. But this only indicates that the ancestor worshipped in cult also approximates to the demon, acquiring a more personal character only in occasional instances in which memory, has preserved with considerable faithfulness the traits of a particularly illustrious ancestor. Here, then, we have the condition underlying the origin of gods. Gods are universally the result of a union of demoniacal and heroic elements. The god is at once demon and hero; since, however, the demoniacal element in him magnifies his heroic attributes into the superhuman, and since the personal character which he borrows from the hero supersedes the indefinite and impersonal nature of the demon, he is exalted above them both: the god himself is neither hero nor demon, because he combines in himself the attributes of both, in an ideally magnified form.

The resemblance of demons to gods is due primarily to the magic power which they exert. The demons of sickness torture and destroy men; the cloud demons bring rain and blessing to the fields, or plot ruin when rain does not relieve the drought of the burning sun. By means of magic incantations and ceremonies, these demons can be won over, or, when angry, reconciled. Their own activity, therefore, is magical, and, as regards the effects that it produces, superhuman. In their fleeting and impersonal character, however, they are subhuman. Since the dominant emotions that call them into being are fear and terror, they are generally regarded as enemies not only of man but even of 
the gods. The struggle between gods and nature-demons is a recurrent theme in the cosmogonies of all cultural peoples. This hostility between demons and gods is connected with the contrast in the feelings evoked by darkness and radiant brightness. Hence the mighty nature-demons are, as a rule, consigned to gloomy abysses, from which they rise to the sky only occasionally, as, for example, in the case of thunder-clouds. The abode of the gods, however, is in the bright celestial realms, and they, themselves are radiant beings upon whose activity the harmonious order of nature and the happiness of mankind are dependent. In the strife which the demons carry on with gods, they occasionally develop into counter-gods, as occurred in the case of the Persian Ahriman and the Jewish-Christian Satan. Yet it is significant of the almost insuperable lack of personality characteristic of the demon, that even these counter-gods of darkness and evil are wanting in one trait which is indispensable for a completely developed personality -namely, changes in motives and the capacity to determine at will the nature of these changes. Herein, again, is reflected the fact that the demon has but a single sourcenamely, fear.

Very different from the relation of the god to the demon is his relation to the hero. The hero, to a greater extent even than the god, is the complete opposite of the demon. For the hero is an idealized man. He is subject to all human destinies, to sickness and death, to afflictions of the soul, and to violent passions. Yet in all these instances the experiences are of a more exalted nature than in the case of ordinary human life. The life as well as the death of the hero are of wide import; the effects of his deeds extend to distant lands and ages. But it is just because the hero is the ideal man himself that he possesses all the more markedly the attribute which the demon lacks-namely, personality. This, of course, does not prevent his character from exhibiting generic differences and antitheses. But herein also the hero is only, the idealized counterpart of man, for, despite all its uniqueness and individuality, man's 
character usually conforms to certain types. Thus, legend introduces the strong, all-conquering hero, and, in contrast with him, the hero who is resourceful and overcomes his enemies through subtle cunning. It tells of the aged man, superior in wisdom and experience, and also of him who, in the unbroken strength of youth and with stormy passion, overthrows all opponents. It further portrays the hero who plots evil, but who is nevertheless characterized by a sharply defined personality.

When we survey these various heroic figures in both their generic and their individual aspects and compare them with the god-personalities, we are struck by the fact that the god was not created directly, in the image of a man, but rather in that of the hero, man idealized. It is the hero who gives to the gods those very characteristics which the demon lacks from the outset. Of these, the most important are personality, selfconsciousness, and a will controlled by diverse and frequently conflicting motives. This multiplicity of motives has a close connection with the multiplicity of gods. Polytheism is not an accidental feature which may or may not accompany the belief in gods ; it is a necessary transitional stage in the development of the god-idea. Folk-belief, which never frees itself entirely from mythology, always retains a plurality of divine beings. Hence true monotheism represent a philosophical development of the god-idea. Though this development was not without influence on the theological speculation which was dominated by traditional doctrines, it was never able to uproot the polytheistic tendency. involved in the god-idea from the very beginning. There are two sources from which this tendency springs. Of these, one is external and, therefore, though of great importance for the beginnings of religious development, is transitory. It consists in the influence exerted by the multiplicity of natural phenomena, through the nature myth, upon the number of gods. More important and of more permanent significance is the second or internal motive, namely, the fact that the psychical needs that come to expres- 
sion in the demand for gods are numerous. There cannot be a single god-ideal any more than a single type of hero. On the contrary, as heroes exhibit the diversity of human effort on an exalted plane, so, in turn, does the realm of gods represent, on a still higher level, the world of heroes. This advance beyond the hero-ideal becomes possible to the mythological imagination only because the very endeavour to exalt the hero above the human itself brought the hero-idea, at the very time of its origin, into connection with the demon-idea. For the demon is a superhuman being, magic-working and unpredictable, affecting in mysterious ways the course of nature and of human destiny. But it lacks the familiar human traits which make the hero an object not only of fear but also of admiration and love. Thus, the fusion of hero and demon results in the final and the greatest of mythological creations, the conception which represents the birth of religion in the proper and ultimately only true sense of the word. I refer to the rise of gods.

The god-idea, accordingly, is the product of two component factors. One of these, the demoniacal, has had a long history, extending back to the beginnings of mythological thought ; the other, the heroic, begins to assert itself the very moment that the figure of the hero appears. This implies that god-ideas are neither of sudden origin nor unchangeable, but that they undergo a gradual development. The direction of development is determined by the relation which its two component factors sustain to each other. The earliest god-ideas are predominantly demoniacal in nature-personal characteristics are few, while magical features are all the more pronounced. Then the heroic element comes to the fore, until it finally acquires such dominance that even the magical power of the god appears to be a result of his heroic might, rather than a survival of the demoniacal nature which was his from the very beginning. In connection with this change, it is significant to note that, as the god loses his original demoniacal character, he comes to be attended by subservient beings 
who remain, in every respect, demons. On the one hand, these beings execute the divine commands; on the other hand, however-as an echo, one might say, of the age of demons which precedes that of gods-they are superior even to the gods in that they possess magical powers. These beings must be regarded as survivals of the age of demons. Between them and the gods proper there are intermediate beings, just as there are between heroes and gods, those of the latter sort being exemplified particularly by such heroes as have been exalted into deities. Inasmuch as all the intermediate forms that arise in the course of this transition continue in existence even up to the culmination of the development, the gods constantly become more numerous. Side by side with the gods, demons maintain their sway. At times, they contend with the gods; in other instances, they are subservient to them; again, as in the earliest periods of mythological thought, they are without any knowledge whatsoever of the existence of gods. The hero also is invariably associated with the god. With the decline of the heroic age, therefore, the realm of gods also disappears. Though the religious developments that ensue have their origin in deity beliefs, they nevertheless discard the original nucleus of these beliefs-namely, the gods themselves-or, at any rate, they retain gods only in a greatly altered form.

That gods belong essentially to the heroic age appears also in the fact that the divine realm mirrors in detail the relations of political society developed subsequently to the beginning of the heroic age. The world of gods likewise forms a divine State. It is at most at an early period that the tribal gods of various peoples betray the influence of the ancient tribal organization that preceded the State. In the supremacy of a single god, however, the idea of rulership, which is basal to the State, is transferred also to the divine realm. This is true whether the ruling deity exercises command over a subservient host of demons and subordinate gods, or whether he has at his side a number of independent gods, who represent, in part, an advisory 
council, such as is found associated with the earthly ruler, and, in part, since the different gods possess diverse powers, a sort of celestial officialdom. Finally, the multiplicity of independent States is mirrored in the multiplicity of the independent realms ruled over by the gods. The differentiation, in this latter case, corresponds with the main directions of human interest. The development is influenced, moreover, by those natural phenomena that have long factored in the capacity of assimilative elements. Over against the bright celestial gods are the subterranean gods who dwell in the gloomy depths. For the inhabitants of the sea-coast and of islands, furthermore, there is a ruler of the sea. The importance of the god of the sea, however, is subordinate to that of the rulers of the celestial and the nether worlds, so that those over whom he holds sway never develop into clearly defined personalities, but always retain more of a demoniacal character. All the more important, therefore, are the contrasts between the celestial and the nether worlds, as the two realms which include the real destiny of man. At death, man must enter the nether world; to rise from the gloom of this realm of the dead to the heaven and immortality of the celestial gods becomes his longing. Thus, deity beliefs enter into reciprocal relations with soul conceptions. The further stages of this development carry us far beyond the heroic age, and reflect the influence of a diversity of motives. The discussion of this point will occupy our attention in later pages.

\section{2. The Hero Saga.}

If the gods be described as personalities, each one of whom possesses a more or less definite individuality, it is at once evident that the conception of an animated natural phenomenon-the idea, for example, that the setting sun is a being which a dark cloud-demon is devouring-cannot in and of itself as yet be called a god-idea. Just as the character of a man may be known only from the manner in which he reacts towards the objects of his experi- 
ence, so also is the nature of a god revealed only in his life and activity, and in the motives that determine his conduct. The character of the god is expressed, not in any single mythological picture, but in the myth or mythological tale, in which the god figures as a personal agent. It is significant to note, however, that the form of myth in which god-ideas come to development is not the deity saga, in the proper sense of the term, but the hero saga, which becomes a combined hero and deity saga as soon as both gods and heroes are represented as participating in the action. The deity saga proper, which deals exclusively with the deeds of gods and demons, is, as we shall see below, only of secondary and of later origin. It is not to such deity sagas, therefore, that we must turn if we would learn the original nature of gods. This circumstance in itself offers external evidence of the fact that gods did not precede heroes, but, conversely, that heroes preceded gods. Or, at least, to be more accurate, the idea of the divine personality was developed in constant reciprocity with that of the hero personality, in such wise, however, that with reference to details the hero paved the way for the god, and not conversely.

But how did the idea of hero arise? Was it a free and completely new creation of this age, based merely on actual observations of individuals who were paragons of human ability? Or did it have precursors in the totemic era? As a matter of fact, this second question must be answered unqualifiedly in the affirmative. The hero was not unknown in the preceding age. At that time, however, he was not a hero in the specific sense which the word first acquired in the heroic age; on the contrary, he was a märchen-hero, if we may use the word 'hero' in connection with the concepts of this earlier period. On the threshold of the heroic age, the märchen-hero changes into the hero proper. The former represents the central theme of the earlier form of myth narrative, the märchen-myth, as does the hero that of the more developed form, the saga. The marks that distinguish the märchen-hero, as he still survives in children's tales, from the hero of saga, 
are important ones and are fraught with significance for the development of myth as a whole. The märchen-hero is usually a child. In the form in which he gradually. approximates to the hero proper, he is more especially, as a rule, a boy who goes forth into the world and meets with adventures. In these adventures, he is aided by various powers of magic, which he either himself possesses or which are imparted to him by friendly magical beings. Opposed to him are hostile, demoniacal beings, who seek his destruction. It is in their overthrow that the action usually consists. Thus, fortune comes to this hero, in great part, from without, and magic plays the decisive rôle in his destiny; his own cunning and skill may be co-operating factors, but they rarely determine the outcome. Not so the hero of the saga. This hero is not a boy, but a man. The favourite theme of the saga is particularly the young man in the bloom of life. In his acts, moreover, this hero is dependent, for the most part, upon himself. True, he, as well as the märchen-hero, is familiar with magic and miracle, but it is primarily by his own power that he overcomes the hostile forces that oppose him. A suggestive illustration of this is Hercules, that figure of Greek saga who is preeminently the typical hero among the most diverse peoples and in widely different ages. Hercules is an entirely selfdependent hero. True, he performs marvellous deeds, but these are never more than extreme instances of what an ordinary man might do were his strength multiplied a hundred or a thousand fold. Hercules is not a magician, but a being of transcendent power and strength. As such, he is able even to carry the weight of the sky on his shoulders; as such, he can overcome monsters, such as the Nemean lion and the Lernæan hydra, or bring Cerberus, the most terrible of these monsters, from the nether world. These are deeds which surpass every measure of human power, but which nevertheless still lie in the general plane of human actions. Thus, just as the magicworking boy was superseded by the man of might, so also does the true magical hero disappear from mythology. Thus, 
the saga differs from the märchen-myth in the character of its hero. The Hercules saga itself, however, is an illustration of the fact that the former may have no connection whatsoever with historical events, any more than has the latter. Moreover, the earliest sagas, particularly, not infrequently still remind one of the märchen in that they are obviously a composite of several narratives. Of this fact also, the saga of Hercules offers a conspicuous example. The deeds of the hero appear to have but an accidental connection with one another. True, later sagas represent these deeds as adventures which the hero undertook at the command of King Eurystheus of Mycene. But even here we obviously have only a loose sort of framework which was at some later period imposed upon the original tales in order to bind the cycle together as a whole. It is not improbable that these various sagas of a hero who vanquished monsters, rendered lands habitable, and performed other deeds, originated independently of one another. Not only may their places of origin have been idifferent, but their narratives may have had their settings in different localities. Possibly, therefore, it was not until later that the sagas were combined to portray the character of a single individual, who thus became exalted into the national hero. But, though the hero saga resembles the märchen in the fact that it grows by the agglutination of diverse legendary materials, it differs from it in the possession of a characteristic which is typical of this stage of development. That which binds together the separate elements of the hero saga is a unitary thought, generally associated with great cultural changes or with historical events.

There is a further differentia of the saga as compared with the märchen. Wherever magic enters into the saga to affect the course of events, the chief vehicle of magical powers is not the hero himself-at most, he has been equipped by others with magical powers and implements. Such demoniacal powers as the saga may introduce into its narrative are usually vested in accessory persons. This fact is closely connected with the self-dependent 
character of the hero-personality, who may, it is true, employ magic in so far as he has received such power from external sources, but who himself possesses none but human attributes. The saga of the Argonauts, for example, is so replete with magic as not to be surpassed in this respect even by the magical märchen. Moreover, the various elements incorporated in the saga are all pure märchen motives-the golden fleece, the talking ship, the closing cliffs, as well as the sorceress Medea and the whole wonderland of Colchis. Those who man the Argo, however, are not magicians, but heroes in the strictly human sense of the word. The same fact stands out even more strikingly in the case of the saga of Odysseus, at any rate in the form in which the Homeric epic presents it. We may here discern an entire cycle of tales, whose separate elements are also to be found elsewhere, some of them in wide distribution. But in the midst of this märchenworld stands the absolutely human hero, contrasting with whom the fabulous events of the narrative run their course as a fantastic show. The hero overcomes all obstacles that block the course of his journey by his own neverfailing shrewdness and resourcefulness. Herein again the märchen-myth gives evidence of being preparatory to the hero saga. At the time when the hero ideal arose, the old märchen ideas were as yet everywhere current. Together with the belief in demons and magic, they, also, found their way into the heroic age. For a long time they continued to be favourite secondary themes, introduced in portraying the destiny of heroes. Nevertheless märchen ideas became subordinate to the delineation of heroic figures, whose surpassing strength was described, very largely, in terms of victory over demoniacal powers. Thus, in the course of the development, the heroic elements gradually increased; the märchen ideas, on the other hand, disappeared, except when some poet intentionally selected them for the enrichment of his tale, as was obviously done by the author of the Odyssey.

The disappearance of the elements derived from the 
märchen-myth, however, must in part be attributed to another factor. This factor, which is closely bound up with the entire culture of the heroic age, consists in the increasing influence of historical recollections. Particularly illuminative, as regards this point, are the Greek and Germanic sagas. The sagas of Hercules and the Argonauts, which, from this point of view, belong to a relatively early stage, are purely mythical creations. So far as one can see, no actual events are referred to by them. The Trojan saga, on the other hand, clearly exhibits the traces of historical recollections; its historical setting, moreover, seems to cause the events that transpire within it to approximate more nearly to the character of real life. Even here, indeed, ancient magical motives still cast their fantastic shadows over the narrative. Occasionally, however, the miracle appears in a rationalized form. The magician of the märchen gives place to the seer who predicts the future. What the miracle effected is now accomplished by the overpowering might and the baffling cunning of the strong and wily hero. In this change, the external accessories may sometimes remain the same, so that it is only the inner motives that become different. Thus, it is not impossible that the wooden horse which was said to have been invented by Odysseus and to have brought into Troy the secreted warriors of the besieging hosts, was at one time, in märchen or in saga, an actual magical horse, or a help-bringing deity who had assumed this form. In this case, the poet may possibly be presenting a rationalistic reinterpretation of an older magical motive, with the aim of exalting the craftiness of his hero. In the account of Achilles' youth, on the other hand, and in the story of Helen which the poet takes as his startingpoint, the märchen-idea of the saga obviously affects the action itself, though it is significant tio note that these purely mythical features do not belong to the plot so much as to its antecedent history. In so far as the heroes directly affect the course of action, they are portrayed as purely human. The same is true of the German Niebelungen saga. Just as Achilles, a mythical hero not at 
all unlike the märchen-hero, was taken over into the historical saga, so also was Siegfried. But here again the märchen motives, such as the fight with the dragon, Siegfried's invulnerability through bathing in its blood, the helmet of invisibility, and others, belong to the past history of the hero, and are mentioned only incidentally in the narrative itself. By referring these specifically märchen miracles to the past, the saga seems to say, as it were, that its heroes were at one time märchen-heroes.

In this course of development from the purely mythical to the historical, the saga may approach no more closely to historical reality than does the purely mythical tale. But while this may be the case, it is nevertheless true that the saga more and more approximates to that which is historically possible. Moreover, it is not those sagas which centre about an historical hero that are particularly apt to be free from elements of the original märchen. Very often the reverse is true. An original märchen-hero may become the central figure of an historical saga, and, conversely, the account of an historical personality may become so thoroughly interwoven with märchen-like tales of all sorts that history entirely disappears. A striking antithesis of this sort occurs in Germanic mythology. Compare the Dietrich saga with the later development of the Niebelungen saga in the form rendered familiar by the Niebelungenlied. Siegfried of the Niebelungen saga originates purely as a märchen-hero; Dietrich of Bern is an historical personage. But, while the Niebelungenlied incorporates a considerable number of historical elementsthough, of course, in an unhistorical combination-the Dietrich of the saga retains little more than the name of the actual king of the Goths. There are two different conditions that give rise to sagas. In the first place, historical events that live in folk-memory assimilate materials of ancient märchen and sagas, and thus lead to a connected hero saga. Secondly, an impressive historical personality stimulates the transference of older myths as well as the creation of others, though these, when woven into a whole, resemble a märchen-cycle rather than a hero saga proper. 
An important intermediate phenomenon of the sort just mentioned, is not infrequently to be found in a specific form of myth whose general nature is that of the hero saga, even though it is usually distinguished from the latter because of the character of its heroes. I refer to the religious legend. Some of these legends, such as the Buddha, the Mithra, and the Osiris legends, border upon the deity saga. Nevertheless, the religious legend, as exemplified also in the mythological versions of the life of Jesus, represents an offshoot of the hero saga, springing up at those times when the religious impulses are dominant. That it is a hero saga is evidenced particularly by the fact that it recounts the life and deeds of a personality who is throughout exalted above human stature, but who, nevertheless, attains to divinity only through his striving, his suffering, and his final victory. In so far, the religious hero very closely resembles the older class of heroes. Nevertheless, instead of the hero of the heroic period, pre-eminent for his external qualities, we have the religious hero, who is exalted by his inner worth into a redeeming god. But it is only because these divine redeemers fought and conquered as men-a thing that would be impossible to gods proper who are exalted from the beginning in supermundane glory-that they constitute heroes of saga, in spite of the fact that they fought with other weapons and in other ways than the heroes of the heroic age. And, therefore, none of these redeemer personalities, whether they have an historical background, as have Jesus and Buddha, or originate entirely in the realm of the mythological imagination, as in the case of Osiris and Mithra, belong to the realm of the saga once they are finally elevated into deities. Even Buddha's return in the endless sequence of ages is not to be regarded as an exception to this rule, for the hope of salvation here merely, keeps projecting into the future the traditional Buddha legend. The redeeming activity of the one who is exalted into a god is to be repeated in essentially the same manner as the saga reports it to have occurred in the past.

Contrasting with the redemption legend is the saint 
legend. The former portrays the fortunes and final victory of a god in the making; the latter tells of the awakening of a human being to a pure religious life, of his temptations and sufferings, and his final triumph. Thus, it has a resemblance to the redeemer legend, and yet it differs from it in that its hero remains human even when he ascends into heaven to receive the victor's crown; the lot that thus befalls him is identical with that of all the devout, except that he is more favoured. This leads to further differences. The hero of the redemption legend is conscious of his mission from the very beginning; in the case of the saint, conversion to a new faith not infrequently forms the starting-point of the legend. Common to the two forms, however, is the fact that suffering precedes the final triumph. The traits that we have mentioned constitute the essential difference between these forms of the legend and the hero saga proper. The latter, also, is not without the element of suffering; the Greek saga has developed the specific type of a suffering hero in the figure of Hercules, as has the German saga in that of Balder. In the case of religious legends, however, the strife-motives of the saga are transferred to the inner life ; similarly, the suffering of the saint, and especially, that of the redeemer, is not merely physical but also mental. . Indeed, the original form of the Buddha legend, which is freest from mythological accretions, is an illustration of the fact that this suffering may be caused exclusively by the evils of the world to be redeemed. The suffering due to a most intense sympathy is so intimate a part of the very nature of the redeeming god-man, that it is precisely this which constitutes the most essential difference between the religious legend and the ordinary hero saga, whose interest is centred upon the actions and motives of external life. And yet the external martyrdom of the redeemer intensifies this difference in a twofold way. In the first place, it directly enhances the impression of the inner suffering; secondly, it gives heightened expression both to the evil which evokes the sympathy of the redeemer, and to the nobility of this sympathy itself. In all of these 
characteristics, however, the redemption legend belongs to the following era rather than to hero saga and the heroic age.

The saint legend exhibits a number of essential differences. It is frequently only through a miracle of conversion, due to external powers, that the saint becomes holy; moreover, it is not, as a rule, through miracles of his own performance that he manifests himself as a saint in the course of his later life and sufferings. The miracles that transpire come as divine dispensations from without, whether they effect his conversion or surround him, particularly at the close of his life's journey, with the halo of sanctity. Thus, to whatever extent the saint may come, in later cult, to supersede the protective undergods and demons of early times, he nevertheless remains human. It is for this very reason, however, that magic and miracle gain a large place in his life. The latter is all the more possible by virtue of the fact that the mythological imagination is not bound by any fixed tradition, and need, therefore, set itself no limits whatsoever either in the number of saints or in the nature of their deeds. Moreover, the legend is almost totally lacking in those factual elements which the hero saga acquires, in its later development, as a result of the historical events that are woven into it. This is not the case with the legend. Here it is at most the name of an historical personality that is retained, while everything else clearly bears the marks of imagination and of myth creation. Hence the saint legend is not to be counted among the factors that underlie the development from the purely mythical tale to the saga, whose content, though not real, is at any rate possible. On the contrary, the tendency of the saint legend is retrogressive, namely, toward a return to the märchen stage of myth. This is all the more true, not merely because elements that are generally characteristic of märchen are disseminated from legend to legend, but also because the saint legend appropriates widely current märchen conceptions. Märchen of very diverse origins found their way into the Christian, as well as the Buddhistic, legends ; moreover, occasional Buddhistic legends, with the clear marks of an Oriental 
origin upon them, were changed into Christian legends. Thus, the saint legend combines two characteristics. As compared with the hero saga, its motives are internalized; moreover, it represents a decided relapse into the pure märchen form of myth. Though apparently contradictory, these characteristics are really closely related, inasmuch as the internalization of motives itself removes any barriers imposed by historical recollection upon the free play, of the mythological imagination.

\section{Cosmogonic and Theogonic Myths.}

In view of the relationship of heroes and gods, not only with respect to origin but also as regards the fact that they both embody personal ideals, it would appear but natural, having treated of the hero saga, that we inquire at this time concerning the corresponding deity saga. A search for the latter, however, will at once reveal a surprising fact. There is no deity saga at all, in the sense in which we have a hero saga that has become a favourite field of epic and dramatic poetry. The reason for this lack is not difficult to see. There can be no real deity saga because, in so far as gods possess characteristics which differentiate them from men, and therefore also from heroes, they have no history. Immortal, unchangeable, unassailable by death or sickness, how could experiences such as befall the hero also be the lot of gods? If we examine the narratives that approach somewhat to the deity saga, we will find that they consist, not of a connected account of the experiences of the gods, but of isolated incidents that again centre about human life, and particularly about the beneficent or pernicious intervention of the gods in the destinies of heroes. We may recall the participation of the Greek gods in the Trojan war, or the interest of Jahve, in Israelitic saga, in the fortunes of Abraham, Jacob, etc. These are isolated cccurrences, and not history ; or, rather, we are given the history of heroes, in which the gods are at times moved to intervene. In so far, therefore, as there are approximations to deity saga, these, in their entirety, are woven into hero saga; 
apart from the latter, the former but report particular actions, which may, doubtless, throw light on the personal character of the god, but which of themselves do not constitute a connected history. Greek mythology offers a clear illustration of this in the so-called Homeric hymns. These hymns must not be ascribed to Homer or merely to singers of Homeric times. They are of later composition, and are designed for use in cult. Their value consists precisely in the fact that they portray the god by reference to the various directions of his activity, thus throwing light partly on the nature of the god and partly, and especially, on his beneficent rulership of the human world. It is this last fact that gives these poems the character of religious hymns.

Nevertheless, there is one class of myths in which the gods themselves actually appear to undergo experiences. I refer to those sagas and poems which are concerned with the birth of the gods, and with the origin of their rulership over the world and over the world-order which they have created, namely, to the cosmogonic and theogonic myths. These myths relate solely to a world of demons and gods, and they deal, as a rule, with an age prior to the existence of man, or with one in which the creation of man is but a single episode. Again, however, one might almost say that the exception proves the rule. For upon close examination it will be found that the gods who figure in these cosmogonies are not those with whose traits the hero saga, and the hymnology connected with it, have made us familiar. The gods whom the cosmogonic myths portray differ from those who protect and direct human life. They are not real gods, even though they bear this name, but are powerful demons. Except in name, the Zeus of Hesiodic theogony has scarcely anything in common with the Zeus of the Homeric hierarchy of gods. This fact does not reflect any peculiarity of the poet, as it were, but is due to the nature of the subject-matter itself. Even though theogonic myths were not elaborated into poetic form until a relatively late period, they are nevertheless of a primitive nature. Analogues to them had existed among primitive peoples long 
before the rise of the hero saga, hence at an age when the preconditions of god-ideas proper were still entirely lacking. The cosmogonic gods of the Greeks and Germans, as well as those of the ancient Babylonians, are of the nature of purely demoniacal beings. They lack the chief attribute of a god, namely, personality. Moreover, the myths themselves-if we disregard their form, which was the product of later literary composition-are not at all superior to the cosmogonies of the Polynesians and of many of the native tribes of North America. Obviously, therefore, it betokens a confusion of god-ideas proper with these cosmogonic beings, when it is maintained, as sometimes occurs, that the mythology of these primitive peoples, especially that of the Polynesians, is of a particularly advanced character. This should not be claimed for it, but neither may this be said of the Hesiodic theogony or the Babylonian creation myths. It is true that these myths are superior to the earlier forms of demon belief, for they at least develop a connected view of the origin of things. Primitive myth accepts the world as given. The origin of the world-order as a whole still lies beyond its field of inquiry. Though it occasionally relates how animals came into being, its imagination is essentially concerned with the origin of man, whom it regards as having sprung from stones or plants, or as having crept up out of caves. Even when this stage is transcended and an actual cosmogony arises, the latter nevertheless remains limited to the circle of demon conceptions, which are essentially the same in the myths of civilized peoples as in those of so-called peoples of nature. According to a cosmogonic myth of the Polynesians, for example, heaven and earth were originally a pair of mighty gods united in embrace. The sons who were born to these gods strove to free themselves and their parents from this embrace. Placing himself on the floor of mother earth, therefore, and extending his feet toward the heavens, one of these sons pushed father heaven upward, so that ever since that time heaven and earth have been separated. This mistreatment aroused another of the divine sons, the god of the winds. Thus a strife 


\section{THE AGE OF HEROES AND GODS}

arose, whose outcome was a peaceful condition of things. This is a cosmogonic myth whose essential elements belong to the same circle of ideas as the cosmogony of the Greeks. In the latter also, Uranus and Gæa are said to have held each other in an embrace, as the result of which there came the race of the Titans. One might regard this as a case of transference were the idea not obviously a grotesque development of a märchen-motive found even at a more primitive period. According to the latter, heaven and earth were originally in contact, and were first separated by a human being of prehistoric times-an idea undoubtedly suggested by the roofing-over of the hut. The Babylonian myth gives a different version of the same conception. It ascribes the separation of heaven and earth to the powerful god Marduk, who cleaves in two the original mother Thiamat. From one part, came the sea ; from the other, the celestial ocean. As in many other nature myths, heaven is here conceived as a great sea which forms the continuation, at the borders of the earth, of the terrestrial sea. This then suggests the further idea that the crescent moon is a boat moving over the celestial ocean.

In all of these myths the gods are given the characteristics of mighty demons. They appear as the direct descendants of the ancient cloud, water, and weather demons, merely magnified into giant stature in correspondence with their enormous theatre of action. Thus, as regards content, these cosmogonic myths are märchen of a very primitive type, far inferior to the developed märchen-myths, whose heroes have already acquired traits of a more personal sort. In form, however, cosmogonic myths strive towards the gigantic, and thus lie far above the level of the märchenmyth. Though the complete lack of ethical traits renders the gods of cosmogonic myths inferior in sublimity to gods proper, they nevertheless rival the latter in powerful achievement. Indeed, however much cosmogony may fail to give its gods the characteristics requisite for true gods, it does inevitably serve to enhance the divine attribute of power. A further similarity of cosmogonic and theogonic myths to the 
most primitive märchen-myths appears in the fact that they seem directly to borrow certain elements from widely disseminated märchen-motives. I mention only the story of Kronos. Kronos, according to the myth, devours his children. But his wife, Rhea, withholds the last of thesenamely, Zeus-giving him instead a stone wrapped in linen; hereupon Kronos gives forth, together with the stone, all the children that he had previously devoured. This is a märchen of devourment, similar or derivative forms of which are common. For example, Sikulume, a South African märchen-hero, delays pursuing giants by throwing behind him a large stone which he has besmeared with fat; the giants devour the stone and thus lose trace of the fugitive.

But there is also other evidence that cosmogonic myths are of the nature of märchen, magnified into the immense and superhuman. In almost all such myths, particularly in the more advanced forms, as found among cultural peoples, an important place is occupied by two conceptions. The first of these conceptions is that the creation of the world was preceded by chaos. This chaos is conceived either as a terrifying abyss, as in Germanic and particularly in Greek mythology, or as a world-sea encompassing the earth, as in the Babylonian history of creation. In both cases we find ideas of terrible demons. Sometimes these demons are said to remain on the earth, as beings of a very ancient time anteceding the creation-examples are Night and Darkness, described in Greek mythology as the children of Chaos. Other myths represent the demons as having been overcome by the world-creating god. Thus there is a Babylonian saga that tells of an original being which enveloped the earth in the form of a snake, but whose body was used by the god in forming the heavens. As a second essential element of cosmogonies we find accounts of battles of the gods, in which hostile demons are vanquished and a kingdom of order and peace is established. These demons are thought of as powerful monsters. They induce a live consciousness of the terrors of chaos, not only by their size and strength but often also by their grotesque, half-animal, half-human forms, by their 
many heads or hundreds of arms. Obviously these Titans, giants, Cyclopes, and other terrible beings of cosmogony are the direct descendants of the weather demons who anteceded the gods. Does not the idea of a world-catastrophe that prepares the way for the rulership of the gods at once bring to mind the image of a terrible thunderstorm? As the storm is followed by the calm of nature, so chaos is succeeded by the peaceful rulership of the gods. Inasmuch, however, as the gods are the conquerors of the storm demons, they themselves inevitably revert into demoniacal beings. It is only after the victory has been won that they are again regarded as inhabiting a divine world conceived in analogy with the human State, and that they are vested with control over the order and security of the world.

All this goes to show that cosmogonic myths, in the poetic forms in which cosmogonies have come down to us, are relatively late mythological products. True, they represent the gods themselves as demoniacal beings. Nevertheless, this does not imply that god-ideas did not exist at the time of their composition ; it indicates merely that the enormous diversity of factors involved in the creation of the world inevitably. caused the gods to lose the attributes of personal beings. The cosmogonies of cultural peoples, however, differ from the otherwise similar stories of those semi-cultural peoples whose mythology consists exclusively of such cosmogonic märchen. In the latter case, real god-ideas are lacking. The gods have remained essentially demons. In the higher forms of this semi-culture, where political development has had an influence on the world of gods, as was once the case among the peoples of Mexico and Peru, divine beings may approximate to real gods. In cosmogonic myths themselves, however, this never occurs. Thus, these myths invariably constitute a stage intermediate between the mythology of demons and that of gods ; they may originate, however-and this is what probably happens in the majority of cases-through a relapse of gods into demons. An illustration of the latter is the Hesiodic cosmogony. The weather-myth which the poet has elaborated obviously incorporated ancient märchen- 
myths that do not differ essentially from the original märchen as to content, but only as respects their grotesque and gigantic outlines. Compared with the gods of the hero saga, therefore, the cosmogonic myths of cultural peoples are of relatively late origin; to discuss the latter first, as is still done in our accounts of the mythology of the Greeks, Germans, etc., may easily lead to misconceptions. Of course, the creation of the world came first, but it is not at all true that the myth of the world's creation anteceded all others. On the contrary, the latter is a late and sometimes, perhaps, the last product of the mythological imagination. This is particularly apt to be the case where, as so clearly appears in the Biblical account of the creation, there is involved a specific religious impulse that is seeking to glorify the world-creating god. This religious impulse imposes upon the older mythical material a new character. Hence we find that, of the two elements universally characteristic of the cosmogonic myth, it is only the idea of chaos that is retained, while the account of struggles with the monsters of earliest times disappears. Nevertheless, though the creating god has lost his demoniacal character, he has not yet attained a fully developed personality - this is precluded by the enormity of the world, which transcends all human measure. He himself is in every respect an unlimited personal will, and is, therefore, really just as much a superpersonal being as the battling gods of other cosmogonies are subpersonal. That such a cosmogony, unique in this respect, may be original, is, of course, impossible. Indeed, the dominant conviction of Oriental antiquarians to-day is that the Biblical account of the creation rests on older and more primitive ideas derived from the Babylonian cosmogony, whose main outlines we have described above. This may doubtless be true, and yet no compelling proof of the contention can be adduced, for it is precisely those features in which both accounts are identical-namely, chaos, the original darkness, and the separating and ordering activity of the godthat are common property to almost all cosmogonies. The 
Biblical account of the creation, however, may not be classed with myths. It is a religious production of priests who were dominated by the thought that the national god rules over the people of Israel and over the world. Hence alone could it substitute a creation out of nothing for the ordering of a chaos, though the latter feature also persists in the Biblical account. The substitution, of course, dates from a later time than the myth, and represents a glorification of divine omnipotence which is entirely impossible to the latter.

A sort of offshoot of cosmogonic myths, though in striking antithesis to them, is the flood saga. This still retains, in their entirety, the characteristics of the original märchen-myth. It belongs to a variety of widely prevalent myths which, like the creation myths, appear to some extent to have originated independently in various parts of the earth, but also to have spread widely from one region to another. Evidence indicative of the independent origin of many of these sagas is to be found in the fact that, in many tropical regions, accounts of a flood, or so-called deluge sagas (Sintflutsagen), are represented by sagas of conflagration (Sintbrandsagen), according to which the world was destroyed, not by a general deluge, but by fire. In neither word has the prefix Sint any connection with Sünde (sin), with which popular etymology commonly connects it. Sint (old high German sin) is a word that has disappeared from modern German and means 'universal.' A Sintflut, thus, is a universal, in distinction from a merely local, flood. In so far, the sagas of universal flood and conflagration already approximate to the myths relating to the destruction of the world. Now, the Biblical story of the flood has so many elements in common with that of the Babylonians that we are compelled to assume a borrowing, and hence a transference, of material. The rescue of a single man and his household, the taking of animals into the ship, its landing upon the summit of a mountain, the dispatching of birds in quest of land - of these elements, some might possibly have originated independently in different parts of the earth. The rescue of individuals, 
for example, is included in almost all flood and conflagration legends, the direct source of the idea being the connection between the antediluvian and postdiluvian worlds. Of the combination of all of these elements into a whole, however, we may say without hesitation that it could not have arisen twice independently. The universal motive of the flood saga and that which led to its origin in numerous localities, without any influence on the part of foreign ideas, is obviously the rain as it pours down from the heavens. For this reason flood sagas are particularly common wherever rain causes devastating and catastrophic floods, whereas they are lacking in such regions as the Egyptian delta, where there are periodic inundations by the sea, as well as in the Arabian peninsula and in the rainless portions of Africa. As a rule, therefore, they are both rain sagas and flood sagas. They naturally suggest, further, the idea of a boatman who rescues himself in a boat and lands upon a mountain. According to an American flood myth which has preserved more faithfully than that of western Asia the character of the märchen, the mountain upon which the boatman lands rises with the flood and settles again as the flood subsides.

The flood sagas of cultural peoples, however, combine these very ancient märchen elements with a projection of the cosmogonic myth into a later event of human history. The flood deluging the earth is a return to chaos; indeed, often, as in the sagas of western Asia, chaos itself is represented as a mighty abyss of water. This is then connected with the idea of a punishment in which the god destroys what he has created, preserving from the universal destruction only the righteous man who has proved worthy of such salvation. Thus, the universal flood (Sintflut) actually develops into a sin flood (Sündflut). This change, of course, represents an elaboration on the part of priests, who projected the religious-ethical feature of a divine judgment into what was doubtless originally a purely mythological saga, just as they transformed the creation myth into a hymn to the omnipotence of the deity. But this prepares the way 
for a further step. The counterpart of these cosmological conceptions is projected not merely, into a past which marks the beginning of the present race of men, but also into the future. Over against the transitory world-catastrophe of the universal flood, there looms the final catastrophe of the actual destruction of the world, and over against a preliminary judgment of the past, the final judgment, at which this life ends and that of the yonder world begins.

Thus, we come to the myths of world destruction, as they are transmitted in the apocalyptic writings of later Israelitic literature and in the Apocalypse of John, who betrays the influence of the earlier writers. At this point we leave the realm of myth proper. The latter is always concerned with events of the past or, in extreme cases, with those of the immediate present. No doubt, the desires of men may reach out indefinitely into the future. Myth narrative, however, in the narrower sense of the term, takes no account of that which lies beyond the present. In general, moreover, its scene of action is the existing world, however much this may be embellished by the imagination. Myth reaches its remotest limit in cosmogonies. Even here, however, no absolute limit is attained, for the world-creation is represented as having been preceded by chaos. The idea of a creation out of nothing, which dislodges the idea of an original chaos, arises from religious needs and is not mythological in character. Similarly, the apocalyptic myth of world-destruction has passed beyond the stage of the myth proper. It is a mythological conception, which, though combining elements of the cosmogonic myth with fragments of märchen and sagas, is, in the main, the expression of a religious need for a world beyond. These myths, therefore, are not original myth creations, as are the cosmogonic myths, at least in part. They are the product of religious reflection, and, as such, they are dominated primarily by the desire to strengthen the righteous in his hopes and to terrify his adversary. Thus, the history of the cosmogonic myth here repeats itself in a peculiarly inverted form. With the exception of occasional 
survivals, the religious hymn, which is the ripest development of the cosmogonic myth, excludes the struggles of demons and wild monsters of the deep; the myth of the destruction of the world, on the other hand, constantly seeks, by its fantastic imagery, to magnify fears and punishments, as well as blessed hopes. As a result, all these accounts clearly bear the traces of a laborious invention seeking to surpass itself and thus to atone for the lack of original mythological imagination. We may call to mind the monster which the Book of Daniel describes as coming forth from the sea, provided with enormous iron teeth, and bearing on its head ten horns, among which an eleventh horn appears, which possesses eyes, and a mouth that speaks blasphemous words: Such things may be invented by the intellect, but they are impossible as natural creations of the mythological imagination. The motives underlying such exaggerations beyond the mythologically possible are to be found in factors which, though extending far back into the beginnings of mythology, nevertheless attain their development primarily in this age of gods and heroes. These factors are the ideas of the beyond.

\section{4. The Belief in Souls AND IN A WORLd Beyond.}

Closely connected with the cosmogonic myth are the ideas of a world beyond into which man may enter at the close of the present life. Before such ideas could arise, there must have been some general world-conception into which they could be fitted. The ideas of a beyond, therefore, are but constituent elements of cosmogonic conceptions; indeed, they are confined to relatively advanced forms of the latter. This is indicated by the fact that the earlier mythological creations contain no clearly defined notions of a beyond. Where there is no definite world-view, such conceptions, of course, are impossible. Thus, the two ideas mutually reinforce each other. The cosmogonic myth gives a large setting to the ideas of a beyond; the latter, in turn, contribute to the details of the world picture which the cosmogonic myth has created. At any rate, when poetry and philosophy, in their endeavour to construct a 
coherent cosmogony, began to appropriate celestial myths, ideas of a life after death and of a world beyond were already in existence. Some of these ideas, indeed, date back to an early period.

It is an extremely significant fact that, wherever we can trace their development at all, these ideas of a beyond follow the same definite and orderly course. The direction of this development is determined not only by the cosmogonic myth but also by the ideas regarding the soul. The formation of ideas of a beyond is impossible without a world-view transcending the limits of earthly existence ; the latter, however, results from the need of ascribing to the soul a continuance after death. This need, of course, is not an original one, but is essentially conditioned by the age of gods. Among primitive peoples, the beginnings of a belief in a life after death are to be found chiefly in connection with the fear of the demon of the dead, who may bring sickness and death to the living. But just as the fear is of short duration, so also is the survival after death limited to a brief period. On a somewhat more advanced stage, as perhaps among the Soudan peoples, most of the Melanesian tribes, and the forest-dwelling Indians of South America, it is especially the prominent men, the tribal chiefs, who, just as they survive longest in memory, are also supposed to enjoy a longer after-life. This conception, however, remains indefinite and of a demoniacal character, just as does that of the soul. In all of these conceptions, therefore, the disembodied soul is represented as remaining within this world. It continues its existence in the environment; as yet there is no yonder-world in the strict sense of the word. It is important, moreover, to distinguish the early ideas of a beyond from the above-mentioned celestial märchen which narrate how certain human beings ascended into heaven. The latter are purely märchen of adventure, in which sun, moon, stars, and clouds, as well as the terrestrial monsters, dwarfs, gnomes, etc., are conceived of as belonging to the visible world. Indeed, these celestial travellers are not infrequently represented as returning unharmed to 
their terrestrial home. Thus, these tales generally lack the idea which, from the outset, is essential to the conception of a yonder-world-the idea, namely, of the sojourn of the soul at definite places, whether these be thought of as on, under, or above the earth. Here again it is characteristic that at first this region is located approximately midway between this world and the one beyond. The belief takes the form of a spirit-village, a conception prevalent especially among the tribes of American Indians. Inaccessible to living beings and in some secret part of the earth, there is supposed to be a village. In this village the spirits of the dead are thought to assemble, and to continue their existence in precisely the same manner as before death, hunting and fighting just as they did in their earthly life. The spirit-village itself is described as exactly like an ordinary village. Characteristic of the totemic setting which all of these ideas still possess, is the fact that among many of the Indians of the prairies there is thought to be not only a spirit-village but also a buffalovillage, where the dead buffaloes congregate, and into which, according to the märchen, an adventurous youth may occasionally stray. Sometimes, moreover, these tales give more specific accounts of the way in which such villages are rendered inaccessible. A river spanned by an almost impassable bridge, or a dense, impenetrable forest, separates the spirit-village from the habitations of the living. Ravines and mountain caves may either themselves serve as the dwellingplaces of the spirits or form the approaches to them. In addition to these conceptions, there are also others, which have, in part, found a place even in later mythology. The dead are represented as dwelling, not in some accessible part of the earth, but on remote islands. Such ideas are common in Polynesia, and also in other island and coast regions. Even in Homer we come upon the picture of a distant. island. It is here that Menelaus found rescue on his return from Troy. The island is described as a place of happiness, where only the privileged among mortals are granted a blessed future.

A second and, on the whole, an obviously later form of 
ideas of a beyond, are the myths of the nether world. These for the first time tell of a beyond which is by its very nature inaccessible to human beings, or which is visited by only a few divinely privileged heroes, such as Hercules, Odysseus, and Æneas. As a third and last form of ideas of a beyond, we may mention those of a heaven, where dwell the dead, in the presence of the gods. As a rule, however, this heavenly beyond does not lead to the disappearance of the nether world. Rather are the two worlds set over against each other, as the result of the enhancement of an antithesis which arose even in connection with the realms of the nether world. The heaven becomes the abode of the blessed, of the devout and righteous, the favoured of the gods ; the underworld continues, at the outset, to be the lot of the majority of human beings. The growing desire to participate in the joys of blessedness, then causes the privilege which was at first enjoyed only by a minority to become more universal, and the underworld is transformed into the abode of the guilty and the condemned. Finally, heaven becomes possible even for the latter, through the agency, more particularly, of magical purification and religious ecstasy.

Of the various ideas of the beyond that successively arise in this development, those regarding the underworld are the most common and the most permanent. This is probably due in no small measure to the custom of burying the corpse. Here the entrance into the underworld is, to a certain extent, directly acted out before the eyes of the observers, even though the mythological imagination may later create quite a different picture of the event. The custom of burial, however, cannot have been the exclusive source of these ideas, nor perhaps even the most important one. In the Homeric world, the corpse was not buried, but burned. And yet it is to Homer that we owe one of the clearest of the older descriptions of the underworld, and it can scarcely be doubted that the main outlines of this picture were derived from popular conceptions. As a matter of fact, there is 
another factor, purely psychological in character, which is here obviously of greater force than are tribal customs. This is the fear of death, and the terror of that which awaits man after death. This fear creates the idea of a ghostly and terrible region of the dead, cold as the corpse itself and dark as the world must appear to its closed eyes. But that which is thought of as dark and cold is the interior of the earth, for such are the characteristics of mountain caves that harbour uncanny animals. The underworld, also, is stocked with creations of fear, particularly with subterranean animals, such as toads, salamanders, and snakes of monstrous and fantastic forms. Many of the terrible beings which later myths represent as living on the earth probably originated as monsters of the underworld. Examples of this are the Furies, the Keres, and the Harpies of the Greeks. It was only as the result of a later influence, not operative at the time of the original conceptions of Hades, that myth permitted these beings to wander about the upper world. This change was due to the pangs of conscience, which transforms the ghosts of the underworld into frightful, avenging beings, and then, as a result of the misery visited even upon the living because of the crimes which they have committed, transfers them to the mundane world. Here they pursue particularly the one who has committed sacrilege against the gods, and also him whose $\sin$ is regarded as especially grievous, such as the parricide or matricide. Thus, with the internalization of the fear impulse, the demoniacal forms which the latter creates are brought forth from the subterranean darkness and are made to mingle with the living. Similarly, the joyous and hopeinspiring ideas of a beyond are projected still farther upward, and are elevated beyond the regions of this earth into heavenly spaces that seem even more inaccessible than the underworld. Prior to the age, however, which regards the heaven as the abode of the blessed, many peoples-possibly all who advanced to this notion of two worlds-entertained a different conception. This conception represents, perhaps, the surviving influence of the earlier ideas of spirit-islands. 
For the underworld was itself regarded as including, besides places of horror, brighter regions, into which, either through the direct favour of the gods or in accordance with a judgment pronounced upon the dead, the souls of the pure and righteous are received. As a result of the division which thus occurred, and of the antithesis in which these images of the beyond came to stand, pain and torment were added to the impressions of horror and hopelessness which the original conceptions of the underworld aroused. The contrasts that developed, however, did not prevent the underworld from being regarded as including both the region of pain and that of bliss. This seems to have been the prevalent notion among Semitic as well as Indo-Germanic peoples. The Walhalla of the Germans was also originally thought to be located in the underworld, and it is possible that it was not transferred to the heavens until the advent of Christianity. For, indeed, we are not familiar with Germanic mythology except as.it took form within the period in which Christianity had already become widespread among the German tribes.

An important change in the ideas of the beyond now took place. The separation of the abodes of spirits gradually led to a distinction between the deities who were regarded as the rulers of the two regions. Originally, so long as only the fear of death found expression in the unvarying gloom, of the underworld, these deities were but vaguely defined. The conceptions formed of them seem to have reflected the ideas of rulership derived from real life, just as was true in the case of the supermundane gods. Indeed, the origin of the more definite conception that the underworld is a separate region ruled by its own gods, must probably be traced to the influence of the ideas of celestial gods. But there is a still more primitive feature of myths of the beyond, one that goes back to their very beginnings, and that long survives in saga and märchen. This is the preference shown by myths of the nether world for female beings, whether as subordinate personifications of fear or as deities. Not only is the ideal of beauty and grace thought of as a female 
deity, an Aphrodite perhaps, but the psychological law of the intensification of contrasts causes also the fearful and terrifying sorts of deities to assume the feminine form. Such a gruesome and terrible goddess is exemplified by the Norse Hel, or, widely remote from her in time and space, by the Babylonian Ereksigal. In the Greek underworld also, it is Persephone who rules, and not Pluto, her consort. The latter seems to have been introduced merely in order that the underworld might have a counterpart to the celestial pair of rulers, Zeus and Hera. If the fear-inspiring attributes are not so pronounced in the Greek Persephone, this is due to the fact that in this case agricultural myths have combined with the underworld myths. To this combination we must later recur, inasmuch as it is of great significance for cult. The dominant place given to the female deity in the underworld myth, again brings the nether world into a noteworthy contrast with the supermundane realm of gods. In the latter, male gods, as the direct embodiments of a superhuman heroideal, are always predominant.

It is not alone the inner forces of fear and horror that cause the realm of the dead to be thought of as located in the interior of the earth. There is operative also an external influence imparted by Nature herself, namely, the perception of the setting sun. Wherever particular attention is called to some one entrance to the underworld, or where a distant region of the earth is regarded as the abode of the dead, this is located in the west, in the direction of the setting sun. We have here a striking example of that form of mythological association and assimilation in which the phenomena of external nature, and particularly those of the heavens, exert an influence upon myth development. It would, of course, be incorrect to assert that the setting sun alone suggested the idea of an underworld. We must rather say that this phenomenon was obviously a subordinate and secondary factor. Its influence was not clearly and consciously apprehended even as affecting the location of the underworld, though this location was determined solely by it. Because of its connection with approaching night, the setting sun came to be associated 


\section{THE AGE OF HEROES AND GODS $40 \mathrm{I}$}

with all those feelings that caused the underworld to be regarded as a realm of shadows and of terrifying darkness. It was the combination of all these factors, and not any single one of them-least of all, a relatively secondary one, such as the sunset-that created and so long maintained the potency of this most permanent of all the ideas of a beyond.

Mention should also be made of the influence exerted, even at an early time, by soul-ideas. At the beginning of the heroic age, it was almost universally believed that after death all human beings lead a dull, monotonous life under the earth, or, as Homer portrayed it, heightening the uniformity, that all lapse into an unconscious existence. Obviously these ideas were determined, in part, by the phenomena of sleep and dreams. Just as death seemed a protracted sleep, so did the dream come to foreshadow the life after death. The characteristics of dream images, therefore, came to be attributed to the souls of the underworld. The latter, it was thought, are visible, but, like shadows, they elude the hand that grasps them and move about fleetly from place to place. This shadow-existence is a fate that is common to all. It is only exceptionally flagrant transgressions against the gods that call forth punishments which not merely overtake the guilty in this world but may also continue in the next. Such figures, therefore, as are described in connection with Odysseus' journey to HadesSisyphus, who must unceasingly roll uphill a stone that is constantly rolling back, and Tantalus, who languishes with hopeless desire for the fruits suspended above his headare not as yet to be regarded as expressing ideas of retribution, even though they may be anticipatory of them. Perhaps, also, it is not without significance that these accounts are probably later accretions, of which the Homeric poems contain a considerable number, particularly the Odyssey, which is so rich in märchen elements.

Gradually, however, that which at first occurs only in occasional instances becomes more universal ; the distinction in destinies comes to be regarded as applying generally. The earlier and exceptional cases of entrance into a world 
of the blessed or of particular punishments in Hades were connected with the favour or anger of the gods. Similarly, that which finally makes the distinction a universal one is religious cult. The object of cult is to propitiate the gods; their favour is to be won through petitions and magical acts. The gods are to grant not merely a happy lot in this world but also the assurance of permanent happiness in the next. Before this striving the shadows of the underworld give way. Though the underworld continues, on the whole, to remain a place of sorrow, it nevertheless comes to include a number of brighter regions in which the righteous may enjoy such happiness as they experienced in this world, without suffering its distresses and evil. It was this that early led to the formation of cult associations. Even during the transition of totemic tribal organization into States and deity cults, such religious associations sprang up out of the older totemic groups. During this period, the conditions of descent and of tribal segregation still imposed limitations upon the religious associations. These limitations, however, were transcended on the stage of deity cults, as appears primarily in the case of the Greek mysteries and of other secret cults of the Græco-Roman period, such as the mysteries of Mithra, Attis, Osiris, and Serapis. No doubt, the extreme forms of the cults prevalent in an age thoroughly. conscious of a deep need for salvation were bound up with the specific cultural conditions of that age. And yet these cults but bring out in particularly sharp relief certain traits which, though they are not clearly apparent until later, are quite universally characteristic of the deity-worship of the heroic era. These cults arise only when the early heroic ideal, embodying certain external characteristics, has disappeared, having given way more and more to inner ideals, connected with religion and morality. This, however, occurs at the very time when minds are beginning to be more deeply troubled by the terrors of the underworld, and when, in contrast with this, the imagination creates glowing pictures of the future, for whose realization it turns to the gods. Thus arises the 
idea of a special region of the underworld, allotted to those cult-associates who have been particularly meritorious in the performance of religious duties. These will enter into Elysium, a vale of joy and splendour which, though a part of the underworld, is nevertheless remote from the regions of sorrow. Here the blessed will abide after death. This Elysium is no longer a distant island intended as a refuge for occasional individuals, but belongs to the established order of the underworld itself. In the sixth book of the Æneid, Virgil has sketched, with poetic embellishments, a graphic picture of this abode of the blessed as it was conceived, in his day, under the confluence of ancient mythical traditions and new religious impulses-a portrayal which forms perhaps the most valuable part of the whole poem. For, in it, the poet presents a living picture of what was believed and was striven for by many of his contemporaries.

In closest connection with this separation of realms in the underworld, is the introduction of judgeship. It devolves upon the judge of the underworld to determine whether the soul is to be admitted to the vale of joy or is to be banished into Orcus. It is significant that, in his picture of the underworld, Virgil entrusts this judgeship to the same Rhadamanthus with whom we are familiar from the Odyssey as the ruler of the distant island of the blessed. Obviously the poet himself recognized that these later conceptions developed from the earlier idea that salvation comes as a result of divine favour. After the separation of the region of the blessed from that of the outcasts, a further division is made ; the two regions of the underworld are partitioned into subregions according to degrees of terror and torment, on the one hand, and of joy and blessedness, on the other. Gradations of terror are first instituted, those of blessedness following only later and in an incomplete form. The subjective factor, which precludes differences in degree when joy is at the maximum, is in constant rivalry with the objective consideration that the merits of the righteous may differ, and, therefore, also their worthiness to enjoy the presence of the deity. In contrast with this, is the much stronger 


\section{4

influence exerted by the factor of punishment. The shadowy existence of souls in Homer's Hades is not regarded as a penalty, but merely as the inevitable result of departure from the circle of the living. Only when the hope of Elysium has become just as universal as the fear of Hades, does the latter become a place of punishment, and the former a region of rewards. Just as language itself is very much richer in words denoting forms of suffering than in those for joy, so also does the mythological imagination exhibit much greater fertility in the portrayal of the pains of the underworld than in the glorification of the Elysian fields. All the horrors that human cruelty can invent are carried over from the judicial administration of this world into that of the beyond. Gradations in the magnitude of punishments are reflected in the location of the regions appointed for them. The deepest region of the underworld is the most terrible. Above this, is the place where those sojourn who may enter Elysium at some future time, after successfully completing a period of probation.

The contrast which first appears in the form of a separation of the realms of torment and blessedness, of punishment and reward, is then carried to a further stage, again by the aid of ideas of a spacial gradation. No longer are all mortals compelled to enter the underworld; this not only loses its terrors for the blessed, but the righteous and beloved of the gods are not required to descend into it at all. Their souls ascend to heaven-a lot reserved in olden times exclusively for heroes who were exalted into gods. With this, the separation becomes complete: the souls of the righteous rise to the bright realms of heaven, those of the godless are cast into the depths. Among both the Semitic and the Indo-Germanic peoples, the antithesis of heaven and hell was established at a relatively. late period. Its first clear development is probably to be found among the ancient Iranians, in connection with the early cosmogonic myths. Here the battle which the creationmyths of other cultural peoples represent as being fought between gods and demons is portrayed as the struggle, 
of two divine beings. One of these is thought to rule over the regions of light above the earth and the other over the subterranean darkness. True, this contrast is also brought out in the battles described by other peoples as between gods and demons, and this surely has been a factor leading to the incorporation of the Iranian myth into the ideas of the beyond elsewhere entertained. The distinctive feature of Iranian cosmogony and that which gave its dualism an unusual influence upon religion and cult is the fact that the original cosmic. war was restricted to a single hostile pair of gods, Ormuzd (Ahuramazda) and Ahriman (Angramainju). Here also, however, Ahriman is the leader of a host of demons-a clear indication that the myth is based on the universal conception of a battle with demons. This similarity was doubtless all the more favourable to the influence of the Iranian dualism upon other religions, inasmuch as the separation of ideas of the beyond had obviously already quite generally taken place independently of such influence, having resulted from universal motives of cult. The fact, however, that the battle was not waged, as in other mythologies, between gods and demons, but between two divine personalities, led to a further essential change. The battle no longer takes place on the earth, as did that of Zeus and the Titans, but between a god of light, enthroned on high, and a dark god of the underworld. This spacial antithesis was probably connected by the ancient Iranians with that of the two ideas of the soul, the corporeal soul, fettered to earth, and the spiritual soul, the psyche, soaring on high. Herein may possibly lie the explanation of a curious custom which markedly distinguished the Iranians from other IndoGermanic peoples. The former neither buried nor burned their dead, but exposed them on high scaffolds, as food for the birds. It almost seems as though the 'platform-disposal,' commonly practised in totemic times and mentioned above (p. 216), had here been taken over into later culture; the only change would appear to be that, in place of the low mound of earth upon which the 
corpse was left to decompose, there is substituted a high scaffolding, doubtless designed to facilitate the ascent of the soul to heaven. Furthermore, many passages in the older Avesta point out that the exposure of the corpse destroys the corporeal soul, rendering the spiritual soul all the freer to ascend to heaven. This is the same antithesis between corporeal soul and psyche that long continues to assert itself in later conceptions. Indeed, it also occurs, interwoven with specifically Christian conceptions, in many passages of the Epistles of the Apostle Paul, where the corporeal soul survives in the idea of the sinfulness of the flesh, and where, in the mortification of the flesh, we still have a faint echo of the Iranian customs connected with the dead.

Thus, the ideas of a twofold beyond and of a twofold soul mutually reinforce each other. Henceforth the heavenly realm is the abode of the pure and blessed spirits; the underworld, that of the wicked, who retain their sensuous natures even in the beyond, and who must, therefore, suffer physical pain and torment in a heightened degree. The thought of a spacial gradation corresponding with degrees of merit, though first developed in connection with the pains and punishments of the underworld, then comes to be applied also to the heavenly world. In this case, however, the power of the imagination seems scarcely adequate to the task of sufficiently magnifying the degrees of blessedness. Hence the imagination is forced; it becomes subservient to reflection, which engenders an accumulation of apocalyptic imagery that completely defies envisagement. In Jewish literature, one of the earliest examples of such apocalyptic accounts of the beyond is to be found in the Book of Enoch. The idea of a journey to the underworld, developed in ancient history, here apparently suggested a journey to heaven; as a result, the celestial realm was divided into various regions, graded according to height, as were those of the underworld according to depth', and leading to places of greater blessedness, as did those of the latter to increasing torment. We here have one of those dream- 
journeys to which dream association readily gives rise in the expectant and excited consciousness of the sleeper. Indeed, it is not improbable that the narrative is based on actual dream images. Had not the appearance of the dead in dreams already led to the belief in a shadow-soul, which now journeys to this distant world? The division of the celestial realms, in these mythical works, fluctuates between the numbers three and seven-the two numbers held sacred par excellence. In the Second Epistle to the Corinthians, the Apostle Paul tells of a dream-vision in which, years before, he was caught up to the 'third heaven' of paradise.

Under the influence of expiatory rites, which were zealously practised even by the ancient mystery cults, these two worlds, the subterranean hell and the celestial paradise, were supplemented by a third region. This development was also apparently of Iranian origin. The region was held to be a place of purification, where the soul of the sinner might be prepared, through transitory punishments and primarily through lustrations, for entrance into the heavenly realm. Purgatorial lustration, after the pattern of terrestrial cult ceremonies, was believed to be effected by means of fire, this being regarded as the most potent lustrical agency, and as combining the function of punishment with that of purification. Dante's "Divine Comedy" presents a faithful portrayal of these conceptions as they were finally developed by the religious imagination of mediæval Christianity out of a mass of ideas which go back, in their beginnings, to a very ancient past, but which continually grew through immanent psychological necessity. Dante's account of the world beyond incorporates a further element. It tells of a guide, by whom those exceptional individuals who are privileged to visit these realms are led, and by whom the various souls are assigned to their future dwelling-places. The first of the visitors to Hades, Hercules, was accompanied by deities, by Athena and Hermes. Later it was one of the departed who served as guide. Thus, Virgil was conducted by his father, and Dante, in turn, was led by Virgil, though into the realms of blessedness, closed to the 
heathen poet, he was guided by the transfigured spirit of Beatrice. The rôle of general conductor of souls to the realms of the underworld, however, came to be given to Hermes, the psychopomp. Such is the capacity in which this deity appears in the Odyssey, in an exceedingly charming combination of later with very ancient soul-conceptions. After Odysseus has slain the suitors, Hermes, with staff in hand, leads the way to the underworld, followed by the souls of the suitors in the form of twittering birds.

These external changes in the ideas of the beyond, leading to the separation of the two realnis, heaven and hell, and finally to the conception of purgatory, an intermediate realm, are dependent also on the gradual development of the idea of retribution. This is not a primitive idea. It arises only in the course of the heroic age, as supplementary to the very ancient experiences associated with the fear of death and to the notions concerning the breath and shadow souls. Moreover, it is especially important to notice that at the outset the idea was not ethical in character, but purely religious-a striking proof that morality and religion were originally distinct. The transference of the idea from religion to morals represents the final stage of the development, and occurred long after other-world mythology had reached its zenith. The first traces of the retributive idea are to be found in connection with those unusual dispensations of favour by which a hero who has won the favour of the gods is either taken up into their midst or is granted admittance to some other region of blessedness; the conception may, however, also take the form of punishments attached to certain particular offences directed against the gods. These latter exceptions already form a prelude to the more general application of the retributive idea in later times. But, even at this stage, the idea did not at once include all men within its scope, but found expression only in the desire to gain some exceptional escape from future suffering or some peculiar claim to eternal joy in the future. True, the natural impulse toward association, and the hope that united conjurations would force their way to the ears of the gods 


\section{THE AGE OF HEROES AND GODS 409}

more surely than individual prayers could do, early led to cult alliances, whose object it was to minister to these other-worldly hopes. None of these alliances, however, was concerned with obtaining salvation for all; on the contrary, all of them sought to limit this salvation to a few, in the belief that by such limitation their aim would be more certain of realization. These cults, therefore, were shrouded in secrecy. This had a twofold purpose. On the one hand, it increased the assurance of the members in the success of their magical incantations-a natural result of the fact that these rites were unavailable to the masses; on the other hand, it augmented the magical power of the incantations, inasmuch as, according to an associative reaction widely prevalent in the field of magical ideas, the mysterious potency of magic led to a belief in the magical effect of secrecy. The influence of these ideas had manifested itself in much earlier times, giving rise, on the transitional stage between totemism and the deity cults, to the very numerous secret societies of cultural and semicultural peoples. At this period, these societies were probably always the outgrowth of the associations of medicinemen, but later they sometimes included larger circles of tribal members. As is evident particularly in the case of the North American Indians, such societies frequently constituted restricted religious groups within the clans-groups which appear to have taken the place of the earlier totemic associations. In harmony with this, and, perhaps, under the influence of the age-groups in the men's clubs, there was originally a gradation of the members, based on the degree of their sanctification and on the extent of their participation in the mystic ceremonies. In peculiar contradiction to the secrecy of such associations, membership in one of its classes was betrayed, during the festivals of the cult groups, by the most striking external signs possible, such as by the painting of the body or by other forms of decoration. Moreover, on the earlier stages of culture, the interest of all these secret societies was still centred mainly on things connected with this world, such as prosperity of 
crops, protection from sickness, and success in the chase. Nevertheless, there was also manifest a concern regarding a future life, especially wherever a pronounced ancestor worship or an incipient deity cult had been developed.

It is the idea of the beyond, however, that gradually crowds out all secondary motives and that gives to the mystery cults proper their characteristic stamp, bringing them into sharp contrast with the dominant ideas of the early heroic age. In the earlier period, the idea of the beyond had been enveloped in hopeless gloom; now, it fills the mystic with premonitions of eternal happiness. In striving for this experience, the mystic wishes for a bliss that is not granted to the majority of mortals. Once more all the magic arts of the past are called into play in order that the initiate may secure entrance into the portals of the yonder world; it is thither that he is transported in the ecstasy induced by these magical means. No longer is admiration bestowed upon the heroes of the mythical past, upon a Hercules and a Theseus, as it was in ancient times. The change came about slowly, and yet at the great turning-point of human history, marked by the Hellenistic age, it spread throughout the entire cultural world. Radiating far beyond the Eleusinian and Orphic mysteries, which these hopes of a yonder-world raised to new life, the same idea was appropriated by the cults of Osiris, Serapis, Attis, and Mithra. The idea of redemption, born of the longing to exchange this world, with its sufferings and wants, for a world of happiness in the beyond, took possession of the age. It is the negation of the heroic age, of the heroes which it prized, and of the gods which it revered. Along with this world, these cults of the beyond repudiate also the previously existent values of this world. The ideals of power and of property fade. Succeeding the hero ideal, as its abrogation and at the same time its consummation, is the ideal of humanity.

At first it is only religious ideals that manifest this shift in values. The enjoyment of the present gives way to hopes for the future, the portrayal of which welds religious feelings into a power that proves supreme over all other 
impulses. It is for this very reason that the future, which the mystic already enjoys in anticipation, comes to be exclusively the reward of the devout. It is not vouchsafed to the moral man who stands outside the pale of these religious associations, for his activity centres about this world. At a much earlier period, however, these ideas became combined with ethical motives of retribution. If, accordingly, the two motives again become entirely distinct at this decisive turning-point of religious development, this only signifies that, in themselves, they are of different origin, and not that from early times forward there were no forces making for their union. These forces, however, were not so much internal as external in character. They did not spring from the religious experiences themselves, nor, least of all, from the ideas of the beyond. Their source is to be found primarily in a transference of the relations of the earthly State to the divine State, as a result of which the ruler of the latter was exalted to the position of lawgiver in the kingdom of men no less than in that of the gods. Proofs of this transference are to be found in the most ancient customs and legal enactments of all regions. Either the ethical and religious commandments are, both alike, supposed to be the very utterances of the deity, as in the case of the Mosaic decalogue, or, as is illustrated by the Babylonian code of Hammurabi, an earthly ruler expressly promulgates his law in the name of the deity, even though this law is essentially restricted to legal and ethical norms. Thus it came about that every ethical transgression acquired also a religious significance. The ethical norm was not, at the outset, religious in sanction, as is usually believed; it acquired this character only through the medium of the world-ruling divine personality. Nevertheless, the union of the ethical and the religious gradually caused the idea of retribution, which originally had no ethical significance whatsoever, to force its way into the conceptions of the beyond. It was essentially in this way that ethical transgressions came to be also religious offences, whereas, on the other hand, the rewards 
of the other-world continued to be restricted to the devout, or were granted to the moral man only on condition that he be devout as well as moral.

In conclusion, we must consider an offshoot of otherworld ideas-the belief in the transmigration of souls. This belief is ultimately grounded in the more general ideas of soul-belief, even though its developed form appears only as a product of philosophical speculation, and has, therefore, found only a limited acceptance. In its motives, the belief most closely resembles the conception of purgatory, in so far as the latter involves the notion that the occupation of animal bodies is a means, partly of transitory punishment, and partly of purification. The idea of lustration, however, is not involved in that of metempsychosis. In its place, there is a new and unique element. It consists in the thought, expressed in Plato's "Republic," that it is proper that man should retain after death the character manifested during life, and that he should therefore assume the form of the animal which exhibits this character. There is thus manifested the idea of a relationship between man and the animal. In the distant past this idea gave rise to the animal totem; in this last form of the animal myth, it leads to the conception of the transmigration of souls. Thus, a complete inversion of values has here taken place. The significance of the totem as an ancestral animal and as an object of cult caused it to be regarded as superior to man. The animal myth, on the other hand, represents transformation into an animal as degrading, even as a severe punishment. It is precisely this difference which makes it probable that the idea of transmigration was not a free creation of Hindoo philosophers-for it was they who apparently first developed it, and from whom it passed over to the Pythagorean school and thence to Plato-but that it, also, was connected with the general development of totemic conceptions. Of course, it is not possible to trace a direct transition of the totem animal into the animal which receives the soul of a human being who is expiating sins that he has committed. It is not probabie, moreover, that such a transition occurred. 
Doubtless, however, the idea of transmigration is connected with the fact that, beginning with the totemic age and extending far down into the period of deity beliefs, the value placed on animals underwent a change. For the Australian, the animal is an object of cult, and the totem animal is frequently also regarded as the incarnation of an ancestor or of some magical being of antiquity; the American Indian calls the animals his elder brothers ; Hercules, the hero of the heroic age, is honoured because, among other things, he was instrumental in exterminating wild animals. This change, moreover, is reflected in animal myths even more than in these general evaluations. Indeed, transformation into animals is a dominant characteristic of these myths. Tracing the conception of this magical process, however, we find, step by step, a progressive degradation of the animal. In Australian legends, animal and man are either absolute equals or the animal is the superior, being endowed with special magical powers. In American märchen-myths also, we still frequently find the same conception, although transformation into an animal is here sometimes regarded as a disgrace. Finally, in many African myths, and, particularly, in those of the cultural peoples of the ancient world, such a transformation is regarded either as a serious injury resulting from evil magic or as a punishment for some crime. We may well suppose, therefore, that the Brahmans, who first incorporated this idea into the religious conceptions of retribution, were influenced by the ideas current in popular belief, which, on their part, represented the last development of earlier totem conceptions. These ideas may also have been reinforced by the belief (not even yet entirely extinct) in soul animals, into which the psyche disappears at the moment of death. Whether the Brahmans had as yet come to the notion that transformation into an animal is a simpler and more natural way of conceiving the future of the soul than ideas of a supermundane and a subterranean beyond, need not concern us. In any event, it is noteworthy that, after science had closed the path to heaven as well as that to Hades, 
Lessing and, in a broader sense, taking into account nature as a whole, Goethe himself, regarded metempsychosis as the most probable hypothesis concerning the way in which the desire for an endless survival of the soul will be satisfied.

\section{5. The Origin of Deity Cults.}

Psychologically, myth and cult are closely interrelated. The myth is a species of idea. It consists of ideas of an imaginary and an essentially supersensuous world that constitutes a background for the phenomena of sensuous reality. This supersensuous world is created by the imagination exclusively from sensuous materials. It finds portrayal throughout the various stages of myth development, first in the märchen-myth, then in the heroic saga, and finally in the deity saga. In the latter, there are interwoven ideas of the origin and destruction of things, and of the life of the soul after death. Cult, on the other hand, comprises only actions. These relate to the demons or the gods whose lives and deeds are depicted by mythology, at first only in fragmentary sketches, but later, especially in the deity saga, after the pattern of human life. Now, inasmuch as action is always the result of feeling and emotion, it is these subjective elements of consciousness that are dominant in cult, whereas cognition plays its rôle in connection with myth. This contrast is important because of its close bearing on the development of myth as well as on that of religion, and on the essential differentiæ of the two. Not every myth has a religious content. In fact, the majority of the myths prevalent, or once prevalent, in the world, have absolutely no connection with religion, if we give to the latter any sharply defined meaning at all. At the setting of the sun, a flaming hero is swallowed by a dusky demonthis conception of nature mythology may possibly be incorporated in religious conceptions, but, in itself, it possesses no religious significance whatsoever, any more than does the idea that the clouds are demons who send rain to the fields, or. that a cord wound about a tree may magically transfer 
a sickness to it. These are all mythological ideas, yet to call them religious would obviously leave one with a most vague conception of religion. Similarly, moreover, not every cult relating to things beyond immediate reality is a religious cult. Winding a cord about a tree, for example, might constitute part of a magic cult which aims at certain beneficent or pernicious results through the aid of demons of some sort. There is no ground, however, for identifying these cult activities with deity cult. From the very beginning, of course, every cult is magical. But there are important differences with respect to the objects upon which the magic is exercised. The same is true with respect to the significance of the cult action within the circle of possible magic actions and of the derivatives which gradually displace the latter. In view of this, it is undeniable that, in deity cult, the cult activity, in part, assumes new forms, and, in part, and primarily, gains a new content. Prior to the belief in gods, there were numerous demon cults, as well, particularly, as single, fragmentary cult practices presupposing demoniacal powers. Moreover, these demon cults and the various activities to which they gave rise, passed down into the very heart of deity cult. The question therefore arises, What marks shall determine whether a deity cult is religious in character? These marks, of course, may be ascertained only by reference to that which the general consensus of opinion unites in calling religious from the standpoint of the forms of religious belief prevalent to-day. From this point of view, a religious significance may be conceded to a deity conception if, in the first place, it possesses by its very nature-that is, objectively-an ideal worth, and, since the ideal transcends reality, a supersensuous character; in the second place, it must satisfy the subjective need of man for an ideal purpose of life. To one outside of the particular cult community, the value of this ideal may be but slight; to the community, however, at the time when it is engaged in the cult practices, the ideal is of highest worth. As the embodiments of the ideals just mentioned, the gods are 
always pictured by the mythological imagination in human form, since it is only his own characteristics that man can conceive as magnified into the highest values in so absolute a sense. Where the deity does not reach this stage, or where, at the very least, he does not possess this ideal value during the progress of the cult activities, the cult is not religious in nature, but prereligious or subreligious. Thus, while myth and cult date back to the beginnings of human development, they acquire a religious character only at a specific time, which comes earlier in the case of cult than in that of the myth. The gods are created by the religious emotion which finds expression in cult, and myth gives them the character of ideal personalities, after the pattern of the heroic figures of actual life. The entire life of man, with all its changes of destiny, is placed in their hands. Their cult, therefore, is no longer associated merely with special circumstances or various recurrent events, as were primitive magic and the conjuration of demons, but is concerned with the whole of life, which is now subordinated to a divine legal order fashioned after the political government. Thus, the god is soon succeeded by the divine State, and by the cult festivals dedicated to the latter. As an idealized counterpart of the human institution peculiarly characteristic of the heroic era, religious cult appears, from this point of view also, as the most distinctive creation of the age of heroes and gods.

If a conception proves to be too narrow to cover all the phenomena which fall within its sphere, it is legitimate, of course, to broaden it, to a certain extent, to suit our needs. Nevertheless, once we admit that not every mythological conception or magical practice is religious in character, we can no longer doubt that there was never a more significant change in the development of these phenomena than occurred in the case of the myths and cults directly connected with the heroic age. Primarily, therefore, it was the cults of the Babylonians, Egyptians, Israelites, and also those of the Greeks, Romans, Aryans, and Germans, that were religious in the full sense of the word. In the 
Old World, the Semitic and Indo-Germanic peoples must be regarded, to say the least, as the most important representatives of religious ideals ; in the New World, prior to the coming of the Europeans, this distinction belongs to the cultural peoples of the Andes, the Mexicans and the Peruvians. Though the religion of these latter races, no less than the other phases of their culture, was of a cruder sort than that of the former peoples, it frequently throws a remarkable light upon the initial stages of many forms of cult. Of course, there is never a sharp separation of periods; intermediate stages are always to be found. The latter result, particularly, from two conditions. On the one hand, a deity cult may be inaugurated by the introduction of elements of a celestial mythology into the still dominant magical cults. In this case, it is important to note, deity myth is usually far in advance of deity cult. This is exemplified in Polynesia, where we find a rich theogony alongside of cults that have not advanced essentially beyond the stage of totemic magic beliefs. On the other hand, however, a people whose civilization is still, on the whole, totemic, may be influenced by the deity cults of neighbouring cultural peoples, and, as a result, fusions of various sorts may occur. Of this, also, the New World affords instructive examples, namely, the Pueblo peoples of New Mexico and Arizona, who were influenced by Mexican culture.

In the soul-life of the individual, action, together with the feelings and emotions fundamental to it, have the primacy over ideation. The same psychological fact universally accounts for the superior importance of deity cult over deity myth. It is action that constantly influences ideas, changing and strengthening them, and thus arousing new emotions which stimulate to further activities. Thus, the elevation of the gods into ideal beings must be ascribed, in great part, to religious cult, for it came about as a result of the influence which the emotions associated with cult exercised upon the ideas of the gods. Even less than the mythological thought from which it develops does religious reflection consist simply of ideas. The mythical 


\section{I8 ELEMENTS OF FOLK PSYCHOLOGY}

tales and legends into which ideas are woven excite primarily the feelings and emotions. These it is that cause the exaltation of the religious consciousness, giving rise to action, which, in turn, enhances the emotions. If anywhere, therefore, it is in the psychology of religion that intellectualism is doomed to failure. The intellectualist is unable to explain even the fact of cult, to say nothing of those effects upon religion by virtue of which cult becomes religion's creative force. While, therefore, there are cults -namely, those of magic and demons-which, for specific reasons, we may call prereligious, there is no religion without some form of cult, even though, in the course of religious development, the external phases of cult may diminish in significance. In so far, cult is to be regarded as moulding, rather than as permanently expressing religious emotions; and it is not merely an effect, but also a source of religious ideas. It is in cult that deity ideas first attain their full significance. By giving expression to his desires in prayer and sacrifice, man enjoys a foretaste of their satisfaction, and this, in reaction, enhances not only the desires but also the mythological conceptions fundamental to them. It is precisely this relationship of myth to cult that extends far back into the totemic age and that causes the dominant magic cults of this period to be displaced by deity cults as soon as gods have arisen through a synthesis of heroes and demons. This accounts for the fact that, in the beginnings of religion, the worship of gods always contained elements that derived from the age of demons. But even the demon cults frequently exhibit one feature, particularly, that remains characteristic also of religion: in the cult the individual feels himself one with the object of worship. This is clearly shown in the case of primitive vegetation festivals. Those who execute the orgiastic cult dances regard themselves as one with the spirits of vegetation, whom they wish to assist, by their actions, in increasing the productive forces of nature. Such vegetation festivals have already been described in our account of totemic cults. Inasmuch, however, as they represent not only the highest 
of the totemic cults but even partake, in part, of the character of deity cults, it was necessary to refer to them again at this point. Vegetation festivals still prevail in richly developed forms among some of the tribes of North and Central America. It is clear that they represent primarily a transitional stage, for, in addition to totemic ideas, demon and ancestor beliefs are everywhere mingled with elements of a celestial mythology. Spirits of ancestors are thought to be seated behind the clouds, urging the rain demons to activity. Above them, however, are celestial deities, whose abode is in the heavens, and to whom is attributed the supreme control over destiny.

Even these relatively primitive vegetation cults manifest still another trait, which later comes more and more to characterize all cult, namely, the union of many cult motives. The great vegetation festivals of Central America attract not only those in health but also the sick. The latter are in search of healing. Hence there come to be special cults alongside of those that serve more universal needs. Moreover, the initiation of youths into manhood is also celebrated during these great festivals. Finally, the individual seeks to expiate some sin which he has committed in the past. Thus, numerous supplementary and subsidiary cults cluster about the great cult festivals. This was true even of the cults that reach far back into the age of magic and demon beliefs, when gods still played a secondary rôle, and conditions remained the same up to the time of the highest forms of deity cult. Furthermore, the incentive, or impelling motive, which originally brought cult members together for these comprehensive festivals seems everywhere to have been the same. The aim in view was to secure the prosperity of the crops, for, on the threshold of this higher civilization, these formed man's chief food-supply. The prominence of this motive in the earliest deity cults, moreover, indicates that the latter were genuine products of the general culture of this period. The roving hunter and nomad were giving place to the settled tiller of the soil, who utilized the animal for the services of man, and thus engaged more systematically 
in the breeding of domestic animals, though also perfecting, in addition to the arts of peace, the agencies of war. The motives that gradually elevated vegetation cults to a higher plane consisted in every case of those that at the outset found expression in the subsidiary cults. The concern for the spiritual welfare of mankind finally supplanted materialistic purposes. This is clearly shown by the history of the Greek mystery cults. These, however, were obviously influenced, particularly at a later time, by the similar cults of the Egyptians, as well as by the Babylonians and other peoples of western Asia. Among all these peoples, the chief cults were vegetation cults, and, as such, they occurred at stated seasons. In the Orient, particularly, the festivals were held at the solstices. Surviving remnants of seedtime and harvest festivals-which were solstice festivals and were prevalent throughout the entire Oriental world -allow us to conclude, even with respect to many regions in which a complete historical tradition is lacking, that agricultural festivals probably represent the earliest deity cults. Hence it is that these remnants still contain so many elements characteristic of demon beliefs.

It is the contrast of spring, of newly awakened Nature and its sprouting and growing crops, with winter and its dying vegetation, that first finds expression in the deity myths which inspire the vegetation festivals. The more permanent significance of these cults, however, is due to the fact that the gods of vegetation gain an increasing sphere of influence. The reason for this is obviously to be found in the fact that subsidiary motives come to be incorporated into the main cults of the earliest cultural peoples. One factor is of particular importance. Though inconspicuous in the earliest of these cults, it becomes increasingly prominent as the cults become more highly developed. I refer to hopes of a beyond. Of course, many phases of the cult remain hidden to us. Due to the combinations already mentioned and to the incorporation, in this case, of magical and mystical elements, these cults acquired a secret nature in proportion as they concerned themselves with the riddle of 
the beyond. The more carefully the individual cult member guarded the secrets of the group, the richer the blessings that he might hope to receive. Nevertheless, the general psychological motives underlying this development enable us to supplement the historical tradition. In this way it is possible to gain a fairly positive knowledge of the process by which, with an apparently almost universal uniformity, vegetation cults came to combine with soul cults. The ideas of changing seasons, of summer and winter, of the budding and the withering of grain, are naturally associated with those of life and death. Winter and bleak nature resemble death; and, just as lifeless nature is again resuscitated in the spring, so also will the soul awaken to a bright and joyous existence in the future. The connection is so obvious that poetry and even myth itself everywhere refer to it. Hence also it could not have been overlooked by the mythologists. Generally, however, this has been regarded as an ingenious allegory by means of which man sought to gain a vivid realization of the resurrection of the soul. In fact, such allegorical reinterpretations occur in later cult legend itself. Particularly characteristic of this is the legend of the Eleusinian mysteries. Persephone, the daughter of Demeter, goddess of the crops, is stolen by Pluto, ruler of the underworld, and the goddess-mother wanders about on the earth seeking her child. Resentfully she withdraws from the heavens and avoids the assemblages of the gods. During this period of mourning, however, she devotes all of her care to mankind. She protects not only the vegetation but also the germinating human life, the child. Thus she becomes a benefactress upon earth. The gods, however, mourn her absence, and Zeus makes a compact with the lord of the underworld. Persephone is to remain in the underworld with her husband, Pluto, during only one-half of the year; during the other half she is to return to her mother. Appeased, Demeter herself returns to the heavens. The allegorical significance of this legend cannot fail to be recognized, nor the fact that it was probably only as a result of a poetical elaboration of the mythological material that 
this allegorical character was acquired. The same is true of all other similar cult legends, from the descent into hell of the Babylonian Ishtar down to the legends of Dionysius and Osiris, and other vegetation legends of the Hellenistic period. In the form in which these have come down to us, they are all products of priestly invention, replete with a conscious symbolism such as cannot be ascribed to the original mythical material upon which they were based. Nevertheless, it is customary not only to regard all of this original content as allegorical, but also to surpass even the traditional legend itself, if possible, in allegorical interpretation. In the legend of Demeter, for example, Demeter is supposed to be the mother earth, and Persephone the seed that is thrown into the earth to grow up and blossom. Analogously, he who participates in the cult hopes that, while his soul, similarly, is at first buried in the earth with his body, it will later ascend to heaven as did Demeter. Back of the myth, therefore, there is supposed to be a symbolical allegory, and to this is attributed the original union of the soul cult with the vegetation festival. When, then, the former lost its influence, the symbolism it thought to have remained as the chief content of the mystery. No original cult, however, shows the least sign of connection with such subtle allegories. On the other hand, there are many indications that the vegetation cults developed into these higher forms of soul cults in an entirely different way. Soul cults of a lower order had, of course, long been prevalent. But these were absolutely distinct from any vegetation myths that may have existed. They pictured souls as demons, against whom it was necessary to be on one's guard, or, at a later stage, as beings whom one might conciliate and win over as helpful spirits. Now, the cults of Demeter practised in Eleusis had as their aim, not only an increased productiveness of the soil, but also success in the interests and activities of this world. Since they related to happiness in general, it was but natural that, as soon as the ideas of a beyond reached a point of development at which the yonder-world became the focus 
of desires and hopes, the cults also should necessarily concern themselves with happiness in a life after death. Thus, interest in the beyond came to be one of the further cult motives that linked themselves to the dominant vegetation cults. The latter, however, held the primacy, as is still clearly apparent by reference to the vegetation festivals of the semi-cultural peoples of America. It is only natural that this should have been the case. When agriculture was in its beginnings, the most pressing need of life was that of daily bread. For the tiller of the soil, moreover, the changes of seasons marked by seedtime and harvest, represent sharply defined periods, suitable above all others for the festivals to which tribal associates assemble from near and far. The later allegories connected with these cults had nothing to do with their transition into soul cults, but, as their whole character indicates, were creations of the priestly imagination. As a result of the reaction of cult activities upon the emotions, however, concern for the future happiness of the soul finally came more and more to overshadow the desires connected with this world. Thus, the cults of Demeter eventually passed over, in all essentials, into cults of the beyond. The same is true of the Dionysius cults of the Greeks, of the Egyptian worship of Isis and Osiris, of the Persian Mithra cult, and of many other mystery cults of Oriental origin. All of these express the same passion for a future bliss that shall begin at the close of earthly life and endure endlessly.

The character of these cults is shaped, in a decisive measure, by other influences, whose source is to be found in the hopes of a beyond. Even in the vegetation festivals of the semi-cultural peoples of America, with their elements of totemism and ancestor worship, an important place is occupied by ecstatic features-by the orgiastic dance, and by the ecstasy that results from sexual excitement and from narcotic poisons, such as tobacco. Conjurations, prayer, sacrifice, and other cult ceremonies aid in stirring the emotions. Doubtless it was due to these ecstatic elements that the cult of Dionysius gained supremacy over the older 
cults of Demeter in the Greek mysteries, and that Dionysius himself was eventually given a place in the Demeter cult. For is he not the god of wine, the most potent of all the means for creating a condition of bliss that elevates above all earthly cares? In the mystery cults, however, the central feature of the cult activity was the vision experienced in the ecstasy. The mysterious equipment of the place, the preliminary ascetic practices, the liturgic conjurations and sacrifices, the wine, which originally took the place of the blood sacrifice, and, among the Hindoos, the soma, which was itself deified-all of these served to transport consciousness to another world, so that the cult became increasingly concerned with the world beyond, and finally devoted itself exclusively to this interest. As a result of this change, the hopes centring about the beyond forced their way overpoweringly into cult, whereas the cult, in turn, reacted in an important measure to enhance these hopes.

Over against the tendency toward unification inherent in vegetation cults and in the other-world cults which sprang from them, the increasing diversity of needs and interests now introduces influences toward a progressive differentiation of cults. Separate deity cults come to be fostered by the various social groups and classes, just as had occurred in the case of the totem cults of the preceding age, which differed according as they were practised by the tribe, the sex, or the individual. The desire for protection against dangers and for security in undertakings gives rise to guardian gods no less than it did to guardian demons. Since, however, this more general desire branches out into a considerable number of special desires, advancing culture results in a progressive differentiation of cults. The foundation of cities and the separation into classes and occupations lead to special cults for each of these divisions of society. The personal characteristics of the gods and the purposes of the cult come to be affected, each by the other. Each specific cult chooses from among the members of the pantheon that god who best suits its purpose, and it then modifies his character according to its needs. The characteristics of the 
gods thus undergo a change of significance analogous to that of the forms of speech and custom. This change, however, is due mainly to cult, and to the fact that the human beings who practise the cult have need of protection and aid. The influence of saga and poetry is only secondary, being, at best, mediated through cult.

In addition to the increasing diversity of human interests, and interplaying with it in various ways, are two further factors that tend toward the differentiation of cult. In the first place, divine personality as such awakens man to the necessity of establishing a cult. As a personal being who transcends human stature, the god calls for adoration by his very nature, even apart from the special motives which are involved in the specific deity cults and which, in the further course of development, give to the latter their dominant tone. Pure deity cults, thus, are the highest forms of cult, and give best expression to ideal needs. Outstanding examples of this are the Jahve cult of the Israelites, and the cults of Christ and Buddha. The latter, in particular, show the great assimilative power of cults that centre about an objective ideal, in contrast with those that are subjective in nature, springing entirely from human desires and hopes, and especially with that most subjective of all cults, the cult of the beyond. Moreover, this idealizing impulse may also create new cults, by deifying heroes who were originally conceived as human. Besides the ancient hero cults, the most prominent examples of such cults are again those of Christ and of Buddha. For there can be no doubt that Christ and Buddha alike existed as human beings and that originally they were also regarded as such. The fact that their heroic character consists entirely in the spiritual qualities of their personalities does not preclude them from consideration in this connection. These qualities proved all the more effective in bringing about the exaltation of the human into the divine. Thus, they enable us to understand how it was possible for the cult of the original deities to be crowded into the background by that of those who later came to be gods. This is 
emphatically brought out in the Buddha legends, many of which represent the ancient Hindoo gods of the Veda as the servants of the divine Buddha.

In addition to the fact that divine personalities call forth homage by their very nature, the multiplication of cults results also from the fusion of the gods of various peoples. This is the most external factor, and yet it is by no means the least potent one. It not infrequently happens that cults gain their supreme importance only in the territory into which they have been transplanted. Dionysius, for example, was a god introduced from elsewhere into Greece. Through his connection with the mystery cults, however, he later came to surpass all other Greek gods in religious significance. The original cults of the native Italian deities, with their numerous elements carried over from the age of demoniacal and ancestral spirits, were but few in number. Through the assimilation of Greek deities, however, and later, at the time of the empire, of Oriental gods, differing widely in character, Rome acquired a multiplicity of cults to which history doubtless affords no parallel. Yet we must not overlook the fact that in certain other cases-such, for example, as the BabylonianAssyrian and the Egyptian cults-the fusions may perhaps have become more complete at an early period, and thus have precluded the juxtaposition of the many separate cults that existed in the Rome of the Empire.

\section{i6. The Forms of Cult Practices.}

This multiplicity of cults, increasing with the advance of civilization both as regards the ends that are desired and the gods who are worshipped, is by no means paralleled by the number of cult agencies. The only possible exception might be in the case of the means which the cults of the beyond employed for arousing ecstasy. Even here the difference lies not so much in the means themselves as in the extent to which they were used. Moreover, the secrecy surrounding these cults is itself an ex- 
ternal indication of the fact that they differed from the cults concerned with the things of this world, for the latter generally sought publicity. And yet there was no form of cult in which ecstatic features were altogether lacking; such features are inherent, to a certain extent, in cult practices as such and, in so far, are absolutely universal. Differences in the specific purposes of the cults and in the deities to whom the acts were dedicated did indeed cause certain variations. These, however, we may here neglect, inasmuch as they do not affect the essential nature of cult itself. From early times on, there were certain activities that were universally characteristic of deity cults, and their fundamental purposes remained the same, namely, to gain the favour of the deity and thereby to obtain the fulfilment of personal wishes. As regards this motive, the three cult agencies-prayer, sacrifice, and sanctification-are absolutely at one. In this order of sequence, moreover, these agencies represent a progressive intensification of the religious activity of cult.

In the records of ancient civilized peoples we meet with a great number of prayers, representing all the forms developed by this simplest and most common of the means of cult. The most primitive form of prayer is conjuration. Conjuration passed over from demon cult into the beginnings of deity cult, and is intermediate between a means of magic and a petition. This also indicates the direction of the further development of the prayer. Conjuration is succeeded by the prayer of petition, whose essential differentia consists in the fact that, however earnestly the suppliant may strive for the fulfilment of his desires, he nevertheless ultimately commits them to the will of the deity. The development of the prayer of petition out of conjuration becomes possible only because gods possess a characteristic which demons lack-namely, personality. Once this personality attains to its ideal sublimity, the exercise of magical power over the deity ceases to be possible, or is so only under the presupposition that the will of the deity is in itself favourably inclined toward the suppliant. The idea underlying conjuration nevertheless 
continues for a time to remain a supplementary factor in the prayer of petition; even where no clearly conscious trace of it appears, it survives in the depth of emotion that reinforces the petition. That conjuration blends with petition is particularly evident in the case of one characteristic, whose origin must be traced to magical conjuration. I refer to the fact that the words of the petition are repeated in the same or in a slightly changed form, and that, at a later stage of developments, there is a constant recurrence of the same content, even though this is variously expressed. This as a derivative characteristic of the prayer of petition. Originally, it was thought that repetition brought about an intensification of the magical effect, particularly in the case of word-magic. We are already familiar with conjurations of this sort as elements of totemic cults. With but few changes, they recur in the older songs of the Avesta and Veda, as well as in some of the Biblical Psalms. In these cases, however, the repetitions are somewhat more extensive, for there is a more detailed statement of that which is desired. And yet the Biblical Psalms, particularly, are an illustration of the fact that, with submission to the will of the deity, the petition becomes less urgent in tone. Even when the petition is repeated the expression more and more assumes a somewhat altered form. It is probably this enhancement through repetition-itself, in turn, due to the dynamic character of the emotions of desire-that accounts for the so-called 'parallelism of members,' characteristic especially of Hebrew poetry. The view, once entertained, that this is a sort of substitute for the rhythm arising from emphasis and sentence arrangement is doubtless incorrect, for recent investigations demonstrate the ingenious rhythm of Hebrew poetry. We would not, of course, deny that the repetition of the thought in a changed form intensifies the rhythmic expression. The real basis of the repetition, however, lies not in this fact but in the motive underlying petition. This is clear, above all, from the fact that repetition is most pronounced particularly in those psalms and prophetic songs which are of the nature of a prayer of petition 
and of the praises closely connected with it. Later, repetition was also employed in other forms of religious expression. In the case of the hymn of praise, particularly, the tendency to repetition is augmented, by virtue of the enthusiastic exaltation of the divine personality whom the hymn extols.

Besides the prayer of petition we find the prayer of thanksgiving. Petition and thanksgiving are properly correlative, the one expressing a wish to the deity and the other acknowledging its fulfilment. Not infrequently, therefore, they are combined, particularly in the more advanced forms of the prayer cult, into a single prayer of thanksgiving and petition. He who prays returns thanks for the blessings which he has received and adds a request for further divine aid. This combination occurs very frequently in the Psalms, but it is to be found also in other hymnodies. The extent to which the request for further favours is subordinated to the thanksgiving for past aid, is a measure of the humility. involved, and represents a fair criterion of the maturity of the religious feeling underlying the prayer. Nevertheless, it may also be noticed that he who prays always aims first to gain the divine favour through his thanksgiving, in the hope that the gods may thereby be rendered more disposed to grant his request. Typical examples of this are to be found, not only in the Biblical Psalms, but also in the ancient Babylonian texts which recent discoveries have brought to light. That the prayer of thanksgiving is a higher form of prayer than is petition, is shown by the very fact that it occurs in deity cult alone. More clearly even than petition does thanksgiving presuppose a personal being, capable of appreciating the feeling of gratitude. It is at most in the fact that the prayer of thanksgiving still seeks to obligate the deity to future favours, that demonconjuration has left its traces upon it. And yet deity cult is characterized precisely by the fact that the compulsion of magical conjuration has entirely disappeared in favour of the free volition of the deity. That prayer is regarded as imposing an obligation upon the god no less than upon man, is extremely well brought out in the conception that 
the relation of the two is that of a contract, or of a covenant sealed in the cult. This idea, reinforced by the national significance of the deity, is fundamental in the Jahve cult of the Israelites.

Praise, or, as it is called in its poetic forms, the hymn, is an even more pronounced feature of deity cult than is the prayer of thanksgiving. The hymn is not usually classified as a form of prayer because, when externally regarded, it may entirely lack the motive of petition, and it is from the latter that the prayer has derived its name. In view, however, of the continuity of the development of the cult forms which find expression in speech, we cannot escape including also the song of praise. Indeed, it generally adduces the blessings conferred by the god as an evidence of his glory; not infrequently, moreover, it concludes with a hope for the future favour of the deity. Artistically perfect examples of such prayers are the compositions known as the Homeric Hymns, which, of course, belong to a much later age than the Homeric epics. They are pæans in praise of Demeter, Apollo, Dionysius, and Hermes, in which the laudation of the beneficent activity of these deities takes the form of a recital of some incident in their lives, followed by a prospective glance at the favour which they may be expected to bestow in the future.

In these cases, the song of praise clearly represients a development of the prayer of thanksgiving. The final and most mature form of prayer, however, the penitential prayer, or, as it is usually called, the penitential psalm, may in a certain sense be called a subform of the petitional prayer. In it, either external need or the consciousness of personal guilt leads the individual to call upon the gods for mercy and for forgiveness of the committed sin. Typical examples are again available in the Hebraic and Babylonian psalms. These psalms contain, in the first instance, prayers of cult, which were offered on the occasion of national disasters and needs, such as crop failure or drought, or, as in the case particularly of the Israelites, were repeated at stated times in penitence for the 
sins of the community. Such being the motives, the most universal form of prayer, that of petition, may here also be discerned in the background. Not only is the penitential psalm in and for itself a particular form of petition, containing as it does a plea for the forgiveness of committed sins, but it is frequently combined with a direct prayer for the favour of the deity and for renewed manifestations of grace through a fortunate turn of destiny. In spite of this egoistic strain, however, which, just as in the case of the song of praise, is seldom absent, the penitential prayer is, religiously speaking, the highest form of prayer, and may be found only at an advanced stage of deity cult. Above all other forms of prayer, its emphasis falls on the inner life; where it comes to expression in its purity, it seeks not external goods, but only peace of conscience. Moreover, more than anywhere else, we find in it a resignation to the will of the deity. This resignation, in turn, draws its strength from the belief that human destiny is in the absolute control of the gods, everything experienced by the individual or by the cult community being interpreted as a divine punishment or reward. Thus, the penitential prayer is closely bound up, on the one hand, with the idea of a divine providence and, on the other, with ideas of retribution. Neither the idea of providence nor that of retribution is to be found in early deity cult ; both are products of the subsequent religious development. Moreover, the issue is not changed by raising the question whether the retribution is regarded as occurring here or in the beyond. As a matter of fact, the retributive idea is far from being implicated with other-world hopes. The conviction that punishment will overtake the guilty man even in this world, because of the direct connection between present fortune and misfortune and the worship of the gods, is itself the immediate source of the idea of a divine power ever controlling the destinies of mankind.

In addition to prayer, however, and usually bound up with it, there is a second important form of cult practice, namely, sacrifice. The usual conception of sacrifice is alto- 
gether too narrow-just as is the case with prayer. Hence the origin and significance of sacrifice have been misunderstood. In view of one of its prominent features in the more highly developed cults, sacrifice is usually regarded as a gift to the deity, and the various meanings that a gift may have are then simply held to apply to sacrifice itself. Accordingly, the purpose of sacrifice is limited either to disposing the god favourably toward the sacrificing individual or community, or to obtaining forgiveness for committed sins. In the Priests' Code of the Israelites, this second form of sacrifice-the trespass or sin-offering-also served the former purpose, thus acquiring the significance of an act of reconciliation which at the same time blotted out any transgressions of the past. The sin-offering, on the other hand, was concerned with purification from a single, definite sin for which the forgiveness of the deity had to be obtained. The peace-offering, therefore, was a cult that was celebrated in common and on a specific day, whereas the sin-offering was brought only on special occasions, when an individual or a restricted group felt the burdens of conscience because of a committed sin. Corresponding to the different purposes indicated by the words 'reconciliation' and 'forgiveness' was the manner in which the sacrifice was brought. The peace-offering was taken to definitely established centres of cult, primarily to the temple at Jerusalem. Those bringing the sacrifice shared its enjoyment with the deity in the sacrificial meal, which was an expression of the covenant concluded with the deity for the future. The sin-offering was made whenever occasion demanded, and the sacrifice was designed for the deity alone. After the removal of the portion reserved for the priesthood, the remainder was burned-those making the sacrifice could enjoy none of it. If we regard both kinds of sacrifice as forms of gift, the peace-offering would correspond more closely to an actual gift with a certain tinge of bribery, though this conception is rendered less crude by the fact that the sacrifice represents also a covenant which receives expression in the sacrificial meal. The sin- 
offering, on the other hand, is more of the nature of a penalty, similar to that which a judge imposes in satisfaction of a crime.

It must be granted that there is a stage in the development of sacrificial cult in which the gift motive is dominant. Nevertheless, even here there are concomitant phenomena which clearly indicate that the sacrifice cannot originally have had the significance of a gift. On the contrary, there has been, in part, a change in meaning and, in part, an arbitrary reinterpretation of phenomena. The Jewish peace-offering was not a true gift. This is evidenced by the fact alone that one of its chief features was the sacrificial feast, which involved the idea of the deity's participation in the meal. In connection with this idea of communion with the deity, the offering of parts of the consumed sacrifice was manifestly only a secondary motive. Nor was the renunciation required of the sacrificer in connection with the Jewish sin-offering a feature which had anything in common with a gift. It was similar rather to punishment. Moreover, all resemblance whatsoever to a gift disappears when we call to mind the earliest forms of sacrifice, as well as the objects that were offered. One of the oldest sacrifices, found even within totemic culture, was that offered to the dead. In its broadest sense, this comprehends everything that was given over to the deceased, or that was burned with him, in case cremation was practised. Such objects originally included some of the belongings of the deceased, particularly his weapons and personal decorations. After despotic forms of government arose, the death of a chief or of a person of influence demanded also the sacrifice of his animals, slaves, and wives. We are already familiar with the change of motives that here occurred. At first, the aim was to keep the deceased from approaching the living ; later, it was to equip him with whatever might be of service in his future life. The sacrifice then became an offering to the demon of the deceased, designed to win his aid for the living. Finally, it was devoted to the gods, whose favour was sought both for the deceased and for the survivors. 
A survey of the development as a whole shows that the gift motive was at first entirely lacking, and that even later it was of relatively little importance. The idea of magic was predominant. The aim was to bring the power of magic to bear upon the deceased and his demon, and finally upon the gods. The demon was to be kept at a distance, just as in the case of burial and of the binding of the corpse, and the gods were to be won over to a friendly attitude. This appears even more clearly when we consider the objects that were sacrificed. In this respect, there was an important change, first mediated, probably, by the cult of the dead, and thence carried over to sacrifice in general. The sacrificer offered such parts of his own body as were held to be the specific vehicles of the soul. Homer tells us that Achilles deposited the two locks of hair, which he had once promised to his native river god, upon the dead body of Patroclus. The use as a sacrifice to the dead of a gift dedicated to a god, clearly indicates that the two forms of sacrifice possessed an identical significance. The deceased takes with him into the underworld part of the person of the sacrificer. Similarly, it was believed that the psychical powers of the deity are, on the one hand, strengthened through the soul which he receives in sacrifice, and are, lon the other hand, inclined toward the one who brings the offering. In animal sacrifice, the blood was poured out beside the sacrificial stone for the enjoyment of the god. Of the inner parts of the bloody sacrifice, it was again those that were in ancient times regarded as the chief vehicles of the soul, the kidneys with the surrounding fat, that were particularly set aside for the god. Closely connected with this is the sacrifice which, through self-mutilation, the priests and temple servants offered in the case of ecstatic cults (pp. $294 \mathrm{f}$.). In all of these instances the ideas of magic and of gift intermingle. The soul-vehicles which are offered are also gifts to the deity, intended for his enjoyment. In partaking of them, however, a magical influence is released by means of which the will of the deity is controlled, or, in the view of a more advanced age, is favourably inclined toward the sacrificer. 
The same idea prevails when public sacrifice demands a human being, instead of an animal, as a vicarious offering for the sacrificing community. Indeed, human sacrifice also has its prototype in the sacrifice to the dead, though the sacrificial idea is in this case kept in the background, inasmuch as the dominant purpose is to equip the deceased with that which he requires for his further life. Human sacrifice proper, therefore, is at most connected with faint survivals of this older practice. In contrast with the latter custom, the individual sacrificed to the deity serves as a substitute for the community. In this form, however, human sacrifice does not antedate animal sacrifice, as has been believed, but follows upon it. Still later, of course, it was again displaced by the latter, as is graphically portrayed in the Biblical legend of Abraham and Isaac. The priority of animal sacrifice is attested, first of all, by its incomparably wider distribution. Human sacrifice, and traditions indicative of it, appear to be altogether restricted to the great agricultural festivals and solstice-cults in which the one who is sacrificed serves, on the one hand, as a substitute for the sacrificing community which offers itself to the deity in his person, and, on the other hand, as the representative of the god himself. Convincing proof of this is furnished by the traditions regarding the seasonal cults of the ancient Mexicans, as these have been reported by K. Th. Preusz. Prior to the sacred festival at which an individual was offered in sacrifice, he was himself reverenced as a god. The twofold significance of the human sacrifice becomes perfectly intelligible in the light of the above-mentioned fusion of the ideas of gift and of magic. Dedication to the deity and union with him merge so completely that they become a single conception. Even the blood poured out upon the sacrificial altar was not merely an offering, but, as a vehicle of the soul, was supposed to transfer to the deity who received it the desires of the offerer. What was true of the blood was quite naturally pre-eminently true when the object of sacrifice was the person himself. 
In this case, all the organs were offered, and, therefore, the entire soul. This is the most extreme form of the sacrificial idea, and occurs only in the sacrificial cult of fairly large political and religious communities. As is characteristic of legend, the 'Abraham and Isaac' story individualizes the ancient tradition, construing the latter as an account of a test of obedience to the god-an interpretation very obviously to be regarded as an invention of later priestly wisdom. On the other hand, the Roman Saturnalia, the Persian festival of Sacæa, and other agricultural cults of the ancient world, exhibit traces of the sacrifice of a human being who represents the deity himself. Along with these we might probably mention also the Babylonian festival of Tammuz and the Jewish feast of Purim. Finally, the Christian conception of the sacrificial death of Jesus combines the same ideas, though their religious significance is transformed and reinforced by the thought of redemption, which has displaced the older protective and fortune-bringing magic. The sacrificial community has here become the whole of mankind, and the one who by his death brings about a reconciliation with the deity is himself the god. For this reason dogma insists-with a logic that is perhaps unconscious and mystical in nature, yet all the more compelling-on the unity of the divine personality with that of the redeemer who died the sacrificial death. This fusion of sacrificial conceptions thus gave rise to the most impressive and effective story that the human mind ever conceived.

Herewith we reach the culminating point in the development of the idea of a gift offered to the deity, and here also the sacrificial object attains its highest worth. That the sacrificer, however, is little concerned with the value of the objects which he brings, is obvious from the fact that these are frequently without any objective value whatsoever. Such, for example, are the small pictures offered in Chinese ancestor cult, and also the miniature representations of desired objects which are placed on votive altarsinstances in which, of the two ideas combined in sacrifice, that of the gift again entirely vanishes, leaving as the sole 
motive the more primitive idea of magic, which never completely disappears. Wherever sacrifice is dominated by the idea of a gift offered to the deity, the sacrificer, in turn, seeks to gain certain ends in return for the value of his gifts. The scale of values may be either quantitative or qualitative, or both combined. Even in the case of the bloody sacrifice both criteria are, as a rule, involved. At the great festivals of Athens and other Greek cities, one hundred steers were sacrificed to the gods, the greater part of the sacrifice, of course, serving as food for the people. In Israel, the rich man sacrificed his bullock, the poor man, his young goat. It was the conception of value that caused especially the fruits of the field, as well as the products of the cattle industry, milk' and butter, to become objects of sacrifice. Later, sacrificial offerings were also made in terms of jewels and money. These were brought to the temple for the decoration of the house of the god and for the support of the cult or the relief of the poor. This development was influenced by another change, connected with the transition from the earlier bloody sacrifice to the bloodless sacrifice. Prior to the influence of the sacrificial customs, the bloody sacrifice involved the loss of the sacrificial animals. These were either entirely burned and thus given to the gods, or their flesh was consumed by the cult miembers at: the sacrificial feast, the god receiving only those parts that were prized as the vehicles of the soul. Now, bloodless sacrifice belongs to a higher stage both of culture and of cult. In general, it presupposes an advanced agricultura! and cattle industry, as well as the existence of more extensive cult-needs whose satisfaction the sacrifice is designed to secure. Thus, the two conditions mutually reinforce each other. The products of agriculture cannot be directly offered to the deity as can the burnt offering, which ascends to heaven in the smoke. On the other hand, the cult cannot dispense with certain means, and these are obtained by utilizing in its interests the economic foresight which has been acquired by the agriculturist and the cattle-raiser in the course of their work. In place of 
the direct products of husbandry, the succeeding age more and more substitutes costly jewels and money. Thus, the development which began with the burnt offering concludes with the money offering. This later offering is no longer made directly to the deity, or, at most, this occurs in the accompanying prayer; the offerer bestows his gifts upon the temple, the priests, or the poor. By so doing he hopes to win the divine favour indirectly, through the merit which such gifts possess or through the cult activities which are purchased by means of them.

The earliest forms of sacrifice are thus more and more displaced by cult agencies which, to a certain extent, themselves approximate to purification ceremonies. This transformation, however, cannot suppress the original sacrificial purpose, which was solely that of exercising a direct magical influence upon the deity. We now meet with phenomena in which this purpose asserts itself all the more potently because of the above development-phenomena from which the idea of a gift possessing objective value is entirely absent. We refer particularly to votive and consecration gifts. These very names, indeed, are evidence of the confusion which a one-sided emphasis of the gift-idea has introduced into the interpretation of sacrifice. For votive and consecration gifts generally consist of artificial objects which are ordinarily devoid of any artistic or other value. They are deposited on the altars of the gods, or, in the Catholic cult, on those of the saints, either to make known a wish, as does the 'gift of consecration,' or, less frequently, to render thanks for the fulfilment of a desire, as in the case of the 'votive offering.' Although these offerings, even in their beginnings, are inseparable from a fairly developed deity cult-since they presuppose altars upon which they are placed, and, therefore, temples consecrated to the gods-it is practically the amulet alone that may be said to rival them in extent of distribution. They occur in ancient Egypt, as well as in Greece and Rome. They were known also to Germanic antiquity, from whence they probably found their way into the Catholic cults of Mary 
and the saints. The consecration gift corresponds to the prayer of petition, the votive offering to the prayer of thanksgiving; these prayers, accordingly, are spoken when the object is placed upon the altar. The gift of consecration is the earlier and more common, just as the prayer of petition precedes that of thanksgiving. The peculiarity of this cult, however, consists in the fact that the object offered as a sacrifice is an artificially fashioned image, usually reduced in size, of the object in connection with which aid is sought. This obviously gives it a certain relationship with the fetish, on the one hand, and with the amulet, on the other. As a matter of fact, the so-called 'consecration gifts' are not in the least real gifts. The sick man presents a figure of the diseased part of his body, fashioned of clay, bronze, or wax, and the peasant who has suffered a loss of cattle brings a representation of the animal. In themselves, these objects are valueless; nor can they be of service to the deity to whom they are brought, as was doubtless believed by the sacrificers to be true in the case of the animal that was slaughtered, as well as of the blood, and doubtless also of the fruits which were offered. The significance of such a gift of consecration lies solely in its subjective value, just as does that of the primitive amulet, which is likewise an article without any objective worth. To believe, however, that this value consists in the fact that the consecration gift symbolizes the submissive reverence of the offerer would be to read back a later stage of religious thought into an age to which such symbols are entirely foreign. Moreover, the purposes of this sacrifice make such an interpretation impossible. The vast majority of consecration sacrifices have another similarity to amulets, in addition to that just mentioned ; those who bring them seek healing from disease. Hence, in ancient times, such offerings were brought chiefly to the temple of Æsculapius. Just as the amulet, in its most common forms, is designed as a protection against dreaded sicknesses, so also does the consecration gift aim at relief from actual suffering. The amulet, however, may be traced far back into the period of demon-cult, and its 
characteristic types, therefore, are patterned on the more prevalent expressions of demon-belief, such as cord magic. The consecration gift, on the other hand, is associated with deity cult, and takes the form of sacrifice. Moreover, it reverts to the most primitive kind of sacrifice, to the purely magical offering. The leg of wax offered by the lame is simply a means of magic. Since it possesses no objective value, it is worthless as a gift, and, as a means of magic, it is again of the most primitive sort. The sacrificial object is regarded as having a soul, quite in the sense of early animism. Through its immanent psychical power it is to exercise magical coercion over the soul of the god or the saint. Its potency is precisely the same as that which the soul of the sacrificial animal or human being is supposed to possess. The only difference is that the external characteristics of animistically conceived objects ordinarily force into the background the idea that the sacrifice magically becomes identical with the deity who receives it, whereas this conception comes out with especial clearness when the offering consists of an animal or of a human being. This is strikingly shown by the abovementioned sacrificial festivals, in which, prior to being offered as a sacrifice, the individual was himself reverenced as the god to whom he was to be offered. True, the fact that the human individual, as well as the animal, possesses a value for those who bring the sacrifice, also introduces the idea of a gift ; added to this, moreover, in the case of human sacrifice, is the further thought that the sacrifice is a substitution for the sacrificial community.

Thus, the idea of a magical effect upon the deity is combined with that of a gift designed to gain his favour. This appears also in connection with the sacrifice of the first-fruits of the harvest or, with what is only a transference from the fruits of the field to the animal used in its cultivation, that of the first-born of the cattle. From the standpoint of the gift theory, such an offering is regarded as a particularly valuable gift. But this greater value is again exclusively of a subjective nature. Objectively 
speaking, the mere fact that it is the first of the fruits or the first-born of the cattle that is offered does not give the sacrifice any additional value. Very probably the decisive factor is the preference which man gives the gods in the enjoyment of the fruits of the field. It certainly cannot be denied that this motive is operative, particularly in later development. That it was the original notion, however, is improbable. Obviously, this offering is closely related to the custom, common even to-day, of leaving the last sheaf in the harvestfield. This custom, which $\mathrm{W}$. Mannhardt was able to trace from ancient times down to rural festivals that are still prevalent, is also of the nature of a sacrifice. On such occasions, an egg, a piece of bread, or the picture of a human being or of an animal, is sometimes tied to the first or to the last sheaf of the harvest and left upon the field. Such acts are obviously due to the need of attributing to the garnered grain life and a soul, as well as the ability to influence by its soul the vegetation demons of the field, and, in later times, the gods who protect the cultivated soil. The custom could scarcely have originated except for the presence, from the very outset, of the idea of a psychical power resident in the sprouting seed. Later, the idea of a gift here also forced the magical motive into the background. Indeed, it may well be that this caused the sacrificial usages which originally, as it appears, marked the end of the harvest, to be put forward to its beginning.

It is only ideas of magic, furthermore, that can account for the practice of divination. Connected with sacrifice are various phenomena that are accidental in nature and unforeseeable on the part of the sacrificer. These phenomena are such as to be sometimes regarded as indications of the acceptance or the rejection of the sacrifice on the part of the deity, while at other times they are interpreted from a different point of view, as general prophetic signs. In the case of the burnt offering, for example, the direct ascent of the smoke to the heavens was regarded as a sign that the deity graciously accepted the offering. Similarly, the examination of entrails, common among Oriental as well as Occi- 
dental peoples, originally, doubtless, had the purpose of discovering whether the animal possessed a nature pleasing to the gods. Later, however, it became one of a large class of general prophetic signs (prodigia), such as the flight of birds, lightning, clouds, and other incalculable phenomena of nature by which the future was predicted, particularly in respect to the success or failure of enterprises about to be undertaken. Because of the general relationship of magic and divination, the sacrificial cult borders upon the oracle. In the oracle, man wishes to read the future; in the sacrifice, he wishes to influence it by his action. This of itself implies that sacrifice occupies the higher plane. The belief in prophetic signs passed over from demon cult to deity worship with relatively little change, except that it became connected with particular gods or priesthoods and was therefore more strictly regulated. The hopes of a beyond, which were involved in the ecstatic practices of the orgiastic cults, opened up a new field to prophecy, and supplied divination with additional methods-the dream and the vision. Though connected in various ways with sacrificial cult, these phenomena are far from containing the wealth of religious motives involved in the former. Nor do they develop any common cult. This is due particularly to the fact that ecstatic visions are dependent upon a certain psychological predisposition, a fact which also enables us to understand the influence exercised by the individual seer and prophet upon religion and cult.

A third, and the highest, form of cult practice consists in sanctification ceremonies. Just as sacrifice is bound up with the various forms of prayer-conjuration, petition, thanksgiving, and penitence-so, in turn, is the sanctification ceremony closely connected with both sacrifice and prayer. On the one hand, it is reinforced by accompanying prayers : on the other, it results directly from sacrifice, particularly whenever the latter takes the form of a cult practice that brings mankind into association with the deity. In this event, the ceremony of sanctification represents an activity supplementary to sacrifice. The impulse to sanctification 
gains the dominance over the sacrificial idea as soon as the desires relating to the personal worth of the sacrificer himself gain ascendancy over the external motives which at first prevailed. This subjective interest, of course, appears only after the religious life has become relatively mature ; at the outset, moreover, it is still everywhere combined with sacrificial practices that centre about external possessions. Once it has finally freed itself, and has become purely a sacrifice designed to enhance personal worth, it becomes a means of sanctification. When sacrifice has reached this highest stage, however, the idea of a gift presented to the deity by the sacrificer completely disappears-in so far, there is a resemblance to the very earliest sacrifices, which were of a purely magical nature and were in no sense intended as gifts. If, therefore, the sacrifice of self-sanctification retains any connection at all with the conception of a gift, the sacrificer must not only be said to offer himself to the deity but the deity must likewise be regarded as giving himself to the sacrificer.

Nevertheless, the origins of sanctification ceremonies and of sacrifice are essentially diverse. At the outset, moreover, these cult practices adopt different paths, meeting only at the height of their development. True, the sanctification ceremony is rooted in magic belief, just as is sacrifice. In primitive sacrifice, however, the magic is directed externally; in the case of sanctification, on the other hand, the object of the magic is the human being himself who performs the cult action or who permits it to be performed upon him. Even in the earliest stages of these practices, therefore, the sanctification ceremony occupies the higher level ; hence, also, this ceremony is subsequent in origin to sacrifice. And yet practices presaging sanctification may be found in much more primitive cults, in the purification ceremonies, whose beginnings may be traced far back into the totemic age. We have already mentioned the fact that water and fire were used as means of magical purification even in the period of demon-belief (pp. $201 \mathrm{ff}$ ). So long as they retain this significance, they may both be classed as 
agencies of counter-magic. Their function is to counteract the evil spells that result from contact with a corpse or with some other object that is regarded as taboo. Purification by fire has the same significance. Because of the more elaborate preparations which it requires, however, such purification tends, from the very beginning, to take the form of a public cult celebration. As a result, it passes over directly from the field of counter-magic into that of magic proper-a reversal common in the field of magical usage. At this point, purification becomes sanctification. For, the original purpose of the means which the latter employs is always that of affording protection against future attacks on the part of the demoniacal powers that threaten man from without, or, in a later and a religiously purified interpretation, against personal transgressions resulting from man's inner nature. Herewith the development reaches the stage of the sanctification ceremony proper. The belief that sanctification is necessary for the individual can arise only in connection with deity beliefs, for it is bound up with ideas of retribution. The latter, in turn, depend upon the feeling of the personal guilt of the individual no less than upon the belief in the existence of personal gods who avenge the sins that are committed. Precisely the same change that takes place in the development of purification by fire transpires also in the case of water, the second and more common means of lustration. Here this transition is most clearly evident in connection with baptism. True, even Christian baptism still partly retains the idea of lustration. For, though the newborn child who is baptized is not himself conscious of any wrongdoing, he is nevertheless tainted, according to the doctrine of inherited guilt, by the original sin from which he must be cleansed. Baptism thus incorporates the meaning both of purification and of sanctification. The latter conception, however, asserts its dominance. And yet the Anabaptists, though insisting that man is unworthy of the sacred act unless he submits to it of his own free will, have also wished to preserve, along with the idea of sanctification, the idea of purification, which 
is both more original and, for sense perception, more real. Moreover, baptism also occurs with this twofold meaning outside the pale of Christianity, not only among the Hebrews, to whom the Christian religion is indebted for the cult, but even elsewhere, particularly among Semitic and African peoples. Sometimes it occurs alongside of another very common custom, that of circumcision; sometimes, as in Christendom, it is found where the latter is lacking ; in still other regions, circumcision is practised, whereas there is no real baptism aside from the ordinary rites of lustration. This diversity itself testifies to the essential difference between the two cult practices-for that circumcision also must be classed as such there cannot be any doubt. Circumcision, however, is not a means either of purification or of sanctification, but is of the nature of a sacrifice. Along with the offering of hair in the cult of the dead and with the pouring out of blood in connection with deity worship, it belongs to that form of sacrifice in which the sacrificial object gains its unique value by virtue of its being the vehicle of the soul. Thus, the object of sacrifice, in the case of circumcision, may perhaps be interpreted as a substitute for such internal organs as the kidneys or testicles, which are particularly prized as vehicles of the soul but which can either not be offered at all, on the part of the living, or whose sacrifice involves serious difficulties.

Originally, sanctification and lustration not only employed the same means but also followed identical methods. The need frequently came to be felt, however, of an external distinction between these two cult practices. Ablution thus came to be regarded as the proper method of actual purification, whereas sprinkling was adopted in connection with sanctification. This also indicates the antithetical positions which the two hold with respect to magic and counter-magic. Lustration aims to remove moral, or, in the last analysis, demoniacal impurity ; sanctification furnishes him who seeks its blessings with water possessed of magical powers. For this reason purification water fell into disuse with the disappearance of belief in demoniacal impurity. On the other hand, it was believed 
that sanctification water must remain as available as possible to him who stands in need of its virtues. Just as baptism is a cult agency whose purpose is intermediate between purification and sanctification, so also does the priest who conducts it lay emphasis, now on the one, and now on the other of these phases. When sprinkling comes to be employed as a means of sanctification, the magical significance of the act leads to a further change. Ordinary water, such as is generally used in lustration, no longer suffices-the water itself needs sanctification if it is to serve the purpose for which it is designed. Even in the ancient mystery cults, therefore, one of the chief elements in the ceremonies of sanctification consisted in sprinkling the members with water from sacred springs. The Jordan festival of the Greek Catholic Church still employs water from the river after which it is named, or ordinary water that has magically. been converted into Jordan water. The relation of the burning of incense to lustration by fire is the same as that of sprinkling to lustration by water. And yet, in the case of incense, the idea of sanctification has almost entirely suppressed the earlier aim of purification. The purpose of sanctification finds its specific expression in the belief that the smoke cannot have a sanctifying effect without the addition of certain other elements. Balsamic substances were therefore used. First and foremost among these, even in ancient times, was incense resin, whose exciting and narcotic odour enhances the magical effect. The herbs and resins that were thrown into the flames, however, were also generally regarded as sacrificial gifts to the gods, whose delight in the ascending odours would, it was thought, render them favourably disposed toward the offerer.

Thus, sanctification ceremony and sacrifice become merged. The highest form of sanctification, moreover, originates in sacrifice itself. It appears as soon as the idea of intercourse with the deity becomes elevated to that of communion with him. This occurs especially in the sacrificial feast. When the sacrificial food is sanctified by virtue of the fact that the deity partakes of it, this sanctification is im- 
parted to those human individuals who receive a share of the sacrifice. In proportion as the worth of the sacrifice increases, so does also the degree of sanctification. The latter reaches its culmination in human sacrifice, where the person sacrificed is the representative both of the sacrificial community and of the deity himself. Sanctification here becomes deification for every participant in the sacrifice. Following the disappearance of human sacrifice, this idea was maintained in connection with the sacred animal that was substituted for man, and finally, after bloody sacrifice was entirely abandoned, in connection with the bread which constituted the sacrificial food. In the most diverse cults of the Old and of the New World, this bread was moulded into the form, sometimes of a human being and sometimes of an animal. In this case again, the sacrificial cult of Christianity unites the various elements. When taken as a whole, the different interpretations that have been given to sacrifice in the Christian world include conceptions representing all the various stages of development. The bread and wine of the sacrament perpetuate the memory of the most exalted human sacrifice known to religious tradition, since, in this case, the idea of the unity of the sacrificial person with the deity continues to survive in the cult of the redeeming deity. In this sacrificial meal, moreover, elements of related sacrificial cults survive-the idea of the paschal lamb, borrowed from the Jewish Passover, and the substitution of wine, as in the Dionysian mysteries, for the blood of the sacrificed god. To the Christian, moreover, this sacrificial sanctification has had three distinct meanings, though these, of course, have frequently been intermingled. There have been magical, mystical, and symbolical interpretations-a series of stages through which all sanctification ceremonies pass. To the uncritical mind, he who receives the bread of the sacrament partakes of the actual body of Christ. Following upon this stage of miracle and magic, is the idea that the cult act effects a mystical union with the Redeemer, a union that is not corporeal but spiritual. At the third stage, the cult action finally becomes the symbol of a religious exaltation of spirit. 
This exaltation is regarded as possible in itself without the external manifestation; nevertheless, it is reinforced by the latter, in accordance with the general relationship that obtains between inner needs and external actions. Moreover, in each of these three cases, participation in the common sacrificial meal is evidence of membership in the religious society-a feature common to all firmly organized religious associations. Such membership must be attested by participation in the cult celebrations. Of the ceremonies in which expression is given to one's religious affiliations, the sacrificial meal has been regarded, from early, times on, as the most important. The end of the development thus returns to its beginning. The meal, enjoyed in common at fixed times, differentiates cultural man from the man of nature. Among all meals in which a relatively large community unites, however, the sacrificial feast is probably the earliest, just as the cult festival is the earliest festival celebration.

\section{I7. The Art of the Heroic Age.}

A survey of the various phases of human interest will show that they are all present from the very beginning in the mental organization of man. Moreover, they are throughout so interconnected that an advance in one field of interest will lead to progress in general. Nevertheless, we are unable to escape the further observation that, in the life of the individual, certain capacities develop earlier than others. Precisely the same is true of the life of humanity. The phenomena in which the character of ages and peoples receives its chief expression differ in each of the periods through which the development of mankind passes. 'The secondary phenomena, in each case, either occur only in their beginnings or, where we are dealing with later stages of culture, are being perfected along lines already established. In this relative sense, we may doubtless say of the three eras following that of primitive man, that totemism is the age of the satisfaction of wants, the heroic age, that of art, and the succeeding period of the development to humanity, that of 
science. Of course, there were many art productions, some of them admirable, even in the totemic age-we need mention only the artistic cult dances, or the high perfection to which the semi-cultural peoples of the period attained in the decoration of the body and of weapons. It must be admitted also that the heroic age already laid imperishable foundations for science. Nevertheless, the main achievements of the totemic age relate exclusively to the satisfaction of the external needs of life. The modes of procuring and preparing food, and the forms of clothing, adornment, implements, and weapons-all originated in the totemic age, and, however great may have been the advances made by succeeding eras along these several lines, the beginnings had nevertheless been made. A manner of dress suitable to the climate had been developed. The preparation of food by means of fire, the manufacture of the fundamental and permanent implements and weapons-the hammer, the axe, the saw, the chisel, the knife-and, finally, the differentiation between weapons of close and of long range, had all been introduced. Moreover-and this is perhaps most significant of all-art itself was governed absolutely by the motive of satisfying. needs. Articles of adornment, tattooing, the dance, song, and music, were first of all means of magic, and as such they served the most urgent needs, such as man by himself was unable to satisfy. These needs were protection against sickness and success in the chase and in war. Only gradually, through a most remarkable heterogeny: of ends, were many of these agencies of magic transformed into pure means of adornment. Such transformations, of course, occurred also in the heroic age. But by this time the necessities of life had in part changed and, of the new interests, those connected with cult and with political organization gained an increasing importance. Esthetic value came to be more and more appreciated as an independent feature of objects. As a result, articles were produced of a nature such as to minister both to the needs of life and to æsthetic enjoyment. But, again, this occurs pre-eminently within the field of spiritual 
needs, particularly in connection with deity cult, on the one hand, and in the glorification of human heroes, on the other. The construction of the temple, the plastic reproduction of the human form and its idealization into the divine image, and, finally, the forms of literature-the epic, the hymn, and the beginnings of the religious drama, with their accompanying music-all of these spring from the spiritual needs of this age, among which needs cult is the foremost. With these various activities, art begins an independent development, gaining a value of its own, and conquering fields that had previously been untouched by æsthetic influences. This conquest of new fields by the higher forms of art is indicative also of an increasing appreciation of the æsthetic, and, along with this, of a spiritualization of life as a whole, such as results, in a particular measure, from art, and only partly, and at a much later period, from science. The first subjects of this art are heroes and gods-that is, those figures which the imagination creates at the threshold of the heroic age, under the influence of the new conditions of life. Gradually art then concerns itself with the human personality and with the objects of man's environment. In correspondence with a change which transpired in the totemic age, in which means of magic were transformed into articles of adornment, the objects of nature and culture are now more and more stripped of their mythological significance and elevated into pure objects of æsthetic appreciation. Thus, the heroic age includes the two most important epochs in the entire history of art. These are the origin of a true religious art, and the attainment of an æsthetic independence which allows art to extend its influence to all departments of human life. Religious art made its appearance with the beginning of the heroic age ; æsthetic independence represents a later achievement. This explains why the totemic age seems to us a vanished world, no less with regard to its art than in other respects. It can arouse our æsthetic interest only if we attribute the final product of this period-namely, decoration freed from its original magical significanceto the motives that really underlie artistic activity. The 
art with which we are still familiar and whose motives we can all still appreciate, begins only with the heroic age. The tattooing of the man of nature and the amulet about his neck are to us adornments of low æsthetic value. A Greek temple, however, may even to-day arouse the mood of worship, and the battles of the Homeric heroes and the tragedy of a Prometheus overtaken by the wrath of the gods may still impress us as real. However remote the age may be which these products of art represent, the general spirit which animated it has not vanished. 'The greatest turningpoint in the spiritual history of man consists in the stupendous achievement which inaugurates the heroic age. I refer to the creation of the ideal man, the hero, and of the god in whom heroic characteristics are magnified into the superhuman and demoniacal. Here lies the beginning of a real history of art; everything earlier is prehistoric, however important it may be for a psychological understanding of art-an importance greater than is generally supposed, since it is only these earliest phenomena that can disclose the conditions underlying the first manifestations of the artistic imagination. Since we may assume that the facts of the history of art are generally familiar, it may here suffice to consider these originating factors and their relation to the general character of the heroic age.

The first and most striking characteristic of the new era is the development of architecture. This is a new art, not to be found in the preceding age, or at most only in very meagre beginnings. The gabled and the conical hut, as well as the tent and the wind-break from which they developed, are not artistic creations, but are products of the most urgent needs of life. The impulse to erect a building for any higher purpose than this, manifested itself first of all when, here and there, the need of the living was attributed also to the dead. For the shelter of the dead, soul and ancestor cults demanded the erection of more permanent structures. Hence there appeared the burial chamber, built of solid stone. Its walls, designed to afford protection from without, were likewise constructed of stone, 
and constantly became more massive. This stimulated a sense of the sublime and eternal, which reacted on the construction of the monuments and gave them a character far transcending the need that called them into being. The development of the gigantic Egyptian pyramids out of the simple walled tomb, the mastaba, tells us this significant story in pictures that impress the imagination more vividly than words. But the cult of the dead, which this history records, was itself intimately connected with deity cult. The preservation of the mummy involved every possible protection of the corpse from the destructive agencies of time. This fact reveals a concern relating to incalculable ages, and thus gives evidence of an idea of a beyond into which the deceased is supposed to enter. Besides the house of the dead, therefore, there is the house belonging to the deity, and this is even more directly and universally characteristic of the age. This edifice, into which man may enter and come into the presence of the deity, stimulates the incomparably deeper impulse to build a structure worthy: of the deity for whom it is erected. Thus, then, we have the temple, designed at the outset for the protection of the sacrificial altar, which had originally been erected in the open, upon consecrated ground. Since it is located at the seat of government, at the place where the citizens assemble for the conduct of political affairs and for purposes of trade, the temple is indicative also of the city and of the State. Secular interests likewise begin to assert themselves. Hence there appears a second mark of the city, the castle, which is the seat of the ruler and of the governing power, and is generally also the final defence, when hostile attacks threaten the city and State. Closely connected with the castle, in all regions in which the ruler lays claim to being a terrestrial deity-as he did, for example, in the ancient realms of the Orient-is the royal palace. In harmony with the twofold position of the ruler, his dwelling is architecturally intermediate between the castle and the temple. Thus, it is the temple, the castle, and the palace, whose development not only awakens the æsthetic sense for 
architectural forms, but also gives impetus to the other arts, especially to sculpture and to ornamentation. The latter had previously found material for its expression in the utensils of daily use. Enriched through its connection with architectural forms, it now recurs to the miniature work of utensils and implements, where it more and more serves a purely æsthetic need. Of the works of architecture belonging to the early part of this period, it is the temple which proves the greatest æsthetic stimulus. This is due not only to its more exalted purpose, but also to the impetus derived from the fact of the multiplicity of gods. The castle represents the unity of the State. Hence the State contains but one such structure, erected, whenever possible, upon a hill overlooking the city. The temple, from early times on, is the exclusive possession of a single deity. The idea of harbouring several deities in a single structure could arise only later, as a result of special cult conditions and of the increasing size of the sacred edifices. Even then, however, the need for unity in the cult generally caused each temple to be dedicated to a specific deity, the chief god of the temple. Hand in hand with this went a striving for richness and diversity in architecture. The temple, therefore, expresses in a pre-eminent degree not only the character of the religious cult, but also the mental individuality of the people to whom the gods and their cult owe their origin.

Closely connected with temple construction is sculpture, for, in it, the importance which the human personality receives in this age finds its most direct expression. Sculpture, moreover, clearly exhibits the gradual advance from the generic to the individual, from a value originally placed on man as such to absorption in the particular characteristics of the individual. The early, 'generic' figure is generally a representation of the divine personality who has inspired the artist to create an image for the sacred shrine. Art does not aim at the outset to copy man himself; it transfers his characteristics to the deity, and only thus, and after laborious efforts, does it attain its mastery over 


\section{4

the human form. True, the gods are conceived as human from the very beginning. So long, however, as the sacrificial stone and the altar stand in the open field, this humanization leads but to inartistic images, similar to fetishes. While these images indicate the presence of the gods at the sacred places, they are not intended as likenesses of the deities themselves. In their external appearance, therefore, the fetishes of early deity cult still impress one as survivals of the totemic age, even though the gods are no longer represented after the fashion of demons, namely, as subhuman, possessing animal or grotesque human forms. The conditions obtaining in life generally were repeated in the realm of art. For the transference of purely human characteristics to the image took place in the case of the hero-or, what amounts to the same thing in the great Oriental civilizations of antiquity, in that of the ruler-earlier than in the case of the deity. The ruler is glorified by means of drawings which represent processions of the hunt and of war, and which are executed on the walls of his palaces. Similarly, the religious impulse expresses itself in the erection of an anthropomorphic image of the deity. This image is placed either in the temple, which is regarded as the dwelling-place of the deity, or in some commanding part of the city which reverences the god as its protector. Here, however, we come upon a noteworthy proof of the fusion of the hero with the demon as described above. From Babylonian and Egyptian monuments we learn that the ruler and his retinue were already represented in human form at a period when deity cult still retained hybrid forms of men and animals, sometimes of the nature of animal demons with human faces, or again as human figures with animal heads. Thus, art strikingly confirms the view that the gods arose from a fusion of the hero personality with the demon. When these external characteristics, due to the past history of gods and their connection with demon beliefs, came to be superseded, the divine image at first reproduced only the typical features of man. In addition to overtowering size, external marks, such as dress, weapons, and sacred animals, were the only evidences of deity. 


\section{THE AGE OF HEROES AND GODS 455}

The first step in the transition from the generic figure to the gradual individualization of personality occurs in connection with the facial expression. It is surprising to note the uniformity with which, in all the civilizations of the Old World, the images of the gods, as well as those of the heroes and rulers, acquire an expression of kindliness and gentleness. This trait, however, is again of a generic nature. The stiff, expressionless form has indeed disappeared, but the expression that supervenes is uniform. Though we have referred to this transition as universal, this is true at most as regards the fact that, on the one hand, the expression of complete indifference gives way to one manifesting emotion, and that, on the other, this emotion, though pronounced, again exhibits uniformity. In the quality of this feeling, differences in the character of peoples may come to light, just as they do in myth and religion, with which sculpture in its first stages is closely connected. In the two great cultural regions of the New World, Mexico and Peru, there is a similar transition. The cults of these peoples, however, emphasize the fear-inspiring character of the gods. Hence, in their art, the terrifying grimace of the earliest divine images becomes moderated into an expression of gloomy, melancholy seriousness-a change such as the art of the Old World approximates only in occasional productions that fall rather within the province of the demoniacal, such as the image of the Egyptian sphinx or the gorgon's head of the Greeks. Thus, the transition from features that are entirely expressionless to such as are generic, and then to those that characterize the individual personality, occurs in connection with a change in the quality of the emotions. To illustrate the relative uniformity of this development we might likewise refer to the early Renaissance. Here again it was necessary to seek a path to the concrete wealth of personality that had been lost. Art reached this goal by way of the pathetic expression of humble submission. As soon as plastic art departs from the typical form, we find not only that a change occurs in the expressions of the face, but also that the entire 
body becomes more lifelike. Along with this, the themes of plastic art pass from the gods, rulers, and heroes to the lower levels of every-day life. Even here art at first continues to be fascinated by the great and conspicuous, though it later gains more and more interest in the significant. This striving for reality in its wealth of individual phenomena is characteristic not only of sculpture, however, but also of painting. Disregarding the bodily form in favour of the portrait, painting first acquires new means of characterization in colour and shading ; then, passing from man to his natural environment, it wins from nature the secrets of perspective, and thus gains a far greater mastery over the depths of space than was possible to sculpture. Landscape painting, moreover, unlocks for art that rich world of emotions and moods which man may create from the impressions of nature, and which attain to purity of expression in proportion as man himself disappears from the artistic reproduction of his environment. Thus, the final product of pictorial art, together with such paintings as those of still life and the interior, all of which are psychologically related inasmuch as they express moods, represent the most subjective stage of art, for they dispense with the subject himself whose emotions they portray. All the more, therefore, are these emotions read into nature, whose processes and activities now constitute the content of personal experience. Once it attains to this development, however, landscape art is already far beyond the borders of the heroic age. Indeed, the Renaissance itself advanced no farther than to the threshold of this most subjective form of pictorial art. This art represents the hero-however broad a conception of him we may form-as in all respects a human individual. Thus, art again returns to the being whose ideal enhancement originally gave rise to the hero.

The changes which the forms of æsthetic expression undergo within the field of formative art, are paralleled, on the whole, by those of the musical arts. By this term, as above remarked, we wish to designate all those arts which depend from the outset upon the external factors 
of tone and rhythm ultimately employed most freely in music (cf. p. 262). In the preceding age, only one of these arts, the dance, really reached any considerable development. Of the two elements of the musical arts, rhythm was as yet predominant. The dance received but little melodic support from the voice; noise instruments had the ascendancy over musical instruments. The further development of these arts leads to continued progress, particularly with respect to the melodic forms of expression. These begin with the language of speech, and gradually pass on to the pure clang formations produced solely by manufactured instruments. Corresponding with this external change is an inner change of motives, influenced, of course, by the varying materials which enter into the creations of the musical arts. From the very beginning, the character of this material is involved in constant change, as is also language, which is the basis of all these arts, and whose rhythmical-melodic forms cannot be arrested at any moment of its living development. The attempt to render permanent some of the movements of this flowing process, by means of literary records or definite symbols, is but an inadequate substitute for the enduring power with which the mute creations of sculpture and of architecture withstand the destructive influences of time. Just because of this plasticity of their working material, however, the musical arts are enabled all the more faithfully to portray the thoughts and feelings that move the artist and his age. Particularly where these thoughts and feelings are directly reproduced in language, the work, even though coming down from a long-departed past, has an incomparably greater power to transport us to its world than is ever possible to plastic art. How much more vividly do we not experience the life of the Homeric heroes while reading the Iliad than when viewing the Mycenian art of that period !

Of all the products of the verbal arts, it is the epic that most faithfully mirrors the character of the heroic age as a whole. The human hero here stands in the forefront of action. His battles and fortunes and a laudatory descrip- 
tion of his qualities constitute the main themes of the poem. In the background, appears the world of gods. It receives no attention apart from its relation to the action. The gods, it is true, take a hand in the destinies of the heroesthey quarrel about them, or, when the need is greatest, descend to the earth and, though unrecognized, assist them in battles. As for the rest, however, their life lies outside the sphere of the epic narrative; it appears to be an even and undisturbed course of existence into which change enters only in so far as there is a participation in the affairs of the terrestrial world. Such is the epic at the zenith of its development and as it receives expression in the Homeric poems. Though such poetry be traced back to its beginnings, the gods will not be found to play any greater rôle, as we should be led to expect were the theory of many mythologists true that the hero saga developed out of the deity saga and, correspondingly, the heroic epic out of the deity epic. In confirmation of our assertion, we might point to the Russian and Servian romances, and also to the songs of the Kara-Kirghiz and to the Finnish Kalewala, though the Kalewala has not come down to us in quite its original form. The Norse Edda, which has been at the basis of certain misconceptions regarding this question, should not here be drawn into consideration, though, were it examined, it would substantiate, if anything, the opposite of what is supposed. It dates from a later period, which no longer believed, as we may assume that the Homeric rhapsodists did, in the gods and heroes of which it sang. The Norse skalds dealt, in their songs, with a departed world, whose memory they endeavoured to renew; they drew their material from märchen-myths and from folksagas. If, now, we turn to that poetry of the Slavic and Turkish tribes which is really preparatory to epic poetry, we find certain radical differences. Here also, of course, there are imaginary beings who either take a hand in the battles and destinies of the heroes or, through the magic over which the human hero as yet still frequently disposes, come to identify themselves with heroes. These beings, however, 
are not gods, but demons. They possess no personal traits whatsoever. Such traits are lacking also to the hero in proportion as he makes use of magical powers rather than of an enhanced measure of human ability. Thus, it is the world of demons, not that of gods, which forms the background of the early epic. As regards the hero himself, it is apparent from his characteristics that he is on the border-line between the hero of märchen and the epic hero. This development of the epic again mirrors the development of the hero saga described above. But, since epic poetry gives permanence to the unstable characters of the folk-saga, and thus, in turn, reacts upon the saga itself, its development is all the more capable of presenting a clear picture of that fusion of demon with human hero which gave rise to the god. It is by virtue of his human characteristics that the hero of the early epic is distinguished from the demons whose world as yet always forms his scene of action. These human characteristics are then more and more transferred to the demons. Throughout all these changes of environment, the hero remains the central figure of epic poetry, and continues to develop purely human characteristics. Hence it is that, at a later period, the gods again completely disappear from the action, and the destinies of human heroes come to be the exclusive concern of the epic. At this stage, it is no longer external factors that determine the destiny of the hero, as they did when demons and, later, gods were supreme ; inner motives, whose source lies within the hero himself, are of paramount importance. When this occurs, however, epic poetry has already passed beyond the boundaries of the heroic age.

At one time it was held that the Homeric epic, so far from marking the climax of a development in which the world of heroes was brought into relation with that of the gods, really inaugurated epic poetry. During this period, the rhythmic-melodic form of Homer was regarded as the beginning of all narrative. Indeed, at times it has been thought to represent the beginning of language. Following the view of Jacob Grimm, it was 
maintained that poetry was the earliest form of speech, and that prose came through a process of deterioration analogous to that by which prehistoric deity and hero sagas passed into the märchen. This theory, of course, is just as untenable for the history of language and poetry as it is for that of the saga. The original narrative is the märchen-myth that passes artlessly from mouth to mouth. The transition to a form which is at first loosely constructed and then more strictly metrical, is clearly bound up with the transition from the hero of the märchen to the hero of the saga. Coincident with this, gods also gradually gain a place in epic poetry. This development is accompanied by two important external changes. The first of these involves the transformation of the every-day prose, in which the märchen-myth had been expressed, into rhythmic-melodic forms. These are reinforced by a simple musical accompaniment that gives to the diction itself the character of a recitative melody. The second change consists in the fact that separate narratives are joined into a series, the basis of connection being, in part, the heroes who participate in the action and, in part, the content of the action itself. Thus, a romance-cycle arises, which, when supplemented by connecting narratives, finally develops into a great epic. As might be supposed, it is primarily the first and the last stage of this development that are accessible to direct observation-the romances of the early epic, preserved in folk-poetry, and the perfected poems, such as the Homeric epics and the Niebelungenlied. As regards the formation of these epics out of their separate elements, we can do no more than to frame hypotheses on the basis of somewhat uncertain inferences relating to differences in style and composition. There can be no doubt, however, that the more important step as regards the form of the epic, namely, the development of rhythmicmelodic expression, was directly bound up with its very first stage, namely, with the appearance of the earliest form of the heroic narrative-a form resembling the romance.

But how may we account for this origin? Does the narrative of itself rise to song because of the more exalted 


\section{THE AGE OF HEROES AND GODS 46I}

character of its content? Or, is the rhythmic-melodic form imposed upon it from other previously existing types of poetry? Such poetry exists. The simple songs of primitive man we have already come to know ; besides these, there are the cult-song, whose conjurations and petitions were addressed to demons prior to the advent of gods and heroes, and, finally, the work-song. This at once indicates that we must postulate a transference from the lyric type of song, taken in its broadest sense, to the narrative. Nevertheless, the first of the above-mentioned factors must not be disregarded. The heroic hero, of course, arouses far greater admiration and enthusiasm than did the märchen-hero. Here, as in the case of the song, the intensification of mental excitement causes its verbal expression to assume rhythmic forms, precisely as the dominance of festive and joyous emotion in the dance transforms the external movements of the body into rhythmical pantomime. Doubtless, therefore, it was primarily from the cult-song, and under the influence of a related poetic ecstasy, that a sustained rhythmical form was carried over to the portrayal of the hero personality and his deeds. And so, as is clearly shown by the romance-like beginnings of epic composition, the metrical form of the epic first follows current songforms, and then gradually adapts these to the specific needs of the narrative. Now, the earliest characteristic of the song, and that which at a primitive stage constitutes almost its only difference from ordinary speech, is the refrain. In the epic, the rhythm becomes smoother. The refrain disappears entirely, or occurs at most in the case of regularly recurring connective phrases or of stereotyped expressions relating to the attributes of the gods and heroes. These aid the rhapsodist in maintaining an uninterrupted, rhythmic flow of speech, and also continue to be used as means for intensifying the rhythmic impression.

Epic poetry thus develops out of the earlier forms of lyric composition, through a process by which the exalted mood of the song is transferred to the portrayal of the hero personality. Finally, however, the epic itself reacts upon the lyric. 
Here again the cult-song occupies the foreground. When it reaches the stage of the hymn, its most effective content is found in narratives that centre about divine deeds which far transcend human capacities, or about the beneficent activity of the deity toward man. The tendency to incorporate such narratives is particularly marked in the song of praise and thanksgiving, which comes to occupy the dominant place in religious cult for the very reason that the mood which it expresses is at the basis of the common cult. At this point, cult acquires a further feature, the preconditions of which, however, date back to the age of demon cults. Even in the case of demons, aid was sought not merely by means of conjurations but also by means of actions that imitated, in dances and solemn mask processions, the activities of demons. In the great vegetation festivals of New Mexico and Arizona, which are intermediate between demon and deity cults, there were imitative magical rites connected with the subterranean demons of the sprouting grain, with the rain-giving cloud demons above the earth, and also with the bright celestial gods who dwell beyond the clouds. After having originated in this sequence, these elements became united into a cult dance whose combination of motives resulted in the mimetic play, the imitative and pantomimic representation of a series of actions. Thus, the mime itself is the original form of the drama, which now takes its place beside the epic as a new form of poetry. What the epic portrays, the drama sets forth in living action. This accounts for the fact that, even in its later independent development, dramatic literature draws its material principally from the epic, or from the saga which circulates in folk-tradition as an epic narrative. Moreover, as may be noticed particularly in the history of the Greek drama, the transition was made but slowly from the individual rhapsodist, who sufficed for the rendering of the epic song, to the additional players necessary for setting forth the narrative in action.

How essentially uniform this transition is, in spite of widely divergent conditions, is illustrated by, the origin of 
the religious plays which grew out of the Christian cult. In reading the gospel, the priest assigned certain passages, originally spoken by participants in the particular event, to sacristans or priests associated in the ceremony, and the chorus of worshippers represented the people present at the event. In spite of, or, we might better say, because of their more recent origin, these Easter, Passion, and Christmas plays represent an early stage of development. In them, we can still follow, step by step, the growth of dramatic art out of church liturgy, and the resultant secularization of the religious play. Heightened emotion results in an impulse to translate the inner experience into action, and thus dramatic expression is given to certain incidents of the sacred narrative that are particularly suited for it. This tendency grows, and finally the entire scene is acted out, the congregational responses of the liturgy passing over into the chorus of the drama. Common to the responses of the congregation and the chorus of the dramatic play, is the fact of an active participation in that which is transpiring. Though this participation is inner and subjective, in the one case, and objective, in the other, the response of the congregation to the priest in the liturgy is nevertheless preparatory to the chorus of the drama. It is inevitable, however, that this change should gradually lead to a break with liturgy. The portrayal of the sacred action is transferred from the church to the street; the clergy are supplanted by secular players from among the people. Even within the sacred walls folk-humour had inserted burlesque episodes-such, for example, as the mimic portrayal of Peter's violence to the servant Malchus, or the running of the Apostles to the grave of Christ. These now gained the upper hand, and finally formed independent mimetic comedies. The serious plays, on their part, also drew material, even at this time, from sources other than sacred history. The newly awakened dramatic impulse received further stimulus from various directions. 'The old travelling comedy, wandering from market to market with its exhibitions, now of gruesomely serious, now of keenly 


\section{ELEMENTS OF FOLK PSYCHOLOGY}

humorous, action, was a factor in the creation of the modern drama, no less than were the amusing performances of the accompanying puppet-show. Added to these, as a new factor, was the short novel, a prose narrative cultivated with partiality particularly since the Renaissance; there was also its elder sister, the imaginary märchen, as well as the epic of chivalry in its popular prose versions, and, finally, that which more clearly approximates to the religious starting-point, the saint legend-all of these united in giving impetus to the modern drama.

Now, the similarity of this development to that of the ancient drama is so marked that, even where details are lacking, we may regard the nature of the transitions as identical so far as their general features are concerned. Indeed, we should doubtless be justified in assuming that in whatever other localities a dramatic art was perfected, as, for example, in India, the course of development was essentially the same as that which has been described. True, the development cannot proceed to its termination apart from an advance in cult and poetry such as was attained but rarely. Its sources, however, are always to be found in universal human characteristics which were operative in the very beginnings of art and cult. The two factors upon which the later drama depends may be detected even in the corroboree of the Australians. The corroboree is a cult dance whose central feature is a regulated imitation of the actions of totem animals, accompanied by song and noisy music. This imitation of animals also leads to the insertion of humorous episodes. Indeed, even in the corroboree, these episodes are frequently so numerous as to crowd out completely the cult purpose-an early anticipation of the secularization which everywhere took place in the art that originated in cult. In numerous other details as well, the continuity of development is apparent. Suggestions of the animal dance occur in the satyric plays of the Greeks. This same satyric drama took over the phallus-bearing choral dancers from the vegetation festival. In striking correspondence, as $\mathrm{K}$. Th. Preusz 
has pointed out, and indicative of analogous customs, are the phallephoric representations found in ancient Mexican cult pictures. The puppet-show, which was perhaps not the least among the factors leading to the secularization of the drama, was not only universally to be found during the Middle Ages, but in India it made its appearance at an early period. It occurs even among peoples of nature, as, for example, among the Esquimos. Among these peoples, the doll and its movements always represent an imitation of man himself and of his pantomimes. But, though the tendencies to dramatic representation and, in part, even the beginnings of the drama, reach back to the early stages of art, the developed drama was the product of a later period, and was dependent for its rise upon almost all the other verbal and mimetic arts. The drama, however, may always be traced back to deity cult. The religious hymn which extols the deeds of the gods is a direct incentive to the translation of these deeds into personal action. The motives for the dramatic elaboration of liturgy were present particularly in those deity cults which combined soul cults with ideas of a beyond, and which centred about the life, the sufferings, and the final salvation of the gods, and the transference of these experiences to the human soul. The development of the mediæval Easter and Passion plays may be traced, step by step, from their origin. It is this development, particularly, that throws clear light upon early Greek and Indian drama, whose beginnings in the mystery cults are rendered obscure by the secrecy of the cults.. These latter dramas, in turn, clearly indicate that the original source of dramatic representations is to be found in the very ancient vegetation ceremonies, which, in part, were transmitted to the heroic age from a period as early as that of demon cults. After the dramatic performance has been transferred from the temple to the market-place and the drama has become secularized, the further course of development naturally differs both with the conditions of the age and with the character of the culture. Nevertheless, however, the epic 
narrative, the mimetic representation, and the older forms of the song may have coöperated in the development of the drama, the latter, like the epic, steadily descends from the lofty realms of the heroes and gods, down to the dwellings of men. In the portrayal of human strivings and sufferings, moreover, the centre of interest shifts from the mysterious course of external events to the secrets of the human soul. But herewith again the drama transcends the boundaries of the heroic age. Its beginnings grow out of early deity cult. In its final stages, dramatic art, with its insight into human life as it is directly lived, becomes the vehicle of the idea of humanity in the entire scope of its meaning, comprehending both the heights and the depths of human life.

Closely bound up with the psychological motives underlying the development of the drama is the last of the musical arts-namely, music. We may refer to it as the last of these arts for the reason that it attained to independence later than any of the others. As a dependent art, however, accompanying the dance, the song, or the epic recital, it dates back to the age of primitive man. Musical art, also, received its first noteworthy stimulus from cult, as an accompaniment of the cult dance and the cult song. The strong emotions aroused by the cult activity caused a constantly increasing emphasis to be placed on the musical part of the ceremony, leading particularly to the development of melody. The polyphonic song of the many-voiced chorus of the cult members, and the music of the accompanying instruments which gradually assumed the same character, eventually developed into harmonic modulation. This introduced musical effects of a novel sort, such as were not possible for the accompaniment of the reciting rhapsodist and were attained only imperfectly by the common song. Thus, dramatic and musical art both sprang from the same religious root, the liturgic ceremonial, thence to pursue different directions of development. Later they again united in the case of certain particularly emotional parts of the dramatic action, first of all in the choral song, which is thus reminiscent of their common origin in liturgy. 
With this exception, however, the emancipation of dramatic and of musical art from their common cult origin was succeeded by a long period in which they remained distinct. Hence it is certainly not without significance that the creator of the modern art-synthesis, the music drama, himself felt his achievement to be religious in character. Whether or not this may be affirmed as regards the content of the music drama, it is true so far as the fact of combining the two arts is concerned. But it is no less noteworthy that in this case also the separation of itself engenders the motives for the reunion. When the drama was transferred from the temple to the public market-place and then descended from the sphere of gods and heroes to the reality of everyday life, it lost, first its musical-melodic form, and then its elevated rhythm, thus giving way to prose. The liturgic song that survived in the cult, however, entered into reciprocal relations with the secular forms of the song, and a copious interchange of melodic motives ensued. With the same justification, perhaps, as in the case of the origin of the dramatic play in general, we may interpret the older developments by reference to the interchange between sacred and secular songs that took place in Christendom during the Middle Ages. The endeavour to combine dramatic with lyric and musical enjoyment gave rise to hybrid forms of art, to the musical play and the opera. This prepared the way for the further attempt to transcend these composite forms of art by creating a new unity of drama and music. Thus, the aim was to restore the original synthesis on a higher plane, not limited to particular religious cults but taking into account universal human emotions. Yet the entire development of this later art, as well as that of its component elements, the drama and the song, again carries us far beyond the limits of the heroic age. It extends over into a period in which, on the one hand, man supplants the hero and, on the other, the religious advance to a superpersonal god displaces those deities who suffer from the defects which they have inherited from their human 
prototypes and their demon ancestors-namely, the personal gods.

Along with the above-mentioned development of musical art there is also a second change, which appears on the surface to be antithetical to the former, but which in reality supplements it. This change consists in the separation of musical expression from the various elements with which it was originally connected, and in its entrance upon a free and independent development. In the recitative of the rhapsodist, in the liturgy of the temple service, in dance and song, the rhythmic-melodic elements are, to a certain extent, limited by the rhythmic-melodic possibilities of language. In part, it is true, they have freed themselves from this limitation-namely, in the instrumental accompaniment-and yet they fail to attain to independence so long as they are but means for intensifying the expression which emotion receives in language and mimicry. From this double bondage to the rhythmic-melodic powers of human expressive movements and to the thought content of language, musical art finally frees itself. While the musical instrument was at first a means designed to assist man in his endeavour to give direct expression to his emotions, man's activity in the case of 'absolute music' becomes limited to the mastery of the instrument itself. This renders available a wealth of new tonal possibilities, and adds an inexhaustible supply of new motifs for the expression of feelings and emotions. Musical art thus becomes purely a language of emotions. Free from connection with specific ideas, it in no wise restricts the experiences which the hearer may enjoy. It affects these experiences only in so far as the musical production is itself a portrayal of pure emotions. Inasmuch as music is not bound by concepts or ideas, its effect upon the hearer will be the purer and the more intense according as he is the more receptive to the particular emotions in question. In the form of the instrumental composition, therefore, music is the most subjective of the musical arts, as are landscape-painting and its related forms, though not in so pronounced a degree, of the plastic 


\section{THE AGE OF HEROES AND GODS 469}

arts. Like these arts, and even more so, music is the expression of purely subjective feelings. Hence, it, as well as they, far transcends the boundaries of the heroic age, whose fundamental characteristic is attachment to the objective world. In the heroic age, the individual may indeed transfuse the outer world with his emotions, but he is never able to isolate his emotions from objects. Consequently, though art places its media at his disposal, he is unable to utilize them in giving expression, in its independence, to the inner life of personality. 


\section{CHAPTER IV}

\section{THE DEVELOPMENT TO HUMANITY}

\section{The Concept 'Humanity.'}

THE question, Do we live in an enlightened age? was answered by Kant, with reference to his own time-which, as is well known, laid claim to the distinction-flatly in the negative. He added, however, that the age was doubtless one of increasing enlightenment. One might, perhaps, be even more justified in raising a similar question with reference to the relation of our own and of preceding ages to a universally human culture, and in answering: We are on the way to this goal, but are still far from having actually reached it. Indeed, in view of human imperfection, it may be doubted whether we will ever be able to reach it, unless the imperfection itself be included as an element in such a culture. The ambiguity of the word 'humanity' is such that it may signify human weaknesses as well as human sympathy and other virtues. It was in the latter, the more favourable, sense of the term that Herder, even in his day, attempted, in his "Ideas," to portray the history of mankind as an "education to humanity." This expression suggests that history manifests only a ceaseless striving toward true humanity; the goal itself lies beyond the reach of possible experience.

Now, a survey of the course of progress described in the preceding chapters may well cause us to doubt whether the presupposition from which Herder set out in his reflections on the philosophy of history is correct. The assumption that factors preparatory to the development 
to humanity are already to be found in the original nature of man-indeed, even earlier than this, in the general conditions of his natural environment-is not beyond question. Neither primitive nor totemic man shows the faintest trace of what we should, strictly speaking, call humanity. $\mathrm{He}$ gives evidence merely of an attachment to the nearest associates of horde or tribe, such as is foreshadowed even among animals of social habits. In addition, he exhibits but occasional manifestations of a friendly readiness to render assistance when danger threatens at the hands of strangers.

It is not until the heroic age that we encounter phenomena such as might properly be interpreted to indicate the gradual rise of feelings of humanity. But if we take into account the entire character of this age, we are more inclined to contrast it, precisely when it reaches its zenith, with all that we to-day understand by humanity. Consider, for example, the sharply demarcated State organizations of the heroic era, its depreciation of strange peoples, and its repudiation of universal human ties, brusquely expressed during times of war in its treatment of the enemy and, during times of peace, in slavery. The question as to whether and in how far the beginnings of our ideas of humanity reach back into the past and prevail at lower levels of culture, is confronted with a serious difficulty. Conceptions such as these are obviously themselves products of a long development and have been in constant flux. The concept 'humanity' suffers from an ambiguity which has attached to it ever since the time of its origin, and which has in no wise diminished as the word has acquired broader meanings. The word humanitas, which in later classical Latin was practically equivalent to our concept ' human nature,' in both its good and its bad connotations, acquired an additional meaning in the language of mediæval scholars. During this period of strong partiality for abstract word formations, the term came to be used also for the collective concept 'mankind,' that is, the Roman genus hominum-a concept independent of value judgments of any sort. Thus, the word passed 
over into our more modern languages with a twofold significance. Although the German language developed the two words Menschlichkeit and Menschheit, corresponding to the conceptual distinction just indicated, the two meanings were again combined in the foreign word Humanität. This is exemplified by Herder's phrase, Erziehung zur Humanität (education to humanity). For, in using this phrase to sum up the meaning of history, Herder meant that the striving which underlies all history was not merely for the development of the qualities of humanity (Menschlichkeit), in the highest sense of the term, but also essentially for their gradual extension to the whole of mankind (Menschheit).

But, whatever our opinion concerning the possible success of such striving and concerning the relation of its two phases, there can be no doubt that the concept 'humanity,' which has become common property among civilized peoples, combines an objective with a subjective aspect. On the one hand, 'humanity' means the whole of mankind, or, at any rate, a preponderant part of it, such as may be regarded as representative of the whole. On the other hand, 'humanity' is a value-attribute. It has reference to the complete development of the ethical characteristics which differentiate man from the animal, and to their expression in the intercourse of individuals and of peoples. This latter thought incorporates in the term 'humanity' the meaning both of 'mankind' and of 'human nature,' although it ignores the secondary implication of human imperfection which 'human nature' involves and takes into account only its laudable characteristics. Humanity, when predicated of an individual, means that he transcends the limits of all more restricted associations, such as family, tribe, or State, and possesses an appreciation of human personality as such; in its application to human society, it represents a demand for an ideal condition in which this appreciation of human worth shall have become a universal norm. This ideal, however, is subject to growth, and, like all ideals, is never completely realizable. Hence the following sketch of the con- 
ditions which succeed the age of heroes and gods cannot undertake to do more than point to the phenomena that give expression to the new motives that dominate this later period. Sharp demarcations are in this instance even less possible than in the case of the earlier stages of human development. The more comprehensive the range of human strivings and activities, the more gradual are the transitions and the more fully are the underlying motives-precisely because they involve the universally human-foreshadowed in the natural predispositions and impulses of man. Tendencies to esteem man as man, and a willingness to render him assistance, are not foreign even to the primitive mind. Even at the beginnings of human culture there are present, dimly conscious, those tendencies out of which the idea of humanity may finally develop. Moreover, every later advance seems to lead in the direction of this conception. The transition from tribe into State, the changing intercourse of peoples, and the spread over wide regions of the mental creations of a single people, of language, religion, and customs-all these phenomena are obviously steps on the way to the idea of humanity and to its permanent incorporation into all departments of human endeavour. Neither in its rise nor in its further changes, moreover, does this new idea entail the disappearance of previous conditions or of the psychical factors involved in their development. On the contrary, humanitarian culture takes up into itself the creations of preceding eras, and allows them to take firmer root. Thus, the idea of a cultural community of peoples has not weakened, but, so far as we may conclude from the past course of history, has strengthened and enriched, the self-consciousness of separate peoples and the significance of the individual State. The dissemination of cultural products has not resulted in their decrease. National differences have led rather to the increase of these products, and have thus enhanced the value attaching to the spiritual distinctiveness of a people and of the individual personality. That we may here, even more than in the case of the earlier periods of cultural 
history, speak only of relative values, needs scarcely be remarked. Humanitarian development includes à vast number of new conditions, in addition to those that underlie the preceding stages of culture. Since, moreover, the synthesis at which this development aims is everywhere still in the process of becoming, the way itself is for the time being the attainable goal. We may neither be said to be on the way to humanity, if we mean by this a condition in which none but humanitarian interests prevail, nor does a humanitarian age, in the sense of the exclusion of more restricted human relations, appear at all within the field of vision disclosed to us as a result of past history. As a legacy from the primitive era, man has permanently retained not only the general needs of individual life but also the most restricted forms of family and tribal organization. In like manner, it will be impossible for an age of humanity ever to dispense with the more limited articulations of State and society that have arisen in the course of cultural development. Scarcely any general result stands out as more certain, in a retrospective survey of our investigations, than the fact that, while every period discards as worthless a vast number of products; some of which were valuable to an earlier age, there are other products which prove to be imperishable. From this point of view, that which precedes is not merely preparatory to the further course of development but is itself the beginning of the development. The immediate beginning, however, is veiled in obscurity. The earlier age is ever unconsciously preparing the way for one that is to come. The clan of primitive tribal organization had no idea of a coming State, nor had the ancient demon worshipper any notion of a cult of rewarding and punishing celestial deities, yet State and deity cult could not have arisen except for clan and demon-belief. Similarly, the earlier modes of collective life possessed the idea of humanity only in the form of a hidden germ'. Hence we may not properly describe these preparatory stages, which exhibit phenomena of a different and, in part, an entirely dissimilar sort, as 
a development to humanity. The term applies rather to an age in which the idea of humanity, having come to clear consciousness, exercises an influence upon the various phases of culture, and is entertained by a sufficiently large portion of mankind to insure its permanent effectiveness. But even with this limitation the development may not be regarded as one of uninterrupted progress. However widely disseminated the humanitarian idea may come to be, there will remain localities and levels of culture to which it has not penetrated. But, inasmuch as peoples of very different cultural stages enter into relations with one another, the possibility is open for such a turn of events as will obscure the idea of the development to humanity for long periods. That such deviations from the path of progress have frequently occurred in the past is certain; that they are never to occur in the future is scarcely probable. For this reason one can scarcely hope to do more than to show that, in spite of such retrogressions, the development to humanity forms a generally connected whole, and that here also psychological law is regnant.

That such law prevails is at once evident from the fact that of the two conceptions which we have found to be involved in the idea of humanity, the external and objective concept expressed by the collective term 'mankind' is historically the earlier; the concept referring to inner characteristics, and associated in the consciousness of the individual with clearly defined value-feelings, follows only gradually. We might express this relationship by the phrase, Mankind must prepare the way for human nature. This does not imply that isolated manifestations of the latter might not long precede the rise of the idea of mankind-indeed, must necessarily have preceded it, in so far as a predisposition is concerned. It means merely that human nature did not, as a matter of fact, attain to its complete development, nor was it able to do so, until after the idea of the unity of mankind had progressed beyond the stage of vague impulses or of recognition on the part of but a few individuals in advance of their 


\section{ELEMENTS OF FOLK PSYCHOLOGY}

age. In other words: The collective concept ' mankind,' as representing, not merely a generic term created by the intellect, but a real totality ultimately uniting all its members in a social whole, preceded the concept 'human nature,' as connoting a recognition of universal human rights to which each of the members of the human race may lay claim, and of duties which he, in turn, owes to human society. The case could not be otherwise. Unless the idea of mankind were already present in some form, even though this be at the outset inadequate, the requirement that an individual give expression to humanitarian sentiments would be impossible, since there would be no object of the activity. If we consider the sequence of the various phenomena involved in the development to humanity, we find a striking agreement between history and the results to which our analysis of the concept 'humanity' has led us. The earliest of the phenomena here in question dates far back to the beginnings of the events known to us through historical monuments, and consists in the rise of world empires. Though the term 'world empire' is sometimes used to refer merely to a great kingdom that results from the absorption of a number of separate States, such a use of the word does not do justice to its meaning. The idea of world empire really comes into existence only at the moment when such a kingdom lays claim to embracing the terrestrial part of the universe, and therefore the whole of mankind, however much this claim may represent a mere demand which has never, of course, actually been realized. The very fact of the demand, however, itself involves the conscious idea of a unity embracing the whole of mankind. Moreover, the endeavour to realize this ambition follows with inner necessity in the case of all political organizations that call themselves world empires, particularly at the period of their zenith and of an increasing consciousness of power. This leads to further important results, which, though at first doubtless not consciously sought, nevertheless later increasingly become the object of voluntary endeavour. Though externally retaining the traditional political organization, the world. 
empire required an extension of the institutions of law and of administration that had thus far prevailed in the more limited State. A similar change gradually took place in connection with intercourse and its fostering agencies, and subsequently in connection with language, customs, and religious beliefs. Thus, it was the world empire that first prepared the way for world culture, only meagre beginnings of which existed in the period of a more restricted political life. The extension of wants and of the means of their satisfaction was first evident in the field of commerce, though a similar tendency came more and more to prevail in the various departments of mental life. Pre-eminent among these interests was the one which is the most universal and is based on the most common needs, such as are experienced by all members of human society, namely, religion. Thus, as one of the last of the creations possessing universal human significance, world religion makes its appearance. The preceding age did not progress beyond national religions. However much the mythological elements of cult, in particular, may have travelled from one people to another, these elements were assimilated by the national religions. Inasmuch as these religions continued, on the whole, to preserve their own identities, the fact that any elements were of foreign origin very soon disappeared from the folk-consciousness. Not until the period which we are now discussing do we find religions that lay claim to being universal. Even though this claim may remain a mere demand, just as in the case of the world empire, it is precisely as such that every historical world religion has asserted its influence. This striving for universality is far keener in connection with world religion than it is in the case of world empire and world culture. In comparison with this endeavour to become universal, the fact that no period ever witnessed merely a single world religion is relatively unimportant, though not to be overlooked in considering the spiritual needs of mankind. Disregarding subordinate religions and such as are of less significance for culture as a whole, there are at least 
two great world religions, Christianity and Buddhism. These have asserted themselves side by side, and will presumably continue further to maintain themselves, inasmuch as they correspond to sharply defined characteristics of universal world culture. Finally, world culture and the world religions form the basis of world history, a third element in the collective consciousness of mankind. If we understand by 'world history,' not the political or cultural events that simultaneously run their independent courses, but the historic consciousness of mankind itself, combining the idea of mankind as a unity with that of the development of this unity in accordance with law, then world history, in this, the only accurate meaning of the term, is the last of all the factors involved in the idea of humanity. Since the individual who is developing in the direction of the ideal of humanity mirrors all other aspects of human nature, world history ultimately becomes for him the gradual realization of the idea of humanity. Thus, world empires, world culture, world religions, and world history represent the four main steps in the development to humanity.

\section{WORLD EMPIRES.}

Even in the midst of the spiritual forces dominating the heroic age there are phenomena that foreshadow a development transcending the limits of this period. Of these phenomena, none is more prominent than the striving for world dominion. The first battles of early political organizations, and the victories over conquered peoples, led to an enhanced consciousness of power on the part of the individual State. This consciousness found expression, first in strife between neighbouring dominions, and later, as soon as one of these had gained the supremacy, in the establishment of an empire including many separate States. Such an impulse to transcend the limits of the single State is so natural and so directly prefigured in the motives to individual action that we come upon it wherever any historically active political 
organizations have arisen. In the realms of western Asia, such attempts are to be found from the time of the Sumerian and Accadian States down to the struggle of Babylon and Assyria for the rulership of the world. Egypt had a succession of dynasties which at first glance might seem to simulate a unified history, but which in reality represents the transference of supreme power from one State or city to another, and along with this the growing ambition for a single all-embracing dominion. The same phenomenon appears in the struggle of the Greek and Latin tribes for hegemony, and also in the foundation of the great Persian kingdom of the Achæmenidæ; the latter gave way to the world empire of Alexander, which, though of short duration, was never again equalled in magnitude; succeeding it, came the world empire of the Romans, the last that could properly lay claim to the name.

It is in Egypt, on the one hand, and in the succession of West-Asiatic kingdoms, on the other, that the first stages of this development of a world kingdom out of the dominance of one powerful State over a number of vassal States are clearly exhibited. The struggle for supremacy, in which vassal might elevate himself to the position of ruler and lord be reduced to vassal, and in which newly immigrant peoples of ten took' a decisive part, immeasurably enhanced the striving to extend the sphere of dominion. This development reached its culmination when the supreme ruler of a power that dominated a very considerable number of vassal States expressly asserted the claim of being ruler of the world. The fact that such a claim was made wherever a supremacy of this sort came into existence under conditions of relatively limited intercourse, testifies to the immanent necessity of the development. Wherever the domain of such an empire approximated the limits of the known world, the universal State was conceived as including also the rest of the inhabited earth. This conception came to expression in the title which the ruler regularly assumed. $\mathrm{He}$ laid claim to being the king of kings, the overlord of the world, the ruler of the 'four quarters of the earth.' 
Through a reversal of that process of transference by which the characteristics of the terrestrial State were carried over, in deity cult, to the divine State, the ruler of the terrestrial State now himself became a god. This accounts for the surprising uniformity with which the idea of a god-monarch arose wherever that of a world monarch was developed. In the pre-Babylonian realms of the Euphrates and Tigris valleys, the ruler erected his own image, as an object of worship, in the temple; in the land of the Pharaohs, the heads of the sphinxes placed in front of the temples bore the features of the monarch. Even Alexander the Great commanded that the Egyptian priests greet him as a son of the god Amon $\mathrm{Re}$; after acquiring the authority of the great Persian kings, he demanded from those about him the external signs of divine adoration. Similarly, the Roman emperors of the period from Diocletian down to Constantine. In spite of their inclination toward republican offices and customs, which by their very nature militated against such ceremonial, these emperors accepted the idea that the world ruler should be worshipped in cult. As the god-idea gained increasing power, however, deity cult itself presented a counteracting influence to the fusion of the ideas of world ruler and deity. A rivalry arose between god and ruler. The king whose omnipotence led to his deification repelled the ruler of heaven, and the ruler of heaven and earth, on his part, refused to tolerate any rival of earthly origin. This led to a temporary compromise in which the ruler, though not himself regarded as a deity, was nevertheless held to be the son of a god, as well as the agent who executed the divine will. Or, after the pattern of hero myths, and in remote resemblance to ancestor cult, the ruler was believed to enter into the heaven of gods upon his death, so that it came to be only the deceased ruler who received divine adoration. The later rulers of Babylon, for example, called themselves the sons of Marduk, who was the chief god of Babylonia, and the features of this deity were given to the image of Hammurabi. The Roman emperors, on the other hand, from the time of Augustus on, were accorded divine reverence after death. When 
the king, realizing the exalted character of divine majesty, finally came to feel himself entirely human, these practices vanished. The emperor now became either the mere representative of the deity or one who was divinely favoured above other men. Hence the development terminates in a formula of royalty which has even yet not disappeared-the formula, "by the grace of God."

The development, which we have described, progressed continuously from beginnings that were almost contemporary with those of States until it eventuated in the world State. What, we must now ask, were its motivating forces? We cannot ascribe it to a craving for power which overmasters the ruler of the single State as soon as he has successfully conquered a foreign territory and a foreign people. Doubtless this factor was operative, yet it was obviously an effect rather than a cause, although an effect which, in the reciprocal relations of impulses, itself forthwith became a cause. But the immediate and decisive factors that led to the idea of establishing a world State, are to be found only partly in the motives underlying the extension of the single State into a world State, and in the results connected with the attainment of this ambition. These motives and results were, in the first instance, of an external nature. They consisted in the fact that the world State enjoyed increased means of subsistence and power by reason of the tribute which it received from subjugated provinces or from vassal States. Tributes of grain and cattle, of precious stones and metals, and especially of valuable human material, were placed at the command of the Pharaoh, or of the Babylonian or Persian monarch, for the building of his canals, his temples, and his palaces, for military services, and for an officialdom more directly subject to his will than were freeborn natives. Everything which the single State required for its maintenance was demanded in a heightened degree by the world empire. Thus, it was the concentration of the means of subsistence and power that led to the displacement of the single State by the world empire, just as it was the same influence, on a smaller scale, that gave to the State its 
ascendancy over the earlier tribal organization. In extending its authority over wider and wider territory, the world empire itself finally perished as a result of the increasing difficulty in unifying its forces. It either broke up into separate States or a similar process of expansion started anew within the same boundaries, beginning now with one of the erstwhile vassal States and now with a new tribe that migrated into the territory. The first of these changes is illustrated by the Babylonian-Assyrian empires; the other, by the catastrophes suffered almost contemporaneously by the realm of the Pharaohs, through the influx of the Hyksos, and by Babylon, at the hands of the conquering hordes of the Hittites. The same phenomena recur in the partition of the empire of Alexander the Great and in the downfall of the Roman world empire. Unless world empires degenerate into a mere semblance of universal dominion, as did the Holy Roman Empire, they obviously become the more short-lived in proportion as history comes to move the more rapidly. Hence the Napoleonic attempt to revive the old idea in a new form became a mere episode. The single State finally triumphed over the world empire, and everything goes to show that the idea of an all-embracing world empire is little likely to recur unless the continuity of history is to be seriously interrupted.

It thus appears that the idea of establishing a world empire is not to be accounted for solely in terms of a constant striving to augment the means of power. Such endeavour prevails now, no less than formerly, in every State that has in any way attained to an independent development of its power. At the present time, however, none but at most an occasional Utopian dreamer adheres to the idea of creating an all-inclusive world State. Even where this occurs the idea is completely antithetical to that of earlier times. The ideal which is at present proposed for the distant future involves, not the extension of any single State into a world State, but rather the dissolution of existing States and the establishment of a society of universal peace among nations, such as would render entirely superfluous any 
instruments of power on the part of the State itself. But we have further evidence that the impulse to increase the means of power could not have been the only, nor even the decisive, factor in the development of the idea of a world empire. This evidence is to be found in the fact that, while a world empire never existed except as an idea, the age in which this idea dominated history regarded the world empire as a reality. Hence there must have been other motives, of an ideal nature, to bridge over the chasm between idea and reality in such wise as to identify the former with the latter. Though it is possible to urge, in explanation, that the knowledge of the real world was at that time limited, this does not solve the problem. Even though the Babylonian king might have felt satisfied to call himself the ruler over the four quarters of the earth because practically all countries of which he had knowledge in the four directions of the wind paid tribute to him, this of itself is not adequate to account for the fact that he regarded the universality as absolute and not relative. Over and above the fact of a limitation of knowledge, there was requisite particularly the idea of the unity of the world, and the application of this idea to the reality given in perception. This idea of unity is similar to that of the absolute unity of the world-order whose centre is the earth, an idea that dominated the astronomical conceptions of antiquity. Both ideas, that of a world empire embracing the whole of mankind and that of a universe whose centre is the earth and whose boundary is the crystal sphere of the heaven of fixed stars, sprang from the same mythological world-view that also found expression in the conception of a divine State projected from earth into heaven. To these gods, with a supreme deity at their head, belonged the rulership of the world. Whenever a change in the city that formed the centre of the terrestrial world empire resulted in a new supreme deity, the conditions of the earthly kingdom were all the more faithfully mirrored in the divine kingdom, for the other gods became, as it were, the vassals of this supreme deity. This mythological picture, projected 
from the earth to heaven, was necessarily reflected back again to earth. Herein lies the deeper significance of the idea that the ruler of the world empire is himself a god, or, at the least, a person of divine lineage and the representative of the supreme guardian deity of the kingdom. It is precisely because of this connection with mythological conceptions that world empires were but transitory. The period of their zenith and, more particularly, the period in which they possessed a fair degree of stability, coincided absolutely with the time at which deity myth was at its height. In the age of a waning deity belief, it was only the influence of numerous elements of secular culture, combined with a high degree of adaptability to the conditions of individual States, such as the Roman mind acquired under the conjunction of unusual circumstances, that enabled the idea of a world empire to be again carried into realization, within the limits which we have set to the term. Proof of the inner connection between the idea of a world empire and a mythological conception of the world, is to be found even in the case of Diocletian, the last powerful representative of the idea of a world kingdom. Diocletian not only invested the Roman emperor with the attributes of the Oriental world ruler of ancient times, but also claimed for himself the worship due to an earthly Jupiter.

\section{WORld CUlture.}

Inasmuch as the world empire belongs essentially to the age of deity cults, it is not so much a realization of the idea of humanity as a preparation for it, presaging a development beyond that of the single State. That this is the case manifests itself even in the temporal sequence of the phenomena. For it is at mast anticipatory elements of the idea of humanity that are embodied in the world empire. With the disintegration of world empires, however, partly as their after-effect and partly as the result of their dissolution, we find phenomena of a new sort-those com- 
prehended under the term world culture. In so far as the rise of world empire involves factors that lead to world culture, these affect primarily the material aspect of the life of peoples-world intercourse, the resulting multiplication of needs on the part of peoples, and the exchange of the means for the satisfaction of these needs. The spiritual phases of culture, which outlast these external and material phases, make their appearance more particularly at the time when the world empire is approaching its end. Since, however, it is these spiritual phases that are of predominant significance, world culture as a whole is to be regarded as an after-effect of world empire rather than as a direct result toward which the latter has contributed. The reason for this is not far to seek. It lies in the one-sided striving for the acquisition of external means of power, and in the consequent despotic pressure which the world empire, particularly in ancient times, brought to bear upon its separate members. It is also connected, however, with the fact that the dissolution of world empires usually brings in its wake migrations and a shifting of peoples. Even within the culture of the ancient Orient, the spread of the elements of myth and saga, as well as of the products of art and science, came especially with the destruction of earlier world empires and the reconstruction of others. The empire of Alexander the Great led to what was perhaps the greatest epoch of world culture in the history of civilization, yet the latter was conditioned, not so much directly by this empire, as by its disintegration at the time of the Diadochi. Similarly, the downfall of the last world empire that may properly lay claim to the name-the Græco-Roman kingdom-likewise resulted in a great cultural movement, due in part to the shifting of peoples which took place at this time, though more especially to the spread of Christianity. Here, again, the fact that the world empire was preparatory to world culture is substantiated. For the dying world empire employed even the last powers over which, in its final agony, it still had control, to pave the way for the world religion that was taking its rise. 
Nevertheless, as a result of the tremendous resources which, in the beginnings of a higher civilization, were possessed by the world empire alone, there was one field in which the period of such empires was directly creative and in which it set an example to future ages. I refer to the technique of mass and to the monumental art connected with it. The streets, viaducts, and magnificent edifices of the period of the Roman emperors have long aroused the wonder and admiration of later generations, as monuments of a power that had unlimited means at its command. The constructions of the Egyptian, BabylonianAssyrian, and Persian world empires lacked the artistic execution which the influence of Greek art made possible to the constructions of the Romans. We have now come to know, however, that the former were not surpassed by the latter in the immensity which resulted from the consciousness, on the part of the builders, that they had countless human forces at their disposal. The canals and roadways of the Egyptian and Babylonian monarchs, moreover, also give clear evidence that the needs of agriculture and commerce were provided for in a way that would have been impossible, in these early stages of world culture, except through the resources at the command of a world State. The extension of intercourse resulting from world empire is to be regarded as at least a partial factor in the transition to the institution of money. It exercised an influence also toward the development of a system of writing, whose purpose it was to communicate the decrees of government to officials and vassals, and to preserve a record of the deeds of rulers and of the laws enacted by them. In this wise, the material aspects of world culture exerted an influence upon the mental aspects, whose direct expressions are speech and writing.

As regards the relation of speech and writing, the two fundamental elements of all culture, the culture of individuals and world culture show an important difference. In the culture of individuals, of course, speech long precedes writing, verbal expression being crystallized into writing only after a relatively high level of culture has been 
attained. In world culture, on the other hand, writing paved the way for verbal intercourse. The reason for this difference lies in the fact that speech is a natural product of the direct intercourse of individuals who are sharing a common life. Writing, however, is an invention by which individuals seek to disseminate and to preserve the ideas embodied in speech far beyond the spacial and temporal bounds that limit oral communication. Hence, communication in writing is the first step from folk culture to world culture. The simplicity of the characters which it employs enables it to pass from one people to another and from one generation to the next even more readily than does the speech of commerce. For though the latter is of a more universal character than the many separate mother tongues, it asserts itself only with difficulty in competition with them. The history of cuneiform writing is especially instructive as regards the point under present discussion. The Semitic people, whose migration to Babylonia succeeded that of the Sumerians, lost all knowledge of the Sumerian language, but they preserved the written texts as sacred. In the course of folk migrations, cuneiform writing likewise penetrated to the coast regions of Asia Minor, although in this instance it was continually used to express new idioms not to be found in the land of its origin. Letters have been found representing a correspondence between certain Babylonian kings and Egyptian Pharaohs, and dating from the fifteenth century before Christ. These letters, called Tel-el-Amarna letters after the place of their discovery, are a remarkable testimony to the fact that the demands of commerce gradually cause speech to follow in the wake of writing, even though the means which the Babylonian employs to make his cuneiform writing intelligible indicates that his Egyptian correspondent possessed only a slight acquaintance with the Babylonian language.

It was not until a much later time that any language of intercourse and literature became sufficiently widespread to be called a world language, even in that relative sense which attaches to all universal terms of this sort. This 
occurred in the case of the Greek language, under the rule of the Diadochi. In this instance, again, the first advance in the direction of world culture followed, in the main, upon world empire. For, though we must admit that the empire of Alexander was of altogether too brief a duration for such a purpose, it is nevertheless true that it witnessed only the beginnings of a world dominance of Greek language and culture. Taking into account the narrow limits of the cultural world of that period of history, there has been no age since that of the Diadochi concerning which we would be prepared to say that it attained to so widespread a dissemination of a uniform culture. The striving beyond a national to a world culture which took place at that time was, of course, the fruition of far earlier tendencies. The fact that the Greek colonies retained the language and customs of the mother country was itself a preparatory step. Following the train of colonists were individual travellers, whose desire for knowledge led them beyond the regions where the Greek language was known. Even in that early day, Pythagoras and Xenophanes, Herodotus and Xenophon, Democritus and Plato made extensive travels throughout the lands bordering on the Mediterranean. Alexander's expedition to India, a country which had up to that time been regarded as a marvellous fairyland, marked the culmination of the journeys to remote regions which had, at the outset, been undertaken by individuals. Nevertheless, the spread of the impulse to wander remains of primary significance for the Hellenistic period. The warrior, the tradesman, and the physician share this impulse with the scholar and the artist. In the age of tribal organization, it was the tribe or clan that travelled to distant places, its object being to escape the pressure of want and the need threatened by the exhaustion of the hunting-grounds or the soil ; in the heroic age, it was the people as a whole who left their homes, either because they were crowded out by enemies or because they were eager to assert their power by establishing cities and States; in the age under present consideration, it is the individual who is seized with the 
longing for travel, his purpose being to find elsewhere more favourable opportunities for the exercise of his vocation, or, perhaps, to see the world, and thus to enlarge his field of experience and his knowledge. The large and rapidly growing cities that spring up into centres of the new world culture attract the people of all lands, as do also the ancient and far-famed seats of intellectual culture. In Alexandria, Pergamus, Athens, and, finally, in Rome, there mingle representatives of all races-of the Greek, Egyptian, Syrian, Persian, and Italic peoples. Greek is the language of common intercourse. Alexandria, however, gradually displaces Athens as the chief seat of science. The latter comes to be fostered, not by Greeks, but, in large part, by individuals of other nationalities, particularly those of the Orient.

This new world culture possesses two distinctive characteristics. The first of these consists in a growing indifference to the State as such. The second, antithetical to the former and yet most closely related to it, is a high appreciation of the individual personality, connected with which is a tendency on the part of the individual to develop his own personality and to assert his rights. That which the public values undergoes a change. The emphasis shifts, on the one hand, from the State to a culture which is universally human, and thus independent of State boundaries ; it passes, on the other hand, from political interests, in part, to the individual personality and, in part, to universal spiritual development. Thus, world culture is at once cosmopolitan and individualistic. As respects both these characteristics, however, the interest in humanity finds expression in a transcendence of the limits of a single people. Here, again, preparatory stages will be found far back in Greek culture. As early as the time of the Sophists, individuals, wandering from city to city as travelling teachers, proclaim the spirit of personal freedom and the dependence of all social institutions and ties upon the will of the individual. When we come to the Epicurean and Stoic schools, which reach over into the period of early world culture, the 
idea of humanity in both its aspects receives its classic expression, though with difiering emphases, conditioned by the ethical and religious needs as a whole. Similar conditions prevail in the positive sciences. In natural science, which reached its first classical development in the Alexandrian period, an interest in universal natural laws, as discovered in astronomy and mechanics, occurs side by side with an absorption in descriptive observations of the most detailed sort. History fluctuates between attempts at an abstract schematization of the epochs of political development, after the pattern of the Aristotelian classification of the forms of the State, and biographical accounts of dominating personalities and their deeds. Similarly, philology combines the grammatical disputes of the Peripatetic and Stoic schools-disputes as yet unfruitful in their abstract generalities-with that minute pursuit of literary studies which has since given the period the discreditable name of 'Alexandrianism.' Art also manifests this coincidentia oppositorum. The monumental edifices of this epoch exhibit a tendency toward the colossal, whereas sculpture is characterized by a painstaking and individualizing art of portraiture; the drama portraying the pompous action of ruler and State, appears alongside of the play of civic intrigue and the mime.

As the result both of inner dissolution and of the aggression of new peoples who were just entering upon their political development, Hellenistic world culture underwent disintegration. It first split up into Greek and Roman divisions, in correspondence with the partition of the Roman world empire and that of the Christian Church connected with it. Except the fact of the separation itself, nothing shows more significantly how far both divisions were from possessing a world culture than does the decline of that indispensable means of common culture, language. The West preserved meagre remnants of the Latin civilization, the East, fragments of the Greek civilization. In the course of the centuries, the clergy of the West developed a class of scholars who were out of sympathy with the prevailing tendencies toward national culture. In the East, the 
barbarian nations, which the Church barely succeeded in holding together, exercised a benumbing influence upon culture ; cultural activity, therefore, sank into a dull lethargy. The ancient world empires, whose last brilliant example, the monarchy of Alexander, had formed the transition to the first great world culture, gave place, at this later time, to world religion. As the result of struggles which, though long, were assured of ultimate success, world religion subjected the political powers to its authority. Destined, in the belief of peoples, to be imperishable, this religion outlived the changing forms of the secular State, and was the only remaining vehicle of world culture, fragmentary as this may have been. But the inner dissolution to which the last of the great world empires, that of Rome, succumbed, overpowered also the Church as soon as the latter endeavoured to become a new world State and insisted on the duty of believers to render obedience to it. When this occurred, the world culture fostered by it necessarily proved too weak to assimilate the new tendencies which were beginning to manifest themselves. Conditions were ripe for the striving to achieve a new culture. In contrast with the ideal of the Church, this culture was concerned with the actual world, and therefore felt itself related to the cultural idea of antiquity. Thus arose the culture of the Renaissance. In it, we again have a world culture in the true sense of the word, even though it was shared, at the outset, only by the ambitious and the educated, as had, indeed, also essentially been the case with its prototype.

The culture of the Renaissance formulated its ideal by reference both to the past and to the future. It sought to revive the world culture of the Græco-Roman period, but yet to give to the latter a content suited to the spirit of the new age and to the tasks awaiting it. Hence the Renaissance was not merely a rebirth, as its name might suggest, but a new world culture. Though possessing many traits in common with the older culture of Hellenism, it bore, in an even greater measure, its own peculiar stamp. The most noteworthy feature common to the two 
was their combination of universalism and individualism-a feature that is, perhaps, characteristic of world culture as such. Apparently both universalism and individualism become more prominent with the course of time. During the period of the Renaissance, the cultivation-one might almost say the cult-of the individual personality probably reached the highest point that it had as yet attained. The human monster, who violated without compunction all laws of propriety and custom, and the ascetic zealot, who sacrificed himself for a visionary ideal, could both alike arouse admiration because of the uniqueness of their characters. Along with this emphasis of individual personality, there flourished social ideals of a religious and a political nature. It was under this influence that the reformation of the church began its work and that new political theories and Utopian accounts of a happy future for the human race made their appearance. In still another respect does the age of the Renaissance appear to be a genuine revival, in an enlarged world, of the Hellenistic period. Again the individual is overpowered by the impulse to travel, and, as a consequence, the age of great geographical discoveries is inaugurated. The voyages of the great discoverers-of Columbus, Vasco da Gama, and Magellan-were the result, for the most part, of personal initiative. And, though other motives may have lurked in the background, the discoverers themselves were chiefly inspired by that desire to wander which, more than a century earlier, had led the Venetian Marco Polo to travel alone in the distant lands of eastern Asia.

But, in certain essential particulars, the later period of world culture possessed a character all its own. The basis of culture was no longer a world State, but a world Church. No longer, moreover, was there an indifference to the State, as had been so generally the case in Hellenistic times. A heightened political interest was everywhere beginning to be manifest. That which long continued to give this period its unique stamp was the struggle between State and Church. The social impulses tended in the direction of a new political 
order, and to a certain extent, even at this time, toward a social reconstruction. The world culture of this period, moreover, sustained a completely altered relation to language, that universal vehicle both of mental life and of the material culture which grows up out of the intercourse of peoples. It was not a world language, such as results naturally from the authority of a world empire, that constituted the basis of the new cultural unity. On the contrary, the latter was dependent upon a multiplicity of languages, which gave expression to the mental individuality of peoples just as did the national States to the diversity of particular political and social interests. The influence of more extensive educational activities made itself felt. The forms of commerce and of the interchange of the mental products of nations were manifold, yet education rendered the means of material and intellectual intercourse common property so far as this was possible and necessary. Thus, world culture itself acquired a new foundation. A world language must of necessity be an active and a living language, and, in view of the fact that all social institutions are historically conditioned, it can attain its supremacy only through the influence of a world empire. Hence every world culture whose basis is a unity of language, in the sense of a world language, is doomed to be transitory. Fragments of such a culture may survive, but it itself must perish along with the language by which it is sustained and, more remotely, with the political power by which the language is upheld. All this is changed as soon as world culture is established on the basis of a multiplicity of national tongues as well as of national States. Then, for the first time, may world culture become more than merely an occasional epoch of history ; thenceforth it may enjoy a permanent development. With this in mind, one may say that the period of the Renaissance laid the foundation for a new form of world culture, whose characteristic feature is that combination of humanistic and national endeavour which is still prevalent throughout the civilized world. 


\section{WORLD RELIGIONS.}

One of the most significant mark's of the heroic age is the existence of national religions. Just as each race possesses its own heroes, so also does it have its own gods, who are reverenced as its protectors in wars with foreign peoples. True, gods and their cults may occasionally pass over from one people to another. Wherever there is an assimilation of foreign cults, however, all traces of origin disappear ; the gods who are taken over from other peoples are added to the company of native gods, and enrich the national pantheon. So far as these conditions are concerned, world empires bring few changes. At most, they expressly subordinate the gods of conquered lands to the god of the ruling city, and thus prepare for the idea of an all-comprehensive divine State corresponding to the universal terrestrial State. The decisive step in the completion of this development is taken only under the influence of the world culture that grows up out of the world empire. The special national deities that represent the particular interests of individual peoples then inevitably recede in favour of gods and cults sustained by universal human needs, in which case the cults are, on the whole, identical, even though the deities bear different names.

It is of importance to note the motives that led to the first steps toward the realization of a universal human religion. They were identical with the very earliest incentives to religion, such as prevailed among all peoples on the very threshold of the belief in demons and gods. For, after the disappearance of political interests, to which the national gods owed their supremacy, it was again two experiences that occupied the foreground-sickness and death. During the period of Hellenistic world culture, the occupation of the physician was held in especial esteem. Connected with this was the fact that the cult of Æsculapius, the god of healing, grew from small beginnings into a cult whose influence extended over distant lands. Even more marked was the increase in the influence of those cults that centred about a world after 
death and the individual's preparation for it. The origin of these cults was connected both with the needs of this life and with the desire for endless joy in the beyond. In view of their identical development, how could it have escaped notice that, whatever formal differences there might be, the Grecian Demeter, the Phrygian Cybele, and the Phœnician Astarte were alike in nature? Even more than was the case with the Greek mysteries, these Oriental cults carried over into the cults of the beyond, into which they developed, certain ecstatic and orgiastic elements of ancient vegetation cults. All the more readily, therefore, were the latter cults incorporated into the deity cults, inasmuch as these had as their concern the satisfaction of human needs generally. But conditions were ripe for a still further advance. As has been suggested, the national and State interests which fettered man to the actual world of his environment gave way to interests transcending this world. In proportion as this occurred, however, did the life of the present, deprived of its former values, relinquish all cherished desires in favour of that heavenly world possible to all men regardless of class, calling, or nationality. This change was antithetical to the innate fear of death, and yet was its own final product. All these cults thus became redemption cults. To be redeemed from the evil of the world-the desire of deeper religious minds-or, after the enjoyment of the good things of this life, to receive still greater happiness after death-a hope doubtless entertained by the majority then as now-such was the primary object of the cults of these supranational gods. National cults had fashioned the gods in the image of man, even though exalting them with all the power of the mythological imagination into the superhuman and the unapproachable. At this later period, all efforts were directed toward bringing these anthropomorphic gods nearer to man as regards the activities in which they engaged, and particularly as regards the experiences which they underwent. No figure in the later Greek pantheon better lent itself to such a purpose than did Dionysius. Like the female deities representing Mother Earth, this male deity originated in the ancient 
field and fertility cults. Later, however, he became more and more transformed by legend into the ideal of a striving and suffering deity, who, after a horrible death, arose to new glory. Related to Dionysius were other deities who likewise became supreme in the Hellenistic age-Mithra, Attis, Osiris, and Serapis. All of these were gods who had been redeemed from pain and anguish, and were therefore capable, in their sympathy, of redeeming man.

In its beginnings, Christianity also was one of these religions of redemption. Over five hundred years before its rise, moreover, there had already appeared in the Far East a religion in which the same thought occupied the foreground. I refer to Buddhism. With reference to the steps by which Buddhism attained its supremacy, our only data are the controversies of the philosophical schools that participated in the development. 'These controversies make it probable that the basal motives involved were similar to those that were later operative in the cultural world of the Occident. There were also essential differences, however, traceable to the fact that the various Brahmanic systems had a common religious substratum, and that Hindoo thought had attained to a fairly advanced stage of philosophical development. One fact is doubtless universal-the appearance of a redemptive religion marks the decadence of an old and the rise of a new period of culture. Beginning with the Hellenistic period, therefore, and continuing with increased strength during the Roman world empire, there was a transition from a national to a humanistic culture. World religion was a more decisive indication of this crisis than were any of the other elements of world culture, or than was even world empire, which prepared the way for world culture. The old gods could no longer satisfy the new age, unless, at any rate, they underwent marked transformations. The age required new gods, in whom national traits were secondary, as they were in life itself, and universal human characteristics were supreme. It was particularly the unique worth of the individual human personality, without regard to birth, class, and occupation, which this 
period of transition from the national to the humanistic ideal emphasized. Hence the obstacles which the surrounding world placed in the way of personal endeavour were inevitably felt the more deeply in proportion as the values of the narrower community life disappeared. A change in mood took place within the consciousness of the age, as it so often does within that of the individual, and this change was enhanced by the contrast of emotions. The world lost the values which it had thus far held, and became a place of evil and suffering. In contrast with it, there loomed up a yonder world in which the desired ideals were believed to meet fulfilment. This mood, of course, did not continue permanently. World religion was of inner necessity forced to adapt itself. to the earthly life in proportion as State and society again acquired a more fixed organization. But, just as the strata of the earth's crust retain the effects of a geological catastrophe long after it has passed, so spiritual life continues to exhibit the influence of upheavals that have occurred in the transitions from age to age, even though the spiritual values themselves have undergone many changes. In this respect, world religion manifests a conserving power greater than that of any other product of mental life.

There are only two world religions, in the strictest sense of the term, Buddhism and Christianity. Confucianism, which might perhaps be included so far as the number of its adherents is concerned, is a system of ethical teachings rather than a religion. Hence, when we take into account the vast number of Chinese peoples, Confucianism will be found to embody a great number of different religious developments, the most important of which are the ancient ancestor cult and Buddhism, the latter of which penetrated into China from elsewhere. The faith of Islam is a combination of Jewish and Christian ideas with ancient Arabian and Turanian traditions. As such, it has brilliantly fulfilled the mission of bringing a cultural religion to barbarian or semi-barbarian peoples, but it cannot be credited with being an original religious creation. Judaism finally formed a supremely important element of Christianity, one whose 
influence would appear to have been absolutely indispensable. In itself, however, it is not a world religion, but is one of those vanquished cults which struggled for supremacy in the pre-Constantinian period of the Roman world empire.

But what, let us ask, were the powerful forces that gave these two great world religions their supremacy? Surely it was not merely their inner superiority, though this be in no way disputed. Nor was it simply propitious external circumstances, such, for example, as the fact that Constantine made Christianity the State religion. Doubtless there were a great number of co-operating factors, foremost among them being the desire for a purely humanistic religion, independent of nationality or external position in life. And yet this also could not have been of decisive significance-precisely such a longing was more or less characteristic of all the religious tendencies of this transitional period. Moreover, this leaves unexplained the peculiarities of each of the two great world religions. These are in complete accord as regards their universal, humanistic tendency, but are just as different in content as is a Buddhistic pagoda from a Gothic cathedral. As a matter of fact, these world religions are also cultural religions. Back of each of them is a rich culture, with characteristics peculiar to itself, even though its basal elements are universally human. Hence it is that these two world religions are not merely expressions of a striving for a universally valid religious and moral ideal, in the sense in which such a striving is common to mankind as a whole ; it should rather be emphasized that they reflect the essentially different forms which this striving has assumed within humanity. Buddhism, in its fundamental views, represents the highest expression to which the religious feeling of the Orient has attained, while Christianity, as a result of the conditions which determined its spread, has become the embodiment of the religious thought of the Occidental world. To appreciate this fact we must not allow our minds to be diverted to the tangled profusion of beliefs in magic and demons which Buddhism 
exhibits, nor to the traditional and, in part, ambiguous sayings of the great ascetic himself. If we would discover the parallels between Buddhism and Christianity, we must hold ourselves primarily to the ideas that have remained potent within the religion of Buddha. True, the worlds which these religions disclose to our view differ, yet in neither case had religions feeling up to that time received so exalted an expression. In Buddhism, as in original Christianity, human life is regarded as a suffering, and this underlies both the irresistible impulse to asceticism and repentance, and the hope for unclouded bliss in the future. The Christian of the primitive church looks forward to the speedy return of Christ, and to His inauguration of an eternal, heavenly kingdom. In contrast with this, it is as a prolonged migration through animal bodies, alternating with rebirth in human form, that the Hindoo thinker conceives that great process of purification by means of which sense is finally to be entirely overcome and man is to partake of an undimmed knowledge of the truth, and, with this, of supreme and never-ending bliss. This is the true Nirvana of Buddha. Nirvana does not represent the nothingness of eternal oblivion, but an eternal rest of the soul in pure knowledge, a peace which puts an end to all striving, just as does the heaven for which the Christian hopes. The difference between Nirvana and the Christian heaven is merely that, in the one case, the emphasis falls on knowledge, whereas, in the other, it is placed on feeling. This distinction, however, is not absolute. Buddha, also, preaches love of one's neighbour-indeed, sympathy with every suffering creature ; and the Christian, as well as the Buddhist, seeks the knowledge of God. Moreover, ideas of purification are necessarily involved in redemptive religions, and hence are to be found in Christianity no less than in the world religion of the Orient, though in a different form. The Occidental Christian, swayed by his prompter emotions, images in the most vivid colours the agonies of the damned and the purification of the sinners in need of redemption. The patient and peaceseeking Oriental entertains the conception of a prolonged 
suffering that leads gradually, through the light of knowledge, from the debasement of animal existence to a state of redemption.

A further feature which differentiates these kindred religious developments is their relation to the contemporary philosophy which affected them. Buddhism grew out of philosophy and then became a folk religion. In its spread, it became transformed from an esoteric into an exoteric teaching, continually absorbing older elements of folk belief. Its ethical basis never entirely disappeared, yet it became more and more obscured by a multitude of miracle-legends and magical ideas. Christianity, on the other hand, began as a folk religion and, in so far, as an exoteric teaching. But, in entering into the strife of religions and into the controversies of the thought-systems of the HellenisticRoman period, Christianity passed under the control of philosophy. Precisely because it lay outside the realm of philosophy, it was subjected to the influence of the various schools, though it was most decisively affected by Platonism and Stoicism. Inasmuch as philosophy itself had its setting in a superstitious age, it was the less able to purify Christianity from the belief in demons, miracles, and magic which the latter, as a folk religion, embodied from the very outset. Nevertheless, philosophical thought supplemented the real meaning of religious statements with an idealized interpretation. This gave birth to dogma, which consisted of a peculiar combination of esoteric and exoteric elements, and for this very reason assumed a mystical character. Hence it is that Buddhism, which sprang from philosophy, never possessed any real dogmas in the sense of binding norms of faith, whereas Christianity, which originated as a folk religion, fell a prey in its dogmatization to a theology which prescribed the content of belief.

These two world religions, which dominate the main centres of spiritual culture, do not, surely, owe their supremacy over other religious cults to the external conditions of their origin. Indeed, these conditions differ in the two cases. To account for the pre-eminence of the two 
religions we must look to the religious and moral nucleus which they possess in the sayings and teachings as well, also, as in the ideal lives of their founders. In spite of all differences, there is a similarity of character between the prince who wandered about as a beggar, preaching to the peoples the salvation which pure knowledge brings to him who renounces all external goods of life, and the man of the common people who pronounced blessings on the poor and the suffering because they are prepared above others to find the way to heaven. Another remarkable coincidence is the fact that the religious communities which they inspired sought to deprive them of the very characteristic which opens human hearts to them; they were real persons who lived and to whose deeds and sufferings their contemporaries bore testimony. What, as compared with them, are the redeeming gods in the pantheon of the various nations-Dionysius, Mithra, Osiris, or even Serapis, whose worship was established by the Ptolemies under the driving power of ideas of extensive political authority? The need of a living god whose existence was historically attested led irresistibly to the elevation of the man into a god. Thus, though in an entirely different world-setting and with a completely changed hero-personality, the process through which deities were created at the beginning of the heroic age was repeated. At this later period, however, it was not the universal type of idealized manhood that was regarded as the incarnate deity, but a single ideal personality. This purely human deity was no longer bound by national ties; he was not a guardian of the State and a helper in strife with other peoples, but a god of mankind. For every individual he was both an ideal and a helper, a saviour from the imperfections and limitations of earthly life. With this process of deification, the religions whose central object of cult was the suffering individual who secures for himself and for mankind redemption from suffering, opened their doors also to the gods and demons of earlier ages. Thus, there penetrated into Buddhism the Hindoo pantheon, together with the beliefs 
in magic and spirits which were entertained by the peoples converted to Buddhism. The Christian Church did not finally supersede the earlier heathen folk belief until it had assimilated the latter in the conceptions of demons and the devil, in the cult of saints, and in the worship of relics, the last-mentioned of which also constituted an important element of Buddhism.

In the case of Christianity, there was still another factor which prepared the soil for the new religion. This factor was due either to a direct transference or, as is probable so far as the main outlines of the history of the passion are concerned, to the real similarity of this event with the legends, prevalent in all parts of the earth, of the death and resurrection of a deity. Such legends everywhere grew up out of vegetation cults, which date back to the beginnings of agriculture. The hopes centred about a world beyond caused the cults based on these ideas to incorporate the soul cults. The latter then displaced the original motives of vegetation cults. In this way, higher forms of soul cult were developed, as exemplified by the ancient mysteries and by the related secret cults of other peoples. The exclusive aim now came to be the attainment of salvation from the earthly into a heavenly world. It was thought that this goal would be the more certain of attainment if, yielding to the old association of the mystical and secret with the magical and miraculous, the circle of initiated cult companions were narrowly limited. But how different is the form which this very ancient legend of a god who suffers, dies, and rises again assumes in the suffering and death of Christ! Jesus was a real person, whose death on the cross many had witnessed and whose resurrection his disciples had reported. Moreover, the cult of this crucified Saviour was not enveloped in a veil of secrecy. The redeeming god did not wish to win heaven merely for a few who had gained the privilege through magical ceremonies. The Christian heaven was open to all, to rich and poor, though especially to the poor, who were to receive in the beyond a rich compensation for the 
good things denied them upon earth. It is but natural that this new cult, with its vastly deeper and more vital significance, and with the strength which it nevertheless continued to draw from the old traditional legends, won for itself the allegiance of the new world with its strivings for a greater security in life as in death. Even some of the Roman soldiers, coming from their Saturnalian or Sacæan festivals, may, perhaps, have felt strangely moved upon seeing re-enacted, as a terrible reality, that which in their country was a playful custom, representing a survival of a once serious cult and ending in the mimic death of the carnival king. It was obviously in recollection of these very prevalent festivals that the coarser members of the crowd gave to him who was crucified the name "King of the Jews." The appellation was exactly suited to heighten the contrast between the joyous tumult of such mimic cults and this murderous reality.

The above scene was prophetic of the entire subsequent development of the new religion. That Christianity became a world religion was not due merely to the depth and sublimity of its spirit-these were hidden under a cover of mythological elements, from which Christianity was not free any more than were other religions. Christianity gained its supremacy, just as did Buddhism, in its own way, through a capacity to assimilate auxiliary mythological conceptions to an extent scarcely equalled by any of the previous religions. The very fact that the latter were national religions precluded them, to a certain extent, from incorporating alien ideas. It was not only mediæval Christianity that took over a large part of the earlier belief of heathen peoples. Even presentday Christianity might doubtless be called a world religion in this sense, among others, that, in the various forms of its beliefs and professions, it includes within itself, side by side, the most diverse stages of religious development, from a monotheism free from all mythological elements down to a motley collection of polytheistic beliefs, including survivals of primitive ideas of magic and demons.

But there is another phenomenon in which the spirit of 
Christianity comes to expression even more significantly than in its capacity to adapt itself to the most diverse stages of religious development. Here, again, there is a similarity between Christianity and the other great world religion, Buddhism. The belief of Hindoo antiquity in a populous heaven of gods was very early displaced, in the priestly wisdom of India, by the idea of " the eternal, unchangeable" Brahma. We here have an abstract deity-idea from which every trace of personality has disappeared. It was under the influence of this priestly philosophy that Buddha grew up, and his esoteric teaching, therefore, did not include a belief in a personal deity. Meanwhile, the ancient gods had continued to maintain their place in popular belief, though their original character was obscured by rankly flourishing ideas of magic and demons. This state of affairs was due to the fact that there was no longer a supreme deity who could give to mythology a religious basis. In the religious movement which began with Buddha, however, the latter himself came to be a supreme deity of this sort, the old nature gods and magic demons becoming subservient to him. The god-idea had been etherealized into the abstract idea of a superpersonal being, but its place was taken by the human individual exalted into a deity. Christianity underwent the same crucial changes, though in a different manner. In the philosophy of the Greeks, the personal deity of popular belief had been displaced by a superpersonal being. Plato's "idea of the good," the Aristotelian Nous, which, as pure form, holds sway beyond the boundaries of the world, even the Stoic Zeus as the representative of the teleological character of the world order, and, finally, the gods of Epicurus, conceived as indefinite forms dwelling in nebulous regions and unconcerned with the world-all manifest the same tendency either to elevate the personal deities of the heroic age into superpersonal beings, or, as was essentially done by Epicurus, to retransform them into subpersonal, demon-like beings. In contrast with this tendency, Jesus, as the representative of a religious folk belief, holds fast to the god of ancient 
tradition, as developed in the Jahve religion of the Israelites. Indeed, it is in the conception of Jesus that this god receives his deepest and most personal expression, inasmuch as he is conceived as a god of love, to whom man stands in the relation of son to father. This conception of the relation of God to man Christianity sought to retain. But history is not in accord with this traditional view. Cult and dogma alike testify that in this case also the deity came to be superpersonal from an early period on. To cult, which is always concerned with personal gods, Christ became the supreme deity ; in the Catholic Church, there came to be also a large number of secondary and subsidiary gods, who sometimes even crowded the Christ into the background, as is exemplified particularly by the cult of the Virgin Mary. Dogma, on its part, cannot conceal the fact that it originated in philosophy, which is destructive of personal gods. For dogma ascribes attributes to the deity that are irreconcilable with the concept of personality. The deity is represented as eternal, omnipotent, all-good, omnipresent-in short, as infinite in all attributes that are held to express his nature. The conception of the infinite, however, contradicts that of personality, for the latter demands a character that possesses sharply defined attributes. However comprehensive our conception of personality may be, limitation is necessarily implied ; the concept loses its meaning when associated with the limitless and the infinite. Even though dogma may continue to maintain that belief in a personal God is fundamental to Christian faith, such a belief is nevertheless selfcontradictory ; the union of the ideas 'personal' and 'god' must be understood as a survival within the era of world religions, where many such survivals occur, of the godidea developed by national religions.

The truth is that the transformation of the personal god into a superpersonal deity is probably the most important mark of world religion. National religion displaced the subpersonal demon in favour of the personal god; in world religion, the personal god is exalted into a superpersonal deity. At this point there is a very close connection 
between world religion and world culture. As the idea that the universe is bounded by a sphere of fixed stars must give way to the conception of the infinitude of the universe, so also does world culture transcend the limits imposed upon it by the preparatory world empire, whose own origin was the State. World Culture, as we have seen, comes to signify a cultural unity of mankind, such as includes the national States. Similarly, world religion strives toward the idea of a deity who is superpersonal, and who, though only in so far as he is superpersonal, transcends the world of experience. The foundations of this concluding stage in the development of religion had long been laid by philosophy. In religion itself, the culmination was actually attained with the recedence of the deity in cult ; in theology, it came with the ascription to the deity of attributes of absoluteness and infinitude, even though the deity-conception did not clearly emerge from a mystic incomprehensibility rendered inevitable by the combination of contradictory ideas.

Though the transition from a personal god to a superpersonal deity is the decisive characteristic that marks a world religion, there is closely connected with it a second distinctive feature. In Christianity, indeed, it was the latter that prepared the way for the idea of the non-personal character of God. The fact to which I refer is that, in addition to the non-personal deity, there is believed to be a personal god in the form of an exalted human individual. Cult continues to require a personal being to whom man may come with his needs and desires. And by whom could his trouble be better understood than by a deity who himself lived and suffered as a man? In Buddhism, therefore, as well as in Christianity, the god-man became the personal representative of the non-personal deity, not as the result of any external transference, but in consequence of the same inner need. The god-man is a representative in more than one respect. Cult honours him as the deity who dwelt upon earth in human form, and who represents the godhead; it turns to him also as the human individual who represents mankind before God. Back of these two ideas of representativeness that 
dominate belief and cult, there is still a further, though an unrecognized, need for a representative. The religious nature requires that there shall be a personal god as the representative of him who has been exalted into a non-personal deity and has become inaccessible. The infinite god posited by the religious intellect is unable to satisfy the religious nature that is pressed by the cares and sufferings of finitude. Herewith the way is opened for a development whose course is determined by the changing relations into which the two aspects of the concept 'god-man' enter with one another. On the first stage, the divine aspect of the god-man overshadows the human character. At this period, it might appear as though world religion merely substituted a new god for the older gods. Though the superpersonal deity receives recognition in dogma, and the development, therefore, marks an important religious advance over the age of gods, the cult is directed to the person of the god-man. Then comes a second stage, in which the human aspect of the concept 'god-man' occupies the foreground. The god-man becomes an ideal human being who succours man in the afflictions of his soul, but who does so not so much by his divine power as by the example of human perfection which he represents. At the third stage, the god-man finally comes to be regarded as in every respect a man. It is recognized that, through the religious movement which bears his name, he indeed prepared the way for the idea that the deity is a non-personal source of being, exalted above all that is transitory. Nevertheless, the god-man is conceived as an ideal man only in the sense in which one may speak of any ideal as actual. Hence, the world religion derives its name from him not so much because of what he himself was as because of that which he created. From this point of view, it is eventually immaterial even whether or not Jesus or Buddha ever lived. The question becomes one of historical fact, not one of religious necessity. Jesus and Buddha live on in their religious creations. That these creations, to say nothing of any other proofs, point back to powerful religious personalities, the unbiased will regard as certain, though from this third point of view the question is of subordinate importance. 
A world religion may lay claim to being such not merely on account of its wide acceptance, but also because of its ability to incorporate the elements of other religions. In a similar manner, and more particularly, a world religion is one that includes within itself elements representing past stages of its own development. Historically considered, religious elements are juxtaposed in such a manner that the religious life of the past is mirrored in the present. Hence the religion can at no time emancipate itself from its historical development. It is just as impossible to return to the religious notions of earlier times as it is to transform ourselves into the contemporaries of Charlemagne or even of Frederick the Great. The past never returns. Nevertheless, it is universally characteristic of mental development, particularly within the sphere of religion, that the new not only continues to be affected by the old, but that the more advanced stages of culture actually embody many elements of the past. That these be permitted to exist side by side with higher conceptions, and that there be no limiting external barriers in either direction, is all the more demanded by world religion inasmuch as the independence of State and society, which its very nature implies, presupposes, first of all, the freedom of personal belief.

Inasmuch as it possesses a universal human significance, religion cannot escape the change to which everything human is subject. This appears most strikingly in the undeniable fact that the fundamental idea of the two great world religions, Buddhism and Christianity, has in both cases changed. I refer to the idea of salvation. We do not, of course, mean to deny that an individual may either permanently or temporarily return to the religious ideas of the past with a fervour which again reinstates in him impulses that have long since disappeared. Nevertheless, the present-day idea of salvation is no longer identical with that which animated the primitive Christian Church when it looked forward to the return of its Saviour. Christianity is a religion of humanity. Precisely for this reason, it, in every age, took up into itself the feelings and aspirations 
representing the ideal spiritual forces of that age. All that was permanent in the midst of this change was really the religious impulse as such, the feeling that the world of sense belongs to an ideal supersensuous order-a feeling for which world religion seeks external corroboration in the development of religion itself. In distinction from national religions, which sprang from an infinitely large number of sources, a world religion requires a personal founder. To this personality is due also the direction of the further development of the religion. Thus, the final and most important characteristic of world religion is the fact that it is preeminently an historical religion. It is historical both in that it has an historical origin, and in that it is constantly subject to the flux of historical development.

\section{WORLD HISTORY.}

The meaning attached to the term 'world history' clearly shows how firmly rooted is the anthropocentric view of the world in connection with those matters that are of deepest concern to man. World history is regarded as the history of mankind-indeed, in a still narrower sense, as, in the last analysis, the mental history of mankind. If facts of any other sort are taken into account, this is not because they are an essential part of the subjectmatter, but because they represent external conditions of historical events. The justifiability of this point of view may scarcely be disputed. If the purpose of all historical knowledge is to understand the present condition of mankind in the light of its past, and, in so far as we also attribute tio this knowledge a practical value, to indicate the probable course of the future, then the history of mind is the immediate source of historical knowledge. If this be true, it follows that the essential content of history consists in those events which spring from the psychical motives of human conduct. Moreover, it is the nexus and change of motives underlying such conduct that lends to events the inner continuity which is universally demanded of history. 
But the very meaning which is universally associated with the term 'world history' itself includes two very different conceptions. For, even when the field of history is limited to the events connected with mankind, as those which are of greatest importance to us, there remains a further question. Is history to deal with the whole of mankind, or is it to be restricted merely to those peoples that have in any way affected the course of the mental history of humanity? As is well known, most of the works on world history have been confined to the more restricted field. For them, world history is an account of cultural peoples, whose activities are shown by a continuous tradition and by existing monuments to form a relatively connected whole. But there have also been more comprehensive works, which have felt it necessary to include at least those cultural and semi-cultural peoples who attained to some independent mental development, as did the peoples of the New World prior to the time of Columbus. Back of this uncertainty arising from the ambiguity of the concept ' mankind' lies a deeper-going confusion due to the no less ambiguous meaning of the concept ' history.' However much we may associate the word 'history' primarily with the traditional limits of historical science, we may not entirely put aside the broader meaning, according to which it includes everything which may at all be brought into a connected order of events. For we also speak of a history of the earth, of the solar system, of an animal or a plant species, etc. Now, with this wider connotation of the idea in mind, we cannot fail to recognize that the conditions that still prevail among certain races, and that doubtless at one time prevailed among all, are such that, while they would not concern historical science in its more restricted and familiar sense, they would demand consideration if the term were taken in its broader meaning. From the latter point of view, the condition of a primitive people of nature is no less a product of history than is the political and cultural condition of present-day Europe. But there is nevertheless a radical difference 
between the two cases. The historically trained European understands, to a fairly great extent, the external circumstances that have led to present conditions. $\mathrm{He}$ is conscious not merely of the present but also of its preceding history, and he therefore looks forward to the future with the expectation of further historical changes. The man of nature knows only the present. Of the past he possesses merely fragmentary elements, legendary in character, and much altered by the embellishments of a myth-creating imagination; his provision for the future scarcely extends beyond the coming day. Hence, we should scarcely be justified in unqualifiedly calling peoples of nature ' peoples without a history.' In the broader sense of the term, they have a history, as well as have the solar system, the earth, the animal, and the plant. But they lack' a history in the narrower sense, according to which historical science includes among ' historical' peoples only such as have had some special significance in the development of mental culture. That even this limitation is variable and uncertain need scarcely be mentioned. The past shows us many instances in which hordes that were previously unknown, and were thus, in the ordinary meaning of the term, peoples without a history, suddenly stepped into the arena of the cultured world and its history. The colonial history of the present, moreover, shows that the characteristics and the past development of races occupying regions of the earth newly opened to cultural peoples, have not been, and are not, without influence upon the course of history. It should also be remembered that between an historical tradition comprehending the entire cultural world and recollection limited to the immediate past, there are a great number of intermediate stages. These stages are dependent primarily upon the forms of social organization, though also upon other cultural factors. Peoples that have failed to advance beyond a tribal organization may frequently have traversed wide regions of the earth and yet have preserved at most certain legendary elements of the history of these migrations, although retaining myths, cults, and customs indefinitely. On the other hand, wherever a national State 
has arisen, there has developed also a national tradition, intermingled with which, of course, there have long continued to be mythological and legendary elements. But the tradition, even in this case, relates exclusively to the particular people who entertain it. Strange races are as yet touched upon only in so far as they have directly affected the interests of those who preserve the tradition. Indeed, such races continue to have but an inconspicuous place in tradition until the establishment of world empires and of the partly anticipatory colonial and trade interrelation of peoples. Hence it is not until the rise of world empires that we find the transition to world history in the sense in which the term is most commonly employed to-day. In so far as world history involves a transcendence of the history of a single people but nevertheless a limitation to the circle of cultural peoples who are more or less generally interrelated, it. is a direct product of world culture. Such a history includes all peoples who participate in world culture and excludes all those who have no share in it.

Considered from a psychological point of view, the different meanings of the concept 'history' in its relation to the various stages of mental culture, clearly show a fluctuation between two ideas which, though opposite, nevertheless mutually imply each other. On the one hand, there is the purely objective conception of history. History, in this case, is regarded as a course of „events of such a nature that the specific occurrences may be brought by an external observer into an orderly sequence of conditions and results. On the other hand, history has been conceived as a course of events, which not only exhibits an orderly sequence from an objective point of view, but which is also subjectively experienced as a nexus by the individuals concerned. In the one case, history is a reconstruction, on the basis of external observation, of the inner connection of phenomena ; in the other, it is the conscious experience of the latter connection. Mankind exemplifies all possible transitional stages between these 
two extremes-history as merely objectively given, and as experienced both objectively, and subjectively. Indeed, it is even true to say, that, as a matter of fact, none but such transitional stages actually, occur. Even the horizon of primitive man includes a narrow circle of consciously experienced history. On the other hand, man is ever far from attaining to a self-conscious grasp of his own history in its entirety. Thus, that which is in a high degree characteristic of world religion is true also of world history. Within the conscious horizon of each individual very different levels of historical consciousness are represented, even in the case of the cultural peoples who participate more or less actively in the course of .world history. Here, as in world religion, we find that what was developed in a sequence during the course of ages continues to remain, at any rate roughly speaking, in juxtaposition. Moreover, even apart from this, we never survey more than a segment of the entire nexus of historical factors. One of the most important tasks of the historian consists in tracing the chain of events back to motives which are, in part, inaccessible to superficial observation, and, in part, indeed, remain of a problematical nature even when we believe that, through inference, we have gained an approximately true conception of them. Nevertheless, it is not necessary that immediate knowledge be complete in order that there may be a consciously experienced nexus of events such as is demanded for the content of history proper. It is merely necessary that some interconnection be actually experienced and that its relations be directly, apprehended. This knowledge, moreover, must possess sufficient power to influence decisively, the actual course of events.

This narrower conception of history brings historical events into relation with the human will. The will is really a phase of conscious experience. It is necessary, however, to single it out for special discussion, because of the fact that popular opinion either regards it as the exclusive factor in history or else stresses it so one-sidedly that the causal view, required in principle even for individual conscious- 


\section{I 4 ELEMENTS OF FOLK PSYCHOLOGY}

ness, threatens to vanish entirely from the conception of historical life. Naturally, the will does not become an influence definitely affecting the course of events until individuals have become consciously aware of the interconnectedness of historical life. Whitenever, therefore, an exaggerated importance is attached to the function of volition, the conscious intervention of individual personalities in the course of events readily comes to appear as the decisive feature that distinguishes the historical from the prehistorical stages of human development. But this is erroneous in both its implications. Even the life of primitive peoples of nature is not entirely unaffected by individual personalities, whose influence may be more or less permanently, operative even after they themselves have been forgotten. On the other hand, the will acts of individuals constitute but one factor among the many which determine historical life. Moreover, inasmuch as every particular volition is conditioned by motives inherent in the general constitution of individual consciousness, it is subject to the same psychical causality that dominates human consciousness in general. The criterion for differentiating historic from prehistoric existence, therefore, is not the influence of a personal will upon the life of the group, but rather the fact that the conscious experience of historical continuity includes a recognition of the effect of individual personalities upon the destinies of peoples. The advance to such an insight is inaugurated by world empires, in which the vicissitudes of peoples first begin to form a unified history; it reaches its completion in world culture, which creates a common mental heritage for mankind, and thus engenders the consciousness of a universal community.

Of the various elements of world culture that give impetus to this development, the world religions occupy the foremost place. In extent and permanence they surpass not only the world empires but also all other forms of material and spiritual interchange between peoples. However much the traditions associated with world religions may be interwoven with mythological and legendary elements, they nevertheless 
constitute a bond whose primary effect is to arouse among peoples who may otherwise be widely different in culture and history, the idea of a universal human community. The peoples of Eastern Asia, for example, though exhibiting marked political differences, were united by Buddhism into a community of religious thought, in which they became conscious that ${ }_{2}$ in spite of differences of race and of history, they possessed a similar religious and ethical temper. If we compare the Brahmanic doctrines with the sayings of such teachers as Confucius and Lao-tsze, we are struck particularly by the similarity of ethical trend as well as by the divergence of this trend from that of Occidental thought. In its idea of a community of faith, Islamism likewise brought the consciousness of unity to numerous peoples of barbaric culture-to a more limited extent than Buddhism, it is true, but for this reason all the more forcefully. Of Christianity, it is even more true that, from the very beginning, it took as its guiding principle the belief that in the eyes of God there is no distinction either of race or of class and occupation. Hence it has regarded missionary activity among heathen peoples as a task whose purpose it is finally to unite the whole of mankind beneath the cross of Christ. Thus, world religion destroyed the barriers erected by the preceding national religions, and took as its aim the unification of men and races into an allembracing community. To the adherent of a national religion, the race that believed in a different god was strange and hostile; both characteristics, strangeness and hostility, were included by the Greek' in the term 'barbarian.' The Christian speak's of heathen who have not as yet beheld the light of pure truth, but for him there are no barbarians. The god to whom the Christian prays likewise rules the heathen world, and to the heathen, also, the gospel is preached. True, we find a recurring limitation in that it is only the Christian who is a brother to Christians. Nevertheless, it is prophesied of the heathen that they will at one time be received into the brotherhood of the disciples of Christ. At the end of time, there is to be but one 


\section{ELEMENTS OF, FOLK PSYCHOLOGY.}

shepherd and one flock upon earth. Thus, in the missionary activity which the Christian recognizes as his calling, the assertion, All men are brothers, is based on the two ideas, All Christians are brothers, and All men are destined to become Christians.

It was on the basis of the Christian tradition that science first attempted to treat history, not as the history of a single people or, at best, as a number of histories of successive or contemporaneous races and States, but as true world history. At the outset, world history, was objective in character. The underlying thought was that the whole of mankind was controlled by a single idea which governed all events, and that the task of humanity. consisted in carrying this idea into realization. Augustine's Civitas Dei was the first attempt at a world history, based on the idea of the religious vocation of mankind. That this exposition is limited to the legendary history of the Israelitic people, supplemented by the history of Jesus as transmitted in the Gospels, and by the Apocalyptic prophecies of a future world, should not cause surprise. The limitation is due to the fact that the idea of humanity is considered solely from the religious point of view. The Church, as the institution about which religion centres, is glorified by. Augustine's work as the divine State. The adoption of this religious viewpoint causes the history. of mankind to appear as a record, not of human experiences that come as a result of human striving: and activity, but of events that are from the very beginning divinely foreordained.

Nevertheless, Augustine's remarkiable work long contimued to determine the general direction of conceptions relating to the history, of mankind. $U p$ to the eighteenth century, religious development was regarded as establishing the only connection between the various periods of history. The sole exception to this occurred in the case of Giambattista Vico. In his New Science (I725), Vico sought to combine the development of language and of jurisprudence with that of religion. True, the question regarding the origin of the State and the causes of changes 
in constitutions had concerned men from the time of the early Sophists on. Particularly during the Hellenistic period and at the time of the Renaissance, such inquiries were of focal interest, as a result of the great political changes that were then taking place. Yet, whenever the underlying laws of such changes were sought, it was the single State that formed the basis of investigation; by comparing its vicissitudes with those of other States, the attempt was made to arrive at a general law along some such line as the Aristotelian classification of States into monarchies, aristocracies, democracies, etc. There was hardly ever a suggestion that the historical sequence of civilizations and of States was a connected process intelligible in causal or teleological terms. Religion alone was conceived as a phenomenon which was, on the one hand, independent of the limits of a single people, and yet, on the other, subject, in its development, to law. The idea that Christianity was destined to be a world religion, together with the fact that it had originated historically and had spread widely, did not admit of any other interpretation. Within this Christian circle of ideas, moreever, the historical development and growth of religion were, quite naturally, brought into connection with the world beyond, in which the development was thought to await its completion. The religious philosophy of history thus terminated in a prophecy whose culmination was the final triumph of Christianity. The Age of Enlightenment, after effecting a unification of Christianity with the religion of reason, again made the world of historical experience the scene of triumph. This triumph was held to consist in the ultimate development of Christianity into a religion of reason-a conception in which the idea of the destiny of Christianity to become a world religion undergoes a philosophical transformation which recurs even in the writings of Kant.

Apart from this transformation, which was only partially complete even in the Age of Enlightenment, the idea of religious development that grew up in connection with 
Christian thought involves two presuppositions. The first of these is that the pathway of mankind was determined by God, and not voluntarily chosen by man himself. It is not to religious thought that the characteristic features of the development must be ascribed, nor is the development immanent in religion-it is the result of external causes. The second presupposition is that this development follows a preconceived plan; it embodies a purpose-indeed, it expresses purpose in the very highest degree precisely because it proceeds from the will of God. Even the co-operation of individuals in the fulfilment of this plan is but the result of divine predetermination, or happens because God has made known $\mathrm{His}$ purposes to these individuals. Thus, this course of thought leads with inner necessity to the conception of revelation. This conception combines two essentially irreconcilable ideas, offsetting each by the other. The religious destiny of man is thought to lie outside his own control : it is imposed upon him from without, and is communicated to him in the form of an illumination which he receives from the supersensuous world. Thus, religious development itself becomes a supersensuous process, which falls beyond the possibilities of the ordinary means of human knowledge. As its goal lies in the supersensuous, so also is the development itself a supersensuous process that extends over into the world of sense.

But at this point the religious view of world history necessarily came into sharp conflict with the philosophical view, though the latter had in certain respects appropriated the idea, developed by the former, of a teleological direction of human destinies. The philosopher, always trusting the guidance of his own reason, might admit both a goal and a plan, but that these should be inaccessible to the lux naturalis, as the philosophy of the Enlightenment called rational knowledge in distinction from lux supranaturalis, or revelation, he could not concede. The logical outcome of this course of thought was an auxiliary concept which appeared to surmount the difficulty, and also possessed the happy characteristic of leaving every one free to retain, 
along with the natural light, as much or as little of thei supernatural thought of an earlier period as he might deem wise. This auxiliary concept was that of education-a 'conception that would readily suggest itself to an age vitally interested in pedagogical questions. The thought here involved represents merely a special application to this particular instance of the idea that the world is governed by a personal deity. Thus it came about that, from the time of Locke and Leibniz down to that of Lessing and Herder, the favourite conception of history was that of an education of mankind. But it is significant that the very work whose title incorporates this idea, Lessing's Education of the Human Race, really ends by displacing it. True, as a result of Biblical tradition, the idea of education is here brought into connection with the thought that the Jewish race is the chosen people of God. Freed from this connection, however, and applied to mankind in general, the idea of education, in Lessing's work, becomes that of self-education, or, what is the same thing, that of a development determined by the general laws of mental life. Hence conditions were ripe for the further advance made by Herder, in his Ideas on the Philosophy of the History of Mankind. Though frequently lapsing, in his discussions of details, into the transcendent teleology of the preceding period, Herder nevertheless did away in principle with the restriction of the history of mankind to religious 'development, substituting for the latter the development to humanity.

Thus was determined the programme which historical science, at about the same time, accepted as its own-the programme of a universal history, whose task did not consist in presenting a loosely connected series of the histories of separate States, but in describing the common participation of peoples and States in the development of a universal culture. Furthermore, the way was cleared for the philosophical position that history is not, as was once thought, the expression of a predetermined plan whose purpose is that of a divine education, but that it is the result of laws immanent in historical life itself, Though variously expressed and 
partly obscured by surviving ideas of the preceding period, this is the fundamental conviction common to the nineteenthcentury philosophers of history. It received its most complete expression in the writings of Hegel, not merely in his Lectures on the Philosophy of History, but in his entire philosophy, which reflects throughout a broad historical outlook. History had by this time come to be regarded as a strictly self-dependent development of ideas in which each advance proceeds with rigid logical necessity from that which went before. In other. words, it was thought of as a development of reason in time, or, in the phraseology of a religious world-view, as the living development of God himself. God is no longer conceived as a transmundane being who guides the destinies of mankind according to a preconceived plan. On the contrary, $\mathrm{He}$ is represented as immanent in the world. His innermost nature is described as the worldreason, and this is said to be unfolded particularly in the history of mankind.

However superior this conception may be to the preceding semi-mythological and semi-rationalistic theory of a divine education, it is clearly apparent that it was the outcome of a continuous development, characterized, we may doubtless say, by strict logical necessity. Antecedent to it were, first, the conception that this world' is a preparation for the kingdom of God, and, later, the thought that life is an education in accordance with a predetermined plan. That the Hegelian conception is the result of such a development is evident from the very fact that it continues to regard the destinies of mankind as guided by a plan. This plan has, from stage to stage, merely passed from transcendence to immanence, inasmuch as it is finally thought to be present to the mind of the philosopher who interprets the meaning of history. Hence this later philosophy of history resembles the earlier in still another respect. Ultimately, both are more concerned with the future than with the past, thus being at once history and prophecy. Even at the later period, the central question to whose answer everything else is preparatory concerns the final goal toward which man- 
kind is striving. Hence it is that the philosophers of this age are led time and again to divide the total life of humanity into periods inclusive of past, present, and future, precisely, as did the world-plan of Augustine, whose basal conception was the idea of redemption. Since these periods are not derived from the progress of events, but are for the most part imposed upon it in conformity to the dictates of logic, the course of history is mapped out by reference to logical categories. Each of the great cultural peoples is portrayed as representing a specific idea, and, disregarding everything that might disturb their sequence, these ideas are arranged in a logical series. Thus, Hegel begins his reconstruction of history, with an account of the Chinese as the people who possessed the earliest civilization. He does so, however, not because Chinese culture was as a matter of fact the earliest, but because it has apparently been more stable than other cultures, as well as more closely bound up with rigid external forms. Correspondingly, all succeeding stages of history are arranged by Hegel according to the principle, on the one hand, of a progress from bondage to spiritual freedom, and, on the other, of a transition from finite limitation to a striving for the infinite. This philosophy of history should not be criticized for its lack of knowledge concerning the beginnings of culture. Its fundamental error lies in the fact that, in tracing the development of mankind, it is guided, not by, the rich concrete actuality of events but by a logical schematism which is in large measure imposed upon history, and only to a far less degree abstracted from it. That which was once a plan prescribed by God for mankind here at length becomes a plan elaborated by philosophers.

Without question, therefore, a philosophy of history must henceforth adopt a different course. True, it cannot dispense with principles that are in a certain sense external to history itself. Yet the function of such a philosophy would appear to consist in considering historical life from the point of view of the purposes that come to realization 
within it, and of the values that are created on the various levels of historical culture. Such a teleology of historyindeed, in the last analysis, every teleology-must be preceded by a causal investigation, which begins, here as everywhere, by entirely ignoring purposes and values. Now, history is really an account of mental life. As such, it gives consideration to physical factors only in so far as they furnish the indispensable basis of mind. Hence the direct approach to a philosophy of history which aims, not to acquire a knowledge of reality from a priori concepts but, conversely, to derive ideas from reality, is a psychological account of the development of mankind. Although the concrete significance of the particular, as such, precludes the historian from disregarding it, everything that is merely particular should be ignored by one who is giving a psychological account of events. The aim, in this latter case, should be that of discovering the determining motives of historical life and its changes, and of interpreting these by reference to the universal laws of mind. Supplementing: this aim should be the endeavour to gain, so far as possible, an insight into the laws that are immanent in history itself. Our first three chapters have attempted to give an account of the development of folk consciousness during the periods that, for the most part, preceded self-conscious historical life. But neither this account nor the bare outline which our final chapter gives of the beginnings of the development to humanity must pretend to be a substitute for, or in any way to represent, a philosophy of history. The difference between an investigation such as ours and a philosophy of history is precisely the same as that which distinguishes a psychological description of mental life in general from a philosophical interpretation. But, if anywhere, it is especially in the field of history that a psychological analysis, concerned primarily to understand life in its actual occurrence, must precede questions regarding the meaning of events and the value which individual historical characters possess as respects both themselves and their permanent influence. In other words, we may henceforth 
demand that any philosophy of history which seeks to contribute to our understanding of the questions just mentioned, should be based on a psychological account of the development of mankind.

The point that we would emphasize is not that the philosophy of history has failed, in the past centuries, to find a satisfactory solution of its problem, and that its failure was inevitable. To the historical mind there is a far more important consideration. This consists in the fact that, when freed from its original mythological and teleological connections, the general conception of a history of mankind developed during these centuries has given clear definition to the idea of humanity in its most universal form. Humanity, it has been shown, included within itself all antecedent social phenomena-peoples and States, religion and culture. This entire social complex has been subsumed under the principle that law is immanent in all history. 



\section{INDEX}

Prepared by Dr. 'Alma de Vries Schaub on the basis of the German Index compiled by Dr. Hans Lindau.

Abraham, 45, I54, 355, 36r, 384, 435 ; and Isaac, 435

Adornment, 2I, 86, I00, I05, I10, I20, I3I, $449 \mathrm{ff}$.

Adventure, Märchen of, 279 f., 395

Esculapius, 439

Agamy, 36, 169, 181

Age, of the development to humanity, $470 \mathrm{ff} . ;$ of heroes and gods, 28I ff.; of personalities, 320 ; of primitive man, II ff.; the totemic, $1 \mathrm{I} 6 \mathrm{ff}$.

Age-groups, 4I, 5I, I3I

Agricultural ceremonies, I 35

Agriculture, I26 f., I40, 486 ; Animals in, $120 \mathrm{f} ., 124$

Alexandrianism, 490

Allegories, $42 \mathrm{I}$ ff.

Amulets, 86, 227 ff., 292, 439, 45 I

Anabaptists, 444

Ancestor, Animal, II7, I32 f., 204, 230 ff.; Demon, 467; Human, 204, 2I4, 230 ff. ; Totem, I86

Ancestor cults, 205, $230 \mathrm{ff}$.

Ancestor theory, $36 \mathrm{r}$.

Ancestor worship, I I7, I 86 f., 204, 214, 410, 480

Ancestral spirits, 4 I9

Animals, Breeding of, I20, $289 \mathrm{ff}$., 420 ; Domestic, I20, 289 ff., 420 ; Sacred, I2I ; Soul, 83, I90 ff., 2I4, 368, 4I 2 f. ; Totem, II7 ff., I3I ff., I43, I 88 ff., I93, 200, 260, 412 f.; Transformations into, I33, 272 ff., $4 \mathrm{I} 2 \mathrm{f}$.

Animal cult, II7, 136
Animal dance, 464

Animal-fable, 272

Animal-mask, 95, 105, 135

Animal names, $187 \mathrm{f}$.

Animal sacrifice, 2 Io $\mathrm{f}$., $433 \mathrm{f}$.

Animal totem, II7, I38 f., I86, 2 I4

Animism, I39, I93, $204 \mathrm{f}$.

Anthropology, Prehistoric, $\mathrm{I}_{4} \mathrm{f}$.

Anthropophagy, 3I, 209 f.

Arbitrator, 331

Architecture, 26I, 45I ff.

Art, 94 ff., 104, 256 ff., 322, 448 ff., 490 ; Formative, roo ff., 256 f.; Imitative, ro7 f. ; Memorial, 23 f., I07 f.; Miniature, 453; Musical, 262 ff., 456 ff.

Aristotle, I 2 f., 19, 350, 504, $5^{\text {I }} 7$

Asceticism, I98

Augustine, 516, 52 I

Aversion, 194

Awe, 194

Bachofen, $\mathscr{F}$., $34 \mathrm{ff}$.

Baptism, $444 \mathrm{f}$.

Barter, I68 ; Secret, Io, 2I, 3 I ff., 55, I 20 ; Marriage by, 157

Beyont, Belief in a, 394 ff., 4r2, 420 1., 423 ff., 43I, 495, 502

Blessedness, 396, 403 f., 406

Blood, Relation of soul to, I9 I, $206 \mathrm{ff}$., 2 I3; Taboo of, 200, 2 ro

Blood-magic, I9I

Blood-relationship, $208 \mathrm{f}$.

Blood-revenge, I63, 314, 333, $339 \mathrm{ff}$, $344 \mathrm{ff}$.

Blowpipe, roo f., 104 
Boat, 129

Boomerang, 27 f., 125,177

Bow and arrow, 16, $26 \mathrm{ff} ., 33,49$, I 12, 124

Breath, Relation of soul to, $192 \mathrm{f}$. 205 ff., 212 f., 242

Bïcher, Karl, 267

Buddha, 381 ff., 425 f., 498 f., 504, 507

Buddhism, ro, 478, 496 ff., $5^{\text {I } 5}$

Bull-roarer, 99 f., I8I, 266

Burial, 2 I 6 ff., 397

Bush soul, 232

Capture, Marriage by, 154 ff., 163 , I68

Castle, 324 f., 327, $45^{2}$

Castration, 290 f., 294 f.

Cattle-raising, 120, I24, $137 \mathrm{f}$.

Causality, $92 \mathrm{f}$.

Cave, 22 ff., ro6, 108

Celestial cults, $25 \mathrm{I}$

Celestial märchen, $275 \mathrm{f}$.

Celestial mythology, 76, 80, 91, r $30 \mathrm{f}$., I34 ff., I 40, I 89, 220, 246, 258, $355^{\mathrm{ff} .,}$ 419

Celestial phenomena, $304 \mathrm{ff}$.

Ceramics, 30, 8o, 135, 259 f.

Ceremonies, Intichiuma, $185 \mathrm{f}$., $188 \mathrm{f}$., 244 ff. ; Sanctification, 442 ff.; Vegetation, I35 f., 189, 249, $418 \mathrm{ff}$. Chaos, 388, 390, 392

Chief wife, $45 \mathrm{f}$., $168 \mathrm{ff}$., $3 \mathrm{r} 6$

Chieftain, 12 I, 134, I95, 233

Chieftainship, I19, 125, 233, 314, 332

Christianity, ro, 478, $496 \mathrm{ff}$., 5 I $5 \mathrm{f}$.

Church and State, 49r $\mathrm{f}$.

Churingas, I77, I81, I85, 190, 204, $221,224 \mathrm{ff}$.

Circumcision, 445

Cities, Foundation of, $3 \mathrm{I}$ I, $33 \mathrm{f}$.

Clan names, I4I ff.

Classes, Differentiation of, I25, $31 \mathrm{I}$, 316 if.

Club, Men's, 4I, 47, I I9, I3I, I73 f., 255, 312, 409

Coat of arms, 143, 232

Colonization, $300 \mathrm{f}$.

Common property, $248,317 \mathrm{ff}$.

Community labour, r36, $247 \mathrm{f}$.
Compurgator, 335

Conception totemism, I76, I80 ff., r89f., 191, 193

Conjuration, $269,427 \mathrm{f}$.

Conscience, 329, 431

Consecration gift, $43^{8} \mathrm{ff}$.

Constitution, $349 \mathrm{ff}$.

Contract, Marriage by, $15^{8 \mathrm{f}}$.

Cord magic, 86 f., 202, 4I 5,440

Corporeal soul, 82, 19I f., 205 ff., 2 I Iff., 2I6, 22 I f., 406

Corrvboree, 184,464

Cosmogony, 370, 385 ff., 393, 404

Cosmopolitanism, 489

Counter-gods, 370

Counter-magic, 84, 105, 201, 203, 444 f.

Counting, Systems of, $304 \mathrm{ff}$.

Couvade, 198

Creation-myths, $388 \mathrm{ff}$.

Cremation, 218 ff., 397

Crouching graves, 218

Cults, Ancestor, II7, $204 \mathrm{f} ., 230 \mathrm{ff}$., Celestial, $25 \mathrm{If}$.; of the dead, $45^{2}$; Deity, 205, 325, 4I $4 \mathrm{ff}$., $424 \mathrm{ff}$. ; Demon, 249 ff. ; Hero, 204 ; Magic, $416 \mathrm{f}$; Mystery, $420 \mathrm{ff}$., 502 ; of Saints, $178 \mathrm{f}$; ; of the soil, $245 \mathrm{ff}$; Soul, 42I f., 502 ; Totemic, $236 \mathrm{ff}$. ; Vegetation, 135, 243 ff., 250 f., 294. $4 \mathrm{I} 8 \mathrm{ff}$.

Cult associations, I $19,136,143,161$, I 79 f., 255

Cult ceremonies, 90

Cult practices, $426 \mathrm{ff}$.

Cult songs, $96,267 \mathrm{ff}$., $46 \mathrm{r}$

Custom, $35^{\circ}$

Dance, 90, 95 f., I04, 249, 262 ff., 449, 457 ; Ceremonial, 264; Ecstatic, $249,264,418,423$

Dance-song, $95 \mathrm{f}$.

Dead, Disposal of the, $8 \mathrm{r}, 2$ r $5 \mathrm{ff}$., $234 \mathrm{f}$., $238 \mathrm{f}$., 397, 405; Realm of the, $398 \mathrm{ff}$; Sacrifice to the, $23^{8} \mathrm{ff}$., 253 f., 433 f.

Deaf and aumb, The, $59 \mathrm{f}$.

Death, 8 r f., 494

Debt, 343

Degeneration theory, 225, 353

Deity cult, 205, 325, 414 ff., 424 ff. 
Deity saga, $384 \mathrm{f}$.

Demon battles, $370,404 \mathrm{f}$.

Demon cult, $249 \mathrm{ff}$.

Demons, 75 ff., 8 r ff., 105, 196, 20r, 203, . 217 f., 22 If., $224,236,263$ f., 284 f., $35 \mathrm{Iff} ., 36 \mathrm{r}$ ff., 387 ff., $4 \mathrm{I} 8 \mathrm{ff}$; ; and the epic, $45^{8} \mathrm{f}$. ; and heroes, $283 \mathrm{ff}$., 369,372 f., 454 ; Vegetation, 44I

Destiny, 366

Development, Theory of, $353 \mathrm{ff}$.

Devourment, Märchen of, $276 \mathrm{ff}$.

Differentiation of classes, 125, 3II, $316 \mathrm{ff}$; of vocations, 3 II, 32 Iff.

Digging-stick, 26, I00, I 20, I24, I $26 \mathrm{f}$.

Dionysian mysteries, 447

Discoveries, Geographical, 492

Divination, 44I $\mathrm{f}$.

Divine State, 329, 373, 388, 4II, 416, 494

Dog, 22 f., 124, 290

Domestic animals, Breeding of, $120 \mathrm{f}$., $289 \mathrm{ff}$.

Drama, 9, 462 ff., 490

Dreams, I 89 f., 193, 205 f., 401,407

Dress, 21 ; Origin of, $85 \mathrm{ff}$., 120,126 , I31, 133, 449

Duel, 336

Dwelling, 2I ff., 106

Dwarf peoples, 19, 77 f., II 5, 353

Eclipse of the sun, 8I

Ecstasy, 249, 397, 423 f., 434

Education and history, 519

Elysium, $403 \mathrm{f}$.

Emotion, 81, 92 f., 105, 114, 264, 268, $356,367 \mathrm{ff} ., 423,466,468 \mathrm{f}$. ; as related to magic, 93

Endogamy, II8, 149, 151, 166

Enlightenment, II, 470, 517

Epic, 9, 280, 450, $457 \mathrm{ff}$.

Ethnology, 5 f., 122

Eunuchs, 294

Evil magic, 274

Exogamy, 46, I18, $144 \mathrm{ff} ., \mathrm{I}_{3} \mathrm{ff} ., \mathrm{I} 83$, $196,289 \mathrm{f}$.

Family, I 2 f., 34 ff., 235, 3 I ff. ; Joint, I53, 312 ff.; The original, 12; Single, 313,315

Father-right, 36, 314
Fear, 81, 92, 194, 200, 224, 370, 400

Fetish, 186f., 214, 220 ff., 352 f., 439, 454

Fetishism, 139, 186 f., 204, $35^{2}$

Fire, $3 \circ \mathrm{f}$., I 24 ; Acquisition of, 30 ff ; Kindling of, 49, 292 ; Lustration by, 20 r f., $218 \mathrm{ff} ., 243,338,407$, 443 f., 446 ; Solstice, 202 ; Trial by, 243,338

First-fruits, Sacrifice of, $440 \mathrm{f}$.

Flood, Universal, $39 \mathrm{r}$ ff.

Flood saga, 39r ff.

Flute, 97, 266

Folk psychology, History of, I ff. ;

Methods of, $6 \mathrm{f}$. ; Problem of, $3 \mathrm{f}$. ; relation to ethnology, $5 \mathrm{f}$; ; relation to general psychology, 3 ; relation to philosophy of history, $522 \mathrm{f}$.

Food, of primitive man, $24 \mathrm{ff}$; Prohibitions on, $199 \mathrm{f}$.

Forest-dwellers, 19, 122, 395

Formative art, $99 \mathrm{ff}$., $256 \mathrm{f}$.

Fortitude, 242 f., 247

Foundation of cities, $31 \mathrm{Ir}, 323 \mathrm{ff}$.

Frazer, $\mathcal{F}$. G., 38, 152, 189 f.

Fusion, Racial, III, $288 \mathrm{f}$.

Gathering of food, 24 f., I24, 140, 144 Genetic psychology, 4

Gesture language, $5^{8} \mathrm{ff}$., 69

Gestures, Graphic, 62 f. ; Pointing, 6I f. ; Significant, 63

Gift, $432 \mathrm{ff}$; Consecration, $438 \mathrm{f}$. ; Marriage by, $158,163 \mathrm{f}$; Votive, $438 \mathrm{f}$.

Gift theory of sacrifice, $240,432 \mathrm{ff}$.

Gillen, Messrs. Spencer and, 18, 38, 188 Gods, Abode of, 364, 366; Age of heroes and, $8 \mathrm{f}$., I2I, $235 \mathrm{f}$., $28 \mathrm{Iff}$. ; Battles of, 37o, $388 \mathrm{f}$., $404 \mathrm{f}$.; Belief in, $285 \mathrm{f}$.; Characteristics of, $282 \mathrm{ff}$., $362 \mathrm{ff}$; ; Cult of, 205, 325, $414 \mathrm{ff}$, $424 \mathrm{ff}$; ; Decline of, 365 ; and demons, 366 f., 369, 459 ; Development of, 362 ff. ; Images of, 223 f., 247,450 , $453 \mathrm{f}$.; Judgment of, 337 ; of the moment, $3^{62} \mathrm{ff}$; Origin of, $35^{\circ} \mathrm{ff}$., $3^{6} 4$ if., 369 ; Particular, 362 ff.; Perfection of, $3^{6} \mathrm{f}$.; Personality of, 236, $366 \mathrm{ff}$.; of the present, 234; 
Saga of, 228, 374 f., 384 f.; Superpersonal, $390,467,504 \mathrm{ff}$.

God-man, $506 \mathrm{f}$.

Greek language and culture, $488 \mathrm{ff}$.

Grimm, Facob, 459

Graves, Crouching, 218

Group-marriage, 38, 4 I f., 44 f., 48, $168 \mathrm{ff} ., 316$

Guardian animal, 190, 232

Guardian deity, 325, 501

Guardian spirits, 178,369

Guide, 407 f.

Guilt, 203, 253, 430

Gynocracy, $35 \mathrm{f}$.

Hades, 398, 40I, 404

Hammurabi, Code of, 330, 338, 343, 347,4 I I

Harvest, Sacrifices in connection with, $440 \mathrm{f}$.

Heart and soul, 207

Heaven, 39.5, 404

Heavens, Mythology of the, 76,80 , 9If., I30 f., I34 f., I40, 189, 220, 246, 258, 355 f., 419; Phenomena of the, $304 \mathrm{ff}$.

Hegel, $520 \mathrm{f}$.

Helios, $35^{8} \mathrm{f}$.

Hercules, 376 f., 382,407

Herd, 52, 121

Herder, 52, 470, 472, $5^{19}$

Hermes, $407 \mathrm{f}$.

Hero, 9, 28I ff.; Cult of the, 204; and demon, 283 ff., 369,372 f., 454 ; and god, $282 \mathrm{ff}$., $364,369 \mathrm{ff}$., 454

Hero saga, 228, 356, $374 \mathrm{ff}$.

Heroic age, 28I ff.

Heroic song, 9

Hillebrand, Karl, I

Historical consciousness, 478

Historical religion, 509

History, 5 ro ff.; and saga, $377 \mathrm{ff}$.

Hobbes, Thomas, II f., 34, 36, III

Hoe, 120, I $26 \mathrm{f}$., I34

Hoe-culture, 134, I38, 246, 248, 250, 289

Horde, 52, 120, 145, I80, 237, 302, $47 \mathrm{I}, 5 \mathrm{II}$

Horse, 293

Hospitality, 34I
Hostage, 343

Howitt, $A$. $W$., I 8, 37 f., I42, 188

Human nature, 47 I f., 475

Humanity, 9, $470 \mathrm{ff}$; ; Ideal of, $4 \mathrm{IO}$

Hunting, 24 f., I40, I44; Use of dog in, $22 \mathrm{f}$.

Hut, Conical, 26r, 45I ; Pole, 26I ; Spherical, 26r

Hymns, 385, 393, 430, 46I, 465

Ideals, Religious, 4 10

Ideas, of a beyond, $393 \mathrm{ff}$., $420,423 \mathrm{ff}$., 43I, 495 ; Concrete, 72 ; Mythological, 74

Idols, 131

Images, Divine, 223 f., $447,450,453$ f. Imitation of animals, 95

Immortality, Belief in, 233, $394 \mathrm{ff}$., 412, 420 f., 423 ff., 43I, 495, 502

Imprisonment, $342 \mathrm{ff}$.

Individual rulership, 287, 313

Individualism, 489, 492

Infanticide, 43 f., 175,237

Infinitude, $505 \mathrm{f}$.

Instruments, of concussion, 265 ; Musical, 97 ff., 265 f., 457,468 ; Stringed, $97 \mathrm{f}$., 266 ; Wind, $265 \mathrm{f}$.

Initiation ceremonies, 202, 24I ff., 247 Intelligence of primitive man, $109 \mathrm{ff}$. Intichiuma ceremonies, $185 \mathrm{f}$., $188 \mathrm{f}$., $244 \mathrm{ff}$.

Islamism, 10, 316, 497

Javelin, $\mathrm{I} 24 \mathrm{f}$.

Joint family, $153,312 \mathrm{ff}$. Jordan festival, 203,446 Judaism, 497

Judge, 33I ff., 347 ; Appointed, 331 ; in the underworld, 403

Judgment of the gods, 337

Judicial functions, Division of, $348 \mathrm{f}$. Justice, Administration of, $33 \mathrm{Iff}$.

Fus prima noctis, 46, 168

Fus talionis, $345 \mathrm{ff}$.

Kant, 470,517

Kern, H., 55

Kidneys, as vehicles of the soul, 209, 2 I I f., 22I, 434 f., 445

Kiss, 242 
Klaatsch, Hermann, 15

Knife, I3I, 449

Kollman, Fulius, 77

Labour, Community, 136, $247 \mathrm{f}$; ; Degradation of, $32 \mathrm{I}$. ; Division of, $49 \mathrm{f}$., 300 ; Equalization of, $322 \mathrm{f}$.

Landscape painting, $45^{6}$

Lang, Andrew, 153, 187

Language, $53 \mathrm{ff}$., 137 ; Gesture, $58 \mathrm{ff}$., 69

Lawgivers, $307 \mathrm{f}$.

Lazarus and Steinthal, Messrs., 2

Legal system, $327 \mathrm{ff}$.

Legends, 38I ff., $42 \mathrm{I}$ f. ; Mura-mura, $23 \mathrm{I}$; of redemption, $382 \mathrm{f}$; ; Religious, $38 \mathrm{I}$; of saints, $38 \mathrm{Iff}$., 464

Lessing, 414, 519

Lie, 63, II4

Lippert, Fulius, 205, 23 I

Liturgy, $463,465 \mathrm{ff}$.

Loin cord, $85 \mathrm{ff}$.

Lustration, 20I ff., 219 f., 252 f., 338 , $407,412,443 \mathrm{ff}$.

Magic, Belief in, $75 \mathrm{ff} ., 8 \mathrm{r}, 84 \mathrm{ff}$., 92, 94 f., ro5, $376 \mathrm{f}$., 434 ff. ; Cord, 86 f., 202, 415, 440; Evil, 274 ; Imitative, 354 ; Protective, 85, 449

Magic staff, $335 \mathrm{f}$.

Magic test, $337 \mathrm{f}$.

Magical offering, $44^{\circ}$

Magical transference, $20 \mathrm{If}$.

Magician, 84 f., 330, 378

Man, E. H., 79

Mankind and human nature, $47 \mathrm{I} \mathrm{f}$., 475

Mannhardt, W., 249, 292, 44I

Märchen, $27 \mathrm{off}$; of adventure, $279 \mathrm{f}$., 395 ; Celestial, 275 f., 395; of devourment, $277 \mathrm{ff}$.

Märchen-cycle, 380

Märchen-hero, 356, 375 ff., 387,459

Märchen-myth, $270 \mathrm{ff}$., $387 \mathrm{ff.}, 4 \mathrm{r} 3$, $45^{8 \mathrm{f}}$.

Mark community, $309 \mathrm{f}$.

Market, 327, 463

Marriage, I2, $34 \mathrm{ff}$., 89 ; by barter, I57; of brother and sister, II8, I48 ff.; by capture, I53 ff., I63,
I67 f. ; by contract, $158 \mathrm{f}$.; by gift, 158 f. ; Group, 38, 4r f., 44 f., 48 , $168 \mathrm{ff}$., 316 ; Modes of contracting, I 55 ff., I 72 f. ; Pirrauru, 168 ff. ; by purchase, $158 \mathrm{f}$. ; Single, $5^{\mathrm{I}}$

Mask, 95, 105, 135, $262 \mathrm{ff}$.

Maternal descent, $35 \mathrm{ff}$., $47,146 \mathrm{ff}$., I65, I73 f., I96 f., 314

Maternal rule, 35, 3I4

Martin, Rudolf, 50

McLennan, $\mathcal{F}$. F., I45, 153

Meal-times of primitive man, 24

Medicine-men, 83 f., 89, 105, 180, 223, 233, 254 f., 330, 34I, 409

Memorial art, 24, 107

Men's club, 4I, 47, II9, I3I, I73 f., 255 f., 312, 409

Metempsychosis, $4 \mathrm{I} 2 \mathrm{ff}$.

Migrations, III, $287 \mathrm{f}$; Folk, $126 \mathrm{ff}$., I64, 288f.; Tribal, I20, I38, I9I, 488

Military organization, 310

Milk industry, $\mathrm{x} 37 \mathrm{f}$., $289,296 \mathrm{f}$.

Mimic play, $459,462,490$

Monogamy, 34, 36, 43, $46 \mathrm{ff}$., 89, II 4, I67, 169 ff., 3 I I ff.

Monotheism, 77, 225, 23I, $353 \mathrm{ff}$.

Monumental edifices, $45^{2}, 490$

Morality, Primitive, II 4 f.

Morgan, Lewes, $38,{ }^{52}$

Mother-right, 34 ff., $3^{14}$

Müller, Max, 225

Mummy, 207

Mura-mura legends, $176 \mathrm{f}$., $23 \mathrm{I}$

Murder, 339 f., 346

Music, 95 ff., 264 ff., 449, 456 f., 464, $466 \mathrm{ff}$; ; Absolute, 468

Musical instruments, 97 ff., 265 f., 457 , 468

Mystery cults, $420 \mathrm{ff}$., 502

Myth, 75 f., 375 f., $384 \mathrm{ff}$., $4 \mathrm{r} 3 \mathrm{ff}$. ; Celestial, 76, 8o, 9r, 130 f., r34ff., 140, r89, 220, 246, 258, 355 ff., 4r9 ; Cosmogonic, 385 ff., 404 ; and cult, $4 \mathrm{r} 4 \mathrm{ff.}$; Märchen-, 270 ff., 387 ff., 4I3, $458 \mathrm{f}$.; Theogonic, $384 \mathrm{ff}$.; of the underworld, $397 \mathrm{ff}$; of world destruction, 39r $\mathrm{f}$.

Mythical hero, 379

Mythology, Nature, 76 
Narrative, $270 \mathrm{ff}$.

Nature, Man of, I I ff.

Nature-demons, 370

Nature-mythology, 76

Neanderthal skull, $\mathrm{I}_{4} \mathrm{f}$.

Nirvana, 499

Nomads, 120, 138, 419

Novel, Short, 464

Numbers, Sacred, 305, 407 ; Social organization and, $304 \mathrm{ff}$.

Oath, $335 \mathrm{f}$.

Offering, $432 \mathrm{ff}$.

Oracle, $44^{2}$

Ordeal, $336 \mathrm{f}$.

Orders, 255

Organization, Military, 3 Io ; Political, $302 \mathrm{ff}$; ; Tribal, II $7 \mathrm{ff}$., I32, I40 ff., 152

Ornamentation, $100 \mathrm{ff}$.

Other-world ideas, $394 \mathrm{ff}$., 4 ro, $420 \mathrm{ff}$., 43I, 495, 502

Painting, I06 ff., 456, 468

Palace, Royal, 452, 454, 48I

Pasha, Emin, II4

Passion plays, 463,465

Particular gods, $362 \mathrm{f}$.

Paternal descent, 37, I46ff., I 73 f., $196 \mathrm{f.}, 314$

Paternal rule, 35,314

Patriarchal family, 3 I3

Patriarchal period, $35 \mathrm{f}$.

Penal law, $338 \mathrm{ff}$.

Penitential psalm, $430 \mathrm{f}$.

Personalities, Age of, 320

Personality, 489, 505

Phallus cult, 212

Philology, 2, 53, 490

Philosophy, 354, 496, 504, 518; of history, 5 I 9 ff.

Pirrauru marriage, $168 \mathrm{ff}$.

Plant totem, 134, 176, 184, $188 \mathrm{ff}$., 192, 199, 214, 245

Platform disposal of the dead, 216, 405

Plough, 134, 138, 248, 289 ff., 298

Poison, Arrow, 26 ; Plant, 25 f.

Poetry, 267 ff., 457

Pole-houses, 26I

Political organization, $302 \mathrm{ff}$.
Polyandry, 42 ff., 167,267, 17 I f., $3^{12}$

Polygamy, 4I f., 47, I66 ff., 312

Polygyny, 42 ff., 139, 167, 170 ff., 312, $315 \mathrm{f}$.

Polytheism, 80, 355, 357, $37 \mathrm{I}$

Pottery, 30, 80, 135, $259 \mathrm{f}$.

Praise, Hymns of, 430

Prayer, 427 ff.; penitential, $430 \mathrm{f}$. ; of petition, 427 f., 439 ; of thanks. giving, 429f., 439

Prehistory, I3 f., 45I

Preusz, K. Th., 242, 435, 464

Priesthood, 321, 330, 332

Priests' Code, 200, 210, 329, 345, 432

Primitive man, Discovery of, I I ff.

Property, 47, II4, I20, 138, I73f., I95 f. ; Common, 248, 3 I 7 ff. ; Private, 298, 300, $317 \mathrm{ff}$.

Prophetic signs, $44^{2}$

Promiscuity, 36, 38, 169, 181

Prohibition of certain foods, $199 \mathrm{f}$.

Protection, Right to, $340 \mathrm{ff}$.

Protective magic, 85

Psyche, 205 f., 212 ff., 217, 220, 24I, 405

Punishment, 338, 342, 404, 406 f., 43r; and sacrifice, 433

Puppet show, $464 \mathrm{f}$.

Purgatory, 407 f., 412

Purification, 20I f., 2 I9f., 499 ; Rites of, 20I, $443 \mathrm{f}$. Cf. Lustration.

Pygmies, I9, 77 ff., II 5, 353

Rain-magic, 253, 268

Rain priests, 249, 263, 268

Rattle, I00, 266

Ratzel, Friedrich, 5

Realm of the dead, $396 \mathrm{f}$., 400

Reconciliation, 432

Redemption, 4IO, 447, 495 f. ; Legends of, 381 ; Religions of, 496

Reformation, 492

Refrain, 96 f., 104

Relationship, Malayan system of, $38 \mathrm{ff}$.

Religion, Origin of, $75 \mathrm{ff}$., $282 \mathrm{ff}$.

Religious ideals, 410

Renaissance, 455 f., 49 I f., 5 I7

Retribution, Idea of, $40 \mathrm{I}, 408,4 \mathrm{II}$, 413

Revelation, $5^{18}$ 
Rhythm, 103 f., $268 \mathrm{f}$.

Rights, Equality of, 320

Rings, Exchange of, 87

Root languages, $68 \mathrm{f}$.

Roskoff, G. G., 75

Rousseau, $\mathcal{F} \cdot \mathcal{F}$., $\mathrm{I} 2$

Rulership, Individual, 287, 313

\section{Sacredness, 195 f., 199}

Sacrifice, 253 f., 295 f., 427 , 43 Iff. ; Animal, 2 rof., $433 \mathrm{f}$. ; to the dead, $238 \mathrm{ff}$., 253 f., $433 \mathrm{f}$; Human, 2 ro, $433 \mathrm{ff}$., 440,447 ; of reconciliation, 432

Sacrificial animal, 2 Io $f$.

Sacrificial feast, $446 \mathrm{f}$.

Saga, Deity, 384 f. ; Flood, 391 ff. ; Hero, 228, 356, $374 \mathrm{ff}$.

Saints, Legends of, $38 \mathrm{rff}, 464$; Worship of, $178 \mathrm{f}$.

Sanctification, 427 ; Ceremonies of, 442

Sanctuary, 34r f.

Sarasin, $F$. and $P$., 19, 49, 75, 90

Satisfaction of wants, $448 \mathrm{f}$.

Satyric play, 464

Scapegoat, 203

Scarab, 229

Schmalz, E., 60

Schmidt, Wilhelm, 78 f., II4, 353

Schultze, Leonard, 88

Schweinfurth, Georg, 18 f., 77

Science, 449, $489 \mathrm{f}$.

Scott, W. R., 60

Sculpture, 26r, 453 ff., 490

Secret barter, I0, 21, 3 I ff., 55, I 20

Secret societies, $254 \mathrm{ff}$.

Secondary wives, $45,168 \mathrm{ff}$., 316

Self-education, 5 I9

Self-mutilation, 294 f., 434

Sex totemism, I19, I76, I82 f., 186f., 190, 193

Sexual organs and the soul, 2 I I, 434, 445

Shadow soul, $192 \mathrm{f}$., 205 f.

Shamans, 84

Shame, feeling of, 88

Shield, 125, 131

Sickness, 81, 83 ff., 90, 494 ; Demons of, 82 f., ro5, 236
Sin offering, $432 \mathrm{f}$.

Single marriage, $5^{1}$

Skull, 217 ; Neanderthal, $14 \mathrm{f}$.

Slave, 154,156

Slavery, 139

Smoke, 220

Snake society, 256, 269

Social psychology, 4

Society, Primitive, $50 \mathrm{ff}$

Soil, Cults of the, $245 \mathrm{ff}$.

Solstice festivals, 420

Solstice fire, 202

Song, 95 ff., I04, 267 ff., 449, 458, $460 \mathrm{ff}$. ; of praise, 430 ; Work, $268 \mathrm{f}$., 461

Soul, Breath, 192 f., 205 ff., 212 f., $242 \mathrm{f}$.; Corporeal, 82, I9I f., $205 \mathrm{ff}$., 2 IIff. ; 216,221 f., 406 ; Ideas of the, I90 ff., $394 \mathrm{ff.}$; and kidneys, 209, 2 I If.; Shadow, I92 f., 205 f. ; Vehicles of the, $207 \mathrm{ff}$., $21 \mathrm{If}$., 22I, 434 f., 445

Soul animals, 83 , Igo ff., 214, 368, $4 \mathrm{I} 2 \mathrm{f}$.

Soul belief, $204 \mathrm{ff}$.

Soul cults, $421 \mathrm{f}$., 502

Souls, Exchange of, 242 ; Transmigration of, $412 \mathrm{ff}$.

Sound and meaning, $65 \mathrm{ff}$.

Spear, 125

Speech, $496 \mathrm{f}$.

Spencer and Gillen, Messrs., 18, 38, 188

Spencer, Herbert, 187, 205, 231

Spirit villages, 396

Sprinkling, 203, $445 \mathrm{f}$.

State, 8 f., I I9, 285 f., $287,303,472$ ff. ; Church and, $49 \mathrm{I}$ f.; Divine, 329, $373,388,411,416,494$; Forms of the, 349,5 I7

Steinen, Karl von den, I0?

Steinthal, H., 2, 68

Stipulation, 334

Stringed instruments, $97 \mathrm{f}$., 266

Stuhlmann, Franz, I 14

Substitute, 435

Sun, Eclipse of the, $8 \mathrm{I}$

Sweat-lodges, 252

Sword, I3I, 299

Symbolism, 334, 422, 447

Symmetry, $103 \mathrm{f}$. 
Taboo, I3I f., 193 ff., 203, 219, 34I ; on foods, $199 \mathrm{f}$.; on relations by marriage, $196 \mathrm{ff}$.

Talisman, 89, 104, $227 \mathrm{ff}$.

Tattooing, 21, 87, r3 I, 135, 255, 257 ff., $45 \mathrm{I}$

Teleology, 522

Temple, r95, 324f., 45o, $45^{2}$ f., 465, $467,48 \mathrm{r}$

Theft, II4; of women, 46

Theogony, 384 ff., 417

Thinking, Primitive, $68 \mathrm{ff}$.

Tippamalku, r68 ff.

Torture, 344

Totem, 8, rr6 ff., 203 f., 4r2 f. ; Inanimate, $177, \mathrm{r} 85 \mathrm{ff}$.

Totem animal, II7 ff., I3I ff., I43, I 88 ff., 193, 200, 260, 412 f.

Totem friendships, $162 \mathrm{ff}$.

Totem poles, I43 f., $232 \mathrm{ff}$.

Totemism, I 6 ff. ; Animal, II7 ff., 131 ff., 138 f., r75 ff., 193, 214, 245, 412 f.; Conception, I76, 180 ff., 189 f., I9I, I93 ; Individual, II9, I75, 178 ff., I87, r89 f. ; Plant, r34, I76, 184, 188 ff., 192, 199, 214, 245 ; Sex, r r9, r76, r8o, r82 f., r 86 f., r9o, I93 ; Tribal, 177 ff., 187

Trade, r21, 300 f., $45^{2}$

Transference, Magical, $201 \mathrm{ff}$.

Transformation into animals, 133, $272 \mathrm{ff} .4^{\mathrm{r}} 2 \mathrm{f}$.

Transmigration of souls, $412 \mathrm{ff}$.

Tribal division, r I 7 f., r4I, r43, r59ff. Tribal migrations, 120, 138 , 191, 488

Tribal organization, I 7 ff., I32, I $40 \mathrm{ff}$. 152

Tribal warfare, I19 f., 123, I25

Tylor, Edward, 205

Underworld, 397 ff., 402 ff.

Unity of the world, 483
Universalism, 492

Usener, Hermann, $36 \mathrm{r}$.

Vegetation ceremonies, 135 f., 189 , 249, $418 \mathrm{ff}$.

Vegetation cults, I35, 243 ff., 250 f., 294, $418 \mathrm{ff}$.

Vegetation demons, 44I.

Vessels, 30, 49

Vico, G., $5 \mathrm{I} 6$

Vision, 407, $44^{2}$

Vocations, Differentiation of, $3 I I$, $321 \mathrm{ff}$.

Votive offering, $43^{8}$

Wagon, $292 \mathrm{ff}$.

Wants, Freedom from, IIo, II 4 ; Satisfaction of, $448 \mathrm{f}$.

Warfare, 33, III, 209; of the gods, 370,388 f., 404 f.

Water, Lustration by, 201 ff., 2 rg f., 252 f., 338, 443 ff. ; Trial by, $33^{8}$

Weapons, 26 ff., 120, I24 f., I3I,' 133 , 299

Week, 305

Wergild, 163, 339

Westermann, D., 58, 68

Wheel, $29 \mathrm{f}$.

Wife, Chief, 45 f., 168 ff., 316;

Secondary, 45, I68 ff., 316

Wind instruments, $265 \mathrm{f}$.

Witchcraft, 338

Work-songs, $268 \mathrm{f}$., $46 \mathrm{r}$

World, Unity of the, 483

World culture, 477,484 ff., 512

World destruction, Myths of, $39 \mathrm{I} \mathrm{f}$.

World empires, 476,478 ff., 484 ff., 493, $5^{12}$

World history, 474 f., 478,509 ff.

World language, 487,493

World religions, 10, 477, 491, $494 \mathrm{ff}$.

Writing, $486 \mathrm{f}$. 


\section{Library of Philosophy}

General Editor: Professor J. H. MUIRHEAD, LL.D.

ANALYTIC PSYChOLOGY. By G. F. Stout. Two Vols.

APPEARANCE AND REALITY. BY F. H. BRADLEY.

Attention. By Prof. W. B. Pimlsbury.

CONTEMPORARY PSYChOLOGY. By Prof. G. Villa.

HISTORY OF ESTHETIC. By Dr. B. BOSANQUET.

HISTORY OF ENGLISH UTILITARIANISM. By Prof. E. Albee. Ios. $6 d$. net. HISTORY OF PHILOSOPHY. By Dr. J. E. ERDMANN.

Vol. I. ANCIENT AND MEDIfeval. Third Edition.

Vol. II. MODERN. Fifth Edition.

Vol. III. SINCE HEGEL. Third Edition.

I5s.

155.

I2s.

history OF PSYchology : Ancient and Patristic. By G. S. Bretr, M.A.

Ios. 6d. net.

MATTER AND MEMoRY. By Henri Bergson. Translated by N. M. Paul and W. S. PALMer.

Ios. $6 d$. net.

NATURAL RIGHTS. By Prof. D. G. RItchie.

Ios. $6 d$. net.

PHILOSOPHY AND POLITICAL ECONOMY. By Dr. J. BONAR. IOs. $6 d$. net. rational Theology SinCE Kant. By Prof. O. Pflembrer. Ios. $6 d$. net. THE PHENOMENOLOGY OF MIND. By G. W. F. HEgel. Translated by J. B. Baillie. Two Vols.

2Is. net.

Thought and Things; OR, Genetic Logic. By Prof. M. Baldwin.

Vol. I. Functional Logic.

Vol. II. EXPERIMENTAL Logic.

Vol. III. Real Logic (I., Genetic Epistemology).

10s.6d. net per vol.

TIME AND FREE WILl. By Henri Bergson. Translated by F. L. Pogson. ros. 6d. net.

valuation: the theory of value. By Prof. W. M. Urban. Ios, $6 d$, net.

THE PSychology OF the REligious Life. By G. M. Stratton. Ios. $6 d$. net.

THE GREAT PROBLEMS. By Prof. Bernardino Varisco. Translated by Prof. R. C. LODGE. ros. $6 d$. net.

kNOW THYSELF. By Prof. Bernardino Varisco. Translated by Dr. Guglize Mo SALVADORI. ros. $6 d$. net. 


\section{The Elementary Forms of the Religious Life}

BY PROFESSOR EMILE DURKHEIM

Medium 8vo. Translated by J. W. SWAIN 15s. net.

"This book is of sufficient value to warrant its translation. The highest praise is due to Prof. Durkheim for his treatment. His thought is clear and his style lucid."-Athenceum.

\section{Group Theories of Religion and the Religion of the Individual}

By CLEMENT C. J. WEBB, M.A.

Fellow and Tutor of Magdalen College, Oxford, late Wilde Lecturer in Natural and Comparative Religion.

Large Crown 8vo.

5s. net.

This book contains the substance of a course of lectures, delivered at Oxford in the summer of 1914, on the Theories of the Nature of Religion put forward in "L'Année Sociologique," with some reference of the kindred speculations of Miss Harrison and $\mathrm{Mr}$. Cornford in this country. Especial attention is paid to M. Lévy Bruhl's theory (which is not shared by M. Durkheim) of a "prelogical mentality," and to that of a social origin of the categories, which is common to this whole group of writers. It is contended that these theories, as stated by the French sociologists and their followers, fail to do justice to the facts of individual religion; and that the cause of this failure is to be sought in an imperfect appreciation of the philosophical problem of individuality.

\section{Anthropomorphism and Science}

A Study of the Development of Ejective

Cognition in the Individual and the Race

By OLIVE A. WHEELER, M.Sc.

Formerly Fellow of the University of Wales

Large Crown 8vo.

5s. net.

This book is a study in genetic psychology, conducted with a view to solving the problem of the relation that exists between theological and scientific interpretations of the world. The work should appeal to all those who are interested in the problem of the apparent opposition between the auxiliary concepts of Science and Theology. 


\section{Text-Books}

INSANITY AND OTHER MENTAL DISEASES. By CHARLES ARTHUR MERcier, M.D., F.R.C.P., F.R.C.S., etc. Second Edition, enlarged and entirely re-written. Crown 8vo.

7s. 6 d. net.

"The most comprehensive, well-reasoned, and instructive review of the whole subject of insanity ... that has yet been produced in any country."-British Medical foumal.

PETROLOGY. Vol. I : Igneous Rocks. By F. H. HaTch, A.M.I.C.E. Seventh Edition Revised. Crown 8vo. Illustrated. 7s. 6d. net.

PETrology. Vol. II : The Sedmentary Rocks. By F. H. Hatch and R. H. RASTALL. Crown 8vo. Illustrated.

7s. 6d. nel.

ORGANIC CHEMISTRY. By J. WADE. Second Edition Revised. Illustrated. Crown 8vo. 8 s. $6 d$. net.

ELEMENTARY BIOLOGY. By Dr. H. J. Campbell. Illustrated. Second Edition. Crown 8vo.

$6 s$.

PHYSIOLOGICAL PSYCHOLOGY. By Prof. Th. Ziehen. Edited by Dr. O. BEYER and C. C. VAN LIEw. With Diagrams. Fourth Edition. Crown 8 vo.

$6 s$.

EMBRYOLOGY OF MAN AND MAMMALS. By Dr. O. HerTwig. Edited by Prof. E. L. MARK. Illustrated. Third Edition. Demy 8 vo.

2 Is.

COMPARATIVE GEOLOGY. By Prof. E. KAysar. Edited by Phillip LAKe. Illustrated. Second Edition. Demy 8 vo.

IOS. $6 d$.

ZOOLOGY. By Prof. Adam Sedgwick. Illustrated. Three Volumes. Demy 8vo. 15 s. net each.

PRACTICAL BOTANY. By Prof. E. Strasburger. Edited by Prof. W. Hillhouse, M.A. Illustrated. New and Enlarged (Sixth) Edition. Demy 8vo. IOS. 6d.

STUDENT'S BOTANY. By Prof. S. H. VINES. Illustrated. Fourth Edition. Demy 8vo.

I5s.

ELEMENTARY BOTANY. By Prof. S. H. Vines. Illustrated. Demy 8vo.

9s.

ELEMENTARY ENTOMOLOGY. By W. F. KIRBY.

Illustrated. Large 8vo.

IOS. $6 d$. 


\section{The Schopenhauer Series}

Crown 8vo, Cloth.

I. THE WISDOM OF LIFE.

2. COUNSELS. AND MAXIMS.

3. RELIGION, A DiALOGUE, Etc.

4. THE ART OF LITERATURE.

5. STUDIES IN PESSIMISM.

2s. 6 d. each.

6. OUTLINES OF A PHILOSOPHY OF RELIGION.

BY H. Lotze. Edited by F. C. ConYBEARE.

7. THE PROBLEM OF REALITY. By E. BELFORT BaX.

8. FiRST STEPS IN PHILOSOPhy. By W. M. Salter.

By A. SCHOPENHAUER.

Translated by

T. BAILEY

SAUNDERS.

9. RELIGION. By S. DE MOLINARI.

[Out of print.

10. THE SEXES COMPARED. By E. voN HARTMANN.

Translated by A. VENNER.

II. THE ART OF CONTROVERSY, ETC.

BY A. SCHOPENHAUER.

12. ON HUMAN NATURE.

By A. SCHOPENHAUER.

Translated by

T. BAILEY

SAUNDERS.

THE BASIS OF MORALITY. By A. SchopenhaUer.

Translated by A. BRODRICK BuLLock, M.A.

4s. $6 d$. net.

\section{Ethical Library}

Edited by J. H. MUIRHEAD, M.A., LL.D., Professor of Philosophy in the University of Birmingham.

AN ETHICAL SUNDAY SCHOOL. By W. L. Sheldon. Crown 8vo, cloth.

$3 s$.

CIVILIZATION OF CHRISTENDOM, AND OTHER STUDIES.

By Dr. B. Bosanquet. Crown 8vo, cloth. $45.6 d$.

DARWIN AND THE HUMANITIES. By Prof. J. Mark

BaLdwin. Crown 8vo, cloth. 35.

LeCtURes ON HUMANism. By Prof. J. S. Mackenzie. Crown 8vo, cloth.

PRACTICAL ETHICS. By Prof. H. SIDGwick.

Crown 8vo, cloth. Crown 8vo, cloth. 4 s. 6 d.

SOCIAL RIGHTS AND DUTIES. By Sir Lesle Stephen.

Two Vols. Crown 8vo, cloth.

95.

STUDIES IN POLITICAL AND SOCIAL ETHICS. By Prof. D. G. RITCHIE. Crown 8vo, cloth.

4s. $6 d$.

THE TEACHING OF MORALITY. By Dr. S. BRYant. Crown 8vo, cloth.

THE WILL TO DOUBT. By A. H. Lloyd. Crown 8vo, cloth.

$3 s$.

4s. $6 d$.

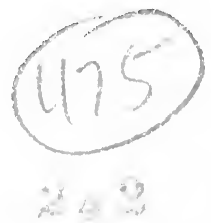






\section{PLEAse do NOT REMOVE}

CARDS OR SLIPS FROM THIS POCKET

UNIVERSITY OF TORONTO LIBRARY 
